\title{
EVOLUTIONARY DYNAMICS OF THE
}

\section{YERSINIA ENTEROCOLITICA COMPLEX}

\author{
SANDRA REUTER
}

A thesis submitted in partial fulfilment of the requirements of Nottingham Trent University for the degree of Doctor of Philosophy

Collaborating Establishment: Wellcome Trust Sanger Institute, Cambridge 
This work is the intellectual property of the author. You may copy up to $5 \%$ of this work for private study, or personal, non-commercial research. Any re-use of the information contained within this document should be fully referenced, quoting the author, title, university, degree level and pagination. Queries or requests of any other use, or if a more substantial copy is required, should be directed in the first instance to the owner(s) of the Intellectual Property Rights. 


\section{Abstract}

The genus Yersinia consists of a heterogeneous collection of organisms, comprising the highly pathogenic species Yersinia pestis, enteropathogens Yersinia pseudotuberculosis and Yersinia enterocolitica as well as environmental species. The evolutionary history of Y.pestis has been well documented, but information on the evolutionary relationship between the other Yersiniae is less characterized. $Y$. enterocolitica is a diverse species classed into six different biotypes (BT), but only a single genome sequence for high-pathogenic BT $1 \mathrm{~B}$ was available at the start of the project.

This project looked into the dynamics shaping the pathogenic lineages in Yersinia. For each of the Y. enterocolitica BTs, a reference genome was sequenced, annotated and analysed in detail. Analysis of biochemical characteristics was carried out using a phenotypic microarray. A further 98 Y. enterocolitica, 36 Y. pseudotuberculosis/Y. pestis isolates and 81 environmental species were sequenced and analysed to investigate the evolutionary dynamics of the genus Yersinia.

The Y. enterocolitica BTs form three distinct groups according to non-, low-, and high-pathogenic BT, each with a different set of accessory genes. The $Y$. enterocolitica core genome comprises $\sim 3,300$ CDSs. The non-pathogenic BT contains genes involved in environmental survival, whereas the high-pathogenic BT has a high-pathogenicity island and the unique Ysa type III secretion system. The low-pathogenic BTs share a second flagella cluster and an insect pathogenicity island. Apart from the virulence plasmid pYV, only $40 \mathrm{CDSs}$ are shared in all pathogenic BTs including the adhesion invasion locus ail. The phenotypic microarray confirmed higher metabolic flexibility of the non-pathogenic BT, and decreased biochemical abilities in the low-pathogenic BTs, mirroring an increased number of pseudogenes.

Looking at the whole genus, pathogenic lineages are visible at opposite ends of an evolutionary tree. Y. pestis has evolved out of Y. pseudotuberculosis, and both are distinct from $Y$. enterocolitica which developed out of the environmental Yersiniae. Fish pathogen $Y$. ruckeri forms a third independent pathogenic lineage. Despite the large evolutionary distance, comparing dynamics in Y. enterocolitica to Y. pestis it is clear that independent acquisition of the virulence plasmid and metabolic streamlining to specific hosts drove pathogen evolution. Whilst this has concurrently happened in Y. pestis, this development has been weighted differently in lineages of $Y$. enterocolitica leading to a high-pathogenic, human-adapted biotype and lowpathogenic biotypes, which can be adapted to human or animal hosts. 


\section{Contents}

Abstract

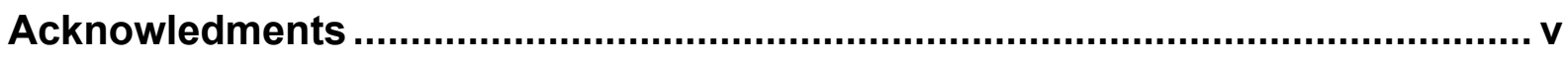

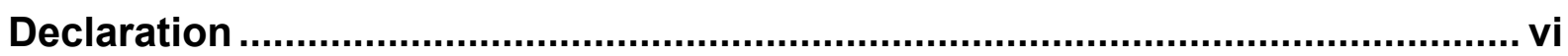

Abbreviations.................................................................................................. vii

List of tables ................................................................................................ ix

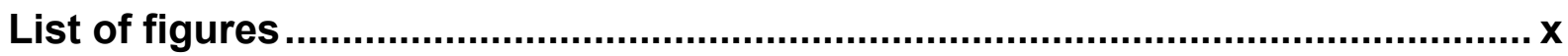

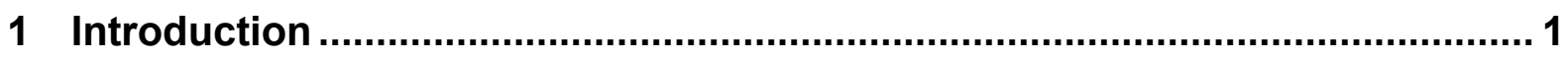

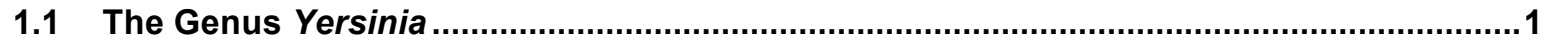

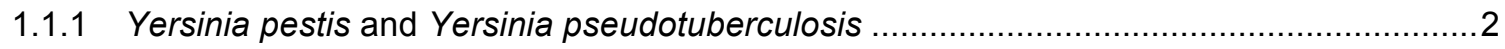

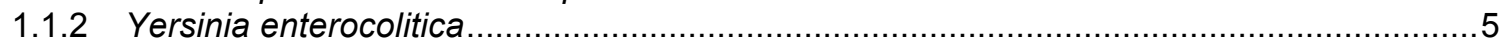

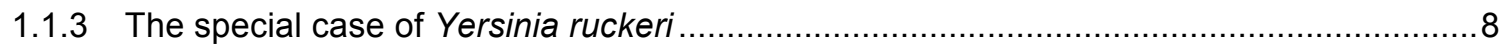

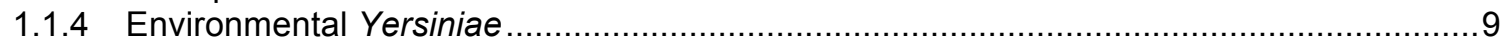

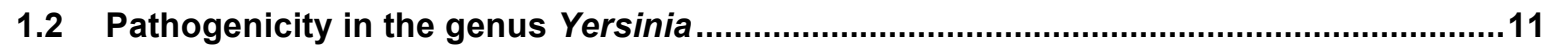

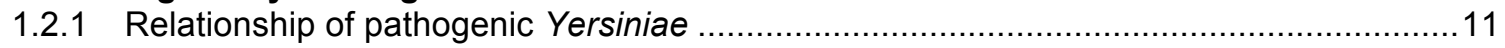

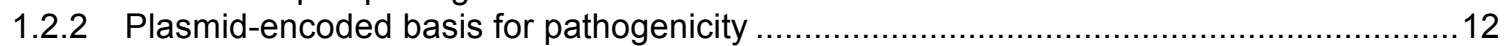

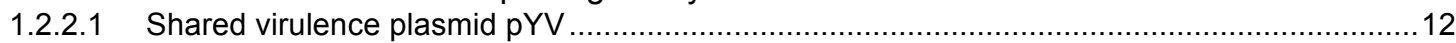

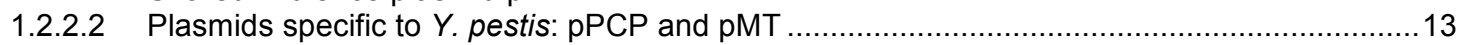

1.2.2.3 Plasmids specific to Y. pseudotuberculosis: pVM82 and pIB .........................................15

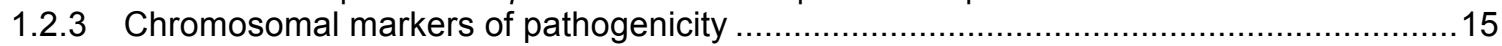

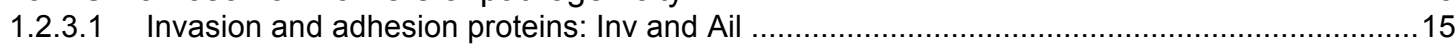

1.2.3.2 Yersinia adhesion pathogenicity island (YAPI) ……………………………………....... 16

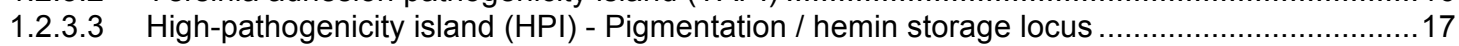

1.2.3.4 Yersinia pseudotuberculosis superantigen YPM .......................................................... 18

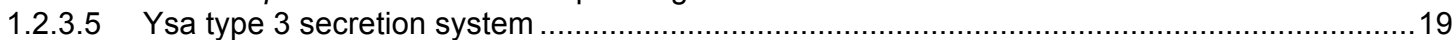

1.2.3.6 Further chromosomally encoded virulence factors ......................................................... 19

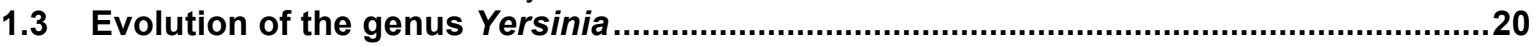

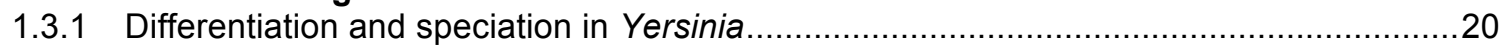

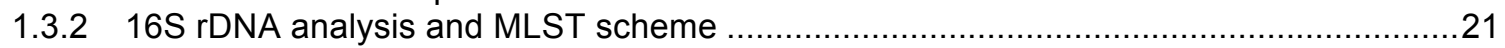

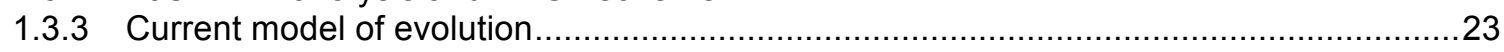

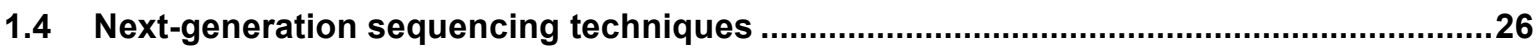

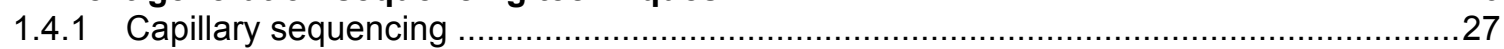

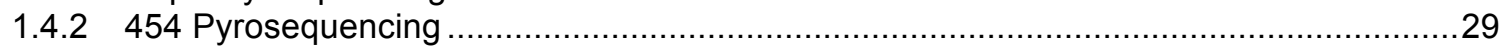

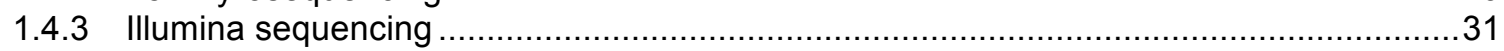

1.4.4 Comparison of capillary, pryo- and Illumina sequencing and applications .......................34

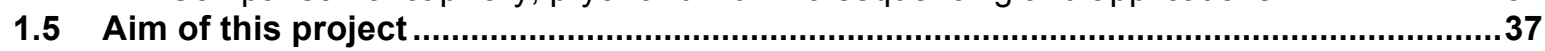

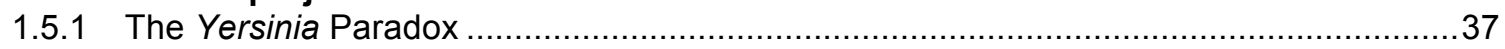

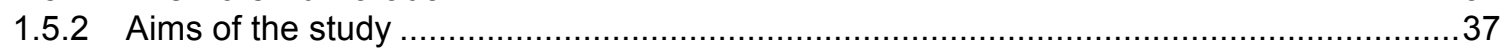

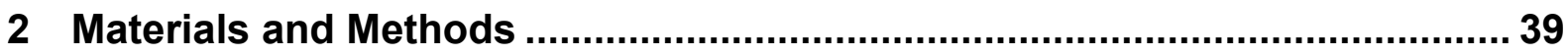

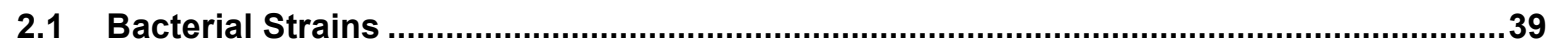

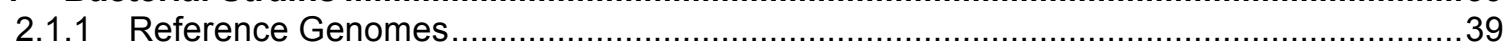

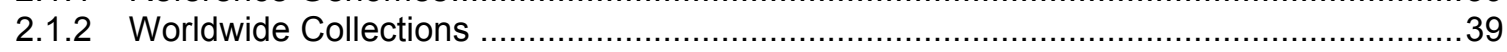

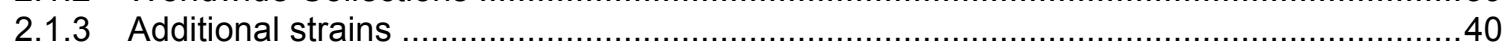

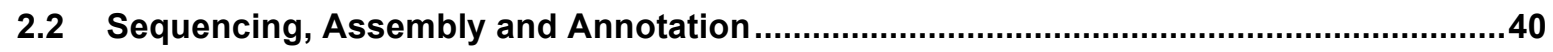

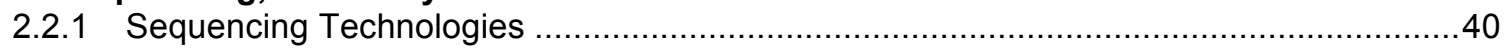

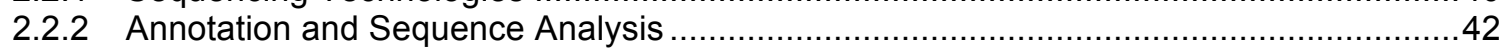

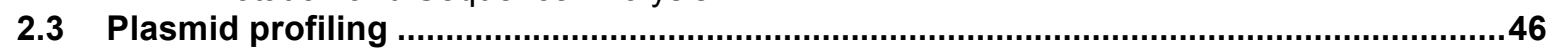

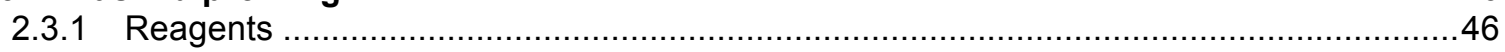

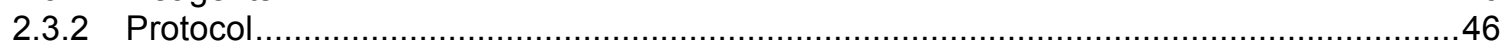

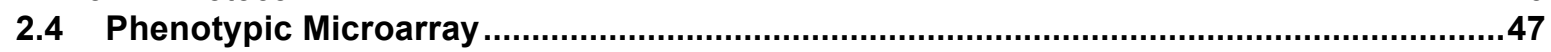

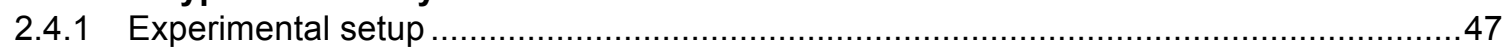

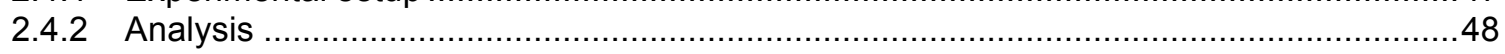

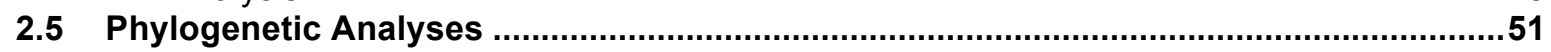

2.5.1 Phylogenetic reconstruction of $Y$. enterocolitica: whole genome alignment .....................51

2.5.2 Phylogenetic reconstruction of the genus Yersinia: extended MLST scheme ..................51 


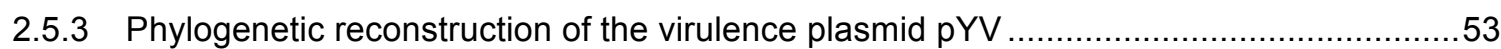

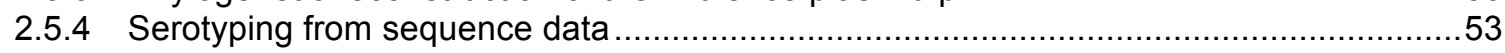

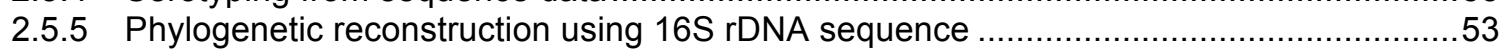

2.5.6 Phylogenetic reconstruction including Y. entomophaga and Y. nurmii ...........................53

3 Sequencing and Analysis of the Reference Genomes for Y. enterocolitica 55

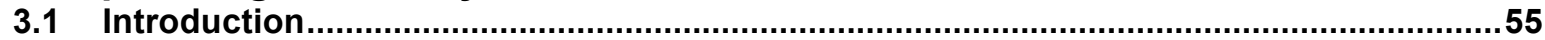

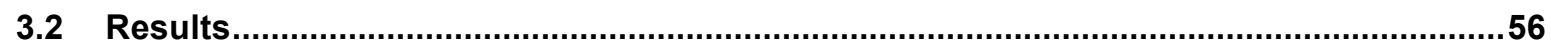

3.2.1 Sequencing, assembly and sequence improvement of the Y. enterocolitica biotypes

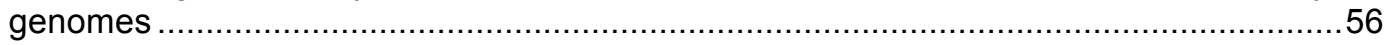

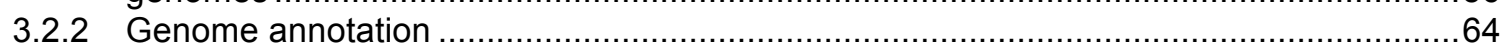

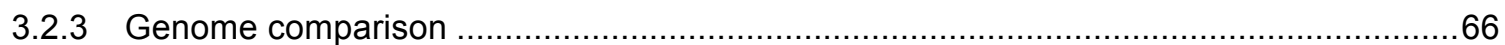

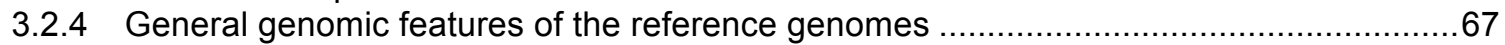

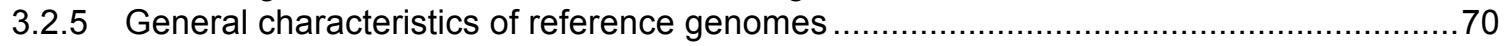

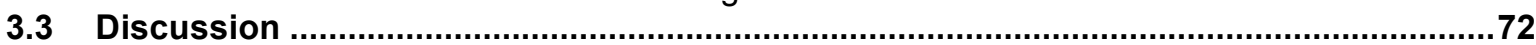

3.3.1 Generation of Improved High-Quality Draft genome sequences ................................... 72

3.3.2 General genomic composition of a Y. enterocolitica genome ...................................... 74

4 Comparative analysis of the Y. enterocolitica biotypes...............................77

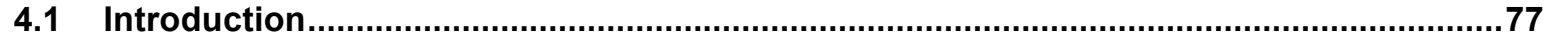

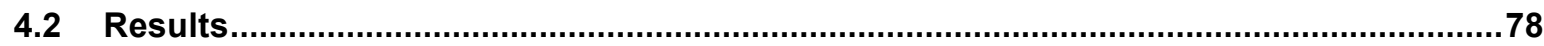

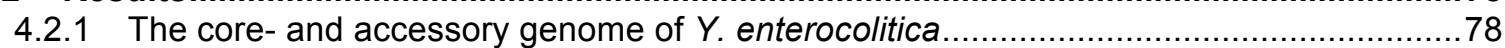

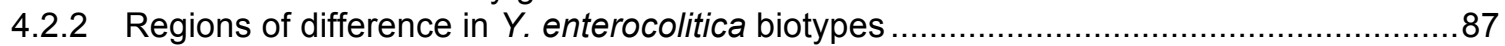

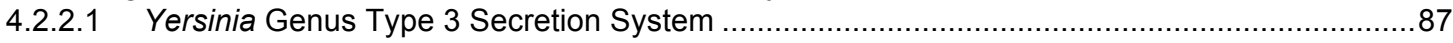

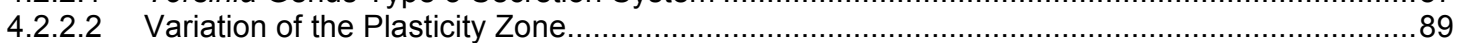

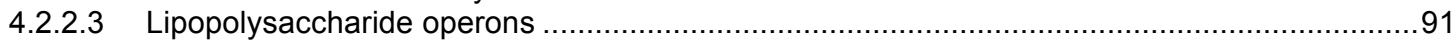

4.2.3 Pseudogene distribution amongst the $Y$. enterocolitica biotypes ..............................95

4.2.4 The role of IS elements and recombination in Y. enterocolitica biotypes .......................103

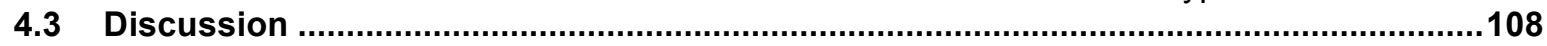

4.3.1 High-, low-, and non-pathogenic biotypes have distinct genomic features .....................108

4.3.2 Metabolism, pseudogenization and IS elements are important evolutionary forces in

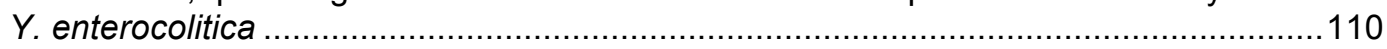

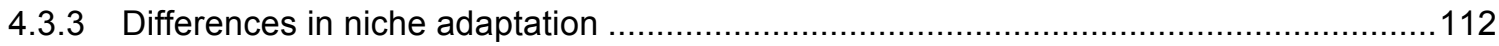

5 Plasmid Variety and Variability in Y. enterocolitica Biotypes ....................115

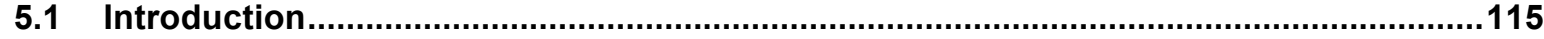

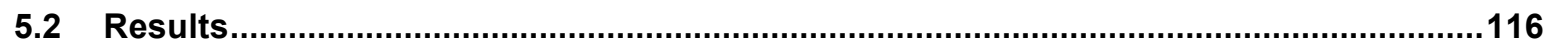

5.2.1 Genetic Organization of the virulence plasmid pYV in Y. enterocolitica BT .......................116

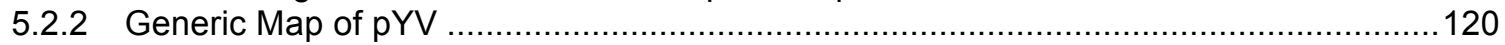

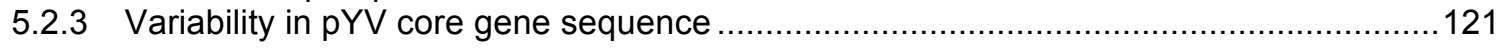

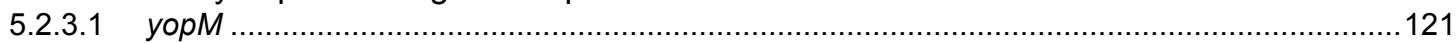

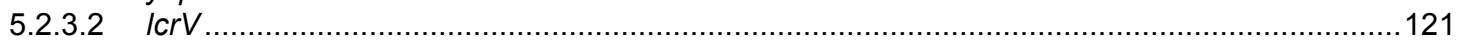

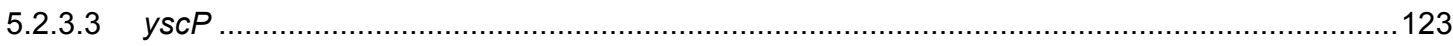

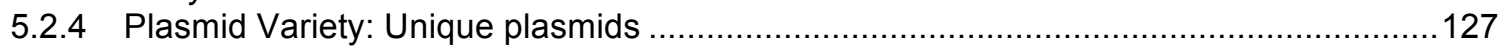

5.2.4.1 Unique plasmids in the reference strain for BT1A: pSR2-1A and pSR3-1A ........................127

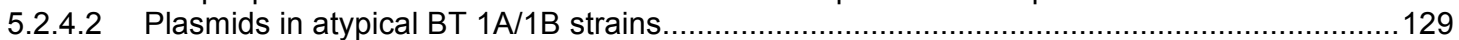

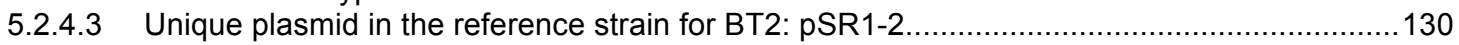

5.2.4.4 Unique plasmid in the reference strain for BT5: pSR4-5 ................................................134

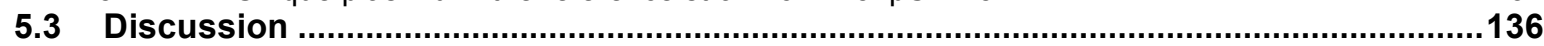

5.3.1 The virulence plasmid differs in high- and low-pathogenic BTs................................136

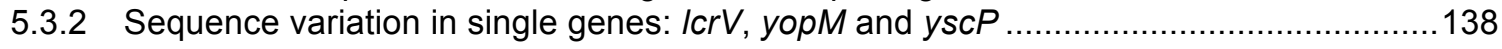

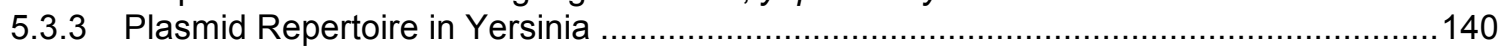

6 Metabolic profiling of the $Y$. enterocolitica biotypes................................... 141

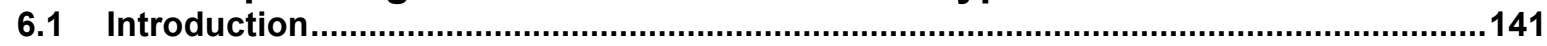

6.1.1 Biotyping and speciation based on biochemical properties ......................................141

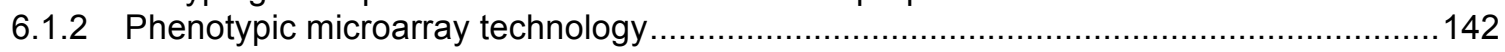

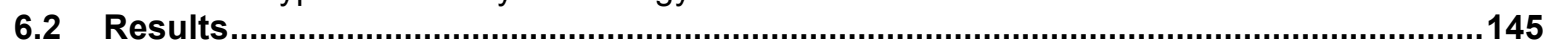

6.2.1 Metabolic activity in Y. enterocolitica biotypes at optimum temperature ........................ 145

6.2.1.1 Metabolism of carbon sources ................................................................................ 148

6.2.1.2 Utilization of essential nutrients nitrogen, phosphorus and sulphur .....................................149

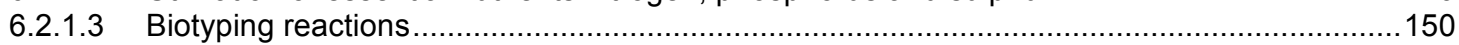

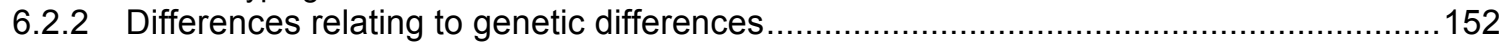

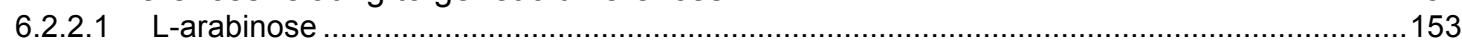

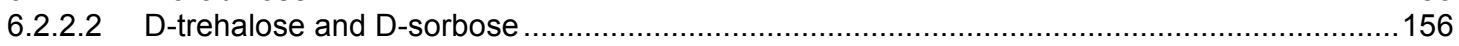

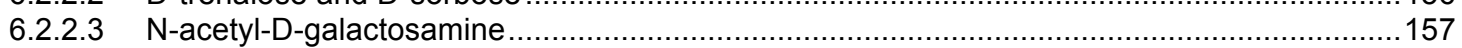




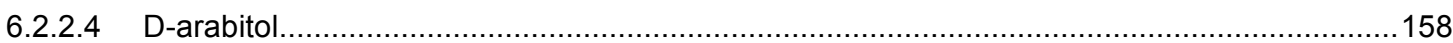

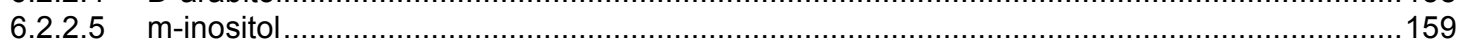

6.2.3 Metabolic activity of $Y$. enterocolitica biotypes at human body temperature ................162

6.3 Discussion ...............................................................................................................164

6.3.1 Metabolic profiling of $Y$. enterocolitica confirms genomic analysis .............................164

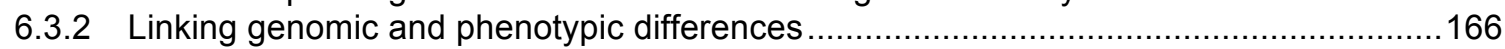

6.3.3 The influence of temperature on metabolism in Y. enterocolitica ..............................168

7 Phylogeny of the Genus Yersinia and the species Y. enterocolitica ........... 171

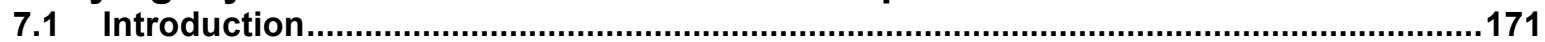

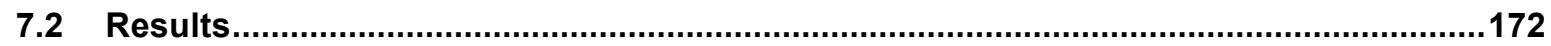

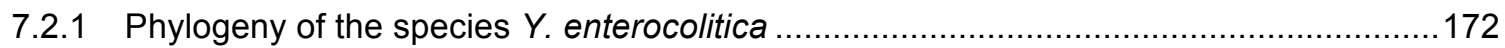

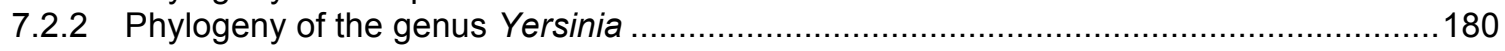

7.2.3 Phylogeny of the genus Yersinia based on 16S rDNA sequences...........................186

7.2.4 Acquisition of the virulence plasmid $p Y V$ in pathogenic Yersiniae ............................188

7.3 Discussion ..................................................................................................................191

7.3.1 The evolution of pathogenic $Y$. enterocolitica lineages......................................... 191

7.3.2 The genus Yersinia and the virulence plasmid pYV - independent evolution of

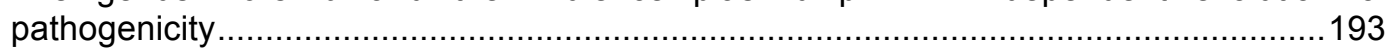

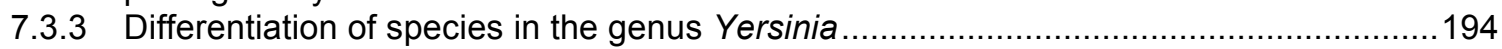

8 Overall Discussion and Future Work ........................................................196

8.1 Analysis of reference genomes and plasmids ...........................................................196

8.2 Analysis of phenotypic microarray..........................................................................197

8.3 Revised model for the evolution of pathogenicity in the genus Yersinia ........................198

8.4 Future Work ....................................................................................................201

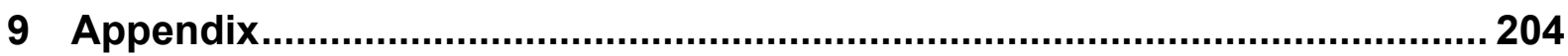

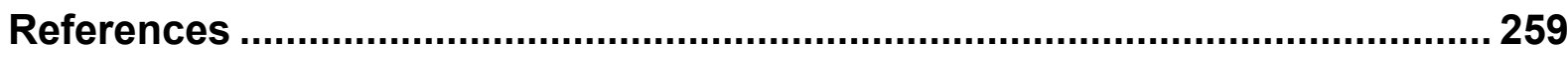




\section{Acknowledments}

I would like to thank my supervisors Dr Alan McNally and Dr Georgina Manning, Nottingham Trent University, and Dr Nicholas R Thomson, Wellcome Trust Sanger Institute, for the opportunity to carry out this $\mathrm{PhD}$ project and for their excellent support and encouragement throughout this project. Their enthusiasm for the project was infective and I very much enjoyed working with them.

Many thanks go to Nico Petty, Matt Holden, Simon Harris, Maria Fookes, Nick Croucher and Tom Connor at the Wellcome Trust Sanger Institute for answering all my questions and for a lot of bioinformatics support. Thanks to Theresa Feltwell for help with the phenotypic microarray and for providing a British experience including Sunday roast dinners and apple crumbles; Danielle Walker for all the Ts shared, and the Gs, Cs, and As as well; and Lars Barquist for implementing the phenotypic microarray data into a visually nice heatmap.

I would also like to thank Prof Brendan Wren, Dr Mike Prentice, and Dr Thilo M Fuchs for stimulating and thoughtful discussions.

Thanks also go to past and present members of the office ED104 at Nottingham Trent University, the Microbiology research team and the prep room for an enjoyable working environment.

Special thanks go to my parents for their never-ending support and encouragement throughout all my studies.

This research was funded by a Nottingham Trent University Vice Chancellor scholarship and core funding to the Wellcome Trust Sanger Institute. 


\section{Declaration}

The conclusions reached in this thesis are my own based on the experiments reported herein. Experiments were performed in the Pathogen Research Group at Nottingham Trent University and in the Pathogen Genomics group at the Wellcome Trust Sanger Institute.

Dr Timothy Hetherington at Nottingham Trent University designed a six-way Venn diagram. Implementation of the phenotypic microarray data in Limma and generation of a heatmap was carried out by Lars Barquist at the Wellcome Trust Sanger Institute. Phylogenetic studies were carried out in collaboration with Drs Simon Harris and Thomas Connor at the Wellcome Trust Sanger Institute. Bayesian Analysis of Population Structure was performed by Dr Thomas Connor. 


\section{Abbreviations}

\begin{tabular}{|c|c|}
\hline ACT & Artemis comparison tool \\
\hline$b, b p$ & bases, base pairs \\
\hline BT & biotype \\
\hline CDS & coding sequence \\
\hline DNA & deoxyribonucleic acid \\
\hline dNTP & deoxynucleoside triphosphate \\
\hline ddNTP & di-deoxynucleoside triphosphate \\
\hline g & gram \\
\hline $\mathrm{HPI}$ & high-pathogenicity island \\
\hline IS element & insertion sequence element \\
\hline $\mathrm{kb}$ & kilo bases $=1,000$ bases \\
\hline $\mathrm{kDa}$ & kilodalton \\
\hline I & litre \\
\hline LB agar/broth & Luria-Bertani medium \\
\hline LPS & lipopolysaccharide \\
\hline M & Molar $=\mathrm{mol} / \mathrm{l}$ \\
\hline $\mathrm{mg}$ & milligram $=10^{-3} \mathrm{~g}$ \\
\hline $\mathrm{mM}$ & millimolar $=10^{-3} \mathrm{~mol} / \mathrm{l}$ \\
\hline $\mathrm{ml}$ & millilitre $=10^{-3}$ litre \\
\hline MLST & multi-locus sequence typing \\
\hline $\mathrm{Mb}$ & mega bases $=1,000,000$ bases \\
\hline NTU & Nottingham Trent University \\
\hline $\mathrm{O}$ & outer antigen \\
\hline PCR & polymerase chain reaction \\
\hline $\mathrm{pl}$ & picolitre $=10^{-12}$ litre \\
\hline PM & phenotypic microarray \\
\hline pMT / pTox / pFra & murine toxin plasmid in Yersinia pestis \\
\hline pPCP / pPst / pPla & plasminogen activator plasmid in Yersinia pestis \\
\hline PTS & phosphotransferase system \\
\hline pYV / pCD1 & Yersinia virulence plasmid \\
\hline PZ & plasticity zone \\
\hline RE & restriction enzyme \\
\hline rDNA & ribosomal DNA \\
\hline
\end{tabular}




$\begin{array}{ll}\text { RNA } & \text { ribonucleic acid } \\ \text { rpm } & \text { rounds per minute } \\ \text { SC } & \text { species cluster } \\ \text { SNP } & \text { single nucleotide polymorphism } \\ \text { SPI } & \text { Salmonella pathogenicity island } \\ \text { ST } & \text { serotype } \\ \text { T2SS } & \text { type II secretion system } \\ \text { T3SS } & \text { type III secretion system } \\ \text { tc PAI } & \text { toxin complex pathogenicity island } \\ \text { V } & \text { volts } \\ \text { WTSI } & \text { Wellcome Trust Sanger Institute } \\ \text { YAPI } & \text { Yersinia adhesion pathogenicity island } \\ \text { YGI } & \text { Yersinia genomic island } \\ \text { YGT } & \text { Yersinia genus T3SS } \\ \text { Yop } & \text { Yersinia outer protein } \\ \text { YPM } & \text { Yersinia pseudotuberculosis mitogen } \\ \mu l & \text { microlitre = 10-6 litre }\end{array}$




\section{List of tables}

Table 1.1: Selection of biochemical characteristics of members of the genus Yersinia.....

Table 1.2: Biotyping scheme according to Wauters, Kandolo and Janssens (1987) and Bottone (1997).

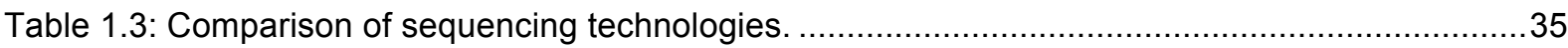

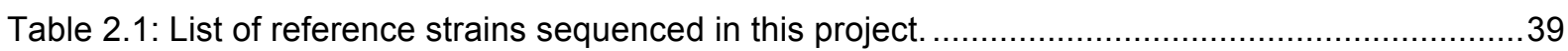

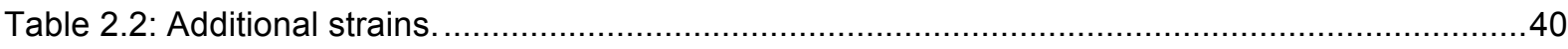

Table 2.3: Function and basis of the algorithms used for gene prediction and characterization. .........42

Table 2.4: Housekeeping genes used for construction of the phylogenetic relationship within the genus

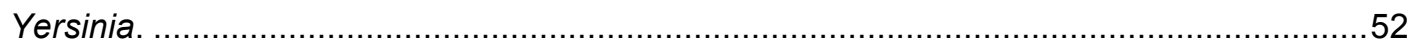

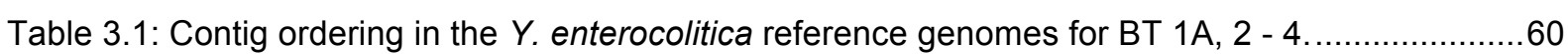

Table 3.2: Contig ordering in the $Y$. enterocolitica reference genome for BT 5.............................61

Table 3.3: General properties of the sequenced reference genomes of $Y$. enterocolitica .................68

Table 4.1: Regions of interest identified in the pangenome of $Y$. enterocolitica (Figure 4.7) ..............86

Table 4.2: IS elements in the $Y$. enterocolitica BT .................................................. 104

Table 4.3: Syntenic blocks identified in the reference genomes of $Y$. enterocolitica. ......................106

Table 4.4: Distribution of important regions and islands of $Y$. enterocolitica in reference genomes...109

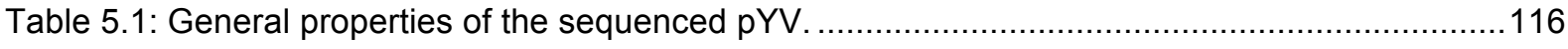

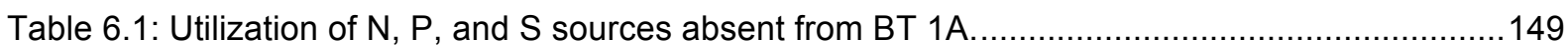

Table 6.2: Comparison of biotyping and phenotypic microarray reactions. .................................. 150

Table 7.1: Numbers of SNPs between different lineages of $Y$. enterocolitica..............................174

Table 7.2: Pseudogenes in the core genome of $Y$. enterocolitica lineages. ...................................176

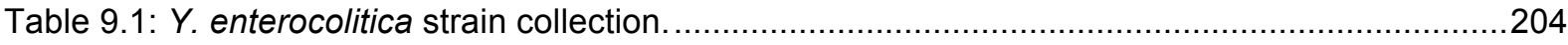

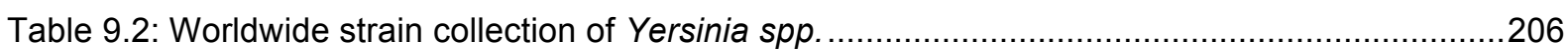

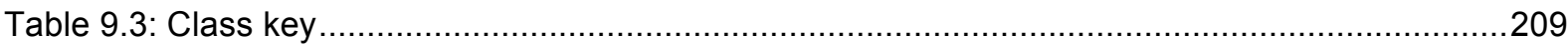

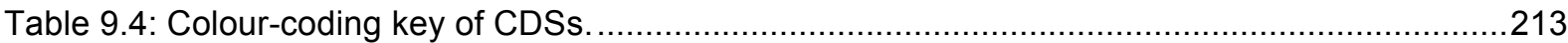

Appendix 9.5: perl script for identification of sets of orthologous genes in seven reference genomes for

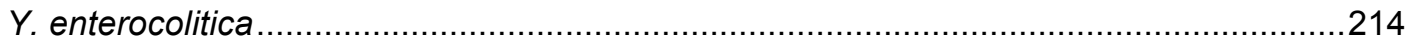

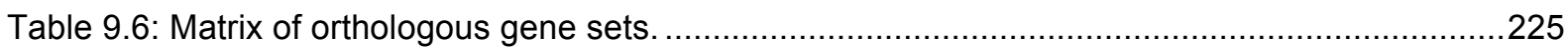

Table 9.7: Pseudogenes and their orthologs in the $Y$. enterocolitica reference genomes. ...............227

Table 9.8: Number of total CDSs, pseudogenes, and partial genes per group for the $Y$. enterocolitica

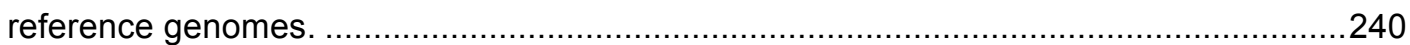

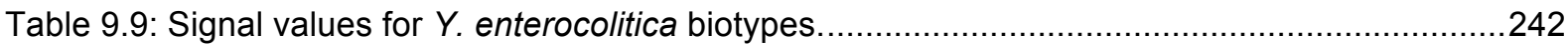

Table 9.10: Positive signal values and significant changes compared to BT $1 \mathrm{~B}$ strain 8081 for Y. enterocolitica biotypes. 


\section{List of figures}

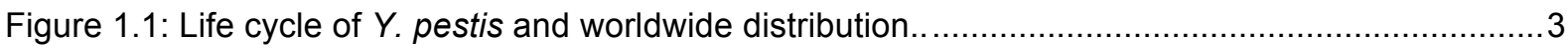

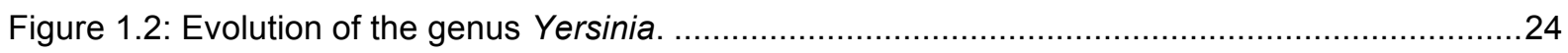

Figure 1.3: Workflow of capillary, pyro- and Illumina sequencing.

Figure 1.4: Preparation of paired-end fragments for pyrosequencing............................................. 31

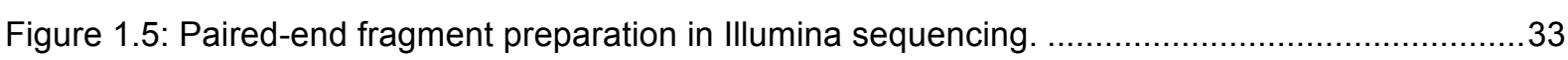

Figure 1.6: Multiplex preparation of DNA fragments for Illumina sequencing . ..................................... 34

Figure 2.1: Example of coverage of $Y$. enterocolitica strains sequenced as part of the worldwide strain

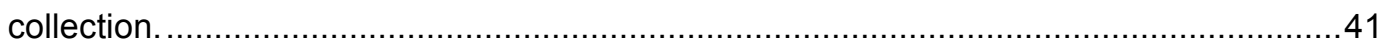

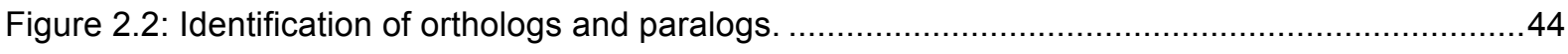

Figure 2.3: Example of a 3-way comparison for identification of orthologous gene sets. .....................45

Figure 2.4: Preparation of fluids for phenotypic microarray. ............................................................ 47

Figure 2.5: Example for utilization of carbon sources in BT 1A and BT 1B. .................................. 49

Figure 3.1: Sequencing timeline for the $Y$. enterocolitica reference genomes...................................57

Figure 3.2: Initial assembly based on single-end 454 reads and comparison of the six $Y$. enterocolitica

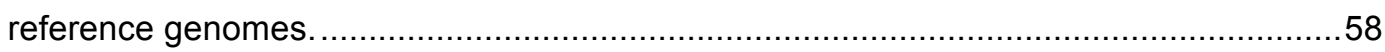

Figure 3.3: Pairwise comparison of final assemblies after gap closure of $Y$. enterocolitica reference

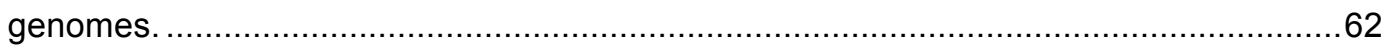

Figure 3.4: Flow chart of the preparation of a genome sequence for annotation and analysis............64

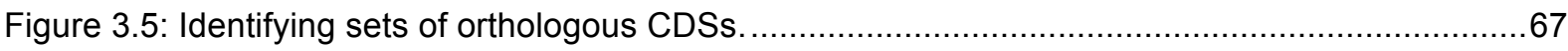

Figure 3.6: Distribution of classes amongst CDSs of all reference genomes. ......................................71

Figure 4.1: Connections between individual reference genomes based on analysis of orthologous

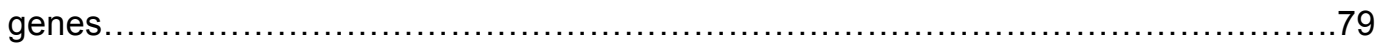

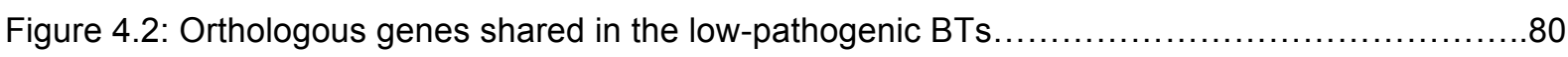

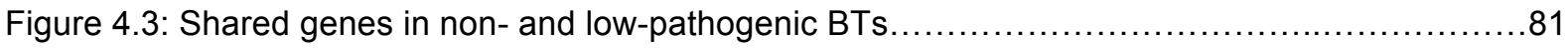

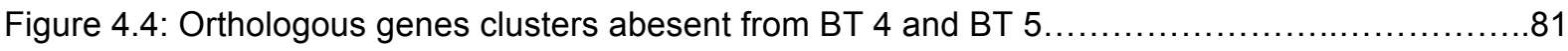

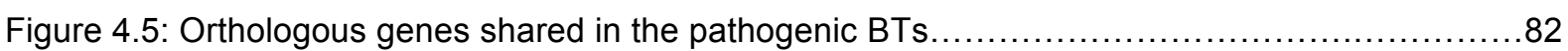

Figure 4.6: Combined comparison of orthologous accessory gene sets in the reference genomes for

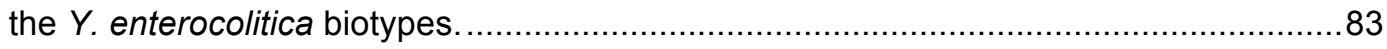

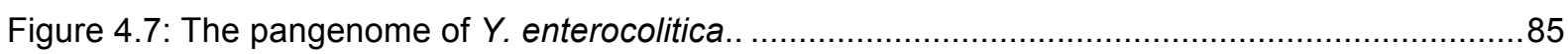

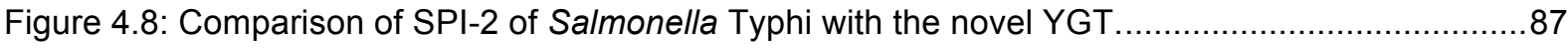

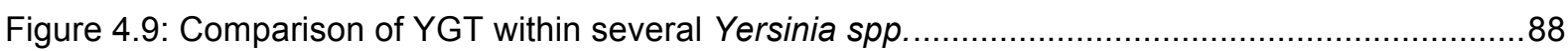

Figure 4.10: Arrangement of the plasticity zone in the different biotypes. ........................................ 90

Figure 4.11: Lipopolysaccharide structure of $Y$. enterocolitica serotypes............................91

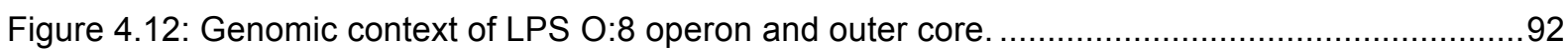

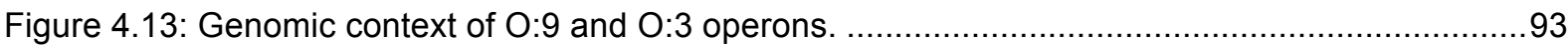

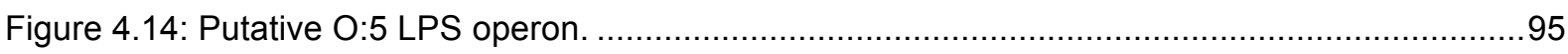

Figure 4.15: Pseudogene and partial gene distribution in the $Y$. enterocolitica reference genomes....98

Figure 4.16: Top 3 pseudogene categories for Y. enterocolitica BTs. ..........................................103

Figure 4.17: Position of the predominant IS element IS1667 in the reference genome assemblies...105

Figure 4.18: Exemplar position of six syntenic blocks. 107 


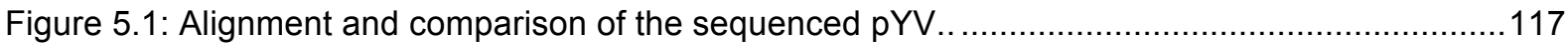

Figure 5.2: Backbone of $\mathrm{pYV}$ and variable regions in different $Y$. enterocolitica biotypes.................120

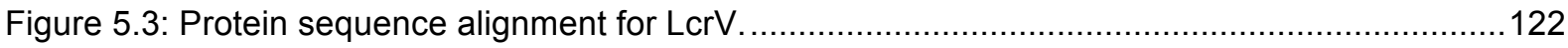

Figure 5.4: Protein sequence alignment of YscP protein sequences of different Yersinia spp...........125

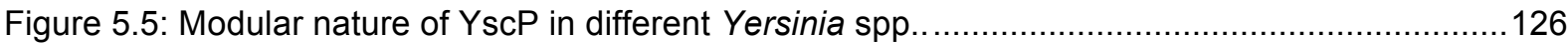

Figure 5.6: Linear plasmid map of pSR2-1A found in $Y$. enterocolitica BT 1A 0:5 strain 5303........128

Figure 5.7: Linear plasmid map of pSR3-1A found in $Y$. enterocolitica BT 1A 0:5 strain 5303.........128

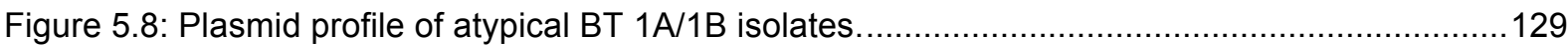

Figure 5.9: Circular plasmid map of pSR1-2 found in Y. enterocolitica BT 2 0:9 strain 21202 ........131

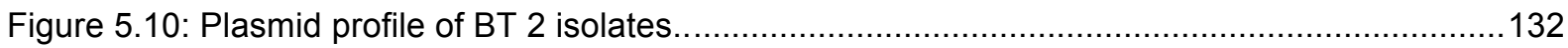

Figure 5.11: Comparison between Salmonella Dublin pMAK-2 and Y. enterocolitica pSR1-2 .........133

Figure 5.12: Plasmid map of pSR4-5 as found in Y. enterocolitica BT 5 O:2a,2b,3 strain 3094........134

Figure 5.13: Comparison between Y. enterocolitica pYE854 and pSR4-5. ..................................135

Figure 6.1: Respiration is coupled to conversion of tetrazolium violet dye ....................................142

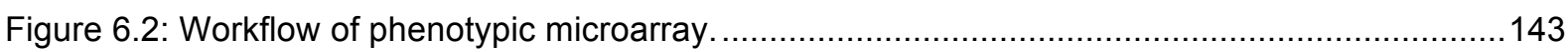

Figure 6.3: Signal values (A) and significant changes towards BT 1B (B) depicted as heatmaps.....146

Figure 6.4: Number of sources that are expressed significantly higher than in BT 1B strain $8081 \ldots . .147$

Figure 6.5: Number of sources that are expressed significantly lower than in BT 1B 8081_............147

Figure 6.6: Kinetic plots for raffinose in $Y$. enterocolitica biotypes.................................. 151

Figure 6.7: Operon for L-arabinose utilization contrasted with signal values and significant changes

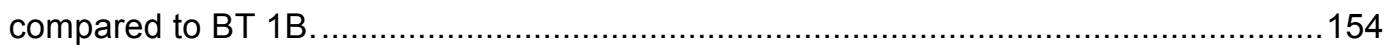

Figure 6.8: L-arabinose utilization kinetic plots over time for $Y$. enterocolitica biotypes................155

Figure 6.9: Mutations specific for biotyping of BT 5 contrasted with signal values and significant

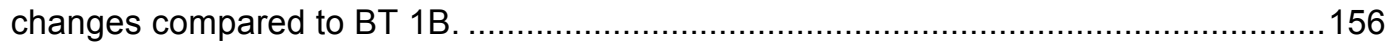

Figure 6.10: Operon structure for utilization of $\mathrm{N}$-acetyl-D-galactosamine in representative BTs contrasted with signal values and significant changes compared to BT $1 \mathrm{~B}$. 157

Figure 6.11: Unique operon for $\mathrm{D}$-arabitol utilization in $\mathrm{BT} 1 \mathrm{~A}$, contrasted with signal values and significant changes compared to BT $1 \mathrm{~B}$. 159

Figure 6.12: Absorbance curves over time for $m$-inositol utilization...............................160

Figure 6.13: Point mutation in the idh gene is the basis for BT 1A negative m-inositol phenotype. ...161

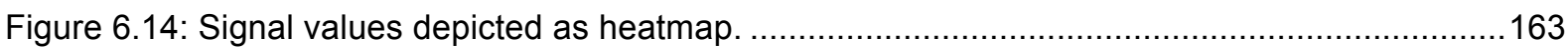

Figure 6.15: Comparison of biotyping reactions and phenotypic microarray. ……..........................164

Figure 7.1: The maximum likelihood phylogenetic tree of $Y$. enterocolitica based on the SNP differences across the whole genome excluding laterally acquired phages.

Figure 7.2: Distribution of the 85 housekeeping genes used for construction of the phylogenetic relationship within the genus Yersinia.

Figure 7.3: The phylogeny of the genus Yersinia and the virulence plasmid pYV ............................183

Figure 7.4: The phylogeny of the genus Yersinia based on $r e c A, g \ln A$, gyrB, and $h s p 60 \ldots \ldots \ldots \ldots \ldots . . . .185$

Figure 7.5: The phylogeny of the genus Yersinia based on 16S rDNA sequences. 187

Figure 7.6: Phylogeny of the plasmid pYV compared to the phylogeny of the chromosome in Y. enterocolitica. 189

Figure 8.1: Revised model of the evolution of pathogenicity in the genus Yersinia. 200 


\section{Introduction}

\subsection{The Genus Yersinia}

The heterogeneous genus Yersinia comprises three human-pathogenic species, Y. pestis, Y. pseudotuberculosis, and Y. enterocolitica, the fish-pathogen Y. ruckeri, and a number of environmental, seemingly non-pathogenic species (Carniel and Mollaret, 1990; Bottone, 1997; Bottone, 1999; Sulakvelidze, 2000; Wren, 2003). The Yersiniae belong to the family of Enterobacteriaceae, and are rod-shaped, facultative anaerobic bacteria (Carniel and Mollaret, 1990; Bottone, 1999; Wren, 2003).

Historically, speciation in Yersinia is based on biochemical differences (Sulakvelidze, 2000). A selection of biochemical tests for species designation is listed in Table 1.1.

Table 1.1: Selection of biochemical characteristics of members of the genus Yersinia [adapted from Bottone, 1997; Sulakvelidze, 2000; Hurst, et al., 2011; Murros-Kontiainen, et al., 2010a,b].

\begin{tabular}{|c|c|c|c|c|c|c|c|c|c|c|c|}
\hline & 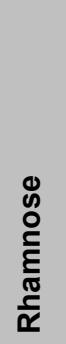 & $\begin{array}{l}\text { め } \\
\text { ஃ } \\
\text { 은 } \\
\text { க் }\end{array}$ & $\begin{array}{l}\text { ळ } \\
\text { 응 } \\
\text { 응 } \\
\overline{0}\end{array}$ & 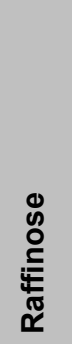 & 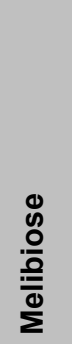 & 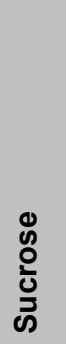 & 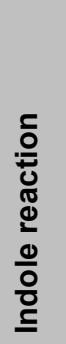 & 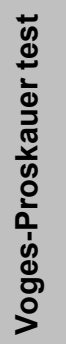 & $\begin{array}{l}\overline{0} \\
\stackrel{+}{0} \\
\stackrel{0}{\circ}\end{array}$ & 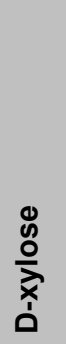 & 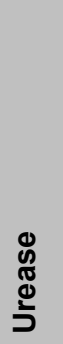 \\
\hline Y. aldovae & + & - & - & - & - & $\overline{-1}$ & $\overline{-1}$ & + & + & + & + \\
\hline Y. aleksiciae & - & + & + & - & - & - & + & - & - & + & + \\
\hline Y. bercovieri & - & - & + & - & - & + & - & - & - & + & + \\
\hline Y. enterocolitica & - & v & + & - & - & + & v & - & + & v & + \\
\hline Y. entomophaga & - & - & + & + & + & + & - & $?$ & - & - & + \\
\hline Y. frederiksenii & + & + & + & - & - & + & + & v & + & + & + \\
\hline Y. intermedia & + & + & + & + & + & + & + & + & - & + & + \\
\hline Y. kristensenii & - & + & + & - & - & - & $\mathrm{v}$ & - & - & + & + \\
\hline Y. massiliensis & - & + & + & v & - & + & + & - & + & + & + \\
\hline Y. mollaretii & - & + & + & - & - & + & - & - & + & + & + \\
\hline Y. nurmii & - & - & $(+)$ & - & - & + & - & + & - & - & - \\
\hline Y. pekkanenii & - & - & + & - & - & - & - & - & - & + & + \\
\hline Y. pestis & $v$ & - & - & - & v & - & - & - & - & - & - \\
\hline Y. pseudotuberculosis & + & - & - & - & + & - & - & - & - & + & + \\
\hline Y. rohdei & - & + & + & + & $v$ & + & - & - & - & + & + \\
\hline Y. ruckeri & - & - & - & + & - & - & - & - & - & - & - \\
\hline Y. similis & + & - & - & - & - & - & - & - & - & + & + \\
\hline
\end{tabular}

+ - positive; (+) - delayed positive; v - variable; - - negative; ? - information unavailable 
There is no one typing scheme used for Yersinia, and the most commonly used key tests are presented (Bottone, 1997; Stock, Henrichfreise and Wiedemann, 2002). The description of further species generally relies on new tests being added, resulting in data that is then not available for all species. Differences in DNA-DNA hybridization values are used to furthermore classify the environmental Yersiniae (Bottone, 1997). Most recently differences in the 16S rDNA sequence have been employed alongside biochemical signatures and DNA-relatedness to define new species (Sprague and Neubauer, 2005; Hurst, et al., 2011).

\subsubsection{Yersinia pestis and Yersinia pseudotuberculosis}

$Y$. pestis is the causative agent of plague, the notorious Black Death of the middle ages, responsible for the death of millions of Europeans (Brubaker, 1991; Perry and Fetherston, 1997; Wren, 2003). The life cycle of Y. pestis is depicted in Figure 1.1A. $Y$. pestis is a host-dependent organism, and has limited abilities to survive outside either the flea vector or a warm-blooded host (Perry and Fetherston, 1997; Parkhill, et al., 2001a; Wren, 2003). In the flea vector, the bacteria block the foregut. The flea cannot feed on a new host, but in trying will regurgitate the bacteria directly under the skin and into the blood stream of the mammalian host (Wren, 2003). This mammalian host can be wild living rodents, such as rabbits or gerbils (Carniel and Mollaret, 1990). This is called the sylvatic cycle, and the ability to hibernate and circulate in wild rodent populations precludes eradication. Y. pestis may cause disease in wild rodents as well, and its persistence might be explained either by hibernation in the ground of abandoned rodent burrows, which may subsequently become re-inhabited, or reduced virulence in rodents due to mutations (Carniel and Mollaret, 1990). 


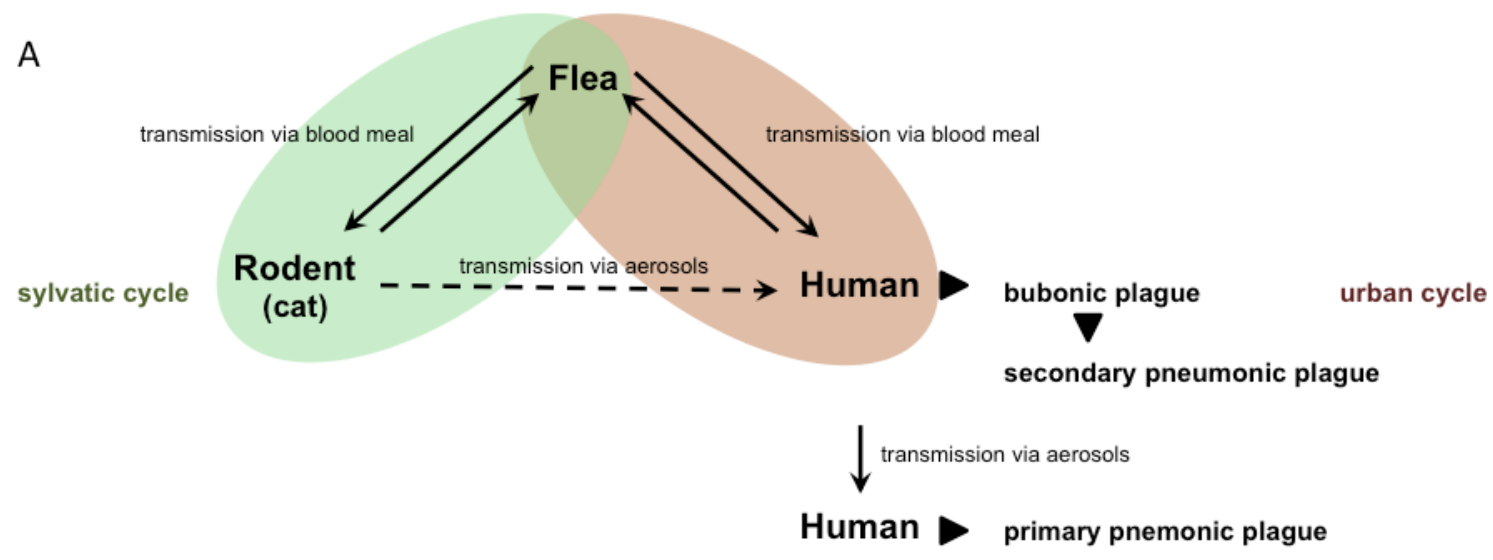

B

World Distribution of Plague, 1998

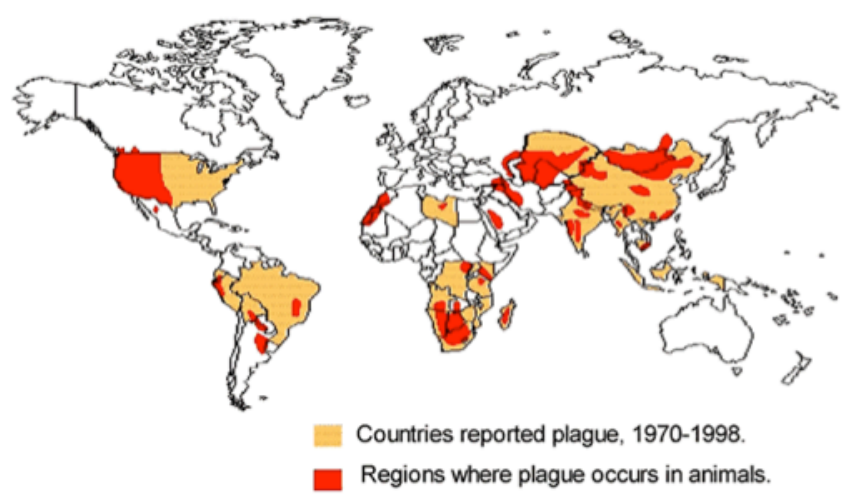

Figure 1.1: Life cycle of $Y$. pestis and worldwide distribution. A. Sylvatic and urban transmission routes of Y. pestis. B. Current endemic foci of plague [according to CDC, 2009].

In the urban cycle, humans are infected as accidental hosts of the flea. Once inside the human body, the bacteria will spread to the regional lymph nodes, either by surviving inside macrophages or through tissue infection (Wren, 2003). Once there, $Y$. pestis multiplies and gives rise to the classic symptoms of inflamed lymph nodes of primary bubonic plague (Carniel and Mollaret, 1990; Perry and Fetherston, 1997). The incubation time of infection is 2 to 6 days after the bite (CDC, 2005). Once the bacteria have entered the lymph nodes of the human host, they then progress to the lungs, liver and spleen (Carniel and Mollaret, 1990; Wren, 2003). The bacteria enter the blood stream causing septicaemia (Carniel and Mollaret, 1990). The flea feeding on an infected, bacteraemic individual closes the cycle from mammalian host back to the flea. A transmission from cats to humans via aerosols has been reported, but is very rare (CDC, 2009). In crossing sylvatic and urban cycles, rats play an important role as hosts to infected fleas and their proximity to towns and settlements (Carniel and Mollaret, 1990; Perry and Fetherston, 1997). 
The terminal phase of $Y$. pestis is associated with lodging and multiplication of the bacteria in the lung (Carniel and Mollaret, 1990). Upon reaching this site of infection, secondary pneumonic plague ensues with a fatality rate of over 50\% (Wren, 2003; CDC, 2005). Additionally at this stage, an arthropod host is redundant as transmission via aerosols to other humans is possible and highly effective (Carniel and Mollaret, 1990; Wren, 2003). Following this infection route, the bacteria can rapidly spread to the bloodstream, circumventing the need for prior establishment in lymph nodes, and death occurs within a few hours (Carniel and Mollaret, 1990).

Today, Y. pestis is still present in sylvatic foci found in North and South America, sub-Saharan Africa, and Asia with a sufficient rodent population (Figure 1.1B; CDC, 2005). It is still a relevant human disease with 1,000 to 2,000 cases a year worldwide (CDC, 2005), and multidrug resistant strains have been identified (Wren, 2003). It also poses a bioterrorism threat due to extremely efficient person-to-person spread, and delayed onset of symptoms enabling a geographic spread (Carniel and Mollaret, 1990; CDC, 2005). The infective dose is also very low, at 10 cells or less (Brubaker, 1991).

Three classic biovars of $Y$. pestis have been associated with the three main pandemics (Achtman, et al., 1999; Wren, 2003). Bacteria that gave rise to the Justinian plague are related to Biovar Antiqua, and those that gave rise to the Black Death in the Middle Age relate to biovar Mediaevalis. Modern plague is associated with biovar Orientalis (Achtman, et al., 1999). These are distinguished on the basis of glycerol fermentation and nitrate reduction.

Y. pseudotuberculosis is closely related to Y. pestis (Chapter 1.3), but has very different ecology. It is found widely in animals and the environment. Humans are infected via the fecal-oral route following the ingestion of contaminated food sources (Carniel and Mollaret, 1990; Carniel, 2003; Wren, 2003). In animals, the bacteria invade the small intestine and spread from lymphatic vessels to distal lymph nodes and subsequently to liver and spleen (Carniel and Mollaret, 1990). At these sites, abscesses form, and the bacteria can enter the bloodstream with fatal outcome (Carniel and Mollaret, 1990). In human infections, the bacteria enter the small intestine as well, but disease is usually milder (Carniel and Mollaret, 1990). Y. pseudotuberculosis targets microfold cells ( $\mathrm{M}$ cells) for invasion of the gut epithelium (Carniel and Mollaret, 1990; Wren, 2003). It then disseminates widely, and causes mesenteric lymphadenitis (Wren, 2003). Mild diarrhoea can be observed, but 
abdominal pain is the most frequent and constant symptom (Carniel and Mollaret, 1990). No further colonization of deeper tissues or secondary organs ensues though, and usually the disease is self-limiting (Carniel and Mollaret, 1990; Carniel, 2003). Seasonality of the disease is suspected, as the susceptible animal hosts appear to shift from being healthy carriers to infected hosts after exposure to stresses such as cold, humid weather and starvation periods (Carniel and Mollaret, 1990).

In countries of the Far East, infection with Y.pseudotuberculosis occurs with additional systemic symptoms such as skin rashes and toxic shock syndrome, which is also known as Far East scarlet-like fever (Fukushima, et al., 2001; Eppinger, et al., 2007). It has been proposed that the presentation of $Y$. pseudotuberculosis as Far East scarlet-like fever arises predominantly in isolates that carry the superantigen Yersinia pseudotuberculosis mitogen (YPM), which is absent in strains causing enteric disease (Fukushima, et al., 2001; Eppinger, et al., 2007).

In Y. pseudotuberculosis, 21 different serogroups are known which are combined in six serotypes (Carniel and Mollaret, 1990). There is no correlation between serotype and disease severity, in contrast to Y. enterocolitica (Carniel and Mollaret, 1990). Y. pseudotuberculosis infections are less common than Y. enterocolitica infections (Carniel and Mollaret, 1990).

\subsubsection{Yersinia enterocolitica}

Similar to Y.pseudotuberculosis, Y. enterocolitica causes enteric disease. $Y$. enterocolitica infections are most commonly due to ingestion of contaminated food products although cases of infections through contaminated blood transfusion have been reported (Bottone, 1997). Survival and proliferation at refrigeration temperatures make it a significant food-borne pathogen (Bottone, 1997; Bottone, 1999). Following ingestion, the bacteria colonize the gastrointestinal tract, specifically the terminal ileum and proximal colon, by attaching and invading $M$ cells in the Peyer's patches (Carniel and Mollaret, 1990; Bottone, 1997; Bottone, 1999). They then multiply and spread horizontally through the mucosal epithelium (Bottone, 1997). This manifests in inflammatory symptoms such as enteritis, enterocolitis, acute mesenteric lymphadenitis, and terminal ileitis, and is also associated with fever and diarrhoea, which can occasionally be bloody (Carniel and Mollaret, 1990; Bottone, 1997; Bottone, 1999). It is thought that serotype 0:8 strains present with more aggressive disease than 0:3 infections (Bottone, 1999). Often, the infection is 
self-limiting, as these symptoms subside on their own account after 2 weeks in adults and longer periods in infants (Pepe and Miller, 1993; Bottone, 1997; Carniel, 2003).

The bacteria are able to invade deeper tissue layers as well, and may enter the bloodstream with subsequent spread to liver, spleen, and mesenteric lymph nodes (Bottone, 1997; Bottone, 1999). Arthritis and erythema nodosum, an inflammation under the skin, have been described as the most common sequelae of Y. enterocolitica infections (Bottone, 1997; Bottone, 1999).

The species $Y$. enterocolitica is very heterogeneous with respect to both biochemical differences and causing disease. Some strains are able to cause severe enteritis in humans, other strains are responsible for milder symptoms, whilst a third group of strains is not associated with any disease in humans (Carniel and Mollaret, 1990; Bottone, 1999; Sulakvelidze, 2000; Virdi and Sachdeva, 2005). Therefore, the species is differentiated into six different biotypes (BT) based on biochemical differences (Table 1.2; Wauters, Kandolo, and Janssen, 1987).

Table 1.2: Biotyping scheme according to Wauters, Kandolo and Janssens (1987) and Bottone (1997).

\begin{tabular}{llcccccc}
\hline & Biotype & BT 1A & BT 1B & BT 2 & BT 3 & BT 4 & BT 5 \\
& Pathogenicity potential & No & High & Low & Low & Low & Low \\
\hline \hline 1 & Salicin AP & + & - & - & - & - & - \\
2 & Pyrazinamidase activity & + & - & - & - & - & - \\
3 & Esculin hydrolysis & + - & - & - & - & - & - \\
4 & Lipase activity & + & + & - & - & - & - \\
5 & Indole production & + & + & v & - & - & - \\
6 & Xylose AP & + & + & + & + & - & v \\
7 & Trehalose AP & + & + & + & + & + & - \\
8 & Sorbose AP & + & + & + & + & + & - \\
9 & Nitrate reduction & + & + & + & + & + & - \\
10 & Ornithine decarboxylase & + & + & + & + & + & $+(+)$ \\
11 & Voges-Proskauer test & + & + & + & + & + & $+(+)$ \\
12 & Inositol AP & + & + & + & + & + \\
\hline AP
\end{tabular}

$\overline{\mathrm{AP}}$ - acid production, + - positive, - - negative, $\mathrm{v}-$ variable, $(+)$ - delayed positive.

There are several reactions, which are unique to BT $1 \mathrm{~A}$, whereas there is only one reaction to distinguish BT 4 from $\mathrm{BT} 3$, and one variable reaction to separate BT 2 and BT 3. Generally, biotyping identification is based on the absence of utilization of a nutrient source compared to BT $1 \mathrm{~A}$ rather than unique properties specific for each biotype. This results in a "step-wise" appearance of positive reactions. 
In line with its heterogeneity there are also approximately 60 serogroups in $Y$. enterocolitica, but only certain bio-serotypes are frequently associated with human infections, which are BT 1B O:8, and O:21, BT 2 O:9, and O:5,27, BT 3 O:1,2,3, O:9, and O:5,27, BT 4 O:3, and BT 5 O:2,3 (Carniel and Mollaret, 1990; Bottone, 1997; Bottone, 1999; Sulakvelidze, 2000; Virdi and Sachdeva, 2005). In early studies, these bio-serotypes have been associated with invasion of epithelial cells, autoagglutination and conjunctivitis in guinea pigs (Pedersen, Winblad and Bitsch, 1979; Schiemann and Devenish, 1982). BT 5 potentially presents an exception and does not invade epithelial cells (Pedersen, Winblad and Bitsch, 1979). It was also noted specifically that BT $1 \mathrm{~B}$ O:8 and $0: 21$ serotypes generate more severe disease in the guinea pig model (Schiemann and Devenish, 1982).

In a mouse infection model, the biotypes also exhibit differences in disease severity. $B T 1 B$ is considered the high-pathogenic BT, as it kills mice in infection models, and BTs 2 - 5 are the low-pathogenic BTs that cause infection in mice but are not lethal. Because it does not cause disease in mice, BT 1A is designated the non-pathogenic BT (Wren, 2003). Recently, two subspecies have been proposed (Neubauer, et al., 2000). Based on differences in the 16S rDNA sequence, it has been suggested to refer to the high-pathogenic BT $1 \mathrm{~B}$ as $Y$. enterocolitica subsp. enterocolitica, and the non- and low-pathogenic BTs 1A, 2 - 5 as $Y$. enterocolitica subsp. palearctica (Neubauer, Hensel, Aleksic and Meyer, 2000; Neubauer, et al., 2000; Neubauer and Sprague, 2003).

$Y$. enterocolitica is a ubiquitous organism, found in the environment as well as the gastrointestinal tract of animals (Bottone, 1999). Pigs are a common reservoir for nearly all $Y$. enterocolitica, and this is considered a major route of infections for humans although a clear link has not yet been discovered (Carniel and Mollaret, 1990; Bottone, 1999; Neubauer and Sprague, 2003). The non-pathogenic BT $1 \mathrm{~A}$ is also found in a range of environmental sources such as animals, food, and water, and is also isolated from faeces of healthy and sick humans (Bottone, 1999; Tennant, Grant and Robins-Browne, 2003; Tennant, Skinner, Joe and RobinsBrowne, 2005). BT 5 is a rarely isolated biotype, and its main ecologic distribution is in hare and goats. It is therefore commonly referred to as the "hare-biotype", and isolation from humans has never been reported (Swaminathan, Harmon and Mehlman, 1982; Carniel and Mollaret, 1990; Wuthe and Aleksic, 1997; Bartling, et al., 
2004). In hares, infection can cause acute septicaemic disease leading to epidemic outbreaks (Bartling, et al., 2004).

\subsubsection{The special case of Yersinia ruckeri}

Amongst the Yersiniae, Y. ruckeri is a special case. Compared to other members of the genus, it is the most distantly related species, and it has been questioned whether it is indeed a member of the genus (Bottone, 1997; Sulakvelidze, 2000). Studies on DNA relatedness indicated distant relationships to both Serratia and Yersinia, and a designation into the genus Yersinia was based on similarity in biochemical reactions as well as the GC content (Sulakvelidze, 2000; Fernández, Méndez and Guijarro, 2007).

Y. ruckeri causes redmouth disease in salmonid fish, especially in rainbow trout, which presents as acute or chronic septicaemia with internal and external haemorrhages (Tobback, et al., 2009). Y. ruckeri has also been implicated in fatal septicaemia in carp (Sulakvelidze, 2000; Fernández, Méndez and Guijarro, 2007). As such, it is considered an important economic factor in the fish industry (Tobback, et al., 2009; Ryckaert, et al., 2010). An effective vaccine is available, yet outbreaks under stress conditions can spread quickly from faeces of ill and carrier fish through water (Fernández, Méndez and Guijarro, 2007). Recently, new strains have been reported, which are unaffected by vaccination, possibly due to changes in surface antigens (Fernández, Méndez and Guijarro, 2007).

Little is known about the molecular mechanisms of pathogenesis. Both abilities to form biofilms and to survive for prolonged time in aquatic, nutrient-restricted environments are thought to play a role, as well as an iron-acquisition system (Fernández, Méndez and Guijarro, 2007). A large plamid that is in common to American and French isolates has also been reported (Toranzo, Barja, Colwell and Hetrick, 1983; Guilvout, et al., 1988). This plasmid could be correlated with pathogenicity as the French isolates were from a redmouth disease outbreak, and because it is found in the most pathogenic serovars of Y. ruckeri, but a link with disease has not been established (Lesel, Lesel, Gavini and Vuillaume, 1983; Garcia, Dominguez, Larsen and Pedersen, 1988). Expression of virulence genes is potentially regulated by the amount of available iron and temperature changes, and a decrease in temperature from $28^{\circ} \mathrm{C}$ to $18^{\circ} \mathrm{C}$ might be crucial (Fernández, Méndez and Guijarro, 2007). It was shown that gills could be an important entry point for Y. ruckeri into the host (Tobback, et al., 2009). The bacteria attach to the gills, which carry a large number of blood vessels for respiration. This opens up a direct route for 
dissemination through the host via the blood stream (Tobback, et al., 2009). Y. ruckeri can also persist in macrophages, similar to the human pathogenic Yersinia, as means of protected environment and immune evasion (Ryckaert, et al., 2010).

Y. ruckeri is a very homogeneous, clonal species, in contrast to the human pathogens $Y$. enterocolitica and Y. pseudotuberculosis, which are both considered heterogeneous (Sulakvelidze, 2000). It can be differentiated into different bioserotypes, with the most virulent strains found in biotype 1 0:1 (Fernández, Méndez and Guijarro, 2007).

\subsubsection{Environmental Yersiniae}

Environmental species of the genus Yersinia are found in various environments, such as water and aquatic ecosystems, sewage, wild and domestic animals as well as foods (Sulakvelidze, 2000). They have not been associated with human infections, although some species have been isolated from sick and healthy individuals (Sulakvelidze, 2000). The environmental Yersiniae are also collectively referred to as 'Y. enterocolitica-like' species, as they were speciated from Y. enterocolitica (Sulakvelidze, 2000). They do present individual species although the extent of their distinction may be underestimated (Sulakvelidze, 2000). 14 species have been described. Of these, eight species could be considered classical, established lineages speciated from Y. enterocolitica between 1978 and 1995 (Sulakvelidze, 2000). The other six species have been portrayed from 2005 onwards and are therefore less well characterized (Sprague and Neubauer, 2005; Murros-Kontiainen, et al., 2010a,b; Hurst, et al., 2011; Merhej, et al., 2008; Sprague, Scholz, Amann and Busse, 2008).

The eight species classically recognized as $Y$. enterocolitica-like species are Y. aldovae, Y. bercovieri, Y. frederiksenii, Y. intermedia, Y. kristensenii, Y. mollaretii, Y. rohdei, and Y. ruckeri (Chapter 1.1.3). In contrast to other environmental species, little is known about $Y$. aldovae.

$Y$. frederiksenii is a heterogeneous species consisting of four genomospecies. These show differences in DNA-hybridization, 16S rDNA sequence comparison and when comparing the electrophoretic properties of specific enzymes (Sulakvelidze, 2000; Virdi and Sachdeva, 2005). As they do not differ on the biochemical level, these genomospecies can currently not be distinguished (Sulakvelidze, 2000).

The naming of $Y$. intermedia arises from the fact that it shows common biochemical properties with both $Y$. enterocolitica and $Y$. pseudotuberculosis, and therefore was 
thought to be 'midway' between the two (Sulakvelidze, 2000). Several different biotypes have been described based on differential utilization of Simmons citrate and fermentation of different sugars (Martin, Leclercq, Savin, and Carniel, 2009).

Y. kristensenii was originally designated for trehalose-positive, sucrose-negative strains. This comprised a heterogeneous collection, with some isolates showing a closer genotypic similarity to Y. mollaretii (Sulakvelidze, 2000). These were later speciated into Y. aleksiciae (Sprague and Neubauer, 2005).

Y. mollaretii and Y. bercovieri were originally grouped as biotype $3 \mathrm{~A}$ and $3 \mathrm{~B}$ of Y. enterocolitica. They were then separated from that species based on differences in ecology, DNA hybridization, and antigen patterns (Sulakvelidze, 2000). $Y$. bercovieri is known to be genetically heterogeneous based on experiments distinguishing several ribotyping and pulse-field gel electrophoresis patterns (Sulakvelidze, 2000).

$Y$. rohdei is a homogeneous group of organisms thought to be most closely related to Y. frederiksenii (Sulakvelidze, 2000).

Recently described species are Y. aleksiciae, Y. entomophaga, Y. massiliensis, Y. nurmii, Y. pekkanenii, and Y. similis. Similarly to the classical environmental species, these have been isolated from various sources including water, symptomatic and asymptomatic humans, food products and animals such as reindeer and pigs.

Y. aleksiciae was speciated as atypical isolates from Y. kristensenii (Sprague and Neubauer, 2005). They differ from Y. kristensenii in 16S rDNA sequence and lysinedecarboxylase phenotype.

Both Y. nurmii (Murros-Kontiainen, et al., 2010a) and Y. entomophaga (Hurst, et al., 2011) are newly described species in which the closest relative appears to be Y. ruckeri. This is interesting as Y. ruckeri is thought to be the most distant member of the genus (Bottone, 1997; Sulakvelidze, 2000). Y. entomophaga was isolated from a diseased insect larva (Hurst, et al., 2011), and Y. nurmii was isolated from meat (Murros-Kontiainen, et al., 2010a). It is unknown how these two species relate to each other. Out of 19 biochemical tests with results available for both species, they differ in raffinose and melibiose utilization. Cellobiose and lactose give weak positive reactions for Y. nurmii, whereas Y. entomophaga is clearly positive (Hurst, et al., 2011; Murros-Kontiainen, et al., 2010a).

Y. massiliensis was initially isolated from water (Merhej, et al., 2008), but can be found in food sources as well (Souza, Falcão, and Falcão, 2011). The species is 
thought to be closely related to Y. bercovieri, Y. mollaretii, and Y. frederiksenii and can be distinguished from these in indole, inositol, rhamnose, and citrate utilization patterns (Merhej, et al., 2008; Souza, Falcão, and Falcão, 2011).

Based on commercially available biochemical tests, Y. pekkanenii cannot be distinguished from Y. pseudotuberculosis (Murros-Kontiainen, et al., 2010b). It was shown though that classical virulence determinants are absent, and that these environmental isolates form a unique cluster related to $Y$. mollaretii and $Y$. aldovae (Murros-Kontiainen, et al., 2010b).

Y. similis isolates were also originally attributed to Y.pseudotuberculosis but phenotypic characterization as well as $16 \mathrm{~S}$ rDNA analysis and DNA-DNA hybridization suggested a separate species (Sprague, Scholz, Amann and Busse, 2008).

\subsection{Pathogenicity in the genus Yersinia}

\subsubsection{Relationship of pathogenic Yersiniae}

The pathogenic Yersiniae have a number of characteristics that are thought to be involved in pathogenicity and virulence. Despite the difference in the diseases caused by Y. pestis, Y. pseudotuberculosis, and Y. enterocolitica some of these genes and islands are shared in all pathogenic Yersiniae (Revell and Miller 2001; Carniel, 2002). There is a common theme in the infections caused by pathogenic Yersiniae, in that iron overload of the host can increase pathogenicity, and there is marked tropism in all three species for lymphatic tissues: Y. pestis targets the lymph nodes, Y. pseudotuberculosis the lymphatic chain, and Y. enterocolitica infects the Peyer's patches and lymphoid follicles (Carniel and Mollaret, 1990; Carniel, 2003).

It is thought that $Y$. pestis expresses antiphagocytic, invasive, and toxic activities to cause an acute infection and effective transmission because it is less adapted to environmental survival than the enteropathogenic Y. enterocolitica and Y. pseudotuberculosis, which favour a chronic infection by production of adhesins and invasins (Brubaker, 1991).

A considerable number of genes and regions have been implicated with a role in pathogenesis in Yersinia, yet not all of them present essential genes in the infection process. It is also becoming clear that some pathogenicity markers or islands that were thought to provide survival advantages under host infection conditions are in fact also present in environmental isolates and non-pathogenic BT 1A strains (Grant, 
Bennet-Wood and Robins-Browne, 1998; Chen, et al., 2010). Some genes identified on the basis of essentiality in infection are housekeeping genes rather than specific virulence factors, therefore only genes involved in specific steps of the infection process are reviewed here.

\subsubsection{Plasmid-encoded basis for pathogenicity}

\subsubsection{Shared virulence plasmid pYV}

The Yersinia virulence plasmid, pYV, is common to all pathogenic Yersinia, and varies in size between 40 to 48 kDa (Portnoy, Moseley and Falkow, 1981), equivalent to 60 to $70 \mathrm{~kb}$. Hybridization experiments have indicated gene content variations suggesting the pYV plasmids are a "closely related family" of plasmids (Portnoy, Moseley and Falkow, 1981). In Y. enterocolitica, pYV is only present in high- and low-pathogenic strains, never in BT 1A (Carniel, 2002; Wren, 2003). It is also found in Y.pseudotuberculosis and Y. pestis; in the latter, it is called pCD1 for calcium dependency as expression of proteins encoded for on the plasmid is triggered under low-calcium conditions (Carniel, 2002; Chain, et al., 2004).

Portnoy, Moseley and Falkow (1981) were among the first to link pathogenicity to the presence of pYV and showed that strains cured of the plasmid had reduced lethality in gerbils. The lethal dose of plasmid-cured strains was higher than $10^{6}$ organisms compared to plasmid-bearing strains with a lethal dose of $10^{3}$ organisms. Using the Sereny test it has been shown that infection of guinea pig conjunctiva is dependent on the presence of pYV, as cured strains did not cause an infection (Heesemann, et al., 1983). The lack of $\mathrm{pYV}$ in BT $1 \mathrm{~A}$ is the main reason why it is considered to be non-pathogenic (Carniel, 2002).

The pYV encodes a type III secretion system (T3SS) secreting Yersinia outer proteins (Yops) at $37^{\circ} \mathrm{C}$ in low calcium conditions (Cornelis, 2002; Carniel, 2002). The functionality of this replicon is conserved in all pYVs, although the overall organization may vary (Carniel, 2002). This system as been extensively studied as a model system for type 3 secretion in the past (Portnoy and Falkow, 1981; Portnoy, et al., 1984; Cornelis, et al., 1987; Cornelis, et al., 1998).

The concerted action of Yops leads to prevention of phagocytosis and resistance to the immune response through three steps of adhesion to host cells, injection of 
effectors using the T3SS, and modulation of target cell function (Cornelis, 2002; Carniel, 2002). The chromosomally encoded invasin Inv (1.2.3.1) and plasmid encoded YadA both mediate adhesion (Carniel, 2002). YadA is an outer membrane adhesin only expressed in enteropathogenic Yersiniae due to a frameshift mutation in Y. pestis (Brubaker, 1991; Cornelis, 1994). It is known to be involved in autoagglutination and erythrocyte agglutination as well as collagen and fibronectin binding (Brubaker, 1991; Cornelis, 1994). Contact with the host cell activates expression of structural and effector proteins of the T3SS, which is known as contactdependent secretion (Carniel, 2002; Cornelis, 2002). The apparatus genes are encoded by the ysc (Yersinia secretion) injectisome, whose 27 proteins make up the channel structure and protruding needle (Cornelis, 2002). The Yop effector proteins (YopEHMPT and YpkA) are then transported from the bacterial cell through the needle and injected straight into the target host cell where they have an effect on cellular pathways (Carniel, 2002). YopEHT and YpkA act on the cytoskeleton of the host cell and thus prevent phagocytosis (Cornelis, 1994; Carniel, 2002; Cornelis, 2002). YopP and $\mathrm{YopH}$ down-regulate the immune response by inhibition of transcription activators resulting in decreased secretion of signal molecules (Carniel, 2002; Cornelis, 2002). This also promotes intracellular survival of Yersinia inside macrophages (Cornelis, 2002). The exact nature of action for YopM is not known, but putatively involves influence on gene transcription (Cornelis, 2002).

\subsubsection{Plasmids specific to $Y$. pestis: $\mathrm{pPCP}$ and pMT}

Apart from the virulence plasmid $\mathrm{pYV}, Y$. pestis has two additional unique plasmids that are implicated in pathogenicity; pPCP and pMT (Brubaker, 1991; Wren, 2003). The plasmid PPCP has a size of $\sim 10 \mathrm{~kb}$ and can be referred to as pPla for plasminogen activator, pPst for pesticin, or pPCP1 for pesticin-coagulaseplasminogen activator (Brubaker, 1991; Perry and Fetherston, 1997; Wren, 2003). Pesticin has a limited range of activity, and is restricted to acting on atypical Escherichia coli, Y. pseudotuberculosis serogroup 1A and 1B, and Y. enterocolitica serogroup O:8 (Brubaker, 1991). An essential role in disease has not been established and pesticin might be more important in competing with other bacteria in the same niche (Brubaker, 1991; Carniel, 2002). The plasminogen activator has fibrinolytic activity, and can coagulate rabbit plasma (Brubaker, 1991). This protein is involved in dissemination of $Y$. pestis in the infected host (Brubaker, 1991; Achtman, et al., 1999; Carniel, 2002). 
The pMT plasmid encodes the murine toxin Ymt and has a size of $96 \mathrm{~kb}$. It is also known as pTox or pFra, because it encodes the fraction 1 capsule-like antigen (Brubaker, 1991; Carniel, 2002; Wren, 2003).

The fraction 1 capsule protein is an outer membrane protein that has been postulated to be involved in resistance to macrophage uptake during the initial stage of infection (Brubaker, 1991). As it presents an outwardly displayed antigen, it might be important in the interaction between the bacterium and host cells, especially of the immune response, and has been the target for vaccine development against plague (Carniel, 2002; Eppinger, et al., 2010). Capsule-deficient Y. pestis biovar Angola was shown to lack lethality in the guinea pig model after subcutaneous infection, and was also less virulent in the subcutaneous mouse model but not in the aerosol model (Eppinger, et al., 2010). The exotoxin Ymt is responsible for the death of infected mice possibly due to blocking host cell receptors in the terminal, septicaemic stage of disease resulting in hypotension and vascular collapse (Brubaker, 1991; Hinnebusch, et al., 2002). Ymt might be more essential for colonization of the flea (Parkhill, et al., 2001a; Hinnebusch, et al., 2002). It was shown that Ymt acts as an intracellular phospholipase that is involved in neutralizing agents in the flea midgut, which might otherwise lead to cell lysis (Hinnebusch, et al., 2002; Carniel, 2002). Through being able to persist and proliferate in the flea midgut, $Y$. pestis can then spread and block the proventriculus for effective transmission (Hinnebusch, et al., 2002). Without the protection through $\mathrm{Ymt}$, the bacteria cannot establish colonization of the flea and are cleared (Hinnebusch, et al., 2002).

A chimeric plasmid consisting of one pMT copy and two tandemly repeated copies of pPCP called pMT-PCP has been reported in Y. pestis strain Angola (Eppinger, et al., 2010). It is thought that the presence of an insertion sequence (IS) element enabled the co-integration of the plasmids via recombination (Eppinger, et al., 2010). The effect of this is unclear, but there are potentially dosage-related effects, as the chimeric plasmid is present in higher copy numbers than other plasmids (Eppinger, et al., 2010). There are three copies of the genes encoding the plasminogen activator. Conversely, the genes encoding the capsular antigen are absent on the chimeric plasmid, and it has been shown that this affects virulence following subcutaneous infection in the mouse model but not the aerosol route (Eppinger, et al., 2010). A chimeric plasmid might be an advantage in vertical plasmid progression and might ensure maintenance of all plasmid-encoded virulence factors (Eppinger, et al., 2010). 


\subsubsection{Plasmids specific to Y. pseudotuberculosis: pVM82 and pIB}

Both pVM82 and plB have been isolated from strains of $Y$. pseudotuberculosis that have been implicated in Far East scarlet-like fever (Carniel, 2002; Eppinger, et al., 2007). A region on pVM82 has been linked to immunosuppression and ability to resist phagocytosis (Eppinger, et al., 2007). Both plasmids carry genes encoding a pilus similar to the pilin gene cluster found on the Yersinia adhesion pathogenicity island (YAPI) although of different evolutionary origin (Eppinger, et al., 2007). This three-fold presence of pilin gene clusters might explain the host immune response associated with Far East scarlet-like fever (Eppinger, et al., 2007).

Further plasmid-encoded genes that might potentially be involved in pathogenicity include a type 4 secretion system and a putative phytotoxin on pVM82, and a plasmid-transfer system on pIB (Eppinger, et al., 2007).

\subsubsection{Chromosomal markers of pathogenicity}

Whilst the virulence plasmid shared in the pathogenic Yersiniae undoubtedly plays a major role in infection, studies have shown that its loss in $Y$. enterocolitica does not completely abolish virulence, indicating the presence of essential chromosomally encoded virulence factors (Revell and Miller, 2001).

\subsubsection{Invasion and adhesion proteins: Inv and Ail}

Both Inv and Ail have been described as important proteins for promoting the invasion of host cells (Miller and Falkow, 1988; Miller, Farmer, Hill and Falkow, 1989). The invasin protein Inv can confer an invasive phenotype on $E$. coli cells if cloned from the high-pathogenic Y. enterocolitica BT 1B (Miller and Falkow, 1988). Invasin mutants were also shown to be defective in colonization and dissemination in a mouse infection model (Pepe and Miller, 1993; Revell and Miller, 2001). The attachment and invasion locus ail was found to change invasive behaviour, but it appeared to be dependent on the cell type used suggesting a different mode of action of the two invasins (Miller and Falkow, 1988). Ail was shown to be involved not only in invasion, but also in adhesion to cells and in serum resistance (Pierson and Falkow, 1993). It is thought that Inv and Ail act in concert, with Inv important for priming of bacteria at lower temperatures, and Ail being responsible for acting once the infection process has started inside the host (Pepe and Miller, 1993). 
Homologs of the invasin gene inv have been described in all Yersinia sp., but functionality has only been demonstrated in enteropathogenic Yersiniae (Miller and Falkow, 1988; Miller, Farmer, Hill and Falkow, 1989; Pierson and Falkow, 1990; Grant, Bennet-Wood and Robins-Browne, 1998). It is thought to be cryptic in Y. pestis due to disruption (Brubaker, 1991; Revell and Miller, 2001). Functionality in BT 1A could not be established, as it is neither expressed in the non-pathogenic BT, nor does it confer an invasive phenotype on E. coli, although no inactivating mutations were detected (Pierson and Falkow, 1990). The global regulator, rovA regulates the expression of invasion, and modulates the ability to infect and colonize deeper tissue layers in the mouse infection model (Revell and Miller, 2001).

Distribution of the attachment and invasion locus protein Ail is very specific to pathogenic isolates, namely Y.pseudotuberculosis, Y.pestis and the Y. enterocolitica biotypes 1B, and 2 - 5 (Miller, Farmer, Hill and Falkow, 1989; Revell and Miller, 2001). Artificial overexpression of Ail in non-pathogenic BTs of $Y$. enterocolitica leads to transcription and localization to the cell surface, but fails to induce invasive behaviour, possibly due to differences in the cell surface structure of high- and non-pathogenic BTs (Pierson and Falkow, 1993).

\subsubsection{Yersinia Adhesion Pathogenicity Island (YAPI)}

The Yersinia adhesion pathogenicity island YAPI shows varied distribution amongst Y. pseudotuberculosis and Y. enterocolitica BT 1B strains. In Y. pseudotuberculosis it has been found in the strain causing Far East scarlet-like fever and a serotype I strain (Eppinger, et al., 2007). Only two BT 1B strains of $Y$. enterocolitica have been described containing YAPI suggesting independent acquisition events of this mobile genetic element (Thomson, et al., 2006).

The conserved part of YAPI encodes a polycistronic pilin cluster that is involved in adhesion of bacteria to the respiratory epithelium in a mouse infection model (Collyn, et al., 2004; Eppinger, et al., 2007). The variable part of YAPI in Y. pseudotuberculosis contains metabolic genes, a restriction-modification system, IS elements and phage genes, which do not appear to contribute to pathogenicity (Collyn, et al., 2004). In Y. enterocolitica, the island is smaller than in Y. pseudotuberculosis, and apart from the shared pilin cluster encodes for a possible hemolysin, toxin/antitoxin system, and an arsenic resistance operon (Thomson, et al., 2006). 
It is thought that YAPI might have derived from the Salmonella Typhi island SPI-7 due to extensive homology and is also found in other Enterobacteriaceae, such as Photorhabdus luminescens (Collyn, et al., 2004; Thomson, et al., 2006; Eppinger, et al., 2007).

\subsubsection{High-pathogenicity island (HPI) - Pigmentation / hemin storage locus}

The high-pathogenicity island ( $\mathrm{HPI})$ has been described in isolates of Y. enterocolitica, Y.pseudotuberculosis, and Y.pestis (Carniel, Guilvout and Prentice, 1996; Carniel, 2002). In Y. enterocolitica, it is only present in the highpathogenic BT 1B. Its presence is variable in Y. pseudotuberculosis isolates (Carniel, Guilvout and Prentice, 1996; Bach, et al., 1999) and was notably absent in the representative Y. pseudotuberculosis clone held responsible for Far East scarlet-like fever (Eppinger, et al., 2007). The HPI has been shown to play a major role in systemic dissemination during infection (Eppinger, et al., 2007).

There are variations in gene content, and the HPI could be considered as two joined segments (Bach, et al., 1999; Carniel, 2002). The total island in Y. pestis occupies $\sim 100 \mathrm{~kb}$ and is referred to as pigmentation or hemin storage locus (hms / pgm; Carniel, Guilvout and Prentice, 1996). This locus has been described in Y. pestis as the ability to absorb exogenous hemin and form pigmented colonies (Brubaker, 1991; Carniel, Guilvout and Prentice, 1996). hms encodes an acquisition and storage system for the essential nutrient iron, which is limited in the host (Brubaker, 1991; Carniel, 2002). Also located on the pigmentation locus are fyuA-psn, which encodes the double protein FyuA acting as the siderophore yersiniabactin and bacteriocin pesticin, and irp1-5, iron-regulated high-molecular-weight proteins of which protein 2 appears to have special importance (Carniel, Guilvout and Prentice, 1996; Bach, et al., 1999). In experimental studies, pigmentation mutants were shown to have a marked decrease in virulence (Brubaker, 1991; Carniel, Guilvout and Prentice, 1996). The locus is required in flea colonization as well, as it blocks the flea's midgut leading to regurgitation of $Y$. pestis upon feeding on a host and thus ensuring efficient transmission (Parkhill, et al., 2001a).

The HPI is significantly shorter in Y. enterocolitica BT 1B than in Y. pestis, and only stretches over 45 kb (Carniel, Guilvout and Prentice, 1996). The only genes conserved with the $Y$. pestis locus are the genes involved in iron capture: fyuA and irp1-5 (Carniel, Guilvout and Prentice, 1996; Bach, et al., 1999). Instead of the pigmentation locus, the rest of the HPI is occupied by IS and repeat elements in 
Y. enterocolitica BT 1B (Carniel, Guilvout and Prentice, 1996). It is thought that this absence of iron-chelating proteins in the low-pathogenic Y. enterocolitica BTs 2 - 5 contributes to their lowered virulence potential and less invasive disease outcome (Carniel, Guilvout and Prentice, 1996).

The HPI in Y. pseudotuberculosis has the same genetic content as Y. pestis, and differs only in the terminal sites (Bach, et al., 1999). The presence of repeats and functional integrases in Y.pseudotuberculosis and Y.pestis leads to frequent deletion of the HPI, whereas due to a mutated integrase the region is highly stable in Y. enterocolitica (Bach, et al., 1999). Deletions of the HPI in Y. enterocolitica are possible but infrequent and comprise a much larger genomic region, with additional loss of phenotypes such as motility and growth defects (Bach, et al., 1999). The HPI has been described in other Enterobacteriaceae as well, including Klebsiella, Citrobacter, and E. coli, supporting the mobile nature of this locus (Bach, et al., 1999; Carniel, 2002).

\subsubsection{Yersinia pseudotuberculosis superantigen YPM}

The Y. pseudotuberculosis-derived mitogen (YPM) is a superantigenic exotoxin that has been linked with Y. pseudotuberculosis infections presenting as Far East scarletlike fever (Eppinger, et al., 2007). Similar to superantigens found in Gram-positive bacteria such as Staphylococci or Streptococci, the Y.pseudotuberculosis superantigen can activate the host immune system in an excessive, uncontrolled manner. The similar clinical manifestation of Far East scarlet-like fever and scarlet fever caused by Streptococcus pyogenes is probably due to an analogous action of the superantigens (Eppinger, et al., 2007). YPM can present in three different variants encoded by $y p m A, y p m B$, and $y p m C$. Whilst ypmB and ypmC have been correlated with environmental and animal isolates, ypmA is established in clinical isolates (Eppinger, et al., 2007). It has been suggested that the last common ancestor of $Y$. pseudotuberculosis bore an ancestral ypm gene, which subsequently diverged and has been lost in individual lineages of Y. pseudotuberculosis as it is contained in an unstable locus in the genome (Ch'ng, et al., 2011). It has been suggested that YPM+ HPI- strains cause Far East scarlet fever, whereas YPM- HPI+ strains are linked with enteric disease (Fukushima, et al., 2001; Ch'ng, et al., 2011; Eppinger, et al., 2007). 


\subsubsection{Ysa type 3 secretion system}

Y. enterocolitica BT 1B strains possess a second T3SS called the Ysa T3SS for Yersinia secretion apparatus that is absent from non- and low-pathogenic BTs and other Yersinia species and that is thought to be involved in virulence (Haller, Carlson, Pederson and Pierson, 2000; Foultier, et al., 2002). At least eight Yersinia secreted proteins, Ysps, depend on secretion by the Ysa T3SS (Haller, Carlson, Pederson and Pierson, 2000). It was shown that secretion by this system is activated at $28^{\circ} \mathrm{C}$ and high salt conditions, and that this secretion is independent from the plasmid-encoded Yop secretion that is triggered at $37^{\circ} \mathrm{C}$ and low calcium conditions (Haller, Carlson, Pederson and Pierson, 2000). This has prompted speculation that the physiological role of this system is early on in the infection process and helps to prime the bacterial cells (Haller, Carlson, Pederson and Pierson, 2000; Foultier, et al., 2002). A study was undertaken to determine the relatedness of this new, chromosomal T3SS to the established plasmid-encoded T3SS (Foultier, et al., 2002). It was shown that the two systems are very distinct, with the Ysa T3SS closely related to the Shigella Mix/Spatype system and the Salmonella SPI-1 T3SS, and the Yop T3SS related to the Psc T3SS in Pseudomonas aeruginosa and Bsc in Bordetella (Foultier, et al., 2002). It appears that there is a third, different T3SS present in Y.pseudotuberculosis and Y. pestis that is similar to the Salmonella SPI-2 T3SS, but so far no in-depth study into this system has been undertaken (Foultier, et al., 2002).

\subsubsection{Further chromosomally encoded virulence factors}

Other genes or operons have been implicated in a role in disease (Revell and Miller, 2001). Amongst these is yst encoding the heat-stable enterotoxin. It is thought that its action induces diarrhoea, specifically in infants, similar to the heat-stable toxin of E. coli (Cornelis, 1994; Revell and Miller, 2001). Some non-pathogenic BT 1A strain may have homologous sequences to yst as indicated by hybridization experiments (Grant, Bennet-Wood and Robins-Browne, 1998), but their expression and functionality need further study.

The myf operon encodes fimbriae similar to pili of E. coli, one of them being the $\mathrm{pH} 6$ antigen (Cornelis, 1994; Revell and Miller, 2001). The operon is present in all of the pathogenic Yersiniae, and mediates binding to host cells (Revell and Miller, 2001). Similarly to $y s t$, myf-homologous sequences might be present in the non-pathogenic BT (Grant, Bennet-Wood and Robins-Browne, 1998). 
Using in vivo expression technology, IVET (Mahan, Slauch and Mekalanos, 1993), a number of genes were identified that are expressed under stress conditions but not under laboratory conditions. This led to the characterization of host responsive elements, hre (Young and Miller, 1997), regulator of systemic colonization RscR (Nelson, Young and Miller, 2001), and systemic invasion factors, sif, all of which have been attributed an undefined function in the mechanism of disease (Revell and Miller, 2001).

It is possible that a combination of different virulence factors is required for full infectivity and that the different factors contribute to various stages of disease, as individual mutants usually fail to completely attenuate the strain (Revell and Miller, 2001).

\subsection{Evolution of the genus Yersinia}

\subsubsection{Differentiation and speciation in Yersinia}

Correct identification of pathogenic Yersiniae is crucial for disease management. In the past, phenotypic tests and serotyping have been used, with the limitations of strains presenting with atypical biotyping reactions and with serotyping only possible at reference laboratories (Virdi and Sachdeva, 2005). Early on, autoagglutination, calcium dependency, uptake of crystal violet and Congo red, as well as serum resistance have been used as indirect indicators of virulence (Pedersen, Winblad and Bitsch, 1979; Schiemann and Devenisch, 1982; Robins-Browne, et al., 1989). Drawbacks of these techniques are atypical and contradictory results for a number of isolates (Robins-Browne, et al. 1989). Commercially available phenotypic tests were also lacking some of those sugars and tests that more reliably differentiate Yersiniae, leading to decreased sensitivity with respect to identification of environmental species (Neubauer, et al., 1998).

These disadvantages prompted investigation into the use of DNA hybridization using probes for potential virulence genes for the detection of pathogenic isolates (RobinsBrowne, et al., 1989). Probes for the invasin Inv derived from Y. pseudotuberculosis only detected Y.pseudotuberculosis and Y.pestis isolates, but probing with Y. enterocolitica Inv gave positive results in environmental as well as pathogenic species of Yersinia (Robins-Browne, et al., 1989). In contrast, probes for Ail proved to be highly specific, and depending on design to include adjacent DNA regions can 
either detect $Y$. enterocolitica BT 1B or all pathogenic Y. enterocolitica biotypes (Robins-Browne, et al., 1989). Probes designed for detection of the virulence plasmid pYV detected plasmid-bearing strain of Y.pseudotuberculosis, Y.pestis, and Y. enterocolitica (Robins-Browne, et al., 1989), but it has to be considered that the plasmid can be easily lost in laboratory culture and thus could have an influence on virulence assessment of isolates. The detection of virulence-associated genes was further developed into assays using polymerase chain reaction (PCR) to amplify parts of the virulence plasmid or chromosomal fragments including inv, ail, or the enterotoxin yst (e.g. Nakajima, et al., 1992; Ibrahim, Liesack and Stackebrandt, 1992).

New methods such as multilocus enzyme electrophoresis (MLEE), restriction endonuclease analysis of chromosomal and plasmid DNA (REAC, REAP), ribotyping, pulse-field gel electrophoresis (PFGE), and typing based on variable number of tandem repeats (VNTR) have been applied to study heterogeneity of $Y$. enterocolitica and 'Y. enterocolitica-like' species (reviewed in Virdi and Sachdeva, 2005; Souza, Pitondo-Silva, Falcão and Falcão, 2010). It was found that Y. enterocolitica has three consistent lineages of high-, low-, and non-pathogenic biotypes (Virdi and Sachdeva, 2005). For some methods, limited data is available on the environmental species, precluding any deduction of the evolutionary relationship of $Y$. enterocolitica and the environmental species (Virdi and Sachdeva, 2005). Further more, these methods are more suitable for short-term study of isolates during an epidemic outbreak, but are less appropriate to determine the historic evolutionary relationships between the Yersinia species (Kotetishvili, et al., 2005). Nucleotide analysis of 16S rDNA gene or housekeeping genes in multi-locus sequence typing (MLST) is considered a more unbiased approach for speciation (Kotetishvili, et al., 2005; Souza, Pitondo-Silva, Falcão and Falcão, 2010).

\subsubsection{S rDNA analysis and MLST scheme}

Initial phylogenetic studies in the genus Yersinia focussed on the 16S rDNA gene (Ibrahim, et al., 1993). The 16S rDNA gene is present in all bacteria and thus used as a gold standard to establish the relationship between different genera (Janda and Abbott, 2007). For Yersinia, five different lineages were identified (Ibrahim, et al., 1993; Ibrahim, et al., 1997). Y. enterocolitica forms a heterogeneous cluster with the possibility of splitting them into two distinct subclusters of American and European isolates. Y. pseudotuberculosis and Y.pestis together with $Y$. frederiksenii form a 
separate cluster. Y. rohdei and Y. ruckeri each form independent clusters, and the environmental species Y. mollaretii, Y. intermedia, Y. bercovieri, Y. kristensenii, and Y. aldovae form another heterogeneous cluster (Ibrahim, et al., 1993; Ibrahim, et al., 1997). Later, a sixth lineage was discovered which includes atypical Yersinia isolates, which have a different $16 \mathrm{~S}$ signature (Ibrahim, et al., 1997). Biochemically, these belong to $Y$. frederiksenii and have been designated as genomospecies 2 (Ibrahim, et al., 1997). Generally, the sequence similarities vary between 96.9 $99.8 \%$. This is considered too high to infer evolutionary relationships between different Yersinia lineages and a distinction between species is less reliable (Ibrahim, et al., 1993; Janda and Abbott, 2007). To improve speciation, 16S rDNA analysis in conjunction with DNA hybridization and biochemical characteristics was suggested (Ibrahim, et al., 1993; Neubauer, Hensel, Aleksic and Meyer, 2000).

Upon reconstruction of a phylogenetic tree based on 16S rDNA sequences only, it was found that $Y$. ruckeri does not present an outlier as opposed to relationships based on other housekeeping genes (Ibrahim, et al., 1993; Ibrahim, et al., 1997; Souza, Pitondo-Silva, Falcão and Falcão, 2010). This might be due to an exchange of $16 \mathrm{~S}$ rDNA sequences as has been noted in other bacteria before (Asai, Zaporojets, Squires and Squires, 1999; Schouls, Schot and Jacobs, 2003; Souza, Pitondo-Silva, Falcão and Falcão, 2010), and this recombinatorial potential might preclude this gene from use in establishing evolutionary connections.

Recent exploration of use of the 16S rDNA sequence has lead to the proposition that Y. enterocolitica should be grouped into two subspecies (Neubauer, Hensel, Aleksic and Meyer, 2000; Neubauer, et al., 2000). DNA hybridization experiments indicate that there are possibly three groups within $Y$. enterocolitica corresponding to highpathogenic 'New-World' strain, low-pathogenic 'Old-World' strains, and nonpathogenic 'Old-World' strains (Neubauer, et al., 2000). Studies on the 165 rDNA gene have found regions to distinguish between 'American' BT $1 \mathrm{~B}$ strains and 'European' BT 1A, 2 - 5 strains (Ibrahim, et al., 1993; Ibrahim, et al., 1997; Neubauer, Hensel, Aleksic and Meyer, 2000; Kotetishvili, et al., 2005). The comparison of 1367 bases of the $16 \mathrm{~S}$ genes showed a difference in 12 nucleotides between isolates from America and Europe, which forms the grounds for establishing two subspecies: the 'New World' BT 1B designated as Y. enterocolitica subsp. enterocolitica and the 'Old World' BTs 1A, $2-5$ belonging to $Y$. enterocolitica subsp. 
palearctica (Neubauer, Hensel, Aleksic and Meyer, 2000; Neubauer, et al., 2000; Neubauer and Sprague, 2003).

An MLST scheme has been attempted using the 16S rDNA sequence as well as the four housekeeping genes $g \ln A, g y r B$, $\operatorname{rec} A$, and hsp60 (Kotetishvili, et al., 2005). Only the 11 classically described Yersinia species were included, as the new species were proposed after publishing of the scheme. Y. pseudotuberculosis and Y. pestis proved inseparable by the gene fragments investigated, indicating their close genetic relationship that has been noted before (Achtman, et al., 1999; Kotetishvili, et al., 2005). It was found that Y. aldovae, Y. bercovieri, Y. intermedia, Y. rohdei, and $Y$. ruckeri as well as $Y$. pestis and $Y$. pseudotuberculosis are homogeneous species, whereas the other four species $Y$. enterocolitica, Y. kristensenii, Y. frederiksenii, and Y. mollaretii are heterogeneous (Kotetishvili, et al., 2005). The 16S rDNA sequences had less discriminatory power than the proposed MLST scheme, and the biochemical species definition was more in agreement with the MLST scheme than 16S rDNA gene (Kotetishvili, et al., 2005), which could indicate recombination amongst $16 \mathrm{~S}$ genes and thus masking of the evolutionary signal. Some strains were found to be distinct from their respective species based both on MLST and 16S rDNA analysis, and would warrant further investigation as potentially new species within the genus Yersinia (Kotetishvili, et al., 2005). Y. frederiksenii is known to be a heterogeneous species and the atypical isolates might be related to the newly proposed Y. massiliensis (Merhej, et al., 2008; Souza, Falcão, and Falcão, 2011). Atypical Y. kristensenii were later speciated into Y. aleksiciae (Sprague and Neubauer, 2005).

\subsubsection{Current model of evolution}

The current model for evolution of the genus Yersinia is presented in Figure 1.2 (Carniel, 2002; Carniel, 2003; Wren, 2003).

It is thought that the pathogenic Yersiniae have evolved from the environmental species following the acquisition of the virulence plasmid pYV (Carniel, 2002; Wren, 2003). Y. enterocolitica and Y. pseudotuberculosis then developed independently. This split is thought to have occurred $42-187$ million years ago, similar to the distance of 140 million years for the last common ancestor of Escherichia coli and Salmonella enterica (Achtman, et al., 1999). Y. enterocolitica is signified by the acquisition of the heat-stable toxin Yst. It then splits into three different lineages of non-, high-, and low-pathogenic isolates. The non-pathogenic BT 1A loses pYV to 
once again become a predominantly environmental lineage. The New World, highpathogenic BT acquired several pathogenicity islands, including HPI, as well as unique type 2 and 3 secretion systems, explaining the increase in virulence potential. The low-pathogenic lineage includes BTs $2-5$.

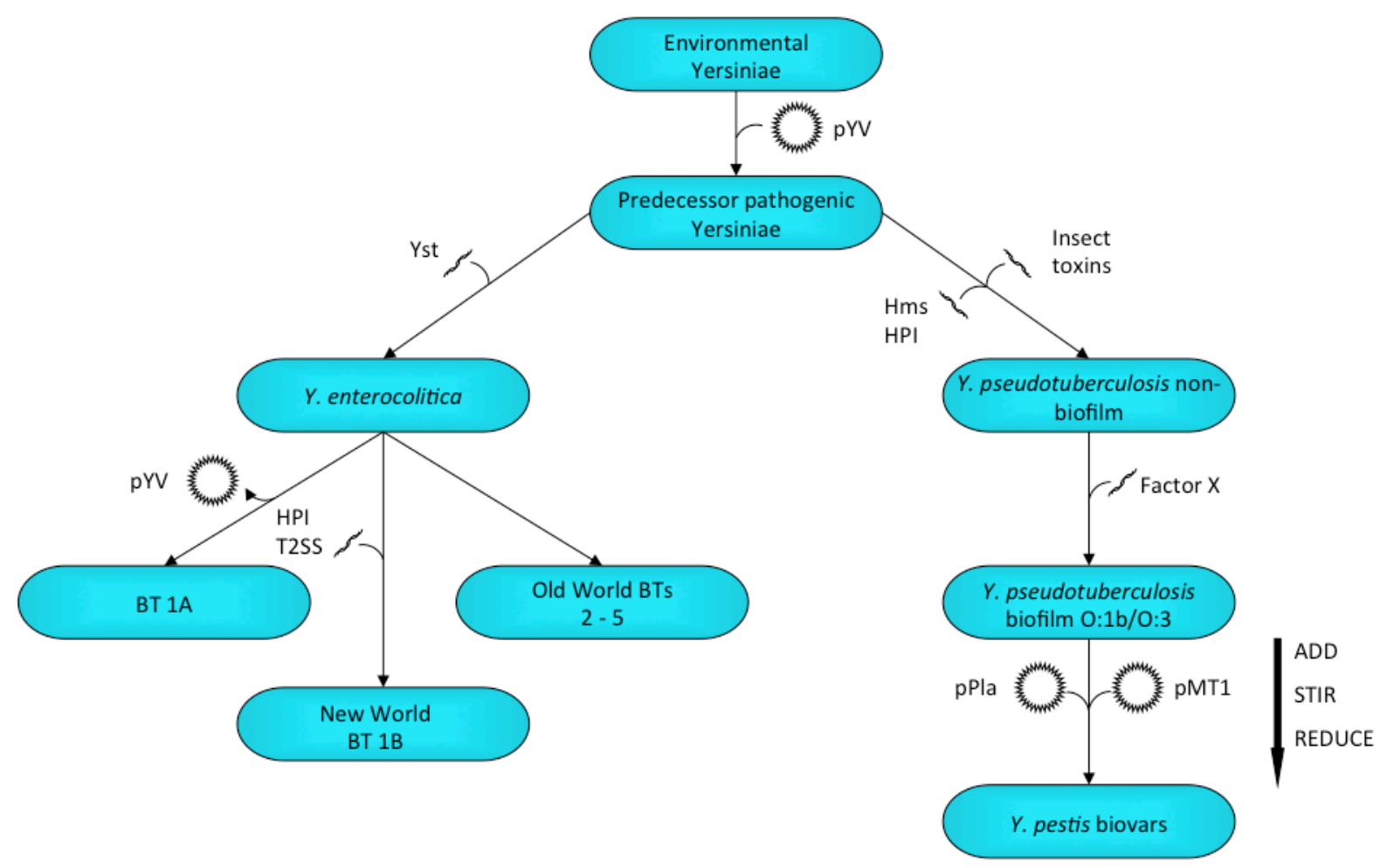

Figure 1.2: Evolution of the genus Yersinia [adapted from Carniel, 2002; Wren, 2003].

Y. pseudotuberculosis also acquired pathogenicity islands. Similar to $Y$. enterocolitica BT 1B, it obtained the HPI, but it also has the hemin storage locus, and the insect toxin encoded on the tcPAl. A subset of $Y$. pseudotuberculosis then evolves the ability of biofilm formation, and the acquisition of the plasmids PPCP and PMT gives rise to the $Y$. pestis lineages. The last common ancestor of $Y$. pseudotuberculosis and $Y$. pestis is thought to have existed $0.4-1.9$ million years ago (Achtman, et al., 1999). The evolution of the $Y$. pestis biovars is thought to have occurred within the last 1,500 to 20,000 years, through a number of bottlenecks (Achtman, et al., 1999).

For the evolution of $Y$. pestis three principles have been identified: add DNA, stir and reduce (Parkhill, et al., 2001a; Wren, 2003; Chain, et al., 2004). The acquisition of large genomic islands, such as the hemin storage locus, and additional plasmids pPCP/pPla and pMT/pFra has enabled the colonization of new niches (Wren, 2003). It is thought that the acquisition of the plasmid pMT occurred first, possibly donated 
by Salmonella, which was important for colonization of and transmission via fleas (Achtman, et al., 1999; Carniel, 2003). Both chromosomal and plasmid factors are required for full virulence potential, acting together to enable $Y$. pestis to colonize the flea and be transmitted by it, and are also needed to avoid the mammalian immune system, and to disseminate in the mammalian host (Carniel, 2003; Wren, 2003).

Compared to $Y$. pseudotuberculosis, the $Y$. pestis genome shows an increase in the number of insertion sequence (IS) elements that are present (Parkhill, et al., 2001a; Deng, et al., 2002; Wren, 2003; Chain, et al., 2004; Eppinger, et al., 2007; Eppinger, et al., 2010). IS elements are DNA sections encoding a transposase flanked by perfect inverted repeats. The transposase can mediate the transposition of the element to a different genomic location. Recombination between IS elements have led to wide-ranged genome rearrangements in Y. pestis, which are still ongoing in culture in vivo (Parkhill, et al., 2001a; Deng, et al., 2002; Chain, et al., 2004; Eppinger, et al., 2010). IS elements have inserted into the coding sequences of other genes, thus disrupting the expression of functional proteins and promoting isolatespecific polymorphisms (Parkhill, et al., 2001a; Chain, et al., 2004; Eppinger, et al., 2010).

The loss of function plays a major part in the evolution of $Y$. pestis, and may be even more important than gene acquisition (Chain, et al., 2004). Some of the genomic islands identified in Y.pestis have orthologs in Y.pseudotuberculosis and thus precede the split of these two lineages, but there is evidence of gene decay in Y. pestis (Parkhill, et al., 2001a; Chain, et al., 2004). For instance, some proteins encoded in the toxin complex pathogenicity island (tcPAl) responsible for insect toxicity have been inactivated in Y. pestis (Parkhill, et al., 2001a). Instead of killing the flea, it might be more important for $Y$. pestis to establish persistence for transmission (Achtman, et al., 1999; Parkhill, et al., 2001a; Wren, 2003). Later studies however showed that the insecticidal toxins are not mutated in other $Y$. pestis biovars, leaving in question the exact role of functionality or non-functionality of the tcPAl in survival in the flea (Chain, et al., 2004). In comparing Y. pestis and Y.pseudotuberculosis it was found that up to $13 \%$ of the $Y$. pestis genome is inactivated (Chain, et al., 2004). These genes account for a number of functions relating to environmental and enteric survival that have become redundant in a hostadapted life-style and include metabolic pathways, motility and lipopolysaccharides (Brubaker, 1991; Achtman, et al., 1999; Parkhill, et al., 2001a; Chain, et al., 2004). This way, Y. pestis has now restricted itself to be reliant on the host for the provision 
of nutrients (Brubaker, 1991; Achtman, et al., 1999). It is also evident that Y. pseudotuberculosis possessed a number of colonization and virulence factors that primed Y. pestis in establishing its pathogenic potential (Parkhill, et al., 2001a).

Recently the grouping scheme for $Y$. pestis has been revised based on genetic relatedness rather than biochemical differences (Achtman, et al., 2004; Morelli, et al., 2010). The high diversity of strains can be a sign of geographical source, indicating Y. pestis originated in Asia (Achtman, et al., 2004; Morelli, et al., 2010). From $Y$. pseudotuberculosis, the evolutionary route develops into a branch 0 , from which biovar Pestoides (0.PE) branches off about 2,600 years ago (Morelli, et al., 2010), confirming the ancestral status of the Pestoides group (Eppinger, et al., 2010). After further evolution along branch 0 , there is a split into branch 1 and branch 2 about 700 years ago (Achtman, et al., 2004; Morelli, et al., 2010). Branch 1 contains isolates of the most recent pandemic sweep of plague, and covers isolates of biovar designation Antiqua and Orientalis, hence 1.ANT and 1.ORI. It could be shown that this clone arose $>200$ years ago, and it subsequently spread out of China and around the world distributed by plague ships (Morelli, et al., 2010). Single introductory events into North America and Madagascar lead to local clonal evolution (Morelli, et al., 2010). Branch 2 of the phylogenetic tree in Y. pestis is evolutionary older than branch 1 (>500 years ago) and is associated with biovars Medievalis and Antiqua (2.MED, 2.ANT; Achtman, et al., 2004; Morelli, et al., 2010). The distribution of clones and their accumulating genomic differences implies that $Y$. pestis was spread along the silk trade route in medieval times (Morelli, et al., 2010). Isolates of biovar Antiqua are found in both branches 1 and 2, indicating that the genomic differences do not relate to phenotypic differences, and that the phenotype possibly arises from independent mutations (Achtman, et al., 2004).

\subsection{Next-generation sequencing techniques}

Recent advances in our knowledge of Yersinia evolution such as tracing plague along the silk trade route (Morelli, et al., 2010) are due to rapid advances in whole genome sequencing capacities. Sequencing of bacterial genomes provides a powerful tool to investigate the complete genetic makeup of a strain and allows for identification of regions unique to particular organisms by comparison of strains against each other. Until recently, sequencing was carried out using automated 
Sanger or chain-termination sequencing (Mardis, 2008a; Ansorge, 2009; Metzker, 2010). As this technique is costly, it was only applied to high-interest representative species of model organisms and pathogens, available at larger genome sequencing centres such as the Wellcome Trust Sanger Institute (WTSI) (Hall, 2007; Mardis, 2008a).

Next-generation sequencing techniques have been available since 2004 (Mardis, 2008a), and have found widespread applications. The term next-generation sequencing is generally used to describe techniques that allow for massive parallel sequencing approaches and circumvent the need for cloning in Escherichia coli. This gives the opportunity to sequence larger strain collections in shorter periods of time compared to traditional Sanger sequencing (Mardis, 2008a; Ansorge, 2009; Metzker, 2010). The different techniques have different strengths and weaknesses, making them more suitable for some scientific questions than others. In the following, the main sequencing techniques used in this study will be described and compared.

\subsubsection{Capillary sequencing}

The traditional sequencing technique is based on the chain termination method published by Sanger, Nicklen and Coulson (1977). DNA is isolated and fragmented into pieces of known size (Figure 1.3). These fragments are then cloned into a plasmid containing universal sequencing primers, thus creating a library of fragments (Mardis, 2008b; Shendure and Ji, 2008). The library is used to transform the vector into E. coli, using the bacterial host for amplification of the vector. Individual clones that have arisen from a single transformation event are then picked and the DNA is isolated for sequencing. The sequencing reaction occurs in several cycles by supplying it with two types of nucleotides: normal deoxynucleotides (dNTPs) that can be incorporated into the growing chain as usual, and di-deoxynucleotides (ddNTPs) which have a modified 3' end (Sanger, 1988). The integration of a ddNTP leads to the termination of the chain, as another base cannot be added due to the blocked 3' end, hence this method is known as chain-termination sequencing. Additionally, the ddNTPs are labelled with different fluorophores corresponding to the different bases. As the incorporation of ddNTPs is competitive with the dNTPs, the resulting chains are of different lengths with the terminal base marked by the fluorescence-labelled ddNTP (Shendure and Ji, 2008). 

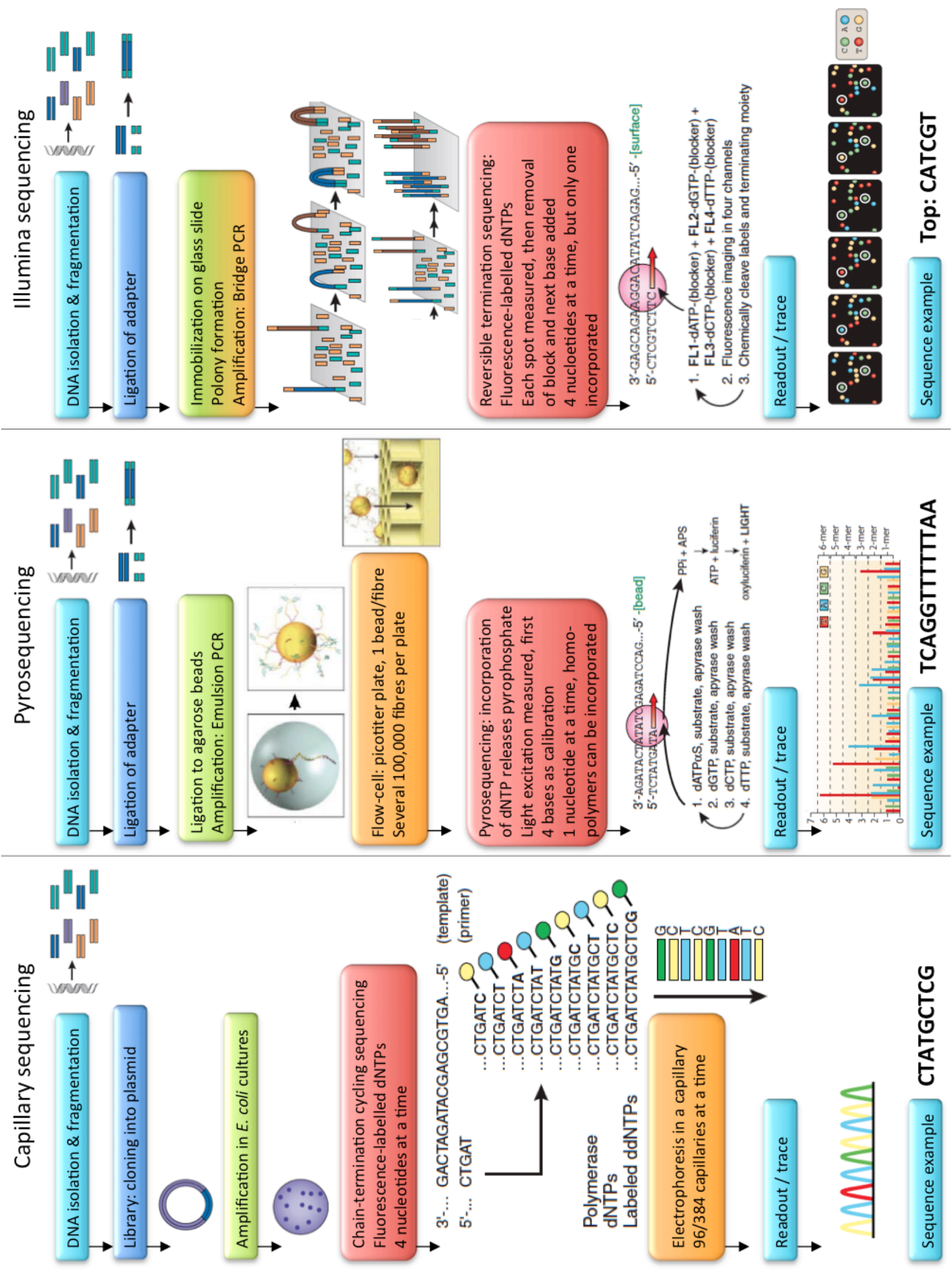

Figure 1.3: Workflow of capillary, pyro- and Illumina sequencing [adapted from Shendure and Ji, 2008; Ansorge, 2009; Meztker, 2010]. 
For visualization of the different chains, separation is carried out in a gel electrophoresis step. In early sequencing, this meant running long gels and photographing them (Sanger, Nicklen and Coulson, 1977). Smaller fragments run faster than longer fragments, and the progression through the gel is proportional to the length of the molecule. A read-out of the 5' to 3' sequence is possible starting from the bottom upward. In more recent times polymer-coated capillaries are used which allow the elution of the chains according to length with high-resolution (Hall, 2007; Shendure and Ji, 2008). The chains are excited with a laser and the fluorophore that terminated the chain can be read out.

Capillary electrophoresis allows for parallelization of 96 or 384 independent capillaries. This limitation is the main drawback of Sanger sequencing, as only a specific number of sequencing reactions can be observed in parallel (Hall, 2007; Shendure and Ji, 2008; Ansorge, 2009).

\subsubsection{Pyrosequencing}

Pyrosequencing was the first commercially available platform introduced in 2004 (Margulies, et al., 2005; Mardis, 2008a). The associated platform is the $454 \mathrm{FLX}$ (Titanium) produced by Roche.

DNA is isolated and fragmented, similar to the traditional sequencing technique (Figure 1.3). Instead of cloning and transformation into E. coli though, the DNA is ligated to specific adapters. These adapters have complimentary sequences located on the surface of agarose beads, so that the adapter-ligated DNA fragments can attach to the beads (Metzker, 2010). A 1:1 ratio of fragments to beads ensures that only one DNA fragment is attached per bead (Margulies, et al., 2005; Mardis, 2008b). The beads are then added to a water:oil mixture containing PCR reagents. Vigorous vortexing results in small micelles, which are beads in an aqueous phase containing PCR reagents and which are surrounded by oil. Universal primer binding sites incorporated in the adapter sequences allow for clonal amplification in vitro of the attached fragments resulting in approximately one million copies per bead (Mardis, 2008b; Metzker, 2010). This has been termed emulsion PCR (Margulies, et al., 2005). By this means a single-stranded fragment library is created (Mardis, 2008b).

In the next step, the beads are added to a flow-cell. This is a picotiter plate consisting of several hundred thousand fibres per plate. The dimensions of the fibre only allow for one bead to be associated with a well in each fibre, thus immobilizing the 
fragments on the plate in a specific position for monitoring of sequencing progress (Margulies, et al., 2005; Mardis, 2008a; Shendure and Ji, 2008; Ansorge, 2009).

The underlying principle for pyrosequencing is that the incorporation of dNTP releases pyrophosphate. This in turn produces energy, which can be used to break down luciferin, resulting in light emittance (Ansorge, 2009). The primer sequence is located within the adapter, and the first four bases that are sequenced correspond to the end of the adapter. As the adapter sequence is fixed, these four bases are always the same and are used for calibration of the detector for signal strength (Mardis, 2008a). Each nucleotide is added separately, and the number of nucleotides incorporated is proportional to the emitted light (Mardis, 2008a,b; Shendure and Ji, 2008). This poses a problem in regions where there are multiples of the same nucleotide, called homopolymeric runs (Mardis, 2008a; Ansorge, 2009). The detector can be saturated, and a differentiation between signal intensities is complicated. An additional or missing nucleotide within a coding sequence will show up as a frameshift in the sequence, implying that the gene would not be encoding a functional protein. This insertion/deletion error is the main inaccuracy in pyrosequencing (Mardis, 2008a; Metzker, 2010). Substitution errors in which the wrong base is incorporated are rare as nucleotides are added individually (Mardis, 2008a,b).

Pyrosequencing produces 250 bp reads on average, although they can be longer (Metzker, 2010).

A way of improving assemblies and counteracting the assembly of a large number of small contigs is paired-end sequencing. The initial fragmentation into $\sim 3 \mathrm{~kb}$ molecules remains unchanged, but before the addition of adapters additional steps are introduced (Figure 1.4).

The ends of the fragments are biotinylated so that circularization is possible. Through this circularization the ends of the $3 \mathrm{~kb}$ fragment come in close proximity to each other and are linked by the biotinylated fragment. The circularized molecules are fragmented into a few hundred base pairs and fragments with the linker can be identified using streptavidin. The adapters are then ligated to the ends of the fragments and are subject to emulsion PCR (454 Life Sciences, 2008). 


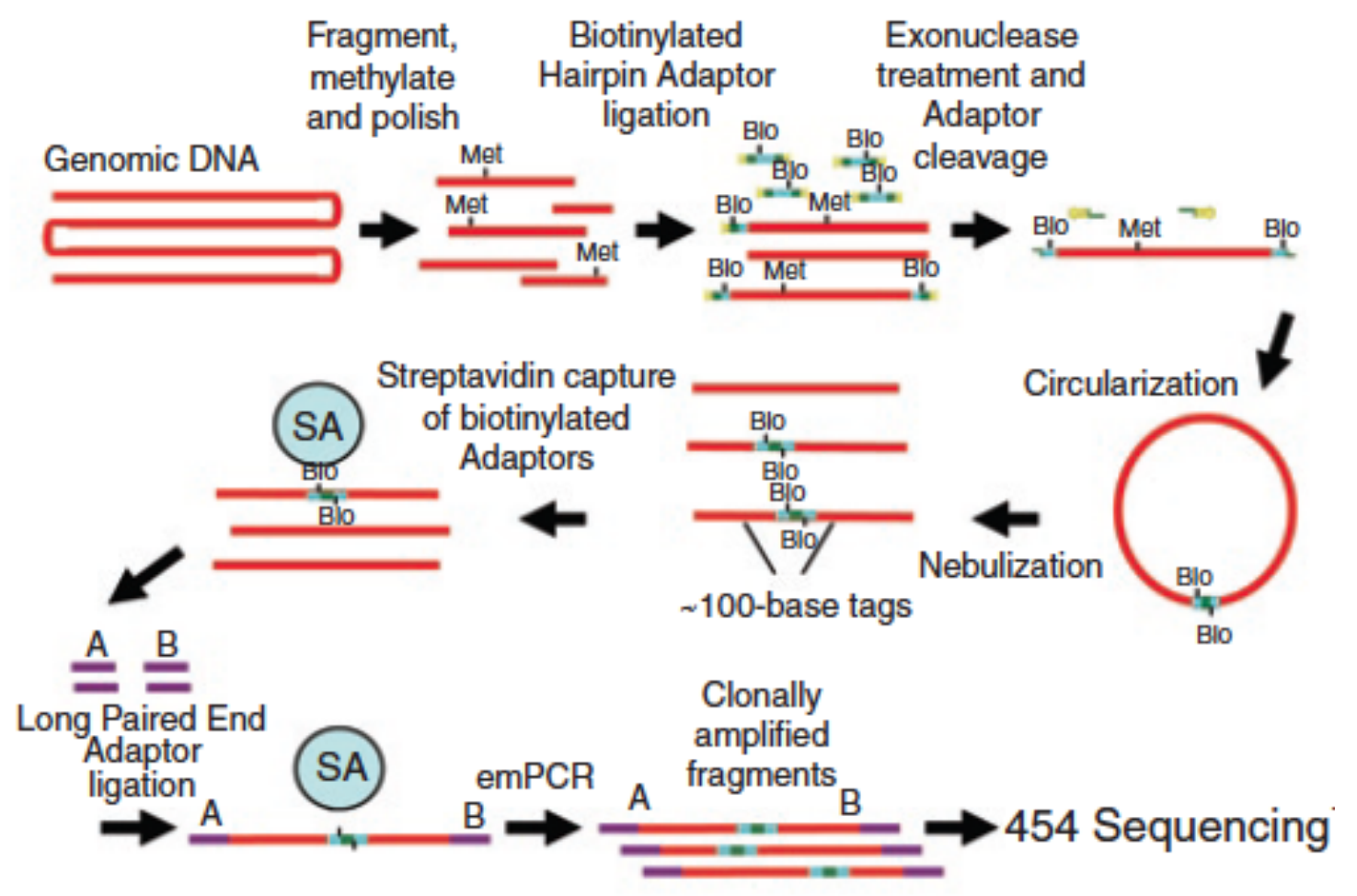

Figure 1.4: Preparation of paired-end fragments for pyrosequencing [taken from 454 Life Sciences, 2008].

This results in paired sequencing reads for which the distance, typically $3 \mathrm{~kb}$, between them is known. This is particularly useful in genomes that have a high number of repeat units such as IS elements (Pop and Salzberg, 2008). A typical IS element including transposase and repeat sequences is about 1,200 bp long. The short reads produced by pyrosequencing are unable to bridge this length with reads with unique end sequence, and the multiple copies of repeat sequences thus make it difficult to find the exact location in the genome. Using the information that mate-pairs are $3 \mathrm{~kb}$ apart, contigs can be linked across the IS element simply because the readpairs can be located on either side of it (Pop and Salzberg, 2008).

\subsubsection{Illumina sequencing}

Illumina produces the Illumina / Solexa genome analyser.

Illumina or Solexa sequencing also starts with DNA isolation, fragmentation and ligation to adapters (Figure 1.3) (Mardis, 2008a). Instead of attachment to beads, the DNA fragments are immobilized on a glass slide in Illumina sequencing, which at the same time acts as the place where sequencing and detection takes place (Mardis, 2008b). The glass slide is covered to a high density with adapters and adapterhomologous fragments (Mardis, 2008a; Ansorge, 2009). Once the fragments have 
been immobilized, PCR reagents are added for amplification. The DNA fragments will bend over to form a bridge, across which PCR can take place (Shendure and Ji, 2008; Ansorge, 2009). The bridge PCR products are then denatured into singlestranded format so that a new round of amplification can take place. Over time a cluster of amplified DNA fragments has grown, which is called a "polony" for a polymerase-triggered, spatially clustered colony (Ansorge, 2009).

The sequencing reaction uses reversible termination technology. ddNTPs are added that contain a reversible block at the 3'end of the base and a fluorophore (Metzker, 2010). All four nucleotides are added at the same time, but only one can be incorporated as the 3'end is blocked from further extension (Mardis, 2008b). Each polony spot is then measured for fluorescence before the fluorophore and the 3 'block are removed thus enabling the addition of a new nucleotide (Mardis, 2008a; Shendure and Ji, 2008; Ansorge, 2009; Metzker, 2010). This technology eliminates the chance of wrong multiple calling in homopolymeric runs as each nucleotide is added singularly. Conversely, the most common error observed is substitution when a nucleotide other than the correct one is incorporated by mistake (Shendure and $\mathrm{Ji}$, 2008; Metzker, 2010).

Illumina sequencing produces very short read lengths, currently between 36 and 54 bp are common, but up to 100bp reads or longer are possible (Metzker, 2010).

Paired-end technology has also been adapted for Illumina sequencing, with distances typically $200-300 \mathrm{bp}$. The process is slightly different as the fragments used for inserts are much shorter (Figure 1.5).

Different adapters with individual primers are attached to either side of the fragmented molecule. Polony formation then enriches both sequences, but supply of only one primer in the first sequencing reaction will lead to sequence readout for one end of the molecule. In a separate sequencing reaction with the second primer the paired-end is read out. The spatial clustering allows sequences to be linked as pairs. 


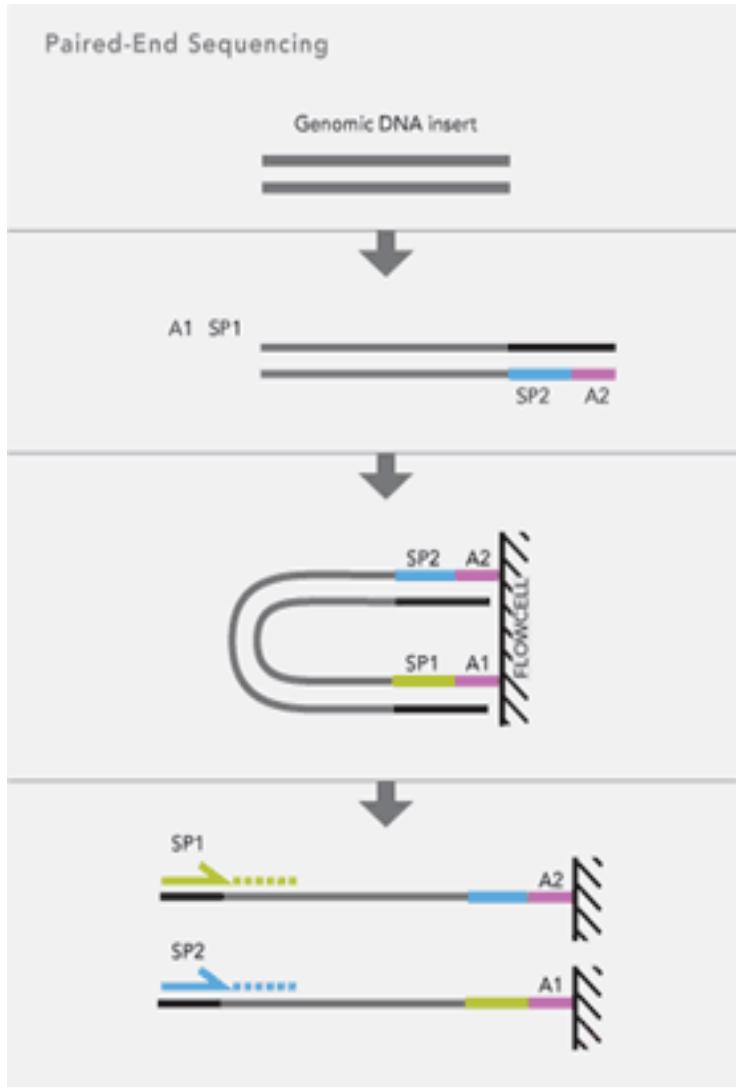

Figure 1.5: Paired-end fragment preparation in Illumina sequencing [taken from Illumina, 2011]. Adapter (A1 and A2) with sequencing primer sites (SP1 and SP2) are ligated onto DNA fragments. Template clusters are formed on the flow cell by bridge amplification and then sequenced by synthesis from the paired primers sequentially.

The paired-end technology as described for pyrosequencing - using larger fragment inserts, biotinylation, and subsequent fragmentation - is also possible with Illumina sequencing, but is termed "mate-pair sequencing". It uses a preparation protocol similar to that used for pyrosequencing.

Usually the glass slide used for Illumina sequencing contains 8 lanes (Mardis, 2008a). Each lane may contain a single DNA species, but it is also possible to sequence up to 12 different species per lane. This process is called multiplexing and involves adding different sequence tags to the adapters (Figure 1.6). 

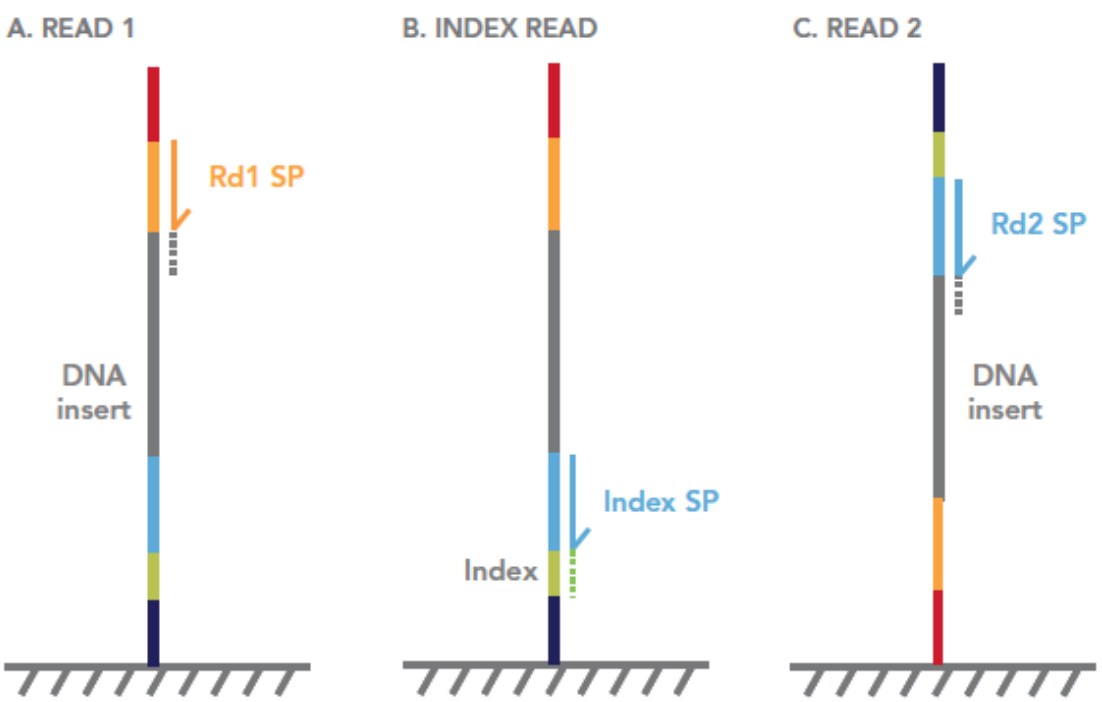

Figure 1.6: Multiplex preparation of DNA fragments for Illumina sequencing [taken from Illumina, 2011].

Each DNA species is treated separately with ligation of adapters for paired-end reads and a specific sequence tag of six bases. The different sequences are then mixed and applied to the lane. Sequencing takes place in three separate steps. The first and third reads supply the paired-end sequences, whilst the second read covers the index sequence. Computational methods then allow for identification of the index sequence and sorting. This increases the number of samples per slide from 8 to 96 , but will decrease the coverage for each of the species, as physically less DNA is present. Because fragment density and thus coverage for each genome is very high in Illumina sequencing, this has minor influence on quality of sequence produced.

\subsubsection{Comparison of capillary, pryo- and Illumina sequencing and applications}

The different technologies described have advantages and disadvantages and are compared in Table 1.3.

The biggest contributor to the need for development of next-generation technology was the time factor. The use of capillary separation presents the most advanced technology that is possible in Sanger sequencing, and does not allow for any more parallelization than the maximum of 384 samples (Shendure and Ji, 2008; Ansorge, 2009). 
Table 1.3: Comparison of sequencing technologies [adapted from Mardis, 2008a,b; Margulies, et al., 2005; Shendure and Ji, 2008; Metzker, 2010].

\begin{tabular}{|c|c|c|c|}
\hline & & Sequencing technolc & \\
\hline & Sanger & 454 & Illumina \\
\hline$>$ time factor & "96/384 reads & $\begin{array}{l}\text { Massive parallel } \\
\text { sequencing array } \\
\text { Several } 100,000 \text { reads }\end{array}$ & $\begin{array}{l}\text { Massive parallel } \\
\text { sequencing array } \\
\text { Tens of millions of reads }\end{array}$ \\
\hline $\begin{array}{l}>\text { time factor } \\
>\text { cloning bias }\end{array}$ & $\begin{array}{l}\text { Vector-based cloning } \\
\text { libraries } \\
\text { E. coli amplification }\end{array}$ & $\begin{array}{l}\text { In vitro fragment library \& } \\
\text { amplification }\end{array}$ & $\begin{array}{l}\text { In vitro fragment library \& } \\
\text { amplification }\end{array}$ \\
\hline$>$ read length & $\sim 1,000 \mathrm{bp}$ & $\sim 250 \mathrm{bp}$ & $36-54 b p$ \\
\hline$>$ base accurarcy & Very high & $\begin{array}{l}\text { Indel error in } \\
\text { homopolymeric runs }\end{array}$ & Substitution errors \\
\hline$>$ error rate & $\begin{array}{l}0.001 \% \\
1 \text { error in } 100,000 \mathrm{bp}\end{array}$ & $\begin{array}{l}0.6 \% \\
6 \text { errors in } 1,000 \mathrm{bp}\end{array}$ & $\begin{array}{l}0.1 \% \\
1 \text { error in } 1,000 \mathrm{bp}\end{array}$ \\
\hline$>$ time factor & Run time $1-2 \mathrm{~h}$ & Run time $12 \mathrm{~h}$ & $\begin{array}{c}\text { Run time: } \mathrm{SE}^{*}: 2 \text { days } \\
\mathrm{PE}: 4 \text { days }\end{array}$ \\
\hline $\begin{array}{l}>\text { reagent cost } \\
>\text { cost per } \mathrm{Mb}^{+}\end{array}$ & $\begin{array}{l}\text { Reagent volume } \mu \mathrm{l} \\
\$ 500\end{array}$ & $\begin{array}{l}\text { Reagent volume pl } \\
\$ 85\end{array}$ & $\begin{array}{l}\text { Reagent volume pl } \\
\$ 6\end{array}$ \\
\hline
\end{tabular}

${ }^{*}$ SE - single-end read, 36 bp, PE - paired-end read, 36 bp; ${ }^{+}$data from Shendure and Ji, 2008; Mardis, 2008a.

Library preparation, transformation and amplification in E. coli and the number of reads that can be analysed present the rate-limiting steps. Furthermore, there may be cloning bias as not all DNA is readily accepted and amplified in E. coli (Hall, 2007; Metzker, 2010). This adds to the comparably high reagent costs and thus the overall cost per megabase.

The next-generation sequencing technologies all share an array approach, allowing massive parallelization of reads (Shendure and $\mathrm{Ji}, 2008$ ). This reduces the time needed to cover the whole genome of an organism. Library preparation time is reduced as well, as the preparation and amplification takes place in vitro. This circumvents the cloning bias given in E. coli, and is also quicker (Shendure and Ji, 2008; Metzker, 2010).

The overall time for a sequencing run is comparable with Sanger sequencing, but gives a higher coverage, that is each base is sequenced and recorded $20-60 \mathrm{X}$, as opposed to usually $2 X$ in Sanger sequencing. The offset is that the new sequencing technologies give shorter sequence reads and have a higher error rate than Sanger sequencing (Mardis, 2008b; Shendure and Ji, 2008). Sanger sequencing gives reads of up to $1,000 \mathrm{bp}$, whereas pyrosequencing currently gives on average $250 \mathrm{bp}$. Ultrashort reads are produced by Illumina sequencing. It should be noted that the read lengths of the next-generation technologies are still under development and are increasing frequently (Shendure and Ji, 2008; Metzker, 2010) so the length limit is not known yet. There have also been studies suggesting that information given by paired-end and mate-pair sequencing might be more important than increasing read 
length (Chaisson, Brinza and Pevzner, 2009). Even in very fragmented assemblies most genes will fall within a single contig and not be split in two different ones. That way discovery and annotation should be possible (Chaisson, Brinza and Pevzner, 2009).

The main errors in pyrosequencing are insertion/deletions (indels) in homopolymeric runs, as mentioned before. Substitution errors are rare as each base is supplied individually. For Illumina sequencing, the opposite is true. Substitution errors are the most common error, as all ddNTPs are competing with each other for incorporation into the growing chain. The reversible termination on the other hand precludes mistakes in homopolymeric runs (Shendure and $\mathrm{Ji}, 2008$ ).

Using an array approach with hundreds of thousands or more parallel reads also allows for a reduced reagent volume, which significantly lowers the cost of sequencing (Shendure and $\mathrm{Ji}, 2008$ ).

The application for which the sequence information will be used very much determines the sequencing technology that will be most appropriate to use (Hall, 2007; Mardis, 2008b).

Higher costs of Sanger sequencing precludes it from being used in large-scale studies, but for the determination of exact sequence it is still considered gold standard (Hall, 2007; Ansorge, 2009). Given the longer reads, pyrosequencing is used in de novo sequencing and assembly, where an appropriate reference genome is not present. Illumina sequencing is used in re-sequencing, when an alignment against a reference is produced and single nucleotide polymorphisms (SNPs) can be determined (Hall, 2007; Mardis, 2008a; Metzker, 2010).

Next generation sequencing technologies have opened up new fields of applications of sequencing data (Mardis, 2008a; Metzker, 2010). A major field is population studies. It is now possible to sequence more strains of single species or even smaller distinctions such as subspecies or multi-locus sequence types. In this way it is possible to determine the normal range of variation within populations and distinguish it from variations that reflect specific adaptations for example in clinical isolates (Fraser-Liggett, 2005; Hall, 2007; Mardis, 2008a). Population studies also aid in mutation discovery, again possibly reflecting adaptational change (Mardis, 2008b; Shendure and Ji, 2008). 
The possibility of sequencing of more representatives of species also helps identification of accessory genes and establishes the core genome of a species. Accessory genes can be unique phages as well as regions acquired by horizontal gene transfer, such as the exchange of antibiotic resistance markers and other socalled "virulence genes" (Hall, 2007; Mardis, 2008a,b). The accessory genes can make up a considerable part of the total genome of a strain, and can vary between strains of a species. Considering this variability in possible genome content supports sequencing of more than one representative for each species to establish the species' core and the overall variety within a species described by the pangenome (Fraser-Liggett, 2005; Hall, 2007). Comparison of the accessory genomes between different eco- and biotypes of a species may also discover mechanisms behind adaptation to specific niches (Fraser-Liggett, 2005).

\subsection{Aim of this project}

\subsubsection{The Yersinia Paradox}

Yersinia is considered a paradoxical organism, as Y.pestis and Y. pseudotuberculosis are nearly identical with respect to genomic content, yet cause very different disease. On the other hand, Y.pseudotuberculosis and $Y$. enterocolitica are very distantly related, but cause the same disease outcome.

From the current hypothesis for the evolution of the genus, there is another paradox: Y.pseudotuberculosis, Y.pestis, and Y. enterocolitica are thought to share a common pathogenic precursor that evolved out of the environmental Yersiniae. Yet, at the same time, $Y$. enterocolitica and the environmental Yersiniae are thought to be closely related, since they were originally part of the heterogeneous group of 'enterocolitica' and are still referred to as 'Y. enterocolitica-like' species.

\subsubsection{Aims of the study}

Studies on the pathogenicity of strains of various biotypes of $Y$. enterocolitica have been undertaken in the past, generally using either random mutagenesis, hybridization techniques or insertional inactivation of genes that were shown to be involved in virulence in Y. pseudotuberculosis or Y. pestis. Although major genetic components were discovered with these methods in a wide range of bacteria, the disadvantages of these approaches are that only a limited number of genes are 
observed - either the ones targeted for identification or those that give strong response signals under selective conditions. Hybridization approaches might also be complicated due to sequence divergence thus leading to false negative results. Also in many instances, single strains of that species or biotype are selected and used in these studies to represent all members of that species or biotype, and most often the high-pathogenic members are selected. In the case of Yersinia, Y. enterocolitica BT 1B has been used extensively as a model for Yersinia pathogenesis.

Little information is currently available on the $Y$. enterocolitica biotypes, although it is apparent that there is additional coding capacity within the BTs (Howard, et al., 2006). Only one representative strain for the high-pathogenic BT 1B has been sequenced and analysed in comparison with $Y$. pestis and $Y$. pseudotuberculosis (Thomson, et al., 2006). This comparison showed that about $68 \%$ of the genes are conserved between the three pathogenic species. This is much higher than the $20.8 \%$ of genes that are shared between all of the $Y$. enterocolitica biotypes as determined by microarray (Howard, et al., 2006), highlighting the sequence diversity inherent in $Y$. enterocolitica and also underlining the limitations of comparative genomic hybridization studies (Thomson, et al., 2006).

This project therefore set out to sequence representative strains for each of the biotypes and comparatively analyse the genomic content. As biochemical traits form an important part of differentiating the $Y$. enterocolitica biotypes, a phenotypic assessment of the representatively sequenced strains was undertaken.

The aims of the projects are:

1) To establish the genetic makeup of each of the biotypes of $Y$. enterocolitica and to define the unique sets of genes for each biotype

2) To characterize what is shared in pathogenic biotypes that is important for mechanism of disease

3) To determine the metabolic capacities of each of the biotype

4) To link phenotypic and genotypic traits for improved annotation and characterization of the $Y$. enterocolitica biotypes 


\section{Materials and Methods}

\subsection{Bacterial Strains}

\subsubsection{Reference Genomes}

The reference strains were selected based on the prevalence of the different bioserotypes of $Y$. enterocolitica isolated from cattle, pigs, and sheep throughout the UK between 1999 and 2000 during a randomised national survey of food pathogens in livestock sent for slaughter (McNally, et al., 2004) and in addition human isolates identified during the Intestinal Infectious Disease study (Tompkins, et al., 1999) carried out over the same time period. BT5 is not prevalent in the UK and poses no health concern. Thus, the reference strain was obtained from Germany from the laboratory of Dr. Thilo M Fuchs. The UK strain collection was extensively characterized genotypically (Howard, et al., 2006; Thomson, et al., 2006) and phenotypically (McNally, et al., 2006) and the strains listed below were chosen as representative strains for each prevalent bio-serotype.

Table 2.1: List of reference strains sequenced in this project.

\begin{tabular}{lllll}
\hline Strain & Biotype & Serotype & Plasmids & Source \\
\hline \hline YE53/03 & 1A & O:5 & $\mathrm{pYV}^{-}$ & Human faecal isolate \\
YE8081* & 1B & O:8 & $\mathrm{pYV}^{+}$ & Clinical isolate \\
YE212/02 & 2 & $0: 9$ & $\mathrm{pYV}^{+}$ & Pig abattoir isolate \\
YE56/03 & 3 & $0: 9$ & $\mathrm{pYV}^{+}$ & Human faecal isolate \\
YE149/02 & 3 & $\mathrm{O}: 5,27$ & $\mathrm{pYV}^{+}$ & Sheep abattoir isolate \\
YE12/03 & 4 & O:3 & $\mathrm{pYV}^{+}$ & Human faecal isolate \\
YE3094/96 & 5 & O:2a,2b,3 & $\mathrm{pYV}^{+}$ & Animal isolate
\end{tabular}

*: Reference strain, sequenced previously [Thomson, et al., 2006]

\subsubsection{Worldwide Collections}

The study of the genus Yersinia and a broader characterization of the species $Y$. enterocolitica were carried out on a worldwide collection of different species and strains from collaborating laboratories. The strains are listed in Table 9.1 (p. 204) and 9.2 (p206) in the appendix. 


\subsubsection{Additional strains}

Some strains examined, which are not part of the sequencing collections, are listed in Table 2.2.

Table 2.2: Additional strains.

\begin{tabular}{llllll}
\hline Strain & Biotype & Serotype & Source & country & Lab \\
\hline \hline YE2103 & 1B & O:19 & Human control & UK & McNally \\
NZ6 & 1B & nd & Human & New Zealand & DuFour \\
YE21402 & 3 & O:9 & Pig & UK & McNally \\
\hline
\end{tabular}

\subsection{Sequencing, Assembly and Annotation}

\subsubsection{Sequencing Technologies}

DNA extraction and genome sequencing was carried out at the Wellcome Trust Sanger Institute (WTSI), Cambridge.

Sequencing for the reference genomes was carried out to improved high-quality draft genome standard (Chain, et al., 2009). The strains were sequenced using three different types of sequencing technologies. Capillary sequencing $(A B I)$, also known as traditional Sanger sequencing, was used for $2 \mathrm{X}$ coverage, and produced long high-quality reads. Pyrosequencing (454/Roche GS20/FLX) and Illumina (Illumina GAll Analyser) technologies produce shorter reads, and thus reach higher coverage, in excess of 30- and 60-fold coverage respectively. PCR fragments were designed to span gaps and close them. This process was computerized by using algorithmic based automatic contiguation of assembled shotgun sequence (ABACAS; Assefa, et al., 2009; http://abacas.sourceforge.net). De-novo assemblies were produced using the Velvet assembler (Zerbino and Birney, 2008; http://www.ebi.ac.uk/ zerbino/velvet) and the GS De Novo assembly software (Roche, 2011). The final assembly was corrected with iCORN (Otto, Sanders, Berriman and Newbold, 2010).

Pseudogenes containing stop codons or frameshifts were checked manually for sequence quality, and if necessary further sequencing of that region in question was carried out.

Sequence for the worldwide collection was generated using the lllumina sequencing platform (GAll instruments) with indexed Illumina runs of 12 samples per lane of 
sequencing, standard 200-300bp fragments and 76 cycles of sequencing. The 12 IP strains were sequenced separately at the Institute Pasteur using single-end Illumina reads.

The genome coverage is a measure of how many times a base is sequenced on average by different reads, and is therefore an indication of sequence quality in nextgeneration sequencing technologies. The coverage can be calculated from the average read length (here $76 \mathrm{bp}$ ), the number of reads, and the average genome size (5 Mbp) using the following formula:

$$
\text { expected coverage }=\frac{(\text { average read length } * \text { number of reads) }}{\text { average genome size }}
$$

The resulting coverage for $Y$. enterocolitica strains is plotted in Figure 2.1. The 12 strains obtained from E. Carniel at the Institute Pasteur were not included in this calculation, as they were sequenced separately.

The average coverage is higher than $20 X$ in the strain collection. Variation arises from the number of reads obtained in a sequencing run.

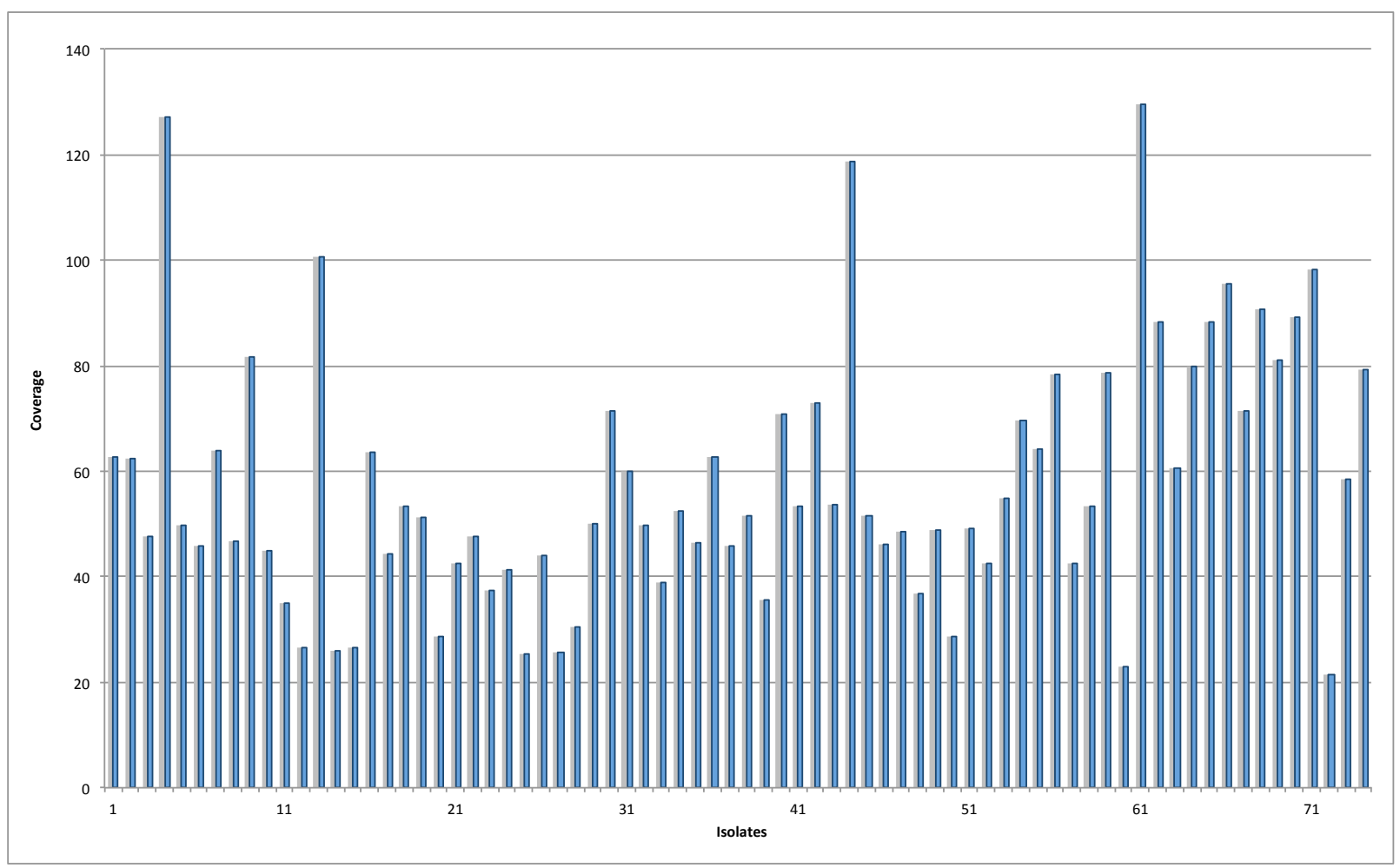

Figure 2.1: Example of coverage of $Y$. enterocolitica strains sequenced as part of the worldwide strain collection. Coverage of strains varies between strains and different runs due to experimental error and DNA quality, but has a minimum of 20X for each strain. 
Data for the worldwide collection was assembled using Velvet de novo assembly for the environmental samples and assembly against reference BT 1B strain 8081 for Y. enterocolitica isolates.

\subsubsection{Annotation and Sequence Analysis}

The reference genomes for each of the biotypes were annotated and analysed in detail. Algorithms used for gene prediction and characterization of CDSs are given in Table 2.3. Glimmer 3 was used for prediction of coding sequences (CDS; Salzberg, Delcher, Kasif and White, 1998; Delcher, Bratke, Powers and Salzberg, 2007), and searches for motifs (Nielsen, Engelbrecht, Brunak and von Heijne, 1997; Brendtsen, Nielsen, von Heijne and Brunak, 2004; Krogh, Larsson, von Heijne and Sonnhammer, 2001; Finn, et al., 2010; Sigrist, et al., 2002; Hulo, et al., 2006) were employed to characterize potential proteins as detailed in chapter 3 .

Table 2.3: Function and basis of the algorithms used for gene prediction and characterization.

\begin{tabular}{|c|c|c|}
\hline Algorithm & Function & Basis \\
\hline Glimmer 3 & $\begin{array}{l}\text { Model for prediction of coding sequences } \\
\text { (CDSs) }\end{array}$ & $\begin{array}{l}\text { DNA sequence contains } 6 \text { reading frames, } \\
\text { probability calculation and modelling of } \\
\text { most likely open reading frame }\end{array}$ \\
\hline TMHMM & Prediction of transmembrane domains & $\begin{array}{l}\text { Transmembrane domains will contain } \\
\text { hydrophobic amino acids for integration } \\
\text { into the lipid bilayer }\end{array}$ \\
\hline SignalP & Prediction of signal domains & $\begin{array}{l}\text { Proteins transported to the cell membrane } \\
\text { contain signals for trafficking and direction }\end{array}$ \\
\hline $\mathrm{HTH}$ & $\begin{array}{l}\text { Prediction of helix-turn-helix motifs (DNA } \\
\text { binding proteins) }\end{array}$ & $\begin{array}{l}\text { Probability calculation that a short given } \\
\text { stretch of amino acids has the properties to } \\
\text { form a tertiary structure in the form of two } \\
\text { linked helices }\end{array}$ \\
\hline Pfam & Prediction of protein families and domains & $\begin{array}{l}\text { Indication for protein functionality by } \\
\text { domain comparison with well-studied, } \\
\text { functionally assigned examples }\end{array}$ \\
\hline Prosite & Prediction of protein families and domains & $\begin{array}{l}\text { Indication for protein functionality by } \\
\text { domain comparison with well-studied, } \\
\text { functionally assigned examples }\end{array}$ \\
\hline
\end{tabular}

Comparisons between BT 1B strain 8081 and the newly sequenced BT reference genomes were done using the Artemis Comparison Tool (ACT; Carver, et al., 2005). Regions differing from $\mathrm{BT} 1 \mathrm{~B}$ received manual annotation in Artemis (Berriman and Rutherford, 2003). Annotation was based on basic local alignment search tool (BLAST) searches (Altschul, et al., 1990) as well as information gained from motif searches. 
Between different sequence versions, the annotation was transferred using an inhouse perl script. This script will perform a blast search with the old FASTA file and compare it to the new FASTA file. Matches within CDSs are transferred into a new annotation file, which can be read into the new FASTA file using Artemis. The annotation transfer can be combined with the Glimmer 3 prediction, so that CDSs are highlighted, which have not yet received annotation.

Homologous genes are called orthologous if they have been present in the last common ancestor before speciation. They are propagated vertically via inheritance from mother to daughter cells. The differences observed relate to the evolutionary distance during evolution. As orthologous genes have been present in the ancestor, their genomic context is conserved between different isolates. In contrast, paralogs arise following duplication of genes, and therefore have a different genomic context and differ with respect to distribution in isolate. One common family of paralogs are insertion sequence (IS) elements, and copy number can vary between isolates.

To identify orthologs between the different reference genomes, another in-house perl script was applied. The script performs a homology search of CDSs with $40 \%$ homology over $80 \%$ of the length of the CDS (Figure 2.2). CDSs are compared pairwise, that is only those are retained as orthologs in which a search of genome 1 against genome 2 yields an ortholog and in which the reciprocal search of genome 2 against genome 1 yields the same ortholog. 


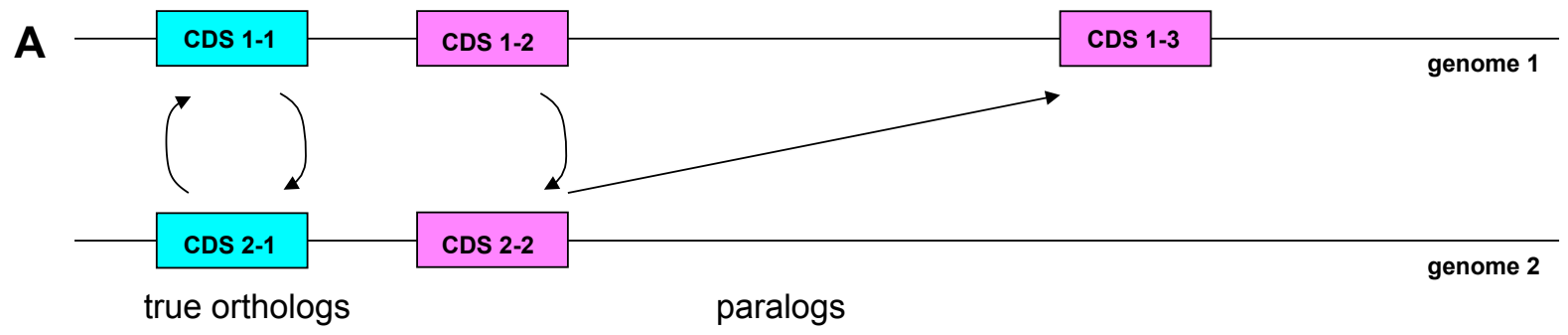

B

orthologs

IS1667 - paralog

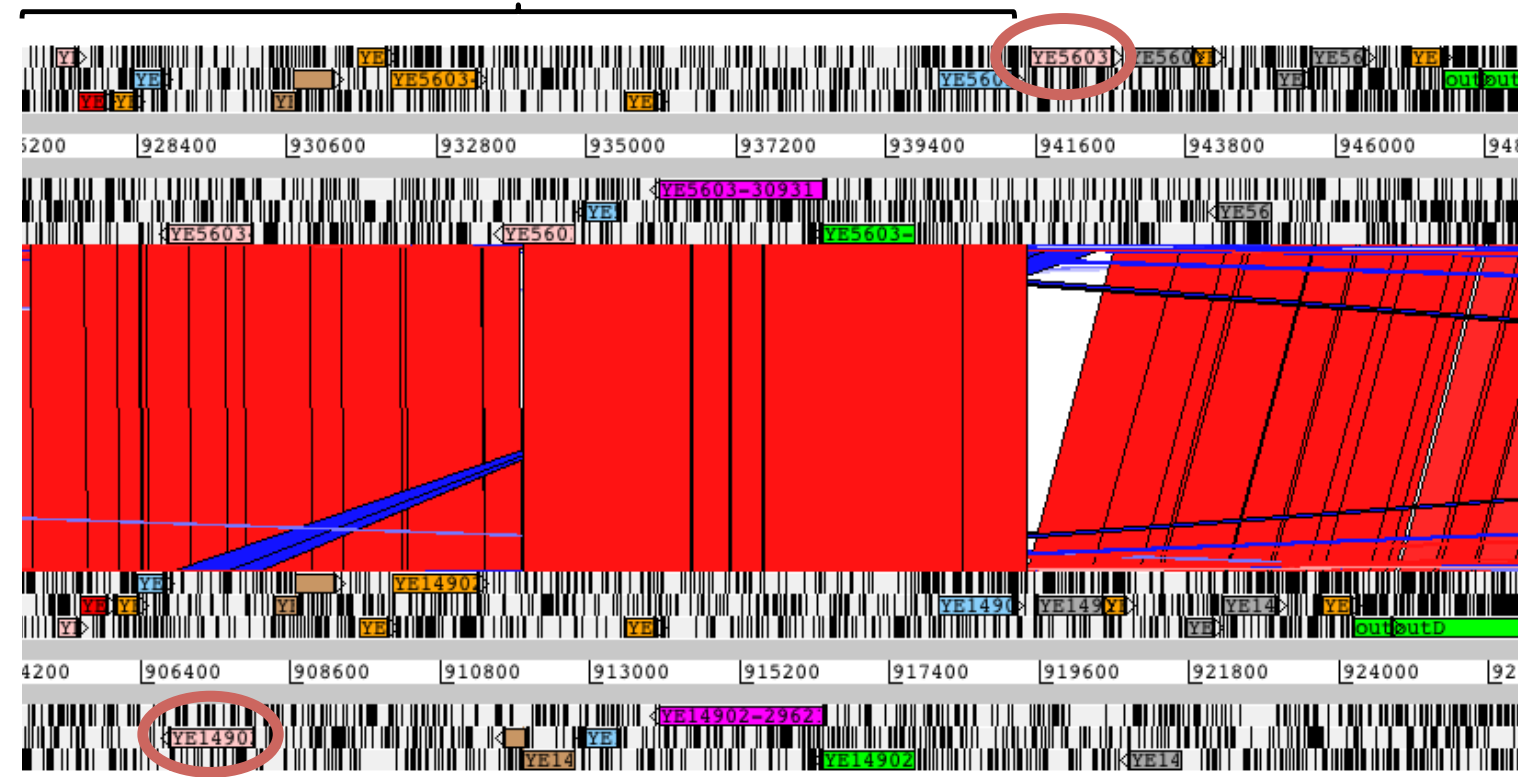

Figure 2.2: Identification of orthologs and paralogs. A. Reciprocal identification of blue CDSs as orthologs. Pink CDSs are not reciprocally identified, CDS 1-2 and CDS 2-2 are orthologs, and CDS 13 is a paralog to CDS 2-2. B. Genome example. Most genes are orthologous in both genomes, except an additional copy of IS1667 inserted in the top genome. This copy is paralogous to IS1667 found in the bottom genome, but is potentially misidentified in the reciprocal FASTA search.

The results are comprised in a format readable in Artemis. The identified orthologs need to be checked manually, as pseudogenes might be below the $80 \%$ length cutoff, and as IS elements may have been identified wrongly as having orthologs. For this, the orthologs of different combinations of reference genomes were firstly combined in a single annotation file. Then, an in-house perl script was employed to look at genomes in 3-way comparisons (Figure 2.3). 


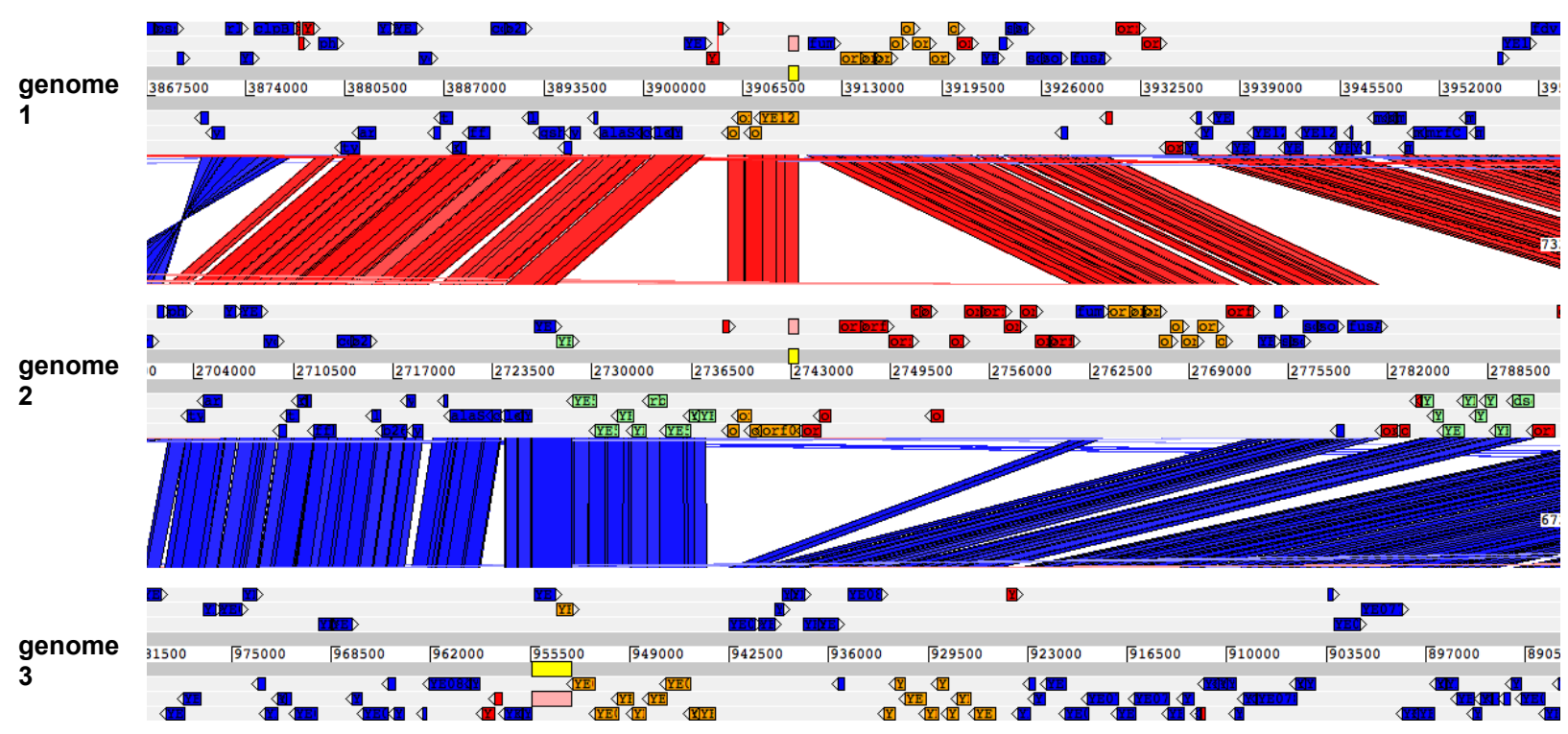

Figure 2.3: Example of a 3-way comparison for identification of orthologous gene sets. Genes present in genomes 1, 2, and 3 are blue. Genes unique to each genome are red. Genes orthologous in genome 1 and 2 are orange in both genomes. Genes orthologous in genome 2 and 3 are green and orange, respectively. Genes shared in genomes 1 and 3 would be green, but are not contained within this comparison.

For each 3-way comparison, CDSs are sorted in four categories and colourhighlighted accordingly: CDSs which are unique to each of the three genomes are highlighted in red, CDSs which are shared in all three genomes are highlighted blue, and CDSs which are shared between only two of the three genomes are highlighted either green or orange. The colouring of CDSs according to the four groups allows for quick identification of those CDSs that have not been coded correctly. For instance, if a red CDS occurs within a region conserved in all three genomes, this can easily be identified and resolved.

The perl script used for identification and colouring of orthologs was then modified to allocate groups instead of colours to specific gene sets (see Appendix 9.5, p. 214). This was done to identify CDSs belonging to the backbone, as well as CDSs that might be shared only within subsets or specific lineages of $Y$. enterocolitica BTs. Sandra Reuter carried out this modification, whereas the Pathogen Genomics group at the Wellcome Trust Sanger Institute provided the other in-house perl scripts.

The information on orthologs was also used to generate circular diagrams, either using the DNA plotter option in Artemis, or an in-house PERL script for highresolution pictures. 
Gene and protein alignments were generated using ClustalW2 (Larkin, et al., 2007) in the Seaview software (Galtier, Gouy, and Gautier, 1996; Gouy, Guindon, and Gascel, 2010).

\subsection{Plasmid profiling}

To establish a plasmid profile selected strains, the method of Kado and Liu (1981) was used.

\subsubsection{Reagents}

E buffer

$40 \mathrm{mM}$ Tris Base $(4.85 \mathrm{~g}$ in $1 \mathrm{I}, 121.14 \mathrm{~g} / \mathrm{mol}$; Fisher Scientific)

$2 \mathrm{mM} \mathrm{Na}{ }_{2}$ EDTA (0.744 g in 1 I, $372.24 \mathrm{~g} / \mathrm{mol}$; Sigma)

Tris base and $\mathrm{Na}_{2}$ EDTA were dissolved in $1 \mathrm{I}$ of distilled water. The $\mathrm{pH}$ was then adjusted to 7.9 using glacial acetic acid.

Lysis buffer

$0.6055 \mathrm{~g}$ Tris Base were dissolved in $40 \mathrm{ml}$ distilled water. $30 \mathrm{ml}$ of $10 \%$ SDS (Sigma) were added. Using $8 \mathrm{~g}$ in $100 \mathrm{ml}$ distilled water, a $2 \mathrm{M}$ solution of $\mathrm{NaOH}$ (Fisher Scientific) was made up. To raise the $\mathrm{pH}, 3.2 \mathrm{ml} 2 \mathrm{M} \mathrm{NaOH}$ were added to the Tris base/SDS solution, and the total volume was adjusted to $100 \mathrm{ml}$.

\subsubsection{Protocol}

A $5 \mathrm{ml}$ overnight culture in Luria-Bertani (LB) broth (Fisher Scientific) was set up for strains to be examined. Y. enterocolitica was grown at $25^{\circ} \mathrm{C}$, Salmonella was grown at $37^{\circ} \mathrm{C}$.

$2 \mathrm{ml}$ of the overnight culture was pelleted for 5 minutes at $4,000 \mathrm{rpm}$ at room temperature using a bench-top centrifuge (Hettich Mikro 2000). The pellet was resuspended in $150 \mu \mathrm{l} \mathrm{E}$ buffer. $300 \mu \mathrm{l}$ lysis buffer were added and gently mixed to observe clearing indicating lysis. The sample was then heated for 60 minutes at $55^{\circ} \mathrm{C}$ using a heat block. After this incubation, $600 \mu \mathrm{l}$ of phenol-chloroform (1:1 ratio saturated with Tris; Sigma) were added and gently mixed. To separate the layers, samples were centrifuged for 30 minutes at $14,000 \mathrm{rpm}$ at room temperature in a bench-top centrifuge. From the upper watery layer, 35 to $45 \mu \mathrm{l}$ of sample was removed and added to $5 \mu \mathrm{l}$ of loading dye (Promega). For the markers, Salmonella 
strains SL1344 and PT4 as well as Y. enterocolitica strains 5303 and 21202 extractions were mixed in 1:1 ratio.

Visualization was carried out on $0.7 \%$-agarose gels with $E$ buffer containing $10 \mathrm{mg} / \mathrm{ml}$ ethidium bromide. Gels were run for one hour at 30V, then a further four hours and 30 minutes at $45 \mathrm{~V}$ for best separation.

\subsection{Phenotypic Microarray}

\subsubsection{Experimental setup}

The phenotypic microarray experiment was carried out with the help of Theresa Feltwell (WTSI). Bacterial strains were cultured on LB agar plates at $25^{\circ} \mathrm{C}$ prior to starting the experiment. Inoculation and preparation of the 96-well plates used for phenotypic characterization was carried out as described by the manufacturer (Biolog Inc., Hayward, U.S.A.). PM1, 2A (carbon), PM3B (nitrogen), and PM4A (phosphorus, sulphur), PM9 and PM10 were chosen.

The workflow is depicted in Figure 2.4.

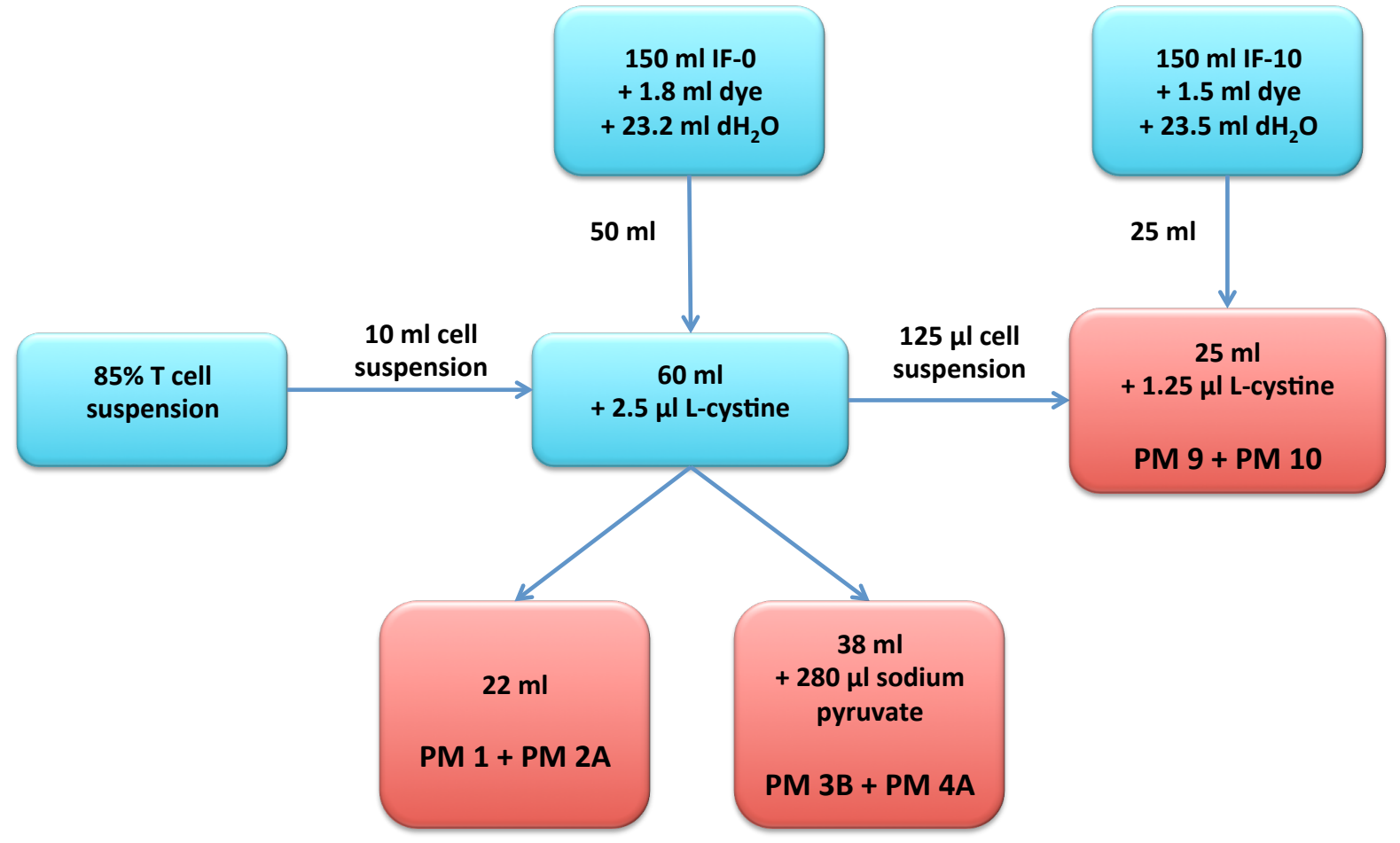

Figure 2.4: Preparation of fluids for phenotypic microarray.

In detail, the inoculation fluids IF-0 for PM $1-4$ and IF-10 for PM 9 and 10 were split into sterile flasks containing $50 \mathrm{ml}$ each, and sterile water and tetrazolium dye A mix 
for Gram negative bacteria was added. Additionally, to each IF-0 flask L-cystine, the homodimer of L-cysteine, $(12.5 \mu \mathrm{M})$ was added because Yersinia needs a reduced from of sulphur for growth. The cell suspension for each strain was prepared by running a sterile swab over the agar plate and transferring the bacteria into a sterile tube containing IF-0. A uniform suspension was created until a turbidity of $42 \%$ transmittance $(T) \pm 2 \%$ in the Biolog turbidimeter was reached. $10 \mathrm{ml}$ of this $42 \% \% \mathrm{~T}$ cell suspension was then added to $50 \mathrm{ml}$ of IF-0 + dye A to yield a final cell density of $85 \%$ T. $125 \mu \mathrm{l}$ of the $85 \%$ T cell suspension was then transferred to a flask containing $25 \mathrm{ml} \mathrm{IF-10+dye} \mathrm{A.} \mathrm{This} \mathrm{suspension} \mathrm{was} \mathrm{then} \mathrm{used} \mathrm{to} \mathrm{inoculate} \mathrm{PM9} \mathrm{and} \mathrm{10,} \mathrm{using}$ a volume of $100 \mu \mathrm{l} /$ well. $22 \mathrm{ml}$ of the $85 \%$ T cell suspension in IF-0 + dye was used to inoculate PM 1 and PM 2A with $100 \mu \mathrm{l} /$ well. To the rest of the $85 \%$ T cell suspension $280 \mu \mathrm{l}$ of $2 \mathrm{M}$ sodium pyruvate was added, and PM 3B and PM 4A were inoculated with $100 \mu \mathrm{l} /$ well. The plates were then placed in the OmniLog incubator/reader, and incubated for $48 \mathrm{~h}$, taking readings every $15 \mathrm{~min}$. The experiment was carried out in triplicate for phenotypic characterization at $28^{\circ} \mathrm{C}$ and $37^{\circ} \mathrm{C}$.

\subsubsection{Analysis}

Evaluation of phenotypic microarray (PM) data has not been standardized. The software that comes with the OmniLog instrument offers a number of variables that can be automatically calculated from the kinetic plots, such as maximum height, average height, area under the curve, and slope. It is also possible to manually select wells that visually stand out.

An example of the current data set is given in Figure 2.5, showing the kinetic plots for the non-pathogenic BT $1 \mathrm{~A}$ and the high-pathogenic BT 1B. Higher metabolic activity in $B T 1 A$ is seen in green, higher activity of $B T 1 B$ in red, and the overlapping areas of the curves are shown in yellow.

One challenge with PM data is to determine a valid point to call metabolism versus non-metabolism of nutrient sources. Considering for instance A08, A09, and E01: is there utilization in these wells, or is the activity so low as to be considered background variation? 


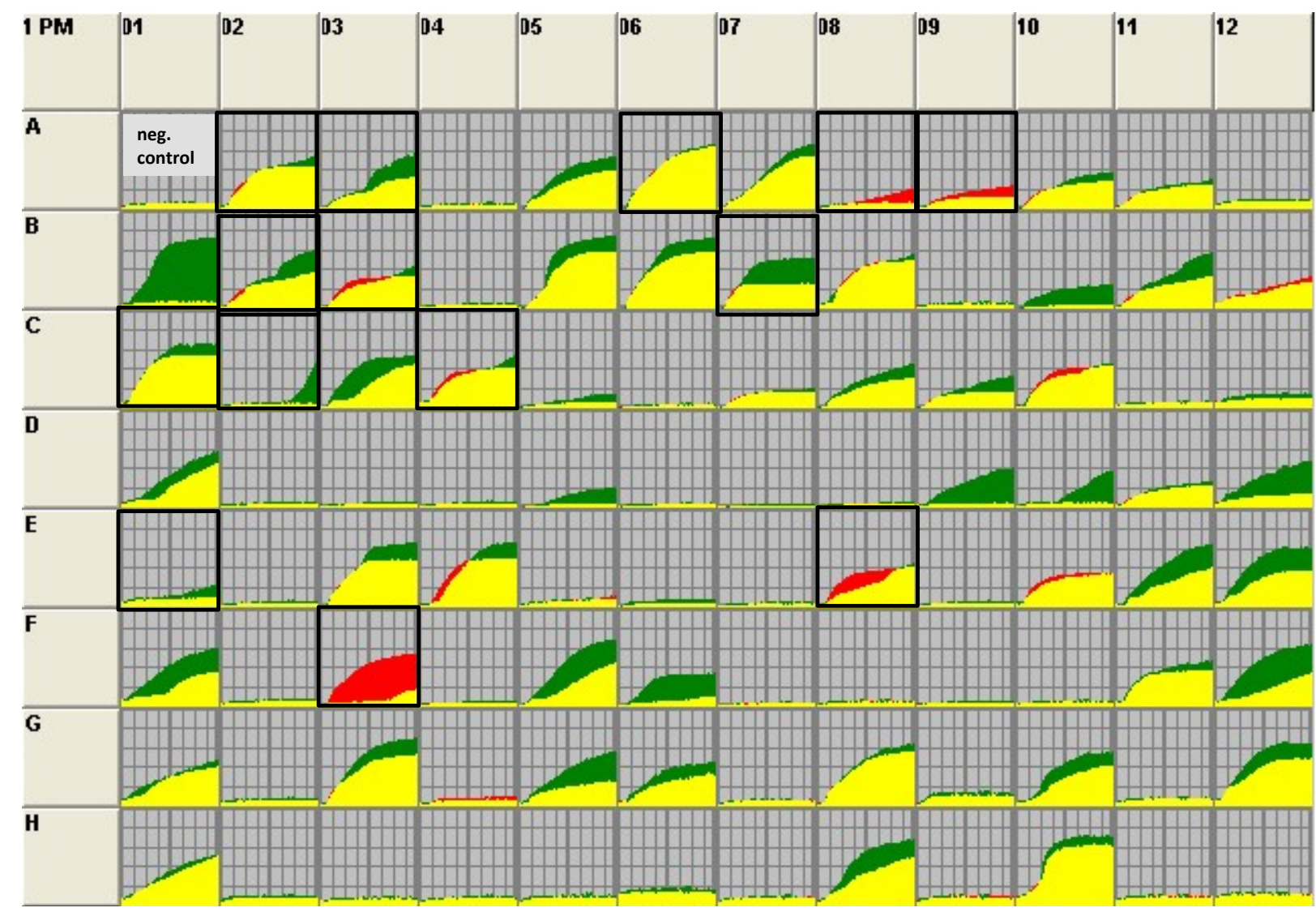

Figure 2.5: Example for utilization of carbon sources in BT 1A and BT 1B. A01 is the negative control. Overlapping curves are shown in yellow, BT 1A is shown in green, BT 1B is shown in red. Highlighted are wells described in the text.

The curves also show very different shapes. Some curves rise steadily right from the start such as A06. Other curves show a sigmoidal progression imitating MichaelisMenten enzyme kinetics, where the curve rises quickly and then levels off as if there is a maximum turnover of this nutrient with a rate-limiting protein present, such as a transporter or a down-stream enzyme involved with conversion of the nutrient (B07, C01). Other curves resemble diauxic growth curves (A03, B02). How do you compare these curves and are the differences seen relevant or significant? One method would be to use the area under the curve, but there can be curves such as in $\mathrm{A} 02, \mathrm{~B} 03$, and $\mathrm{C} 04$ with slightly different progression yet same area under the curve. One strain metabolises the nutrient earlier, the other strain utilizes it to a higher degree. Another method would be to look at the endpoint of the measurements. $\mathrm{C0} 2$ shows a curve that shows no utilization in the strains for most of the measurements and only gives a signal in the last hours. Is this to be treated the same as other curves that show a steady utilization over extended periods of time? Comparison with E08 shows that both curves have the same endpoint, but their progression is entirely different. Also interesting to keep in mind is F03: Is there utilization in BT 1A as the 
signal activity is detected very late, or is there no activity as the signal is not very strong?

We chose to adopt an approach by Homann, Cai, Becker and Lindquist (2008) and modified it to be able to apply statistical tests. This was done in collaboration with Lars Barquist (WTSI) who wrote the scripts to process the data and perform the calculations as well as produce heatmaps.

The data was exported from the Biolog File Manager, and further analysis was conducted in $\mathrm{R}$. For each replicate, the average height and maximum height over $48 \mathrm{~h}$ and the average height over $2 \mathrm{~h}$ was extracted. The background control was then subtracted for each PM plate. The signal value was calculated using the following formula:

signal value $=\left(\frac{[\text { average signal over } 48 h+\text { maximum signal over } 48 h]}{2}\right)-($ average signal over first $2 h)$

This was done to firstly correct the average height for later data points, to take the progression of the curve into account and to reduce any background signals. A single number can therefore reflect the time course of metabolism.

Each plate showed a clear bimodal distribution of signal values. To call wells in which metabolic activity was taking place, normal distributions were fitted to the two peaks ("off" and "on") of the bimodal distribution. From this the log-odds score for each data point could be calculated, showing how likely each well was to have been produced by the "on" distribution. A conservative cut-off of four times more likely was used for further analysis. To examine differential metabolism between strains, the limma R/Bioconductor package was used (Smyth, 2004). All strains were compared to BT 1B strain 8081, and the Benjamini-Hochberg corrected p-values were used to determine statistical significance of differences controlling for a false discovery rate of $5 \%$. 


\subsection{Phylogenetic Analyses}

\subsubsection{Phylogenetic reconstruction of Y. enterocolitica: whole genome alignment}

The phylogeny of $Y$. enterocolitica was reconstructed using an approach from Harris, et al. (2010). Dr Simon R. Harris (WTSI) provided the necessary scripts. Sandra Reuter and Dr Thomas R. Connor (WTSI) both carried out parts of the workflow.

The genome sequence of BT 1B strain 8081 was used as a reference sequence. Regions containing phage were excluded, as they most likely constitute unique acquisition events for the strain and might lead to false positive single nucleotide polymorphisms (SNPs) when compared to related, but independently acquired phages. These SNPs are therefore non-informative in an evolutionary context. The reads obtained from Illumina sequence were mapped against this reference using SSAHA (Sequence Search and Alignment by Hashing Algorithm; Ning, Cox, and Mullikin, 2001). Strains with low mapping percentage $(<50 \%)$ to the reference were excluded from the analysis. Candidate SNPs were compiled. A conservative approach was chosen to minimize false positive and false negative SNP calls by removing SNPs with low quality scores and by filtering for SNPs that are present in at least $75 \%$ of the mapped reads. For each strain, the polymorphisms identified in the previous steps replaced the reference sequence. Thus a SNP alignment was obtained.

For estimation of an evolutionary tree based on maximum likelihood RAxML was used (Stamatakis, 2006). The general time reversal (GTR) model was used to account for differences in rates of nucleotide substitutions, and gamma correction was chosen to model the different positions within a triplet codon. 100 random bootstraps were performed for node support. Again, Dr Simon R. Harris provided scripts for this. In a last step, the SNPs were reconstructed on the tree so that SNPS associated with specific branches can be identified. No outgroup was used for the $Y$. enterocolitica tree, but the tree was rooted in retrospect on the non-pathogenic BT $1 \mathrm{~A}$ as this is the oldest lineage.

\subsubsection{Phylogenetic reconstruction of the genus Yersinia: extended MLST scheme}

For the phylogenetic relationship of the genus Yersinia, a concatenated sequence of 75,651 bases from 85 housekeeping genes (Table 2.4) was used. These genes were 
identified in conserved, syntenic blocks in the $Y$. enterocolitica genome. The genes were chosen by Miquette Hall (NTU) on the basis of exhibiting 10-25\% SNP divergence between Y. pestis and Y. enterocolitica.

Table 2.4: Housekeeping genes used for construction of the phylogenetic relationship within the genus Yersinia.

\begin{tabular}{ll|ll|ll|ll|ll}
\hline ID* $^{*}$ & gene & ID & gene & ID & gene & ID & gene & ID & gene \\
\hline \hline YE0003 & asnA & YE0919 & gloB & YE2176 & ppsA & YE2427 & kdsA & YE3331 & galR \\
YE0027 & glnA & YE0926 & nrdF & YE2209 & trpE & YE2431 & prfA & YE3374 & lysS \\
YE0062 & dfp & YE0927 & nrdE & YE2254 & xthA & YE2432 & hemA & YE3376 & prfB \\
YE0093 & tpiA & YE1009 & nadB & YE2257 & topB & YE2477 & chaA & YE3416 & tktA \\
YE0134 & sthA & YE1082 & guaA & YE2261 & ansA & YE2783 & udk & YE3419 & speA \\
YE0166 & rhIB & YE1281 & aroC & YE2281 & dadA & YE2794 & sfcA & YE3425 & endA \\
YE0168 & rho & YE1538 & aroA & YE2283 & fadR & YE2818 & folE & YE3427 & gshB \\
YE0251 & udp & YE1640 & tmk & YE2284 & nhaB & YE2928 & nadA & YE3656 & parC \\
YE0258 & aarF & YE1643 & ptsG & YE2371 & minC & YE2977 & glnS & YE3673 & rfaE \\
YE0263 & hemB & YE1717 & phoQ & YE2372 & minD & YE3067 & rosA & YE3681 & gcp \\
YE0354 & groEL & YE1718 & phoP & YE2374 & rnd & YE3088 & hemH & YE3708 & uxaC \\
YE0414 & mdh & YE1720 & purB & YE2383 & zwf & YE3089 & adk & YE3766 & pyrB \\
YE0600 & thrA & YE1725 & icdA & YE2386 & msbB & YE3202 & proB & YE4132 & fdhE \\
YE0610 & dnaJ & YE1784 & purT & YE2388 & znuA & YE3308 & argA & YE4133 & fdol \\
YE0746 & pyrG & YE1793 & pip & YE2389 & znuC & YE3317 & thyA & YE4151 & glyQ \\
YE0773 & pcm & YE1887 & kduD1 & YE2390 & znuB & YE3318 & lgt & YE4170 & gyrB \\
YE0829 & recA & YE2114 & tyrR & YE2397 & aspS & YE3324 & tas & YE4206 & atpD \\
\hline
\end{tabular}

${ }^{*}$ systematic ID respective to BT 1B strain 8081 .

The respective gene sequences for the MLST tree were extracted using a script that performs a tblastn search on an assembled query sequence using a translated nucleotide subject to search for a matching amino acid string. The genes were concatenated into a single super-locus for each strain. Similarly to the phylogeny of the species, the alignment of genes was used to estimate a tree with RAxML based on the GTR model with gamma correction and bootstrapping (Stamatakis, 2006). Dr Thomas R. Connor was responsible for this part of the project. Bayesian analysis of population structure (BAPS; Cheng, et al., 2011) was used to identify clusters of homologous sequences within the 85 housekeeping genes, and formed the basis for the proposition of the new species clusters. Dr Thomas $\mathrm{R}$ Connor performed this work as well. 


\subsubsection{Phylogenetic reconstruction of the virulence plasmid pYV}

To determine the phylogenetic relationship of the virulence plasmids, the same approach as for Y. enterocolitica was used. Both pYV8081 and pYV5604 were used as references to map Illumina reads using SSAHA in separate approaches. Alignments were created and trees were estimated using RAxML based on the GTR gamma model with 100 bootstraps. Both approaches gave the same tree topology. Dr Thomas R. Connor and Sandra Reuter both performed parts of this experiment.

\subsubsection{Serotyping from sequence data}

As some of the operons for the serotypes of $Y$. enterocolitica were either known or were identified during the annotation of the reference genomes, this information was used to perform in silico serotyping on the larger strain collection. This was done using an in-house perl script by Nicholas Croucher. This script is based on BWA mapping (Burrows-Wheeler Aligner; Li and Durbin, 2009), and will count the number of Illumina reads that map to a reference sequence - the operon of interest - and will return a percentage of mapping. This gives a good indication whether a specific operon is present or absent given the divergence of the different operons.

\subsubsection{Phylogenetic reconstruction using 16S rDNA sequence}

The $16 \mathrm{~S}$ sequences were extracted from the Illumina reads by mapping against the BT 1B reference strain 8081 . The sequences were then corrected using iterative correction of reference nucleotides (iCORN) to improve sequence quality (Otto, Sanders, Berriman and Newbold, 2010). Sequences for the previously published isolates of the environmental species as well as Y. pestis and Y. pseudotuberculosis were obtained from the National Center for Biotechnology Information webpage (NCBI; www.ncbi.nlm.nih.gov) and are based on high-quality Sanger sequencing.

Sequences were aligned using Seaview (Galtier, Guoy, and Gautier, 1996) and trimmed for same start and stop of the molecules. A phylogenetic tree was then calculated using the PhyML option in Seaview for a maximum likelihood tree, employing the GTR gamma model with 100 bootstraps.

\subsubsection{Phylogenetic reconstruction including $Y$. entomophaga and $Y$. nurmii}

The newly established species $Y$. entomophaga and $Y$. nurmii were not included in the large-scale sequencing approach. To determine their relationship with the other 
Yersiniae, the deposited sequences for four housekeeping genes recA, hsp60, gyrB, and gInA (Hurst, et al., 2011; Murros-Kontiainen, et al., 2010b) were obtained from the NCBI website. These four genes were included in the 85 housekeeping genes, so this sequence information was extracted for the worldwide strain collection. The genes were concatenated into a superlocus and aligned using Seaview. Again, a phylogenetic reconstruction using PhyML in Seaview was carried out. 


\section{Sequencing and Analysis of the Reference Genomes for Y. enterocolitica}

\subsection{Introduction}

At the start of the project, only one genome sequence for the $Y$. enterocolitica highpathogenic biotype 1B had been published (Thomson, et al., 2006). This strain 8081 was isolated from a fatal human septicaemia case in America in 1980 (Portnoy, Moseley and Falkow, 1981). More recently individual genomes of other BTs have been published, and compared to the reference genome of BT 1B (Batzilla, Heesemann and Rakin, 2011; Fuchs, Brandt, Starke and Rattei, 2011; Wang, et al., 2011; Batzilla, et al., 2011a,b). New regions described are a novel T3SS, potential membrane transporters and associated metabolic pathways, a toxin complex pathogenicity island and other potential toxin clusters, and a second flagella cluster Flag-2 (Wang, et al., 2011; Fuchs, Brandt, Starke and Rattei, 2011).

Currently absent from the analyses are BT 5 strains. A comprehensive comparison of "all against all" is also still outstanding but necessary to define the "enterocolitica" core and the accessory genes that define the biotypes. A comparison between the biotypes is also required to determine whether the regions identified as unique for individual strains compared to BT $1 \mathrm{~B}$ are the same as those described in the other studies and whether the regions are indeed functional or show pseudogene acquisition when compared with each other. The genotypic and phenotypic heterogeneity of $Y$. enterocolitica justifies further in-depth comparison non-, low- and high-pathogenic strains with each other. Strains in this study were chosen as representatives based on the prevelance of bioserotypes in the UK in humans and animals (McNally, et al., 2004; Tompkins, et al., 1999) and have been characterized genotypically and phenotypically (Howard, et al., 2006; Thomson, et al., 2006; McNally, et al., 2006).

Sequencing of representative strains for common $Y$. enterocolitica bio-serotypes of non- and low-pathogenic lineages should allow us to identify general characteristics of a $Y$. enterocolitica genome. The advantage of whole genome sequencing is that it gives information about genome organization and topology. It also not only identifies new regions but gives information on whether shared regions are orthologous, that is of the same evolutionary origin and thus conserved in terms of genomic context, or are paralogous, that is if convergent evolution took place. A comparison of all isolates should give a full representation of the $Y$. enterocolitica species. 


\subsection{Results}

3.2.1 Sequencing, assembly and sequence improvement of the

\section{Y. enterocolitica biotypes genomes}

The genomes of six Yersinia enterocolitica strains $(1203,5603,5303,21202,14902$, 3094; Table 2.1) were sequenced using multiple sequencing technologies. The work was carried out at the core genome sequencing facilities at the Wellcome Trust Sanger Institute (WTSI), Hinxton, UK. The sequencing process, assembly and improvement are outlined in Figure 3.1.

Initially, sequencing started on five representative strains (5303, 21202, 5603, 14902, 1203) of the predominant bio-serotypes $1 A$ and $2-4$ in the UK. The first round of sequencing was carried out using 454/Roche GS20/FLX shotgun pyrosequencing using $3 \mathrm{~kb}$ DNA fragment sizes. This produced in excess of 30 -fold coverage of each genome. Polymerase chain reaction (PCR) products were designed to span gaps in contigs and sequenced to close gaps. This was automated by using algorithmic based automatic contiguation of assembled shotgun sequence (ABACAS; Assefa, et al., 2009), which was under development at the time. This process was supervised and carried out by Danielle Walker at the WTSI. The de novo assemblies for strains 5303 and 1203 were produced using the Newbler software (454 Life Sciences, Roche). Initial analysis and annotation was started at this stage. 

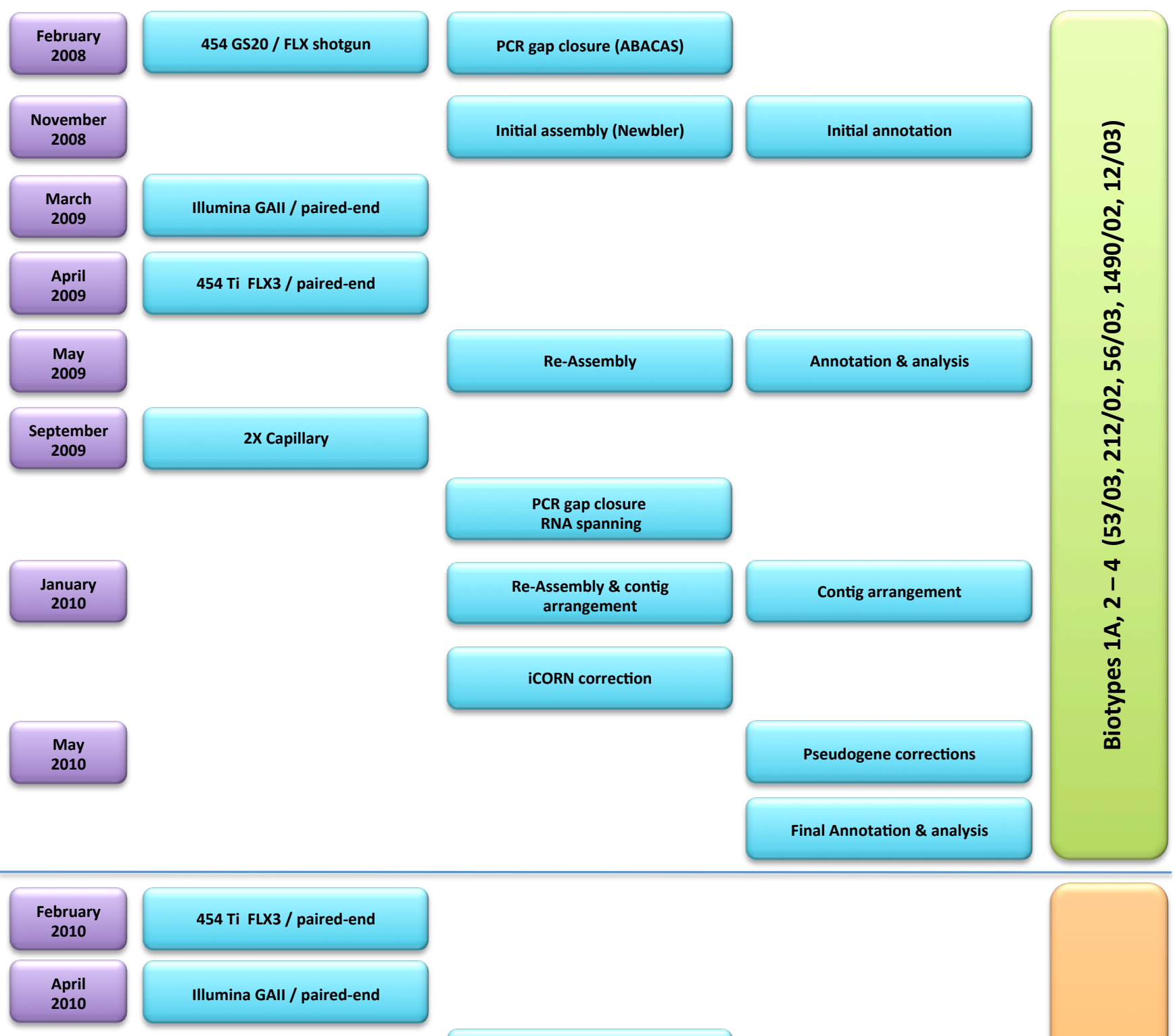

\section{May}

2010

August

2010
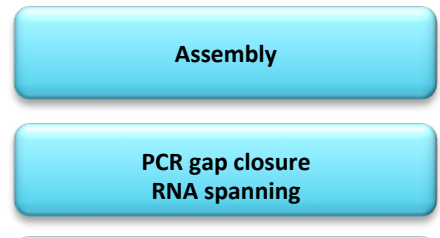

Assembly \& contig arrangement

Contig arrangement

iCORN correction

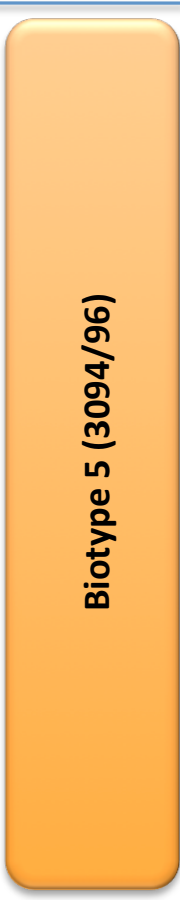

Pseudogene corrections

Final Annotation \& analysis

Figure 3.1: Sequencing timeline for the Y. enterocolitica reference genomes. The timeline is presented on the left and contrasted with the sequencing progress. The work carried out by Danielle Walker and Sandra Reuter is highlighted. The first five reference genomes are split from the last genome due to difference in sequencing progress. 
Analyses were hampered by the fact that some regions of difference compared to the reference genome 8081 were at the end of contigs, thus making it difficult to assess the genomic location as well as the completeness of that region. Also, each of the genomes was present in more than 300 contigs. This was due to a large number of IS elements and repeats as well as several copies of the rRNA operon that are not spanned by a single-ended sequencing technique. The newly sequenced genomes appear o have the same genome architecture as the reference genome 8081 , as the large number of contigs can be arranged in a co-linear fashion against the reference (Figure 3.2). The non-pathogenic BT 1A strain 5303 shows the largest genome size. Some gaps are visible which might be due to lower homology hits being filtered out.

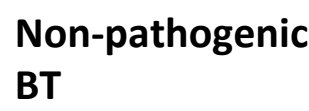

High-pathogenic BT

\section{Low-pathogenic} BT
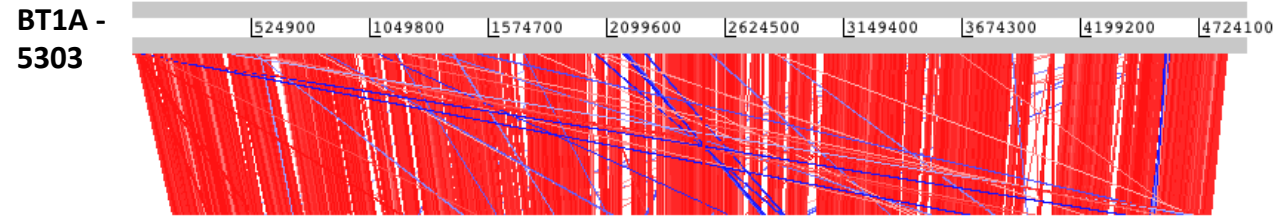

BT1B 8081

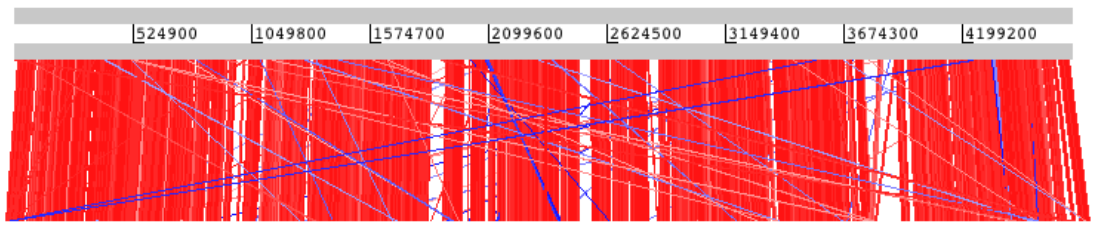

BT2 -

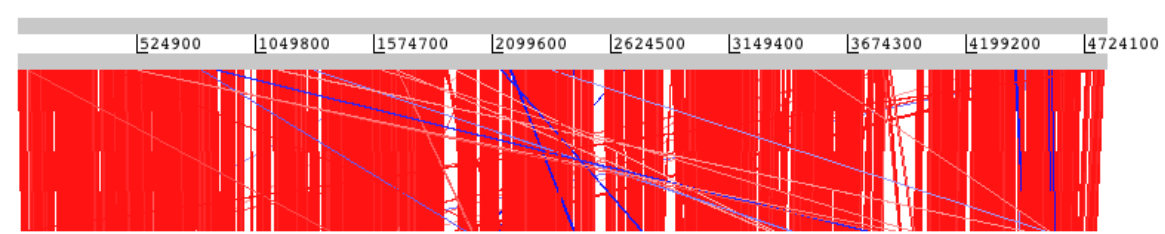

BT3 -

5603

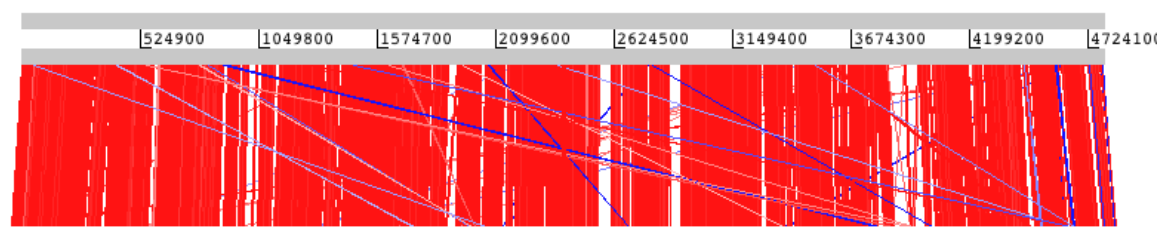

BT3 -

14902

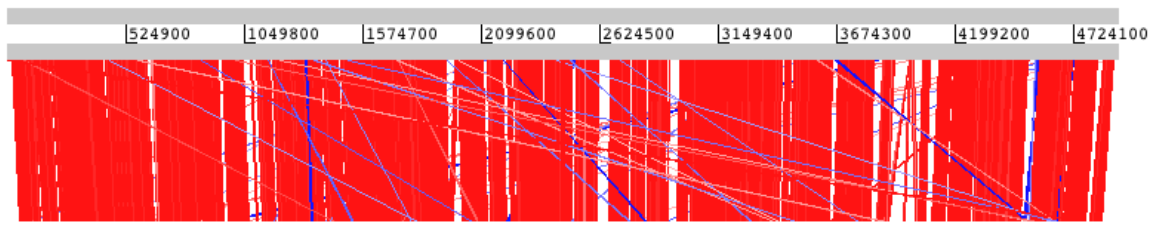

BT4 -

1203

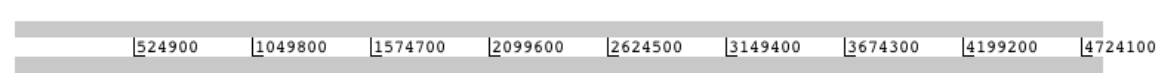

Figure 3.2: Initial assembly based on single-end 454 reads and comparison of the six Y. enterocolitica reference genomes. Genomes are compared in a pair-wise fashion. Red bars - colinear homology >999 bp, blue bars - inverted homology >999 bp. Due to the large number, contigs are not highlighted.

It was then decided to undertake further sequencing. 454/Roche GSFLX Titanium (paired-end) was used to generate sequence in excess of 30-times coverage. It also 
used $3 \mathrm{~kb}$ DNA fragment sizes, but employed long-read chemistry and paired-end technology (454 Life Sciences, 2008), which had not yet been introduced into the WTSI production pipeline when the first round of pyrosequencing had been carried out. To further improve the assemblies, the five strains were sequenced by more conventional Sanger sequencing using big-dye terminator chemistry on $A B I 3730$ automated sequencers to approximately 2-fold coverage from pOTW12 (insert size 5-6 kb) and pMAQ1Sac_BstxI (insert size 9-12 kb) genomic shotgun libraries. These subsequent rounds of sequencing with the additional information on the distance of both paired-end reads and large insert size libraries were used to help link contigs. Thus the total number of contigs was reduced, as was the chance of mis-assemblies, because unambiguous placement of physically linked fragments was possible. Finally to improve accuracy of the sequence and reduce the possibility of generic errors of 454/pyrosequencing in homopolymeric tracts further sequence was generated using paired-end Illumina sequencing platform on the Illumina GAll Analyser with standard 200-300 bp fragments and 37 cycles of sequencing to give a coverage with just this technology in excess of 60 -fold coverage. Danielle Walker again supervised this process.

The Illumina data was assembled, using the de novo Velvet assembler (Zerbino and Birney, 2008) with a kmer length of 31 for the 37 cycle runs. The consensus was then extracted and artificially broken down into $500 \mathrm{bp}$ fragments. The GS De Novo assembly software (Roche, 2011) was used to assemble the 454 reads, capillary reads and the artificially fragmented velvet consensus as well as a round of automated PCR reactions designed on the 454 scaffolds using ABACAS. This was then converted for viewing and analysis in the STADEN - Gap4 software package (Bonfield, Smith and Staden, 1995).

The assembled consensus sequence for strains 5303 and 1203 was annotated and this analysis was also used to inform the gap closure process. With additional PCRbased gap closure and sequencing using a primer walking approach especially over large repeat regions such as rDNA operons, Danielle Walker was able to close gaps and scaffold large regions. However, it was still not possible to close all gaps and so for the 16S operons the consensus sequence of BT 1B strain 8081 was used. Finishing this part of the reference genomes was not a priority, and would have been too resource-extensive.

In addition to the gold standard, finished BT 1B genome, only the genome for BT 1A could be contiguated that is assembled into only one large contig. For all other 
reference genomes the sequencing was finalized at a point were all the genomes were present in less than eight contigs (Table 3.1). The IS elements present had a great impact on the ability to assemble the genomes because IS elements can be present as multiple perfect repeats that are usually around 1,000 bp long and thus not spanned from unique sequence to unique sequence by paired-end sequencing reads. The number of IS elements varied between the biotypes with BT1A having the smalles number of IS elements and the low-pathogenic BT possessing a larger number. In addition there are 3-8 rRNA operons in the sequenced reference genomes. This also impacted on the assemblies, as the operons present a large repeat region that has recombinatorial potential.

The final assemblies of the low-pathogenic BT genomes were based both on information on rRNA operons linking contigs as well as on comparisons towards both $\mathrm{BT} 1 \mathrm{~B}$ and $\mathrm{BT} 1 \mathrm{~A}$. As comparison towards $\mathrm{BT} 1 \mathrm{~A}$ and $\mathrm{BT} 1 \mathrm{~B}$ yields different contig orders, a preference was given towards BT 1A. This biotype contained the least number of IS elements and repeats, and thus gave the impression of having undergone less genome rearrangement and presenting an ancient lineage. Table 3.1 gives the contig order and the basis for that particular order.

Table 3.1: Contig ordering in the Y. enterocolitica reference genomes for BT 1A, 2 - 4.

\begin{tabular}{|c|c|c|c|}
\hline Strain & Contig \# & Size $[b p]$ & Information \\
\hline 5303 & 1 & $4,940,199$ & Contiguated, cut for same start as 8081 \\
\hline \multirow[t]{8}{*}{21202} & 1 & 331,132 & Cut for same start as 8081 \\
\hline & 2 & 34,537 & Flipped to fit with 5303 \\
\hline & 3 & 106,274 & Joins with \#4 by read pair, both flipped to fit with 5303 \\
\hline & 4 & 485,939 & Joins with \#3 by read pair, both flipped to fit with 5303 \\
\hline & 5 & $2,189,202$ & \\
\hline & 6 & $1,011,144$ & Joins with \#7 by read pair \\
\hline & 7 & 6,554 & Joins with \#6 by read pair \\
\hline & 8 & 400,227 & First half from contig \#1 \\
\hline \multirow[t]{8}{*}{5603} & 1 & 19,724 & Cut for same start as 8081 , flipped to fit 5303 \\
\hline & 2 & 143,745 & \\
\hline & 3 & 197,661 & \\
\hline & 4 & 760,938 & \\
\hline & 5 & 776,795 & Joins with \#6 by read pair \\
\hline & 6 & $1,405,148$ & Joins \#5 and \#7 by read pair \\
\hline & 7 & 860,219 & Joins with \#6 by read pair \\
\hline & 8 & 399,855 & Joins with \#1 \\
\hline \multirow[t]{6}{*}{14902} & 1 & 18,110 & Cut for same start as 8081 \\
\hline & 2 & 313,931 & \\
\hline & 3 & 701,464 & Joins with \#4 by read pair \\
\hline & 4 & 360,673 & Joins with \#3 by read pair \\
\hline & 5 & 344,361 & \\
\hline & 6 & $2,857,874$ & Joins with \#1 \\
\hline \multirow[t]{4}{*}{1203} & 1 & 402,778 & Cut for same start as 8081 \\
\hline & 2 & 4,317 & Based on read pairs \\
\hline & 3 & $1,725,059$ & Based on read pairs \\
\hline & 4 & $2,395,791$ & Based on read pairs \\
\hline
\end{tabular}


The $6^{\text {th }}$ strain sequenced in this study, BT 5 strain SZ3094/96 (short: 3094), was sequenced using the 454/Roche GSFLX with long-read GS FLX Titanium chemistry to approximately 25 -fold coverage using $8 \mathrm{~kb}$ DNA fragment sizes. Sequence was also generated using the Illumina sequencing platform on the Illumina GAll Analyser to approximately 200-fold coverage with standard 200-300 bp fragments and 54 cycles of sequencing. A de novo assembly was done for the lllumina reads using Velvet (Zerbino and Birney, 2008) with a kmer length of 45 for the 54 cycle run. Again the consensus was extracted, broken down into 500 bp fragments, and combined with the 454 reads using the GS De Novo assembly software (Roche, 2011). Endsequenced PCR products were used to close gaps and scaffold large repeat regions. This strain was not analyzed until it was in less than 10 contigs, and assembly was based both on information on rRNA operons linking contigs as well as on comparison to both BT 1B and BT 1A. Table 3.2 gives the contig order.

Table 3.2: Contig ordering in the $Y$. enterocolitica reference genome for BT 5.

\begin{tabular}{llrl}
\hline Strain & Contig \# & Size $[\mathrm{bp}]$ & Information \\
\hline \hline 3094 & 1 & $1,118,114$ & Cut for same start as 8081 \\
& 2 & 36.977 & \\
& 3 & 262,567 & \\
& 4 & 27,479 & \\
& 5 & $3,220,765$ & \\
\hline
\end{tabular}

The final assemblies of the reference genomes after gap closure and corrections can be seen in Figure 3.3. There are striking differences to the initial assemblies of the 454/single-end run (Figure 3.2). Each strain shows several extensive inversions and even translocations compared to other strains. The assemblies have been trimmed for a common start, and thus share beginning and end, but apart from that no common theme of genome organization is visible. The genome rearrangements are visible within contigs, not only at the end of contigs, thus can be considered genuine. The differences to the initial assemblies can be explained partially by the reduced number of contigs in the final assembly. The initial assemblies were missing information on read-pairs. Not only did this result in a more fragmented assembly, the short contigs were then aligned and orientated according to the reference genome as information on linking contigs was missing. These mis-assemblies thus give the impression of co-linear organization of the reference genomes visible in the original comparison (Figure 3.2). 


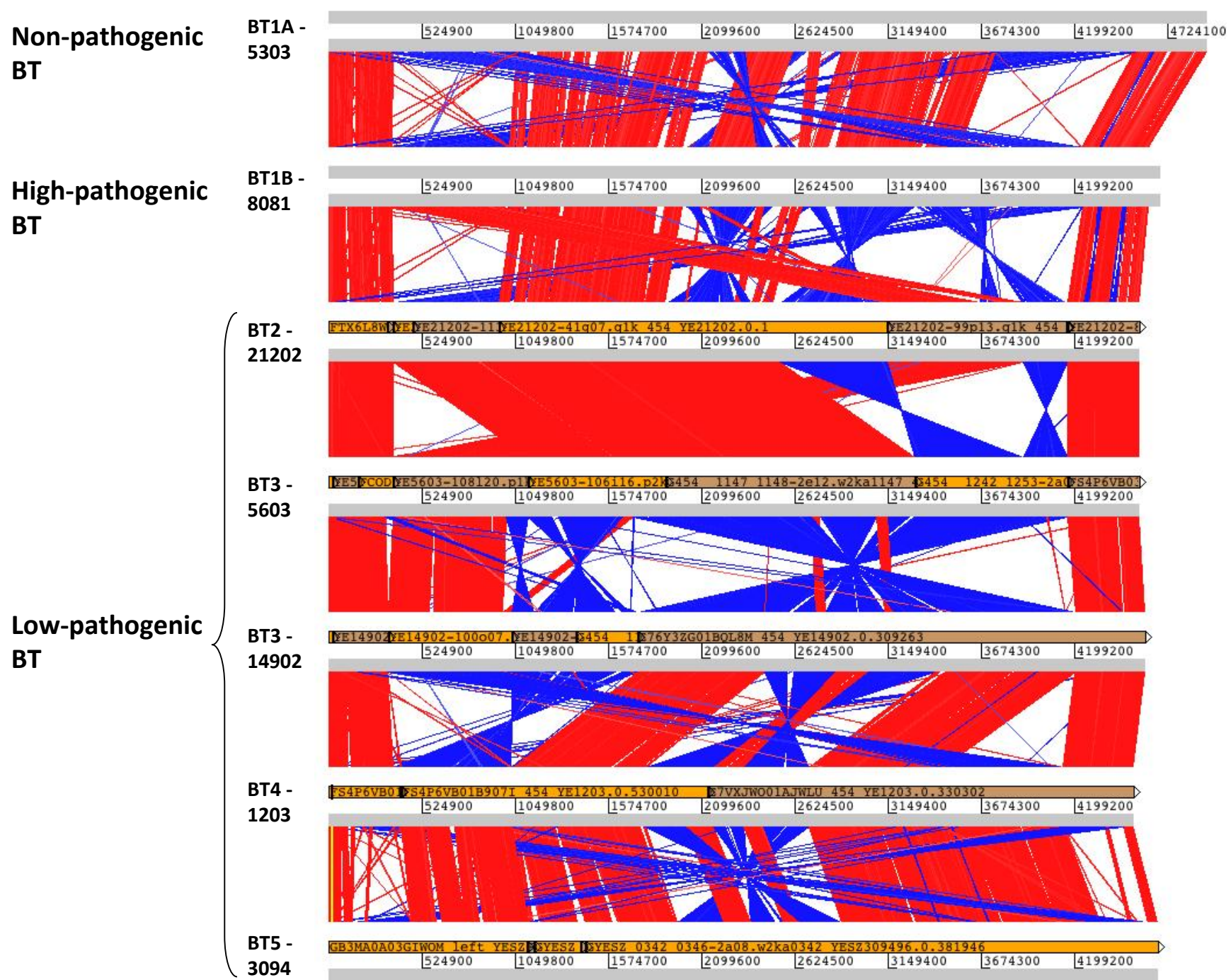

Figure 3.3: Pairwise comparison of final assemblies after gap closure of Y. enterocolitica reference genomes. Red bars - homologous, co-linear matches $>1,200 \mathrm{bp}$, blue bars - homologous, inverted matches $>1,200 \mathrm{bp}$, alternating orange/brown - contig borders. The non- and highpathogenic BTs both consist of only one single contig.

Sequencing of the virulence plasmid pYV contained in BTs $2-5$ as well as sequencing of unique plasmids of individual strains was carried out as for the genome of the respective strain. Danielle Walker and Sandra Reuter both manually designed primers for closing gaps in the plasmids.

Finally all assemblies were corrected using iterative correction of reference nucleotides (iCORN; Otto, Sanders, Berriman and Newbold, 2010). All strains were completed to an Improved High-Quality Draft Standard (Chain, et al., 2009), and consist of a circular genome with several contigs and varying plasmid numbers.

For further sequence improvement, all CDSs with homopolymeric runs resulting in frameshifts and stop codons introduced in the coding sequence (pseudogenes) were 
checked against the original sequencing data for coverage and sequencing technique. If the sequence was questionable in terms of number of reads, or contained data from only one sequencing technique, PCR reactions were designed for improved quality and the sequence corrected accordingly. This was done for all functional classes except those of foreign origin (phage, IS elements) and hypothetical, unclassified proteins. This should present an accurate total number of the pseudogenes as well as give confidence in the nature of the mutations.

During this process, between two and 22 bases were changed in the reference genomes, yielding an error rate beween $0.00004 \%$ and $0.0005 \%$ equivalent to up to 5 wrong base calls in $1,000,000$ bases. This is very much dependent on the sequencing technologies employed. For instance, reference genome YE3094 was sequenced only with pyrosequencing and Illumina technology, and thus has a larger number of errors in the sequence that were identified during subsequent analysis. There is an undetermined error rate with respect to base changes that do not result in immediately visible effects on open reading frames, but judging from the number of changes carried out upon pseudogene inspection it can be assumed to be low.

Other single genomes for $Y$. enterocolitica BT have been made available but vary in sequence quality compared to the reference genomes presented here. Two BT $1 \mathrm{~A}$ strains have been sequenced to draft quality, resulting in 74 and 97 contigs (Batzilla, Heesemann and Rakin, 2011). The published BT 2 0:9 strain W22703 (Fuchs, Brandt, Starke and Rattei, 2011) has been classified as Annotation-Directed Improvement, but gap closure has not been carried out and only one sequencing technique has been employed. Thus the genome is presently in more than 300 contigs, making it difficult to do a full characterization of the genome. The published BT 3 0:9 strain 105.5R(r) (Wang, et al., 2011) might be classified as High-Quality Draft, since two sequencing techniques have been employed and some gap closure has been carried out so that the genome is present in 81 contigs. A detailed analysis of BT 4 O:3 strain Y11 has been published (Batzilla, et al., 2011a,b). Due to gap closure and quality control, this genome may be classified as Improved High-Quality Draft, and so has the same genome standard as the reference genomes generated for this study. 


\subsubsection{Genome annotation}

The individual steps for preparation of a reference sequence for annotation are shown in Figure 3.4.

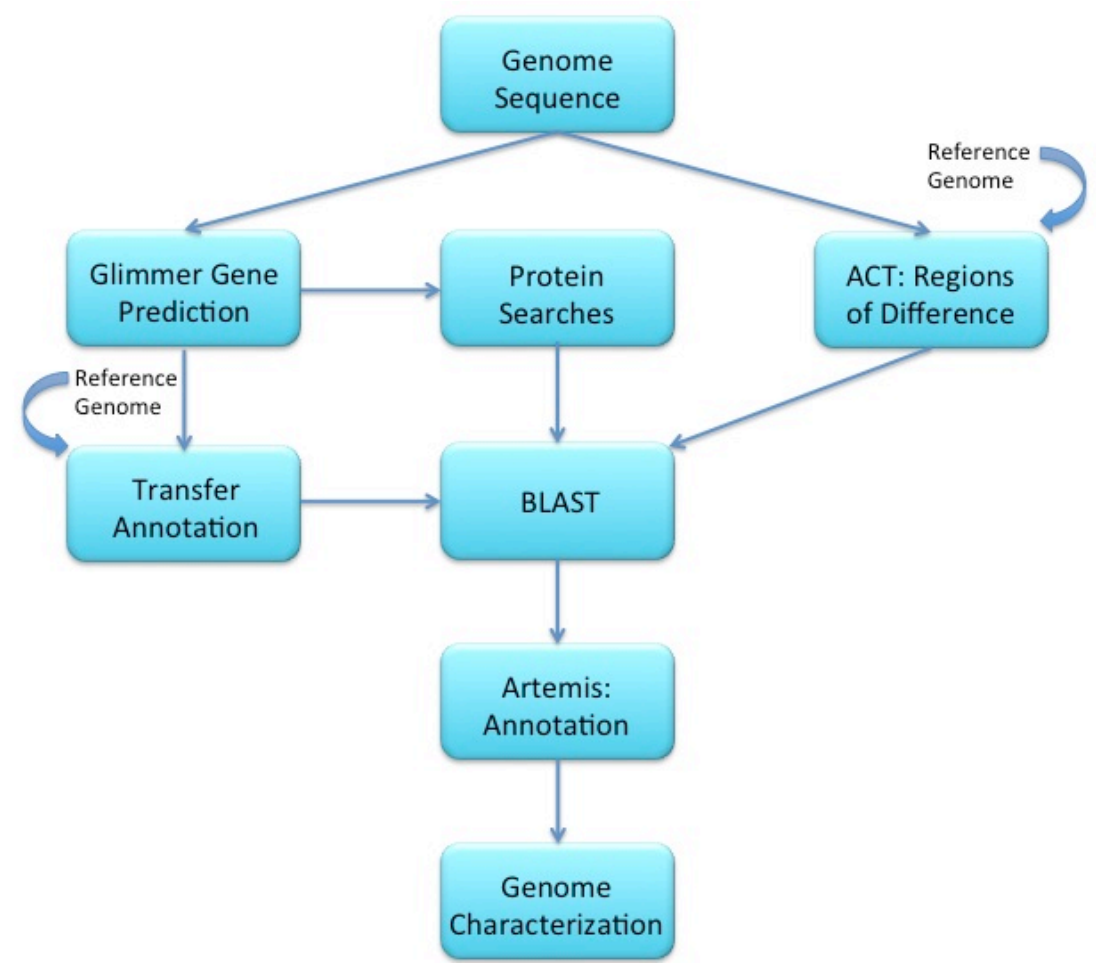

Figure 3.4: Flow chart of the preparation of a genome sequence for annotation and analysis. Gene prediction software is used for finding open reading frames. These are then characterized by protein searches, such as transmembrane domains, signal domains, etc. Annotation is transferred using a reference genome. Both the gene prediction and the glimmer prediction are combined and open reading frames without annotation are blasted and annotated using Artemis. The genome sequence is also visually compared to the reference genome using ACT. Following annotation, the genome can be characterized further with respect to overall composition and orthologous gene sets with other genomes.

First of all, Glimmer 3 was used as a model for prediction of potential open reading frames (Salzberg, Delcher, Kasif and White, 1998; Delcher, Bratke, Powers and Salzberg, 2007). To help characterization of potential new CDSs, searches for transmembrane domains (TMHMM; Krogh, Larsson, von Heijne and Sonnhammer, 2001), signal domains (SignalP; Nielsen, Engelbrecht, Brunak and von Heijne, 1997; Brendtsen, Nielsen, von Heijne and Brunak, 2004), helix-turn-helix motifs (HTH), protein family domains (Pfam; Finn, et al., 2010) and prosite (Sigrist, et al., 2002; Hulo, et al., 2006) were carried out. Using the Artemis Comparison Tool (ACT; Carver, et al., 2005), genomes were compared to the reference BT 1B strain 8081 and to each other to define large-scale regions of difference. The files needed for 
comparing two genomes with each other were generated using an in-house bigger blast script, but can also be obtained from WebACT (www.webact.org).

The reference genomes were annotated by transferring annotation from the reference genome BT1B strain 8081. This script compares the reference genome to the subject genome and transfers annotation to CDSs that have more than $90 \%$ BLAST identity. The Glimmer prediction and the annotation transfer were combined, and unique regions received new manual annotation using Artemis (Berriman and Rutherford, 2003). All CDSs were blasted against prokaryotes using the UniProt knowledgebase (UniProt, 2011). Annotation was equally based on sequence similarity and indications for protein function.

Annotation was also transferred from one annotated genome to the other, since regions were identified that were absent in $\mathrm{BT} 1 \mathrm{~B}$ strain 8081 , but shared in the other genomes. The annotation transfer was also employed when transferring annotation from old sequence versions to updated assemblies.

For annotation, each CDS is given a set of qualifiers for identification. Apart from a unique locus tag or a systematic ID that is allocated automatically to each CDS, each CDS is characterized with a product, potentially a gene name if there are wellcharacterized homologs, a class and a colour. Class and colour qualifiers both reflect the product, but allow easier classification of CDSs into groups (see Table 9.3, p. 209 , and 9.4 , p. 213, in appendix). The 14 colours used reflect the function and subcellular location of a protein, e.g. green proteins are associated with the cell wall and membrane, such as permease or lipoproteins, whereas black proteins are involved in carbon energy metabolism. The class system fulfils a similar purpose, but breaks down the functions in more detail. Thus an exported protein may be classified as being in the inner membrane, or a protein involved in carbon metabolism can be allocated to anaerobic respiration. The product will very specifically give the protein name and function, but the class / colour system allows it to bring proteins of different names together under a common denominator.

Notes were added for further explanations if necessary, and homologs from other bacterial species were added.

Pseudogenes are defined as carrying mutations that would prevent the expression of the gene or would alter the nature of the resultant protein. This includes truncations of $\mathrm{N}$ and/or $\mathrm{C}$ termini, deletions of other parts of the gene, stop codons introduced 
within the coding sequence and frameshifts. All pseudogenes except IS elements and hypothetical proteins were checked for sequence quality and confirmed as described above. IS elements were excluded as they are repetitive. Their function also might not only be in the expression of the transposase itself, but also lies in recombinatorial potential with the same class of IS element in other parts of the genome, which is independent of a functional transposase. Due to the large number of IS elements, their exclusion from confirming pseudogenes can be justified. Similarly, hypothetical proteins were excluded, as their transcription and function is unknown. Pseudogenes were annotated in a different way with labelling the product as pseudogene, partial, or remnant, stating in a note the nature of the mutation, setting the colour to 11 (brown) and adding the qualifiers "/pseudo" and "/partial" where applicable. This gives a set of filters that allows for easy identification of pseudogenes within a given set of CDSs. Pseudogenes are also made to end on the codon before the stop codon ("taken off the stop codon"). These modifications aid in downstream analysis of the genomes and simple tracking of pseudogenes.

RNA searches using Rfam (Griffiths-Jones, et al., 2003; Gardner, et al., 2011) were performed to establish the location of the RNA components. The location of the $16 \mathrm{~S}$ and $23 S$ rDNA operons was established in comparison with the reference genome of BT 1B strain 8081.

The resultant set of CDSs in the reference genomes was then used for detailed gene-by-gene annotation and analysis.

\subsubsection{Genome comparison}

For the six biotypes, it was not only interesting to compare strains in a pairwise fashion, but it was more important to see which sets of CDSs might characterize more than two biotypes. For this, orthologous gene sets were identified by reciprocal FASTA searches. This approach is shown in Figure 3.5. 


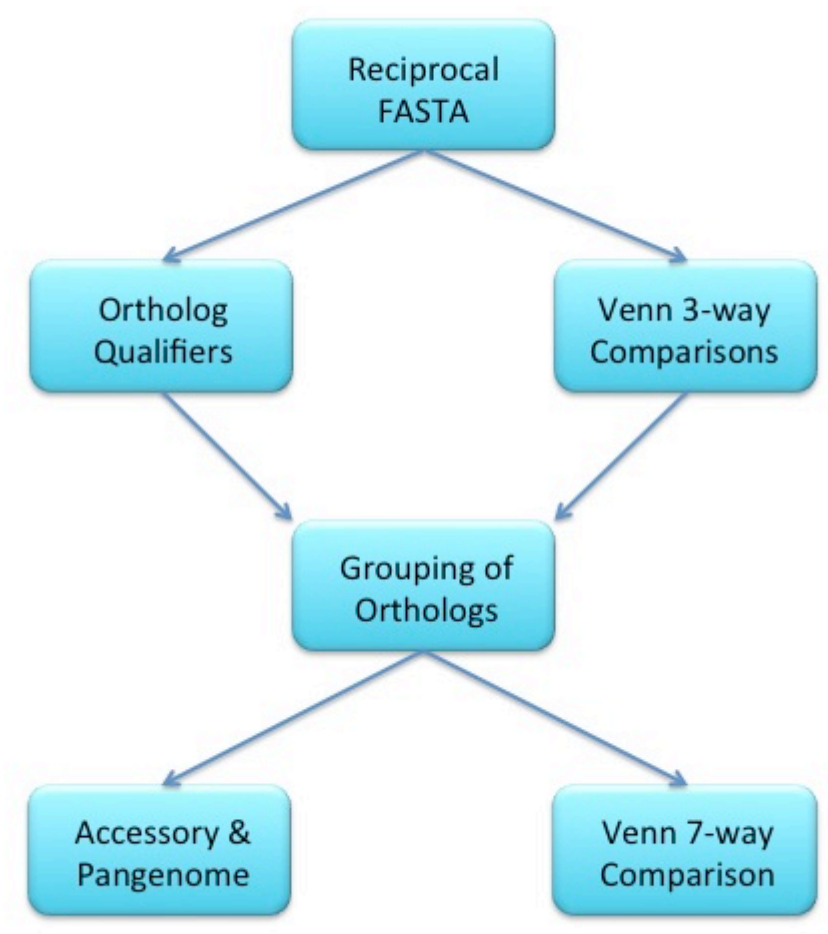

Figure 3.5: Identifying sets of orthologous CDSs. Reciprocal FASTA searches are carried out, the orthologs are combined in a file and manually curated using 3-way Venn comparisons. The identified orthologs can then be grouped. This establishes pan- and accessory genome and allows for a 7-way Venn comparison.

Only those pairs of homologous CDSs were retained for further analysis where the predicted amino acid identity was $\geq 40 \%$ over $80 \%$ of the protein length. These genes were then subjected to manual curation using gene synteny to increase the accuracy of this analysis. 3-way Venn diagrams were employed to facilitate manual curation. This curation is specifically needed for paralogous gene families such as IS elements, which can be numerous in the genome. Sequence divergence can inhibit direct identification of CDSs as orthologs but looking at genome synteny can help identify these. Truncated genes might also not be identified in the automated analysis as their length and identity can fall below the cutoff. This strategy was applied to pairwise comparisons of the genomes of all sequenced biotypes. These identified orthologs were then combined together with the annotation file.

\subsubsection{General genomic features of the reference genomes}

The Table 3.3 gives a general overview of the properties of the sequenced reference genomes for each of the biotypes of $Y$. enterocolitica. These basic figures can be used to characterize genome composition. 
Table 3.3: General properties of the sequenced reference genomes of $Y$. enterocolitica

\begin{tabular}{|c|c|c|c|c|c|c|c|}
\hline Property & $\begin{array}{l}\text { BT 1B } \\
0: 8 \\
(8081)^{*}\end{array}$ & $\begin{array}{l}\text { BT 1A } \\
0: 5 \\
(5303)\end{array}$ & $\begin{array}{l}\text { BT } 2 \\
0: 9 \\
(21202)\end{array}$ & $\begin{array}{l}\text { BT } 3 \\
0: 9 \\
(5603)\end{array}$ & $\begin{array}{l}\text { BT } 3 \\
0: 5,27 \\
(14902)\end{array}$ & $\begin{array}{l}\text { BT } 4 \\
0: 3 \\
(1203)\end{array}$ & $\begin{array}{l}\text { BT } 5 \\
0: 2 a, 2 b, 3 \\
(3094)\end{array}$ \\
\hline Genome size (bp) & $4,615,899$ & $4,940,199$ & $4,565,009$ & $4,564,085$ & $4,596,413$ & $4,527,945$ & $4,665,902$ \\
\hline $\begin{array}{l}\text { Number of genome } \\
\text { contigs }\end{array}$ & 1 & 1 & 8 & 8 & 6 & 4 & 5 \\
\hline $\begin{array}{l}\text { Plasmid name and } \\
\text { size (contiguated) }\end{array}$ & pYV 67kb & $\begin{array}{l}\text { pSR2-1A 8kb } \\
\text { pSR3-1A 4kb }\end{array}$ & $\begin{array}{l}\text { pYV 75kb } \\
\text { pSR1-2 42kb }\end{array}$ & $\mathrm{pYV} 75 \mathrm{~kb}$ & $\mathrm{pYV} 75 \mathrm{~kb}$ & $\mathrm{pYV} 75 \mathrm{~kb}$ & $\begin{array}{l}\text { pYV 75kb } \\
\text { pSR4-5 100kb }\end{array}$ \\
\hline $\mathrm{G}+\mathrm{C}$ content $(\%)$ & 47.27 & 48.42 & 48.09 & 48.10 & 48.16 & 48.22 & 48.24 \\
\hline Number of CDS & 4,053 & 4,365 & 3,922 & 3,917 & 3,978 & 3,893 & 4,051 \\
\hline Coding density (\%) & 84.7 & 86.7 & 85.9 & 82.7 & 83.3 & 80.7 & 79.8 \\
\hline $\begin{array}{l}\text { Average gene size } \\
\text { (bp) }\end{array}$ & 963 & 965 & 981 & 982 & 975 & 976 & 964 \\
\hline $\begin{array}{l}\text { Total number } \\
\text { pseudogenes }\end{array}$ & 84 & 81 & 157 & 141 & 146 & 235 & 322 \\
\hline $\begin{array}{l}\text { Number of partial } \\
\text { genes }\end{array}$ & 57 & 39 & 96 & 86 & 97 & 126 & 129 \\
\hline Prophage regions & 4 & 6 & 2 & 2 & 3 & 2 & 3 \\
\hline rRNA operons & 7 & 6 & 3 & 3 & 4 & 8 & 7 \\
\hline tRNA loci & 81 & 77 & 72 & 72 & 71 & 72 & 67 \\
\hline IS elements & 60 & 31 & 87 & 85 & 79 & 101 & 110 \\
\hline
\end{tabular}

${ }^{*}$ numbers updated from Thomson et al., 2006

The genome sizes of the reference genomes vary between 4.53 and $4.94 \mathrm{Mb}$, with BT1A showing the biggest genome. Relating to the genome size is the number of CDSs, which is higher in larger genomes. The $G+C$ content of all strains is similar and is about $48 \%$. The coding density lies between $80-85 \%$, with BT 5 having the lowest and BT 1A having the highest coding density. The average gene size across all biotypes was $970 \mathrm{bp}$.

Comparing the reference genomes to the other five published $Y$. enterocolitica genomes, one can see that they are similar to the results obtained here. Limited information is available on the two BT 1A strain (Batzilla, Heesemann and Rakin, 2011), but their genome size estimated at $4.7 \mathrm{Mb}$ and GC content of $47 \%$ are similar to BT 1A strain 5303. Due to their status as draft genomes, a detailed comparison of CDS numbers, pseudogenes, phages, etc. is not possible.

Genome size for BT 2 0:9 strain W22703 (Fuchs, Brandt, Starke and Rattei, 2011) is given as $<4.75 \mathrm{Mb}$, which is in the same range as the $4.56 \mathrm{Mb}$ obtained for the BT 2 O:9 reference strain 21202 . The GC content and coding density of W22703 (46.9\%, $84.4 \%)$ are slightly lower than 21202 (48.1\%, 85.9\%). The number of CDSs in 
W22703 exceeds the number of CDSs in 21202 (> 4,003, and 3,922, respectively), but given the high number of contigs it could be that some CDSs are in fact split onto different contigs. It is more likely though that the higher number of CDSs reflects the larger genome size. It is not possible to compare the number of rRNA operons nor the number of IS elements as these are likely to present the gaps between the contigs. Both BT 2 0:9 strains carry two prophages, but it cannot be assessed whether these present the same prophage acquired by the BT 2 0:9 lineage or independent phage infections.

The genome size, GC content, coding density, and number of CDSs of BT 3 0:9 strain 105.5R(r) (Wang, et al., 2011) are all comparable to the BT 3 O:9 strain 5603. The number of tRNAs are exactly the same (71). The number of rRNA operons in $105.5 R(r)$ is higher than in 5603 ( 7 , and 4 respectively), whereas the number of IS elements is lower than in 5603 (67, and 79 respectively). 85 pseudogenes are reported for $105.5 R(r)$, which is considerably lower than the 146 pseudogenes found in the reference strain 5603. It could be the definition of a pseudogene that causes this discrepancy between the values. The number of partial genes and gene remnants in 5603 is 97 , which is closer to the number stated for $105.5 R(r)$. It could be that Wang et al. (2011), did not consider genes with frameshifts and stop codons as pseudogenes as the complete gene sequence is present.

The genome size and GC content of BT 4 O:3 strain Y11 (Batzilla, et al., 2011a,b) are similar to the reference genome for BT 4 O:3 strain 1203. The number of CDSs $(4,355)$ is higher than for $1203(3,893)$. The coding density thus is higher for $Y 11$ than for $1203(85.0 \%$, and $80.7 \%$ respectively). The number of tRNA and rRNA operons in Y11 (70, and 7 respectively) is close to the numbers present in 1203 (72, and 8 respectively). The number of pseudogenes as obtained from the available NCBI entry (accession number FR729477) is considerably lower for Y11 (92) than for 1203 (235), similar to the discrepancy observed between the two BT 3 strains. Again, it could be due to the definition of a pseudogene, but even only considering the 126 partial genes and gene remnants in 1203 , this number is higher than that for $\mathrm{Y} 11$.

Several plasmids were identified in the reference strains with the virulence plasmid being common to all pathogenic BT. The variations in the virulence plasmid and the unique plasmids found are described in more detail in chapter 5 . 
In summary, the reference genomes are similar in general composition, with an average genome size of $4.6 \mathrm{Mb} \pm 0.13 \mathrm{Mb}$, a GC content of $48 \% \pm 0.3 \%$, and $\sim 4,000$ CDSs. Significant variations arise in the number and distribution of IS elements and pseudogenes.

\subsubsection{General characteristics of reference genomes}

Using the annotation information contained in the assigned class qualifier (see appendix classification scheme table 9.1) for each gene the overall distribution of classes for the whole gene set within the genome could be determined for each of the biotypes (Figure 3.6). The classes of CDSs were combined in 20 different functional groups (see Table 9.1 in appendix).

All bio-serotypes show a similar distribution of CDSs according to class. About a quarter $(23.6-26.0 \%)$ are proteins that have no assigned function or that are hypothetical, unconfirmed open reading frames. The predominant assigned functions are related to transport proteins $(11.7-12.3 \%)$, cell envelope $(14.8-16.6 \%)$, foreign DNA such as phages and IS elements $(6.6-9.4 \%)$, regulation $(6.5-6.9 \%)$, and macromolecule synthesis $(5.7-6.0 \%)$. 


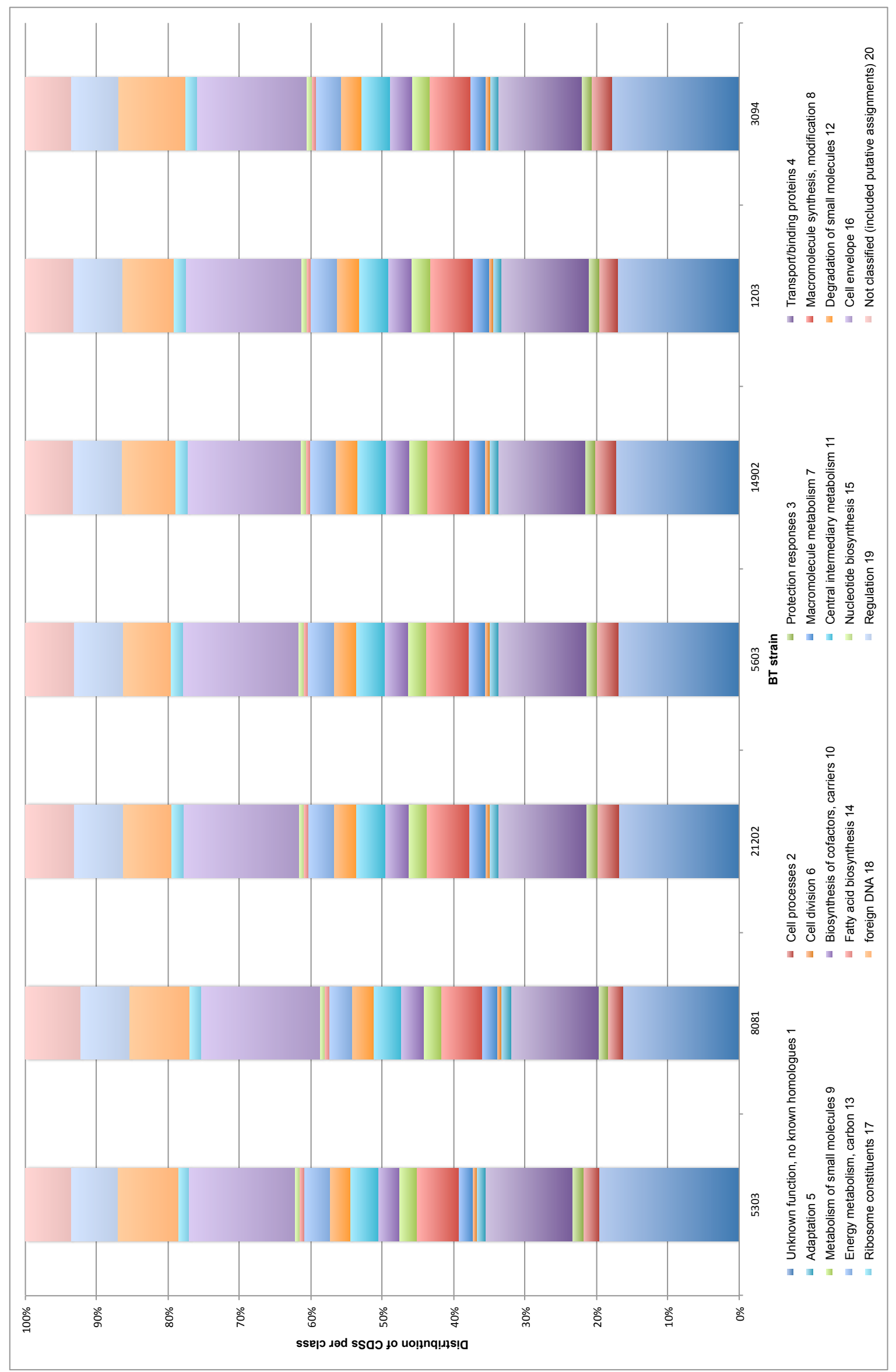

Figure 3.6: Distribution of classes amongst CDSs of all reference genomes. The information on class of a CDS was extracted and individual classes were combined in 20 different functional groups. The functional groups represent the spectrum of activity that can be performed by a bacterial cell. The CDSs in each group were combined to give a percentage of the total number of CDSs. 


\subsection{Discussion}

\subsubsection{Generation of Improved High-Quality Draft genome sequences}

The generation of the reference genomes in this study tracked the technological advances in next-generation sequencing technologies.

The initial sequencing and analysis was based on a single-end run of pyrosequencing. Although the genome could be assembled into contigs, and gave an approximate size for each genome, the assembly was incomplete and often the interesting regions of difference were at the end of contigs making it difficult to determine the true nature of for example pathogenicity islands or to make statements on the completeness of an operon.

One factor that made assembly of the Y. enterocolitica genomes difficult was the presence of large numbers of perfectly repeated IS elements, especially IS1667, in the biotype genomes. At the start of sequencing it was not known that this insertion sequence element was present in over 30 copies in each of the low-pathogenic BT. Other IS elements are also present, plus several copies of the 16S and 23S rRNA operons, which also represent perfect extended repeat regions. Similar numbers of IS elements are also present in the previously sequenced BT 1B strain 8081 as well as in Y. pseudotuberculosis and Y. pestis (Thomson, et al., 2006). Each Yersinia genome contains 6-7 copies of the rRNA operon, and $Y$. pestis has on average more than 100 IS elements of varying classes. Using Y. pestis as an example, Pop and Salzberg (2008) hypothesize that sequencing the plague bacterium with short reads would result in obtaining data for only $93 \%$ of the genome, with a very fragmented assembly. The length of the read will not be able to bridge the repetitive elements, thus the importance of linking information on either side of the repeat reads is stressed (Pop and Salzberg, 2008). In particular de novo assembly is impossible without read-pair information (Chaisson and Pevzner, 2007; Chaisson, Brinza and Pevzner, 2009). Chaisson, Brinza and Pevzner (2007) could even show that the information on read-pairs is more important than an increase in read length.

As a result of this it was then decided to initiate further sequencing. Both Illumina and 454 technologies by that time had the possibility to perform paired-end runs, thus giving additional information on contig linking and scaffolding of sequence reads.

A low coverage Sanger sequencing run was also performed as this was the technology with longest reads and established paired-end technology, thus it was 
hoped to improve sequence quality and assembly. As was later seen in the sequencing of BT 5, this would have been redundant, as a combination of paired-end pyrosequencing and paired-end Illumina sequencing produced the same result. The knowledge on read-pairs was sufficient enough in this case to assemble BT 5 to a high quality. Gap closure was still needed as with the other genomes, but a Sanger sequencing run was not necessary.

Similar findings on the assembly of genomes from next-generation sequencing data have been shown before. In one early study, pyrosequencing was combined with Sanger sequencing (Goldberg, et al., 2006). At this point only single-end reads were available for 454 sequencing as the paired-end protocol was to be released in early 2008 (454 Life Sciences, 2008). They found that pyrosequencing data would supplement $5 X$ coverage Sanger sequencing in terms of increasing coverage, and gap reduction. The pyrosequencing reads also reduced regions with poor sequencing due to avoiding amplification bias that is giving in cloning into a vector (Goldberg, et al., 2006). Thus an even coverage of the genome is given. The Sanger sequence, on the other hand, would have balanced the problems of pyrosequencing in homopolymeric runs.

A later study (Aury, et al., 2008) investigated the combination of Sanger, pyro- and Illumina sequencing. They found that with paired-end 454 technology and Illumina sequencing a Sanger sequencing run is dispensible. They suggest using pyrosequencing with paired-ends as the first strategy for assembly due to it having the longer reads. The Illumina reads are then projected or mapped onto the 454 scaffold and can be used to correct the errors in homopolymeric runs. In this way it is possible to circumvent the use of expensive Sanger sequencing (Aury, et al., 2008). The implication of using several sequencing technologies to balance the individual faults of each is also approached by Harismendy, et al. (2009). They compared error rates, coverage, and variant discovery of 454, Illumina, SOLiD, and Sanger technology in the human genome. They found that although the next-generation sequencing platforms produce large amounts of data, not all of it passes the quality filter, and not all of the data passing the quality control will actually map back to the target genome. They suggest taking this into account for coverage calculation. The coverage, although high, can also vary depending repeat sequences in the target sequence. The next-generation technologies showed variation in coverage, but the non-uniform coverage was different in the three technologies compared 
(Harismendy, et al., 2009). Thus a combination of technologies might counteract the coverage variation of each other. They also found that the sequencing accuracy is high in all next-generation platforms. Pyrosequencing had the lowest false positive rate for variant discovery, whereas the lowest false negative rate was for Illumina technology.

The reference genomes created can be classified as Improved High-Quality Draft Standard according to Chain, et al. (2009). They confirm with manual and automated gap closure, reduction of number of contigs and lowered possibility of misassemblies. In case of BT $1 \mathrm{~A}$ strain 5303, the genome could even be contiguated into a single molecule. Misassemblies for the low-pathogenic BT genomes are possible, but in comparing them to both contiguated genomes attempts were made to find the most likely assembly.

All strains have an unresolved tandem repeat in the Orthologs of YE2684. The repeat consists of six amino acids PTDTPE corresponding to CCG ACC GAT ACG CCA GAG base sequence at the $\mathrm{C}$-terminal end. This gene encodes a conserved hypothetical protein, so that at the current stage further sequencing was deemed unnecessary.

In contrast to a finished, gold standard genome the reference genomes generated have an unknown error rate, and bases were only manually re-checked for sequence quality in apparent pseudogenes.

\subsubsection{General genomic composition of a $Y$. enterocolitica genome}

Detailed manual annotation of the reference genomes gives confidence in further downstream analysis. The general features of the biotypes are very similar with respect to GC content, genome size and number of CDSs, as well as coding density and gene size. The main differences can be seen in the number of pseudogenes which is highest in BTs 4 and 5, and in the number of IS elements which is generally higher in the low-pathogenic BT but again highest in BTs 4 and 5.

Comparison to other published genomes also showed that general composition is similar and that the main differences lie in the number of pseudogenes. As mentioned before, the differences in pseudogenes observed between the BT 3 0:9 and the BT 4 O:3 strain to the reference strains generated in this study could be due to the definition of a pseudogene. It could be that the other studies did not include 
genes with a frameshift or an introduced stop codon in the category of pseudogene as technically the whole reading frame is still present.

The differences in the number of pseudogenes might also arise due to the difference in pseudogene analysis. Once the phylogenetic relationship between the Y. enterocolitica biotypes was established (Chapter 7 ), the pseudogenes were in fact re-examined and compared. The evolutionary relationship gives putative information and a trajectory on loss versus acquisition events of regions, aiding in pseudogene identification. In this way, even small gene fragments were identified such as a 26 aa remnant of $f o c B$ in the low-pathogenic BTs. Automatic gene prediction software miss these small fragments as they fall below the length cut-off. Their correlation score which relates the amino acid composition to the translation of the three reading frames is probably also lowered. Additionally, the comparative analysis of all BT helped to establish whether genes in BT 1B strain 8081 might possibly be new pseudogenes as compared to the other BTs. These detailed analyses contributed to an increase in the number of pseudogenes. The newly created genomes would need to be compared in depth to the reference genomes of this study to compare which genes exactly are affected and how, if the genes are orthologous, as well as which pathways might be deactivated, in order to establish a true set of pseudogenes for each lineage. A larger strain set will also help to distinguish between strain-specific, niche-specific, and lineage-specific pseudogene distribution.

The variability detected with respect to number of pseudogenes might also reflect differences in niche adaptation. In case of the BT 4 strains it can be said that $Y 11$ is a representative of continental European strains, which is the predominant bioserotype isolated of Y. enterocolitica. On the other hand, strain 1203 is a representative of UK strains. In the UK, the predominant strains isolated are BT 3 O:9 and BT 4 O:3 (McNally, et al., 2004). There is no increased prevalence of a single bio-serotype. The higher number of pseudogenes in the UK isolate might thus reflect decreased survival fitness. A quick comparison of the two strains did not reveal any large difference in genomic content, thus extensive acquisition of virulence factors in the European isolate can be excluded.

The overall comparison of gene functions of the reference genomes is very similar. The predominant functional classes are hypothetical, unknown proteins, transport proteins, proteins involved with the cell envelope, regulation, and macromolecule synthesis as well as DNA of foreign origin. 
One could have expected to see an increase in protection responses, adaptation, or DNA of foreign origin in the high-pathogenic BT. These functional classes include proteins such as cell killing, detoxification, drug sensitivity, adaptation to atypical conditions, iron storage, and pathogenicity island-related functions. Such functions could be considered to signify "virulence genes" which could explain the increased virulence potential of high-pathogenic strains. In the overall comparison, this does not seem to be the case, so a closer look into absent, present, and shared regions between the biotypes is necessary and will be done in Chapter 4 . 


\section{Comparative analysis of the $Y$. enterocolitica biotypes}

\subsection{Introduction}

As seen in the previous chapter, the general composition of the Y. enterocolitica genomes are different. Certain genomic islands might only be shared in some lineages, such as the HPI in high-pathogenic BT 1B.

The aim of this chapter is to define sets of orthologous gene clusters in the biotypes and to identify biotype-specific marker regions, thus determining the genetic make-up of each bio-serotype and see how this relates to the pathogenic potential.

The main differences observed thus far relate to the number of pseudogenes and partial genes as well as the number of IS elements. Hence, the aim is also to investigate the nature of the pseudogenes to determine in which genes they occur, and to establish the distribution of IS elements. 


\subsection{Results}

\subsubsection{The core- and accessory genome of Y. enterocolitica}

In order to determine which CDSs or regions are shared among the biotypes, the ortholog qualifiers were taken to group each CDS on the basis of number and combination of orthologs in the other strains. The resulting matrix is listed in Table 9.3 in the appendix and was used as the basis for representing the accessory genes shared in different BTs in a 7-way Venn diagram, which was designed in collaboration with Dr Timothy Hetherington, NTU. The information obtained was also used to characterize the core- and accessory genome of the species Y. enterocolitica.

For the sets of orthologous gene clusters, an in-house script was used (see Appendix 9.5 , p. 214). This script takes one genome at a time and determines which genes have orthologs in other genomes, and the respective combination. Starting with the first genome strain 5303, the other genomes are allocated a genome number, for example strain 8081 is genome1, strain 21202 is genome2, etc (Table. 9.6, p. 225). The different genome combinations are then sorted into different groups. The first group corresponds to the Y. enterocolitica backbone, as it contains CDSs, which have orthologs in all of the other genomes. The second group contains CDSs that have orthologs in all genomes except genome6. In the case of strains 5303, 8081, $21202,5603,14902$, and 1203, this corresponds to strain 3094. In strain 3094, on the other hand, genome6 is strain 1203. This shift in the group numbers has to be taken into account when evaluating table 9.6, and is due to the fact that only orthologs of one genome can be considered at a time.

Theoretically, certain groups might be of special interest. Apart from the backbone, group 2 and group 8 in table 9.3 could be of interest as they exclude BT 5 and BT $4+5$, respectively, and thus could help in identifying the genomic region encoding the biotype reaction. Group 6 excludes BT 1B. Group 7 is of particular interest as this excludes $B T 1 A$, and thus will contain the sets of genes common in all pathogenic BT. Group 22 excludes BT 1A and 1B, giving the shared CDSs of the low-pathogenic BT. The human, low-pathogenic isolates are in group 53, whereas the animal isolates are in group 56. These two groups could indicate sets of genes involved in niche adaptation either to a human or an animal host. Groups 58 to 63 indicate links 
between individual genomes, and group 64 will contain CDSs that are unique for each of the biotypes.

From looking at the results (Table 9.6, p.225), only a limited number of sets of orthologous genes are relevant. Some groups, such as group 11,12,13, and others, do not contain any genes. For better visualization of these groups, collaboration was set up with Dr Timothy Hetherington, NTU, who did the initial design of a 6-way Venn diagram. Later, this was modified by Sandra Reuter to include the $7^{\text {th }}$ strain 3094 . The complete diagram is shown in Figure 4.6, but will be considered in several steps for clarity.

The individual unique connections between strains are observed first (Figure 4.1). BT $1 \mathrm{~B}$ is only directly connected to BT $1 \mathrm{~A}$. Surprisingly, the non- and the highpathogenic BT share 85 CDSs. The arsenic resistance operon that is located outside the plasticity zone in BT $1 \mathrm{~B}$ is shared with BT 1A. Two genomic islands are common to BT 1A and 1B: YGI-2 and YGI-4. YGI-2 encodes a glycolipoprotein that is also found in other Enterobacteriaceae (Thomson, et al., 2006). YGI-4 represents an integrated plasmid. The original $\mathrm{YGl}-4$ appears to be retained in BT $1 \mathrm{~A}$, as the island is $20 \mathrm{~kb}$ larger than in BT 1B. A common theme of the other CDSs shared between BT $1 \mathrm{~A}$ and $1 \mathrm{~B}$ include metabolic regions such as $A B C$ transporters predicted to transport amino acids and sugars, as well as sugar kinases and permeases, exported proteins, and membrane efflux proteins.

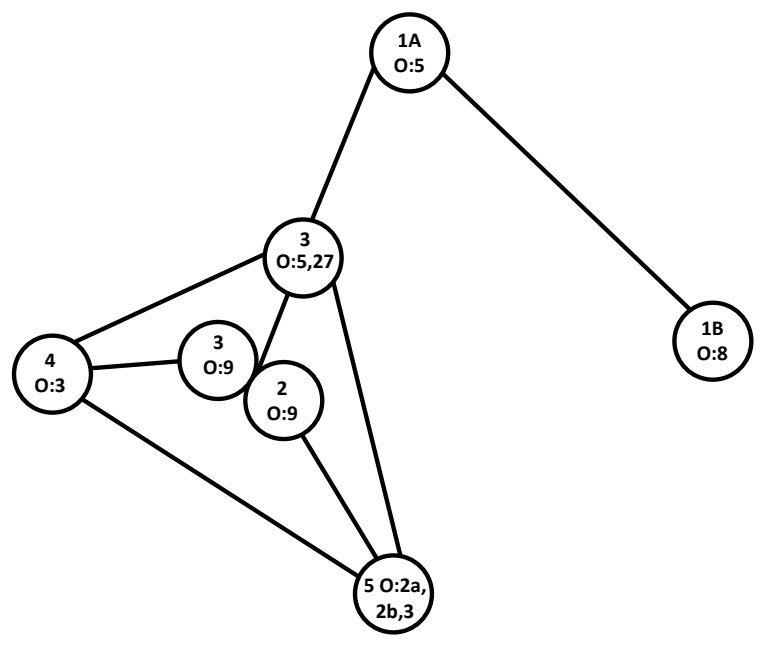

Figure 4.1: Connections between individual reference genomes based on analysis of orthologous genes.

BT $1 \mathrm{~A}$ and $1 \mathrm{~B}$ also share operons encoding a nickel-cobalt efflux system. One of the putative sugar transporters, YE2603-YE2609, appears to have been deleted from the low-pathogenic BT, as YE2604-YE2608 are absent and YE2603 and YE2609 are both truncated.

BT $1 \mathrm{~A}$ and BT 5 share $\sim 30$ CDSs, which correspond to a prophage locus. Although the phage has integrated at the same chromosomal location and thus could be considered orthologous, there are differences within the prophage. As phages are mobile elements, the genome locus might also simply present an integrational 
hotspot, and the phage would be considered paralogous in BT $1 \mathrm{~A}$ and BT 5 . The connection is thus not highlighted in Figure 4.1.

Connections between some isolates are given through shared parts of the operons for O-specific chain synthesis of the outer membrane lipopolysaccharide (LPS). BT1A and BT 3 0:5,27 share 17 CDSs. This region contains genes encoding for sugar conversions and modifications and a sugar export system and will be described in more detail later on (see 4.2.2.3).

BT 2 O:9 and BT 3 0:9 share a considerable number of CDSs relating to two prophage loci, clustered and unclustered hypothetical proteins as well as the 0:9 components of the O:antigen. These two bio-serotypes also share transposases at the exact same genomic positions. BT 3 O:5,27 and BT 4 share transposases and hypothetical proteins, mostly unclustered. BT 4 O:3 and BT 5 O:2a,2b,3 share an operon encoding the $0: 3$ antigen synthesis and transposases.

Moving on to three-way comparisons, the BT 2 and BT 3 isolates have two common prophage loci. The 0:9 isolates and BT 4 also share part of a prophage locus.

It appears in general that BTs 2 - 5, the low-pathogenic isolates, are very closely related with respect to their shared accessory gene content (Figure 4.2). They share $\sim 170$ CDSs with functions that might relate to environmental survival. These are a combination of groups 22 and 40 (Table 9.3) as BT 4 has lost some CDSs independently but did contain the complete set initially. The lowpathogenic BTs have unique fimbrial proteins that are absent from BT $1 \mathrm{~A}$ and $1 \mathrm{~B}$ and there are also two genomic islands, which have been described in the past as specific for low-pathogenic Figure 4.2: Orthologous genes shared in the BT.

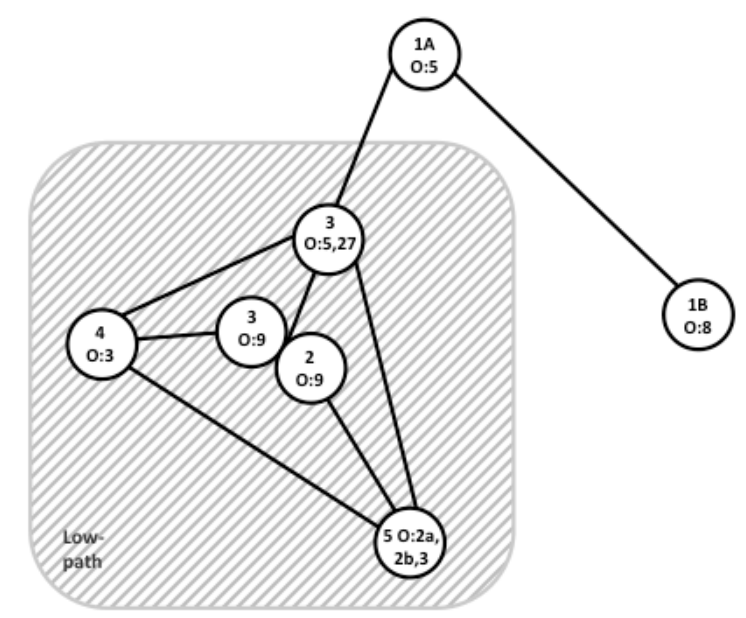

The first is the tc pathogenicity island (PAI) which confers toxicity against insects to Y. enterocolitica (Bresolin, et al., 2006a; Fuchs, et al., 2008). Except for one strain, these have not been found in the non- or high-pathogenic BTs. The other island corresponds to the second flagella cluster Flag-2 (Bresolin, Trček, Scherer and Fuchs, 2008). Part of this cluster has been deleted in BT 4 (group 40) due to the 
action of transposases. Additionally the low-pathogenic BTs share further hypothetical and phage-related proteins. The low-pathogenic BTs are signified by the expansion of IS element IS1667. Some of the IS1667 insertions appear to be ancient, as the position of the copies is conserved in all low-pathogenic lineages.

The non- and low-pathogenic BTs share $~ 120$ accessory CDSs associated with different metabolic traits such as cytochrome c-type biogenesis, utilization of $\mathrm{N}$ acetyl-D-galactosamine, amino acid metabolism, and anaerobic dimethyl sulfoxide reduction (Figure 4.3). These BTs also possess the same LPS outer core region, which has been replaced by the $0: 8$ operon in the highpathogenic BT 1B. There are also some functions that might relate to pathogenesis such as a novel hemolysin and its activator protein, $A$ and $B$ subunits of a cytolethal

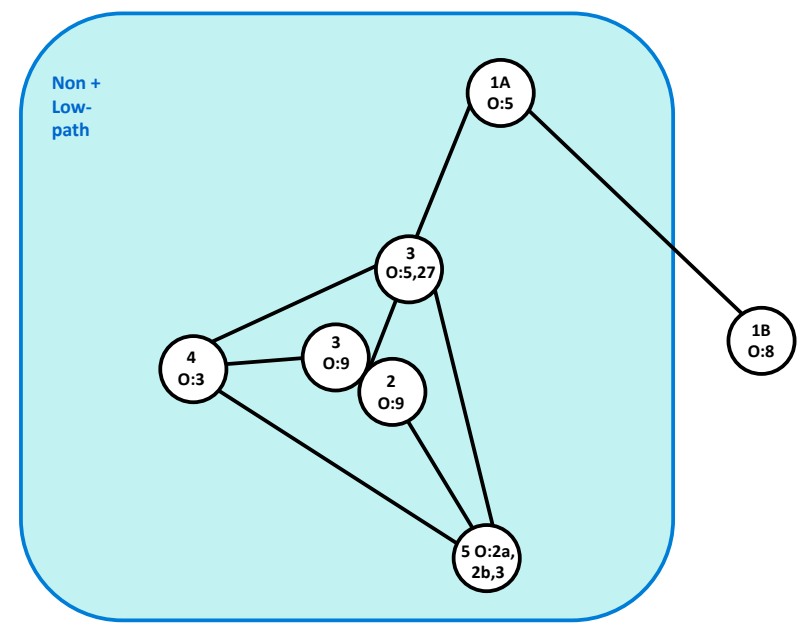

Figure 4.3: Shared genes in non- and lowpathogenic BTs.

distending toxin, a putative repeat toxin RtxA, and an alternative T3SS. A similar T3SS has been identified in the recently published environmental Yersinia (Chen, et al., 2010) and it is also found in Y.pestis and Y.pseudotuberculosis. We thus propose to call it the Yersinia Genus T3SS (YGT) and it will be described in more detail later (4.2.2.1).

Both BT 4 and BT 5 (Figure 4.4) show signs of having undergone independent genome decay. BT 4 has lost the fes-fep iron-uptake operon. A putative amino acid transport system and a phosphotransferase system have been lost from BT 5. Further unclustered CDSs that have been lost from BT 5 are also putatively associated with metabolic pathways and membrane transporters. Both BTs are missing a putative sugar

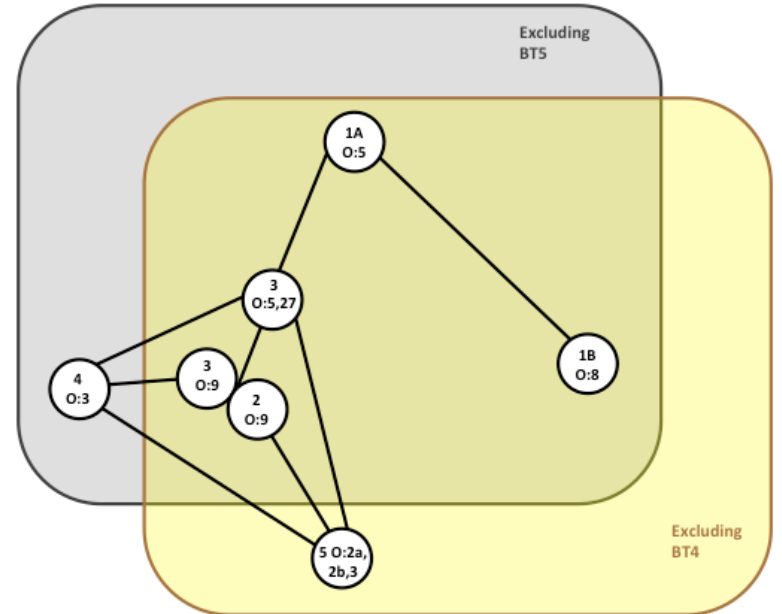

Figure 4.4: Orthologous gene clusters absent from BT 4 and BT 5 . 
transport system that is present in all other biotypes. This loss of metabolic properties is also evident in the biotyping scheme used for differentiation of $Y$. enterocolitica biotypes, with BT 5 testing negative or variable for 11 of the 12 reactions. BT 4 has five of those negative reactions in common with BT 5 and tests positive for the rest. As the absent sugar transporters are missing a definite pathway assignment, a direct link between the biotyping reaction and genome locus is not possible. The bases of the sorbose and trehalose negative phenotype of BT 5 are mutations in sorB and tre $B$, respectively.

One further group is relevant: the "pathogenic core", shared in low- and highpathogenic BTs (Figure 4.5). There are only $\sim 40$ chromosomal CDSs in addition to the virulence plasmid pYV ( 90 CDSs) that are shared exclusively by all pathogenic BTs. These functions include the adhesion gene ail, some clustered exported proteins, non-clustered hypothetical and conserved hypothetical proteins, a putative repeat toxin family protein, and one locus encoding genes involved in threonine and serine metabolism. This last operon however is presumably only functional in $B T 1 B$, as it contains additional genes encoding a dehydrogenase/reductase, an aldo/keto reductase, and a transcriptional regulatory protein. As the hypothetical proteins are not organized in clusters, but are rather scattered throughout the genome, a function is questionable.

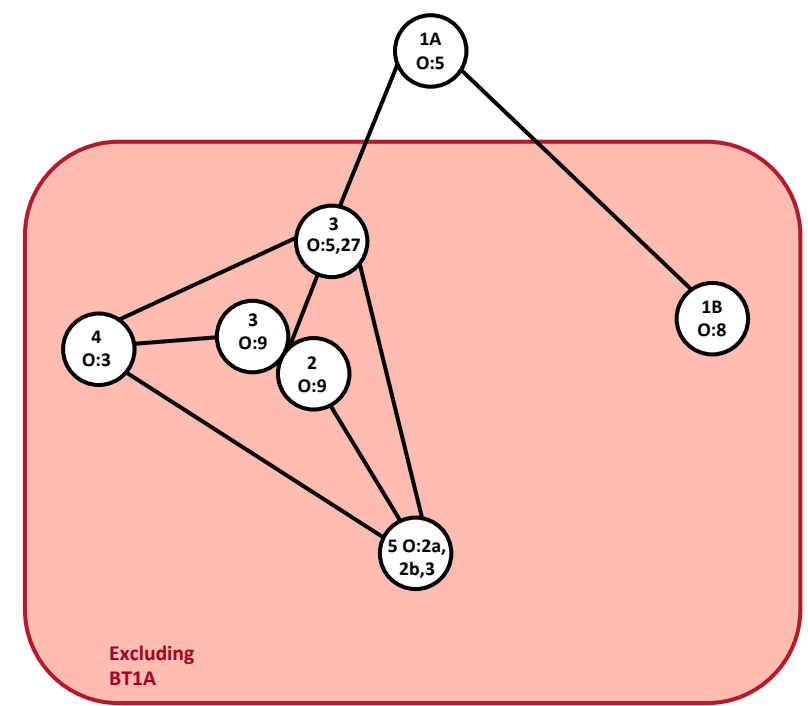

Figure 4.5: Orthologous genes shared in the pathogenic BTs.

The attachment invasion locus gene ail is shared in pathogenic BTs, but it is located in a different genomic context compared to the low-pathogenic BTs. This is likely to be due to IS-mediated transposition, as fragments of IS1328, IS1400 and IS1668 are visible in both high- and low-pathogenic BTs. The virulence plasmid pYV and the chromosomally located ail consequently constitute the only shared, wellcharacterized "virulence factors". None of the other identified CDSs present exclusively in the pathogenic BTs have known functions. 
The individual groups described above are combined in Figure 4.6. It is important to note the number of connections between the different low-pathogenic bio-serotypes based on shared accessory gene content. It is also striking how both the non- and the high-pathogenic BTs stand singled out, with only few, selected connections to the other bio-serotypes.

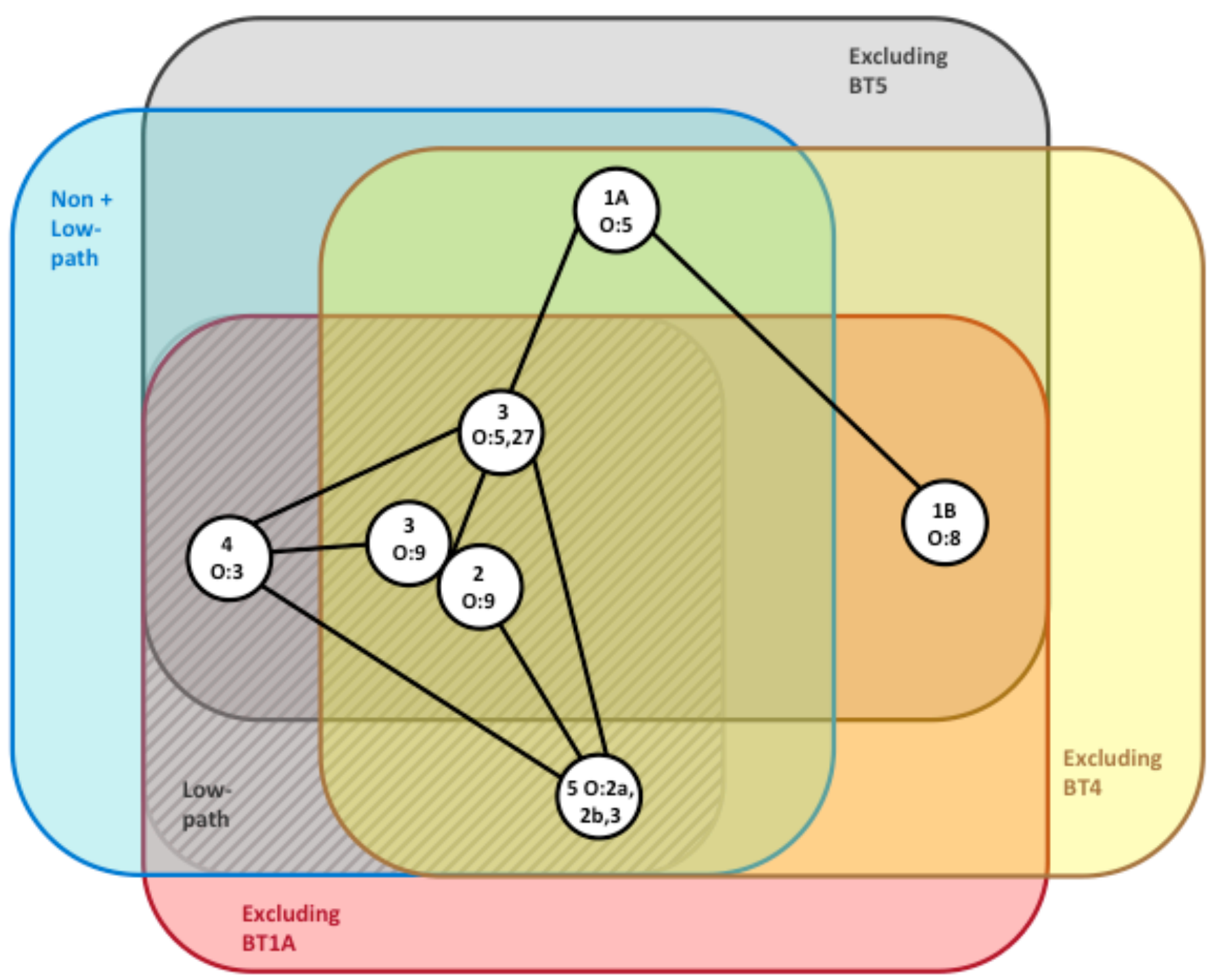

Figure 4.6: Combined comparison of orthologous accessory gene sets in the reference genomes for the Y. enterocolitica biotypes. As described above, the single lines represent connections between isolates. The low-pathogenic BTs are highlighted with a shaded box. Further orthologous gene sets are highlighted with boxes for non- and low-pathogenic BTs (blue), all BTs except BT 4 (yellow), all BTs except BT 5 (grey), and all BTs except BT 1A (red; the pathogenic BTs).

After consideration of sets of orthologous genes, it was also possible to determine the number and type of CDSs that are unique to each bio-serotype.

BT $1 A$ and BT 1B both show a considerable number of unique CDSs, 728 (16.7\% of total CDSs) and $540(13.3 \%$ of total CDSs) respectively. For BT $1 \mathrm{~B}$, these have been 
previously described as being specific for high-pathogenic strains and include the acquisition of the high-pathogenicity island ( $\mathrm{HPI})$, the Ysa T3SS, and the general secretion pathway Yst-1. Also unique to the high-pathogenic BT is the Yersinia adhesion pathogenicity island, YAPI. Many of the CDSs unique to BT 1A appear to extend its metabolic repertoire compared to the other biotypes, including proteins involved in transport and metabolism of sugars. Additionally, the non-pathogenic BT 1A isolate carries several gene clusters involved in environmental survival such as metal resistance and an arsenic resistance operon. The low-pathogenic BT isolates generally have a lower number of unique CDSs compared to BT $1 \mathrm{~A}$ and $1 \mathrm{~B}$. The reference BT 2 0:9 genome carries five genes that are unique to this strain, and reference BT 3 0:9 strain carries no unique genes (Table 9.6 p. 225). Both serotype O:9 isolates of BT 2 and 3 are genetically very close, sharing phage and transposases, thus it is not surprising that they do not have many unique genes. BT 3 $\mathrm{O}: 5,27$, BT $4 \mathrm{O}: 3$, and BT 5 have 171, 134, and 274 unique CDSs, respectively. The majority of these genes are related to several prophage loci, hypothetical and conserved hypothetical proteins and transposases. Prophages thus are the largest contributors to the accessory genome.

All Y. enterocolitica BT have 3,306 CDSs in common which represent the core functions of the species (Figure 4.7, Table 4.1). It has been previously speculated that the Yersinia genomic island 1 (YGI-1) encoding the tight adherence (tad) operon was associated with enteropathogenicity and specific for $Y$. pseudotuberculosis and the pathogenic Y. enterocolitica biotypes (Howard, et al., 2006; Thomson, et al., 2006). However the tad operon is actually part of the core genome being found at the same locus in all isolates including the non-pathogenic BT 1A strain. The presence of the tad operon was also confirmed for the environmental species (Chen, et al., 2010), therefore this cluster is probably ancestral. The same is true of other functions known to be important for virulence including inv encoding invasion, its regulator RovA and the myf fibrillar operon (Badger and Miller, 1998; Ellison, Lawrence and Miller, 2004; Iriarte and Cornelis, 1995). 


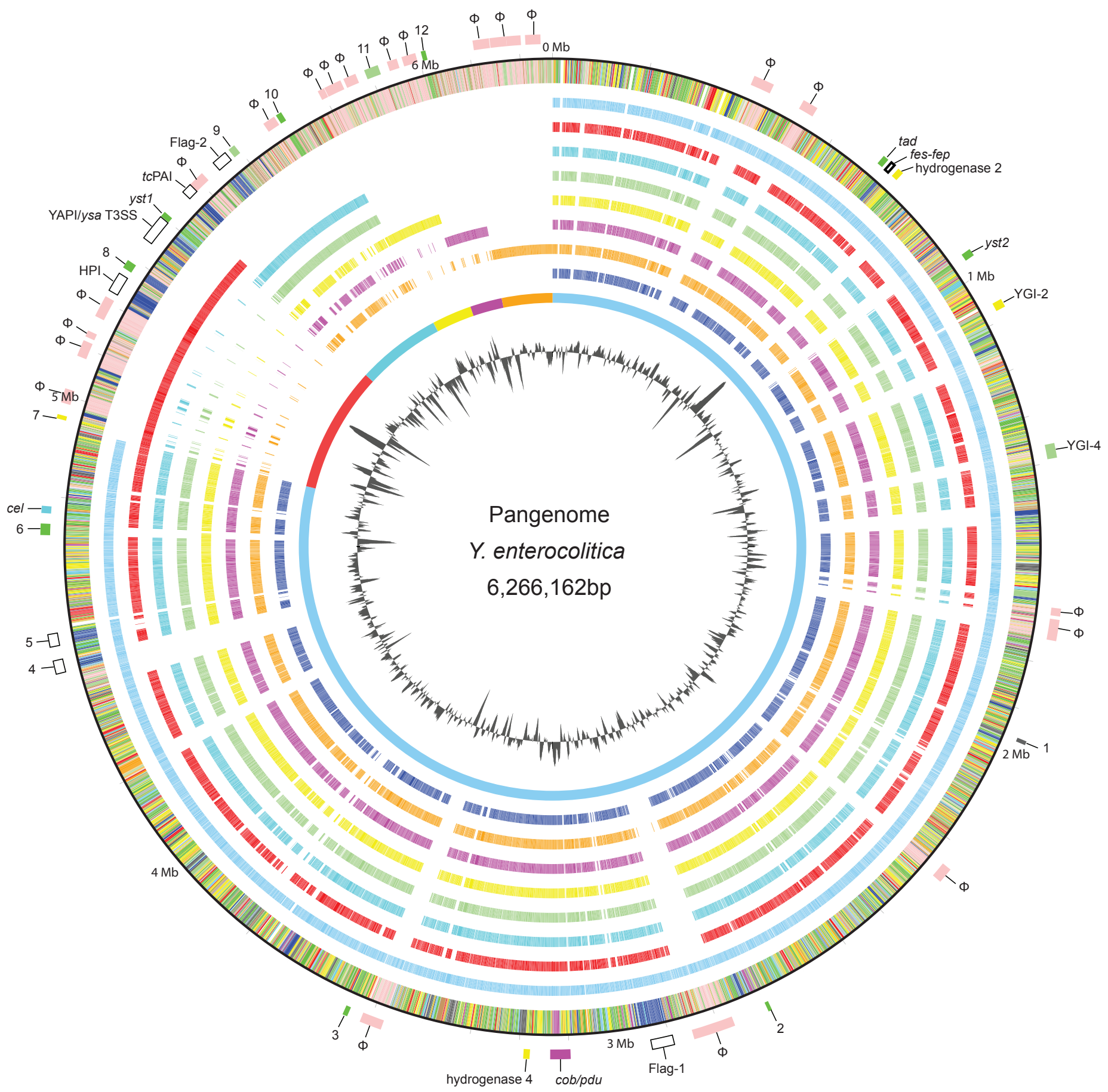

Figure 4.7: The pangenome of $Y$. enterocolitica. The outer scale shows the size in Mbps. From the outside in, circle 1 shows the position of CDSs (for colour codes see below). Circle 2 (CDSs coloured blue) marks the position of Y. enterocolitica strain 5303 / BT1A 0:5 genes. Circle 3 (red) shows the position of strain 8081 / BT1B O:8 genes. Circle 4 (light blue) 21202 / BT2 O:9. Circle 5 (light green) 5603 / BT3 O:9. Circle 6 (yellow) 14902 / BT3 0:5,27. Circle 7 (pink) 1203 / BT4 O:3. Circle 8 (orange) 3094 / BT5 O:2a,2b,3. Circle 9 (purple) highlights genes shared in all Y. enterocolitica biotypes. Circle 10 represents the fasta record from which strain the CDSs were written out. Circle 11 shows a plot of $\mathrm{G}+\mathrm{C}$ content (in a 10-kb window). Genes in circles 1 are colour-coded according to the function of their gene products: dark green, membrane/surface structures; yellow, central or intermediary metabolism; dark blue, pathogenicity island-related functions; cyan, degradation of macromolecules; red, information transfer/cell division; cerise, degradation of small molecules; pale blue, regulators; salmon pink, pathogenicity/adaptation; black, energy metabolism; orange, conserved hypothetical; pale green, unknown; and brown, pseudogenes. The position of important regions (mentioned in the text) are marked and labeled in the outermost ring. See Table 4.1 for a description. 
Table 4.1: Regions of interest identified in the pangenome of $Y$. enterocolitica (Figure 4.7).

\begin{tabular}{lll}
$\begin{array}{l}\text { Region of } \\
\text { interest \# }\end{array}$ & Base position & Content \\
\hline \hline 1 & $1955006 . .1962863$ & cytochrome c-type biogenesis proteins \\
2 & $2691400 . .2698800$ & Phosphotransferase system for N-acetyl-D-galactosamine \\
3 & $3544600 . .3554299$ & outer core LPS biosynthesis operon \\
4 & $4448957 . .4475254$ & silver and copper resistance proteins \\
5 & $4502803 . .4527755$ & Yersinia Genus T3SS (YGT) \\
6 & $4722584 . .4745103$ & putative O:5 LPS biosynthesis cluster \\
7 & $4952491 . .4960977$ & serine/threonine metabolism \\
8 & $5274712 . .5291988$ & O:8 LPS biosynthesis proteins \\
9 & $5583332 . .5597425$ & replaces YGI-3 \\
10 & $5697260 . .5709091$ & O:9 LPS biosynthesis proteins \\
11 & $5890466 . .5918695$ & putative genomic island, similar to Escherichia blattae proteins and \\
12 & & phage proteins \\
\hline
\end{tabular}

The two hydrogenase complexes, hydrogenase 2 (hyb) and hyrdogenase 4 (hyf), were thought to be associated with colonisation and survival of the gut. Both clusters are present in all $Y$. enterocolitica biotypes. The hydrogenase 2 operon shows signs of truncation in the low-pathogenic BTs as the formate transporter focB (YE2812) is fragmented. The hydrogenase 4 complex is truncated in hypB (YE3603) in BT 3 O:5,27 strain 14902 and BT 4 O:3 strain 1203. In contrast these clusters are probably functional in the non-pathogenic isolate.

The cellulose biosynthesis operon is also present in all BTs. In Salmonella an extracellular matrix is formed through the production of cellulose, which could increase the time the bacteria are retained in the gut. A similar idea has been proposed for Y. enterocolitica (Thomson, et al., 2006). Again, this cluster shows signs of mutations. In BT 4 0:3 strain 1203 a stop codon has been introduced in bscC (YE4072). An opal stop codon (UGA) has been introduced in bscA (YE4074) in BT 1 A, which could potentially be decoded by selenocysteine. 


\subsubsection{Regions of difference in Y. enterocolitica biotypes}

Some of the regions identified as regions of difference between the $Y$. enterocolitica bio-serotypes have not been extensively characterized previously. These regions will be discussed in turn below.

\subsubsection{Yersinia Genus Type 3 Secretion System}

The first interesting region of difference is an alternative T3SS we propose to call the Yersinia Genus T3SS (YGT) since orthologs have been described in the environmental species as well as $Y$. pseudotuberculosis and $Y$. pestis. The system is located in in the same genomic context in all species, indicating an ancient acquisition before the split into separate species, justifying the denomination as a common T3SS of the genus. By sequence, this system is most similar to the Salmonella pathogenicity island 2 (SPI-2, Figure 4.8).

\section{SPI-2 Salmonella Typhi}

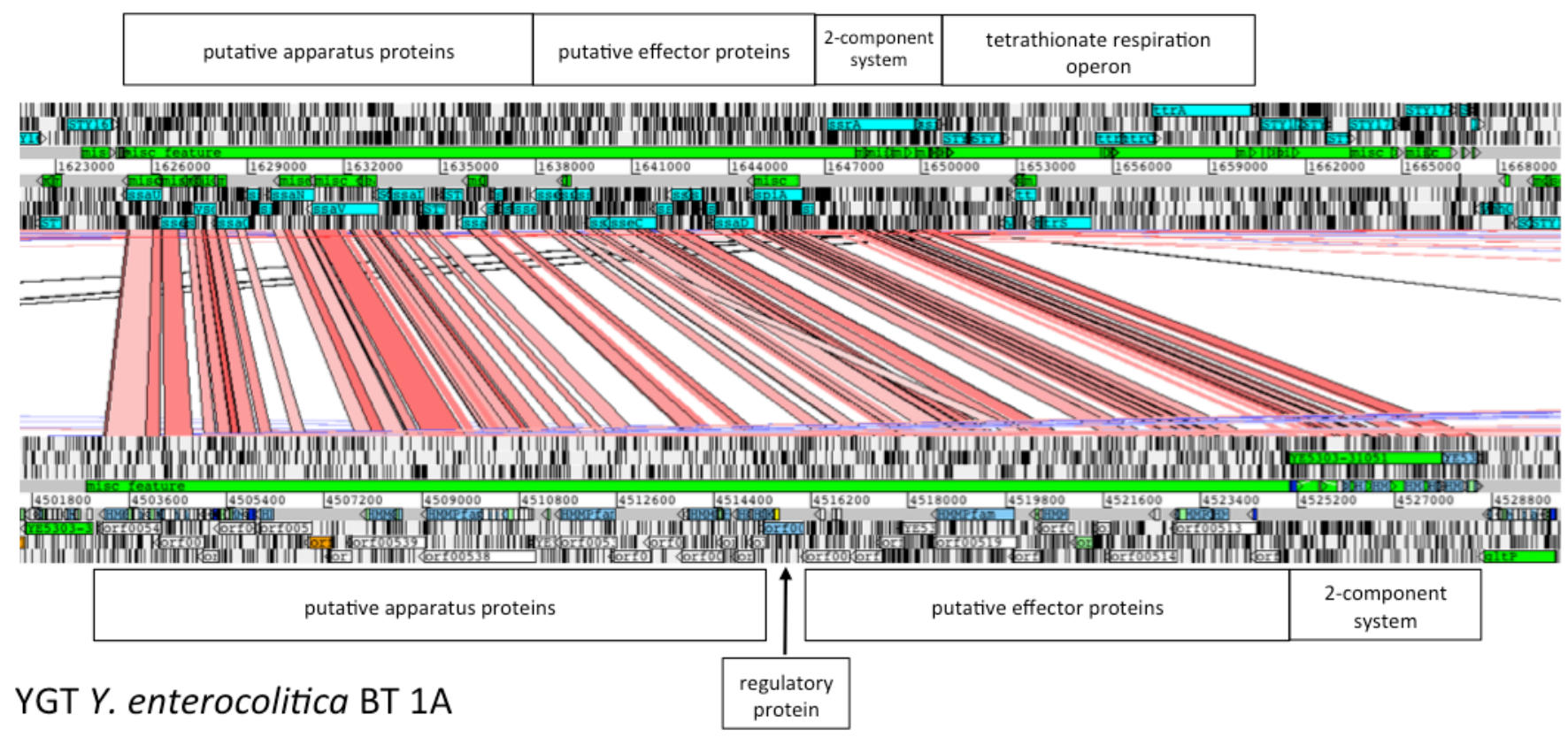

Figure 4.8: Comparison of SPI-2 of Salmonella Typhi with the novel YGT. The apparatus and effector regions are highlighted, as well as the 2-component system located upstream of the T3SS in both cases. In SPI-2, the YGT is preceeded by the tetrathionate respiration operon. In $Y$. enterocolitica, the tetrathionate respiration operon is located in a different genomic context.

The sequence similarity shared between SPI-2 and YGT is low but is consistent across the whole YGT region, and ranges on average around $\sim 40 \%$ similarity. Upstream of YGT and SPI-2 T3SSs is a 2-component system encoding a sensor kinase and a response regulator that might be involved in governing the expression 
of the T3SS. In SPI-2, the tetrationate respiration operon is loacted within the pathogenicity island, whereas for $Y$. enterocolitica, a highly related operon is located elsewhere in the chromosome.

Comparison of the YGT within different assembled Yersinia spp. shows differences in the gene content (Figure 4.9).

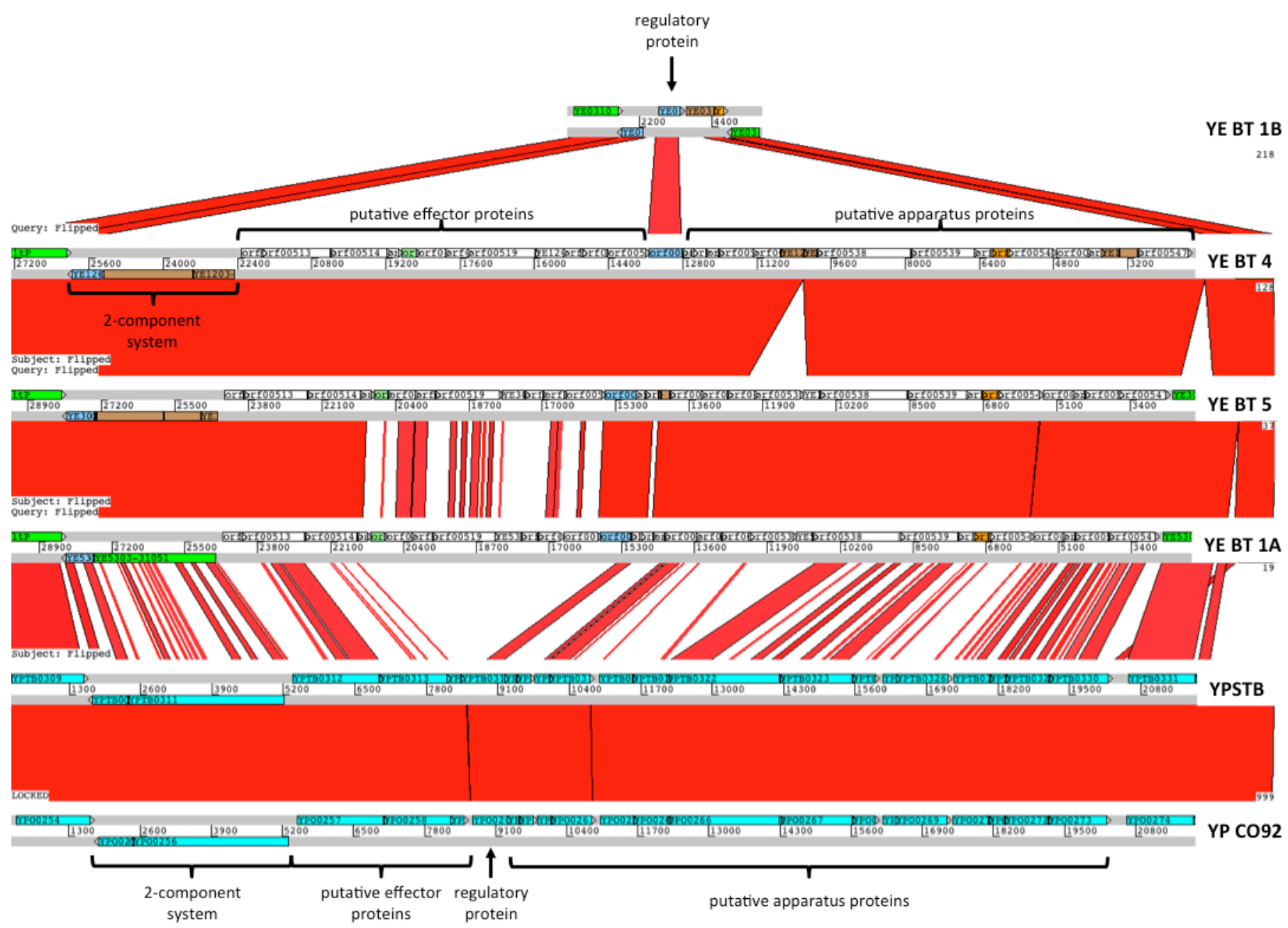

Figure 4.9: Comparison of YGT within several Yersinia spp. Four representative $Y$. enterocolitica BT (YE BT 1B, 4, 5, and 1A) are compared to Y. pseudotuberculosis (YPSTB: IP32953) and Y. pestis (CO92). Effector and apparatus regions are highlighted, as well as the 2-component system.

As mentioned before, the YGT is absent from BT 1B, only three proteins are present: the response regulator (YE0311), the AraC-family regulatory protein (YE0312) in the central part of the YGT, and an inner membrane protein (YE0313) that is missing the $\mathrm{N}$ terminus. As the YGT is present in all other Yersiniae, and as the central regulatory protein has been retained, this indicates a possible deletion in at least two separate steps from BT $1 \mathrm{~B}$. 
The YGT is generally well conserved amongst the non- and low-pathogenic BTs although there is evidence that some genes in YGT are defunct in BTs 4 and 5. Those mutations are different in BTs 4 and 5, with BT 4 having mutations in three apparatus related genes and the deletion of another apparatus protein and BT 5 having only one independent mutation in an apparatus gene. Moreover the sensor kinase of the 2-component system has a stop codon introduced in BT 4 and several frameshifts in BT 5. The inner membrane protein (YE0313) is missing the central part in BTs 2, 3, and 4, and is only functional presumably in BT $1 A$ and BT 5 . Some parts of the effector protein region show reduced sequence similarity between non- and low-pathogenic BTs.

A comparison of YGT in several Yersinia spp. to $Y$. enterocolitica shows that parts of the effector protein region have been deleted in Y. pseudotuberculosis and $Y$. pestis (Figure 4.9). Preliminary studies (Golubov, Heesemann and Rakin, 2003; Pujol and Bliska, 2003; Balada-Llasat and Mecsas, 2006) have been undertaken on YGT in Y. pestis and Y.pseudotuberculosis and have shown that this system plays no obvious role in causing disease, but functionality in Y.pestis and Y. pseudotuberculosis is probably compromised due to the absence of the effector region. The distribution of YGT within the genus suggests that this system is more likely to be required for colonisation outside a human host. Initial investigation into the phylogeny of the T3SS (Foultier, et al., 2001) is in accordance with the finding that this T3SS is most closely related to SPI-2 of Salmonella.

\subsubsection{Variation of the Plasticity Zone}

Another large-scale region of variation distinguishing different BTs is the plasticity zone (PZ). The PZ was first described in the high-pathogenic BT 1B strain 8081 and constitutes a region, which contains several operons associated with increased pathogenic potential. The region shows signs of having been formed through several independent acquisition events, hence being termed plasticity zone (Thomson, et al., 2006). A comparison of this region with the other BTs confirms this locus to be highly variable between BTs (Figure 4.10). The plasticity zone in the high-pathogenic BT shows the largest size. The acquisition of the Yersinia adhesion pathogenicity island YAPI is possibly specific for strain 8081 where it was originally defined, as it was not observed consistently in other BT 1Bs (Thomson, et al., 2006; Carniel, Guilvout, and Prentice, 1996). Furthermore BT 1B acquired the ysa T3SS as well as the general secretion pathway yst1. At the same locus, the non-pathogenic BT possesses heat 
shock proteins, hypothetical proteins, and an additional lac operon. No equivalent region is present in the low-pathogenic BTs. All BTs have an iron transporter and operons for hydrogenase 2 biosynthesis.

The second flagella cluster Flag-2 is only retained by the low-pathogenic BTs and has been independently lost in the non- and the high-pathogenic lineages as indicated by the presence of different fragments of Flag-2. This region is also marked by the presence of a transposase in BT 4 likely to have been responsible for the loss of Flag-2 and additionally the deletion of the fes/fep operon. Both high- and lowpathogenic BTs then have outer membrane efflux proteins, whereas the lowpathogenic BTs show a different set of membrane proteins. These membrane protein operons have no specifically assigned functions so far. As mentioned before, the tigh-adherence operon tad is present in all biotypes.

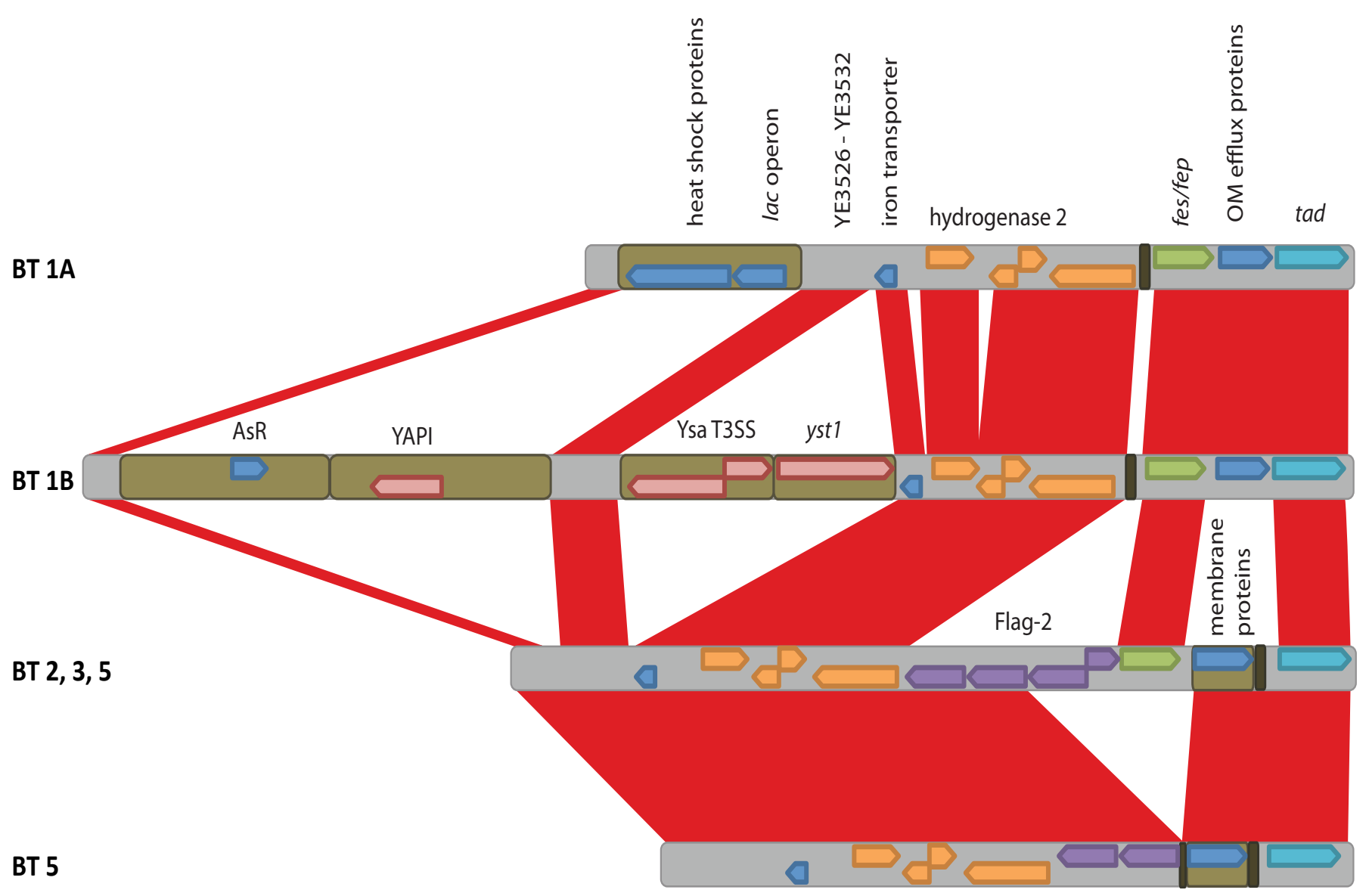

Figure 4.10: Arrangement of the plasticity zone in the different biotypes. Non-pathogenic BT $1 \mathrm{~A}$, high-pathogenic BT - 1B, low-pathogenic BT - 2,3,4,5. Red - colinear homology, modified from an ACT comparison. Light brown - potential inserted region. Dark brown - deleted region. 


\subsubsection{Lipopolysaccharide operons}

The lipopolysaccharide (LPS) of Y. enterocolitica has been proposed as a supporting virulence factor as it confers resistance to polymyxin and antimicrobial peptides and has been associated with virulence in the mouse model (Zhang, et al., 1997; Skurnik, et al., 1999; Skurnik, et al., 2007). The LPS structure for serotypes 0:8, 0:3 and 0:9 is known, and a schematic is shown in Figure 4.11.

All $Y$. enterocolitica serotypes share - with minor variations - the same lipid A and inner core structures (Müller-Loennies, et al., 1999; Oertelt, Lindner, Skurnik and Holst, 2001). It is known that the outer core is absent from the highpathogenic $\mathrm{BT}$ 1B $0: 8$ strains but shared in the low-pathogenic BTs O:9 and 0:3 (Skurnik and Bengoechea, 2003). The O-antigens differ in sugar composition and in linkage of those sugars. The $0: 8$ antigen is a heteropolymeric pentasaccharide in which three saccharides are linked in a chain with a further two saccharides branching off (Zhang, et al., 1997). In 0:3 and

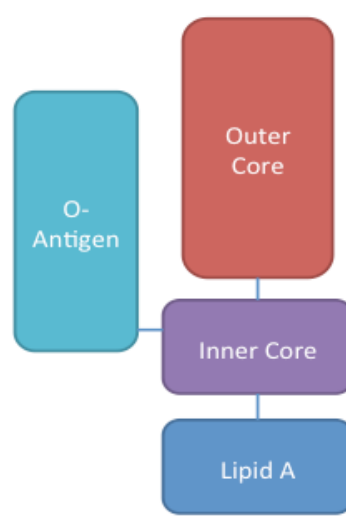

Outer Membrane structure of $0: 9$ and $0: 3$

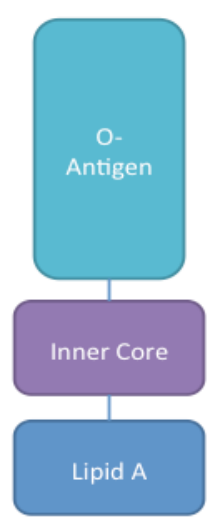

Outer Membrane structure of $0: 8$

Figure 4.11: Lipopolysaccharide structure of Y. enterocolitica serotypes. Structures with shared functions are highlighted with the same colour. Adapted from Sirisena and Skurnik, 2003, and http://www.hi.helsinki.fi/yersinia/Research1.htm.

O:9 O-antigens the chain consists of unbranched homopolymers (Zhang, Toivanen and Skurnik, 1996; Skurnik and Bengoechea, 2003). The outer core of 0:3 and 0:9 serotype strains consists of a branched heteropolymer (Reeves, et al., 1996b; Skurnik, et al., 1999). It has been proposed that the outer core presents an ancestral $\mathrm{O}$-antigen and that it has been supplemented by later acquisition of homopolymeric O-antigens in the low-pathogenic BTs (Müller-Loennies, et al., 1999; Skurnik, et al., 1999; Sirisena and Skurnik, 2003).

It has been shown before that the $0: 8$ encoding locus is in the same genomic context as the outer core operon of serotypes 0:3 and 0:9 (Zhang, Toivanen and Skurnik, 1996; Skurnik and Bengoechea, 2003; Figure 4.12). The outer core is located beween gsk (inosine-guanosine kinase, YE3069) and hemH (ferrochelatase, YE3088). Apart from gsk and hemH, the UDP-glucose 4-epimerase galE has been conserved between the outer core and the O-antigen. The same locus also contains 
genes involved in the synthesis of the heteropolymeric lipopolysaccharides of Y. pseudotuberculosis and Y. pestis, although five genes are inactivated in the latter (Zhang, Toivanen and Skurnik, 1996; Skurnik and Bengoechea, 2003). In a comparison between BT $2 \mathrm{O}: 9$, BT 1B O:8, and the environmental Yersiniae, the outer core region could be detected in Y. frederiksenii, Y. kristensenii, and Y. rohdei. The absence of the outer core in the genome data for the other environmental Yersiniae could be due to assembly problems, therefore a definitive answer on the distribution of the outer core within the genus is not possible.

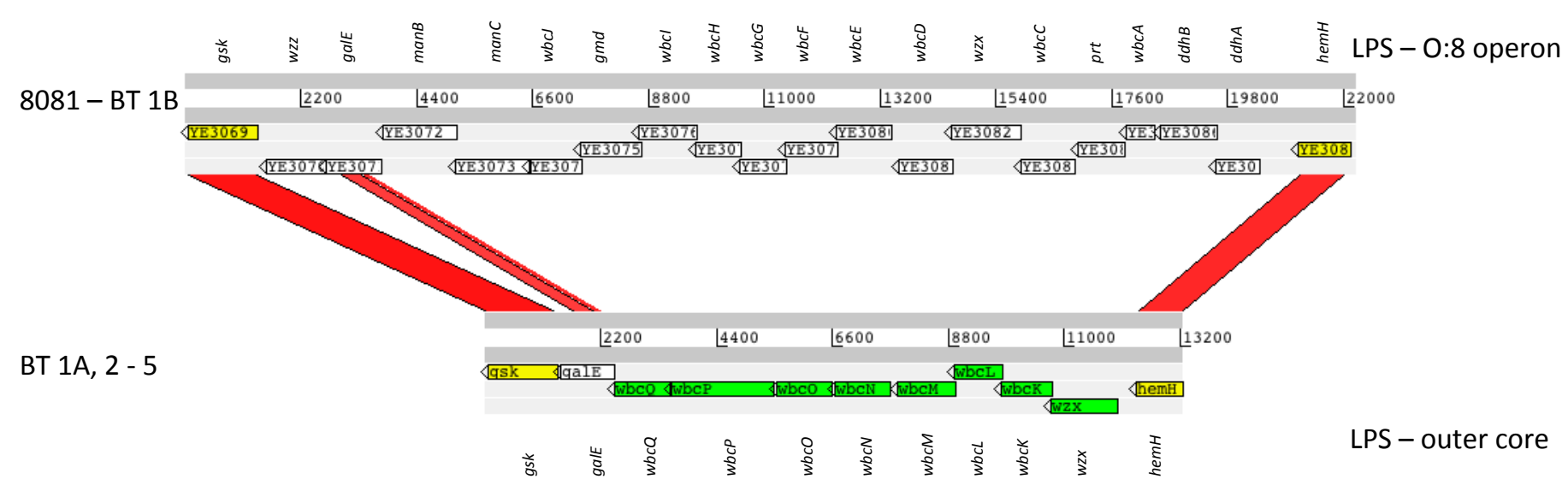

Figure 4.12: Genomic context of LPS 0:8 operon and outer core. Both outer core and 0:8 operon are located between gsk and hemH.

In the comparative analysis it became clear that the outer core is shared in low- and non-pathogenic BTs. Considering that BT $1 \mathrm{~A}$ shows signs of being the historically oldest biotype in that it has fewer IS elements, less genomic reorganization, fewer pseudogenes and an extensive metabolic repertoire as apparent from the unique regions, it is likely that the outer core is the ancestral form. This supports the idea of an exchange of one region for another.

The location of the O-antigen clusters for 0:3 and 0:9 was unknown, although their operon structure has been investigated in the past (Reeves, et al., 1996b; Skurnik and Bengoechea, 2003; Skurnik, et al., 2007). The O-antigen clusters for 0:3 and $0: 9$ are found in the same genomic location between a putative membrane protein (YE2779) and gnd, a phosphogluconate dehydrogenase (YE2773, Figure 4.13).

Both BT 2 O:9 and BT 3 O:9 clusters are identical and are the same O:9 operons described in the past (Skurnik, et al., 2007). There are six CDSs involved in sugar biosynthesis (manCB, gmd, per, wbcTV). Four CDSs are encoding transferases for 
linking the sugars (wbcUW galUF). galU and galF are conserved with the $0: 3$ operon, and are also found in the other biotypes. Two CDSs are then encoding the transport proteins (wzm, wzt).

The 0:3 operon is larger than the 0:9 operon. An IS element for IS1668 has inserted upstream of the locus, and a remnant for IS1400 can be found in the central part of the operon. The LPS 0:3 operon of BT 4 contains one pseudogene, a hypothetical protein carrying a stop codon.

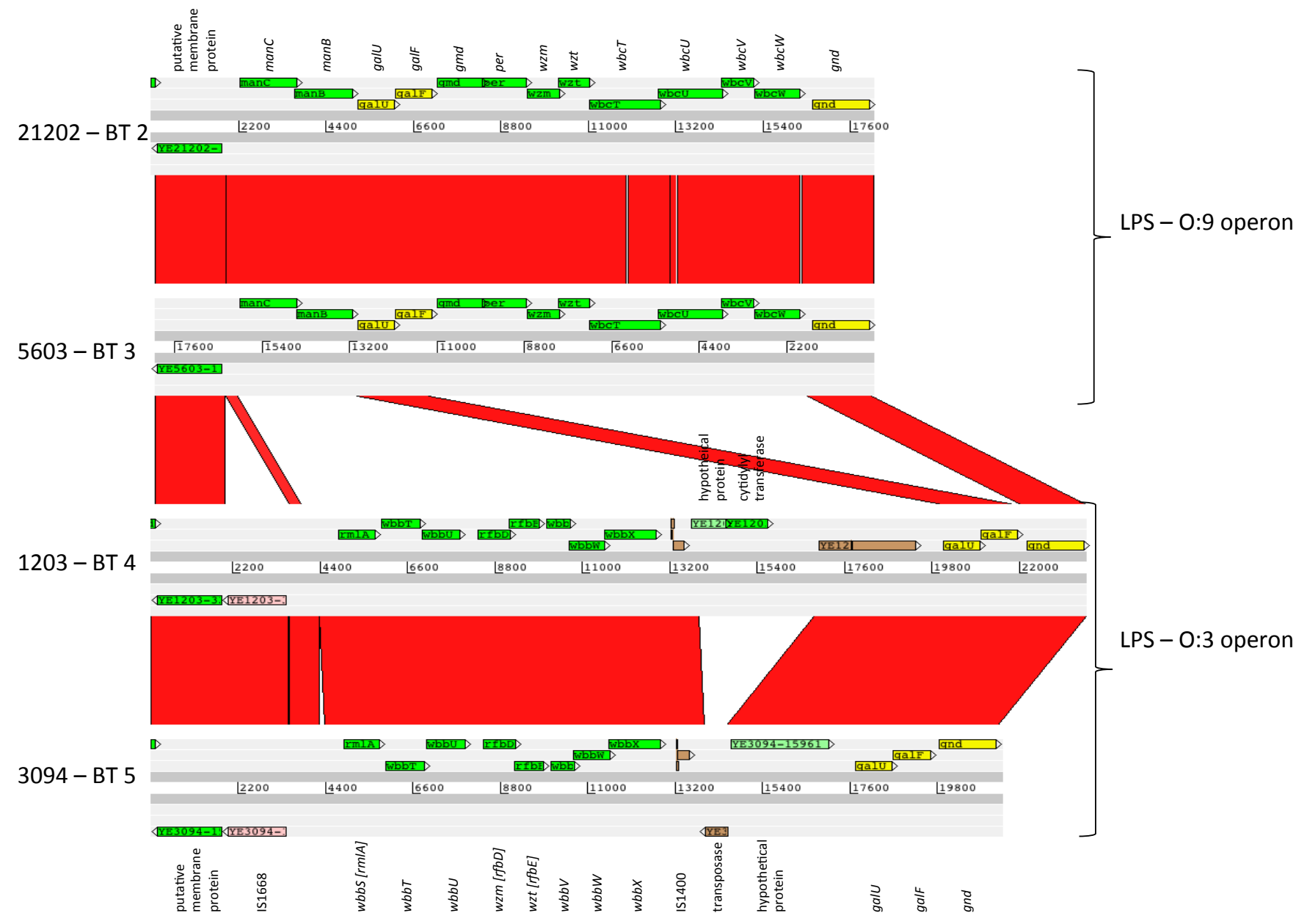

Figure 4.13: Genomic context of 0:9 and 0:3 operons.

The exact serotype of the reference BT 5 isolate is unknown, but the serotype typically described for this biotype is often a mixture of O-antigens $2 a, 2 b$, and 3 (Bottone, 1997; Bottone, 1999; Bartling, et al., 2004). The operon found in BT 5 is similar in sequence to the $0: 3$ operon of BT 4 and is thus thought to contribute to the serotype of BT 5 . There are however differences to the standard 0:3 operon. A hypothetical protein and a cytidylyl transferase are absent, possibly due to the action of transposases, as remnants of these are found upstream and downstream of these 
CDSs. The hypothetical protein, which has a nonsense mutation in BT 4 , is not mutated in BT 5 . These differences could be an explanation for the difference in serotyping. Both $0: 3$ and $0: 2 a, 2 b, 3$ homopolymeric repeat units are reported as 6deoxy-L-altrofuranose, with the difference for $0: 2 a, 2 b, 3$ being that they have $O$-acyl side groups (Reeves, et al., 1996b; Bruneteau and Minka, 2003).

Both 0:3 and 0:9 O-antigen operons encode for homopolymeric polysaccharides and share the same biosynthesis mechanism. The sugars are assembled to chains and then transported across the membrane using ATP-binding cassette (ABC) transporters (Zhang, et al., 1997; Samuel and Reeves, 2003). In the new nomenclature for bacterial polysaccharide synthesis genes, these are designated as wzm and wzt although they do not necessarily share nucleotide homology (Reeves, et al., 1996a,b; Samuel and Reeves, 2003). The synthesis of heteropolymeric polysaccharides as are found in 0:8 differs in that each subunit is assembled and then translocated by a flippase wzx before the units are polymerized by Wzz (Zhang, Toivanen and Skurnik, 1996; Reeves, et al., 1996a,b; Zhang, et al., 1997; Samuel and Reeves, 2003).

The structure of LPS 0:5 has not been published yet, although a putative cluster has been deposited in GenBank for BT 1A 0:5 strain T83 (accession number AY653208; Tennant, S.M., Joe, A. and Robins-Browne, R.M., 2004). The putative O-antigen operon in BT 1A strain 5303 is located between the glutathione reductase gor (YE4057) and the autotransporter protein YapE (YE4059) (Figure 4.14). A comparison between the two BT 1A strains showed a high degree of sequence similarity in this cluster. BT $30: 5,27$ strain 14902 has a similar operon, but in a different genomic context. The putative O-antigen operon is located between YE0502 and YE0513 and is surrounded by IS elements and fragments of transposases, possibly explaining the change in location. This operon is also missing two hypothetical proteins, a DNA binding protein, and an alcohol dehydrogenase of the presumed 0:5 cluster, which could explain the mixed serotyping reaction.

The sugars of the 0:5 serotype have been described as homopolymers of rhamnose (Gorshkova, Kalmykova, Isakov and Ovodov, 1986), explaining the presence of two polysaccharide export proteins that constitute the $A B C$ transport of the homopolymeric chain. Homopolymeric lipopolysaccharide operons are generally smaller in size than heteropolymeric operons, as a higher complexity in the number of sugars and their linkages calls for more genes (Reeves, et al., 1996a). With a size 
of $\sim 18 \mathrm{~kb}$ it compares well to the other homopolymeric clusters of $0: 3(18 \mathrm{~kb})$ and $0: 9(14 \mathrm{~kb})$.

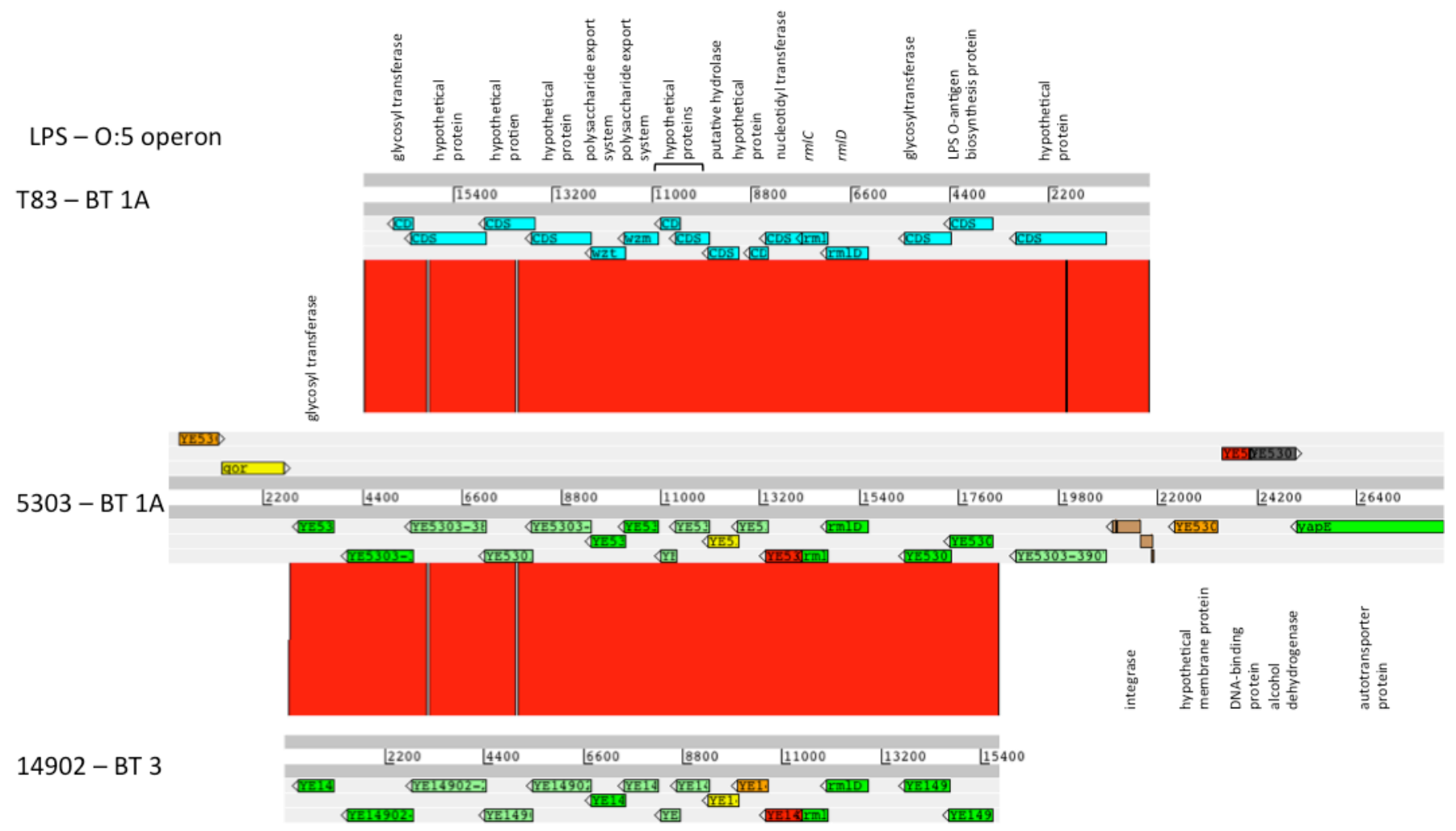

Figure 4.14: Putative 0:5 LPS operon. The putative 0:5 operon has been deposited in GenBank for BT 1A strain T83, but has not been published yet (accession number AY653208). The presumed region in BT 1A strain 5303 is presented. A truncated version of this operon is found $B T 30: 5,27$, but in a different genomic context.

Three genes can be identified that resemble glycosyl transferases, and two rhamnose processing genes, $r m I C D$, are present. Together with the putative export system, the three main classes of genes of processing and linking sugars as well as sugar pathways involved in O-antigen synthesis are thus present (Reeves, et al., $1996 a, b)$. The sugar composition of $0: 5,27$ is very similar to $0: 5$. The LPS structure for O:5,27 also contains rhamnose but is O-acylated (Gorshkova, Kalmykova, Isakov and Ovodov, 1986; Bruneteau and Minka, 2003). This explains the close relatedness of the O-antigen synthesis operons.

\subsubsection{Pseudogene distribution amongst the $Y$. enterocolitica biotypes}

Pseudogenes can be defined as CDSs that show mutations that may inhibit the expression of a fully functional protein. Mutations include the truncation of the protein due to deletion events of the termini or other parts of the protein and the disruption 
due to the insertion of an IS element into the reading frame. This first category can also be described as partial proteins, or fragments/remnants. These might be viewed as truly non-functional proteins. In a second category, the pseudogenes show mutations such as frameshifts and introduced stop codons that interrupt transcription. In this case the complete reading frame is still present and it might be argued that stop codons are read through on some occasions and that in the case of homopolymeric runs the RNA polymerase might easily slip into the other reading frame resulting in a full-length protein. Two examples in the given dataset are YE3376, peptide chain release factor 2, which carries a frameshift mutation in all orthologs, but has been shown to be functional, and YE4135, the major subunit of formate dehydrogenase, which has a read-through of the opal (UGA) stop codon in all orthologs. To take these things into consideration, pseudogenes and partial genes have to be compared separately.

A list of all pseudogenes found in the $Y$. enterocolitica reference genomes and their orthologs can be found in the appendix (Table 9.7, p. 227). In addition to the above described YE3376 and YE4135, only two CDSs are present as pseudogenes in all biotypes. YE0416 is a conserved hypothetical protein, and YE2410 a putative oxidoreductase.

As seen in Table 3.3, there is a difference between the biotypes in the total number of pseudogenes. BT $1 \mathrm{~A}$ and $1 \mathrm{~B}$ have the lowest number of pseudogenes, 81 and 84 respectively, whereas the low-pathogenic bio-serotypes show more than 140 pseudogenes each. BT 4 O:3 (strain 1203) and BT 5 O:2a,2b,3 (strain 3094) show a marked increase in pseudogenes with 235 and 322 pseudogenes in total, respectively. This amounts to $6.0 \%$ and $7.9 \%$ of the total number of CDSs in each genome. In contrast, only about $2 \%$ of the genomes of BT $1 \mathrm{~A}$ and $1 \mathrm{~B}(1.9 \%, 2.1 \%)$ show signs of genome decay. BT 2 and 3 possess less than $4 \%$ of CDSs with potentially impaired function (BT 2 O:9 4.0\%, BT 3 0:9 3.6\%, BT 3 0:5,27 3.7\%). This trend of increased genome decay towards BT4 and 5 can be observed when only considering partial CDSs, too. Both BT show about 130 CDSs that are nonfunctional.

Figure 4.15 shows the split of pseudogenes and partial genes for each of the reference strains according to function. One can see that some classes are absent 
because they constitute essential cell functions. These are genes involved in cell division (6) and ribosome constituents (17).

The highest number of pseudogenes and partial genes in hypothetical proteins (1) is found in BTs 4 and 5. Hypothetical proteins are putative open reading frames with either homologs in E. coli or other bacterial species with unassigned functions or do not have any homologs. Their transcriptional and functional status is thus unknown. As the contribution towards cell survival under various conditions still needs to be assessed, the impact of the pseudogenes in this category is uncertain.

When looking at cell processes (2), it is striking that BT $1 \mathrm{~A}$ has the highest number of partial genes compared to the other BTs. These are found in chemotaxis and motility genes of flagellar cluster 1 and 2. The four copies of the flagellin gene fliC of Flag-1 are missing 40-70 amino acids, and Flag-2 is only present in remnants. Yet, this strain has been studied and was shown to be motile, thus the mutations seen in Flag1 do not have an impact on function (McNally et al., 2007). This might be different in BTs 4 and 5. BT 4 has lost half of the Flag-2 region, and both BTs show mutations and gene deletions in Flag-1 and -2. A similar inactivation of the flagellar clusters is apparent in $Y$. pestis as an adaptation to a niche where motility is not necessary (Parkhill et al., 2001a). 


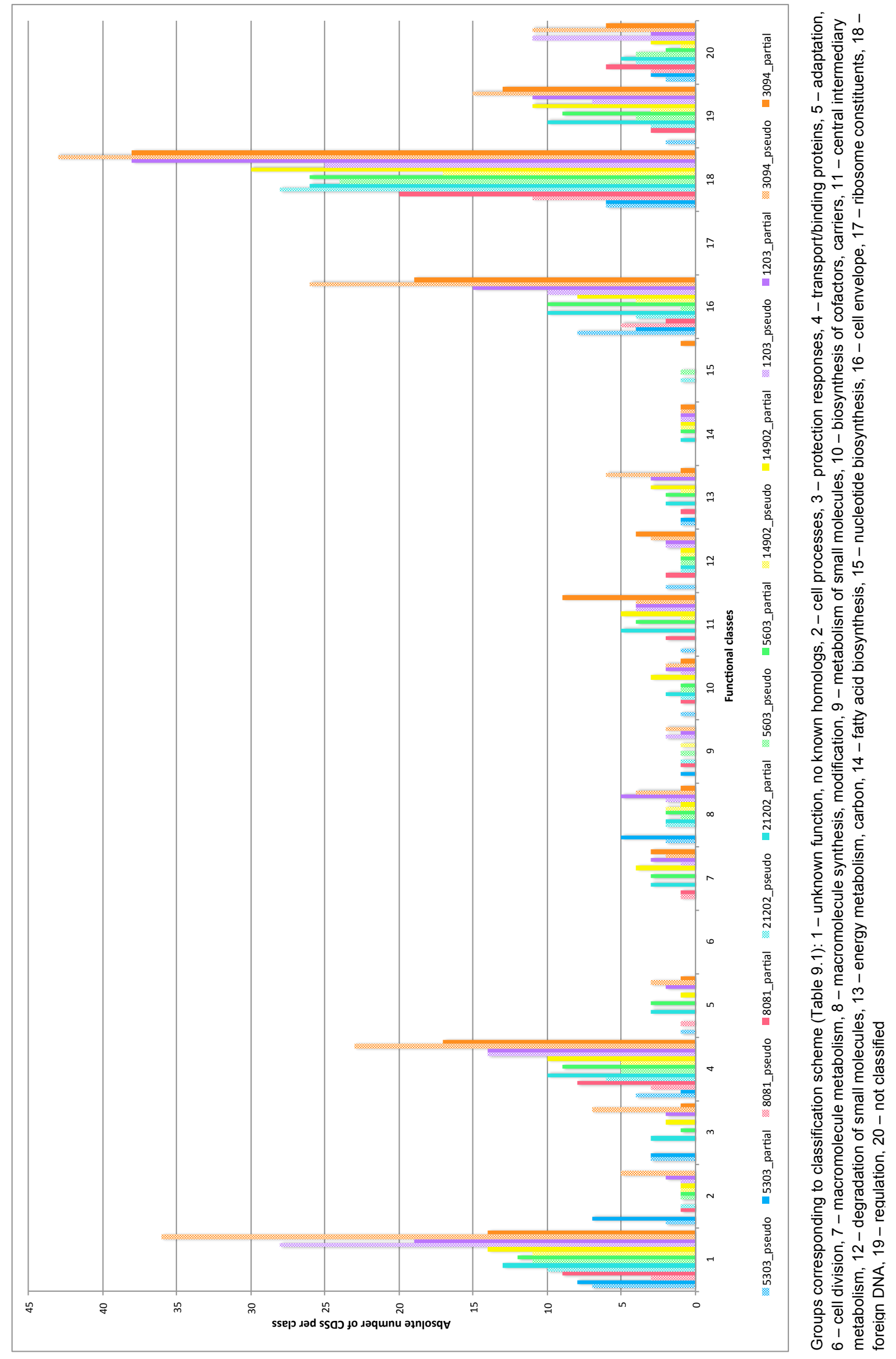

Figure 4.15: Pseudogene and partial gene distribution in the $Y$. enterocolitica reference genomes. 
BT $1 \mathrm{~A}$ also shows a high number of pseudogenes and partial genes in protection responses (3) when compared to the other BTs. Some of these CDSs relate to adhesion/hemagglutinin proteins unique to BT 1A, an enterotoxin-like protein of Y. pestis, and a hemolysin paralog of BT 1B. Furthermore, BT 1A has a stop codon mutation in umuD (YE1733), a UV protection protein. There is a functional unique copy of umuD elsewhere in the genome though. Interestingly, in this second operon, umuC carries a frameshift mutation. A drug resistance operon shared in BTs $1 \mathrm{~A}$ and $1 \mathrm{~B}$ has been deleted from the low-pathogenic BTs. These retain only fragments of two proteins encoded in this region (YE0443, YE0445). A stop codon has been introduced into the BT 1A homolog of YE0444, hence the functionality of this multidrug efflux protein is compromised. Some mutations are found in the tc PAI. The BT $20: 9$ strain has a frameshift mutation in tccC2 due to the fact that this CDS links two contigs. Therefore it could be functional, but sequence information is missing. Irrespective of this, two additional, functional copies of tcc $C$ are present upstream. BTs 4 and 5 also show mutations in tc PAI. Both have mutations in the regulator tcaR2 and the protein tcaC'(2) in common, and BT 5 additionally has mutation in tcaB2. BT 1B has no mutations in protection responses.

The lowest number of pseudogenes in transport and binding proteins (4) is given in BT 1A. The highest number of pseudogenes is given in BTs 4 and 5 . Transport proteins are involved in sugar uptake and metabolism, and a higher number of pseudogenes in pathogenic BTs could reflect the reduced metabolic capabilities apparent in the biotyping scheme. With respect to adaptation (5), both BT 1 A and BT 1B only have one partial gene. The acid shock protein Asr (YE0933) is missing 15 aa in a repeat region, resulting in a shortened protein. This same mutation is evident in the low-pathogenic BTs. Both BT 2 and BT 3 0:9 isolates also show a truncation of another copy of this protein (YE2086) which is functional in the other BTs. The pseudogenes in BT 5 are located in the haemin storage operon. A stop codon has been introduced in hmsF (YE2483), and hmsH (YE2484) shows a frameshift mutation in a homopolymeric run. In BT 4 this last protein also has a frameshift, with 10 amino acids missing.

BT 1A shows no mutations in macromolecule metabolism (7). A beta-glucosidase (YE1236A) has been truncated in both high- and low-pathogenic BTs due to a deletion event. The other pseudogene in BT $1 \mathrm{~B}$ is a putative protease (YE1406). A putative DNA-binding protein (YE1874) and a metalloprotease (YE4052) are commonly fragmented in the low-pathogenic BT. BTs 4 and 5 again share additional 
pseudogenes. A putative serine protease (YE1389) absent from BT 1A has a stop codon introduced in BT 4, and several stop codons plus a frameshift in BT 5 . Further more in BT 5, there is an exported zinc protease (YE0320) with a frameshift in a homopolymeric run.

The partial genes in macromolecule synthesis and modification (8) found in BT 1A are mostly in CDSs that are unique to this strain. Annotation is thus based on comparison with homologs in other bacterial species and functionality is unknown. This applies also to the pseudogenes in BT 4; these are also not found in the backbone of $Y$. enterocolitica CDSs but within the accessory genome. The inactivation of those genes might present niche adaptation. One partial gene in BT 1A, ampM (YE3285) a methionine aminopeptidase, is also mutated in BT 5. Interestingly, BT 1B shows no mutations in this class.

There are only very few pseudogenes and partial genes in the metabolism of small molecules (9). In BT $1 \mathrm{~A}$, this is due to an additional copy of $\operatorname{trp} A$ involved in tryptophan synthesis. This protein is missing 160 amino acids at the $\mathrm{N}$ terminus, but due to a functional gene copy present elsewhere in the genome, this should have no impact on metabolism of tryptophan. In $\mathrm{BT} 1 \mathrm{~B}$ the inactivated gene is a unique possible homoserine synthase (YE1737) that has been truncated by the insertion of an upstream IS element. A common pseudogene in the low-pathogenic BTs is a frameshift in the glutamate synthase precursor gltB (YE3735). One of the pseudogenes in BT 4 is in the tryptophanase tnaA (YE0650). The protein could be responsible for a negative phenotype for indole production in the biotyping reaction, but does not explain why BTs 3 and 5 show a negative phenotype as well.

The group of biosynthesis of cofactors and carriers (10) shows a marked increase in partial genes in BT 3 0:5,27 strain 14902. All pathogenic BTs carry pseudogenes in genes encoding the metabolism of the cofactors cobalamin and cobinamide, cbiG (YE2719) and cobC (YE2708). The exact effect of these mutations is unknown as there are potential alternative pathways. BT 5 also possesses two more pseudogenes in this pathway affecting the cobinamide kinase CobU and a membrane protein (YE2724).

There are few mutations in central intermediary metabolism (11) in the non- and high-pathogenic BTs. BT $1 \mathrm{~A}$ has one mutation in pldB (YE0207), a lysophospholipase. A stop codon has been introduced terminating the protein 7 amino acids early. An impact of this mutation is equivocal. All BTs except BT $1 A$ show various degrees of fragmentation and remnants of YE2586A encoding a CoA 
transferase familiy protein. All low-pathogenic BTs also only have the $3^{\prime}$ end of $s s u D$ (YE1950) encoding an alkanesulfonate moooxygenase. Additionally pseudogenes are found in BT 5 in the backbone of Y. enterocolitica. Both amn (YE2052, AMP nucleosidase) and ushA (YE3066, UDP sugar hydrolase) are missing the 3' end, amn also shows a frameshift mutation.

BT 1A shows two pseudogene mutations in the degradation of small molecules (12). An opal UGA stop codon has been introduced in idh (YE4026, m-inositol dehydrogenase). The other mutation is shared in the non- and low-pathogenic BTs and regards the xylose kinase $x y I B$ (YE4123). Stop codons have been introduced towards the $C$ terminal end of the protein. Both $i d h$ and $x y l B$ could still be functional, as the full coding sequence is still present. $x y / B$ could be linked to xylose metabolism, but the xylose acid production in the biotyping test for $Y$. enterocolitica does not agree with the mutation pattern. BTs $1 \mathrm{~A}, 1 \mathrm{~B}, 2$ and 3 are positive for xylose acid production, only BT 4 is negative, and BT 5 variable. The genetic basis for the biotyping reaction is unknown. An operon involved in N-acetyl-D-galactosamine metabolism has been deleted from BT 1B. This is evident in the gene remnants encoding for AgaS (YE2449) and AgaY (YE2450), which are missing the $N$ and C terminus, respectively. BT 5 also shows mutations in this operon, with frameshifts present in agaS and agaW.

In carbon energy metabolism (13), most mutations again are evident in the lowpathogenic BTs, although BT $1 \mathrm{~A}$ has the highest number of total CDSs in this group. The mutations present in BT 1A are outside of the core genome for $Y$. enterocolitica. The mutation present in $\mathrm{BT} 1 \mathrm{~B}$ is due to a deletion event of a region involved in cytochrome $C$ type biogenesis. Only a remnant of the nitrate reductase nrfG (YE1598) is present. The low-pathogenic BTs also show mutations in this cluster in nrfF and BT 5 also in nrfE, thus a fully functional operon might only be present in BT $1 \mathrm{~A}$. The carbonic anhydrase cah is absent from BT $1 \mathrm{~B}$ and has been disrupted in BTs 2, 3, and 4 by a transposase. It is only functional in BTs $1 A$ and 5 . BT 5 again has additional mutations in pathways involving $\mathrm{NADH}$-quinone reductase ( $\mathrm{ngrC}$, YE3218), formate dehydrogenase ( $f d h F$, YE2810), tetrathionate reductase (ttrA, YE1617), and cytochrome $C$ type proteins (napC, YE1158).

The non- and high-pathogenic BTs have no mutations in proteins involved in fatty acid biosynthesis (14). The low-pathogenic BTs have retained a remnant of the Yersinia genomic island 2 (fabF, YE0911). The $\mathrm{N}$ terminus has been deleted possibly due to the presence of a transposase. No mutations are evident in BTs $1 \mathrm{~A}, 1 \mathrm{~B}, 3$ 
0:5,27, and 4 for nucleotide biosynthesis (15). The two 0:9 isolates have a stop codon in the cytosine deaminase $\operatorname{codA}$ (YE3964). BT 5 has a $\mathrm{N}$-terminal deletion in a protein involved in purine metabolism.

Most of the CDSs mutated in BT 1A in the cell envelope (16) relate to putative exported proteins. The cellulose synthase 1 catalytic subunit gene bcs $A$ has an opal stop codon introduced at the 5' end, thus the function is questionable. The pseudogenes found in the low-pathogenic BTs are often shared and related to exported proteins without specific functions.

The highest number of pseudogenes and partial genes is found in genes encoding products that belong to the functional group of "foreign DNA" (18). This group contains IS elements and phage proteins. These were not corrected or re-checked for their mutations, and the true numbers are probably lower than stated here. Moreover, especially the low-pathogenic BTs show an expansion of IS elements, therefore the number of CDSs contained in this class is higher than in BT 1A and $1 \mathrm{~B}$. Even though the number of pseudogenes is corrected for the total number of CDSs per group, the fact that these CDSs were not re-checked increases their overall contribution.

Most of the pseudogenes in the low-pathogenic BTs involved in regulation (19) are shared in all BTs, and a function has only been retained in the high- and nonpathogenic BTs. For example, the regulator of the tetrathionate reduction operon, $t$ trS (YE1614), has a stop codon introduced in the 0:9 BTs 2 and 3, and a frameshift in BT 5. As mentioned earlier, BT 5 has an additional mutation in this operon.

Unclassified and putative assignments (20) constitute proteins of unknown function and preliminary assignments, similar to hypothetical proteins (1). It appears that the number of CDSs is again highest for pseudogenes found in BT 4 and 5.

To summarize there are certain trends within the pseudogenes. The three lineages of high-, low-, and non-pathogenic BTs described for the shared genome content are also visible when comparing the pseudogenes. It appears that especially BTs 4 and 5 have acquired a considerable number of pseudogenes and partial genes. The predominant groups (Figure 4.16) for pseudogenes in BT 1A are cell processes, macromolecule synthesis and modification, and cell envelope proteins. Pseudogenes in the pathogenic BTs relate to transport/binding proteins, proteins related to the cell envelope, and regulatory proteins. BTs 4 and 5 additionaly show pseudogenes in 
degradation of small molecules, which could reflect the reduced metabolic potential visible in the biotyping scheme.

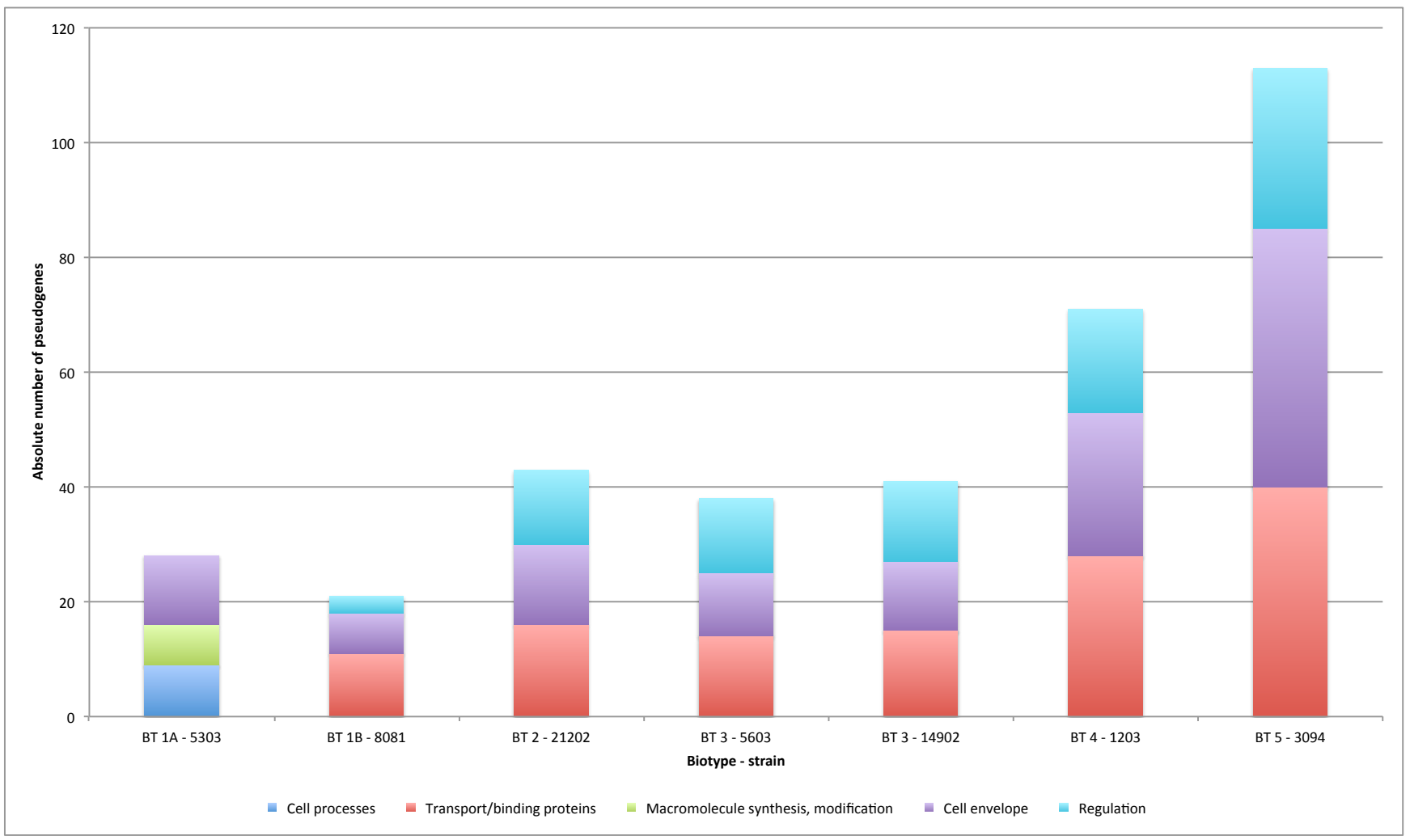

Figure 4.16: Top 3 pseudogene categories for $Y$. enterocolitica BTs. Absolute numbers are given only for top 3 categories, disregarding possible pseudogenes in other categories.

Fewer pseudogenes are observed in the non- and high-pathogenic BTs than in the low-pathogenic BTs. The non-pathogenic BT has more pseudogenes than partial genes, whereas it is the other way round in the high-pathogenic BT. Hence, the highpathogenic BT shows a higher degree of genome decay than the non-pathogenic BT. Quite often the pseudogenes in the low-pathogenic BTs are shared in all of those isolates. BTs 4 and especially 5 have additional mutations. The mutations observed in the same genes in BTs 4 and 5 are not identical, but appear to have arisen independently.

\subsubsection{The role of IS elements and recombination in Y. enterocolitica biotypes} IS elements are believed to have played an essential part in the evolution of $Y$. pestis from Y. pseudotuberculosis through transposition into coding genes and through genomic rearrangements between IS elements (Parkhill, et al., 2001a; Deng, et al., 2002; Chain, et al., 2004). During sequencing and assembly of the Y. enterocolitica 
biotypes, it became clear that there was a predominance of one particular IS element (IS1667). The total breakdown of all IS elements is given in Table 4.2.

Table 4.2: IS elements in the Y. enterocolitica BT

\begin{tabular}{lccccccc}
\hline IS name & $\begin{array}{c}\text { BT1B - } \\
\text { 8081* }\end{array}$ & $\begin{array}{c}\text { BT1A - } \\
\mathbf{5 3 0 3}\end{array}$ & $\begin{array}{c}\text { BT2 - } \\
\mathbf{2 1 2 0 2}\end{array}$ & $\begin{array}{c}\text { BT3 - } \\
\mathbf{5 6 0 3}\end{array}$ & $\begin{array}{c}\text { BT3 - } \\
\mathbf{1 4 9 0 2}\end{array}$ & $\begin{array}{c}\text { BT4 - } \\
\mathbf{1 2 0 3}\end{array}$ & $\begin{array}{c}\text { BT5 - } \\
\mathbf{3 0 9 4}\end{array}$ \\
\hline \hline IS3 & 2 & 0 & 0 & 0 & 0 & 0 & 0 \\
IS285 & 1 & 0 & 0 & 0 & 0 & 0 & 0 \\
IS1222 & 1 & 1 & 0 & 0 & 0 & 0 & 0 \\
IS1328 & 5 & 0 & 8 & 8 & 5 & 4 & 8 \\
IS1329 & 6 & 0 & 0 & 0 & 0 & 0 & 0 \\
IS1330 & 8 & 0 & 0 & 0 & 0 & 1 & 4 \\
IS1400 & 4 & 0 & 2 & 2 & 3 & 1 & 2 \\
IS1541 & 3 & 0 & 0 & 0 & 0 & 0 & 0 \\
IS1660 & 6 & 0 & 0 & 0 & 0 & 0 & 0 \\
IS1664 & 3 & 0 & 0 & 0 & 0 & 0 & 0 \\
IS1665 & 5 & 0 & 0 & 0 & 0 & 0 & 0 \\
IS1666 & 1 & 0 & 1 & 1 & 2 & 0 & 0 \\
IS1667 & 8 & 0 & 37 & 35 & 30 & 53 & 50 \\
IS1668 & 2 & 0 & 10 & 10 & 9 & 10 & 9 \\
IS1669 & 5 & 0 & 2 & 3 & 2 & 6 & 3 \\
unclassified IS & - & 30 & 27 & 26 & 28 & 26 & 34 \\
\hline total & 60 & 31 & 87 & 85 & 79 & 101 & 110 \\
\hline
\end{tabular}

*taken from Thomson et al., 2006

There is an uneven distribution of IS elements in the BTs. The reference genome for BT 1A shows none of the typical IS elements found in the other BTs. A search in other BT 1A isolates showed that this might be atypical and copies of IS1329 and IS1667 could be present in BT 1A. Additionally, IS1222 is present, but not consistently. There are a number of unclassifed IS elements which have homologies to ISEhe3 and IS621 found in E. coli, transposases similar to IS116/IS110/IS902 of Stenotrophomonas, and transposases similar to IS2 and IS3, but these have not been characterized in more detail here.

The IS elements of BT 1B belong to a wide range of different families, with no more than 8 copies of any single IS element. In total, BT 1B carries 60 IS elements. A similar number of IS elements can be found in the low-pathogenic BTs 2 - 5, but here the distribution is skewed towards IS1667, which is present in more than 30 copies per strain. Other IS elements present are IS1328, IS1400, IS1668, and IS1669.

The position of IS1667 (Figure 4.17) indicates that it might be mediating intragenomic recombination, as it is often found at borders of transpositions and inversions. 
Non-pathogenic

BT

High-pathogenic

BT

Low-pathogenic

BT
BT1A -

5303

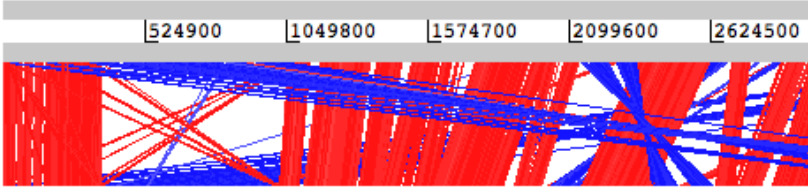

149400

\begin{tabular}{l|l}
4199200 & 4724100
\end{tabular}

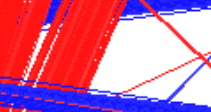

BT1B -

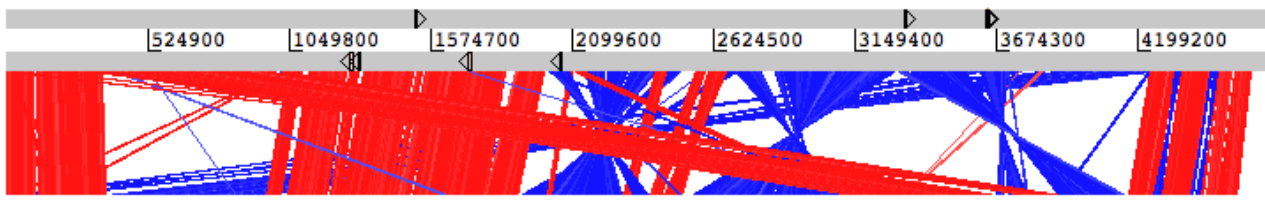

BT2 -

21202

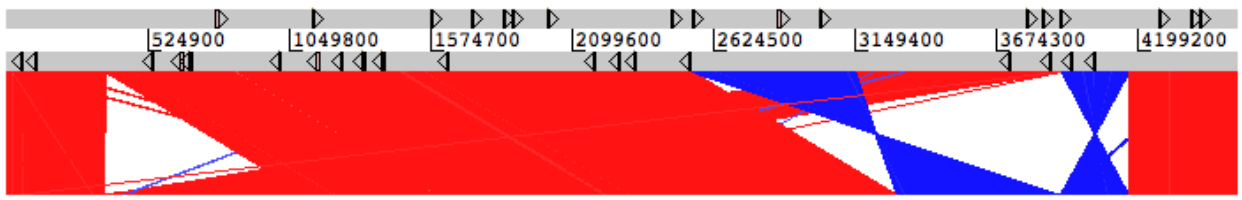

BT3 -

5603

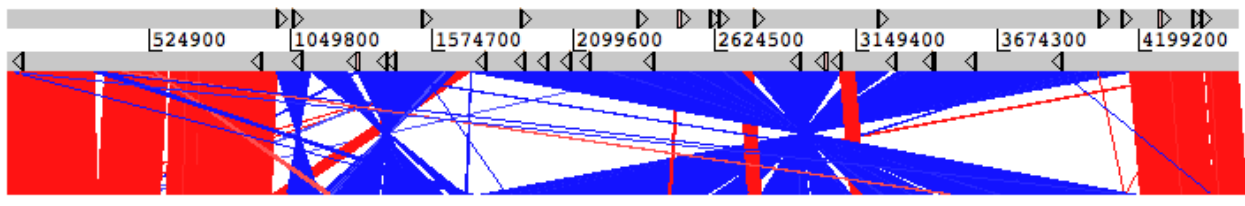

BT3 -

14902

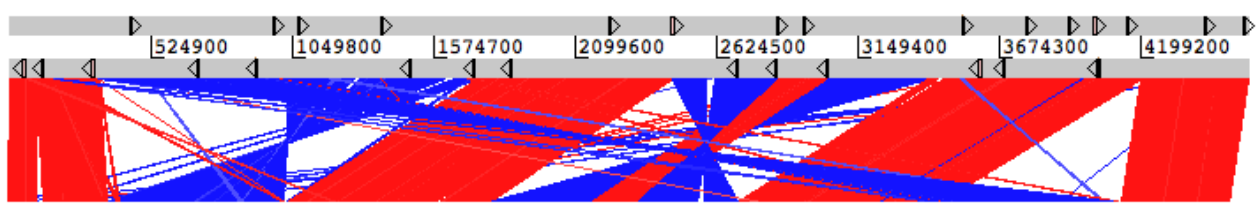

BT4 -

1203

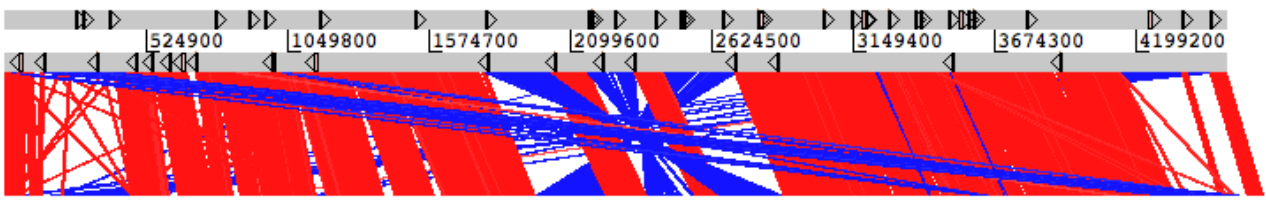

BT5 -

3094

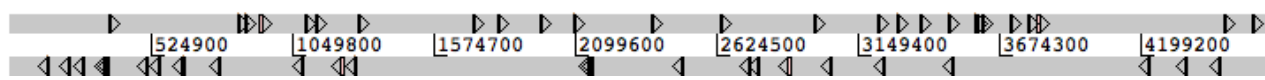

Figure 4.17: Position of the predominant IS element IS1667 in the reference genome assemblies. Genomes are compared in a pair-wise fashion. Red bars - co-linear homology >999 bp, blue bars - inverted homology $>999 \mathrm{bp}$. Contigs are not highlighted. Arrows indicate position and orientation of the IS1667.

As is the case in Y.pestis, IS elements can also be shown to promote genome instability via recombination. There are examples of IS elements which have inserted into CDSs and thus disrupt their expression in the low-pathogenic BTs. One example mentioned above is the carbonic anhydrase cah, which has been disrupted by a transposase in BTs 2, 3, and 4. Another good example as well is found in BT4 where transposase action lead to the loss of half of the flagellar cluster and the iron-uptake operon, as described before.

As can be seen from Figure 4.17 there are some regions in the Y. enterocolitica reference genomes that are not to affected by IS mediated recombination or disruption. These regions retain the same gene arrangements althought their position respective to each other can vary. This concept has been introduced in $Y$. pestis by Deng, et al. (2002), and was used to describe syntenic blocks. Later Chain, et al. 
(2004) tried to deduce the number of recombinatorial events in Y. pestis since the divergence from $Y$. pseudotuberculosis through the investigation of those syntenic blocks.

This concept can also be applied in Y. enterocolitica, and 29 syntenic blocks could be identified through the comparison of the seven reference genomes (Table 4.3).

Table 4.3: Syntenic blocks identified in the reference genomes of $Y$. enterocolitica.

\begin{tabular}{|c|c|c|c|c|c|c|}
\hline Syntenic block & start position $^{\dagger}$ & end position $^{\dagger}$ & CDS start $^{\dagger}$ & CDS end ${ }^{\dagger}$ & size & \# CDS \\
\hline 1 & 0 & 107030 & YE0001 & YE0097 & 107030 & 97 \\
\hline \multirow{3}{*}{$2^{*}$} & 108300 & 191330 & YE0099 & YE0160 & 83030 & 61 \\
\hline & 191500 & 202555 & YE0161 & YE0168 & 11055 & 7 \\
\hline & 282830 & 313630 & YE0169 & YE0273 & 30800 & 104 \\
\hline 3 & 319700 & 353900 & YE0274 & YE0303 & 34200 & 29 \\
\hline 4 & 362987 & 874170 & YE0307 & YE0747 & 511183 & 440 \\
\hline 5 & 879520 & 980830 & YE0754 & YE0852 & 101310 & 98 \\
\hline 6 & 1039400 & 1251800 & YE0911 & YE1115 & 212400 & 204 \\
\hline 7 & 1264600 & 1802600 & YE1124 & YE1602 & 538000 & 478 \\
\hline 8 & 1802900 & 1991400 & YE1603 & YE1798 & 188500 & 195 \\
\hline 9 & 2027865 & 2087750 & YE1846 & YE1901 & 59885 & 55 \\
\hline 10 & 2108500 & 2142600 & YE1933 & YE1962 & 34100 & 29 \\
\hline 11 & 2154500 & 2325300 & YE1975 & YE2124 & 170800 & 149 \\
\hline 12 & 2326000 & 2406630 & YE2125 & YE2196 & 80630 & 71 \\
\hline 13 & 2407700 & 2446400 & YE2198 & YE2234 & 38700 & 36 \\
\hline 14 & 2447240 & 2499700 & YE2237 & YE2285 & 52460 & 48 \\
\hline 15 & 2554700 & 2591553 & YE2365 & YE2402 & 36853 & 37 \\
\hline 16 & 2602500 & 2630230 & YE2409 & YE2439 & 27730 & 30 \\
\hline 17 & 2640950 & 2668185 & YE2454 & YE2477 & 27235 & 23 \\
\hline 18 & 2668285 & 2709585 & YE2478 & YE2513 & 41300 & 35 \\
\hline 19 & 2709700 & 2800900 & YE2514 & YE2602 & 91200 & 88 \\
\hline 20 & 2854263 & 3294700 & YE2636 & YE3022 & 440437 & 386 \\
\hline 21 & 3313400 & 3544778 & YE3048 & YE3248 & 231378 & 200 \\
\hline 22 & 3552700 & 3610900 & YE3252 & YE3308 & 58200 & 56 \\
\hline 23 & 3610900 & 3712864 & YE3309 & YE3399 & 101964 & 90 \\
\hline 24 & 3726200 & 3761260 & YE3413 & YE3447 & 35060 & 34 \\
\hline 25 & 3897560 & 3933900 & YE3581 & YE3609 & 36340 & 28 \\
\hline 26 & 3960000 & 4238800 & YE3631 & YE3882 & 278800 & 251 \\
\hline 27 & 4245400 & 4464400 & YE3883 & YE4083 & 219000 & 200 \\
\hline 28 & 4504400 & 4561500 & YE4118 & YE4162 & 57100 & 44 \\
\hline 29 & 4561800 & 4615788 & YE4163 & YE4215 & 53988 & 52 \\
\hline
\end{tabular}

${ }^{\dagger}$ labelling respective to BT 1B strain 8081; ${ }^{7}$ CDSs present a clean transposition in BT 5 due to the action of transposases; colours correspond to syntenic blocks highlighted in Figure 4.18.

The syntenic blocks vary in size and gene content, ranging from 23 CDSs (17) up to 478 CDSs (7). It is possible that with sequencing of further $Y$. enterocolitica genomes this number could change, but considering the divergence between these isolates gives confidence in the assignment of syntenic blocks. An example for the variable positions of the syntenic blocks is given in Figure 4.18 . 


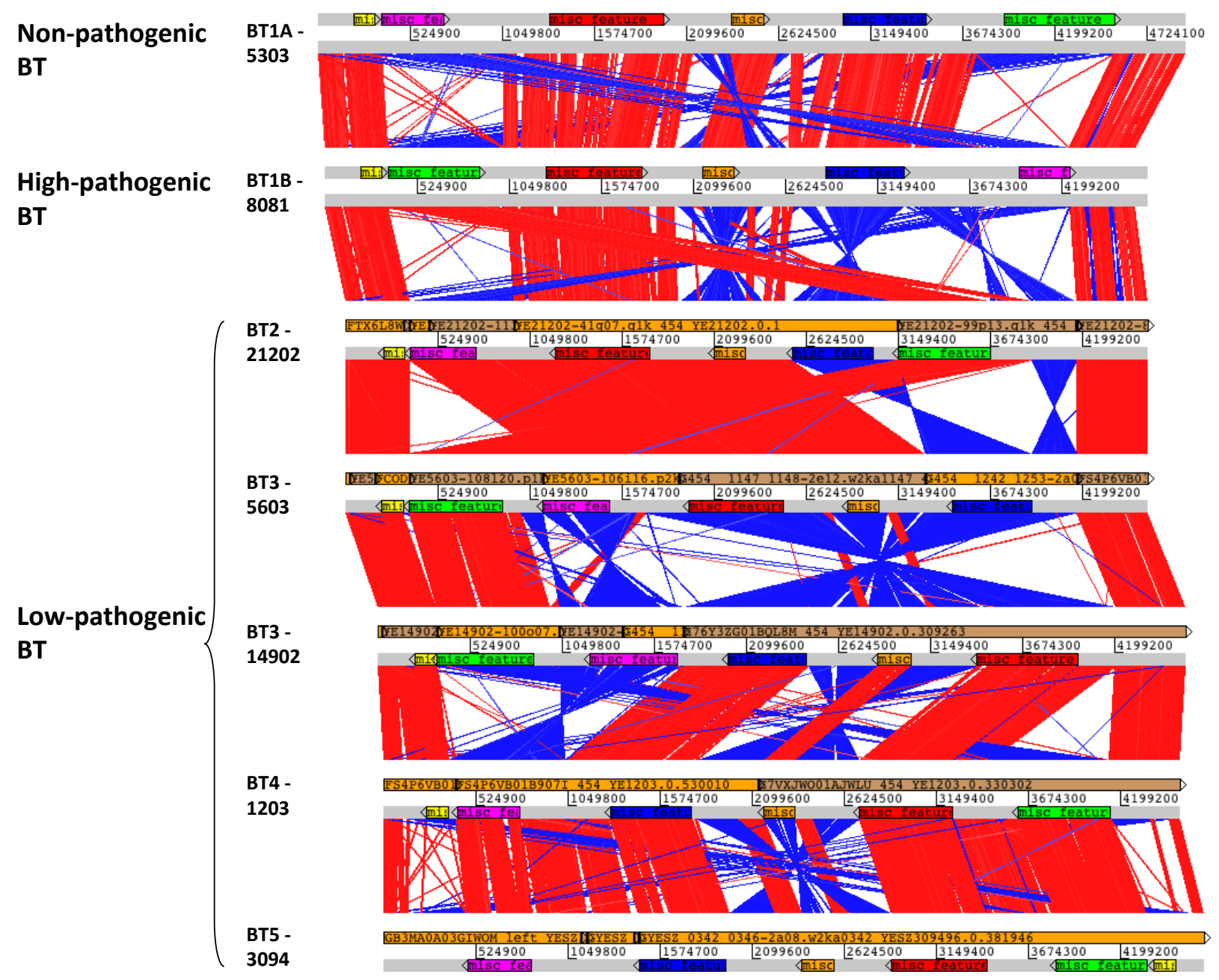

Figure 4.18: Exemplar position of six syntenic blocks. Six syntenic blocks are highlighted in yellow, pink, red, orange, blue, and green in the respective reference genomes to illustrate their variable positions within the genomes relative to the sequence start. Colours correspond to syntenic block colours in Table 4.3. Contigs are highlighted in orange and brown in the low-pathogenic BTs.

As one can see none of the regions appear in the same order and orientation as in any of the other genomes. BT 1 A and BT 2 do have the same order in the six syntenic blocks observed, but the blue and green block show inversions which are not easily visible as the syntenic blocks have been artificially allocated to the reverse strand to allow for visualization of the contigs on the forward strand.

The identification of syntenic blocks could help in the design of a multilocus sequencing typing (MLST) scheme. One of the underlying principles for MLST is that the genes should be evenly distributed around the chromosome to exclude that the genes are linked with respect to evolutionary changes and recombination events (Maiden, et al., 1998). In chosing genes located within major syntenic blocks one can ensure that the genes are unlinked, as the blocks are conserved in structure. Despite genomic rearrangements due to recombination that change the position of the blocks, the syntenic blocks should cover different parts of the chromosome and guarantee an even spread. 


\subsection{Discussion}

\subsubsection{High-, low-, and non-pathogenic biotypes have distinct genomic features}

Through the analysis of orthologous gene sets it is apparent that high-, low-, and non-pathogenic BTs are very distinct in their genomic content. High- and nonpathogenic BTs each have a considerable number of unique CDSs, whereas the lowpathogenic BTs appear as a tight cluster of isolates. The regions specific to the lowpathogenic BTs are shared in all of the isolates, and unique regions only relate to independently acquired phages and LPS operons. It is important to note that BT 2 and BT 3 are genetically indistinguishable from each other. BT 4 has lost the fes-fep iron acquisition operon and half of the second flagellar cluster, and this has been confirmed in larger strain collections for fes-fep operon (Schubert, Fischer and Heesemann, 1999; Thomson, et al., 2006). The loss of half of the flagellar cluster has not been reported before, and could only be detected through in-depth comparison between all of the reference genomes. BT 5 is signified by massive genome decay. The genetic basis for the biotyping reactions could only be determined for trehalose and sorbose, both pseudogenes in BT 5.

It was interesting to see how closely related high- and non-pathogenic BTs appear to be with respect to shared gene content. On the other hand, the non- and lowpathogenic BTs also do have a lot in common. In fact, these two groups incorporate more CDSs than the supposedly more important group of pathogenic BTs. A common gene set of pathogenic BTs is reduced to the virulence plasmid $p Y V$ and the attachment/invasion locus protein Ail. There are also no signatures for humanpathogenic isolates or for animal isolates. A similar finding has been made when comparing pathogenic and commensal E. coli strains (Chaudhuri, et al., 2010). The authors found only $13 \mathrm{CDSs}$ that resembled potential virulence factors absent from the commensal strain. They suggest that this absence of pathogenic signature indicates a phylogenetic heterogeneity of enteroaggregative E. coli (Chaudhuri, et al., 2010). It will be investigated later if this case is similar in Y. enterocolitica (Chapter 7), but the split into high- and low-pathogenic BTs due to heterogeneity resembles the findings in E. coli.

Many genes and operons have been suggested as "virulence markers" or "virulence genes" of pathogenic Yersiniae in the past, only later for homologs to be found in environmental species as well. As classic example of this is the presence of hitherto 
considered Yersinia virulence genes in BT 1A including the invasion gene invA, the tight adherence operon and two YGI (Pierson and Falkow, 1990; Thomson, et al., 2006). Similarly, the host-responsive element rscBACR (Young and Miller, 1997; Nelson, Young and Miller, 2001), thought to be involved in the dissemination of Y. enterocolitica in the spleen, is found in all BTs. These discoveries may help explain why BT $1 \mathrm{~A}$ is increasingly being associated with mild human intestinal infectious disease. The only robust markers for pathogenicity identified in BT $1 \mathrm{~B}$ relate to the HPI, Ysa T3SS, and yst-1.Some important regions and islands of Y. enterocolitica and their distribution within the species are shown in Table4.4.

Table 4.4: Distribution of important regions and islands of $Y$. enterocolitica in reference genomes.

\begin{tabular}{|c|c|c|c|c|c|c|}
\hline & BT 1A & BT 1B & BT 2 & BT 3 & BT 4 & $\overline{\text { BT } 5}$ \\
\hline YGl-1 (tad) & + & + & + & + & + & + \\
\hline YGI-2 & + & + & - & - & - & - \\
\hline YGI-4 & + & $(+)$ & - & - & - & - \\
\hline Ni-Co efflux & + & + & - & - & - & - \\
\hline Ag-Cu efflux & + & - & - & - & - & - \\
\hline tc PAI & $-*$ & - & + & + & + & + \\
\hline Flag-1 & + & + & + & + & + & + \\
\hline Flag-2 & - & - & + & + & $(+)$ & + \\
\hline Outer core LPS & + & - & + & + & + & + \\
\hline YGT & + & - & + & + & + & + \\
\hline Ysa T3SS & - & + & - & - & - & - \\
\hline pYV & - & + & + & + & + & + \\
\hline fes-fep & + & + & + & + & - & + \\
\hline ail & - & + & + & + & + & + \\
\hline HPI & - & + & - & - & - & - \\
\hline$y s t-1$ & - & + & - & - & - & - \\
\hline yst-2 & + & + & + & + & + & + \\
\hline YAPI & - & $+^{\dagger}$ & - & - & - & - \\
\hline inv/rovA & + & + & + & + & + & + \\
\hline myf fibrillar operon & + & + & + & + & + & + \\
\hline hydrogenase-2,4 & + & + & + & + & + & + \\
\hline
\end{tabular}

The regions that were thought to be involved in niche adaptation to the human gut now need to be re-evaluated for their contribution to disease. The distribution of putative virulence factors should also be examined in larger strain collections of $Y$. enterocolitica as well as environmental Yersiniae. 


\subsubsection{Metabolism, pseudogenization and IS elements are important evolutionary forces in Y. enterocolitica}

A theme emerging from the comparison of the different BTs is the potential difference in metabolic properties. Regions that are unique to $B T 1 A$, regions shared between $\mathrm{BT} 1 \mathrm{~A}$ and $1 \mathrm{~B}$, and regions common to $\mathrm{BT} 1 \mathrm{~A}$ and $2-5$ relate to metabolic pathways and transport operons. Similarly, the comparison of the pseudogenes found that especially the BTs 4 and 5 show an increase in pseudogenes in operons associated with metabolism and transport. This trend of reduced metabolic flexibility is also apparent in the biotyping scheme. BT $1 \mathrm{~A}$ is positive for all of the reactions, then BT $1 \mathrm{~B}$ follows, and a reduced number of positive reactions is observed in the lowpathogenic BTs, finally arriving at BT 5 which is negative or variable for all but one of the reactions (Wauters, Kandolo and Janssens, 1987).

Metabolism is also a driver in the host-adaptation in other pathogenic bacteria (Rohmer, Hocquet and Miller, 2011). Metabolic streamlining to a specific niche can be specifically observed in obligate intracellular organisms for instance when comparing Mycobacterium tuberculosis and Mycobacterium leprae (Cole, et al., 2001). Here it was found that $M$. leprae shows signs of extensive genome decay in form of accumulation of a large number of pseudogenes. Predominant functional groups of pseudogenes are, similar to Y. enterocolitica, small molecule catabolism, energy metabolism, regulatory proteins, synthesis and modification of macromolecules, cell envelope proteins, and transport proteins. Similar trends of pseudogene acquisition alongside host or niche restrictions are also observed in Salmonella enterica serovars Tyhpi (Parkhill, et al., 2001b) and Gallinarum (Thomson, et al., 2008). Again, targets for pseudogenization are metabolic pathways. Interestingly, there are certain pathways found to be targets of pseudogene formation in Y. pestis, S. Typhi, and S. Gallinarum (Parkhill, et al., 2001a,b; Thomson, et al., 2006, 2008) that are also affected by mutations in the potentially highly successful, more host-restricted lineages of $Y$. enterocolitica (BTs 1B, 4, 5). These relate to cobalamin biosynthesis, tetrathionate respiration, and mutations in flagella operons. Y. ruckeri was also found to be deficient in cobalamin biosynthesis and tetrathionate respiration (Chen, et al., 2010). These could potentially be pathways essential in environmental survival and which are lost upon host adaptation due to maintenance cost. For tetrathionate respiration it has also been suggested that the loss of the pathway could be advantageous for growth outside the intestine (Thomson, et al., 2008; Rohmer, Hocquet and Miller, 2011). A recent study (De Paepe, et al., 2011) 
investigated the effect of colonization of germ-free mouse intestines with $E$. coli and found a diversification process. The original $E$. coli population split into separate lineages, one acquiring mutations in the flagellar flhDC operon, another showing mutations leading to a maltose-negative phenotype. This microevolution is potentially driven by the presence of bile salts (De Paepe, et al., 2011). The authors also suggest that maintenance of flagellar operons might require extensive energy, and that functionality in the gut is dispensible. A similar dynamic could explain the parallel loss of flagellar operons in other pathogenic bacteria.

It is not known though if the regions uniquely acquired by BT $1 \mathrm{~A}$ or the regions showing pseudogenes in the pathogenic BTs do in fact have a phenotype associated with them. This will be investigated further in Chapter 6. Pseudogenes are also said to be lost rapidly in populations as their truncated transcript could have negative effects on the cell (Kuo and Ochman, 2010). It could thus be that a lot of the pseudogenes in BTs 4 and 5 have been acquired recently and thus have not been completely deleted yet, or that in fact these pseudogenes still fulfil a function and thus are retained. Again, confirmation of pseudogene status is subject to further phenotypic experiments.

IS elements also present an important evolutionary driving force. The expansion of IS1667 in the low-pathogenic BTs mirrors the overall expansion of IS elements in Y. pestis. A similar finding was made in Burkholderia mallei. This horse-pathogen evolved out of the environmental B. pseudomallei. It was shown that there has been an expansion of three different IS elements in $B$. mallei since its divergence from B. pseudomallei (Song, et al., 2010). These have been attributed to genome decay and reduction in overall genome size. Interestingly, the authors found that a large number of IS elements were conserved with respect to genomic location in several unrelated $B$. mallei genomes indicating that these might have been acquired during evolution of this lineage (Song, et al., 2010). Following geographic spread of the bacteria, there have been few further expansions and transpositions of the IS elements. This can also be observed to some respect in the low-pathogenic BTs. Amongst the orthologous genes of the low-pathogenic BTs, there are 35 transposases and IS elements, of which 10 belong to IS1667. On the other hand, transposition appears to be an ongoing process after the separation into different bioserotypes. As an example, some transposase are further shared between BTs 4 and 5 , and BT 5 possesses 35 unique transposases (12 IS1667). Active IS elements 
have also been observed in Y. pestis when one genomic region was found to be present in two orientations suggesting a high genome fluidity (Parkhill, et al., 2001a). A proteomic study also found unique peptides corresponding to active transposases of IS285, demonstrating that genome recombination is an ongoing process in Y. pestis (Payne, Huang and Pieper, 2010).

\subsubsection{Differences in niche adaptation}

It is apparent that the non-pathogenic BT $1 \mathrm{~A}$ is suitably adapted for environmental survival. It possesses a large number of operons associated with transport and metabolism of various sugars and other metabolites.

The pathogenic BTs lack most of these properties and seem to have chosen a different approach. The high-pathogenic BT seems to have found a unique niche that allowed it to get in contact with other bacteria to exchange pathogenicity islands, thus acquiring the HPI and the Ysa T3SS. In turn, it loses the YGT and regions associated with generalist survival. The YGT is present in all Yersiniae but shows signs of fragmentation specifically in more pathogenic lineages. Y.pestis and Y. pseudotuberculosis are missing parts of the effector region, explaining why an initial investigation into functionality showed no role in pathogenicity (Pujol and Bliska, 2003; Balada-Llasat and Mecsas, 2006). This cluster has also been deleted from the high-pathogenic BT 1B and shows signs of pseudogenization in BTs 4 and 5 , which are potentially the most host-adapted low-pathogenic BTs. BT 4 is isolated from animals and the environment as well, but presents the predominant bioserotype in human infections in Europe (McNally, et al., 2004), suggesting some degree of host-adaptation over the other low-pathogenic BTs. This inactivation of YGT in host-adapted lineages and the distribution in the environmental Yersiniae indicate that the YGT possibly has a role in environmental survival rather that in causing human disease. YGT resembles the SPI-2 T3SS of Salmonella. This system was identified together with the SPI-1 T3SS following signature-tagged mutagenesis and isolation of attenuated clones in a mouse infection model (Shea, Hensel, Gleeson and Holden, 1996). SPI-2 was later implied to be involved in intracellular pathogenesis and survival within macrophages (Hensel, 2000). Recently though it has also been associated with survival inside amoebae and it has been suggested that the mechanisms for survival inside amoebae is the same as for macrophages (Bleasdale, et al., 2009). Insect and protozoa could present an important environmental niche not only for Salmonella but also for Yersinia. Further 
investigations could be carried out to determine a role in colonization of amoeba or insects.

The low-pathogenic BTs have kept the second flagella cluster and possess the tc PAI involved in insect toxicity. Instead of a large metabolic repertoire required in the general environment, the low-pathogenic BTs appear to have adapted to an insect and amoebae niche. Salmonella has been described as containing two flagella cluster as well, and it was hypothetized that the second cluster might act as a spare tyre in that the loss results in host restriction whereas a retention enables colonization of environmental niches (McQuiston, Fields, Tauxe and Logsdon, 2008). On the other hand a second flagellar cluster might enable phase variation and thus immune evasion, especially in a new niche (McQuiston, Fields, Tauxe and Logsdon, 2008). No phase variation has been described in Yersinia thus far, and the fact that both flagella clusters are deactivated in Y. pestis might argue the point that Flag-2 is involved in environmental survival in the low-pathogenic BT, possibly due to their reduced metabolic capacities. It has been suggested that a second flagella cluster supports the search for an insect vector (Bresolin, Trček, Scherer and Fuchs, 2008). Mutations in BTs 4 and 5 on the other hand again possibly reflect host restriction to a niche other than insects, similar to findings made in other pathogenic bacteria, namely Salmonella Typhi and Gallinarum as well as Y. pestis (Thomson, et al., 2008; Parkhill, et al., 2001a).

An important part in infection is immune evasion and how the bacteria present themselves to a host through outer membrane proteins. Both core and outer antigen are recognized by the adaptive immune response and trigger the host complement cascade that results in attraction of phagocytes and lysis of bacterial cells, and are also important in bacterial cell membrance integrity and resistance (Prior, et al., 2001; Skurnik, Venho, Bengoechea and Moriyón, 1999). It is therefore interesting to observe the differences in LPS makeup between high-pathogenic BTs on the one hand and non- and low-pathogenic BTs on the other hand. Because the pathogenic and non-pathogenic $Y$. enterocolitica lineages exhibit a large number of different LPS structures, full protective immunity might only be acquired over time upon infection with each lineage. This would impact on vaccine strategies, but are of low impact due to the usually self-limiting nature of the disease.

The fact that the outer core is shared in non- and low-pathogenic BTs indicates that this might be the ancestral LPS structure of Y. enterocolitica. It is interesting to see 
that the high-pathogenic BT 1B abandoned the outer core in favour of a heteropolymeric operon, whereas the low- and non-pathogenic BTs seem to have acquired additional homopolymeric operons. The LPS structure of the highpathogenic BT thus resembles more the structure of $Y$.pseudotuberculosis and Y. pestis (Skurnik and Bengoechea, 2003). It has been suggested that an ancestor of the pathogenic Yersiniae had acquired a common ancestral version of this heteropolymeric O-antigen (Zhang, Toivanen and Skurnik, 1996). Considering that the non-pathogenic BT exhibits signs of being the historically oldest lineage, and that it shared the outer core biosynthesis operon with the low-pathogenic BTs, this scenario is unlikely. It is more likely that the outer core presents the ancestral form in Y. enterocolitica and that it has been replaced in BT 1B upon colonization of a new niche. So far, nothing is known about the genetic organization and location of the Oantigen operons in the environmental Yersiniae, but it has been suggested that the outer core is also present in the environmental species (Skurnik, et al., 1999). A quick search on the published genomes (Chen, et al., 2010) confirmed the presence of outer core in Y. frederiksenii, Y. kristensenii and Y. rohdei, but due to possible assembly problems, no definitive answer on the other isolates is possible. Thus the directionality of the outer core is loss in the high-pathogenic BT. It also has to be considered though that the O-antigens undergo horizontal gene transfer as well. This has been well documented in E. coli and Salmonella as well as for Y. pseudotuberculosis (Samuel and Reeves, 2003; Skurnik and Bengoechea, 2003).

In conclusion, the comparative analysis carried out enabled a deeper look into what is shared and what is different in Y. enterocolitica BTs. This detailed knowled is specifically important with respect to truncation and mutations of regions in view of BTs 4 and 5. A pathogenic core of genes shared exclusively in high- and lowpathogenic BTs as such does not exist except for the virulence plasmid pYV and the adhesion/invasion locus protein Ail. The presence of some genes that were thought to be virulence determinants in the past in BT $1 \mathrm{~A}$ could explain why it can be associated with disease in humans. The lack of markers for increased pathogenic potential such as the HPI explains the lowered pathogenicity of BTs $2-5$ in a mouse infection model. The regions unique to low-paths indicate a potential environmental niche associated with insects. Evolutionary driving forces for pathogenesis can be defined as IS elements and pseudogenization as well as metabolic properties. 


\section{Plasmid Variety and Variability in Y. enterocolitica Biotypes}

\subsection{Introduction}

The only common "virulence factors" shared in pathogenic BTs of Y. enterocolitica are the chromosomally encoded ail as well as the virulence plasmid pYV. It was shown in the past that functionality of $\mathrm{pYV}$ is conserved, despite marked sequence differences and size variations between plasmids of high- and low-pathogenic BTs of Y. enterocolitica as well as Y. pseudotuberculosis (Portnoy, Moseley and Falkow, 1981; Portnoy and Falkow, 1981; Heesemann, et al., 1983; Portnoy, et al., 1984; Kapperud and Nesbakken, 1987; Fukushima, Gomyoda, Aleksic and Tsubokura, 1993). Variability was also shown in the sequence, orientation and position of some genes as well as gene content in general (Roggenkamp, et al., 1997; Boland, Havaux and Cornelis, 1998; Snellings, Popek and Lindler, 2001; Wagner, et al., 2009; Foultier and Cornelis, 2003; Oberhettinger, et al., 2011). The plasmids can consequently be considered as a "closely related family of plasmids" rather than a uniform molecule (Portnoy, Moseley and Falkow, 1981; Portnoy and Falkow, 1981).

A variety of other plasmids are found in the genus Yersinia. Analysis of plasmids in $Y$. enterocolitica strains showed that strains often harbour more than one plasmid of different types and sizes (Kapperud and Nesbakken, 1987). Those plasmids vary in sequence similarity. Plasmids have also been the object of interest in the nonpathogenic BT1A with a considerable number of isolates containing plasmids of varying sizes (Lewin, et al., 1996). Although BT 1A is considered to be nonpathogenic due to the lack of the virulence plasmid, this study showed that some of the plasmid-bearing strains had atypical biotyping reactions; they exhibited autoagglutination, slow and weak Congo Red uptake, and were also resistant to serum (Lewin, et al., 1996).

The aim of this chapter is to investigate the differences in virulence plasmids found in the pathogenic $Y$. enterocolitica biotypes. Differences found will be investigated in terms of distribution in high- and low-pathogenic BTs and are also compared to Y. pseudotuberculosis and Y. pestis. The plasmid repertoire of $Y$. enterocolitica will also be considered as several unique plasmids were identified during sequencing. 


\subsection{Results}

\subsubsection{Genetic Organization of the virulence plasmid $\mathrm{pYV}$ in Y. enterocolitica}

BT

General properties of the sequenced virulence plasmids in the reference genomes are given in Table 5.1 .

Table 5.1: General properties of the sequenced pYV.

\begin{tabular}{|c|c|c|c|c|c|c|}
\hline Property & $\begin{array}{l}\text { BT1B 0:8 } \\
\text { pYVe8081 }\end{array}$ & $\begin{array}{l}\text { BT2 0:9 } \\
\text { pYVe21202 }\end{array}$ & $\begin{array}{l}\text { BT3 0:9 } \\
\text { pYV5603 }\end{array}$ & $\begin{array}{l}\text { BT3 0:5,27 } \\
\text { pYVe14902 }\end{array}$ & $\begin{array}{l}\text { BT4 O:3 } \\
\text { pYVe1203 }\end{array}$ & $\begin{array}{l}\text { BT5 O:2a,2b,3 } \\
\text { pYVe3094 }\end{array}$ \\
\hline Plasmid size (bp) & 67,721 & 75,103 & 75,107 & 72,395 & 72,413 & 72,824 \\
\hline GC content $(\%)$ & 43.92 & 44.16 & 44.16 & 44.24 & 43.98 & 44.40 \\
\hline Number of CDS & 90 & 90 & 92 & 88 & 86 & 91 \\
\hline Coding density (\%) & 73.0 & 70.6 & 74.0 & 71.6 & 71.9 & 78.5 \\
\hline Average gene size (bp) & 618 & 671 & 664 & 659 & 678 & 647 \\
\hline w/o pseudogenes (bp) & 677 & 698 & 678 & 682 & 694 & 665 \\
\hline
\end{tabular}

One can see that the plasmids from the low-pathogenic BT range between 72.4 and $75.1 \mathrm{~kb}$, and thus are considerably larger than the plasmid from the high-pathogenic BT, which is only $67.7 \mathrm{~kb}$. The GC content of the plasmids ranges from 43.92 to $44.4 \%$. Number of CDS, coding density and average gene size can vary, with no clear pattern for high- or low-pathogenic strains. 59 CDSs are conserved between all pYVs, and a further 22 CDSs are present in pYVs of low-pathogenic BTs. The conserved CDSs are part of the Yop T3SS machinery and regulatory proteins and are encoded within the virABGC operons. This can also be seen in the overall organization of the virulence plasmid (Figure 5.1). From the plasmid comparison one highly conserved region is obvious, which corresponds to the type 3 secretion system machinery. This region shows no inversions or deletions at all, but appears to be essentially the same in gene composition and orientation in all biotypes. In addition the plasmids from the low-pathogenic biotypes present only minor variations in gene content, relating to fragments or pseudogenes of transposases, and show few regions of rearrangement. The orientation of an IS element near the origin can vary and is absent in BT 4. 


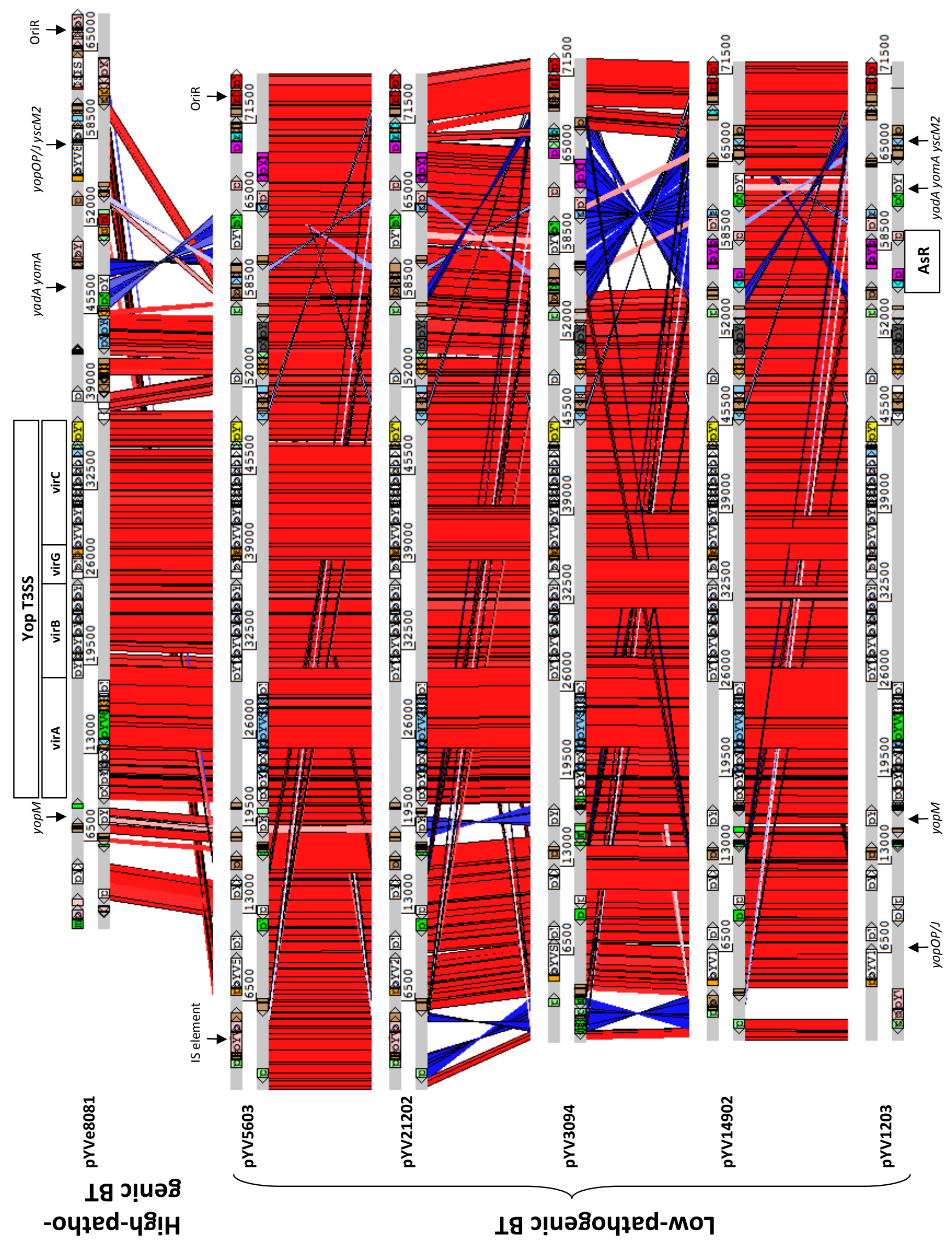

Figure 5.1: Alignment and comparison of the sequenced pYV. The plasmid from the highpathogenic biotype is presented at the top, with the low-pathogenic biotype pYVs aligned below. Regions of specific interest are labelled; OriR - origin of replication. 
Smaller differences are seen in the orientation of yopM and yscM2. These do not show any clear biotype-specific pattern, and this is likely to be explained by the transposase fragments and pseudogenes that surround both genes. The gene yopM is found in the anticlockwise orientation in pYVe8081, pYV21202, and pYV5603, but has the opposite, clockwise orientation in pYV3094, pYV14902, and pYV1203. yscM2 found in pYV14902 and pYV1203 is in the opposite orientation to all other pYVs. This indicates that the rearrangments probably happened independently over time, mediated by adjacent transposases.

The genes for the targeted effector proteins YopO and YopP/J present the only translocations visible in the virulence plasmids. yopOP/J are located upstream of the origin of replication in the high-pathogenic $\mathrm{BT}$, but are located downstream of it in the low-pathogenic BTs. Transposase remnants are present adjacent to these genes, and thus have probably facilitated the translocation. In Y.pestis and $Y$. pseudotuberculosis, yopOP/J are found downstream of the origin of replication, thus resembling the pYV of the low-pathogenic BTs (Snellings, Popek and Lindler, 2001).

It is also obvious that the origin of replication represented at the end of the alignments is different in plasmids of low- and high-pathogenic biotypes. This difference has been noted before in hybridization experiments (Portnoy and Falkow, 1981). The origin of replication of pYV8081 has been allocated to the IncL/M incompatibility group (Snellings, Popek and Lindler, 2001), and it has been noted that this is a feature of other pYVs from high-pathogenic BTs as well (Snellings, Popek and Lindler, 2001; Oberhettinger et al., 2011). Sequence similarity of the origin of replication in high-pathogenic pYVs to pYVs from low-pathogenic BTs is very low with $16 \%$ whereas protein identity is higher than $92 \%$ for the rest of the plasmid (Snellings, Popek and Lindler, 2001). Conversely, the origins of replication of both pYVs of low-pathogenic Y. enterocolitica and of Y. pseudotuberculosis as well as pCD1 of $Y$. pestis are similar and belong to the IncFIIA incompatibility group (Snellings, Popek and Lindler, 2001).

A major difference in gene content between pYVs of high- and low-pathogenic BTs is the presence of an arsenic resistance operon in the low-pathogenic BTs. The orientation of this region and with it the position of $y o m A$, yadA, and $y s c M 2$ plus fragmented transposases can vary amongst the biotypes. 
The arsenic resistance operon has been described as part of the transposon Tn2502 (Neyt, Iriarte, Thi and Cornelis, 1997). It contains a total of six CDSs. The first CDS is the gene for transposase $\operatorname{tnp} A$. The second CDS tnpR encodes the resolvase. The other four CDSs make up the arsenic resistance operon, containing an arseniteinducible transcriptional repressor regulator ArsR, the transmembrane channel ArsB for export of arsenite, and the arsenate reductase ArsC catalyzing the conversion of arsenate to arsenite, which is then exported. The fourth CDS, arsH, is of unknown function, but is essential in the full resistance towards arsenite and arsenate. The presence of this transposon only in plasmids of low-pathogenic strains has been reported previously (Neyt, Iriarte, Thi and Cornelis, 1997). As the transposase is presumed to be non-functional in the described plasmid, it has been proposed that the acquisition event is ancient, and might be related to the worldwide spread of those biotypes where the arsenic resistance is thought to be of importance in environmental survival. In the past arsenic has been used as a growth factor in pigs. As pigs are thought to be one of the main reservoirs of $Y$. enterocolitica, their treatment with arsenic could have selected for plasmids carrying the operon (Neyt, Iriarte, Thi and Cornelis, 1997). Alternative arsenic resistance operons however are also found in BT 1B and BT 1A. The high-pathogenic BT 1B carries one unique copy inside the plasticity zone. Another copy outside the plasticity zone is orthologous to a copy in the non-pathogenic BT $1 \mathrm{~A}$. BT $1 \mathrm{~A}$ also has a unique additional arsenic resistance operon. The chromosome or the plasmid can consequently carry arsenic resistance operons. pYV-independent arsenic resistance has been reported in the high-pathogenic BT 1B strain 8081 (Neyt, Iriarte, Thi and Cornelis, 1997), but nothing is known about functionality of arsenic resistance operons found in the nonpathogenic BT $1 \mathrm{~A}$.

In the presented strains it seems as if the region for the transposon extends beyond that described by Neyt, Iriarte, Thi and Cornelis (1997). Not only the Tn2502 region changes orientation in different plasmids, but also yscM2, yadA and yomA plus fragmented transposases surrounding yscM2. It can be argued that only Tn2502 might change orientation if it was mobile, as opposed to the whole region between positions $58.5 \mathrm{~kb}$ and $71.5 \mathrm{~kb}$ flipping, as seen here. There is no current evidence or experimental data whether these transposition events are ancient or still undergoing recombination in current cultures. 


\subsubsection{Generic Map of pYV}

Figure 5.2 summarises all the genetic variations between $\mathrm{pYV}$ plasmids.

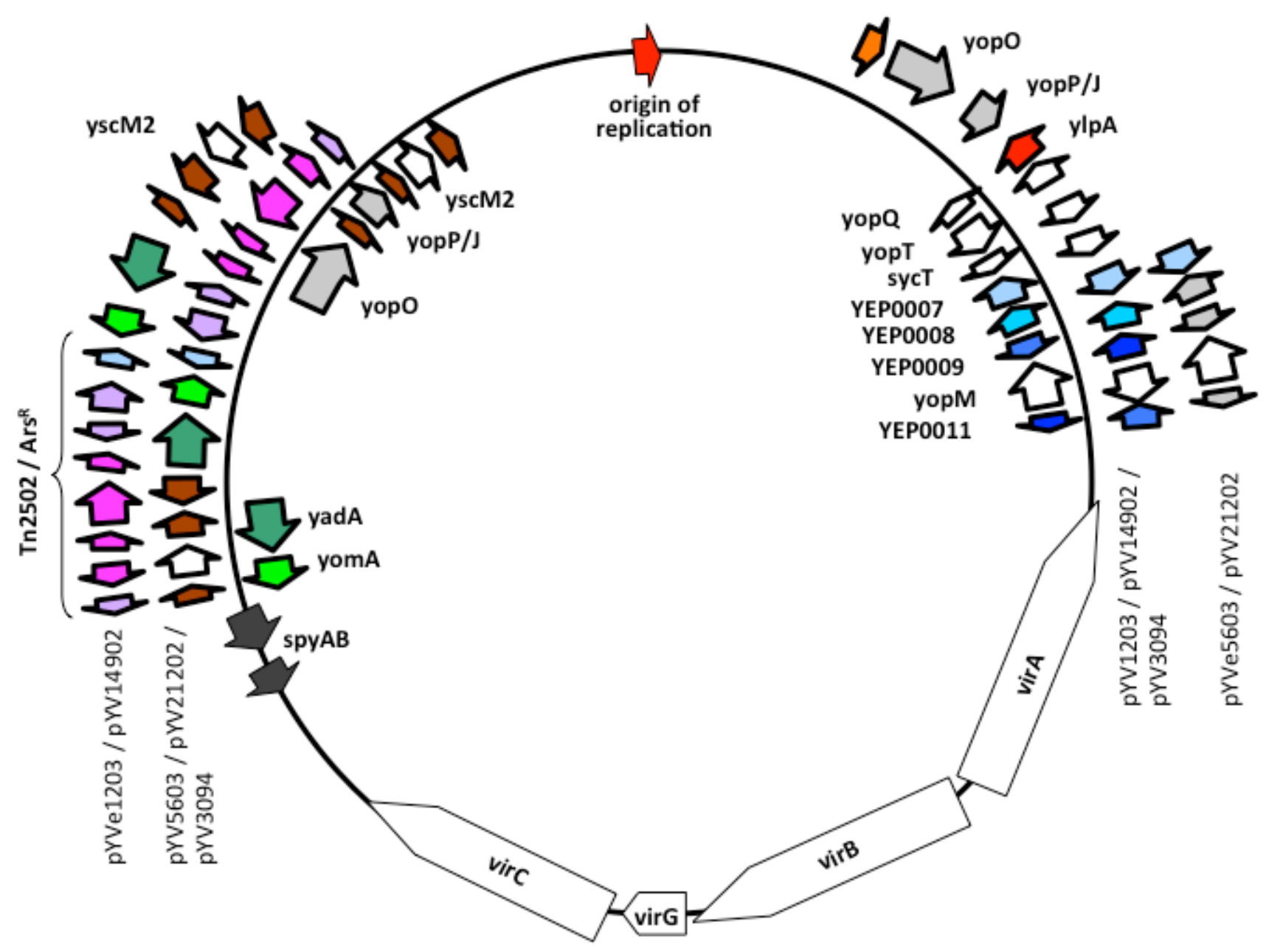

Figure 5.2: Backbone of $\mathrm{pYV}$ and variable regions in different $Y$. enterocolitica biotypes. The unvariable backbone of the virulence plasmid is presented on the circle and consists of the operons virABGC. Inside the circle the genes of the high-pathogenic BT pYVe8081 are depicted, outside the circle are the genes of the low-pathogenic BT pYVs. Orientation of following genes is variable: yopM, yadA, yomA, yscM2. Position of yopO, yopP/J is variable. Position of yadAyomA varies due to insertion of arsenic resistance cassette $\left(\mathrm{As}^{\mathrm{R}}\right)$.

The T3SS machinery and regulatory proteins (virABGC) is the backbone of the virulence plasmid and comprises about half of the plasmid. The other regions are variable. In case of the origin, the region appears to have been swapped for another region in high- or low-pathogenic biotypes. It could be argued that the T3SS machinery, being the backbone of pYV, was inserted into different, pre-existing plasmids in high- and low-pathogenic BTs. This could explain the difference in origin of replication. An argument against this is that there are numerous CDSs outside the T3SS encoding effector proteins, which then would have had to be introduced individually. A more likely scenario is the acquisition and subsequent exchange of the 
origin of replication possibly after a co-integration event with another plasmid in highpathogenic BTs as suggested by Snellings, Popek and Lindler (2001).

Reciprocal fasta searches and analysis of orthologous gene was used to define the core and variable pYV genes comparing the reference plasmid sequences: 38 CDSs of a core set of 59 CDS belong to the T3SS region. In the accessory genes, 22 CDSs are found commonly in the low-pathogenic biotypes. Most of these low-pathogen specific genes are transposase fragments and pseudogenes, $y / p A$, CDSs that represent an alternative origin of replication and the arsenic resistance operon. 28 CDS are unique to pYVe8081, and account for CDSs of the origin of replication and transposase fragments and pseudogenes.

\subsubsection{Variability in pYV core gene sequence}

\subsubsection{1 уорМ}

Heterogeneity has been reported for some of the genes encoded on the virulence plasmid. YopM is one of the T3SS effector proteins. Its size can vary between 367 and 515 amino acids, as the central part contains repeat domains of 60 amino acids, with the number of repeats present leading to a difference in size (Boland, Havaux and Cornelis, 1998; Snellings, Popek and Lindler, 2001). This heterogeneity though does not appear to make a difference in function and does not change the virulence potential of strains expressing an altered YopM (Boland, Havaux and Cornelis, 1998). There is also no apparent link to bio-serotype, as two BT 1B O:8 strains were found to encode YopM proteins of different lengths (Boland, Havaux and Cornelis, 1998; Foultier and Cornelis, 2003). In the plasmids sequenced in this study, the same length of yopM as in pYVe8081 was observed.

\subsubsection{I $\mathrm{crV}$}

Another protein known to be polymorphic is LcrV (Foultier and Cornelis, 2003; Roggenkamp, et al., 1997). This protein is part of the T3SS machinery and encodes a translocator (Foultier and Cornelis, 2003). As LcrV is part of the apparatus of the T3SS and expressed on the cell surface where it is referred to as the V-antigen (Roggenkamp, et al., 1997). Immunization of mice with V-antigen sera conferred protection against infection with Y. enterocolitica (Roggenkamp, et al., 1997). Comparison of LcrV sequences between serotype 0:8 isolates and Y.pestis and Y. pseudotuberculosis showed that a 9-amino-acid insertion is present in some BT 
1B O:8 isolates (Roggenkamp, et al., 1997; Foultier and Cornelis, 2003). Yet, similarly to YopM, LcrV variation is not linked to bio-serotype as other 0:8 isolates were found with sequences identical to those in Y. pseudotuberculosis. A comparison of LcrV sequences of the reference strains of this study to exemplar strains studied in the past (Roggenkamp, et al., 1997) is shown in Figure 5.3. As was shown before, LcrV of BT 1B 0:8 strain WA314 is found to be 9 amino acids longer than BT 1B O:8 strain 8081. There is also variation in LcrV sequences of $0: 9$ isolates. The two isolates sequenced in this study show 5 additional amino acids directly upstream of the WA314 insertion, which corresponds to amino acid position 225 to 232 of Y. pseudotuberculosis.

\footnotetext{
YPS-YPI MIRAYEQNPOHFIEDLEKVRVEQLTGHGSSVLEELVQLVKDKNIDISIKYDPRKDSEVFANRVITDDIELLKKILAYFLPEDAILKGGHYDNQLQNGIKRVKEFLESSPNTQWELRAFMA YE-8081 MIRAYEQNPQHFIEDLEKVRVEQLTGHGSSVLEELVQLVKDKKIDIS IKYDPKKDSEVFAERVITDDIELLKKILAYFLPEDAILKGGHYDNQLQNGIKRVKEFLESSPNTQWELRAFMA YE-WA314 MIRAYEQNPQHFIEDLEKVRVEQLTGHGSSVLEELVQLVKDKKIDISIKYDPKKDSEVFAERVITDDIELLKKILAYFLPEDAILKGGHYDNQLQNGIKRVKEFLESSPNTQWELRAFMA YE-W22703 MIRAYEQNPOHFIEDLEKVRVEQLTGHGSSVLEELVQLVKDKKIDISIKYDPKKDSEVFADRVITDDIELLKKILAYFLPEDAILKGGHYDNOLQNGIKRVKEFLESSPNTOWELRAFMA YE-21202 MIRAYEQNPOHFIEDLEKVRVEQLTGHGSSVLEELVQLVKDKNIDISIKYDPRKDSEVFANRVITDDIELLKKILAYFLPEDAILKGGHYDNQLQNGIKRVKEFLESSPNTQWELRAFMA YE-5603 MIRAYEQNPOHFIEDLEKVRVEQLTGHGSSVLEELVQLVKDKNIDISIKYDPRKDSEVFANRVITDDIELLKKILAYFLPEDAILKGGHYDNQLQNGIKRVKEFLESSPNTOWELRAFMA YE-Y96 MIRAYEQNPOHFIGDLEKVRVDQLTGHGSSVLEELVQLVQDKKIDISIKYDPQKDSEVFADRVITDDIELLKKILAYFLPEDAILKGGHYDNOLQNGIKRVKEFLESSPNTOWELRAFMA YE-14902 MIRAYEQNPOHFIEDLEKVKVEQLTGHGSSVLEELVQLVKDKKIDISIKYDPKKDSEVFADRVITDDIELLKKILAYFLPEDAILKGGHYDNOLQNGIKRVKEFLESSPNTQWELRAFMA YE-Y527 MIRAYEQNPQHFIGDLEKVRVEQLTGHGSSVLEELVQLVQDKKIDISIKYDPKKDSEVFADRVITDDIELLKKILAYFLPEDAILKGGHYDNQLQNGIKRVKEFLESSPNTQWELRAFMA YE-1203 MIRAYEQNPOHFIEDLEKVRVEQLTGHGSSVLEELVQLVKDKKIDISIKYDPKKDSEVFANRVITDDIELLKKILAYFLPEDAILKGGHYDNQLQNGIKRVKEFLESSPNTQWELRAFMA YE-SZ3094 MIRAYEQNPQHFIEDLEKVRVEQLTGHGSSVLEELVQLVKDKKIDISIKYDLKKDSEVFADRVITNDIELLKKILAYFLPEDAILKGGHYDNQLQNGIKRVKEFLESSPNTQWELRAFMA
}

\begin{tabular}{ll} 
YPS-YPI & VIHFSLTADRIDDDILKVIVDSMNHHGDARSKLREELAELTAELKIYSVIQAEINKHLSSGGTINIHDKSINLMDKNLYGYTDEEIFKASAEYKILEKMPQTTI------QX---------X \\
\hline YE-8081 & VMHFSLTADRIDDDILKVIVDSMNHHGDARSKLREELAELTAELKIYSVIQAEINKYLSNSGTINIHDKSINLMDKNLYGYTDEEIFKASAEYKILEKMSQTTI------KX----------X \\
YE-WA314 & VMHFSLTADRIDDDILKVIVDSMNHHGDARSKLREELAELTAELKIYSVIQAEINKHLSSSGTINIHEKSINLMDKNLYGYTDEEIFKASAEYKILKKMPQTTI------KDDELHEVGVI \\
\hline YE-W22703 & VMHFSLTADRIDDDILKVIVDSMNHHGDARGKLREELAELTAELKIYSVIQAEINKHLSNSDTINIHDKSINLMDKNLYGYTDEEIFKASAEYKILEKMPQTTI------KX----------X \\
YE-21202 & VMHFSLTADRIDDDILKVIVDSMNHHGDARSKLREELAELTAELKIYSVIQAEINKHLSNSGTINIHDKSINLMDKNLYGYTDEEIFKASAEYKILEKMPQTTVPPIPGNX---------X \\
YE-5603 & VMHFSLTADRIDDDILKVIVDSMNHHGDARSKLREELAELTAELKIYSVIQAEINKHLSNSGTINIHDKSINLMDKNLYGYTDEEIFKASAEYKILEKMPQTTVPPIPGNX---------X \\
YE-Y96 & VMHFSLTADRIDDDILKVIVDSMNHHGDARGKLREELAELTAELKIYSVIQAEINKHLSNSDTINIHDKSINLMDKNLYGYTDEEIFKASAEYKILEKMPQTTI------KX---------X \\
\hline YE-14902 & VMHFSLTADRIDDDILKVIVDSMNHHGDARSKLREELAELTAELKIYSVIQAEINKHLSNSGTINIHDKSINLMDKNLYGYTNEEIFKASAEYKILEKMSQTTII------KX----------X \\
YE-Y527 & VMHFSLTADRIDDDILKVIVDSMNHHGDARSKLREELAELTAELKIYSVIQAEINKHLSNSDTINIHDKSINLMDKNLYGYTNEEIFKASAEYKILEKMSQTTI------KX---------X \\
YE-1203 & VMHFSLTADRIDDDILKVIVDSMNHHGDARSKLREELAELTAELKIYSVIQAEINKHLSNSDTINIHDKSINLMDKNLYGYTNEEIFKASAEYKILEKMSQTTI------KX---------X \\
YE-SZ3094 & VMHFSLTADRIDDDILKVIVDSMNHHGDARSKLREELAELTAELKIYSVIQAEINKHLSNSDTINIHNNSINLMDKNLYGYTNEEIFKASAEYKILNNMSQTTT------KDKX-------X
\end{tabular}

YPS-YPI GETEKKIVSIKNFLESEKKRTGALGNLKDSYSYNKDNNELSHFATTCSDKSRPLNDLVSOKTTOLSDITSRFNSAIEALNRF IOKYDSVMORLLDDTSGK* YE-8081 GETEKKIVSIKNFLESENKRTGALGNLKNSYSYNKDNNELSHFATACSDKSRPLNDLVSOKTTOLSDITSRFNSAIEALNRF IQKYDSVMORLLDDT--R* YE-WA314 AGAEKOIVSIKNFLESENKRTGALGNLKDSYSYNKDNNELSHFATACSDKSRPLNDLVSOKTTOLSDITSRFNSAIEALNRFIOKYDSVMORLLDDT--RL YE-W22703 GETEKKIVSIKNFLESENKRTGALGNLKDSYSYNKDNNELSHFATTCSDKSRPLNDLVSOKTTOLSDITSRFNSAIEALNRF IQKYDSVMORLLDDT--R* YE-21202 NGSEKKIVSIKNFLESENKRTGALGNLKDSYSYNKDNNELSHFATTCSDKSRPLNDLVSOKTTOLSDITSRFNSAIEALNRFIOKYDSVMQRLLDDT--R* YE-5603 NGSEKKIVSIKNFLESENKRTGALGNLKDSYSYNKDNNELSHFATTCSDKSRPLNDLVSOKTTQLSDITSRFNSAIEALNRFIOKYDSVMQRLLDDT--R* YE-Y96 GETEKKIVSIKNFLESENKRTGALGNLKDSYSYNKDNNELSHLGTTCSDKSRPLNDLVSOKTTOLSDITSRFNSAIEALNRF IQKYDSVMORLLDDT--R* YE-14902 GETEKKIVSIKNFLESENKRTGALGNLKDSYSYNKDNNELSHFATTCSDKSRPLNDLVSOKTTOLSDITSRFNSAIEALNRFIQKYDSVMQRLLDDT--R* YE-Y527 GETEKKIVSIKNFLESENKRTGALGNLKDSYSYNKDNNELSHLGTTCSDKSRPLNDLVSQKTTQLSDITSRFNSAIEALNRF IQKYDSVMORLLDDT--R* YE-1203 GETEKKIVSIKNFLESENKRTGALGNLKDSYSYNKDNNELSHFATTCSDKSRPLNDLVSOKTTOLSDITSRFNSAIEALNRFIOKYDSVMORLLDDT--R* YE-SZ3094 GKTEKKIVSIKNFLESENKRTGALNNLKESYSYNKDNNELSHFATTCSDKSRPLNDLVSQKTTQLSDITSRFNSAIEALNRFIQKYDSVMORLLDDT--R*

Figure 5.3: Protein sequence alignment for LcrV. YPS-YPI - Y. pseudotuberculosis YPIII, YE Y. enterocolitica, 8081 and WA314 - BT 1B O:8, W22703, 21202, 5603, and Y96 - BT 2 O:9, 14902 and Y527 - BT 3 O:5,27, 1203 - BT 4 O:3, SZ3094 - BT 5 O:2a,2b,3.

The difference in sequence between American 0:8 isolates and Y.pestis, Y. pseudotuberculosis, and European $Y$. enterocolitica isolates has been implicated 
in plague epidemiology (Roggenkamp, et al., 1997). The authors suggest that the antibodies raised against the $Y$. enterocolitica $V$-antigen are cross protective in rodents to $Y$. pestis infection. Since the American 0:8 isolates express a different antigen it is thought that this cross protection is not given, explaining the plague foci found in the Americas (Roggenkamp, et al., 1997). Undoubtedly the insertion of additional amino acids can cause a conformational change in the protein and thus leads to the expression of an altered epitope, but this variation is not found in all $0: 8$ isolates. BT 1B strain 8081 was isolated from North America and shows high LcrV sequence identity with Y.pseudotuberculosis and Y.pestis and no amino acid insertions, suggesting that this isolate should theoretically confer immunity against $Y$. pestis. The variation in the $0: 9$ isolates would presumably not give protective immunity against plague, yet these isolates are very common in Europe without associated plague foci. An effect on infectivity, virulence potential or severity of disease has not been correlated with a changed LcrV epitope.

\subsubsection{3 yscP}

YscP forms an essential part of the type III secretion machinery. Deletion mutants showed that abolishing the complete gene leads to a loss of secretion of Yops, and a functional protein is necessary for the delivery of the effectors into target cells (Stainier, et al., 2000). It was noted as well that it shows more variability than any other protein in the T3SS between Y.pseudotuberculosis, Y.pestis, and Y. enterocolitica (Stainier, et al., 2000). YscP from Y. enterocolitica has additional 60 amino acids that appear to be part of a repeat compared to $Y$. pseudotuberculosis and Y.pestis. Later investigations showed that YscP controls the length of the injectisome needles, acting like a ruler to determine optimum length (Journet, Agrain, Broz and Cornelis, 2003). The $\mathrm{N}$ and $\mathrm{C}$ terminal part of the protein are essential for length control in general, as deletion mutants showed a wide range of needle sizes. Deletions or insertions into the central part of the protein resulted in proportionally shorter or longer needles, therefore being the functional domain responsible for fixed needle length (Journet, Agrain, Broz and Cornelis, 2003). Additionally, YscP acts as a specificity switch signalling the apparatus to stop producing needle proteins and change over to late substrates (Journet, Agrain, Broz and Cornelis, 2003; Wagner, et al., 2009). YscP is anchored to the base and the growing tip of the needle so that it is stretched alongside the needle during assembly. The content of helix makers such as alanine and the overall length of the protein YscP determine the needle length 
(Wagner, et al., 2009). Once YscP reaches a breaking point when stretched to its maximum, it detaches and signals back so that late injectisome molecules are produced (Wagner, et al., 2009).

A comparison between YscP protein sequences previously investigated (Foultier and Cornelis, 2003; Wagner, et al., 2009; Oberhettinger, et al., 2011) and sequences generated here shows that YscP contains three different repeat units (Figures 5.4 and 5.5). These repeats are modules that can be duplicated and reorganized in different strains. The red repeat contains 13 amino acids, green 25 amino acids, and blue 46 amino acids, identical to repeats described before (Wagner, et al., 2009). The region of variability begins at amino acid position 219 respective to BT 1B strain YE-8081 (Figure 5.4).

At the beginning of the region of variability there is a first red repeat in all strains except for one BT 1B isolate that shows a 24 amino acid deletion. The red repeat is followed by the first green repeat that is present in all strains except for BT 5 . Instead of a green repeat, one BT 5 strain shows blue and red repeat units. The next repeats are the first blue and the second red repeats in all low-pathogenic BTs and three BT 1B isolates. The BT 3 O:5,27 isolate YE-14902 shows an in-frame deletion of 6 bp (2 amino acids). The three BT 1B isolates are A11/86, WAT-988, and WA314, which then also have a second green repeat. The region of variability ends with a second blue repeat in all strains.

The protein sequences of the strains are shown in Figure 5.5 to highlight the modular nature of YscP. 
YERPE

YPSTB

YE-A1186

YE-WA31

YE-WA314

YE-A 12790

YE-WAT28

YE-W22 703

YE-E 40

YE-14902

YE-SZ 3094
YE-IP17

YERPE

YPSTB

YE-A1186

YE-WAT988
YE-WA314

YE-WA 314

YE-A12790

YE-WAT2 88

$\mathrm{YE}-21202$

YE-W22 703

YE-E 40

YE- 14902

YE-1203

YE-SZ3094

YE-IP17

YERPE

YE-A1186

YE-A1186
YE-WAT988

YE-WA314

YE-WA314
YE-8081

YE-A12790

YE-A12790
YE-WAT288

YE-21202

YE-W22703

YE-E 40

YE-5603

YE-14902

YE-1203

YE-SZ 3094
YE-IP17

M-NKITTRSPLEPEYQPLGKPHHALQACVDFEQALLHNNKGNCHPKEESLKPVRPHDLGKKEGOKGDGLRAHAPLAATSOPGRKEVGLKPOHNHQNNHDFNLSPLAEGATNRAHLYOODSRFDDRV M-NKITTRSPLEPEYQPLGKPHHALQACVDFEQALLHNNKGNCHPKEESLKPVRPHDLGKKEGQKGDGLRAHAPLAATSQPGRKEVGLKPQHNHONNHDFNLSPLAEGATNRAHLYQQDSSRFDDRV MANKITTRSPLEPEYQPLGKPHHDLOARVDFEOALLHN-KDNRHPKEEPRRPVRPHDLGKKEGOKGDGLRAHAPLAATFOPGR-EVGLKPOHNHONNHDFNLSPLAEGATNRKHLYOQDSRFDDRV MANKITTRSPLEPEYQPLGKPHHDLQARVDFEQALLHN-KDNRHPKEEPRRPVRPHDLGKKEGQKGDGLRAHAPLAATFQPGR-EVGLKPOHNHQNNHDFNLSPLAEGATNRKHLYQQDSRFDDRV M-NKITTRSPLEPEYQPLGKPHHDLQARVDFEQALLHN-KDNRHPKEEPRRPVRPHDLGKKEGQKGDGLRAHAPLAATFQPGR-EVGLKPOHNHQNNHDFNLSPLAEGATNRKHLYQQDSRFDDRV M-NKITTRSPLEPEYOPLGKPHHDLOARVDFEOALLHN-KDNRHPKEEPRRPVRPHDLGKKEGOKGDGLRAHAPLAATFOPGR-EVGLKPOHNHONNHDFNLSPLAEGATNRKHLYOODSRFDDRV M-NKITTRSPLEPEYOPLGKPHHDLOARVDFEOALLHN-KDNRHPKEEPRRPVRPHDLGKKEGOKGDGLRAHAPLAATFOPGR-EVGLKPOHNHONNHDFNLSPLAEGATNRKHLYOODSRFDDRV MANKITTRSPLEPEYQPLGKPHHDLQARVDFEQALLHN-KDNRHPKEEPRRPVRPHDLGKKEGQKGDGLRAHAPLAATFQPGR-EVGLKPOHNHONNHDFNLSPLAEGATNRKHLYOODSRFDDRV M-NKITTRSPLEPEYQPLGKLHHDLQARADFEQALLHNNKGNRHPKEEPRRPVRPHDLGKKEGOKGDGLRAHAPLEATFQPGRKEVGLKPQHNHONNHDLNLSPLAEGVTNRAHLYQQDSRFDDRV M-NKITTRSPLEPEYQPLGKLHHDLQARADFEQALLHNNKGNRHPKEEPRRPVRPHDLGKKEGOKGDGLRAHAPLEATFQPGRKEVGLKPOHNHQNNHDLNLSPLAEGVTNRAHLYQQDSRFDDRV 作 作 M-

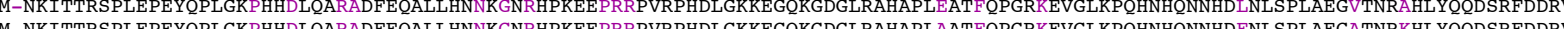
M-NKITTRSPLEPEYOPLGKPHHDLQARADFEQALLHNNKGNRHPKEEPRRPVRPHDLGKKEGQKGDGLRAHAPLAATFOPGRKEVGLKPOHNHONNHDFNLSPLAEGATNRKHLYQODSRFDDRV

ESI INALMPLAPFLEGVTCETGTSSESPCEPSGHDELFVQOSPIDSAOPVOLNSKPTVOPLNPAADGAEVIVWSVGRETPASIAKNORDSROKRLAEEPLALHOKALPEICPPAVSATPDDHLVAR ESIINALMPLAPFLEGVTCETGTSSESPCEPSGHDELFVQQSPIDSAQPVQLNSKPTVQPLNPAADGAEVIVWSVGRETPASIAKNQRDSRQKRLAEEPLALHQKALPEICPPAVSATPDDHLVAR

ESI INALMPLAPFLEGVTCETGTSSESPCEPFGHDELFVQQSPIDSAQPVQLNTKPTVQSLNPAADGAEVMIWSVGRDTLASIAKNQRDSRQKRLAEEPLP--------ESIINALMPLAPFLEGVTCETGTSSESPCEPFGHDELFVQQSPIDSAQPVQLNTKPTVQSLNPAADGAEVMIWSVGRDTLASIAKNQRDSRQKRLAEEPLPLHOEALPEVCPH ESI INALMPLAPFLEGVTCETGTSSESPCEPFGHDELFVQQSPIDSAQPVQLNTKPTVQSLNPAADGAEVMIWSVGRDTLASIAKNQRDSRQKRLAEEPLPLHOEALPEVC ESIINALMPLAPFLEGVTCETGTSSESPCEPFGHDELFVQQSPIDSAQPVQLNTKPTVQSLNPAADGAEVMIWSVGRDTLAS IAKNQRDSRQKRLAEEPLPLHQEALPEVC ESI INALMPLAPFLEGVTCETGTSSESPCEPSGHDELFVQOSPIDSAQPVQLNTKPTVQPLNPAADGAEVMIWSVGRDTLASIAKNOCDSRQKRLAEEPLPLHOEALPEVC
ESI INALMPLAPFLEGVTCETGTSSESPCEPSGHDELFVOOSPIDSAOPVOLNTKPTVOSLNPAADGAEVMISSVGRDTLASIAKNORDSROKRLAEEPLPLHOEALPEVC ESI INALMPLAPFLEGVTCETGTSSESPCEPSGHDELFVQQSPIDSAQPVQLNTKPTVQSLNPAADGAEVMISSVGRDTLASIAKNQRDSRQKRLAEEPLPLHOEALPEVC ESIINALMPLAPFLKGVTCETGTSSESPCEPSGHDELFVQQSPIDSVQPVQLNTKPTVQPLNPAADGAEVIVWSVGRETPASIAKNQRDSRQKRLAEEPLPLHQEALPEVC ESI INALMPLAPFLKGVTCETGTSSESPCEPSGHDELFVQQSPIDSVQPVQLNTKPTVQPLNPAADGAEVIVWSVGRETPAS IAKNORDSRQKRLAEEPLPLHQEALPEVC ESI INALMPLAPFLKGVTCETGTSSESPCEPSGHDELFVQQSPIDSVQPVQLNTKPTVQPLNPAADGAEVIVWSVGRETPASIAKNQRDSRQKRLAEEPLPLHOEALPEVC ESIINALMPLAPFLEGVTCETGTSSESPCEPSGHDELFVQQSPIDSVQPVQLNTKPTVQPLNPAADGAEVIVWSVGRETPASIAKNORDSRQKRLAEEPLPLHOEALPEVCP ES I INALMPLAPFLEGVTCETGTSSESPCEPSGHDELFVQQSPIDSVQPVQLNTKPTVQPLNPAADGAEVIVWSVGRETPASIAKNORDSRQKRLAEEPLPLHQEALPEV ESIINAAMLAPFLKGTCETGTSSESPCEPSGHDELFVQOSPIDSVQPVQLNTKPTVOPLNPAADGAEVIVWSVGRETPASIAKNORDSROKRLAEEPLPLHOEALPEV

WCATPVTE

WCATPVAEV

WCATPVAEV

WCATPVA

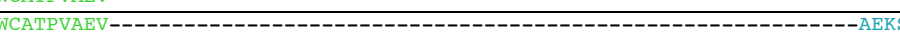

WCATPVAEV
WCATPVAEV
WCATPVAEV
WCATPVAEV -

YERPE

YPSTB

YE-A1186

YE-WAT988

YE-WA314

YE-8081

YE-A12790

YE-WAT 288

YE-W22703

YE-E40

YE-5603

YE-14902

YE-1203

YE-SZ3094

YE-IP17

YERPE
YPSTB

-----------------------------AEKSARFPYKATVQSEQLDMTELADRSOHLTDGVDSSKDTIEPPRPEKLLLPREETLPEMYSLSFTAPVVTPGDHLLATMRATRLASVSEQLIQLAQR PEVCPPAVSTTPDDHLVARWCATPVAEVAEKSARF PHKATVOSEOLDMTELADRSOHLTDGVDSSKDTTIEPPRPEELLLPREETLPEMYSLSFTAPVITPGDHLIATMRATRLASVSEOLIOLLAOR PEVCPPAVSTTPDDHLVARWCATPVAEVAEKSARFPHKATVQSEQIDMTELADRSOHLTDGVDSSKDTIEPPRPEELLLPREETLPEMYSLSFTAPVITPGDHLLATMRATRLASVSEQLIQLAOR

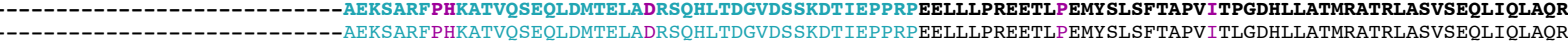
PEV---1 PEV------------------AEKSARF QHKATVQSEQLDMTELAARS QYLTDGVDSSKDTIEPPRPEELLLPREETLLEMYSLSFTAPVVTPGDHLLATMRATRLTSVSEQLIQLAQR PEV---------------AEKSARF PHKATVQSEQLDMTELAARSOYLTDGVDSSKDTIEPPRPEELLLPREETLLEMYSLSFTAPVVTPGDHLLATMRATRLTSVSEQLIQLAQR PEV-------------AEKSARFPHKATVQSEOLDMTELAARSOYLTDGVDSSKDTIEPPRPEELLLPREETLLEMYSLSFTAPVVTPGDHLLATMRATRLTSVSEQLIOLAOR PEV--------------AEKSARFPHKATVQSEQLDMTELAARSOYLTDGVDSSKDTIEPPRPEELLLPREETLLEMYSLSFTAPVVTPGDHLLATMRTTRLTSVSEQLIOLAQR PEV-----------------AEKSARFPHKATVQSEQLDMTELAARSOYLTDGVDSSKDTIEPPRPEELLLPREETLLEMYSLSFTAPVVTPGDHLLATMRATRLTSVSEQLIOLAQR PEV-------------------AEKSARFPHKATVQSEQLDMTELAARSQYLTDGVDSSKDTIEPPRPEELLLPREETLLEMYSLSFTAPVVTPGDHLLATMRATRLTSVSEQLIQLAQR

LAVELELRGGSSOVTQLHLNLPELGAIMVRIAEIPGKLHVELIASREALRILAQGSYDLLERLQRIEPTQLDFOASDDSEQESROKRHVYEEWEAEE*

LAVELELRGGSSOVTQLHLNLPELGAIMVRIAEIPGKLHVELIASREALRILAQGSYDLLERLQRIEPTQLDFQASDDSEQESRQKRHVYEEWEAEE*

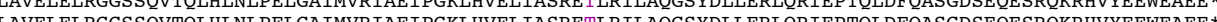

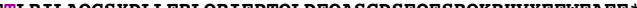

YE-A12790 LAVEETRG

YE-WAT288 IAVELT

YE-21202 LAVELELRGGSSOVTOLHLNLPELGAIMVRIAEI PGKLHVELIASOEALRILAOGSYDLLERLORIEPTOLDFOASGDSEOESROKRHVYEEWEAEE*

YE-W22703 LAVELELRGGSSOVTOLHLNLPELGAIMVRIAE IPGKLHVELIASOEALRILAOGSYDLLERLQRIEPTOLDFOASGDSEOESROKRHVYEEWEAEE* YE-E40 LAVELELRGGSSQVTQLHLNLPELGAIMVRIAEIPGKLHVELIASQEALRILAQGSYDLLERLQRIEPTQLDFQASGDSEQESRQKRHVYEEWEAEE* * YE-5603 LAVELELRGGSSOVTQLHLNLPELGAIMVRIAEIPGKLHVELIASOEALRILAQGSYDLLERLQRIEPTQLDFQASGDSEQESRQKRHVYEEWEAEE* YE-14902 LAVELELRGGSSOVTQLHLNLPELGAIMVRIAEIPGKLHVELIASOEALRILAOGSYDLLERLQRIEPTQLDFQASGDSEQESRQKRHVYEEWEAEE* * YE-1203 LAVELELRGGSSOVTQLHLNLPELGAIMVRIAE IPGKLHVELIASQEALRILAOGSYDLLERLQRIEPTQLDFQASGDSEQESROKRHVYEEWEAEE* YE-SZ3094 LAVELELRGGSSOVTOLHLNLPELGAIMVRIAEIPGKLHVELIASOEALRILAOGSYDLLERLQRIEPTQLDFOASGDSEQESROKRHVYEEWEAEE*
YE-IP17

Figure 5.4: Protein sequence alignment of YscP protein sequences of different Yersinia spp. Repeats in amino acid sequences are highlighted in red (13 amino acids), green ( 25 amino acids), and blue (46 amino acids). Variations in amino acids are highlighted in purple. Vertical bars indicate every 10 amino acids respective to YE-8081 (bold sequence). YERPE - Y. pestis strain IP275, YPSTB Y. pseudotuberculosis strain YPIII, YE- - Y. enterocolitica, BT 1B: A1186 (O:21), WAT988 (O:4), WA314 (O:8), 8081 (O:8), A127/90 (O:8), WAT288 (O:13), BT 2: 21202, E40 (O:9), BT 3: 5603 (O:9), 14902 (O:5,27), BT 4: 1203 (O:3), BT 5: SZ3094 (O2a,2b,3), IP17 (O:2,3). 


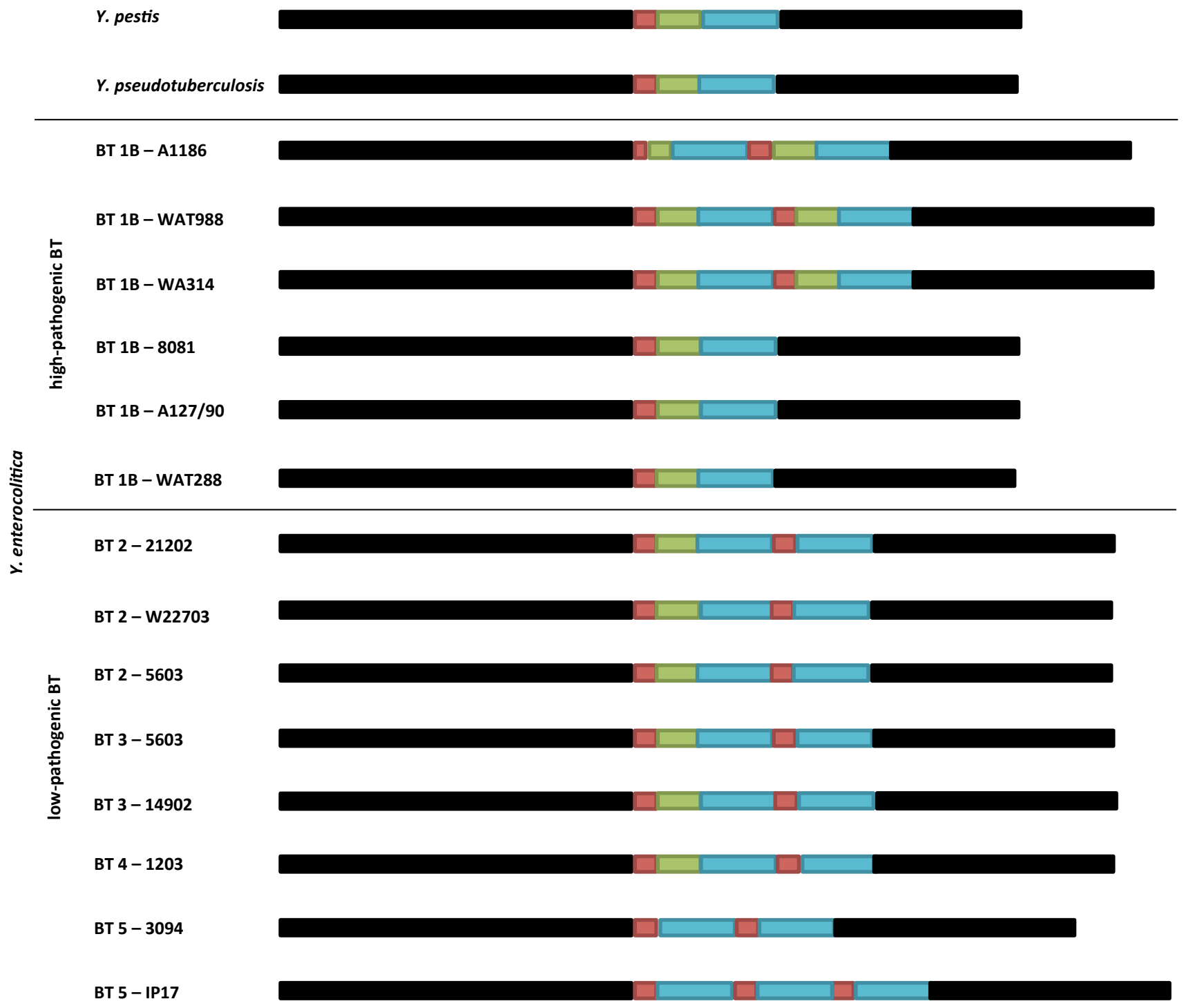

Figure 5.5: Modular nature of YscP in different Yersinia spp. Conserved sequences are shown in black, repeat sequences are red (13 amino acids), green ( 25 amino acids), and blue (46 amino acids). Strains are the same as in Figure 5.4.

From Figure 5.5 one can see that there are certain themes concerning the makeup of YscP in Yersinia spp. First of all, Y.pestis and Y.pseudotuberculosis show an identical YscP protein with a single repeat of each the red, green, and blue module. For the high-pathogenic BT 1B of $Y$. enterocolitica, there are two options. The first option is to have the same YscP sequence as Y. pestis. Presumably evolved from this is the second option of duplicating the red-green-blue modules. Strain A1186 shows a deletion of the parts of the red and green modules. This could have happened after duplication of the whole repeat region, as it is otherwise identical to strains WAT988 and WA314. In the low-pathogenic BTs there are also two possibilities. For BTs 2, 3, and 4, the red and blue modules are duplicated. For BT 5, 
only the red and blue modules are present, and can vary in copy number. A caveat for BT 5 has to be added, as so far only these two isolates have been studied and it is not known whether this is a common phenomenon for BT 5 isolates. It is also unknown whether the differences observed correlate to a change in pathogenicity and whether a change in needle length affects efficiency of effector translocation.

\subsubsection{Plasmid Variety: Unique plasmids}

In the $Y$. enterocolitica strains that were sequenced, four unique plasmids were identified apart from the virulence plasmid. BT 2 strain 21202 and BT 5 strain 3094 both had large additional plasmids, and biotype 1A, though lacking the virulence plasmid, showed the presence of two small plasmids.

\subsubsection{Unique plasmids in the reference strain for BT1A: pSR2-1A and pSR3-1A} Two unique plasmids are present in the reference strain 5303 for BT 1A, which were named pSR2-1A and pSR3-1A. pSR2-1A (Figure 5.6) has a size of $8 \mathrm{~kb}$. The GC content is $48.0 \%$ and therefore remarkably close to the chromosomal GC content of strain $5303(48.42 \%)$. The coding percentage is $81.3 \%$. A total of 12 CDSs were identified, but function could only be assigned to four of these. The other eight CDSs are currently hypothetical proteins with uncharacterized homologs in other bacterial species other than E. coli. These homologs are either distantly related or are not well characterized, thus a function cannot be allocated. It contains three genes mobABC that encode for mobility of the plasmid. There are no transfer genes encoded on this plasmid, which means that this plasmid can be co-mobilized if another plasmid with tra genes for conjugational transfer is present. 
PSR2-1A - 8,329 bp

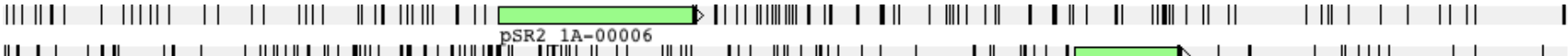

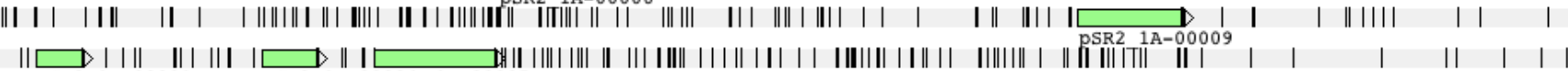

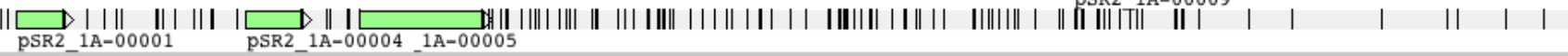

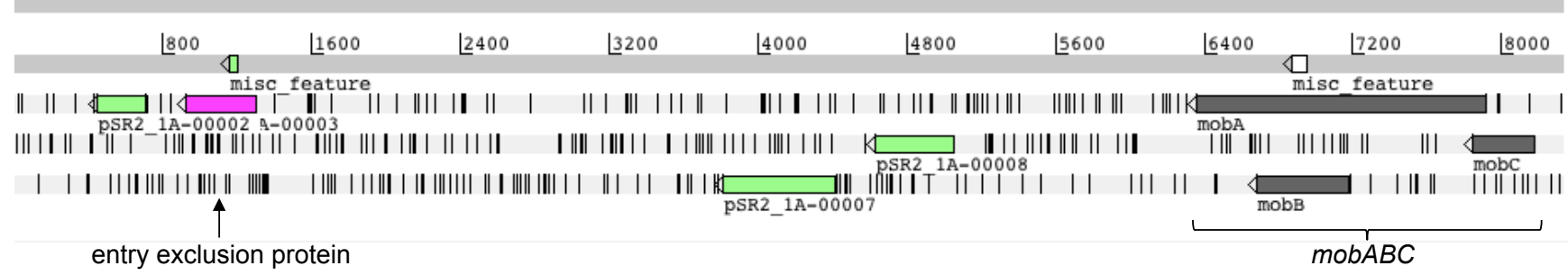

Figure 5.6: Linear plasmid map of pSR2-1A found in Y. enterocolitica BT 1A 0:5 strain 5303.

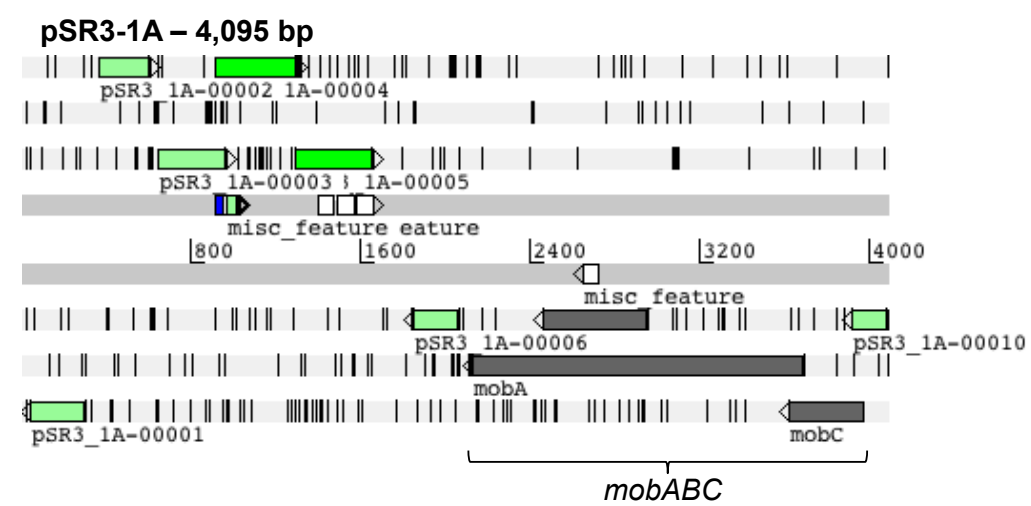

Figure 5.7: Linear plasmid map of pSR3-1A found in Y. enterocolitica BT 1A 0:5 strain 5303.

Similarly, pSR3-1A contains mobABC, but no tra genes (Figure 5.7). This plasmid only has a size of $4 \mathrm{~kb}$. The GC content of pSR3-1A is $53.92 \%$. This is higher than the GC content of pSR2-1A as well as the chromosome of strain 5303 . The coding percentage is $104.3 \%$. It is likely that this is an overestimation as half of the putative CDSs are of hypothetical nature.

Both plasmids appear to be cryptic, as they do not contain any genes of assigned functions. pSR3-1A contains two genes which might encode for membrane proteins, as one of them has a SignalP-domain, and the other shows potential transmembrane domains.

Two cryptic plasmids, pYe4449-1 and pYe4449-2, described in another BT 1A strain (Lepka, et al., 2009) show no similarity to the plasmids described here apart from the mobilization region mobABC. These plasmids add to the overall gene pool in $Y$. enterocolitica, especially the non-pathogenic BT, but their function and impact is unknown. 


\subsubsection{Plasmids in atypical BT 1A/1B strains}

It is know that there are some isolates of $Y$. enterocolitica that biochemically type as BT 1B but which lack all of the determinants of high-pathogenic strains such as the virulence plasmid and the high-pathogenicity island. A plasmid profile of potential strains matching these criteria was established using the approach of Kado and Liu (1981; Figure 5.8).

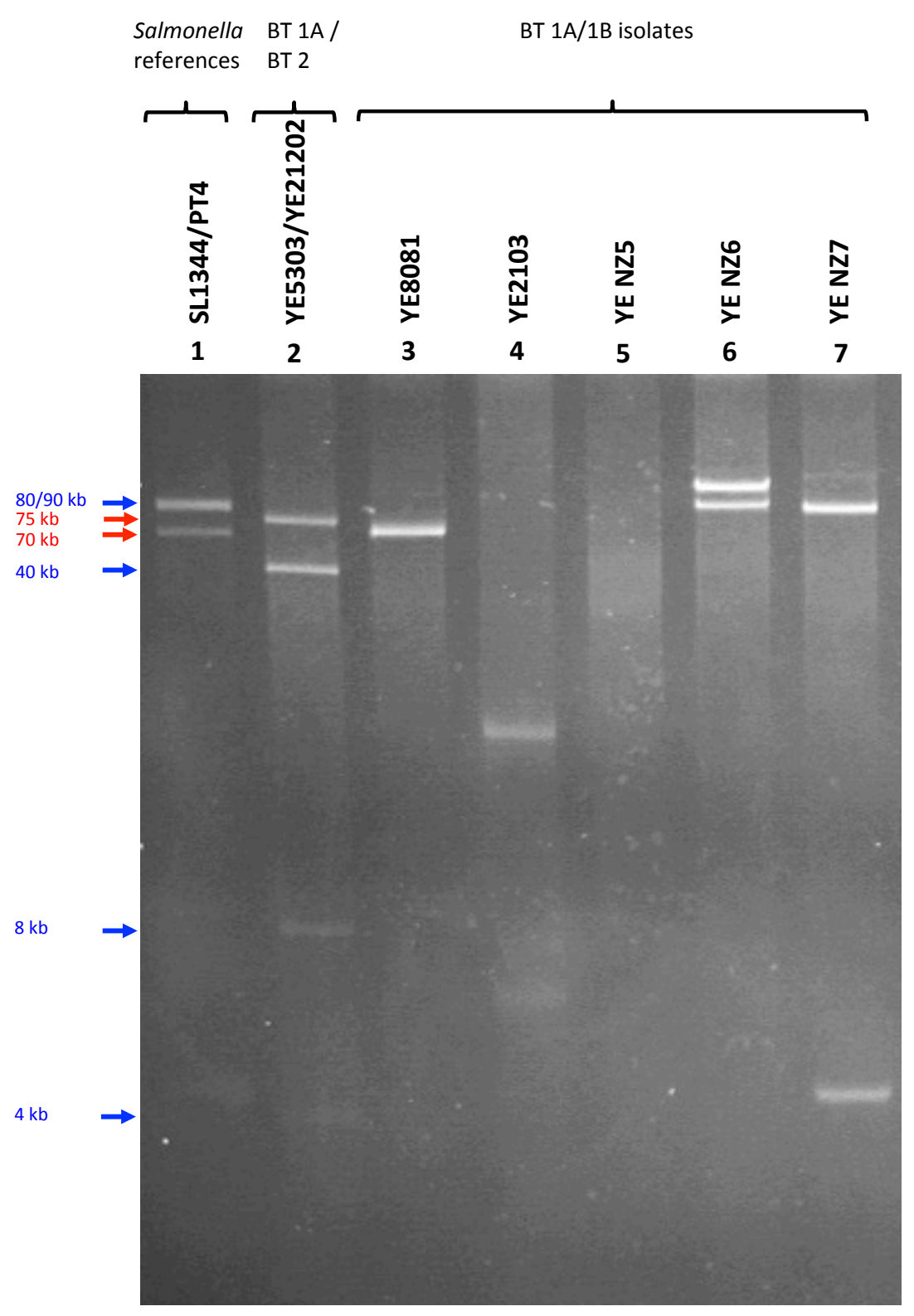

Figure 5.8: Plasmid profile of atypical BT 1A/1B isolates. Lane 1: Mixture of Salmonella plasmids of isolates SL1344 and PT4. Lane 2: Mixture of YE5303 and YE21202 plasmids (pSR1-2, pYV, pSR21A, pSR3-1A). Lane 3-7: BT 1A/1B isolates. YE8081 - BT 1B O:8, YE2103 - BT 1B 0:19, NZ5-7 - BT $1 \mathrm{~B}$, no serotype information. Red arrows indicate size of $\mathrm{pYV}$.

Plasmid size can be estimated by the use of reference plasmids of known size, presented here in lane 1 and 2 . In lane 1 there is a mixture of two Salmonella strains. 
SL1344 has two large plasmids of 80 and $90 \mathrm{~kb}$ that form a single band on this gel, and further smaller plasmids are not visible. PT4 has a plasmid of about $70 \mathrm{~kb}$ size. Lane 2 has a mixture of plasmids from strain 5303 (pSR2-1A and pSR3-1A) and strain 21202 (pYV and pSR1-2). The high-pathogenic BT 1B reference strain 8081 is shown to demonstrate the size difference between high- and low-pathogenic pYVs.

YE2103 is an atypical BT 1B strain that was isolated during the IID study in the UK (Tompkins, et al., 1999). It was obtained from a GP control, i.e. an asymptomatic person matched in age and sex to a case subject. It was non-invasive in tissue culture (A. McNally, personal communication) and it was shown in a microarray study that it is closely related to non-pathogenic isolates (Howard, et al., 2006). One can see that this isolate does not possess the virulence plasmid pYV or any other plasmid of similar size. Instead, it has two smaller plasmids, one of $\sim 20 \mathrm{~kb}$, the other $\sim 6 \mathrm{~kb}$. As this strain was not included in the sequencing project (Chapter 7), no information on the nature of these plasmids is currently available.

NZ5-7 were isolated from humans by collaborators in New Zealand (M. DuFour) and are lacking serotype information. NZ5 shows no presence of plasmids. NZ6 contains two plasmids of $75 \mathrm{~kb}$ and $\sim 95 \mathrm{~kb}$. NZ7 also has two plasmids, one of $75 \mathrm{~kb}$, the other of $4 \mathrm{~kb}$. From the information that these isolates were typed as BT 1B, it was initially assumed that the $75 \mathrm{~kb}$ plasmids of NZ6 and NZ7 are pYV. Two of these strains, NZ5 (NZ523784) and NZ7 (NZ761915), were sequenced as part of a strain collection (Chapter 7 ). Sequencing of NZ7 revealed that $\mathrm{pYV}$ is not present. Both NZ5 and NZ7 phylogenetically group with non-pathogenic BT 1A isolates, and HPI, Ysa T3SS, and YAPI could not be detected. Whilst nothing can be deduced for NZ6, it is clear that NZ5 and NZ7 have been mistyped as BT 1B and that the presence of a large plasmid of similar size to pYV may have influenced this decision in NZ7. It confirms that large plasmids can be present in non-pathogenic strains and that the presence of large plasmids does not indicate the presence of pYV.

\subsubsection{Unique plasmid in the reference strain for BT2: pSR1-2}

BT 2 0:9 strain 21202 carries a unique plasmid of $42 \mathrm{~kb}$ which was named pSR1-2. The plasmid has a GC content of $52.2 \%$, which is higher than the chromosomal GC content of strain $21202(48.1 \%)$. This would indicate an acquisition of this plasmid from a donor outside the genus Yersinia, because the average GC content is $48-49 \%$ (Sprague and Neubauer, 2005). 54 CDSs have been identified (coding percentage 
$87.8 \%$ ). It belongs to the IncN incompatibility group, and a map is shown in Figure 5.9 .

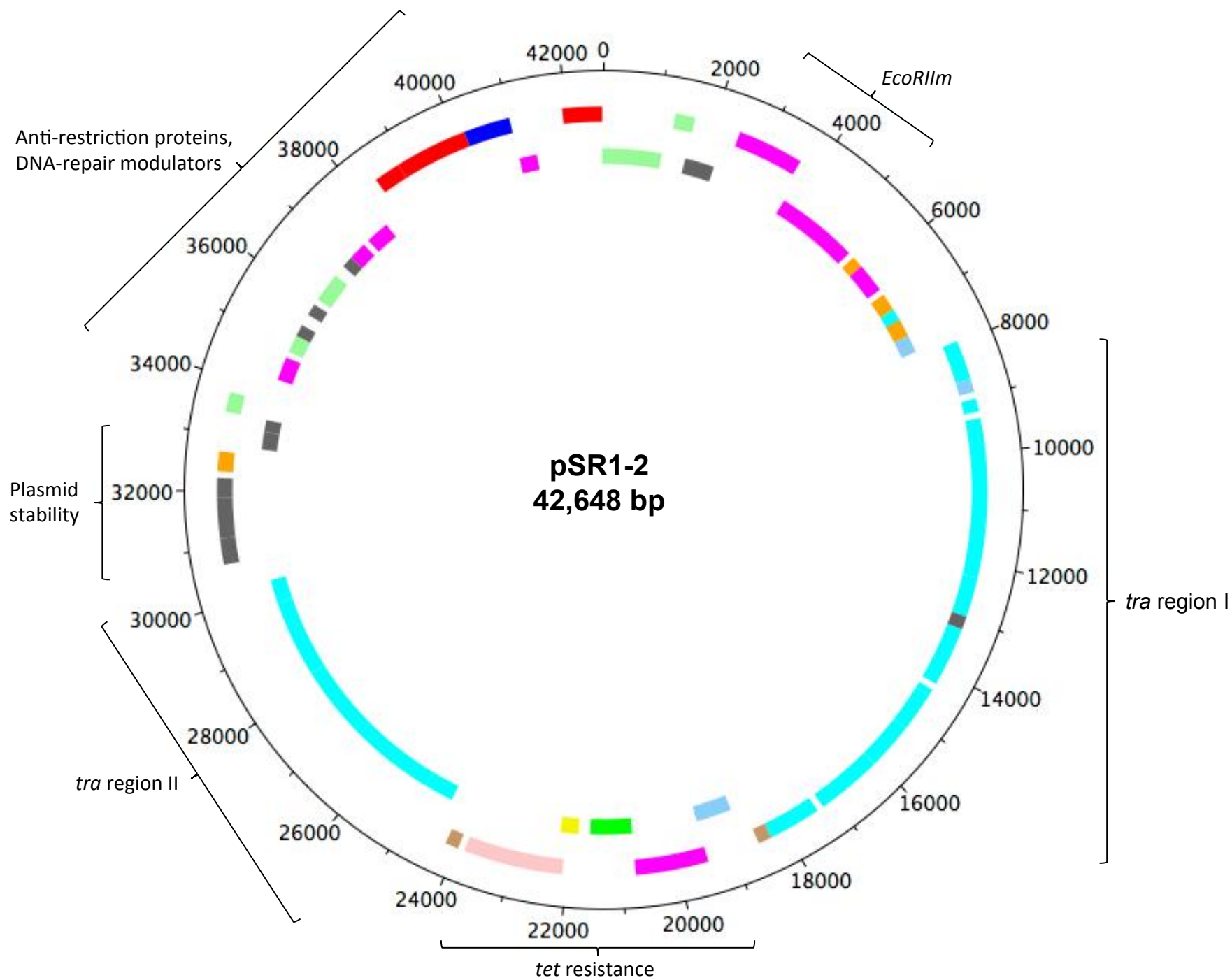

Figure 5.9: Circular plasmid map of pSR1-2 found in Y. enterocolitica BT 2 0:9 strain 21202. Regions of interest are highlighted.

pSR1-2 carries stbABC involved in plasmid stability, as well as anti-restriction proteins, DNA-repair modulators, and an EcoRIIm restriction modification enzyme. In contrast to pSR2-1A and pSR3-1A, there are only five hypothetical genes. The plasmid contains tra genes for pilus formation and conjugational transfer. These genes are split into two operons. In between these two operons there is a tetracycline resistance cassette. The functionality of this antibiotic resistance cassette has been established by growth on LB agar containing 10, 20, and $50 \mu \mathrm{g} / \mathrm{ml}$ tetracycline. Compared to the other reference sequence strains, only BT 2 0:9 strain 21202 was able to grow on this medium. Five other BT 2 strains and one BT 3 0:9 strain 
(YE21402) tested negative for growth on LB supplemented with tetracycline. The same BT 2 strains were examined for the presence of plasmids (Figure 5.10).

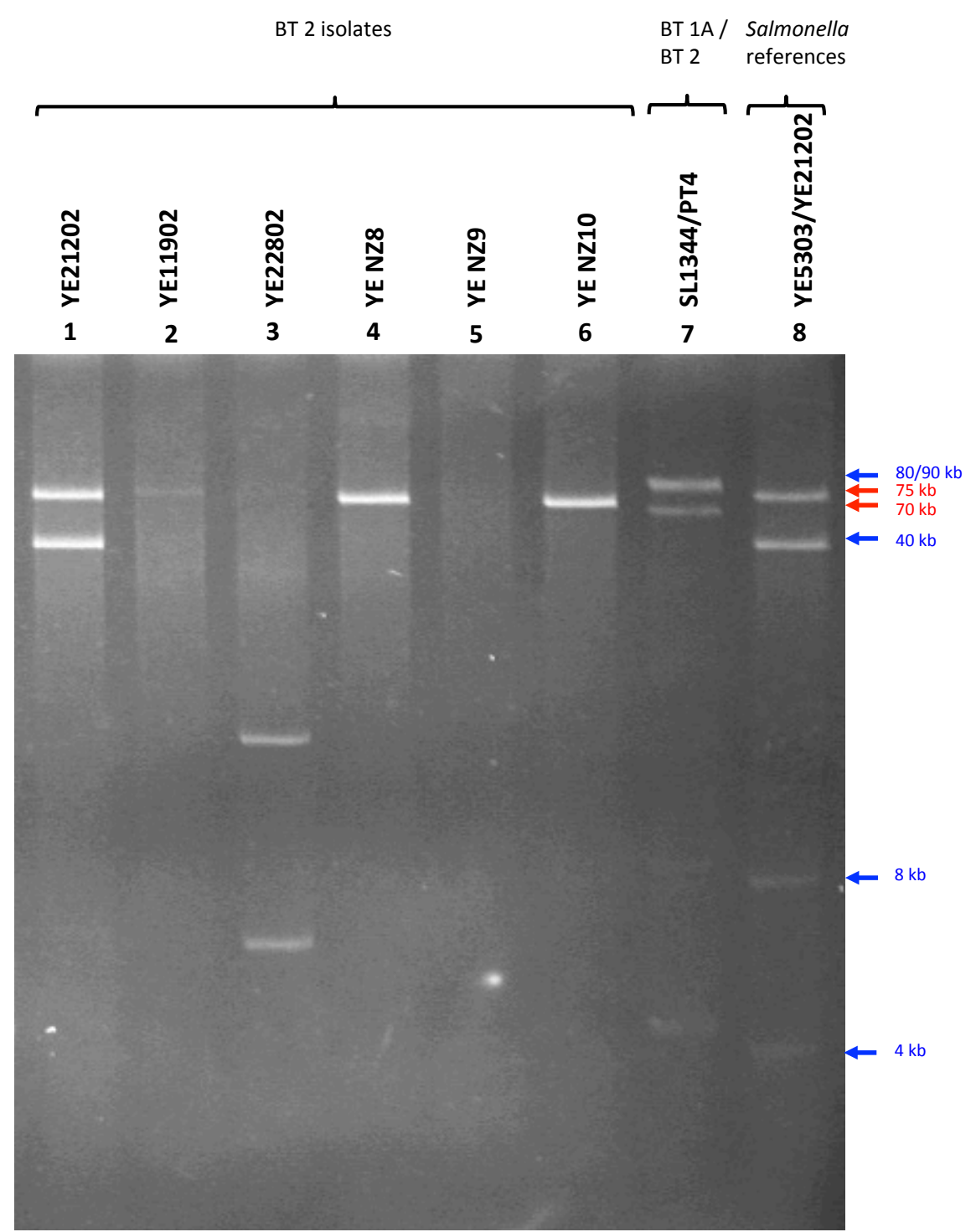

Figure 5.10: Plasmid profile of BT 2 isolates. Lane 1-6: BT 2 isolates. YE21202, YE11902, YE22802 - BT 2 O:9, NZ8-10: BT 2, no serotype information. Lane 7: Mixture of Salmonella plasmids of isolates SL1344 and PT4. Lane 8: Mixture of YE5303 and YE21202 plasmids (pSR1-2, pYV, pSR2$1 \mathrm{~A}, \mathrm{pSR} 3-1 \mathrm{~A})$. Red arrows indicate size of pYV.

Strain 21202 shows the clear presence of pYV at $75 \mathrm{~kb}$ and pSR1-2 at $40 \mathrm{~kb}$. YE11902 has a plasmid of a size corresponding to pYV although in this extraction the band is very faint. YE22802 does not contain pYV but has two smaller plasmids of $\sim 20 \mathrm{~kb}$ and $\sim 6 \mathrm{~kb}$. NZ8 (NZ832501) and NZ10 (NZ1032126) both are positive for the virulence plasmid, and NZ9 (N2982399) does not carry any plasmids.

Similar to arsenic, tetracycline is used as a growth promoter in pigs. The BT 2 0:9 strain 21202 is a pig isolate, whereas the other tested strains are of other origins. This might explain the presence of a tetracycline-carrying plasmid in this strain. The 
BT 3 0:9 pig isolate was not examined for the presence of pSR1-2, as it was sensitive to tetracycline. Sequencing of other isolates of BT 2 O:9, BT 3 O:9, BT 2 O:5,27, and BT $30: 5,27$ (Chapter 7) showed that only one other isolate potentially carries a copy of pSR1-2. That respective strain YE0403 is of BT 3 0:5,27 and was isolated from human faeces. The acquisition of pSR1-2 in 21202 therefore might present a unique event not shared by other isolates of the same niche.

pSR1-2 shows overall similarity to pMAK-2, a plasmid found in Salmonella Dublin (accession number AB366441.1; Akiba, 2007). A comparison between these two plasmids is shown in Figure 5.11.

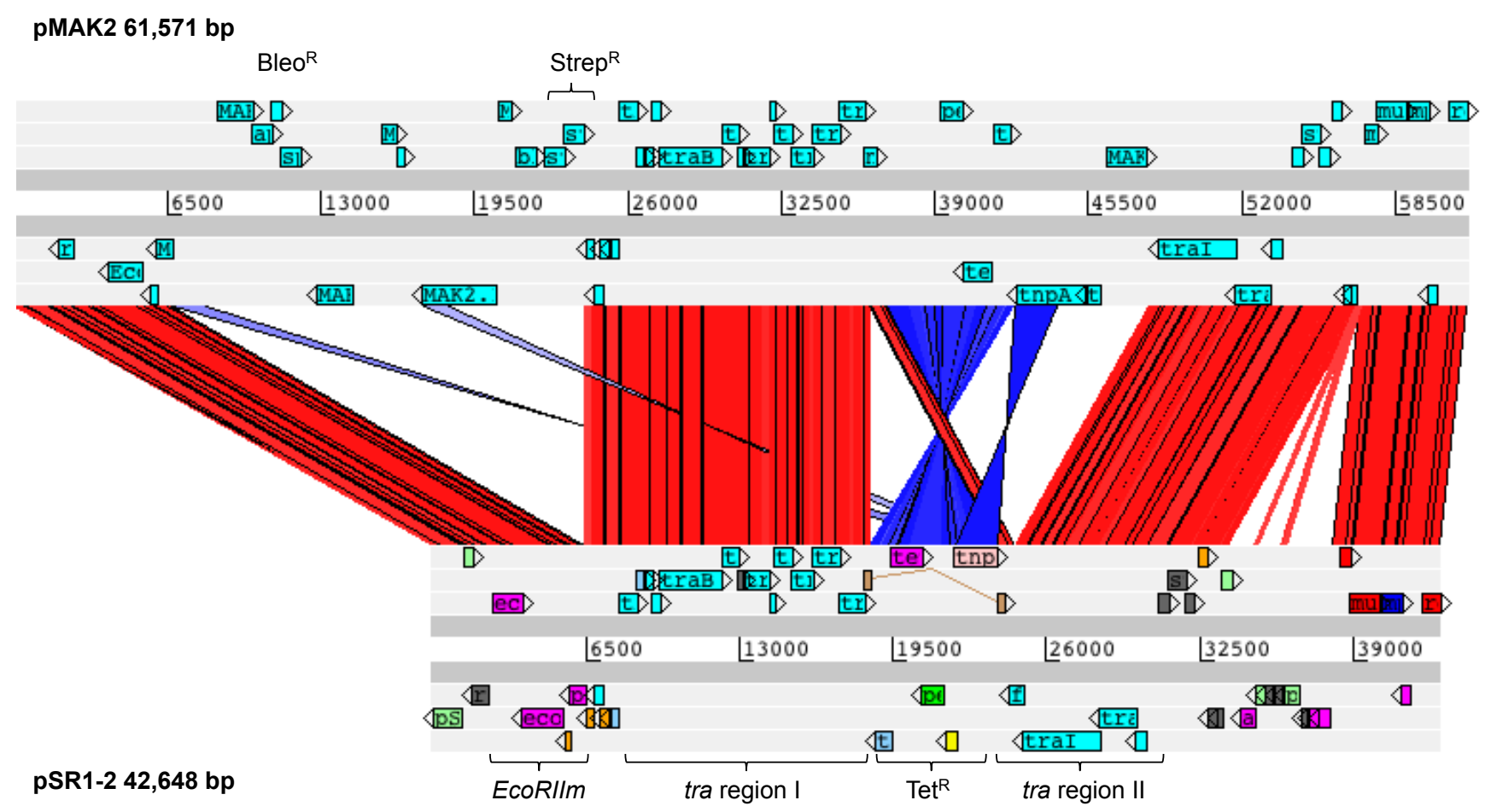

Figure 5.11: Comparison between Salmonella Dublin pMAK-2 and Y. enterocolitica pSR1-2. BleoR - bleomycin resistance, StrepR - streptomycin resistance, TetR - tetracycline resistance.

pMAK-2 is larger in size than pSR1-2, and contains additional genes encoding for bleomycin and streptomycin resistance. Tetracycline resistance is shared in both plasmids, but is inverted in pSR1-2.

Salmonella Dublin is predominantly found in cattle, and tetracycline resistance carried on plasmids has been described before (Frech and Schwarz, 1998). It is unknown whether there is a shared niche between $S$. Dublin and Y. enterocolitica that could facilitate the specific transfer of tetracycline resistance plasmids. 


\subsubsection{Unique plasmid in the reference strain for BT5: pSR4-5}

BT 5 O:2a,2b,3 strain 3094 also carries a unique plasmid which has been named pSR4-5. The GC content of the plasmid is lower than the chromosome $(42.2 \%$ and $48.2 \%$, respectively). This is a large plasmid of $107 \mathrm{~kb}$ (Figure 5.12). 108 CDSs were identified (coding percentage 66.3\%), of which 42 are hypothetical genes. Its replication protein could not be assigned to any incompatibility group. Similar to pSR1-2, this plasmid carries its own tra genes encoding a type IV pilus for conjugational transfer.

pSR4-5 also carries a putative hemolysin and its activator protein, a putative toxin/antitoxin system, an endonuclease and a transglycosylase, a putative invasion protein, and proteins involved in plasmid partitioning.

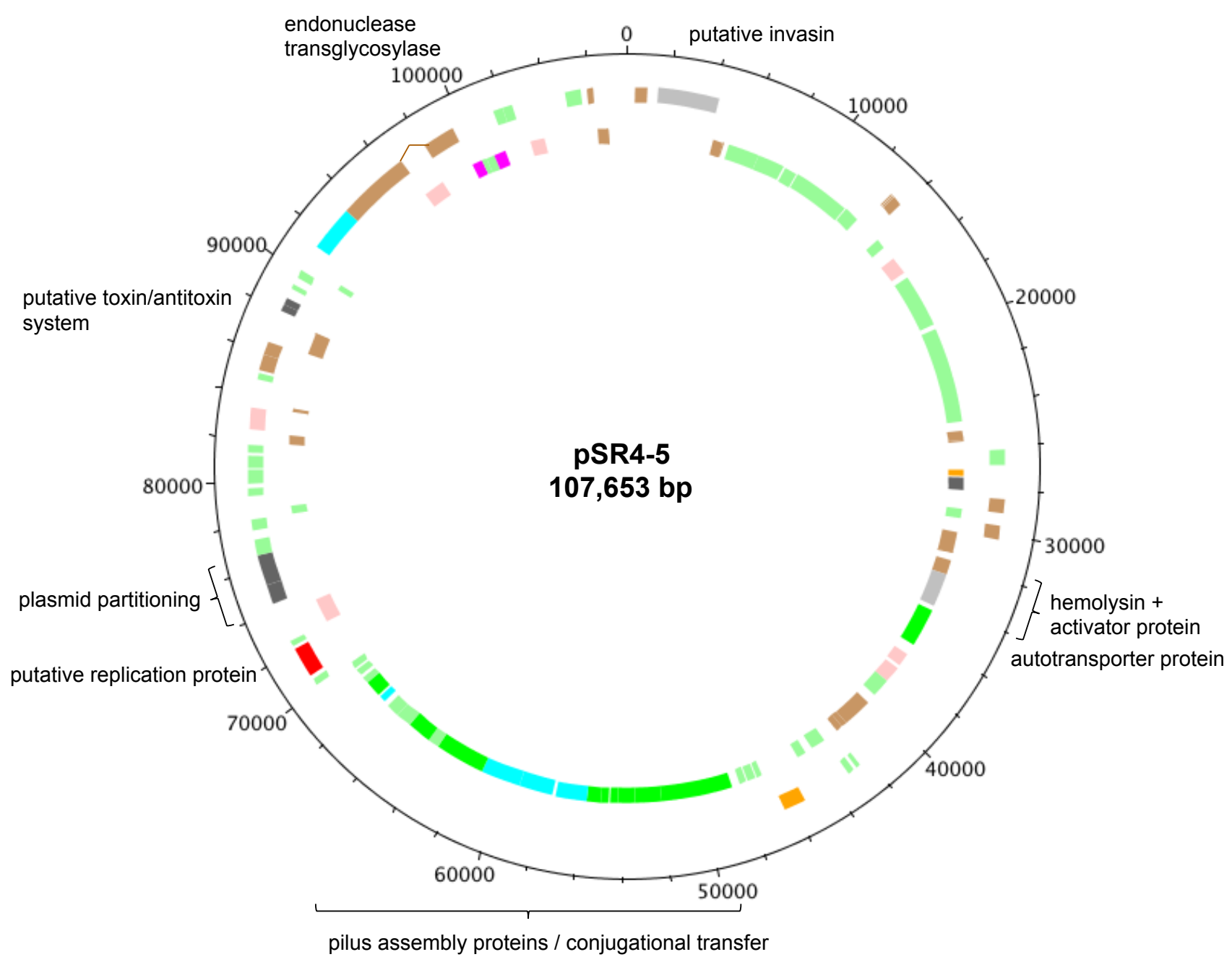

Figure 5.12: Plasmid map of pSR4-5 as found in Y. enterocolitica BT 5 0:2a,2b,3 strain 3094. Regions of interest are highlighted.

pSR4-5 was also found to be present in two other BT 5 isolates sequenced in a larger strain collection. A similar plasmid, pYE854, has been isolated from a non- 
pathogenic BT 1A strain (Hertwig, Klein, Hammerl and Appel, 2003; Hammerl, et al., 2008). A comparison between these two plasmids is shown in Figure 5.13.

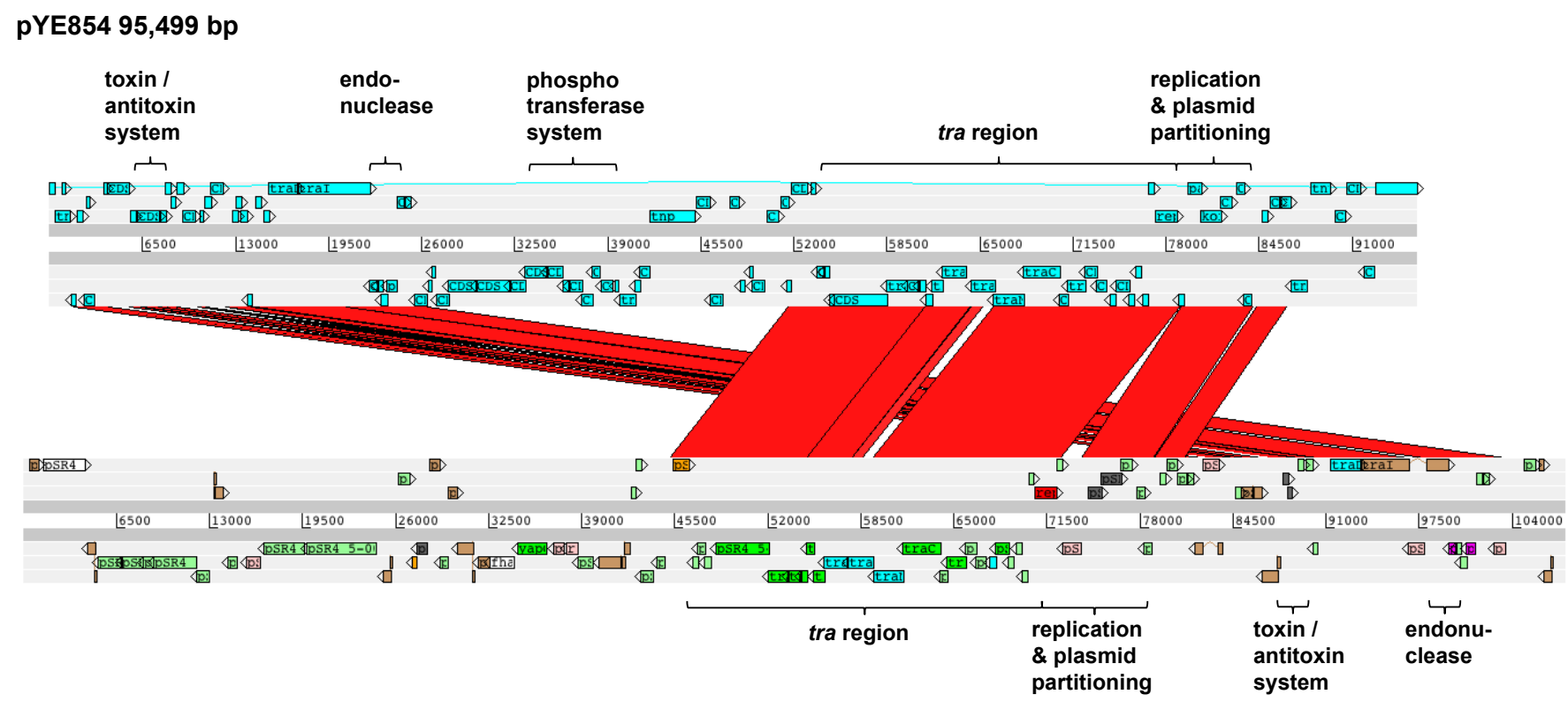

pSR4-5 107,653 bp

Figure 5.13: Comparison between Y. enterocolitica pYE854 and pSR4-5. Regions of interest are highlighted.

Both plasmids have about $60 \mathrm{~kb}$ in common, including the conjugative transfer region, as well as replication and plasmid partitioning region. Additionally, pYE854 carries a phosphotransferase system potentially involved with the transport and metabolism of sugars, although functionality could not be shown (Hammerl, et al., 2008).

pYV854 was shown to be able to transfer not only itself but also mobilize the virulence plasmid pYV in experimental studies (Hammerl, et al., 2008). This is important, as pYV itself is non-conjugative. Interestingly, pYE854 was isolated from a non-pathogenic BT $1 \mathrm{~A}$ strain, which is known never to carry the virulence plasmid (Cornelis, et al., 1987; Lepka, et al., 2009; Wren 2003). The study suggests though that self-transmissible plasmids are common amongst Yersiniae as they also found a plasmid related to pYE854 which could also mobilize pYV (Hammerl, et al., 2008).

pYE854 potentially belongs to an unknown incompatibility group as it showed no homology with the known incompatibility groups (Hammerl, et al., 2008). Furthermore co-carriage of pYE854 with plasmids of various incompatibility groups was shown (Hammerl, et al., 2008). As pSR4-5 shares the replication protein with pYE854, they might belong to the same incompatibility group. 


\subsection{Discussion}

\subsubsection{The virulence plasmid differs in high- and low-pathogenic BTs}

The virulence plasmids of high- and low-pathogenic BTs differ in two important points. The low-pathogenic BTs carry an additional arsenic resistance operon, and the origin of replication differs between high- and low-pathogenic BTs.

Arsenic resistance appears to be an important theme in Y. enterocolitica. The lowpathogenic BTs carry an operon on the virulence plasmid as part of the transposon Tn2502. The high- and non-pathogenic BTs on the other hand show the presence of operons for arsenic resistance on the chromosome, one of which is orthologous between the two BTs. In the last century and largely before World War II, arsenic has been used as a therapeutic agent in veterinary and human medicine, in particular in pigs (Neyt, Iriarte, Thi and Cornelis, 1997). This is specifically relevant as pigs are considered one of the main hosts outside the human and an important source of infections. Arsenic resistance thus might promote survival in an important niche. The presence of the arsenic resistance on pYV together with the fact that the lowpathogenic pYVs show few variations in gene content that only relate to IS elements can explain why human case and pig isolates showed identical restriction enzyme patterns (Kapperud and Nesbakken, 1987; Fukushima, Gomyoda, Aleksic and Tsubokura, 1993).

To carry virulence and resistance genes together on a plasmid is unusual (Neyt, Iriarte, Thi and Cornelis, 1997). It is also interesting because the virulence plasmid is known to be easily lost under non-selective conditions (Heesemann, et al., 1983). It has been suggested that the acquisition of arsenic resistance coincided with the use of arsenic compounds, which then lead to a global spread of a low-pathogenic clone (Neyt, Iriarte, Thi and Cornelis, 1997). The population structure and relatedness of the low-pathogenic BTs cannot be deduced from a virulence plasmid and will be investigated in Chapter 7. Given the fluid nature of plasmid maintenance, arsenic resistance might also be viewed as a dispensable trait in low-pathogenic BTs.

Analysis of the origin of replication showed dissimilarity between high- and lowpathogenic BTs. They carry different proteins of distantly related replicons. It has been proposed that the two plasmids are of an independent origin (Portnoy, et al., 1984; Snellings, Popek and Lindler, 2001). The selective pressure is on the type III secretion system and the secreted effector proteins; these build the backbone of the 
plasmid (Portnoy, et al., 1984). The rest of the plasmid shows divergent, independent evolution (Snellings, Popek and Lindler, 2001). Different plasmids, one carrying the calcium-dependence region, the other carrying a different origin of replication, might have been cointegrated to form the present variety of pYVs (Portnoy, et al., 1984; Snellings, Popek and Lindler, 2001). It could be argued that the T3SS has integrated into pre-existing plasmids as a mobile genetic element. High- and low-pathogenic BTs might have carried different plasmids into which the T3SS inserted, explaining the difference in the origin of replication. The fact that more than $75 \%$ of the plasmid sequence is shared, that the only region of difference is the origin, and that important effector proteins are located outside of the conserved T3SS operons argue against this hypothesis though. The effector proteins located outside the T3SS operons also show some degree of conservation with respect to gene order, and their varying location is explained by the presence of transposases rather than further independent acquisition events. The difference observed in the origins argues for a single source for the T3SS, and that there is a distinct repertoire of plasmids (Portnoy and Falkow, 1981). The pYV should therefore be rather designated as a family of plasmids rather than a clonal molecule that shows no variation (Portnoy and Falkow, 1981). It is difficult to say, which of the plasmids came first, and how they were passed on to other lineages, and how they evolved further. The events leading to the family of virulence plasmids have happened a long time in the past, and intermediates that are potentially found in environmental bacteria would be needed to shed light on the source and evolutionary direction of pYV.

It has been argued that the degree of divergence between plasmids from Y. pseudotuberculosis/Y. pestis and $Y$. enterocolitica BT $1 \mathrm{~B}$ is similar to the divergence found in the chromosome (Portnoy and Falkow, 1981). Together with $16 \mathrm{~S}$ analysis and biochemical typing, this has probably led to the common conception of evolution of pathogenic Yersiniae through a single acquisition event of the virulence plasmid and subsequent speciation into $Y$. pseudotuberculosis and Y. enterocolitica (Carniel, 2002; Wren, 2003). The divergence between the virulence plasmid though is only based on hybridization values, and presumably focuses on the conserved T3SS backbone. Generally the virulence plasmids of Y. enterocolitica appear to be hybrids. Sometimes the variations observed in the high-pathogenic pYV more closely resemble the organization and single amino acid changes of $Y$. pseudotuberculosis and Y.pestis, at other times the low-pathogenic BTs are closer to Y.pestis, for example in the origin of replication. Chapter 7 will investigate the relationship 
between the Yersiniae more thoroughly, and the consequences for plasmid acquisition.

Plasmid variability has also been reported in Y.pestis (Eppinger, et al., 2010). Usually, the two Y. pestis unique plasmids pMT1 and pPCP1 are highly conserved. Eppinger, et al. (2010) describe a chimeric plasmid pMT-PCP consisting of a cointegrate of a single copy of pMT1 and two copies of pPCP1 in Y. pestis biovar Pestoides strain Angola. Some other Pestoides isolates were shown to lack one of the two plasmids, indicating that virulence plasmids play an important role in disease but are not predominant (Eppinger, et al., 2010). Just like in Y. enterocolitica, virulence is more than the acquisition and maintenance of a single plasmid and also involves the interplay with chromosomally encoded factors (Revell and Miller, 2001).

\subsubsection{Sequence variation in single genes: IcrV, yopM and yscP}

Although the T3SS forms a highly conserved part of the virulence plasmid, there are a few variations detected in single genes. These differences could be of physiological interest if they are predominantly present in certain lineages.

The epitope LcrV is also known as the $\mathrm{V}$ antigen of pathogenic Yersiniae (Roggenkamp, et al., 1997). Research has shown that there are two variants (Roggenkamp, et al., 1997). The first variant is found in Y.pestis, Y.pseudotuberculosis and in European Y. enterocolitica strains as well as the biotype $1 \mathrm{~B}$ strain 8081 . The second variant has an additional 9 amino acids in the hypervariable region of the protein and is mostly found in American BT 1B isolates of Y. enterocolitica (Roggenkamp, et al., 1997). Protective immunity is only given for the respective antigen, cross-reactivity in vivo is low; this might help explain why there are natural niches for plague in the Americas, whereas in Europe the wild rodent population is immunized due to $Y$. enterocolitica infection resulting in antibody production against LcrV (Roggenkamp, et al., 1997).

Here, a third variation with 5 additional amino acids present in the low-pathogenic BTs 2 and $30: 9$ is described. As this variation possibly changes the protein structure, a protective immunity would not be given after exposure to this antigen. Yet, BTs 2 and 3 are very common in Europe. A physiological role of LcrV as plague protection is therefore questionable as the presence of the three variants of LcrV does not correlate with the known plague foci. It is unknown though whether this third variant is commonly found in BT2 and 3 isolates, as only two strains were examined 
here. It could also be that these isolates show mutations independent to the rest of the lineage.

YopM is the only protein of the Yop regulon that contains leucine-rich repeats. It could be shown that the copy number of the repeat region containing 60 aa can vary between different strains (Boland, Havaux and Cornelis, 1998). This phenomenon is not linked to a specific biotype, as biotype 1B plasmids pYVe8081 and pYVeA127/90 have different sizes (Boland, Havaux and Cornelis, 1998). In the presently investigated strains, no sequence variation compared to BT 1B was detected. The size and orientation of YopM does not have any visible effect on its function. pYVe8081 and pYVeA127/90 have not only proteins of different sizes, the orientation of said gene is also opposite on these plasmids; it is in clockwise orientation on pYVeA127/90 and in counter clockwise orientation on pYVe8081 (Foultier and Cornelis, 2003; Snellings, Popek and Lindler, 2001).

The needle protein YscP was found to be 60 amino acids shorter in pYVe8081 than in pYVe227 (BT 2) (Snellings, Popek and Lindler, 2001), which made the protein from pYVe8081 more closely related to the ones from Y.pestis and Y. pseudotuberculosis plasmids. It was shown that $\mathrm{YscP}$ functions as a molecular ruler when the needle of the T3SS is formed (Journet, Agrain, Broz and Cornelis, 2003; Wagner, et al., 2009). As the needle is built with YscR building blocks, YscP is stretched alongside the growth of the needle, until a certain breaking point. YscP then has a second function in signalling back to the T3SS to produce late injectisome molecules (Wagner, et al., 2009). The functionality of YscP depends both on the length of the protein (Journet, Agrain, Broz and Cornelis, 2003) and on the helical content determined by amino acid content (Wagner, et al., 2009). It was shown that needles were longer when encoded by a longer CDS, but also when helix maker alanine was substituted by helix breakers proline and glycine (Wagner, et al., 2009). The needle length might relate to the effectiveness of protein transfer into the target cell.

Here, three different repeats were identified. The red repeat contains 13 amino acids, the green repeat 25 amino acids, and the blue repeat 46 amino acids. Y. pestis, Y. pseudotuberculosis, and Y. enterocolitica BT 1B had a single red-green-blue repeat only, except for some BT 1B strains that showed two full red-green-blue repeats. The low-pathogenic BTs had one red-green-blue repeat and an additional 
red-blue repeat, and the low-pathogenic BT 5 strains only showed red-blue repeats in variable number. This has not been correlated with any change in mechanism or outcome of disease so far.

\subsubsection{Plasmid Repertoire in Yersinia}

Several unique plasmids were detected in the sequenced reference strains. The two plasmids of the non-pathogenic BT $1 \mathrm{~A}$ are most likely cryptic, as they are small and only potentially encode for few proteins of undefined nature. Large plasmids can also be present in non-pathogenic BTs as demonstrated by the atypical BT 1B/1A strains. The presence of large plasmid is not indicative for pathogenic potential of a strain and might confuse the distinction of high- and non-pathogenic BT. These large plasmids warrant further investigation into the genes encoded and their biological relevance. The unique plasmid of BT 2 strain 21202 carries an antibiotic resistance cassette for tetracycline. Similar to arsenic, this antibiotic has been extensively used in pigs in the past, and might explain the acquisition of this plasmid. Although an initial search was limited to isolates available in the strain collection at Nottingham Trent University, the hypothesis of presence of pSR1-2 in pig isolates of BT 2 could not be validated and was not further pursued. Compared with larger strain collection it now seems more likely that pSR1-2 is a unique acquisition event in BT 2 0:9 strain 21202. The BT 5-unique plasmid could be potentially involved in conjugative transfer of the virulence plasmid pYV as it shows homology with another plasmid in which such a mobilization event was proven (Hammerl, et al., 2008). The unique plasmids constitute an extended gene pool for $Y$. enterocolitica and selection is driven by environmental factors such as the pig niche. 


\section{Metabolic profiling of the Y. enterocolitica biotypes}

\subsection{Introduction}

\subsubsection{Biotyping and speciation based on biochemical properties}

Biochemical tests such as sugar utilization and fermentation and testing for the presence of enzymes like ornithine decarboxylase traditionally differentiate bacterial species. Given the heterogeneity within Y. enterocolitica and the differences in pathogenic potential, this species was further subdivided into several biotypes (see Table 1.2; Wauters, Kandolo and Janssens, 1987; Bottone, 1997). Biotyping is reasonably robust, and reflects the human pathogenic potential. There have been reports however of strains with atypical biotyping reactions (Guiyoule, et al., 1998) as well as high-pathogenic strains that lack the typical invasive phenotype, and which cluster with non-pathogenic BTs in microarray and AFLP analysis (Fearnly, et al., 2005; Howard, et al., 2006; McNally, et al., 2006; personal communication M. Prentice). This variability arises because biotyping is affected by the media used for culturing of the strain before typing and is subject to incubation time and temperature (Wauters, Kandolo and Janssens, 1987; Bottone, 1999; Cornelis, et al., 1987; Farmer, et al., 1992; Stock, Henrichfreise and Wiedemann, 2002; personal communication E. Carniel).

Metabolism is now being recognized as an important factor in colonization not only in the environment but also in human, animal or insect hosts (Rohmer, Hocquet and Miller, 2011). Transition from environmental lifestyle to a host-adapted niche imposes challenges on bacteria in terms of $\mathrm{pH}$, oxygen levels, and nutrient availability such as free iron. An extensive metabolic repertoire is needed to accommodate these changes. Conversely certain metabolic functions might become redundant with limitation to highly specific niches (Rohmer, Hocquet and Miller, 2011). For Y. pestis, such a change in lifestyle to become reliant on an insect vector and a warm-blooded host has been investigated in the past, and additional nutrient requirements are known (Burrows and Gillett, 1966). Less is known about Y. enterocolitica metabolism and whether some biotypes have adapted to certain niches. 


\subsubsection{Phenotypic microarray technology}

Generally, biotyping is based on a handful of metabolic properties of a cell, and only presents a fraction of the metabolic profile. The phenotypic microarray technology produced by Biolog offers a wider range of metabolites that can be tested in an automated fashion. An overview of the workflow is shown in Figure 6.1 and 6.2.

Bacteria grown on solid medium are suspended in a minimal, defined medium (Bochner, Gadzinski and Panomitros, 2001; Bochner, 2009). The phenotypic microarray is carried out in 96-well plates, with each of the wells containing a different nutrient. For the carbon plates, the minimal medium supplies sulphur, phosphate and other essential nutrients apart from carbon, which will be supplied separately in each of the wells (Bochner, Gadzinski and Panomitros, 2001; Bochner, 2009). For the nitrogen plates, all nutrients except nitrogen are contained in the minimal medium, and so forth.

The phenotypic microarray is based on cellular respiration reflecting cell activity (Figure 6.1; Bochner, Gadzinski and Panomitros, 2001). Transport and metabolism of nutrients results in respiration through the production of energy along the electron transport chain.

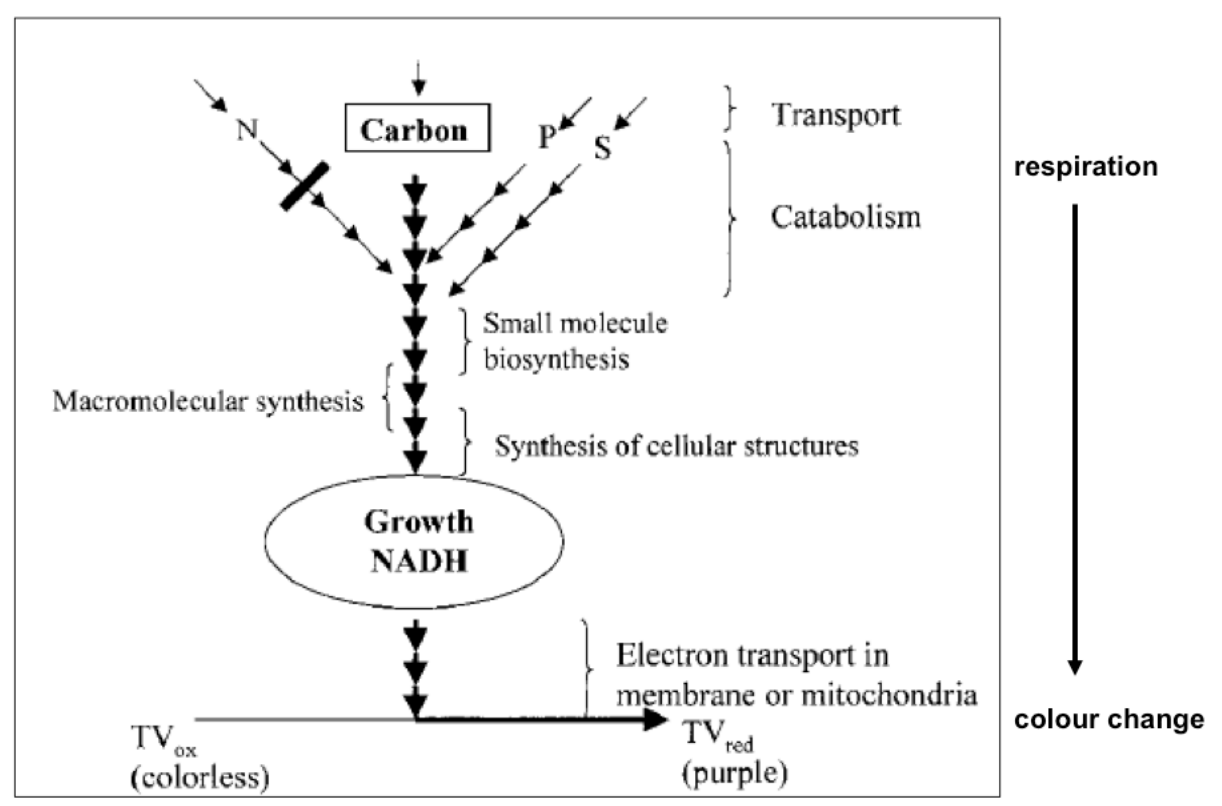

Figure 6.1: Respiration is coupled to conversion of tetrazolium violet dye [taken from Bochner, Gadzinksi and Panomitros, 2001].

Respiration is not necessarily coupled to growth and can therefore also detect phenotypes that do not produce growth but respiration only (Bochner, Gadzinski and Panomitros, 2001; Bochner, 2009). A tetrazolium violet dye added to the reaction is reduced in the presence of electrons causing a colour change from colourless to 
purple (Bochner, Gadzinski and Panomitros, 2001). An increased rate of conversion leads to a quicker colour change, because the dye reduction is proportional to the electron flow (Bochner, 2009).

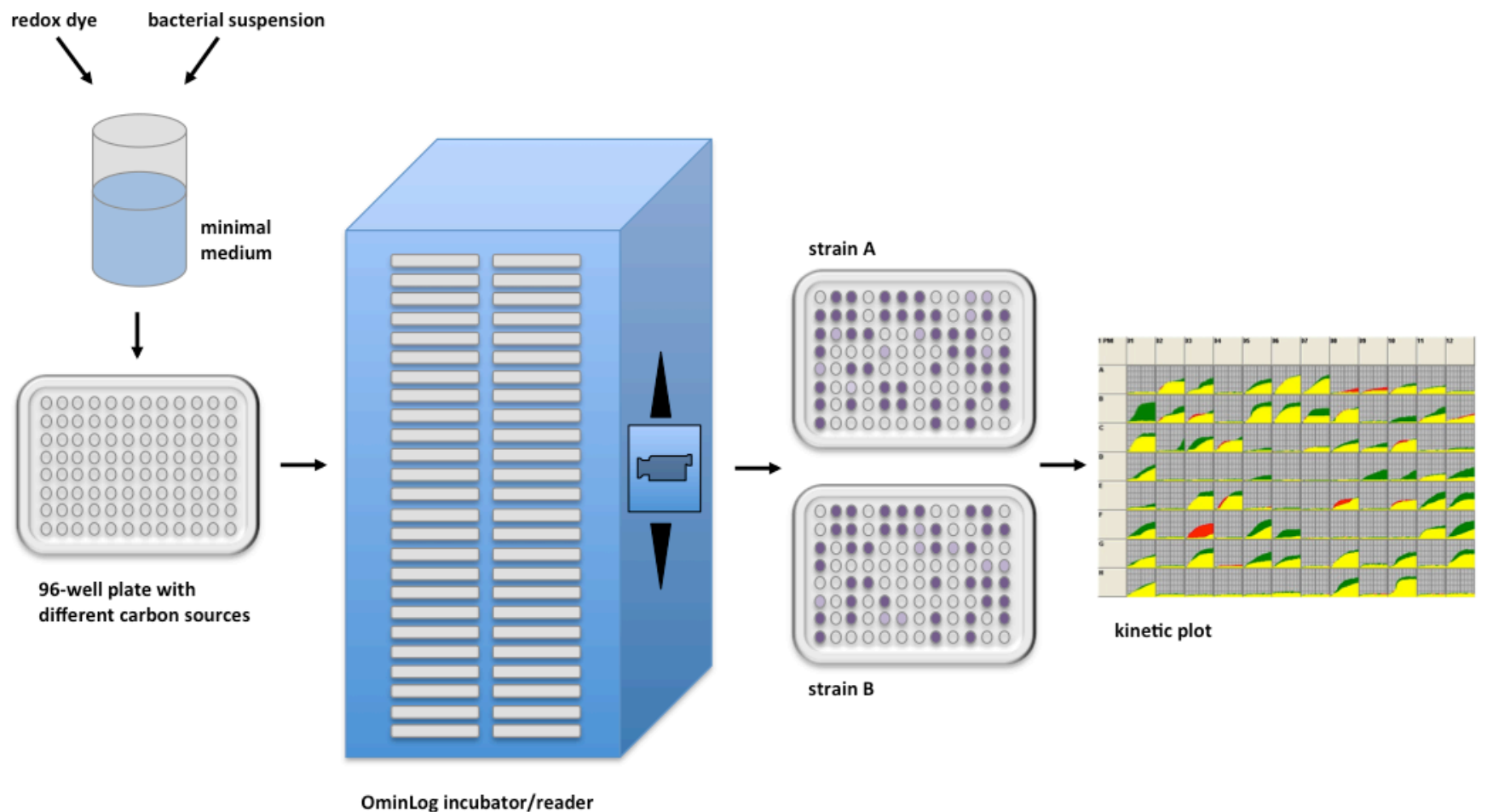

Figure 6.2: Workflow of phenotypic microarray [adapted from Bochner, Gadzinski and Panomitros, 2001; Bochner, 2003]. Redox dye and bacterial suspension are added to the minimal medium. This is used to inoculate 96-well plates containing different nutrient sources in each of the wells. The plates are placed in the OmniLog incubator/reader, which takes up to 50 plates at one time. The camera incorporated in the machine takes readings of the plates. Respiration results in a purple colour change in active wells. Different strains can be compared visually and more in detail in the kinetic plot, showing strain A in green, strain B in red, and the overlay in yellow.

If either transport of the nutrients or metabolism is dysfunctional, there will be no electron flow and consequently no reduction of the dye. Therefore metabolic activity can be monitored and quantified through purple colour production. Accumulation of the dye is harmless to the cells and the signal is intensified as respiration continues (Bochner, Gadzinski and Panomitros, 2001). The 96-well plates are placed in the OminLog instrument, which acts as an incubator and measures dye production in parallel (Figure 6.2; Bochner, Gadzinski and Panomitros, 2001). Data is recorded every 15 minutes over 48 hours.

The phenotypic microarray can be used instead of an API test to identify bacterial species and is potentially more versatile as it can test for 95 different substrates simultaneously (Miller and Rhoden, 1991). It has also been used to study microbial 
communities and the changes in composition upon incubation with different carbon sources (Smalla, et al., 1998).

Chosen for this study were two phenotypic microarray plates with carbon sources, PM1 and PM2A, one phenotypic microarray plate with nitrogen sources, PM3B, one mixed phenotypic microarray plate with phosphorus and sulphur sources, PM4A, and phenotypic microarray plates PM9 and 10 which test for osmolytes and $\mathrm{pH}$. For each strain, triplicates were carried out at optimum growth temperature of $28^{\circ} \mathrm{C}$ and at human body temperature of $37^{\circ} \mathrm{C}$. Data will mainly be presented on PM1-4 at $28^{\circ} \mathrm{C}$. The phenotypic microarray was used to establish the metabolic profile of each sequenced reference strain. The differences observed in the genomic analysis should be reflected in the metabolism of each strain. The phenotypic microarray should aid annotation and establish direct links between phenotype and genotype. 


\subsection{Results}

\subsubsection{Metabolic activity in Y. enterocolitica biotypes at optimum temperature}

Given the apparent role of metabolism in determining the breadth of host range and pathogenic potential and the targeted loss of function as presented in the pseudogenes, the reference strains were assayed using the phenotypic microarray for their ability to utilise different sources of carbon, nitrogen, sulphur, and phosphate at optimum temperature of $28^{\circ} \mathrm{C}$. The signal values are given in the appendix Table 9.6 (p. 242) and the rearranged layout for positive wells plus significant changes towards BT 1B strain 8081 are listed in Table 9.7 (p. 254).

The heat map in Figure 6.3A shows the signal values corresponding to the intensity of utilization for the different nutrient sources. The three replicates are shown for each strain, with blue indicating utilization and red absence of utilization. Figure 6.3B shows the change towards $\mathrm{BT} 1 \mathrm{~B}$, with yellow indicating a significantly stronger activity and blue a significantly reduced activity compared to the high-pathogenic BT. Even from an overview it is apparent that the non-pathogenic BT 1A shows a wider metabolic activity with the highest number of positiv signals (Figure 6.3A). This is consistent with the biotyping scheme in which this $\mathrm{BT}$ is positive for the fermentation of all the sugars tested and maintains functionality of metabolism. The metabolic activity detected is often also significantly higher than in BT 1B (Figure 6.3B), suggesting a more effective metabolism. BT 2 0:9 strain 21202 and BT 3 0:9 strain 5603 were shown to be nearly identical with respect to genomic content and pseudogenes. Looking at their metabolic activity (Figure 6.3A) but specificatlly the change towards $\mathrm{BT} 1 \mathrm{~B}$ (Figure $6.3 \mathrm{~B}$ ) one can see that they exhibit very similar profiles. This confirms their genetic relatedness, and also suggests that there is little difference between BTs 2 and 3 if they are of the same serotype.

Comparing the low- and high-pathogenic BT to the non-pathogenic BT, both have lost metabolic pathways with two extreme cases of loss of function presented by BT3 O:5,27 and BT5. BT 3 O:,27 shows marked reduction of nutrient utilization in nitrogen and phosphorus sources. The metabolism of all nutrient sources appears to be greatly reduced in BT 5 , with only clear positive signals in carbon sources and hardly any signals in nitrogen, phosphorus and sulphur sources. The biotyping scheme shows an indication for this as well, as BT5 is mostly negative for the fermentation of sugars tested. 


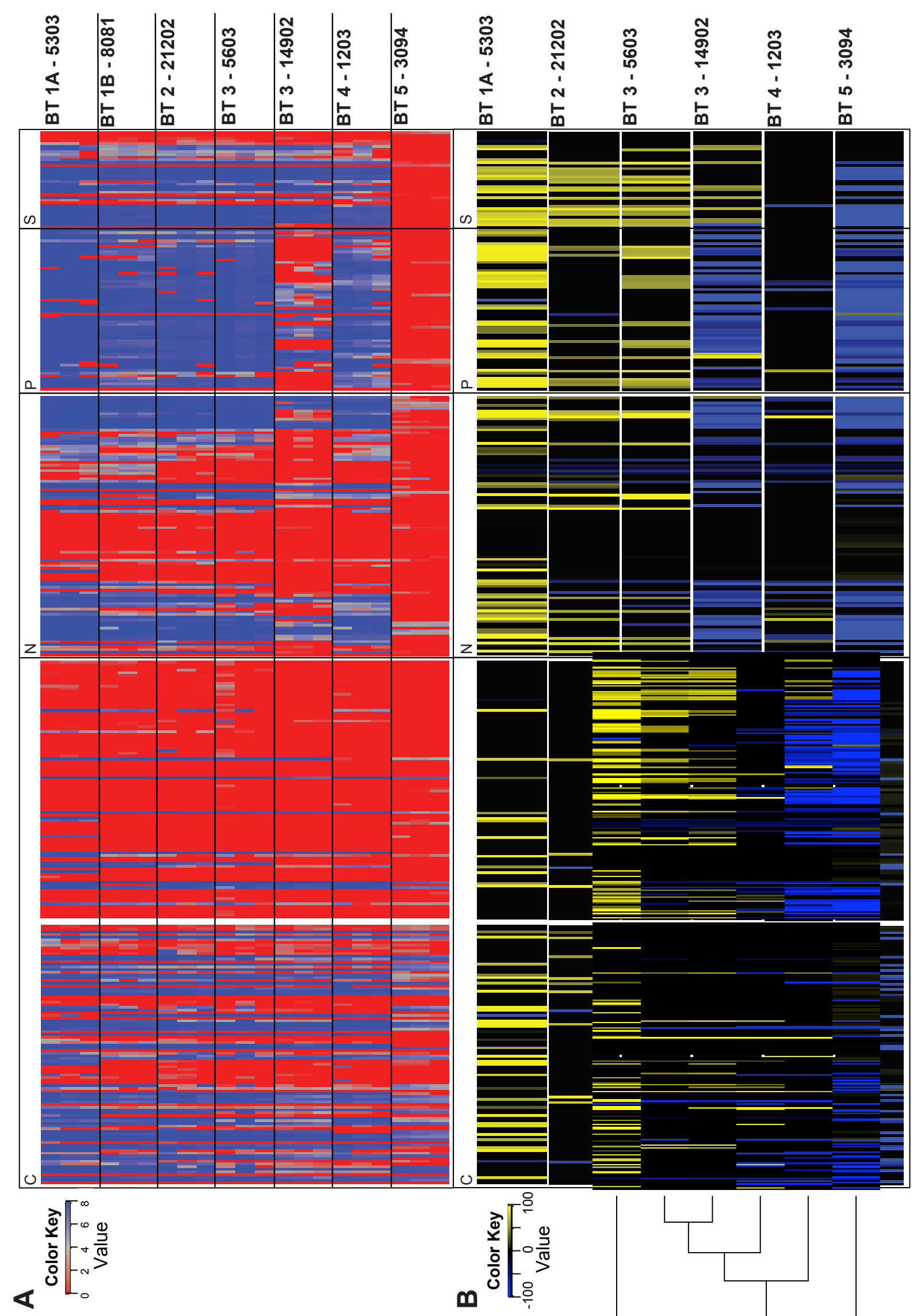

Figure 6.3: Signal values (A) and significant changes towards BT 1B (B) depicted as heatmaps. Triplicates are shown for the signal values. C - carbon sources (190), N - nitrogen sources (95), P phosphorus sources (59), S - sulphur sources (35). 
Determining positive and negative signals, it was also examined whether the change observed posed a significant difference towards the BT 1B reference strain 8081 (Figure 6.3B, Figure 6.4 and 5 for absolute numbers).

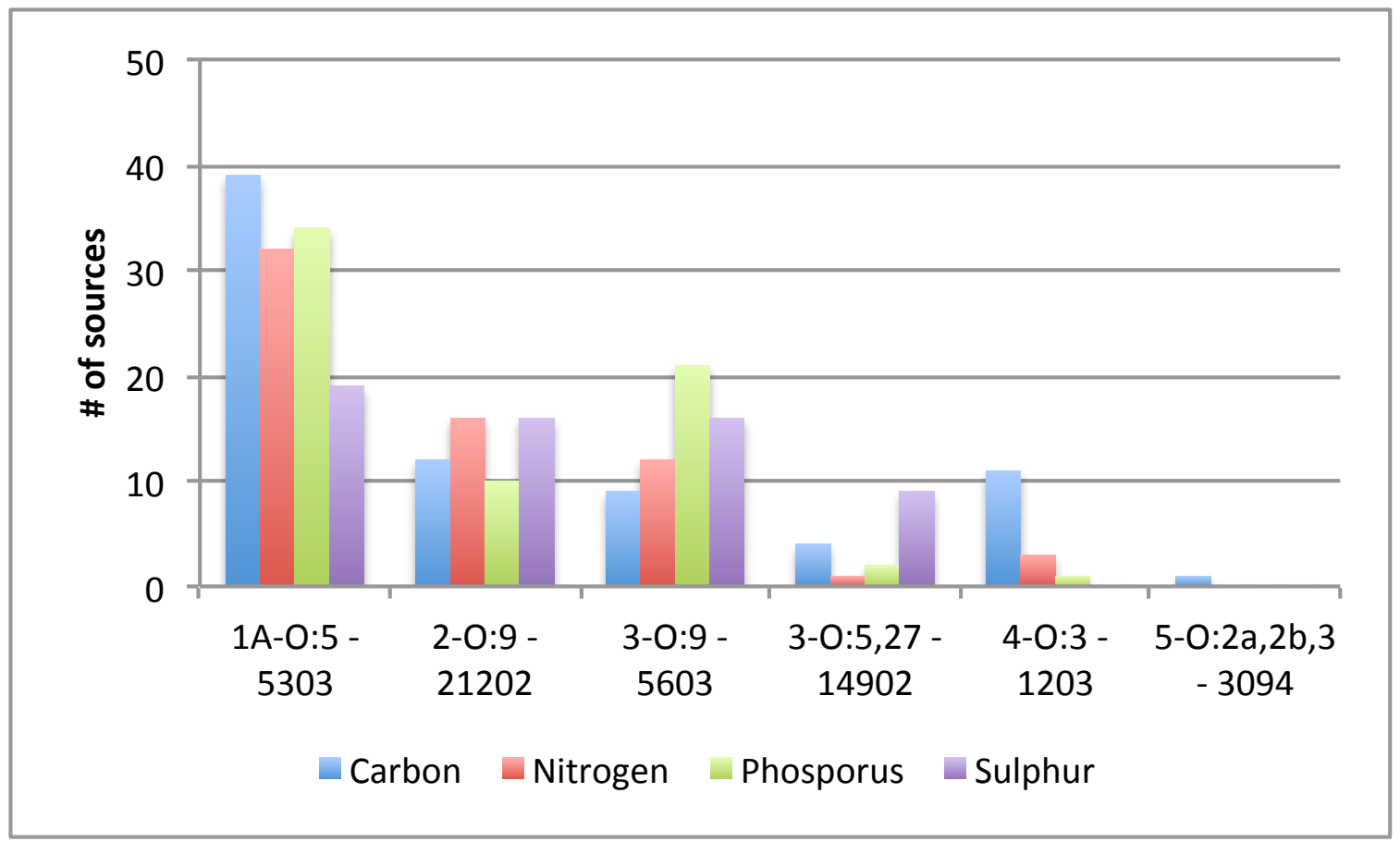

Figure 6.4: Number of sources that are expressed significantly higher than in BT 1B strain 8081.

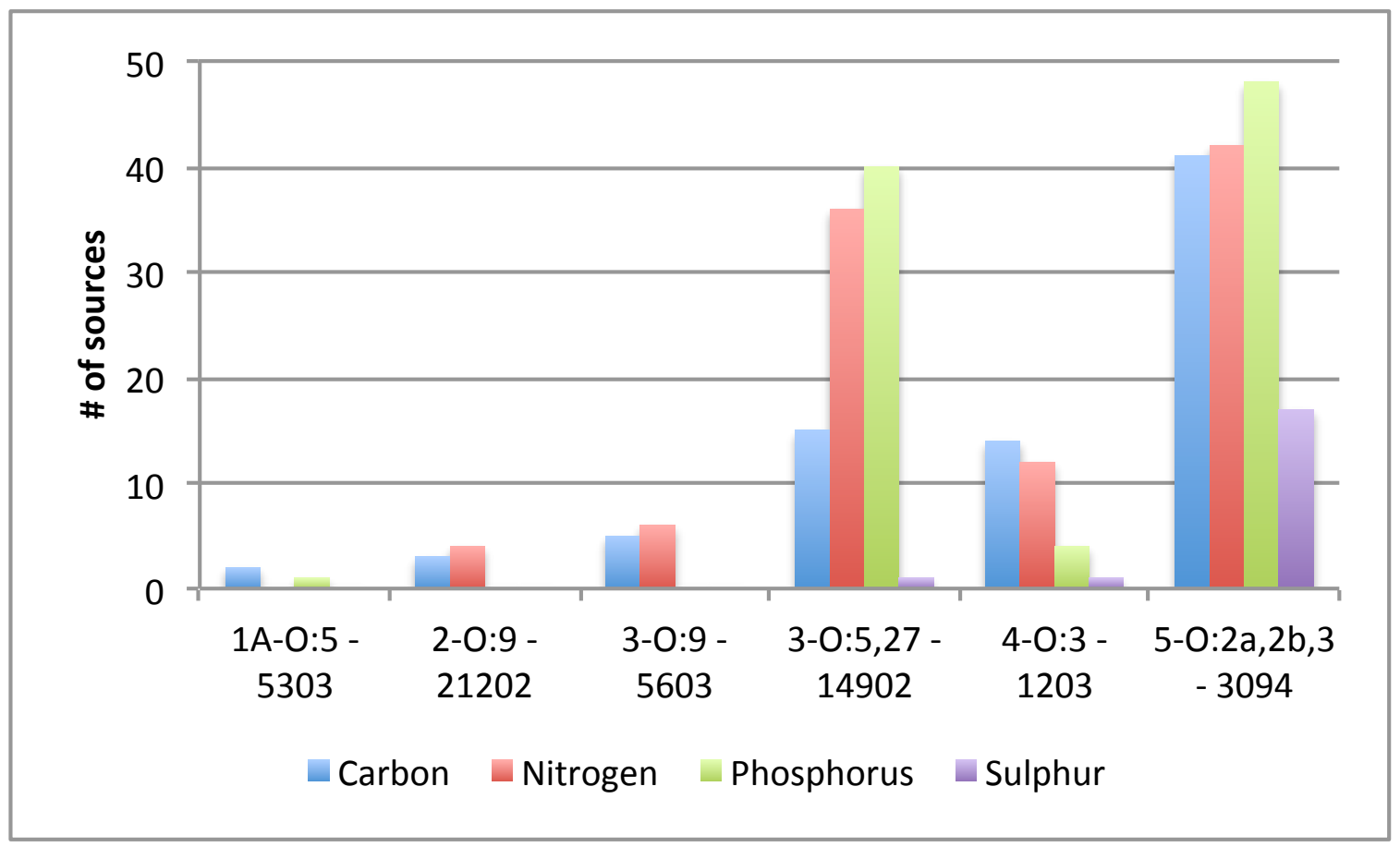

Figure 6.5: Number of sources that are expressed significantly lower than in BT 1B 8081. 
The non-pathogenic BT1A not only shows a wider range of metabolic activity, this activity is also significantly higher than in BT 1B. In contrast there are few sources that show a significantly lower activity, and two out of these three relate to sources that are not utilized in BT 1A. Both BT 2 and 3 0:9 isolates show a significantly higher activity in some sources, but also show a significantly lower activity especially in carbon and nitrogen sources. The other three BTs show only a selected number of sources which are utilized significantly higher than in BT 1B. BT 3 0:5,27 shows a significant reduction in nitrogen and phosphorus sources as was already apparent from the heatmap. BT 4 shows some reduction in carbon and nitrogen sources, similar to the ST 0:9 isolates. The absence of metabolic activity in BT 5 is seen in the high number of significantly reduced sources in all nutrients.

\subsubsection{Metabolism of carbon sources}

Looking in more detail at the carbon sources (phenotypic microarray plates PM1 and $2 \mathrm{~A}$ ), a total of 27 carbon sources (14.2\% of total of 190 tested carbon sources) were found to be utilised in all biotypes, with a further 14 sources $(7.4 \%)$ utilised by all but BT 5 (Table 9.7). In line with the genomic observations there are a significant number of additional metabolic capacities shared by BTs $1 \mathrm{~A}$ and $1 \mathrm{~B}$ strains including the ability to use D-alanine, L-asparagine, dextrin, succinamic acid, and L-histidine as sole carbon sources. Also reflected in the genetic makeup of the BT 1A is a greater metabolic capacity than all the other biotypes with 15 carbon sources, which are utilized only by BT1A. In addition there are a further 12 carbon sources that BT1A shares in combination with one or more of the BT, whereas there are only two carbon sources that are present in any of the other BTs and not in 1A. These two carbon sources are glycogen, which is only utilized in BT 2 O:9 strain 21202, and m-inositol which is metabolized in all BTs except BTs $1 \mathrm{~A}$ and 5.

With the exception of the two mentioned carbon sources there are no other carbon sources unique to the high- and low-pathogenic BTs that are not present in BT $1 \mathrm{~A}$. BT $1 \mathrm{~A}$ is an environmental organism well adapted to a number of niches. Together with the fact that it has the lowest number of pseudogenes, this suggests that BT $1 \mathrm{~A}$ is the oldest lineage from which the other BTs evolved through loss of metabolic functions. 


\subsubsection{Utilization of essential nutrients nitrogen, phosphorus and sulphur}

The utilization of the essential nutrients nitrogen, phosphorus, and sulphur is tested for in phenotypic microarray plates $\mathrm{PM} 3 \mathrm{~B}$ and $4 \mathrm{~A}$. BT1A, 1B, and 2-4 have 13 nitrogen sources ( $13.7 \%$ of total of 95 sources), 2 phosphorus sources (3.4\% of total 59 sources), and 14 sulphur sources (40.0\% of total 35 sources) in common. No metabolic activity can be seen for BT5 for any of these sources.

Similar to the observations made for carbon sources, there are two sources which are exclusively utilized in BTs $1 \mathrm{~A}$ and $1 \mathrm{~B}$. Both BTs metabolize D-asparagine and Lphenylalanine as sole nitrogen sources. There are seven nitrogen sources, two phosphorus sources and one sulphur source, which are uniquely metabolised in BT 1A. Further 27 nitrogen sources, 44 phosphorus sources, and 4 sulphur sources show shared metabolism in BT $1 \mathrm{~A}$ and any of the other BTs, underlining the increased metabolic flexibility of the non-pathogenic BT. There are three nitrogen sources, seven phosphorus, and 2 sulphur sources absent from BT 1A. These are listed in Table 6.1.

Table 6.1: Utilization of $N, P$, and $S$ sources absent from BT 1A.

\begin{tabular}{|c|c|c|c|c|c|c|c|c|}
\hline & Nutrient source & $\begin{array}{c}\text { BT 1A } \\
5303\end{array}$ & $\begin{array}{l}\text { BT 1B } \\
8081\end{array}$ & $\begin{array}{c}\text { BT } 2 \\
21202\end{array}$ & $\begin{array}{l}\text { BT } 3 \\
5603\end{array}$ & $\begin{array}{c}\text { BT } 3 \\
14902\end{array}$ & $\begin{array}{l}\text { BT } 4 \\
1203\end{array}$ & $\begin{array}{l}\text { BT } 5 \\
3094\end{array}$ \\
\hline $\mathbf{N}$ & D,L-a-amino-caprylic acid & - & - & + & - & - & - & - \\
\hline $\mathbf{N}$ & L-tryptophan & - & + & - & - & - & - & - \\
\hline $\mathbf{N}$ & Xanthine & - & + & - & - & - & - & - \\
\hline $\mathbf{P}$ & $\begin{array}{l}\text { Adenosine-5'- } \\
\text { monophosphate }\end{array}$ & - & + & + & + & - & + & - \\
\hline $\mathbf{P}$ & D-glucose-6-phosphate & - & + & + & + & - & + & - \\
\hline $\mathbf{P}$ & Cytidine-3'-monophosphate & - & + & + & - & - & - & - \\
\hline $\mathbf{P}$ & Cytidine-5'-monophosphate & - & + & - & + & - & + & - \\
\hline $\mathbf{P}$ & Uridine-5'-monophosphate & - & + & - & + & - & + & - \\
\hline $\mathbf{P}$ & O-phospho-L-tyrosine & - & - & + & + & - & - & - \\
\hline $\mathbf{P}$ & $\begin{array}{l}\text { Adenosine-2',3'-cyclic } \\
\text { monophosphate }\end{array}$ & - & - & - & + & - & - & - \\
\hline $\mathbf{S}$ & Hypotaurine & - & - & - & + & + & - & - \\
\hline $\mathbf{S}$ & D,L-ethionine & - & - & - & + & - & - & - \\
\hline
\end{tabular}

Adenosine, cytidine, and uridine phosphates are involved in purine and pyrimidine metabolism. Their physiological relevance in BT $1 \mathrm{~A}$ is unknown. An impairement of purine and pyrimidine metabolism is rather apparent in BT 3 0:5,27 and BT 5, as these also lack the utilization of guanosine and thymidine phosphates as well as further adenosine, cytidine, uridine mono- and cyclic phosphates. 
As was seen earlier, BT 3 0:5,27 has a defect in nitrogen metabolism. This relates to amino acids amongst others. Three of the amino acids which BT 3 0:5,27 cannot metabolize are L-arginine, L-glutamic acid, and L-glutamine. These may be grouped together in the glutamate family. Apart from these, the other nine amino acids and diamino acids cannot be grouped chemically or physiologically.

\subsubsection{Biotyping reactions}

The sugars used for differentiation of Yersiniae and for biotyping of $Y$. enterocolitica are also found on the phenotypic microarray. A comparison of results is given in Table 6.2.

Table 6.2: Comparison of biotyping and phenotypic microarray reactions.

\begin{tabular}{|c|c|c|c|c|}
\hline Substrate & Biotyping result * & $\begin{array}{l}\text { Phenotypic microarray } \\
\text { substrate } \\
\text { (PM plate and position) }\end{array}$ & Result & $\begin{array}{l}\text { Agreement biotyping and } \\
\text { phenotypic microarray }\end{array}$ \\
\hline Glucose & All BT + & A-D-Glucose (PM1 C09) & All BT + & Yes \\
\hline Sucrose & All BT + & Sucrose (PM1 D11) & All BT + & Yes \\
\hline Rhamnose & All BT - & L-Rhamnose (PM1 C06) & All BT - & Yes \\
\hline Raffinose & All BT - & D-Raffinose (PM2A D01) & $\begin{array}{l}\mathrm{BT} 1 \mathrm{~A}+, \mathrm{BT} 1 \mathrm{~B}, 2, \\
3,4,5-\end{array}$ & No \\
\hline Melibiose & All BT - & D-Melibiose (PM1 C11) & All BT - & Yes \\
\hline Cellobiose & All BT + & D-Cellobiose (PM1 F11) & All BT + & Yes \\
\hline Sorbose & $\begin{array}{l}\text { All BT +, } \\
\text { except BT } 5-\end{array}$ & L-Sorbose (PM2A D04) & $\begin{array}{l}\text { All BT +, } \\
\text { except BT } 5-\end{array}$ & Yes \\
\hline Salicin & BT $1 \mathrm{~A}+$ & Salicin (PM2A D02) & BT $1 \mathrm{~A}+$ & Yes \\
\hline Xylose & $\begin{array}{l}\text { BT } 1 \mathrm{~A}, 1 \mathrm{~B}, 2,3+\text {, } \\
\text { BT } 4-\text {, BT } 5 \mathrm{~V}\end{array}$ & D-Xylose (PM1 B08) & $\begin{array}{l}\text { BT } 1 \text { A, } 1 B, 2,3+, \\
\text { BT } 4-\text {, BT } 5+\end{array}$ & Yes \\
\hline Trehalose & BT 5 - & D-Trehalose (PM1 A10) & BT $5-$ & Yes \\
\hline Inositol & All BT + & M-Inositol (PM1 F03) & $\begin{array}{l}\text { BT 1A -, BT1B, 2, 3, } \\
4+, \text { BT } 5-\end{array}$ & No \\
\hline
\end{tabular}

*adapted from Bottone, 1997, and Wauters, Kandolo and Janssens, 1987. + positive, - negative, V variable.

There are only two sugars in which the biotyping reaction and the phenotypic microarray disagree.

Raffinose utilization is supposed to be absent from $Y$. enterocolitica but in the phenotypic microarray BT $1 \mathrm{~A}$ gives a positive signal. Looking at the absorbance measurements, one can clearly see that BT $1 \mathrm{~A}$ metabolizes raffinose, although an activity can only be detected about 28 hours after the start of the incubation (Figure 6.6). 


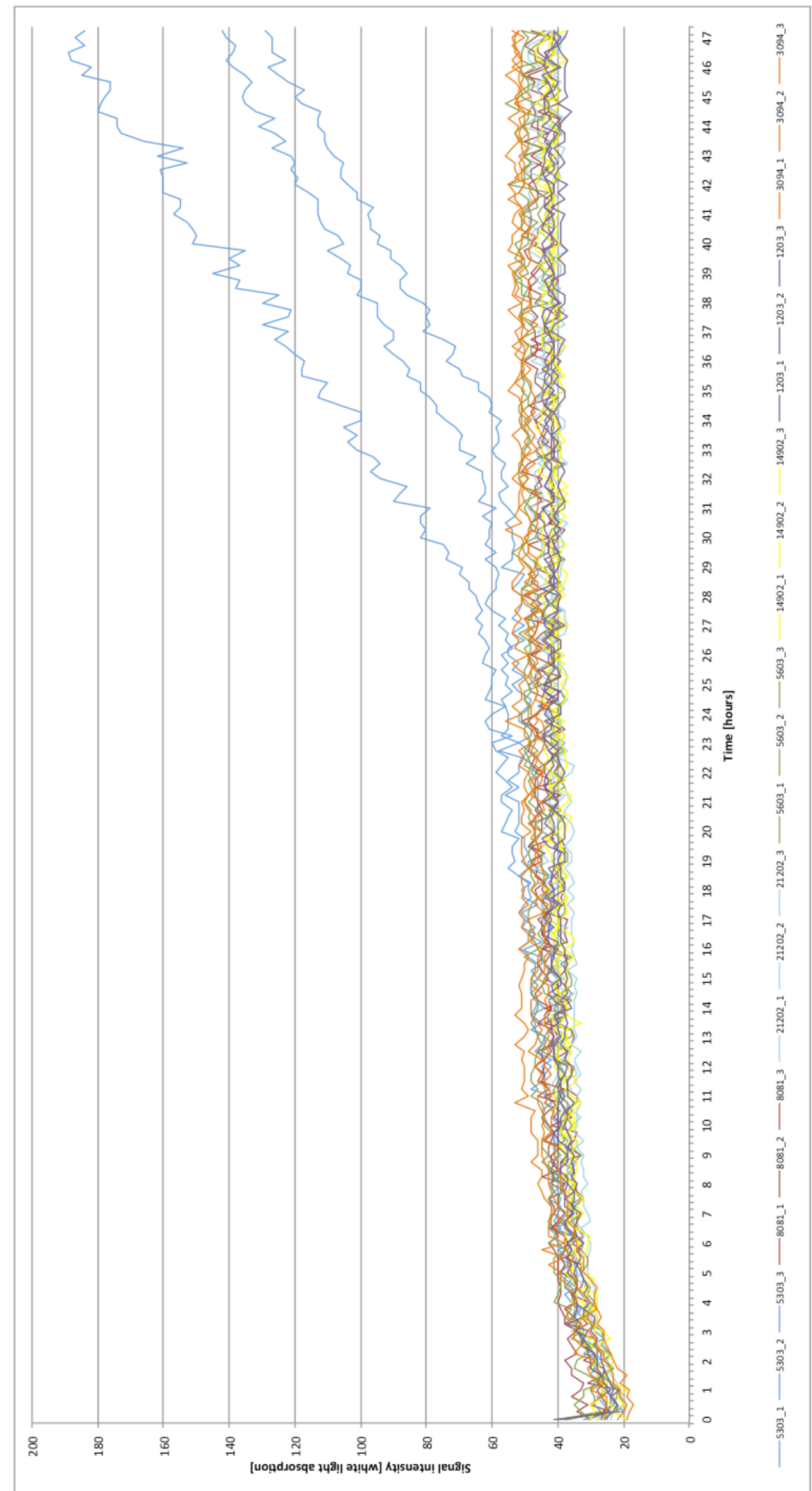

Figure 6.6: Kinetic plots for raffinose in Y. enterocolitica biotypes. Triplicates are shown. BT 1A / 5303 - blue; BT 1B / 8081 - red; BT 2 / 21202 - light blue; BT 3 / 5603 - green; BT 3 / 14902 yellow; BT 4 / 1203 - purple; BT 5 / 3094 - orange. 
It has been reported that raffinose might be metabolized if the lac permease is present conferring lactose uptake as well (Cornelis, Luke and Richmond, 1978). BT $1 \mathrm{~A}$ does utilize $\alpha$-D-lactose as well as lactulose, and a copy of laclZY is present encoding the lactose operon repressor, $\beta$-glucosidase, and permease respectively (YE5303-03921 - 41). Copies of lacl (YE2591) and lacZ (YE2592) are carried in all $Y$. enterocolitica biotypes but a permease is absent. The presence of transposases in some of the strains could potentially indicate a loss of the gene mediated by recombination. A definitive genomic basis for the raffinose-positive phenotype could not be established but it could be that the laclZY operon is involved in the metabolism of this sugar. Another possibility for a different test result is that the mode of the test is different, i.e. utilization under aerobic conditions versus anaerobic fermentation.

The second difference between biotyping reaction and phenotypic microarray result is inositol. All BTs are normally positive for the utilization of this sugar, but the phenotypic microarray only showed metabolism in BT 1B, 2, 3 and 4 . This will be explained in 6.2.2.5.

\subsubsection{Differences relating to genetic differences}

The general trends observed both in the genome comparisons and the analysis of the pseudo- and partial genes highlighted metabolic regions and transport proteins (Chapter 4). BT 1A was found to have additional operons relating to the uptake and conversion of sugars such as D-arabitol, and BT 5 was shown to contain mutations in core genes that might relate to the biotyping reactions.

These trends are reflected in the metabolic profiling. In the following, some examples of linking genotypes to specific phenotypes are given. This task is most straightforward when considering carbon metabolism, because well characterized examples from E. coli and Salmonella can be found. Nitrogen, phosphorus and sulphur metabolism on the other hand is less clear and the metabolites cannot be tied into metabolic pathways easily. This is due to the fact that some metabolites are not naturally occuring intermediates in standard metabolic pathways and constitute synthetic nutrients not found in the environment. Their metabolism is unknown and their physiological relevance is unidentified.

Other difficulties presented in analysing the phenotypic microarray relate to the fact that genomic data alone is insufficient for allocation of pathways, as homology to known enzymes does not indicate specificity of a transporter or an enzyme, as for 
example $A B C$ transporters might transport a range of metabolites from amino acids to sugars. Additionally, the mutations giving rise to the phenotype might be acquired independently in different genes of a pathway. If then a pathway is not clearly defined in terms of regulation, associated transporters, secondary pathways, etc., a clear link between phenotype and genotype is difficult to establish and requires additional experiments with targeted knock-out mutants. The metabolites tested in the phenotypic microarray also do not present complete pathways, but rather individual points in larger networks. It is therefore not possible to obtain a continuous picture of active or disrupted pathways. Nevertheless it is possible to obtain an overall impression of metabolic capacities of a cell.

\subsubsection{L-arabinose}

One clear example for linking phenotype and genotype can be seen in L-arabinose metabolism (PM1 A02). This is a carbon source metabolized in all BTs, and the respective enzymes are encoded by the araABFGHC operon (Figure 6.7). 


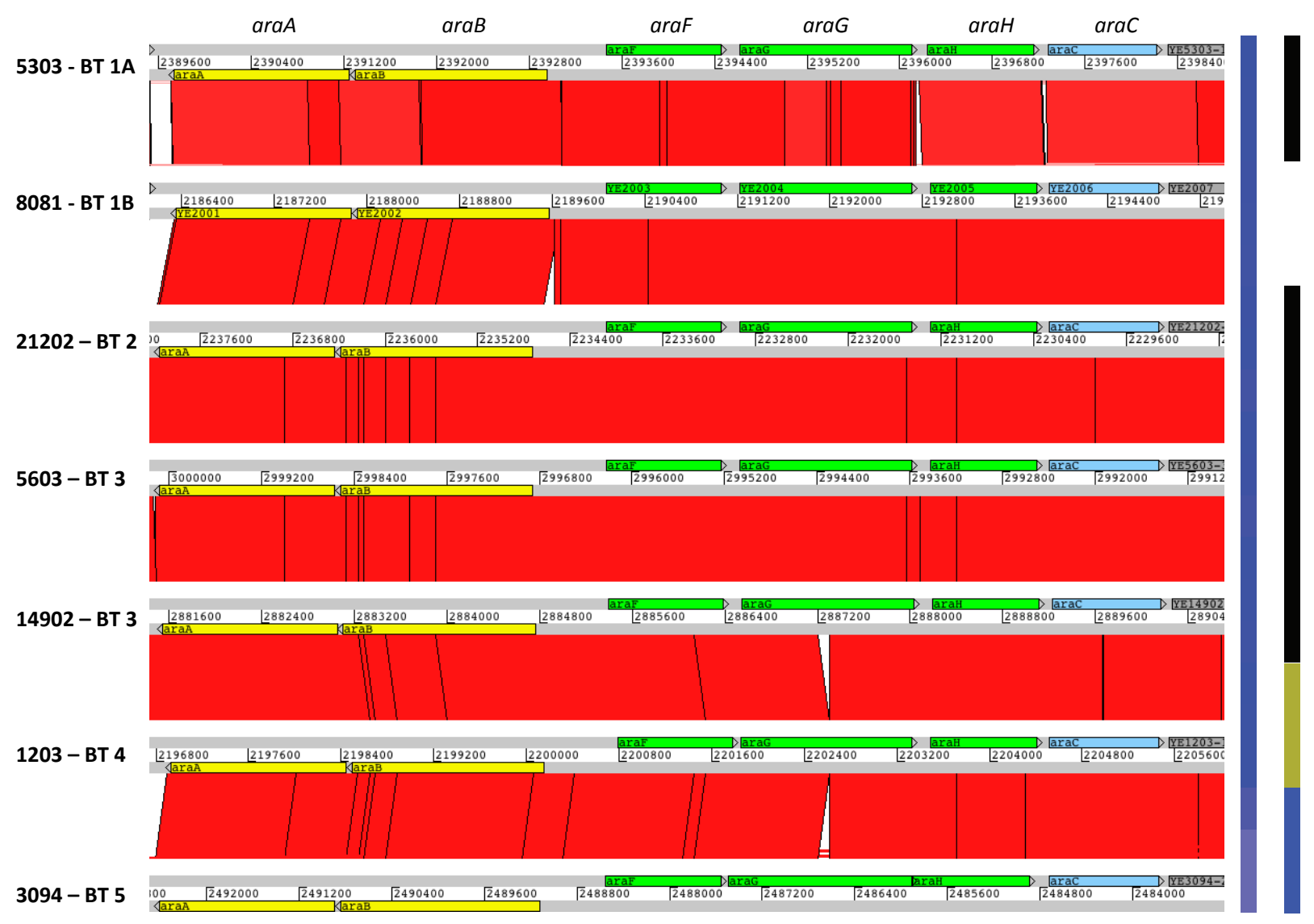

Figure 6.7: Operon for L-arabinose utilization contrasted with signal values and significant changes compared to BT 1B. Utilization (blue +, red -), significant change compared to BT $1 \mathrm{~B}$ (yellow + , black none, blue -).

The transport proteins are $\mathrm{AraFGH}$, $\operatorname{araAB}$ encode for an isomerase and a L-ribulokinase, respectively, and AraC is the regulatory protein for this operon. Although there appear to be slight variations between and within genes (BT 4 deletion in araG), these do not have an effect as utilization could be shown in all BTs. The signal values for all BTs are positive, although there is a significantly higher intensity in BT 4 and a significantly lower intensity for BT 5 compared to BT $1 \mathrm{~B}$. Looking at the absorbance recordings over time (Figure 6.8), one can see that BT 5 shows utilization of L-arabinose, but only to a limited extent. In contrast, BT 4 is very efficient at using L-arabinose. There appears to be a slight delay of 4 hours compared to BTs $1 \mathrm{~A}, 1 \mathrm{~B}, 2$, and 3 in the onset of utilization, but then $\mathrm{BT} 4$ outperformes the other BTs in all three replicates.

Other carbon sources that are metabolized in all BTs and for which unambiguous operons exist are $\mathrm{N}$-acetyl-D-glucosamine (nagEBACD), D-galactose (galDBAM), D-mannose (manZYXA), glycerol (glpKFTABCR), D-glucuronic acid (uxaC), Dmannitol (mt/RDA), D-fructose (fruBKAR), and L-serine (sdaBCA). 


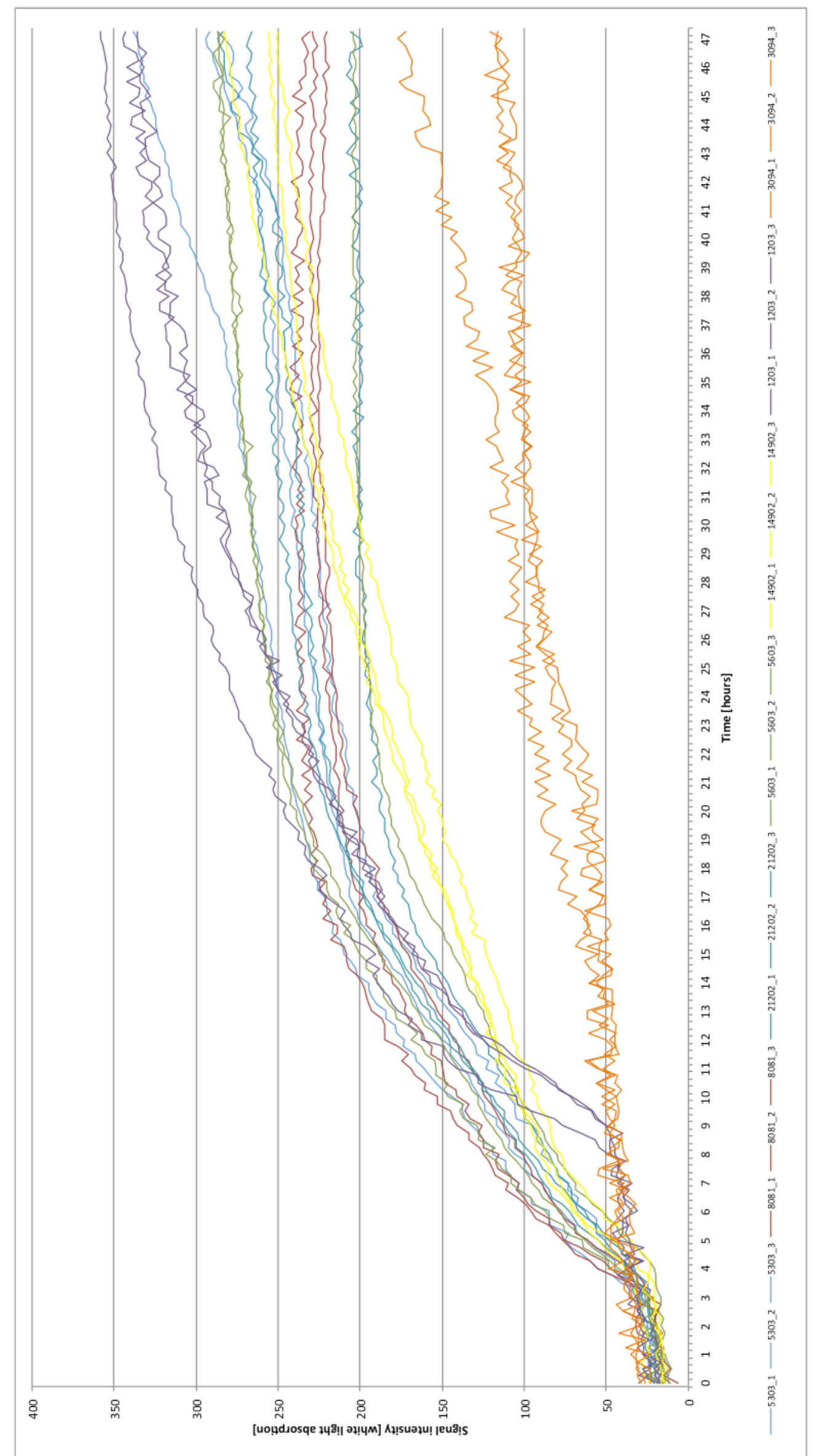

Figure 6.8: L-arabinose utlization kinetic plots over time for $Y$. enterocolitica biotypes. Triplicates are shown. BT 1A / 5303 - blue; BT 1B / 8081 - red; BT 2 / 21202 - light blue; BT 3 / 5603 - green; BT 3 / 14902 - yellow; BT 4 / 1203 - purple; BT 5 / 3094 - orange. 


\subsubsection{D-trehalose and D-sorbose}

Examples for mutations specific for BT 5 are found in D-trehalose and D-sorbose, which are also used in biotyping. The respective tests in the phenotypic microarray are found in PM1 A10 and PM2A D04.

The sorbose operon is shown in Figure 6.9A.

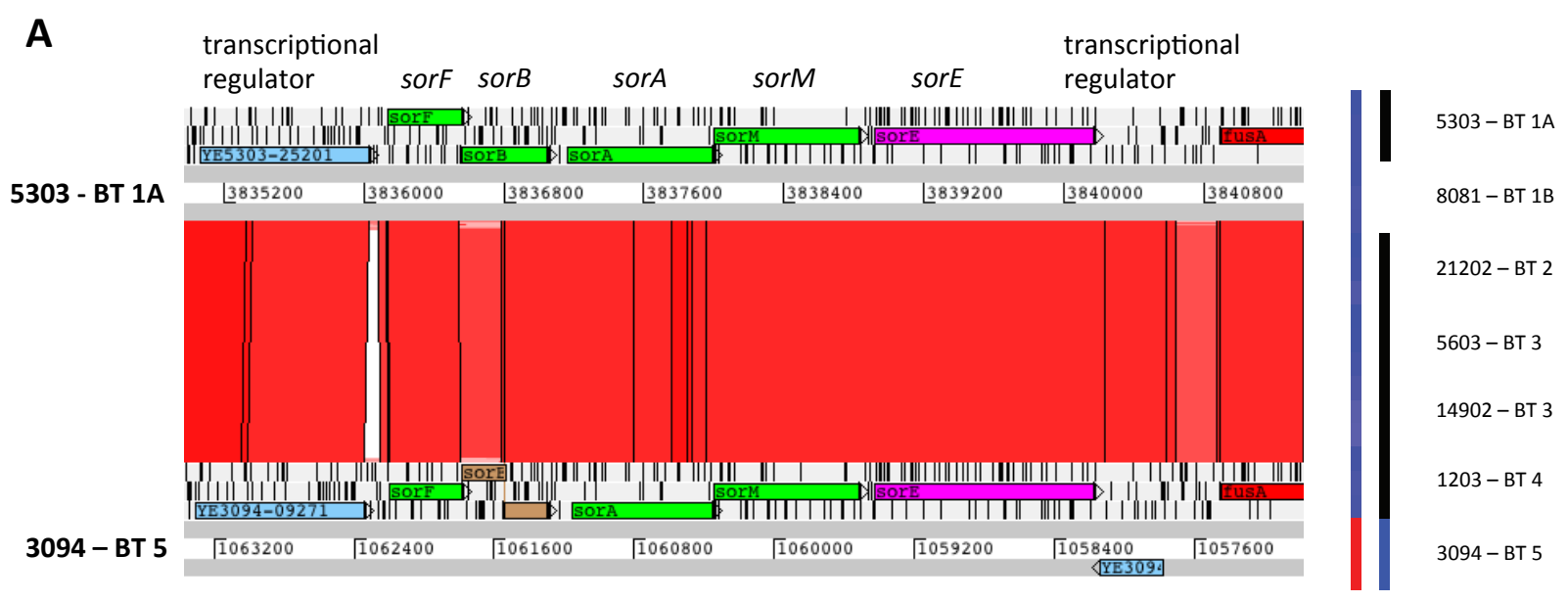

B

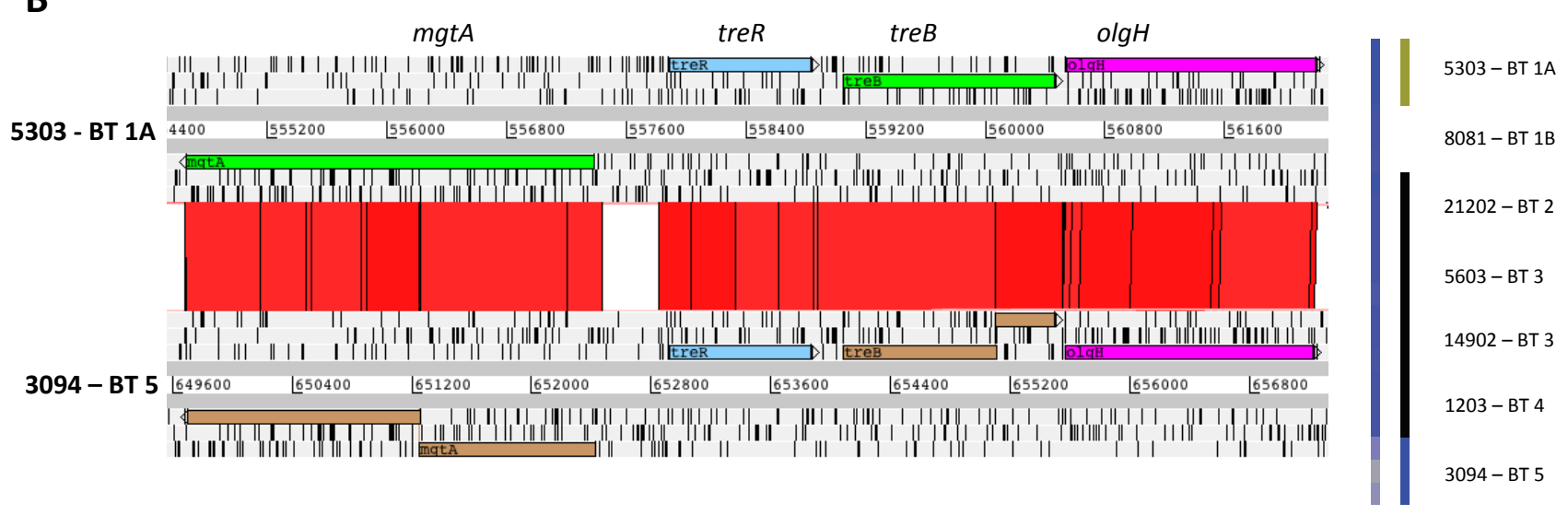

Figure 6.9: Mutations specific for biotyping of BT 5 contrasted with signal values and significant changes compared to BT 1B. Utilization (blue +, red -), significant change compared to BT 1B (yellow +, black none, blue -). A - Sorbose operon. B - trehalose operon.

The sorbose-specific phosphotransferase uptake system (PTS) is made up of several components encoded by sorFBAM. SorE is a L-sorbose-1-phosphate reducatase. There are two transcriptional regulatory proteins upstream and downstream of the operon but it is not known whether any of these or other regulators are involved with governing the expression of this operon. BT 5 is the only biotype with a mutation in this operon. It is missing a TTTA repeat motif resulting in a frameshift mutation in sorB, one of the PTS components. The signal values reflect this mutation. BT 5 is the only biotype without metabolic activity in this well, and this reduction is also 
significant compared to BT 1B. BT 1A shows a significantly higher utilization than BT $1 \mathrm{~B}$.

The trehalose operon is depicted in Figure 6.9B. Similar to the sorbose operon, BT 5 shows a frameshift in the PTS system TreB resulting from an insertion of one A. The trehalose operon repressor TreR and the trehalose-6-phosphate hydrolase OlgH remain intact. Upstream of the trehalose operon is a magnesium transporter encoded by $m g t A$, which also specifically shows a frameshift mutation in BT 5 . There is a further gene possibly encoding a component of the PTS involved in trehalose transport (YE3690). This gene is disrupted by the presence of a transposase in BT 5, indicating further mutations in trehalose metabolism.

\subsubsection{N-acetyl-D-galactosamine}

The utilization of N-acteyl-D-galactosamine (PM2A B01) is encoded by the aga operon (Figure 6.10).

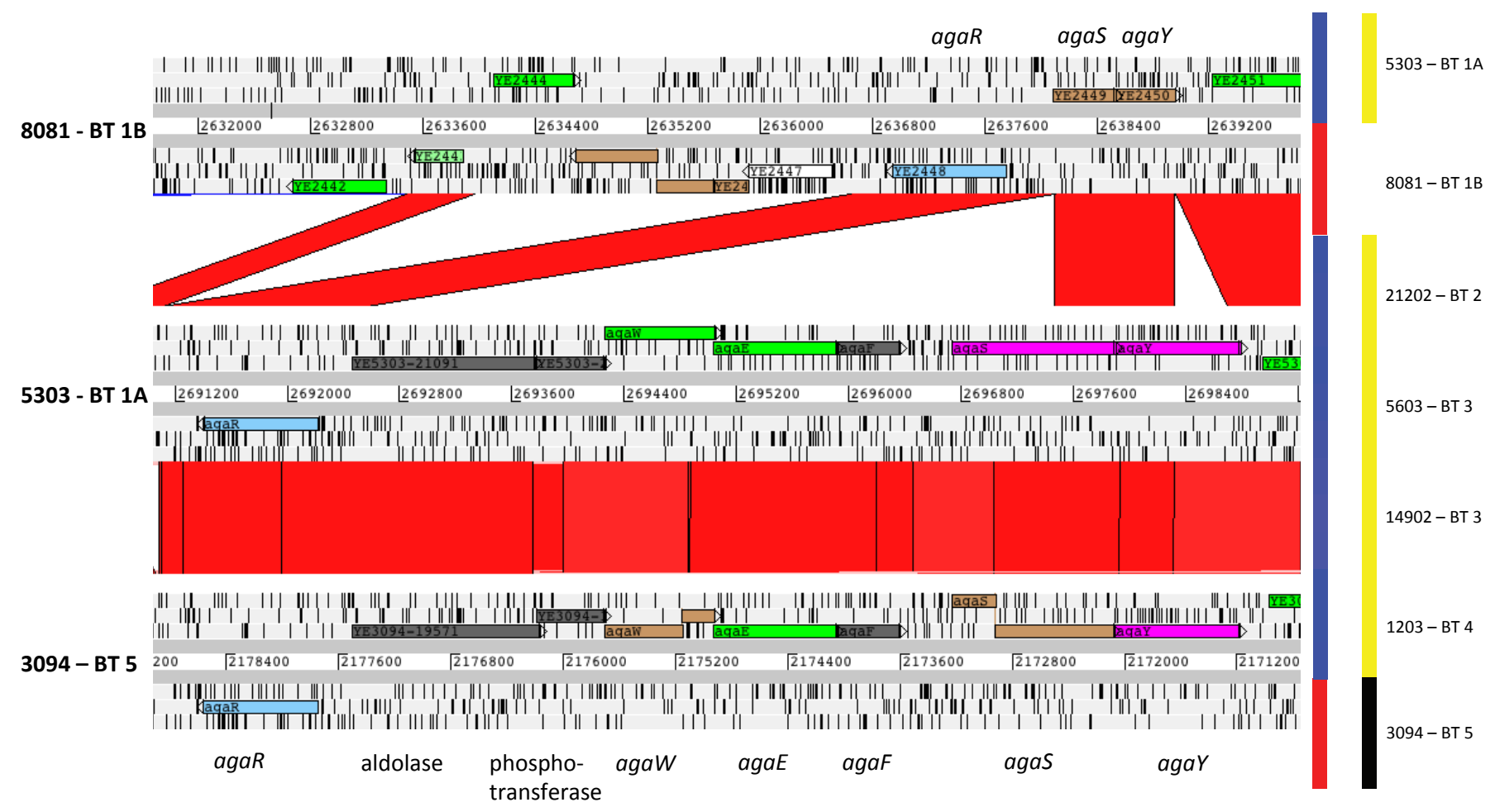

Figure 6.10: Operon structure for utilization of $\mathrm{N}$-acetyl-D-galactosamine in representative BTs contrasted with signal values and significant changes compared to BT $1 \mathrm{~B}$. Utilization (blue + , red -), significant change compared to BT 1B (yellow + , black none, blue -). BT 1B - deletion of most of the operon; BT 1A - representative for BTs $1 \mathrm{~A}, 2$, 3, and 4, fully functional; BT 5 - operon present, but mutations inhibit functionality. 
The operon starts with a DeoR-family regulatory protein AgaR. This is present in all BTs. The D-tagatose-1,6-bisphosphate aldolase, $\mathrm{N}$-acteylgalactosamine-specific phosphotransferase, and the phosophotransferase system encoded by agaWEF have been deleted from BT 1B. BT 5 is the only BT to show a frameshift in a homopolymeric run of $\mathrm{Gs}$ in agaW. The tagatose-6-phosphate ketose/aldose isomerase AgaS, and the D-tagatose-1,6-bisphosphate aldolase AgaY have been retained as fragments in BT 1B. BT 5 has a 4 base deletion resulting in a frameshift mutation in agaS. Both mutations in BT 5 render the utilization of N-acetyl-Dgalactosamine non-functional, as can be seen in the signal values. The biotypes metabolising this carbon source show a significantly higher expression than BT $1 \mathrm{~B}$.

A highly conserved, homologous operon is found in E. coli (Reizer, et al., 1996) and its functionality was confirmed for the phosphotransferase system of N-acetyl-Dgalactosamine (Brinkkötter, Klöß, Alpert and Lengeler, 2000). Functionality of this cluster has been independently studied in a BT 4 0:3 strain (Batzilla, et al., 2011b). Complementation studies showed that this operon could confer the ability to grow on $\mathrm{N}$-acetyl-D-galactosamine onto BT 1B strains (Batzilla, et al., 2011b), confirming that this cluster is responsible for the phenotype.

\subsubsection{D-arabitol}

The utilization of D-arabitol (PM2A B06) is unique to the BT 1A strain studied here. The utilization could be linked to a unique region contained in the BT $1 \mathrm{~A}$ genome (Figure 6.11).

D-arabitol utilization is encoded for by a mannitol dehydrogenase DaID and a xylulose kinase AtlK. A transcriptional regulatory protein of the DeoR2 family guards the expression of the operon, and AtIT constitutes the D-arabitol membrane transporter. The gene $a c p D$ for an acyl carrier protein phosphodiesterase is located upstream of the operon. This region has been deleted from the pathogenic BTs. BT $1 \mathrm{~B}$ retains a fragment of the regulatory protein, but the operon is completely absent from the low-pathogenic BTs.

A similar, conserved D-arabitol operon structure has been described in Klebsiella pneumoniae and function confirmed (Heuel, Turgut, Schmid and Lengeler, 1997). 


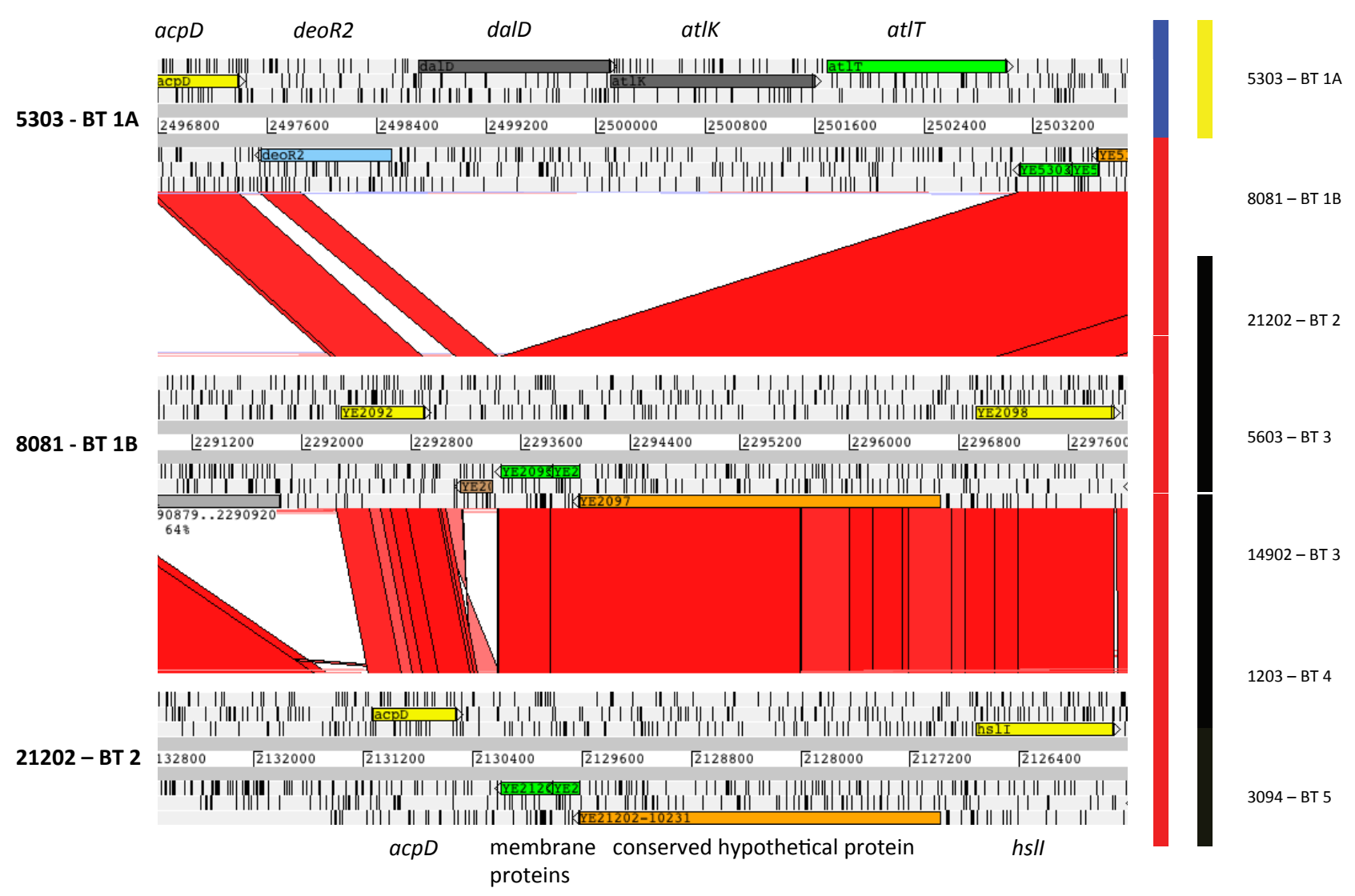

Figure 6.11: Unique operon for D-arabitol utilization in BT 1A, contrasted with signal values and significant changes compared to BT 1B. Utilization (blue + , red - ), significant change compared to BT 1B (yellow +, black none, blue -). BT 2 as representative for low-pathogenic BTs.

D-arabitol has been linked to the non-pathogenic biotype before in comparison to BT 4 0:3 (Shehee and Sobsey, 2004). A positive D-arabitol phenotype is not exclusive to non-pathogenic BTs though (Fantasia Mazzotti, et al., 1985; Fantasia Mazzotti, Mingrone and Martini, 1987), as some BT 1A strains can be negative, just as some pathogenic BT 3 strains were found to metabolize D-arabitol.

\subsubsection{5 m-inositol}

Acid production from inositol is one of the biotyping reactions for which all Y. enterocolitica BT are supposed to be positive. The phenotypic microarray also tests for this in form of m-inositol (PM1 F03). As could be seen from the signal values, with the exception of $B T 1 A$ and $B T 5$, all BTs are positive for the utilization of this carbon source. Looking at the absorbance plot over time (Figure 6.12), one can see some differences in the kinetic plots. 


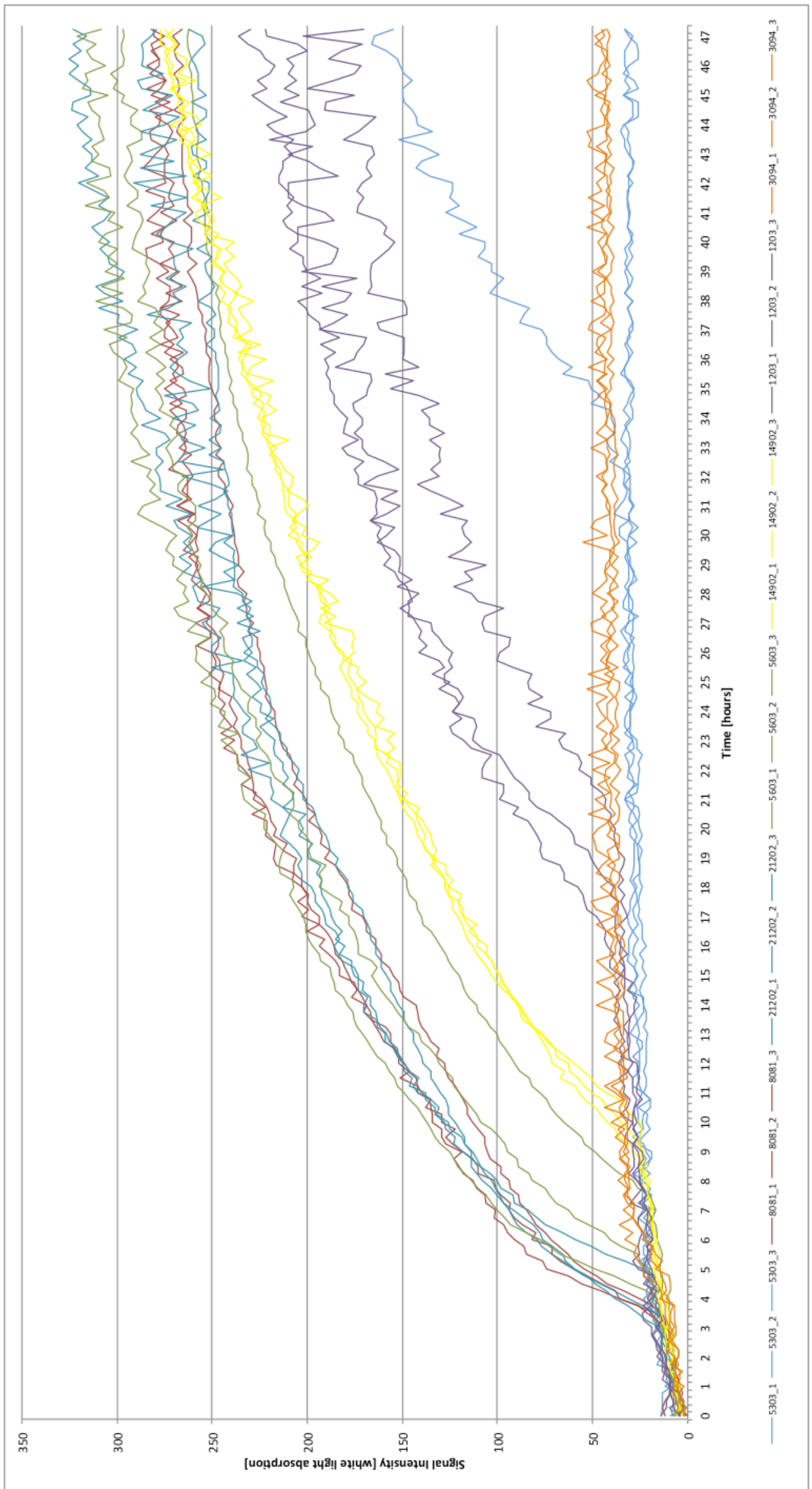

Figure 6.12: Absorbance curves over time for $m$-inositol utilization. Triplicates are shown. BT 1 A / 5303 - blue; BT 1B / 8081 - red; BT 2 / 21202 - light blue; BT 3 / 5603 - green; BT 3 / 14902 - vellow: BT 4 / 1203 - purple: BT 5 / 3094 - oranqe. 
The strains 8081,21202 , and 5603, representing biotypes $1 \mathrm{~B}$ and $2 / 3$ O:9, start to show activity after about 4 hours. BT 3 0:5,27 strain 14902 starts 6 hours later, 10 hours into the incubation time. BT 4 O:3 strain 1203 shows signs of metabolic activity around 18 hours after the start of the incubation. BT 5 strain 3094 shows no activity apart from background noise, but one of the replicates for BT 1A strain 5303 shows activity after 35 hours.

Looking into the genomic data, no basis for the phenotype in BT 5 could be established. In BT 1A, the myo-inositol dehydrogenase gene idh shows a point mutation (Figure 6.13).

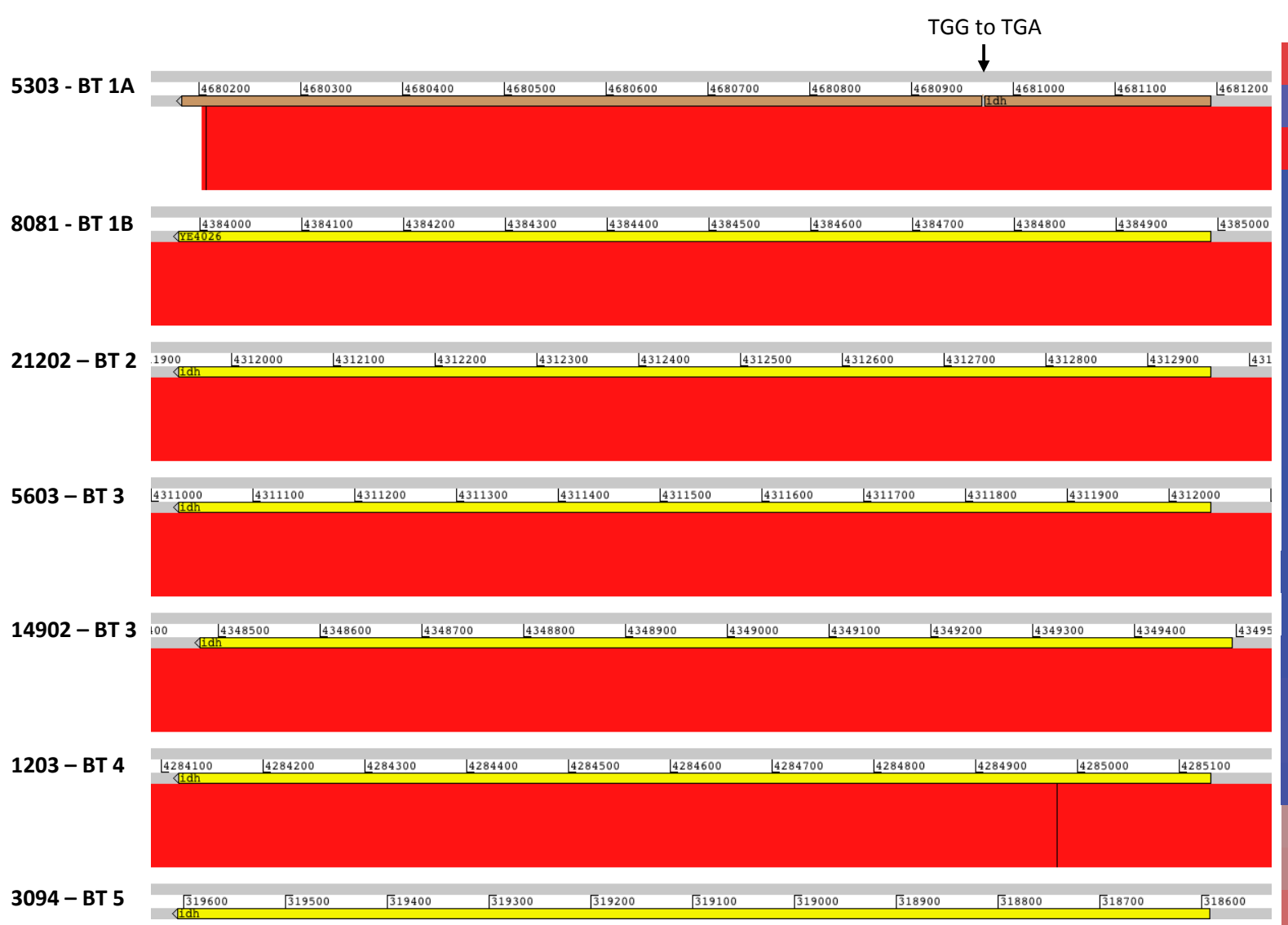

Figure 6.13: Point mutation in the idh gene is the basis for BT 1A negative m-inositol phenotype. Utilization (blue + , red -), significant change compared to BT 1B (yellow + , black none, blue -). Mutation in BT 5 unknown.

The sequence TGG encoding for tryptophan has changed to TGA. The translation into UGA results in a premature stop codon, inhibiting the expression of a fully functional protein. Therefore the utilization of $\mathrm{m}$-inositol is not possible in this strain. The UGA stop codon is peculiar though, as it has been shown to encode for the unusual amino acid selenocysteine occasionally. Y. enterocolitica does have a 
selenocysteine tRNA, so it could be that the stop codon is either read through or decoded by selenocysteine, giving a fully functional protein, albeit in lower concentrations than normal. This would then result in a delayed response to utilization of the carbon source, explaining the metabolic activity observed in one of the BT 1A strain 5303 replicates. In a larger strain collection, this point mutation was shown to be specific for BT 1 A strain 5303 , and not representative for BT 1 A strains in general. It also highlights the problem of strain-to-strain variation in biotyping following random inactivating mutations that lead to misidentification of isolates and potential misrepresentation of their pathogenic potential.

\subsubsection{Metabolic activity of Y. enterocolitica biotypes at human body temperature}

The phenotypic microarray was mainly evaluated at $28^{\circ} \mathrm{C}$. This represents the optimum growth temperature of $Y$. enterocolitica. The metabolic profile for $37^{\circ} \mathrm{C}$ is given in Figure 6.14. One can see a dramatic overall reduction in metabolism in all BTs. BT 5 shows hardly any metabolic activity at all, having lost most of the few carbon sources it was utilizing at $28^{\circ} \mathrm{C}$. In the other BTs, a specific temperature effect can be observed in nitrogen and phosphorus sources. A marked reduction of sulphur metabolism can be observed in BT 4. Generally, the non-pathogenic BT 1A still shows the highest metabolic flexibility as it shows more metabolically active nutrient sources.

This severe change in metabolism with temperature shift was unexpected as $Y$. enterocolitica causes human infections and thus thrives at $37^{\circ} \mathrm{C}$. It could be that oxygen levels or the presence of additional nutrients play a role in this temperature effect. The basis is most likely less genetic in terms of presence or absence of operons, but likely to be of regulatory nature.

The effect of temperature change from $28^{\circ} \mathrm{C}$ to $37^{\circ} \mathrm{C}$ would warrant further in depth experiments such as a transcriptional response following temperature shift. 


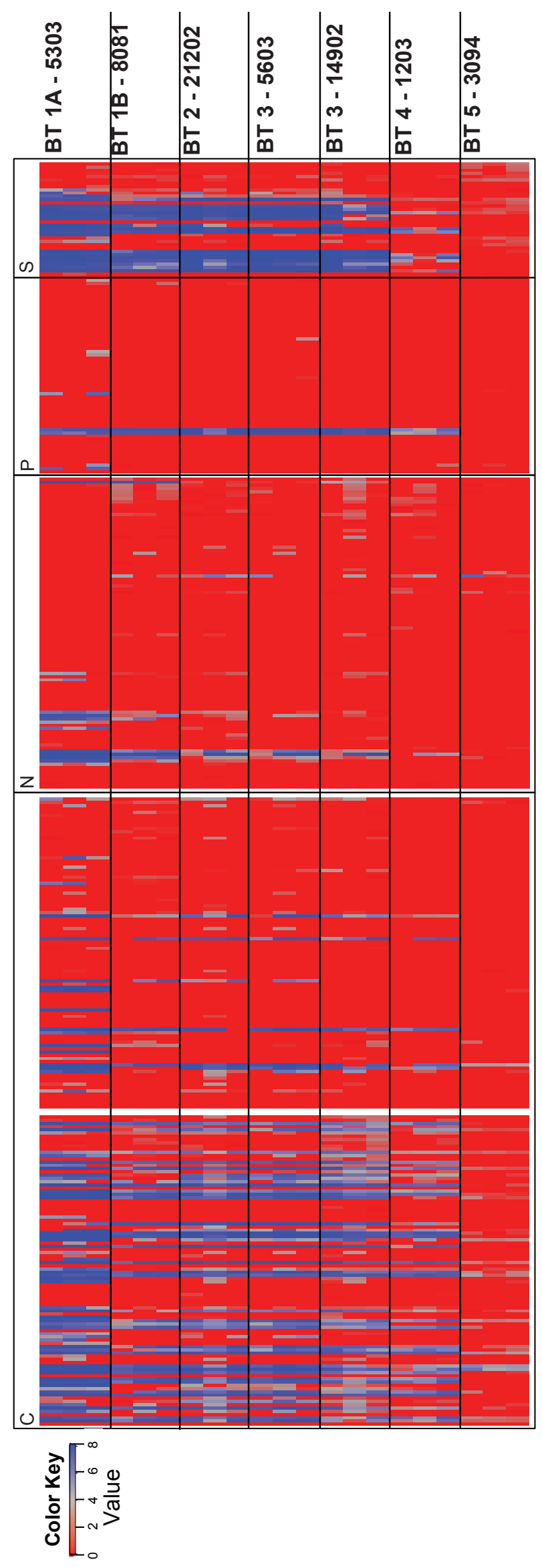

Figure 6.14: Signal values depicted as heatmap. Triplicates are shown for the signal values. C carbon sources (190), N - nitrogen sources (95), P - phosphorus sources (59), S - sulphur sources (35). 


\subsection{Discussion}

\subsubsection{Metabolic profiling of $Y$. enterocolitica confirms genomic analysis}

The phenotypic microarray mirrors both the observations made in the genomic analysis in terms of increased genome decay and loss of metabolic functions in BTs 4 and 5 , as well as the biotyping scheme in that a reduction of metabolism can be observed (Figure 6.15).

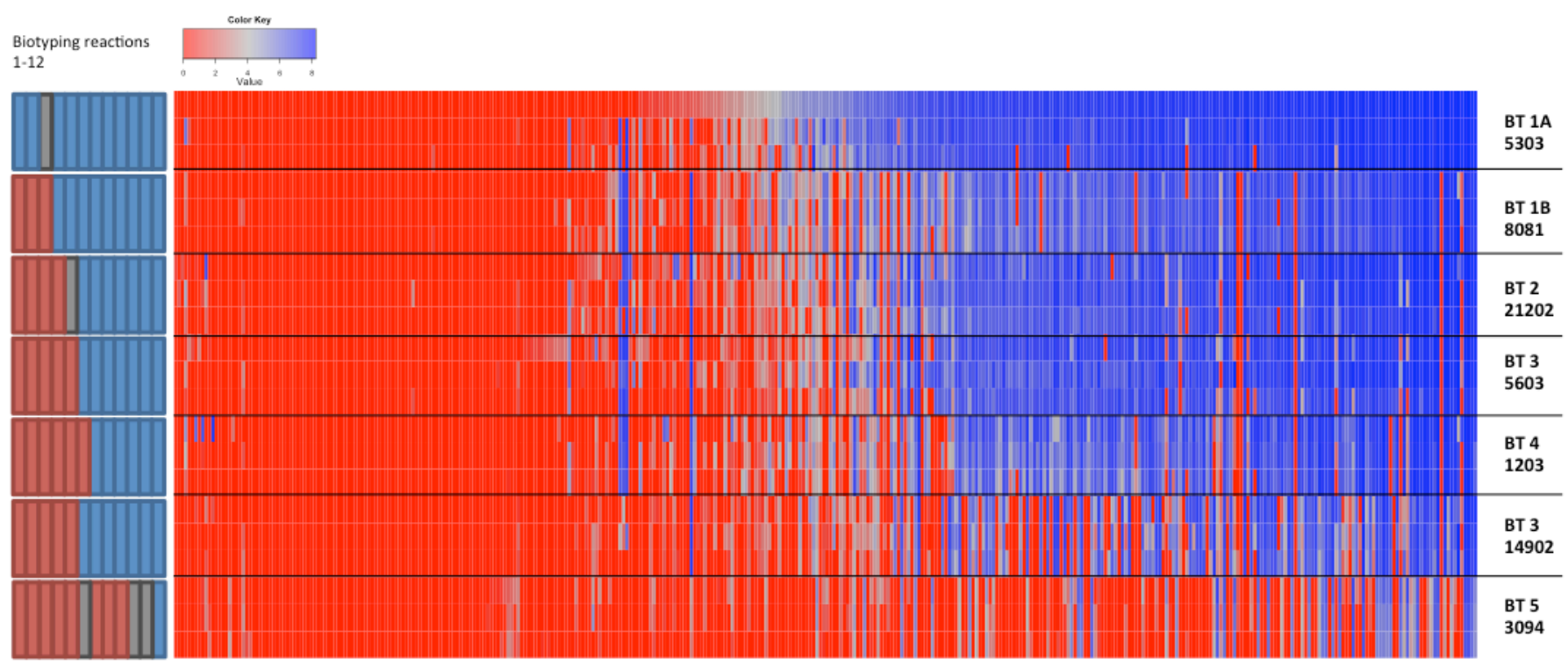

Figure 6.15: Comparison of biotyping reactions and phenotypic microarray. The 12 biotyping reactions correspond to the order given in Table 1.2. The phenotypic microarray data has been rearranged for the first replicate of $B T 1 A$ from negative to positive reactions irrespective of plate and well position. The other replicates are linked to the BT $1 \mathrm{~A}$ ordering so that differential utilization of nutrients is reflected.

The non-pathogenic, environmental BT $1 \mathrm{~A}$ shows the ability to use a wider repertoire of nutrient sources that it can utilize. Of the metabolic capacities it shares with other BTs, it can metabolize these better or more efficiently resulting in higher signal intensity. The high-pathogenic BT 1B and the low-pathogenic BTs 2 0:9, 3 O:9, and 4 O:3 show some reduction in metabolism compared to BT 1A. A marked reduction in metabolic flexibility can be observed in two strains. BT $30: 5,27$ is deficient specifically in nitrogen and phosphorus sources. This strain type is of interest in that it is predominantly associated with asymptomatic carriage in livestock, and is less frequent in human diarrheal samples (McNally, et al., 2004). This isolate specifically was isolated in cattle and represents the UK 0:5,27 isolates. The reduction in metabolism therefore could be a result of adaptation to a cattle host. The metabolism of BT 5 is greatly reduced, very little metabolic activity can be observed in carbon 
sources and no utilization of nitrogen, phosphorus, or sulphur sources is detectable. It might thus be no coincidence that BT5 is the rarest of all biotypes. It is referred to as the "hare-biotype", and has not been isolated from humans (Swaminathan, Harmon and Mehlman, 1982; Wuthe and Aleksic, 1997; Bartling, et al., 2004). It is possible that BT5 has adapted to a very narrow niche that makes survival outside this niche near impossible. It may even present a dying lineage. This resembles the extreme loss of function and degenerate genome of another pathogenic Yersinia: $Y$. pestis. It was shown that $Y$. pseudotuberculosis and $Y$. enterocolitica can both grow on minimal medium at $28^{\circ} \mathrm{C}$ with few extra nutrients, but that $Y$. pestis needs further additives for adequate growth (Burrows and Gillett, 1966; Perry and Fetherston, 1997; Navid and Almaas, 2009). This is due to the biphasic lifecycle of Y. pestis, being present either in a warm-blooded host or in the flea. Y. pestis is not subjected to environmental metabolic challenges, and at the same time the bacterium depends on the host to provide for nutrients (Navid and Almaas, 2009). Both situations lead to accumulation of mutations in metabolic pathways. The genus Yersinia thus presents two examples of extreme niche adaptation to warm-blooded animals - Y. pestis and Y. enterocolitica BT5 - that have evolved independently. It is possible that BT 5 would have needed additives of amino acids or other nutrients to improve metabolic activity. This could potentially be uncovered by incubation on PM 5 , which tests for common biosynthetic pathways via the utilization of nutrient supplements. An addition of a nutrient supplement though would have influenced the kinetic plots making a direct comparison to the other BTs impossible.

In contrast to the biotyping scheme, the phenotypic micorarray also highlighted some nitrogen, phosphorus, and sulphur sources that are absent from BT 1A. These could potentially be used for differentiation resulting in an improved biotyping scheme or maybe the development of new media. Unusual sources might present a possible advantage to also distinguish $Y$. enterocolitica from other enterobacteriaceae. These sources would need to be screened in a wider BT selection.

Metabolic properties thus play an important role in niche adaptation for Y. enterocolitica. BT1A is the metabolically most diverse biotype reflecting its ubiquitous nature and wide range of niches. High- and low-pathogenic biotypes have lost some metabolic flexibility. Especially in the predominant animal isolate BT3 $0: 5,27$ and the rare BT5 there are signs of extreme niche adaptation, reflected in a significant loss of metabolic activities. 


\subsubsection{Linking genomic and phenotypic differences}

The phenotypic microarray has been originally designed to study isogenic mutants, that is the wild-type strain against a single-gene mutant (Bochner, Gadzinski and Panomitros, 2001; Bochner, 2003; Zhou, Lei, Bochner and Wanner, 2003; Johnson, et al., 2008). This way very clean phenotypes could be observed. For instance, a wild-type strain of $E$. coli was compared to a malF mutant, and it could be shown that the metabolism of maltose, maltotriose, and dextrin is coupled to the functionality of that gene (Bochner, 2003). Here, several strains are compared at the same time, with differences in gene content and pseudogene distribution. BT 5 for example differs in more than one pseudogene compared to its nearest neighbour BT 4 , therfore it is difficult to assign phenotypes to a single gene or even operons. It is possible for alternative secondary transporters or enzymes to complement mutations, obscuring possible links. Often, genes thought to be involved in certain pathways are either not mutated in any BT although differential expression is detected or mutations are found in the "wrong" BT, i.e. a BT utilizing the source, whereas no mutation is observed in a phenotype-negative BT. In addition to the principle of "One transporter - one substrate", one needs to consider "One transporter - several substrates" as well as "Several transporters - one substrate". Only very clear-cut phenotypes such as the maintenance of the $D$-arabitol operon in $B T 1 A$ can be observed and annotated unambiguously. For most cases of BT $1 \mathrm{~A}$-unique regions, there are indications of encoding metabolic pathways, but either no high-homologous hits in other bacteria to justify a clearer enzyme assignment or no good reference to base the annotation on. In the phenotypic microarray, there are new indications of possible pathways encoded, but no indication if several pathways could be activated by one and the same operon.

A study used the phenotypic microarray to detect changes in metabolism caused by mutations in 2-component systems (Zhou, Lei, Bochner and Wanner, 2003). 2component systems consist of a sensor kinase and a response regulator. The sensor protein is a membrane-associated protein which detects environmental signals and passes them on to the response protein which is either a transcription factor or acts on sigma factors to modulate gene expression. Zhou, Lei, Bochner and Wanner (2003) confirmed links between NtrBC to nitrogen metabolism and PhoRB to phosphorus metabolism. No mutations in these or other 2-component systems implicated with phenotypic changes are mutated in any of the Y. enterocolitica biotypes except creC (YE3383) in BT 4. This component system is thought to be 
involved in carbon/energy metabolism (Chaudhuri, et al., 2010), but a direct link cannot be established in the $Y$. enterocolitica dataset. BTs 4 and 5 show mutations in regulatory proteins, including potential 2-component systems, thus these could be the reason for the widespread metabolic defects visible especially in BT 5. The 2component systems of Y. enterocolitica as well as of Y.pestis and $Y$. pseudotuberculosis have not been as extensively characterized as in E. coli and regulatory relationships are theoretical and deduced from DNA homology (Marceau, 2004). It is possible that either the E. coli-homologous systems in Y. enterocolitica fulfil a different role, or that one of the uncharacterized 2-component systems is in fact taking the place of one of the well-established E. coli 2-component systems.

The phenotypic microarray has highlighted general metabolic capacities of each strain, and has lowered the possibilities of nutrients that could be encoded for in the unique regions or the pseudogenes affected. In this way, the phenotypic microarray facilitates future annotation.

Variability of phenotypic tests has also been described in Y. pestis. Most notably is rhamnose fermentation. Classically, Y. pestis is negative for rhamnose fermentation; a positive phenotype is associated only with Biovar Pestoides (Achtman, et al., 2004). Rare rhamnose-positive mutants are observed in $Y$. pestis after prolonged incubation (Englesberg, 1957a,b). The rhamnose operon rhaTRSBAD is present in all Y.pestis, and BetB, a betaine-aldehyde dehydrogenase, and RhaS, a transcriptional regulator, have been suggested as the genetic basis for this phenotype (Navid and Almaas, 2009; Eppinger, et al., 2010). This ability of Y. pestis for phenotypic gain of function is known as meiotrophy, and has also been documented for ammonia assimilation, urease, production of inv, fermentation of melibiose, and biosynthesis of certain amino acids (Brubaker, 1991). The respective gene regions are presumably present and inactivated by reversible mutations or highly regulated (Brubaker, 1991). This variability also makes assignments of phenotype to genotype difficult.

Achtman, et al. (2004) demonstrated that biovar designation does not reflect the genetic relationship in Y. pestis. The distinction of $Y$. pestis into different biovars is based on fermentation patterns of glycerol, rhamnose, melibiose, and nitrate reduction and characterizes the three classic biovars Orientalis, Medievalis, and Antiqua, as well as atypical biovars Pestoides and Microtus. Rhamnose, as described above, is known to be variable and strains can show a positive phenotype 
upon prolongued incubation. The phylogenetic relationship in Y. pestis also showed that the inability to metabolize sugars or reduce nitrate can arise independently in several lineages and can thus have a different genetic basis (Achtman, et al., 2004). The same biovar might therefore be allocated to very different lineages. The grouping in $Y$. pestis was revised on the basis of evolutionary relationships rather than metabolic traits, which do not have the correlation with evolutionary origin (Achtman, et al., 2004). It is even suggested that biovars and metabolic traits are unsuitable for distinction into taxonomic groups (Achtman, et al., 2004). Given the metabolic variety, variable biotyping reactions, and inactivating mutations as found in BT $1 \mathrm{~A}$ strain 5303 in the utilization of $m$-inositol, the grouping of $Y$. enterocolitica might need to be re-evaluated likewise, to move away from phenotypic variability.

\subsubsection{The influence of temperature on metabolism in Y. enterocolitica}

It is known that $Y$. enterocolitica displays differential expression of genes upon changes in temperature. Certain genes thought to be involved in environmental survival as well as insect pathogenicity such as genes in the tc PAI and motility and chemotaxis genes as well as genes potentially involved in nitrogen utilization could be shown to be upregulated in low-temperature conditions of $10^{\circ} \mathrm{C}$ (Bresolin, Neuhaus, Scherer and Fuchs, 2006b). Genes such as inv and ail involved in pathogenicity in a human host are upregulated when the bacteria are exposed to $37^{\circ} \mathrm{C}$ corresponding to human body temperature (Straley and Perry, 1995). Most famous is the low-calcium response coupled with temperature increase that triggers the expression of the Yop T3SS on the virulence plasmid (Straley and Perry, 1995; Bochner, 2009; Brubaker, 1991). The optimum temperature of Yersinia though is 25 $28^{\circ} \mathrm{C}$ (Cornelis, et al., 1987).

In the first set of experiments conducted at optimum growth temperature $Y$. enterocolitica showed a wide range of metabolic activities that were dependent on the bio-serotype. Repeating these experiments at $37^{\circ} \mathrm{C}$, one could have expected a possible upregulation of nutrient utilization in pathogenic biotypes as a response to conditions similar to those found in the human body. The results however showed a severe reduction in metabolic activity specifically in nitrogen and phosphorus sources. BT 4 also had a reduction of sulphur metabolism. This might have several explanations. First of all, it could be that the human host provides specific trace elements or other nutrients that are essential for growth under high temperatures. As these are absent under the conditions tested (only L-cystine was added as a reduced 
sulphur source at both temperatures) this could explain the absence of metabolic activity. Secondly, it could also be that certain metabolic pathways are restricted for use under anaerobic conditions. The 96-well plates do not allow for much oxygen circulation, but can still be considered rather aerobic. For the anaerobe Streptococcus pneumoniae it could be shown that sealing of the 96-well plates in bags under anaerobic conditions and subsequent incubation had a great influence on experimental outcome (T. Feltwell, personal communication).

For all Yersinia spp. additional nutrient requirements were demonstrated upon temperature shift (Burrows and Gillett, 1966). Defined minimal medium complemented with sodium metabisulphite and amino acids isoleucine, valine, cystine, methionine, and phenylalanine was enough for growth of $Y$. pestis at $28^{\circ} \mathrm{C}$, but had to be further complemented with glutaminc acid and thiamine and replacing cystine with thiosulphate. Y.pseudotuberculosis and Y. enterocolitica also had additional requirements at $37^{\circ} \mathrm{C}$, although to a lower degree than $Y$. pestis (Burrows and Gillett, 1966; Brubaker, 1991). It is entirely possible that addition of other nutrients at $37^{\circ} \mathrm{C}$ could increase the metabolic activity and possibly show the activation of sources specific to pathogenic BTs. Unpublished results indicate that calcium might play an important role in adaptation to higher temperatures (Bochner 2009; Holtz-Morris, 2005; Fodor, et al., 2005), but unfortunately a direct comparison with the data obtained for Y. enterocolitica is not possible. Other signals could include magnesium concentration, pH, or acidic conditions (Bochner, 2009). One microarray study showed that following temperature shift specific metabolic pathways are differentially regulated (Motin, et al., 2004). They could demonstrate that a full citric acid cycle is present at $37^{\circ} \mathrm{C}$, whereas nitrogen and amino acid metabolism appear downregulated. These differences are not as clear in Y. enterocolitica and might be due to differences in the culture media used thus supplying nutrients that influence metabolic state. Not all metabolic pathways or all intermediates of those pathways are present on the phenotypic microarray either, making a full assessment of metabolic state difficult. A further experiment could be carried out, testing growth at $37^{\circ} \mathrm{C}$ first on PM 5 for nutrient supplements. Depending on the outcome, essential nutrients could be added to the inoculation medium and the experiment repeated at $37^{\circ} \mathrm{C}$. Alternatively, the experiment could be repeated with the present medium, but under anaerobic conditions. Either way, only one of the parameters should be altered at any point to allow for comparison and determine which trigger is decisive. 
It might not only be the acquisition of beneficial factors enabling survival / potential pathogenesis in the human body that is important, it might also be the loss of metabolic flexibility that drives the colonization of new niches and associated putative virulent behaviour. To fully assess all aspects of $Y$. enterocolitica biology, one further interesting experiment to carry out would be to repeat the phenotypic microarray at $10^{\circ} \mathrm{C}$. This would complement the current data set of optimum temperature and human body temperature with temperature found under environmental conditions and conditions associated with potential insect hosts.

Generally, the phenotypic microarray results reflected the regions of difference and pseudogenization noticed in the reference genomes. Comparing the results with traditional biotyping, there were only two discrepancies found, one of which could be related back to a unique mutation in the reference strain chosen for BT 1A. Prediction of metabolic operons from the microarray data is restricted by annotation, i.e. some annotation is potentially too general to relate an operon to a specific metabolic function. The other way round, annotation is limited by the lack of clear phenotypic results that can be unambigiously linked to metabolic pathways. The phenotypic microarray data can be used in future for directed experiments to link phenotype and genotype, and might help identification of new pathways such as the utilization of $\mathrm{N}$ acetyl-D-galactosamine. 


\section{Phylogeny of the Genus Yersinia and the species Y. enterocolitica}

\subsection{Introduction}

From the data presented thus far it appears that the driving forces of pathogen evolution are the same in Y. enterocolitica, Y. pseudotuberculosis and Y. pestis. It is thought that the pathogenic Yersiniae evolved out of a common ancestor that acquired the virulence plasmid pYV, enabling the coloniziation of new niches such as warm-blooded animals including humans (Carniel, 2002; Wren, 2003). Y. enterocolitica and Y.pseudotuberculosis then evolved separately, and Y. pseudotuberculosis gave rise to the $Y$. pestis biovars. The evolution of Y. pestis has been studied in detail (Achtman, et al., 1999; Achtman, et al., 2004; Morelli, et al., 2010) and is characterized by the expansion of IS elements leading to wideranged genome rearrangements and subsequent gene loss (Parkhill, et al., 2001a).

Unlike the Y.pseudotuberculosis/pestis cluster the evolutionary dynamics of $Y$. enterocolitica are understudied and knowledge is currently limited to microarray and amplified fragment length polymorphism studies (Fearnley, et al., 2005; Howard, et al., 2006). As seen in the previous chapters, Y. enterocolitica presents as a heterogeneous groups of organisms with very clear distinctions in gene content. It is necessary to establish the evolution of the $Y$. enterocolitica lineages to obtain a trajectory for acquisition and loss events of genomic islands, and clarify the presence of remnants of genes and islands and their potential influence on the evolution of pathogenesis in Y. enterocolitica.

This chapter aims to elucidate the evolutionary relationships within $Y$. enterocolitica as well as with respect to the whole genus Yersinia and to investigate the interrelationships between the different lineages. This should help to identify any shared ancestry or patterns of evolution for the human pathogenic lineages of this genus.

Sequencing more than one genome for each lineage will also clarify the status of reference sequences amongst a larger strain collection. The assumptions made for the reference genomes will be integrated with their respective lineage and confirm their role as representatives of the respective BT. 


\subsection{Results}

\subsubsection{Phylogeny of the species Y. enterocolitica}

In addition to the seven reference genomes a further $89 \mathrm{Y}$. enterocolitica isolates were sequenced (Table 9.1, p. 204). These were contributed by collaborators from Europe and New Zealand and sequenced using Illumina technology. The strains represent human case isolates as well as isolates from humans without disease association, animal isolates, and environmental isolates. Twenty-four BT $1 \mathrm{~A}$, seven BT 1B, thirteen BT 2, twenty-two BT 3, twenty-one BT 4, and two BT 5 isolates were included. Only two BT 5 isolates were sequenced, as this is a rarely isolated biotype.

The phylogeny of the $Y$. enterocolitica species was obtained from genome-wide single nucleotide polymorphism (SNP) distribution by mapping against the BT1B reference strain 8081 . The four prophage regions were excluded from the analysis as they would result in false-positive SNPs from distantly related phages present in other BTs. This work was carried out in close collaboration with Dr Simon R. Harris and $\mathrm{Dr}$ Thomas R. Connor at the Wellcome Trust Sanger Institute.

It is evident from Figure 7.1 that there are three different $Y$. enterocolitica lineages corresponding to non-, high-, and low-pathogenic BTs. The different lineages show considerable differences in the SNP variation across the tree. This is also evident from the number of SNPs in the different lineages (Table 7.1). Individual biotype 1A strains are differentiated by up to 32,000 SNP sites. Since most of these isolates came from passive sampling from abattoirs and some clinical studies, this diversity is likely to be a significant underestimate of the genuine diversity of this clade as true environmental isolates such as strains isolated from water are currently underrepresented. Within this non-pathogenic clade there are five non-BT 1A strains. Two of these strains were typed as BT $1 \mathrm{~B}$ and were donated to the study from collaborators in New Zealand. Both strains were isolated from humans and had no serological information. 


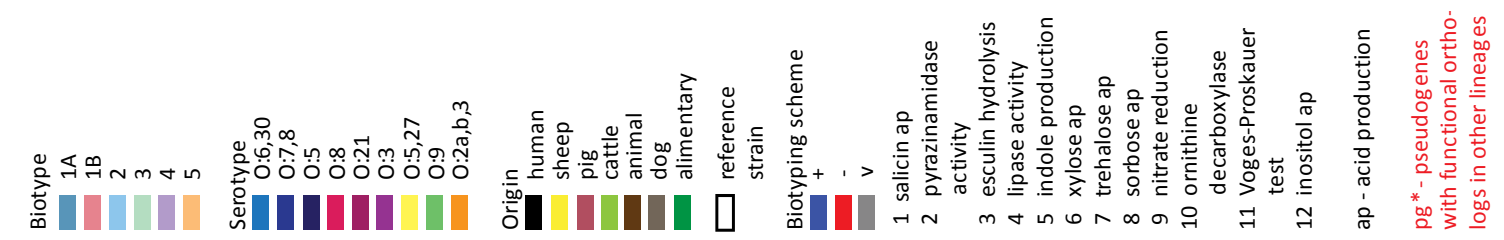
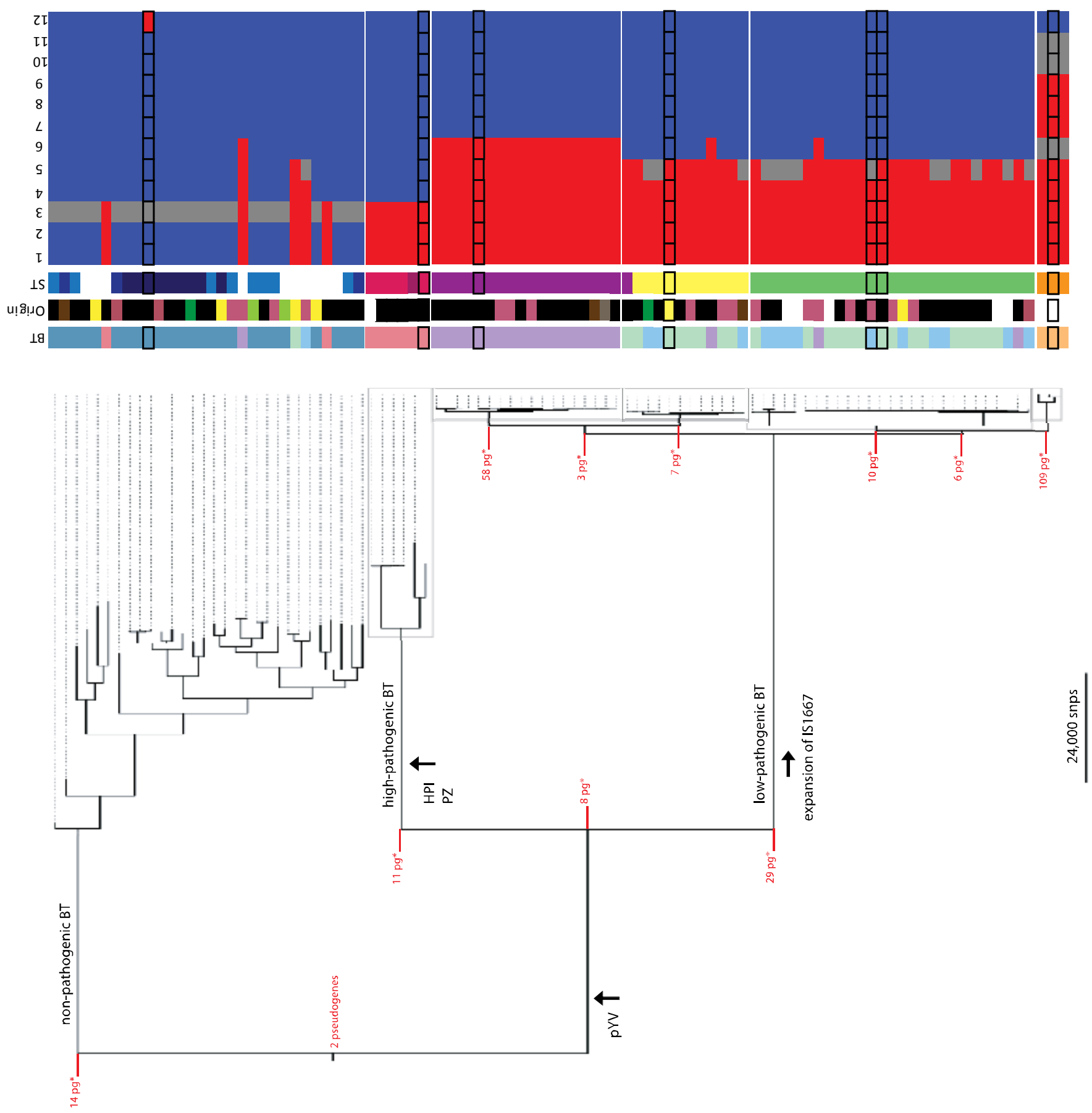

Figure 7.1: The maximum likelihood phylogenetic tree of $Y$. enterocolitica based on the SNP differences across the whole genome excluding laterally acquired phages. The tree highlights the key acquisition events of the virulence plasmid $\mathrm{pYV}$ in the pathogenic lineages of $\mathrm{Y}$. enterocolitica, and the acquisition of markers of high-pathogenic BT. The low-pathogenic BTs are marked by an expansion of the IS element IS1667. The number of pseudogenes that are shared within specific lineages and that have functional orthologs in other lineages is given $\left(\mathrm{pg}^{*}\right)$. The different lineages are characterized by biotype (BT), biological origin, and serotype (ST). The biochemical tests used for biotyping of $\mathrm{Y}$. enterocolitica are presented for each strain. 
Table 7.1: Numbers of SNPs between different lineages of $Y$. enterocolitica.

\begin{tabular}{|c|c|c|c|c|c|c|}
\hline & BT 1A & BT 1B & BT 2/3 0:9 & BT 2/3 O:5,27 & BT 4 & BT 5 \\
\hline BT 1A & $\begin{array}{l}0- \\
31,690\end{array}$ & & & & & \\
\hline BT 1B & $\begin{array}{l}87,039- \\
117,383\end{array}$ & $\begin{array}{l}11- \\
29,511\end{array}$ & & & & \\
\hline BT 2/3 0:9 & $\begin{array}{l}79,461- \\
115,724\end{array}$ & $\begin{array}{l}63,727- \\
92,073\end{array}$ & $\begin{array}{l}0- \\
5,582\end{array}$ & & & \\
\hline BT $2 / 3$ O:5,27 & $\begin{array}{l}81,599- \\
115,965\end{array}$ & $\begin{array}{l}64,399- \\
92,699\end{array}$ & $\begin{array}{l}1,607- \\
7,265\end{array}$ & $\begin{array}{l}1- \\
250\end{array}$ & & \\
\hline BT 4 & $\begin{array}{l}80,828- \\
115,651\end{array}$ & $\begin{array}{l}63,808- \\
91,888\end{array}$ & $\begin{array}{l}4,075- \\
8,202\end{array}$ & $\begin{array}{l}4,637- \\
5,685\end{array}$ & $\begin{array}{l}8- \\
882\end{array}$ & \\
\hline BT 5 & $\begin{array}{l}84,994- \\
114,528\end{array}$ & $\begin{array}{l}72,361- \\
89,850\end{array}$ & $\begin{array}{l}5,406- \\
9,691\end{array}$ & $\begin{array}{l}7,503- \\
10,734\end{array}$ & $\begin{array}{l}8,211- \\
11,461\end{array}$ & $\begin{array}{l}82- \\
2,883\end{array}$ \\
\hline
\end{tabular}

Blue - range of SNPs within a BT/ST lineage; green - range of SNPs between BT 1A and other lineages; red range of SNPs between 1B and other lineages; yellow - range of SNPs within the low-pathogenic BTs.

It has been noted before that BT 1A strains can be mistyped as BT 1B strains, but they commonly lack the virulence factors associated with the high-pathogenic BT (personal communication A. McNally, M. Prentice; Fearnley, et al., 2005). Analysis of these two strains showed that they do not carry the virulence plasmid, and do not have the Ysa T3SS, or the HPI. On the contrary, these two strains have operons for the utilization of D-arabitol and N-acetyl-D-galactosamine, and they also possess the YGT.

Three other isolates also do not group according to their biotyping reaction but cluster with BT 1A. These are a BT 3 O:5,27 isolate, one BT 4 O:3 strain, and a BT 2 strain. The BT 2 strain, YE228/02, is known to give variable biotyping reactions, and which consequently might explain the discrepancy between typing and clustering of this particular strain (personal communication A. McNally, T. Cheasty). Sequences of the other isolates were mapped to the respective serotype operon but did not yield any homologous hits, suggesting difficulties in typing these strains.

Although there is a reduced number of true $1 \mathrm{~B}$ isolates, like BT $1 \mathrm{~A}$, they also show significant genetic divergence with up to 30,000 SNPs defining this lineage. In contrast it is evident from Figure 7.1 that despite the large number of samples, the average divergence between BT $2-5$ isolates is much lower than the BT $1 \mathrm{~A}$ and BT 1B ( 12,000 SNPs), resulting in very short terminal branch lengths within this clade. As the included strains represent a wide temporally and geographically dispersed collection the possibility of this feature being due to a sampling artefact is unlikely. 
Another explanation is that the low genetic diversity is a signature consistent with a relatively recent population bottleneck.

While it is apparent from Figure 7.1 that there is no obvious clustering of the isolates according to human or animal origin, the phylogeny of the pathogenic $Y$. enterocolitica lineages is fully congruent with serotype and partially with biotype. Although there is some variation in the results of these biochemical tests they appear largely phylogenetically robust: BT $1 \mathrm{~A}$ isolates show a much higher metabolic potential whilst, even from these limited biochemical tests, it is clear that metabolic flexibility is sequentially reduced when moving from the high pathogenic 1B lineages across the low pathogenic lineages (Figure 7.1). This trend of reduced metabolic flexibility was observed on a much larger scale in the phenotypic microarray (Chapter 6 ) and is consistent with an increased number of pseudogenes in the low-pathogenic BTs and an expansion of IS1667. The observed variation in the biochemical tests confusing the assignments of BT2, BT3 and BT4 isolates is largely explained by differences in the ability to catabolise D-xylose and the production of indole. The latter test is known to show a high degree of methodological variation. In particular BTs 2 and 3 are distinguishable only by a variable indole reaction, and Chapter 4 illustrated that the two 0:9 isolates are identical apart from three transposases and two putative phage proteins. Both BTs even present the same pseudogene mutations with very few exceptions. Phylogenetically BTs 2 and 3 intermingle and isolates are only clearly separated by serotype.

For the reference strains, the number of pseudogenes in each branch with functional orthologs in other lineages has been highlighted on the tree. The pseudogenes defining a lineage are listed in Table 7.2. 
Table 7.2: Pseudogenes in the core genome of $Y$. enterocolitica lineages.

\begin{tabular}{|c|c|c|c|c|c|c|c|c|c|}
\hline ID & $\begin{array}{l}1 \mathrm{~B} \\
8081\end{array}$ & $\begin{array}{l}1 \mathrm{~A} \\
5303\end{array}$ & $\begin{array}{l}20: 9 \\
21202\end{array}$ & $\begin{array}{l}30: 9 \\
5603\end{array}$ & $\begin{array}{l}30: 5,27 \\
14902\end{array}$ & $\begin{array}{l}40: 3 \\
1203\end{array}$ & $\begin{array}{l}5 \\
3094\end{array}$ & product & mutation \\
\hline YE0416 & $\bar{\Delta}$ & 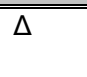 & 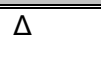 & $\overline{\Delta \Delta}$ & 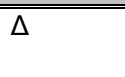 & $\overline{\Delta \Delta}$ & $\overline{\Delta \Delta}$ & $\begin{array}{l}\text { conserved hypothe- } \\
\text { tical protein }\end{array}$ & stop codon \\
\hline YE2410 & $\Delta$ & $\Delta$ & $\Delta$ & $\Delta$ & $\Delta$ & $\Delta$ & $\Delta$ & $\begin{array}{l}\text { putative oxidoreduc- } \\
\text { tase }\end{array}$ & FS deletion \\
\hline YE0207 & + & $\Delta$ & + & + & + & + & + & $\begin{array}{l}\text { putative lysophospho- } \\
\text { lipase }\end{array}$ & stop codon \\
\hline YE0480 & + & $\Delta$ & + & + & + & + & + & $\begin{array}{l}\text { putative exported } \\
\text { protein }\end{array}$ & stop codon \\
\hline YE0688 & + & $\Delta$ & + & + & + & + & + & $\begin{array}{l}\text { putative type II secre- } \\
\text { tion system protein }\end{array}$ & FS deletion \\
\hline YE0972 & + & $\Delta$ & + & + & + & + & + & $\begin{array}{l}\text { conserved hypothe- } \\
\text { tical protein }\end{array}$ & stop codon \\
\hline YE1432 & + & $\Delta$ & + & + & + & + & + & hypothetical protein & FS deletion \\
\hline YE1733 & + & $\Delta$ & + & + & + & + & + & $\begin{array}{l}\text { putative UV protection } \\
\text { and mutation protein }\end{array}$ & stop codon \\
\hline YE1743 & + & $\Delta$ & + & + & + & + & + & $\begin{array}{l}\text { putative AraC-family } \\
\text { transcriptional } \\
\text { regulatory protein }\end{array}$ & stop codon \\
\hline YE1759 & + & $\Delta$ & + & + & + & + & + & $\begin{array}{l}\text { conserved hypothe- } \\
\text { tical protein }\end{array}$ & $\begin{array}{l}\text { disrupted by } \\
\text { IS element }\end{array}$ \\
\hline YE2179 & + & $\Delta$ & + & + & + & + & + & hypothetical protein & stop codon \\
\hline YE2521 & + & $\Delta$ & + & + & + & + & + & flagellin & deletion \\
\hline YE3354 & + & $\Delta$ & + & + & + & + & + & $\begin{array}{l}\text { putative oxidoreduc- } \\
\text { tase }\end{array}$ & $\begin{array}{l}\text { disrupted by } \\
\text { IS element }\end{array}$ \\
\hline YE4026 & + & $\Delta$ & + & + & + & + & + & $\begin{array}{l}\text { myo-inositol 2- } \\
\text { dehydrogenase }\end{array}$ & $\begin{array}{l}\text { opal stop } \\
\text { codon }\end{array}$ \\
\hline YE4074 & + & $\Delta$ & + & + & + & + & + & $\begin{array}{l}\text { cellulose synthase } 1 \\
\text { catalytic subunit }\end{array}$ & $\begin{array}{l}\text { opal stop } \\
\text { codon }\end{array}$ \\
\hline YE4185 & + & $\Delta$ & + & + & + & + & + & $\begin{array}{l}\text { Probable two-compo- } \\
\text { nent sensor kinase }\end{array}$ & stop codon \\
\hline YE0522 & $\Delta$ & + & $\Delta$ & $\Delta$ & $\Delta$ & $\Delta$ & $\Delta$ & $\begin{array}{l}\text { putative exported } \\
\text { protein }\end{array}$ & $\begin{array}{l}\text { deletion } \mathrm{N} \\
\text { terminus }\end{array}$ \\
\hline YE1236A & $\Delta$ & + & $\Delta$ & $\Delta$ & $\Delta$ & $\Delta$ & $\Delta$ & $\begin{array}{l}\text { 6-phospho-beta- } \\
\text { glucosidase }\end{array}$ & deletion \\
\hline YE1400 & $\Delta$ & + & $\Delta$ & $\Delta$ & $\Delta$ & $\Delta$ & $\Delta$ & $\begin{array}{l}\text { Putative type I } \\
\text { secretion protein }\end{array}$ & FS mutation \\
\hline YE2586A & $\Delta$ & + & $\Delta$ & $\Delta$ & $\Delta$ & $\Delta$ & $\Delta$ & $\begin{array}{l}\text { putative coA transfe- } \\
\text { rase family protein }\end{array}$ & FS mutation \\
\hline YE2708 & $\Delta$ & + & $\Delta$ & $\Delta$ & $\Delta$ & $\Delta$ & $\Delta$ & $\begin{array}{l}\text { a-ribazole-5'-phos- } \\
\text { phate phosphatase }\end{array}$ & deletion \\
\hline YE2719 & $\Delta$ & + & $\Delta$ & $\Delta$ & $\Delta$ & $\Delta$ & $\Delta$ & $\begin{array}{l}\text { conserved } \\
\text { hypothetical protein }\end{array}$ & deletion \\
\hline YE3341 & $\Delta$ & + & $\Delta$ & $\Delta$ & $\Delta$ & $\Delta$ & $\Delta$ & prepilin peptidase & FS insertion \\
\hline YE0270 & $\Delta$ & + & + & + & + & + & + & $\begin{array}{l}\text { conserved hypothe- } \\
\text { tical protein }\end{array}$ & stop codon \\
\hline YE0936 & $\Delta$ & + & + & + & + & + & + & $\begin{array}{l}\text { putative MFS family } \\
\text { transport protein }\end{array}$ & FS deletion \\
\hline YE0944 & $\Delta$ & + & + & + & + & + & + & hypothetical protein & $\begin{array}{l}\text { stop codon, } \\
\text { FS mutation }\end{array}$ \\
\hline YE1406 & $\Delta$ & + & + & + & + & + & + & putative protease & FS mutation \\
\hline YE1488 & $\Delta$ & + & + & + & + & + & + & $\begin{array}{l}\text { putative inner mem- } \\
\text { brane protein }\end{array}$ & FS mutation \\
\hline YE1598 & $\Delta$ & + & + & + & + & + & + & $\begin{array}{l}\text { nitrite reductase gene } \\
\text { remnant }\end{array}$ & deletion \\
\hline YE1728 & $\Delta$ & + & + & + & + & + & + & $\begin{array}{l}\text { putative sugar per- } \\
\text { mease }\end{array}$ & FS deletion \\
\hline YE1729 & $\Delta$ & + & + & + & + & + & + & $\begin{array}{l}\text { putative sucrose } \\
\text { operon repressor }\end{array}$ & FS deletion \\
\hline YE2450 & $\Delta$ & + & + & + & + & + & + & $\begin{array}{l}\text { putative D-tagatose- } \\
\text { 1,6-bisphosphate } \\
\text { aldolase subunit }\end{array}$ & $\begin{array}{l}\text { deletion C } \\
\text { terminus }\end{array}$ \\
\hline
\end{tabular}




\begin{tabular}{|c|c|c|c|c|c|c|c|c|c|}
\hline YE2640 * & $\Delta$ & + & + & + & + & + & + & $\begin{array}{l}\text { putative membrane } \\
\text { protein }\end{array}$ & $\begin{array}{l}\text { opal stop } \\
\text { codon }\end{array}$ \\
\hline YE3168 & $\Delta$ & + & + & + & + & + & + & $\begin{array}{l}\text { conserved hypothe- } \\
\text { tical protein }\end{array}$ & $\begin{array}{l}\text { disrupted by } \\
\text { IS element }\end{array}$ \\
\hline YE3180 & $\Delta$ & + & + & + & + & + & + & $\begin{array}{l}\text { phosphate binding } \\
\text { protein }\end{array}$ & stop codon \\
\hline YE0324 & + & + & $\Delta$ & $\Delta$ & $\Delta$ & $\Delta$ & $\Delta$ & $\begin{array}{l}\text { putative ATP/GTP- } \\
\text { binding protein }\end{array}$ & $\begin{array}{l}\text { deletion C } \\
\text { terminus }\end{array}$ \\
\hline YE0443 & + & + & $\Delta$ & $\Delta$ & $\Delta$ & $\Delta$ & $\Delta$ & multidrug efflux protein & $\begin{array}{l}\text { deletion C } \\
\text { terminus }\end{array}$ \\
\hline YE0445 & + & + & $\Delta$ & $\Delta$ & $\Delta$ & $\Delta$ & $\Delta$ & $\begin{array}{l}\text { probable outer mem- } \\
\text { brane efflux lipoprotein }\end{array}$ & $\begin{array}{l}\text { deletion } \mathrm{N} \\
\text { terminus }\end{array}$ \\
\hline YE0483 & + & + & $\Delta$ & $\Delta$ & $\Delta$ & $\Delta$ & $\Delta$ & $\begin{array}{l}\text { putative LysR-family } \\
\text { transcriptional } \\
\text { regulatory protein }\end{array}$ & deletion \\
\hline YE0807 & + & + & $\Delta$ & $\Delta$ & $\Delta$ & $\Delta$ & $\Delta$ & $\begin{array}{l}\text { putative transcriptional } \\
\text { regulatory protein }\end{array}$ & deletion \\
\hline YE0911 & + & + & $\Delta$ & $\Delta$ & $\Delta$ & $\Delta$ & $\Delta$ & $\begin{array}{l}\text { 3-oxoacyl-[ACP] } \\
\text { synthase II }\end{array}$ & $\begin{array}{l}\text { deletion } \mathrm{N} \\
\text { terminus }\end{array}$ \\
\hline YE0934 & + & + & $\Delta$ & $\Delta$ & $\Delta$ & $\Delta$ & $\Delta$ & hypothetical protein & $\begin{array}{l}\text { deletion } \mathrm{N} \\
\text { terminus }\end{array}$ \\
\hline YE0935 & + & + & $\Delta$ & $\Delta$ & $\Delta$ & $\Delta$ & $\Delta$ & $\begin{array}{l}\text { AraC-family transcrip- } \\
\text { tional regulator }\end{array}$ & $\begin{array}{l}\text { deletion } \mathrm{N} \\
\text { terminus }\end{array}$ \\
\hline YE0941 & + & + & $\Delta$ & $\Delta$ & $\Delta$ & $\Delta$ & $\Delta$ & $\begin{array}{l}\text { AraC-family transcrip- } \\
\text { tional regulator }\end{array}$ & FS deletion \\
\hline YE1005 & + & + & $\Delta$ & $\Delta$ & $\Delta$ & $\Delta$ & $\Delta$ & phospholipase A & FS insertions \\
\hline Ye1165 & + & + & $\Delta$ & $\Delta$ & $\Delta$ & $\Delta$ & $\Delta$ & $\begin{array}{l}\text { putative LyxR-family } \\
\text { transcriptional } \\
\text { regulatory protein }\end{array}$ & deletion \\
\hline YE1166 & + & + & $\Delta$ & $\Delta$ & $\Delta$ & $\Delta$ & $\Delta$ & $\begin{array}{l}\text { putative LysR-family } \\
\text { transcriptional } \\
\text { regulatory protein }\end{array}$ & $\begin{array}{l}\text { FS mutation, } \\
\text { deletion C } \\
\text { terminus }\end{array}$ \\
\hline YE1251 & + & + & $\Delta$ & $\Delta$ & $\Delta$ & $\Delta$ & $\Delta$ & $\begin{array}{l}\text { putative regulatory } \\
\text { protein }\end{array}$ & $\begin{array}{l}\text { deletion } \mathrm{N} \\
\text { terminus }\end{array}$ \\
\hline YE1252 & + & + & $\Delta$ & $\Delta$ & $\Delta$ & $\Delta$ & $\Delta$ & $\begin{array}{l}\text { putative exported } \\
\text { protein }\end{array}$ & deletion \\
\hline YE1409 & + & + & $\Delta$ & $\Delta$ & $\Delta$ & $\Delta$ & $\Delta$ & $\begin{array}{l}\text { putative prophage } \\
\text { repressor protein }\end{array}$ & stop codon \\
\hline YE1610 & + & + & $\begin{array}{l}\text { FS } \\
\text { deletion }\end{array}$ & $\begin{array}{l}\text { FS } \\
\text { deletion }\end{array}$ & $\begin{array}{l}\text { FS } \\
\text { deletions }\end{array}$ & $\begin{array}{l}\text { FS } \\
\text { deletions }\end{array}$ & $\begin{array}{l}\text { stop } \\
\text { codon }\end{array}$ & $\begin{array}{l}\text { putative MFS-family } \\
\text { transport protein }\end{array}$ & $\begin{array}{l}\text { independent } \\
\text { mutations }\end{array}$ \\
\hline YE1874 & + & + & $\Delta$ & $\Delta$ & $\Delta$ & $\Delta$ & $\Delta$ & $\begin{array}{l}\text { putative DNA-binding } \\
\text { protein }\end{array}$ & deletion \\
\hline YE1905 & + & + & $\Delta$ & $\Delta$ & $\Delta$ & $\Delta$ & $\Delta$ & hypothetical protein & stop codon \\
\hline YE1944 & + & + & $\begin{array}{l}\text { FS } \\
\text { insertion }\end{array}$ & $\begin{array}{l}\text { FS } \\
\text { insertion }\end{array}$ & $\begin{array}{l}\text { FS } \\
\text { insertion, } \\
\text { stop } \\
\text { codon }\end{array}$ & $\begin{array}{l}\text { FS } \\
\text { insertion, } \\
\text { stop } \\
\text { codon }\end{array}$ & $\begin{array}{l}\text { stop } \\
\text { codon }\end{array}$ & $\begin{array}{l}\text { putative membrane } \\
\text { permease }\end{array}$ & $\begin{array}{l}\text { independent } \\
\text { mutations }\end{array}$ \\
\hline YE1950 & + & + & $\Delta$ & $\Delta$ & $\Delta$ & $\Delta$ & $\Delta$ & $\begin{array}{l}\text { alkanesulfonate } \\
\text { monooxygenase }\end{array}$ & deletion \\
\hline YE2286 & + & + & $\Delta$ & $\Delta$ & $\Delta$ & $\Delta$ & $\Delta$ & $\begin{array}{l}\text { acyl-[ACP]--UDP-N- } \\
\text { acetylglucosamine o- } \\
\text { acyltransferase }\end{array}$ & deletion \\
\hline YE2603 & + & + & $\Delta$ & $\Delta$ & $\Delta$ & $\Delta$ & $\Delta$ & putative sugar kinase & $\begin{array}{l}\text { deletion C } \\
\text { terminus }\end{array}$ \\
\hline YE2609 & + & + & $\Delta$ & $\Delta$ & $\Delta$ & $\Delta$ & $\Delta$ & $\begin{array}{l}\mathrm{ABC} \text { transporter, } \\
\text { substrate binding } \\
\text { component }\end{array}$ & deletion \\
\hline YE2812 & + & + & $\Delta$ & $\Delta$ & $\Delta$ & $\Delta$ & $\Delta$ & $\begin{array}{l}\text { putative formate } \\
\text { transporter }\end{array}$ & deletion \\
\hline YE3630 & + & + & $\Delta$ & $\Delta$ & $\Delta$ & $\Delta$ & $\Delta$ & putative cation efflux & deletion \\
\hline
\end{tabular}




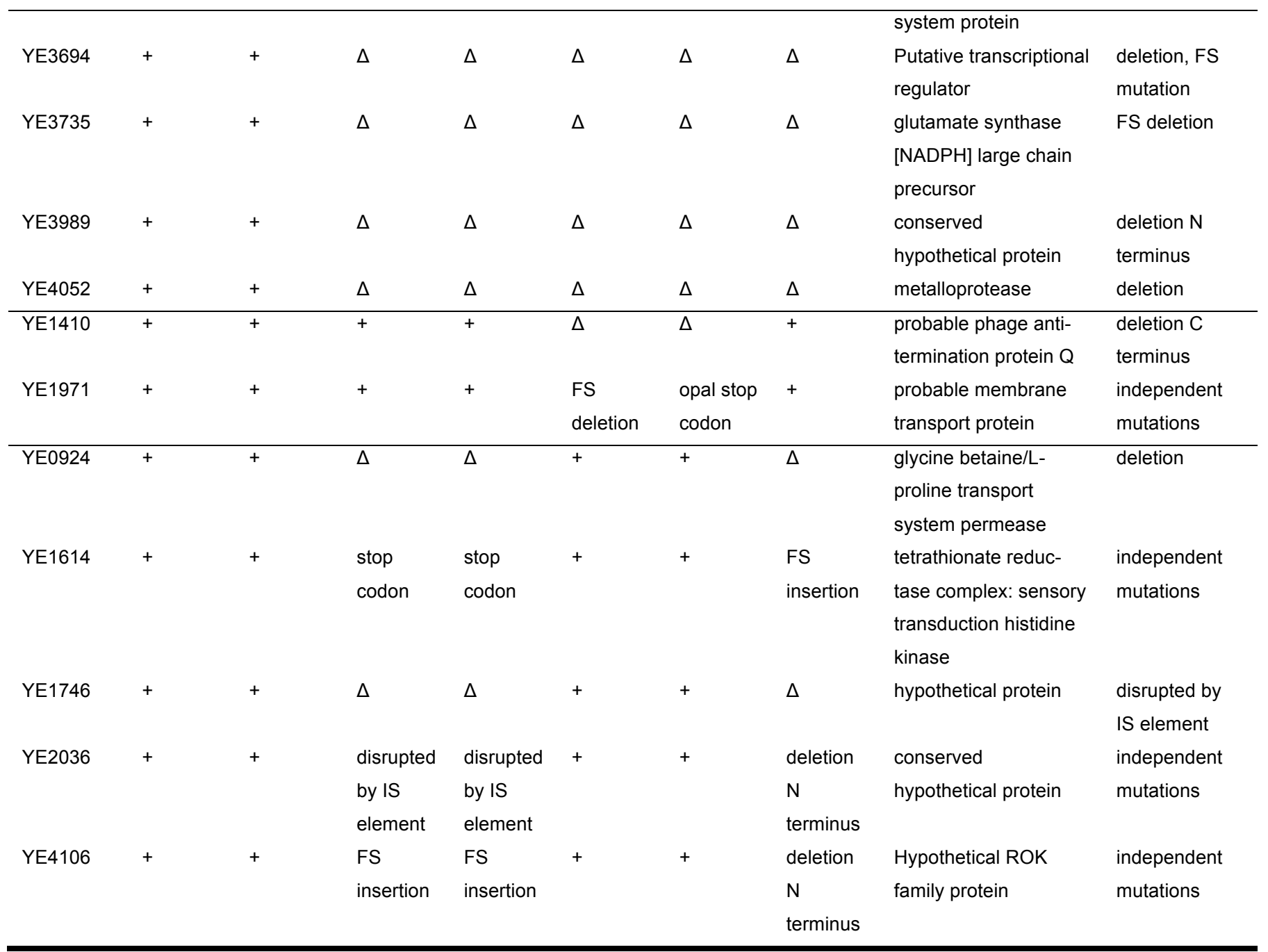

+ - functional ortholog; $\Delta$ - mutation; column mutation lists shared mutational inactivation, further mutations possible for individual isolates; independent mutations are listed within the table.

There are two pseudogenes which are shared in all BTs and which show the same mutational inactivation. The non-pathogenic BT 1A has acquired 14 pseudogenes. There is no predominant class among those pseudogenes, four are in hypothetical proteins and two are in regulatory proteins. All pathogenic lineages share seven pseudogenes in the core genome. These have been acquired in single events before the split of high- and low-pathogenic lineages because the same mutations are discernible. It has to be mentioned though that some of these genes have acquired further mutations following the split of the lineages. The high-pathogenic BT has 12 pseudogenes with functional orthologs in the other lineages, three of which are in hypothetical proteins, and four are involved in metabolism and transport.

A high accumulation of pseudogenes is apparent in the low-pathogenic BTs, again indicating a potential bottleneck evolution of this lineage. The low-pathogenic BTs have 29 pseudogenes of which only two have been acquired independently. These independent mutations target a transport protein and a membrane permease. The 
shared pseudogenes are predominantly found in transport proteins (4 pseudogenes), proteins involved in metabolism (6 pseudogenes), and regulatory proteins (8 pseudogenes). This supports the reduced metabolic flexibility observed in the phenotypic micorarray for these biotypes. The branch leading to the $0: 3$ and $0: 5,27$ lineages has accumulated only two further pseudogenes, one in a shared phage, the other acquired independently in a membrane protein. Five pseudogenes are found in the branch leading to the $0: 9$ and $0: 2 a, 2 b, 3$ lineages. Of these, only two are shared. These are present in a transport permease and a hypothetical protein. The independent mutations are found in the histidine kinase controlling the tetrathionate respiration operon, and in hypothetical proteins. The two 0:9 isolates show identical mutational inactivations, but these mutations were acquired independently in BT 5.

A significant expansion of core pseudogenes in the terminal branches of the lowpathogenic BTs is visible in BT 4 O:3 (58 pseudogenes) and BT 5 O:2a,2b,3 (109 pseudogenes). These two isolates can be considered the two most host-adapted lineages of $Y$. enterocolitica. BT $40: 3$ is the predominant isolate found in human cases, and BT 5 is responsible for disease in hares. BT 5 is very niche-restricted. After accumulation of a massive amount of pseudogenes, it appears to struggle to survive outside its host and is very rarely isolated in general. The increased amount of pseudogenes also again reflects the loss of metabolic flexibility detected in the phenotypic micorarray (Chapter 6).

As mentioned before (Chapter 4), some trends found within the pseudogenes also translate in a broader context. Cobalamin biosynthesis, tetrathionate respiration, and the flagella cluster are not only affected by mutations in $Y$. enterocolitica lineages, but are also targets of pseudogene formation in Y. pestis, S. Typhi, and S. Gallinarum (Parkhill, et al., 2001a,b; Thomson, et al., 2006, 2008). Fish pathogen Y. ruckeri has also been described as lacking cobalamin biosynthesis and tetrathionate respiration (Chen, et al., 2010). Evolution from environmental survival to host adaption has probably driven the loss of these properties.

It has been proposed to sub-speciate BT 1B into $Y$. enterocolitica subspecies enterocolitica and BT 1A, 2-5 into Y. enterocolitica subspecies palearctica (Neubauer, et al., 2000). This distinction of two subspecies is based on variations in 12 SNPs over the full length of the $16 \mathrm{~S}$ rRNA gene (1637 bp). However, this differentiation is not supported by our data. Based on SNP distribution in all core 
genes three distinct lineages can be observed. Very specifically, the proposed subspecies Y. enterocolitica palearctica is based on the worldwide distribution of those isolates but their very distinct genomic content argues against this. Obviously, the resolution power is much greater using the full genome information as opposed to a single, highly conserved gene.

Judging solely from our tree, it appears that the virulence plasmid pYV has been acquired in the pathogenic lineages of $Y$. enterocolitica. Signifying the split into highpathogenic BTs is the uptake of the HPI and operons within the plasticity zone. Indicative for the low-pathogenic BTs is the expansion of IS1667. From this tree, the non-pathogenic BT seems to be the oldest lineage. The second flagella cluster Flag2 , which is only consistently present in the low-pathogenic BTs, has been lost independently in high- and low-pathogenic BTs. The shape of the tree dictates this, but different gene fragments of Flag-2 in those BTs are also suggestive of independent loss events. Further to this, there are some BT 1A isolates that have retained Flag-2, but there is no link to a specific biological origin.

\subsubsection{Phylogeny of the genus Yersinia}

To determine sequence based phylogeny of the Yersinia genus we examined a representative sample of pathogenic isolates composed of $Y$. pseudotuberculosis (33 in total; 29 sequenced in this study) and $Y$. pestis (using the published genomes of CO92/Parkhill, et al., 2001; KIM5/Deng, et al., 2002; Yp91001/Song, et al., 2004) as well as the relatively understudied lineage of $Y$. enterocolitica (98 isolates). To explore the large number of environmental Yersinia spp., 81 isolates from all species except the recently described Y. entomophaga (Hurst, et al., 2011) and Y. nurmii (Murros-Kontiainen, et al., 2010a) were included. These isolates are listed in Table 9.2 (p. 206) in the appendix. In total 199 Yersinia isolates were sequenced which had been conventionally typed using biochemical and serological techniques. Some isolates that had been assigned to species but showed atypical biochemical profiles were included as well.

As the genus Yersinia shows a very high sequence divergence, an approach based on mapping sequence reads to gain information on the whole genome could not be carried out. As seen in Y. enterocolitica, even within a single species of Yersinia $>$ 250, 000 SNPs differentiate members of that species. Instead, the syntenic blocks identified in $Y$. enterocolitica (Chapter 4) were used to sample a set of 85 
housekeeping genes common to all members of the Yersiniae. The housekeeping genes were identified on the basis of showing 10-25\% SNP divergence between Y. pestis and Y. enterocolitica. This work was carried out in collaboration with Miquette Hall, NTU. The genes identified are given in Table 2.3 and their locations with respect to the BT 1B reference genome for strain 8081 is shown in Figure 7.2

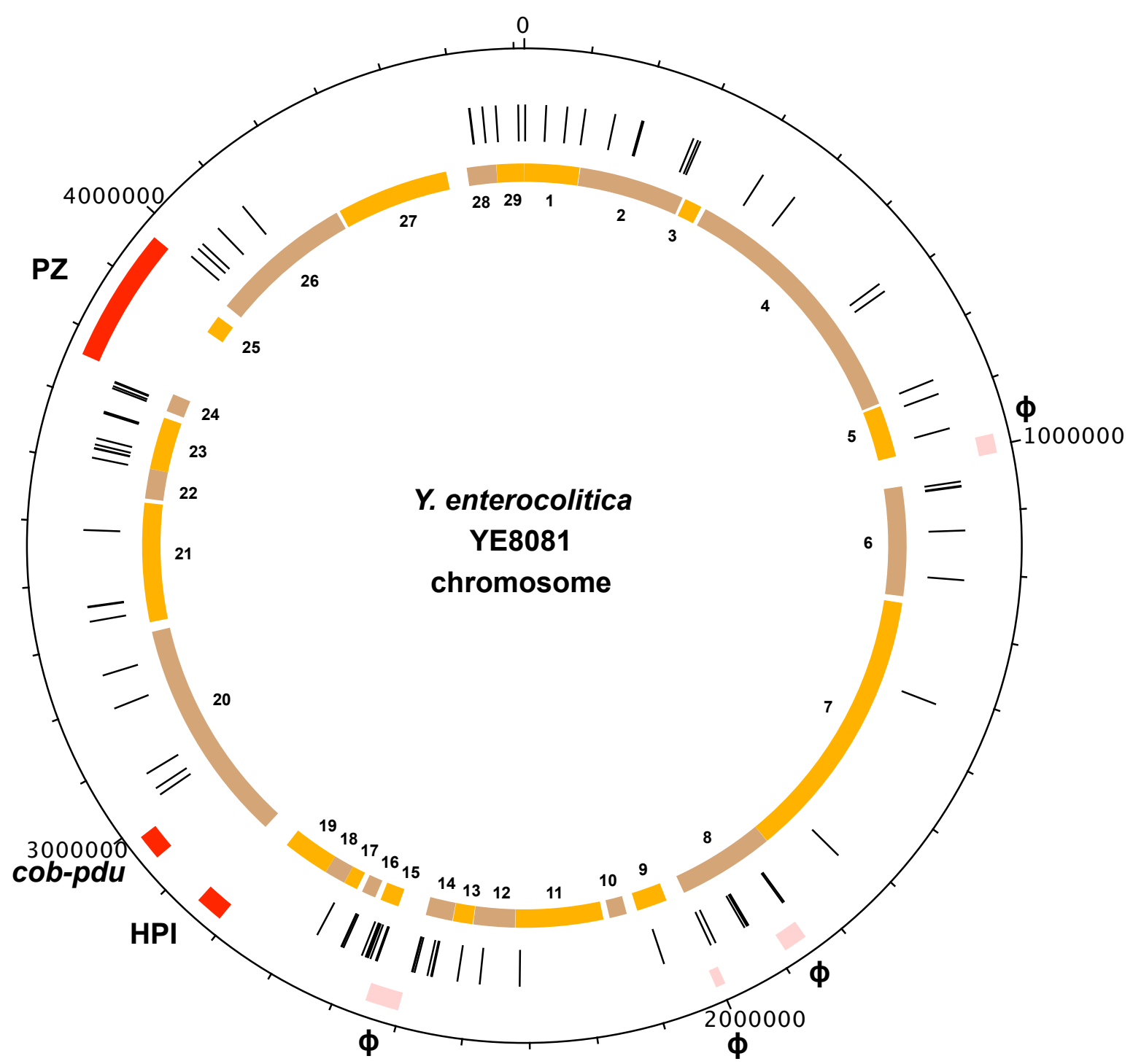

Figure 7.2: Distribution of the 85 housekeeping genes used for construction of the phylogenetic relationship within the genus Yersinia. The first circle shows regions naturally excluding the presence of housekeeping genes, such as phage, and pathogenicity islands. In the second circle, each bar represents a single housekeeping gene. The third circle highlights the 29 syntenic blocks in alternating colours of orange and brown (see Table 4.3).

There are some syntenic blocks that are not represented with genes (\#3, 10, 18, 19, $22,25,27)$. This can be due to several reasons. First of all, the blocks were identified purely on the basis of being a stretch of DNA sequence that did not show any 
recombination in the different $Y$. enterocolitica BT. Some blocks, like \#3 and \#10, are small in size and only contain $\sim 30$ CDSs. This restricts the number of possible genes, and the genes contained in these blocks might not be housekeeping genes to start with, or do not comply with the required divergence levels. The absence of housekeeping genes in larger regions such as \#27 could be explained by the fact that housekeeping genes were identified, but they could not be obtained from sequence reads for Y. ruckeri. This species is arguably part of the genus Yersinia (Sulakvelidze, 2000) and notably very distantly related to the other members of the genus. There were occasions when housekeeping genes were easily identified in all species except $Y$. ruckeri and thus had to be excluded from an initial set of potential genes.

Region \#25 corresponds to a conserved region within the plasticity zone, but does not contain any housekeeping genes.

Bayesian Analysis of Population Structure (BAPS) was used to divide the population into groups of shared sequence similarity. Phylogenetic analyses are usually based on a concatenated sequence. In contrast, BAPS compares individual genes and thus can look at a broader level of relationships, including potential recombination events and linkage between genes. This BAPS clustering resolved the genus into 12 distinct species clusters (SC; Figure 7.3). Definition of Yersinia spp. has been based on biochemical differences, which have been overlayed onto the tree. It is obvious that some Yersinia spp. show a similar biochemical profile, yet are distantly related. This is the case for $Y$. frederiksenii. The type strain is part of SC 7, but a distantly related group can be found in SC 2, together with isolates designated as Y. massiliensis. This phenomenon has been noted before (Sulakvelidze, 2000; Virdi and Sachdeva, 2005; Merhej, et al., 2008). On the other hand, SC 3 groups three species, Y. aleksiciae, Y. bercovieri, and Y.mollaretii, with similar biochemical profiles together. $Y$. mollaretii and $Y$. bercovieri were originally classified as $Y$. enterocolitica BT 3A and 3B, respectively (Sulakvelidze, 2000). They were later speciated based on differential tests of sorbose, sucrose, indole, and inositol (Sulakvelidze, 2000). Here, they are once again grouping together in one heterogeneous lineage. 


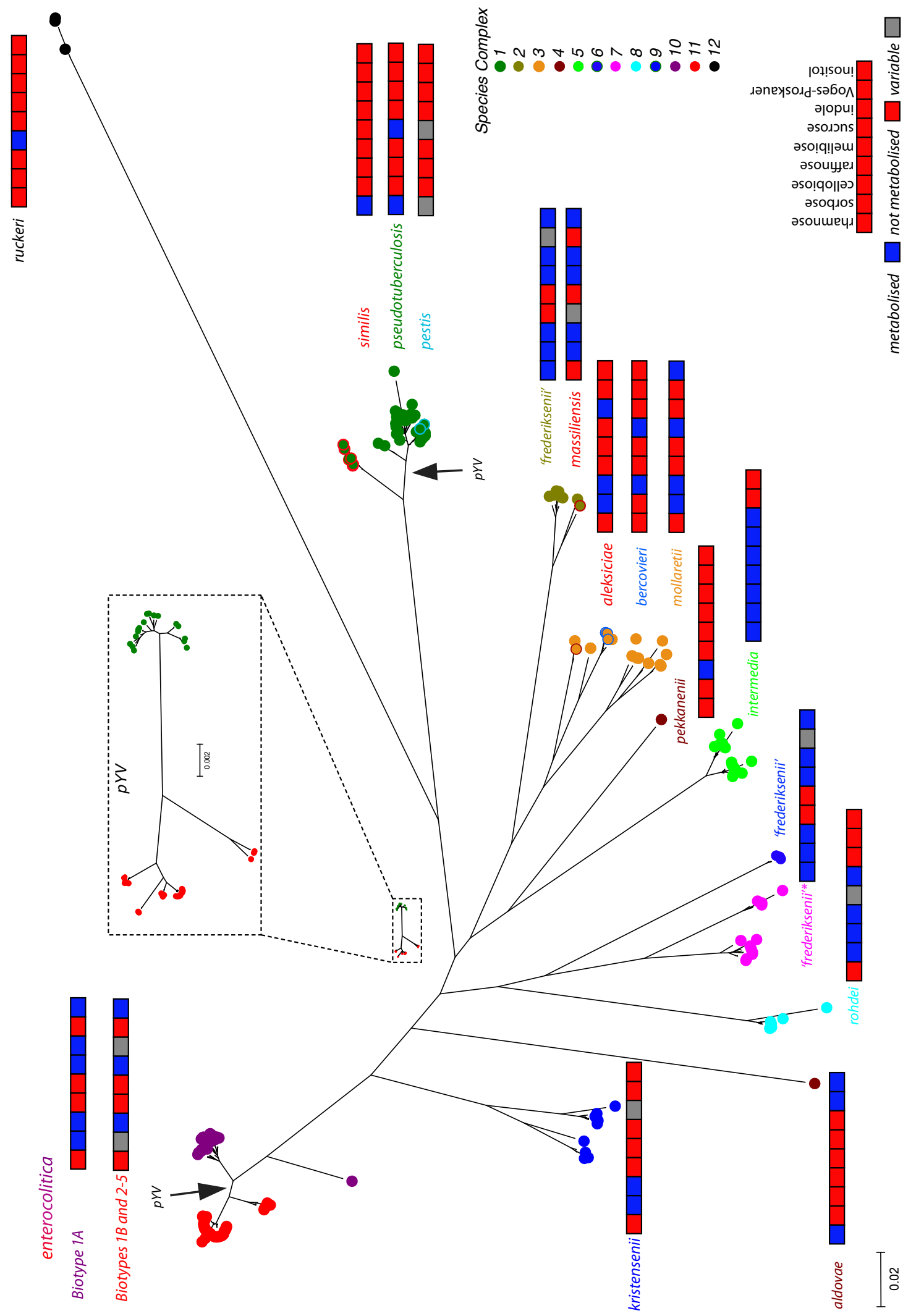

Figure 7.3: The phylogeny of the genus Yersinia and the virulence plasmid pYV. The maximum likelihood phylogenetic tree of the genus Yersinia is based on the concatenated sequence of 85 housekeeping genes. Current species assignments (colour circle borders) are contrasted with the species complexes (coloured circles) as allocated by BAPS analysis and the biochemical typing scheme used for speciation. There are three separate Y. frederiksenii lineages, labelled 'frederiksenii', thus subject to renaming to differentiate them. The type strain (Chen, et al., 2010) is depicted by ${ }^{*}$. An arrow shows the two independent acquisition events of the virulence plasmid pYV. The maximum likelihood phylogenetic tree of $\mathrm{pYV}$ is based on the whole sequence of the plasmid mapping against pYVe8081. The colours are the same as for the species complexes in which the plasmid is detected. 
Y. pestis and Y.pseudotuberculosis form a tight, almost indistinguishable group which is consistent with previously published results (Achtman, et al., 1999; Kotetishvili, et al., 2005). They form a species complex with Y. similis. This nonpathogenic species was recently split off from Y. pseudotuberculosis on the basis of a melibiose-negative phenotype and differences in the 16S rRNA gene sequence (Sprague, Scholz, Amann and Busse, 2008). Furthermore, isolates of Y. similis do not possess the virulence plasmid pYV (Sprague, Scholz, Amann and Busse, 2008). Based on clustering analysis, it shows significant sequence similarity to be still considered part of the Y. pseudoutberculosis/pestis species cluster.

The other environmental species occupy branches between the Y.pseudotuberculosis complex and $Y$. enterocolitica, which is positioned diametrically opposed to $Y$. pseudotuberculosis at the other side of the tree. The only exception to this is Y. ruckeri, which forms an independent, long branch, and is clearly distantly related to the other members of the genus Yersinia. As was seen in Figure 7.1, Y. enterocolitica forms three separate lineages corresponding to non-, high-, and low-pathogenic BTs. As suggested from the genomic data, BT $1 \mathrm{~A}$ is indeed the oldest lineage. One early branching isolate potentially reflects the diversity inherent in this environmental lineage. The pathogenic lineages are signified by the acquisition of the virulence plasmid after their separation from the non-pathogenic BT 1A.

Two new species have been recently described as members of the genus Yersinia: Y. entomophaga and Y. nurmii (Hurst, et al., 2011; Murros-Kontiainen, et al., 2010a). These isolates were not included in the sequencing collections, as they were too recently suggested. Both are described as being closely related to Y. ruckeri. This is interesting, as Y. ruckeri presents the most outlying lineage of the genus, and so far no intermediates to other lineages have been identified. The description of two such species now raises the question whether these are the same species described by two research teams independently and where they can be positioned with respect to the other lineages identified.

For these two species, only the sequences of the four housekeeping genes $g \ln A$, gyrB, hsp60, and recA are currently available. Although these genes might not be informative representatives to re-establish the phylogeny of the genus, as especially $\operatorname{rec} A$ shows a high level of conservation, in combination with the known lineages and 
their evolutionary history it is possible to establish the position of $Y$. entomophaga and Y. nurmii (Figure 7.4).

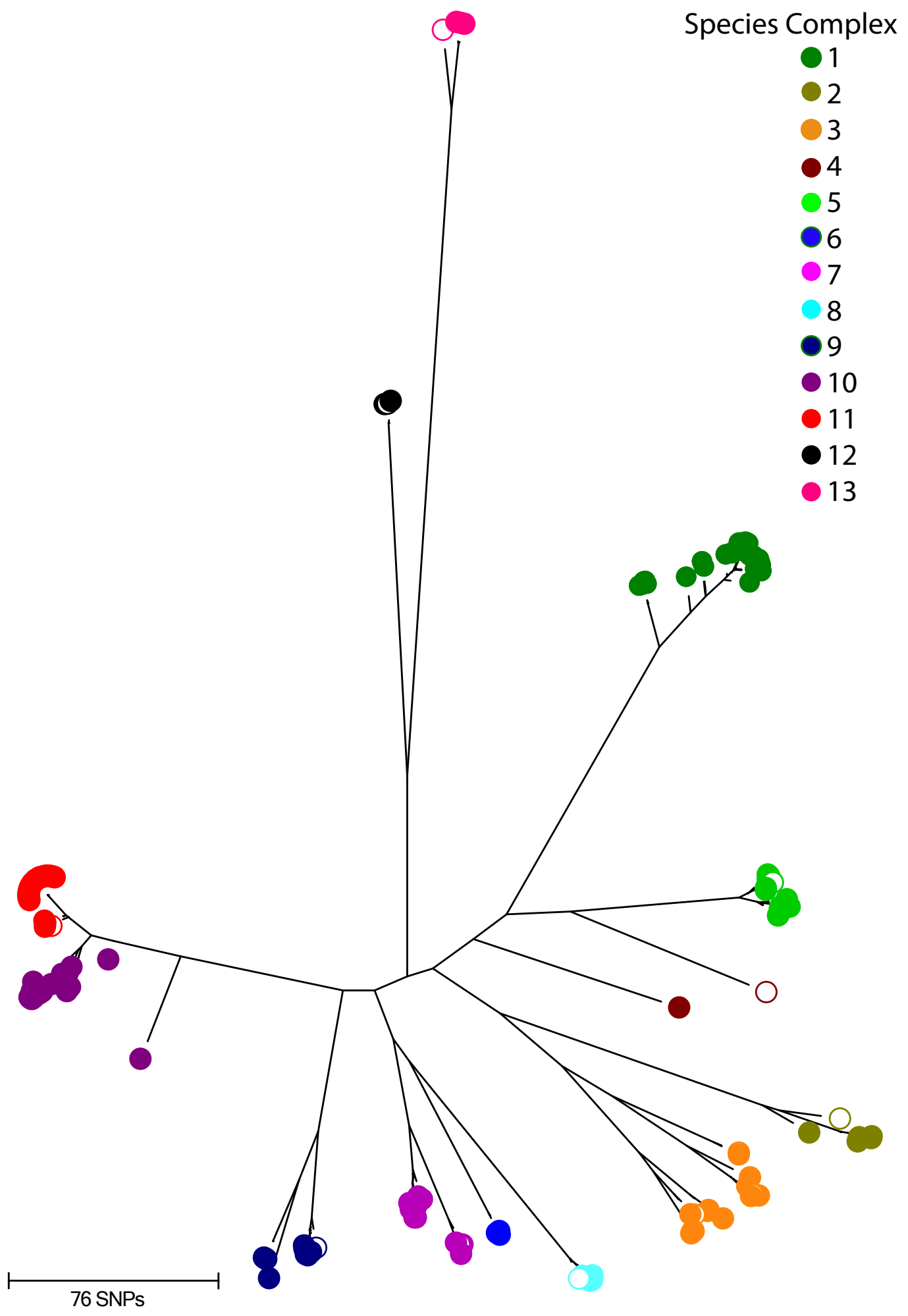

Figure 7.4: The phylogeny of the genus Yersinia based on $\operatorname{recA}, g \ln A, g y r B$, and $h s p 60$. The maximum likelihood phylogenetic tree of the genus Yersinia is based on the concatenated sequence of four housekeeping genes. The same species complexes (coloured circles) as allocated by BAPS analysis (Figure 7.3) are highlighted. The type strains are depicted by open circles. Y. entomophaga and Y. nurmii form SC13, with Y. entomophaga shown as open circle. 
From Figure 7.4 one can see that the branching order differs in these four hosuekeeping genes from the 85 genes, yet the exact same species clusters are formed and can be used to establish the dimension of divergence or clonality of Y. entomophaga and Y. nurmii. It is obvious that $Y$. entomophaga and $Y$. nurmii form a single lineage. Whilst no clustering analysis is available, comparison with the diversity of other lineages makes it likely that these form a single species complex SC13.

The 17 officially described and recognized species of Yersinia therefore from 13 different species cluster. Of the old species definition, eight designations do not agree with the species complexes, and taxonomy and nomenclature will need to be revised to clarify the nature of the isolates.

\subsubsection{Phylogeny of the genus Yersinia based on 16S rDNA sequences}

The independent evolution of pathogenic lineages in Yersinia has not been noticed before, but it was assumed due to their pathogenic potential in humans that they shared a common pathogenic predecessor. The evolutionary distance of environmental, "Y. enterocolitica-like" species (Sulakvelidze, 2000) appears to have been hugely underestimated as well. The study of only few genes or of genes that are not phylogenetically informative or robust might have skewed the image of an evolutionary relationship as well. One example for this is the 16S rRNA gene (Figure 7.5).

Although this representation of the 16S rRNA genes in the genus is based on corrected Illumina reads and not on finished Sanger sequencing, the sequences for the representative type strains of the environmental Yersiniae fall together with other isolates of their species cluster (Figure 7.5). This indicates that the sequencing data is comparable to the Sanger sequencing approach. Overall, there is very little variation within the $16 \mathrm{~S}$ gene. $95.69 \%$ of the gene, or 1398 out of 1461 bases investigated, are invariable and phylogenetically non-informative. One highly variable region is detected between bases 429 and 449 . Based on this data set, one can see that the phylogeny based on $16 \mathrm{~S}$ rDNA is in disagreement with the 85 housekeeping genes. There is still an indication of clustering according to species cluster, and the Y. enterocolitica and Y. pseudotuberculosis/pestis lineages are still at opposite ends of the tree. Notably though, in $Y$. enterocolitica pathogenic and non-pathogenic lineages intermingle. The distinction of two subspecies was based on variation in 12 bases only (Neubauer, et al., 2000), and the corrected Illumina data might not reflect 
this, yet these subspecies are invalid nonetheless with respect to the whole genome phylogeny.

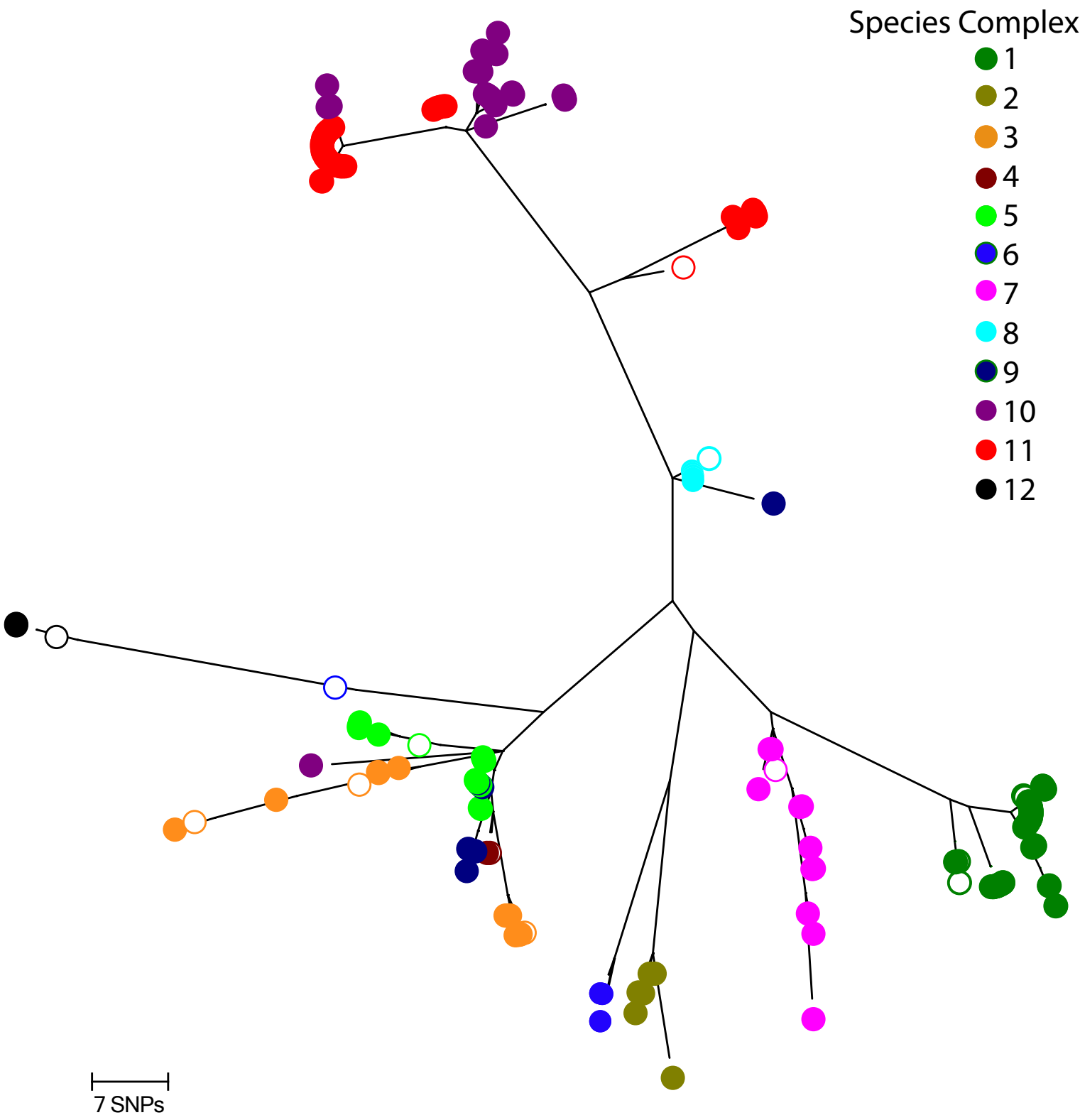

Figure 7.5: The phylogeny of the genus Yersinia based on 16S rDNA sequences. The maximum likelihood phylogenetic tree of the genus Yersinia is based on 1461 bases of the 16S rRNA gene. The species complexes (coloured circles) as allocated by BAPS analysis are shown. The type strains sequenced in previous studies are depicted by open circles.

The clusters formed are the same clusters originally described by Ibrahim, et al. (1993 and 1997). The environmental Yersiniae generally form a heterogeneous cluster. The branching order of individual lineages that was seen in the phylogeny of 85 housekeeping genes cannot be observed, Y. frederiksenii has changed its position to be closer to Y.pseudotuberculosis/pestis, and some isolates of 
Y. mollaretii/bercovieri/aleksiciae as well as Y. intermedia are found on two different branches.

The phylogenetic analysis of 85 housekeeping genes therefore provides much greater resolution of the genus than is possible using the $16 \mathrm{~S}$ gene sequence, which fails to adequately resolve the environmental Yersinia strains. The limitations of species delineation (and assignation) by biochemical tests and $16 \mathrm{~S}$ sequence illustrates the necessity of using a finer scale approach to distinguish between members of the genus, and the need for thorough assessment of the genomic signature of novel species currently being reported in the Yersinia genus.

\subsubsection{Acquisition of the virulence plasmid pYV in pathogenic Yersiniae}

Also shown in Figure 7.3 (inset) is the phylogeny of the virulence plasmid pYV. The acquisiton of this plasmid is thought to be a key element in the evolution of pathogenic Yersiniae lineages. So far, the plasmid has only been found in pathogenic Yersinia spp., and this is confirmed in the present strain collection as pYV was only detected in the Y.pseudotuberculosis/Y. pestis cluster as well as the pathogenic Y. enterocolitica BTs $1 \mathrm{~B}$ and $2-5$. The previous model for the evolution of Yersinia postulated a single acquisition event of pYV in a pathogenic predecessor and subsequent loss in the non-pathogenic Y. enterocolitica BT 1A (Figure 1.2; Portnoy and Falkow, 1981; Carniel, 2002; Wren, 2003). From Figure 7.3 it is evident that this is unlikely. With the environmental isolates positioned between the pathogenic lineages a more parsimonious justification is the acquistion of pYV in two separate events by the pathogenic lineages. Further support to this is given in the evolutionary

distance between virulence plasmids that does not equal the distance of Y. enterocolitica to the Y. pseudotuberculosis/Y. pestis cluster. Drawn to scale, the diversity within the plasmids is considerably less than in the genome (Figure 7.3).

Looking more in detail at the plasmid phylogeny (Figure 7.6) one can see that it relates to the phylogeny seen in the $Y$. enterocolitica lineages. 


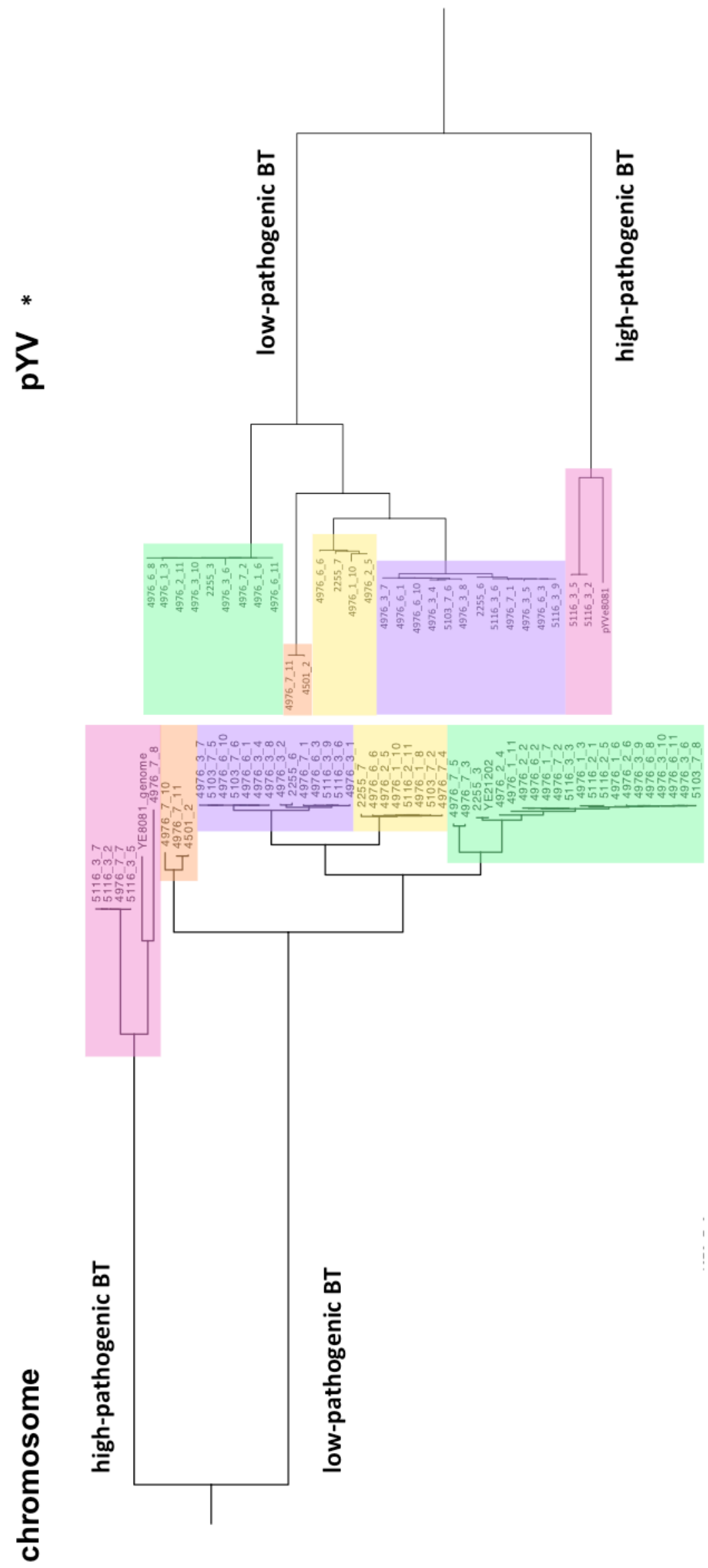

Figure 7.6: Phylogeny of the plasmid pYV compared to the phylogeny of the chromosome in Y. enterocolitica. ${ }^{*}$ not drawn to scale of each other. Red - BT 1B O:8; orange - BT 5 O:2a,2b,3; purple - BT 4 O:3; yellow - BT 2/3 O:5,27; green - BT 2/3 O:9. 
The different lineages of the virulence plasmid exactly reflect the lineages in the pathogenic BT, although the branching order is slightly different. This is consistent with a single acquisition event, but also indicates that the plasmid then co-evolved with a specific lineage and was not transferred between bio-serotypes. It would indicate that the pathogenic strains have adapted to specific niches, which were enabled by the genes encoded on the virulence plasmid. The low-pathogenic BTs show signs of evolving into specific ecotypes, with no exchange of genetic material between them, be it LPS operons, or the virulence plasmid.

The acquisition of the virulence plasmid therefore has added significantly to the pathogenic potential of two different pathogenic species, in independent events and in different genetic backgrounds. 


\subsection{Discussion}

\subsubsection{The evolution of pathogenic $Y$. enterocolitica lineages}

The phylogeny of $Y$. enterocolitica shows that there are three very distinct lineages. $B T 1 A$ is the oldest lineage, from which the pathogenic lineages have evolved through the acquisition of the virulence plasmid on at least one independent occasion. The non-pathogenic BT shows heterogeneity, as indicated by the large number of SNPs between individual isolates resulting in long branch lengths. Due to predominant sampling in a clinical setting and during an abbatoir study, this divergence is likely to be an underestimate. Analysis of the other sequenced isolates might yield a considerable number of unique CDSs other than phage, and the pangenome of BT 1A can be considered open.

The position of BTs $1 \mathrm{~A}$ at the root of the $\mathrm{Y}$. enterocolitica tree explains some of the features of the reference genome. When considering regions of difference between BT $1 \mathrm{~A}$ and the other BTs, it is now apparent that loss in the pathogenic lineages is a driver for evolution rather than acquisition events in the non-pathogenic BT. Inspection for example of the D-arabitol cluster (Chapter 6) shows that most of it has been lost during the evolution of the pathogenic lineages, and that the last gene remnant was lost without trace in the low-pathogenic BTs, whilst the remnant remains in BT 1B. This trajectory also helps to explain the differences in pseudogenes and remnants of the second flagella cluster Flag-2; the operons have been lost independently in $\mathrm{BT} 1 \mathrm{~A}$ and $1 \mathrm{~B}$, and the genome decays has happened in different genes in the cluster. A search for Flag-2 genes in the $Y$. enterocolitica strain collection showed that some BT $1 \mathrm{~A}$ isolates even retain the complete cluster. It is not known, however, whether the clusters are still functional, yet their frequent loss in individual branches indicates a dispensable nature.

The pathogenic BTs have a common evolutionary path, acquiring seven pseudogenes, before splitting into high- and low-pathogenic BTs. The highpathogenic BT is marked by the acquisition of previously mentioned markers of isolates with increased pathogenicity, the HPI, and the Ysa T3SS in the plasticity zone. Considering that only five strains were sequenced, there is still considerable diversity amongst this $\mathrm{BT}$, although it is not comparable with the heterogeneity in BT $1 \mathrm{~A}$. As for the distribution of the pathogenicity islands, the larger strain collection confirmed the Ysa T3SS and the HPI as specific for BT 1B of Y. enterocolitica, 
whereas the YAPI appears to be an acquisition specific to strain 8081. This was found before (Thomson, et al., 2006), and it is similar to Y. pseudotuberculosis, which shows a variable distribution of the HPI and YAPI in different combinations (Fukushima, et al., 2001; Eppinger, et al., 2007).

The low-pathogenic BTs have evolved through genome rearrangements and gene loss, as indicated by the increased number of IS1667 and the marked pseudogenization. The phylogeny of the low-pathogenic BTs of $Y$. enterocolitica is strictly based on serotype. Serotypes $0: 9,0: 5,27,0: 3$, and $0: 2 a, 2 b, 3$ each form their own branch. This is surprising, as the LPS operons can be exchanged in other bacteria, for instance Streptococcus pneumoniae, and serotype switching may occur so that an evolutionary signal based on serotype is not visible (Croucher, et al., 2011). In Y. enterocolitica, the different lineages strictly adhere to serotype, with no indication of serotype recombination. This is also different from the population structure of $Y$. pseudotuberculosis where recombination is frequent, and where parts of the serotype operons appear to undergo exchange and recombination with other clusters (Ch'ng, et al., 2011). The population structure of the low-pathogenic Y. enterocolitica BTs suggest niche adaptation following a bottleneck leading to specific ecovars. This niche could involve different insect vectors suggested by the presence of insecticidal toxin complexes.

One caveat has to be added - the serotypes investigated in this study present the dominant, most prevalent serotypes. The low-pathogenic BTs may exhibit other serotype as well, but these are infrequent and not associated with disease (McNally, et al., 2004; Bottone, 1999). It would be interesting to see where they appear on a phylogenetic tree of the low-pathogenic BTs.

Also in contrast to Y.pseudotuberculosis, the differentiation of BTs in Y. enterocolitica into high-, low-, and non-pathogenic lineages appears to be ancient, with a clear phylogenetic signal and long branches. Although Y. pseudotuberculosis was divided in high- and low-pathogenicity groups as well, these do not present in specific lineages in a MLST tree (Ch'ng, et al., 2011). This population appears to be more dynamic in terms of exchange of pathogenicity islands, whereas Y. enterocolitica seems to have a long history of adaptation, with recent clonal expansion of the low-pathogenic BTs. The short branches in the low-pathogenic BTs, and the fact that unique regions of the reference genomes pertain to phage, this might suggest a closed pan-genome for BTs $2-5$. This means that further sequence 
analysis is unlikely to uncover new pathogenicity islands, similar to the closed pangenome of $Y$. pestis (Eppinger, et al., 2010). This is in contrast to the non-pathogenic BT 1A, and possibly the high-pathogenic BT, which show a reasonable diversity to suggest a pan-genome that is still open for new discoveries.

\subsubsection{The genus Yersinia and the virulence plasmid pYV - independent evolution of pathogenicity}

The phylogeny of the whole genus Yersinia and the virulence plasmid pYV do not agree with the current evolution of pathogenic lineages of Yersinia. The pathogenic lineages are positioned diametrically at opposite ends of the genus tree. The most parsimonious explanation for this is the independent acquisition of the virulence plasmid on at least two separate occasions; this presents $2+$ events as opposed to a single gain in the last common ancestor of Yersinia and 10+ loss events for each environmental lineage. Further to this, not only is the virulence plasmid not present in any of the environmental lineages, the evolutionary distance between the virulence plasmids is too recent to compete with the ancient lineages presented in the analysis of the chromosome. It could be argued that the virulence plasmid is under strict selection and therefore shows less sequence variation. Yet, pYV is only essential inside a warm-blooded host, and the life cycle of Yersinia includes environmental and insect stages. The housekeeping genes on the other hand are under constant selection no matter the environmental condition as they perform essential function for cellular survival. Their divergence should thus give a more accurate estimate and scale of the relationship within the genus.

Interestingly, the virulence plasmid also shows signs of co-evolution with specific lineages in $Y$. enterocolitica. This indicates that the acquisition was a key event that led to a marked change in lifestyle and niche. It implies that the exchange of mobile genetic elements is not important in the low-pathogenic BTs, possibly due to the adaptation to specific niches. This would also explain the strict adherence to serotype in the phylogenetic grouping.

Considering the genus as a whole, it is apparent that both the human pathogenic lineages share patterns of evolution. Their development following the acuqisition of the virulence plasmid is characterised by functional gene loss. In one lineage, this lead to the emergence of the acutely pathogenic $Y$. pestis, and in the other lineage to the low-pathogenic $Y$. enterocolitica biotypes. Functional gene loss is also observed 
in other human pathogens, such as M. tuberculosis and Salmonella, and can thus be considered an emerging pattern on the road to human pathogenicity.

The evolution of $Y$. pestis through gene loss, genomic rearrangements, expansion of IS elements, and metabolic streamlining is exactly mirrored in the low-pathogenic BTs of Y. enterocolitica, especially BTs 4 and 5 . BT 4 is ubiquitous, but is the biotype that is predominantly isolated from human cases in mainland Europe (McNally, et al., 2004; Batzilla, et al., 2011), which indicates some degree of host adaptation, and BT 5 is the hare biotype.

\subsubsection{Differentiation of species in the genus Yersinia}

Several papers have already pointed out that commerically available biochemical tests are insufficient to distinguish Y. enterocolitica from environmental, Y. enterocolitica-like species (Stock, Henrichfreise and Wiedemann, 2002; Merhej, et al., 2008). Especially with identification of new species in the genus, the list of possible phenotypic tests is growing and might become unmanagle, confusing, and impractical. A more useful approach is needed, that unambiguously speciates isolates, without dependency on phenotypes that might vary with reagents, incubation conditions, and are subject to random inactivating mutations.

The 16S rDNA gene that has been commonly used to establish a tree of life is unsuitable for speciation in Yersinia. It has been noted before that the 16S rDNA is only useful up to the genus level, but that sequences most often are too conserved to distinguish individual species (Drancourt and Raoult, 2002; Janda and Abbott, 2007). This is the case for example in Staphylococcus, where for example rpoB, the RNA polymerase gene, and others are used instead of $16 \mathrm{~S}$ gene to speciate isolates (Drancourt and Raoult, 2002; Ghebremedhin, Layer, König and König, 2008; Janda and Abbott, 2007). It has also been shown that, despite the high degree of conservation, and despite the essentiality of the small ribosomal subunit, an exchange of $16 \mathrm{~S}$ rDNA genes between different species is possible and that active recombination between species does not exclude the $16 \mathrm{~S}$ gene (Asai, Zaporojets, Squires and Squires, 1999; Schouls, Schot and Jacobs, 2003). Adding to this the fact that the 16S rDNA gene is commonly present in several copies in a single genome (Schouls, Schot and Jacobs, 2003; Janda and Abbott, 2007), and that sequence variation - however small - are known, makes the 16S rDNA inapt to Yersinia speciation. 
With the 85 housekeeping genes presented here, the development of an MLST scheme reflecting the evolution of the genus is a self-evident proposal. Improvement on the MLST scheme proposed by Kotetishvili, et al. (2005) is currently under way by Miquette Hall to establish a set of seven genes that can be used to differentiate Yersinia isolates exactly, and that will also allow for future identification of potential new species. The Yersinia taxonomy has to be updated to incorporate the findings of this study, and an MLST approach circumvents the reliance on biochemistry. 


\section{Overall Discussion and Future Work}

\subsection{Analysis of reference genomes and plasmids}

The sequencing of the reference genomes highlighted the fact that a single genome might not be representative of a whole species, depending on the heterogeneity of said species. Although the general genome composition is similar in all Y. enterocolitica BTs with respect to overall size, coding density, and functional classes of CDSs, significant differences can be observed on closer inspection relating to pseudogenes and regions that are absent or inactivated in certain lineages.

All BTs have about $80 \%$ of their CDSs in common, signifying the $Y$. enterocolitica core functions. Amongst these are the flagella cluster Flag-1, the tigh-adherence operon, and both hydrogenase operons. A considerable number of CDSs is shared in $B T 1 A$ and $B T 1 B$ as well as $B T 1 A$ and the low-pathogenic $B T s$, indicating differential loss after the split of the pathogenic BTs. The non- and high-pathogenic BTs share two genomic islands, YGI-2, encoding a glycolipoprotein, and YGI-4, an integrated plasmid, as well as regions relating to resistance to heavy metals nickel, cobalt, and arsenic. The non- and low-pathogenic BTs share the Yersinia Genus T3SS, the outer core as part of their LPS, and metabolic properties. On the other hand, only 40 CDSs are shared in all pathogenic BTs apart from the virulence plasmid pYV. Most of these are related to hypothetical or conserved hypothetical proteins and have therefore no allocated or confirmed function in a role in disease, except for the adhesion invasion locus ail. Therefore there is no conserved pathogenic core present in high- and low-pathogenic BTs apart from ail and the virulence plasmid pYV.

The non-pathogenic BT has unique regions involved in arsenic, silver and copper resistance as well as other metabolic operons. The HPI, YAPI, general secretion pathway yst1 and Ysa T3SS were confirmed to be unique to the high-pathogenic BT. The low-pathogenic BTs share the tcPAl and a second flagella cluster. The lowpathogenic BTs are further signified by the expansion of IS1667 leading to wideranged genome rearrangements.

The virulence plasmid pYV shows considerable variation regarding its organization, and should therefore rather be considered a family of closed related plasmids as suggested by Portnoy, Moseley and Falkow (1981). The Yop T3SS forms the 
conserved part of the plasmid, whereas other CDSs can change orientation and position. Nevertheless, the overall functionality of the virulence plasmid is preserved. Smaller variations concerning YscP have been correlated with needle length, but there are no indications that BTs with different needle length show a difference in efficiency of protein transfer into the target cell.

The variations seen between pYV plasmids of high- and low-pathogenic BTs suggest divergent evolution of the virulence plasmid in $Y$. enterocolitica. The origins of replication in low-pathogenic BTs is the same as in Y.pestis and Y. pseudotuberculosis, which could mean that the high-pathogenic pYV evolved through co-integration with a plasmid of a different incompatibility group (Snellings, Popek and Lindler, 2001). The exact evolutionary history and potential exchanges of pYV between Y.pseudotuberculosis/pestis and Y. enterocolitica BTs cannot be established, unless a similar, intermediate plasmid is discovered in an environmental bacterium outside the Yersinia genus.

The unique plasmids add to the overall genetic pool of $Y$. enterocolitica. Whilst the plasmids sequenced from BT $1 \mathrm{~A}$ are cryptic, pSR1-2 is potentially important for tetracycline resistance in the pig host, and pSR4-5 might be able to mobilize the virulence plasmid based on homology with a similar conjugative plasmid.

\subsection{Analysis of phenotypic microarray}

A recurring theme in regions of difference and pseudogene targets are metabolic operons. Metabolism is both a driver and a consequence of host or niche adaptation; it is a driver because the search for nutrients drives bacteria into exploring new environments, and it is a consequence as subsequent metabolic streamlining locks bacteria in a niche. The best example for this are Y. enterocolitica BT 5 and Y. pestis, which exhibit reduced ability to survive outside their respective hosts.

Owing to the fact that $B T 1 A$ is a ubiquitous, environmental organism, it can utilize a wide range of different metabolites, and is adaptable to very different conditions. This provides a survival advantage in the environment, as conditions change with season and with respective current environment such as aquatic or soil environments that pose very different challenges on a bacterium. The unique operons involved in metabolism of nutrients carried in the non-pathogenic BT confirm this. 
The increased number of pseudogenes observed in the low-pathogenic BTs correlates with the decreased metabolic flexibility that is apparent in the phenotypic microarray. The pathogenic BTs have lost part of their metabolic flexibility upon adaptation to human hosts and warm-blooded animals. Both human and animal hosts provide a stable surrounding compared to the outside environment regarding temperature and available nutrients. On the other hand, nutrients might be available that are not found outside the gut environment and that require specialized transport and metabolic operons. Additionally, the availability of other nutrients may be limited and targeted acquisition systems are needed. Examples in the latter category are iron and heme acquisition systems, for example encoded on the high-pathogenicity island of BT 1B, that allow for capturing and utilization of this trace element. The phenotypic microarray thus far only highlighted the loss of functions in the pathogenic BTs rather than the gain of utilizing new nutrients compared to the environmental BT 1A. An in-depth analysis of the $37^{\circ} \mathrm{C}$ data might yield results, but it is possible that the conditions tested do not activate the utilization of those nutrients. Considering the conditions found in the gut, oxygen starvation and change in $\mathrm{pH}$ might be important signalling factors that could be investigated in the future using the phenotypic micorarray.

\subsection{Revised model for the evolution of pathogenicity in the genus Yersinia}

The themes of gene loss, expansion of IS elements, metabolic streamlining and acquisition of mobile genetic elements in the high- and low-pathogenic BTs is reminiscent of the evolutionary path of $Y$. pestis. To investigate the relationships between different Yersinia species, 85 housekeeping genes were chosen for comparison. This extensive set of genes should give a robust representation of this diverse genus. The phylogeny of the genus Yersinia showed that the humanpathogenic lineages evolved independently out of the environmental species, yet employing the same driving forces of "add, stir, and reduce" that have been suggested for Y. pestis (Wren, 2003).

The comparison of the phylogeny of the chromosome of Yersinia spp. against the phylogeny of the virulence plasmid pYV showed that the virulence plasmid has been acquired in at least two instances and more recently than the divergence of the Y. pseudotuberculosis and Y. enterocolitica lineages. This is in disagreement with the 
notion that the pathogenic Yersiniae had a common, pathogenic precursor that acquired the virulence plasmid in a single event.

It also needs to be considered that the environmental species form very distinct lineages from $Y$. enterocolitica and should therefore not be considered 'Y. enteroclitica-like' species purely based on the fact of similar biochemical typing reactions.

The model for the evolution of pathogenicity in the genus Yersinia needs to be revised and one suggestion is presented in Figure 8.1.

From the ancestral Yersinia, several lineages evolved. On opposite ends of the spectrum of diversity are the pathogenic lineages, with the environmental, supposedly non-humanpathogenic species situated in between.

The humanpathogenic lineages have evolved independently, yet employing the same mechanisms. Both have acquired novel genetic material that distinguishes them from environmental species. For Y. pseudotuberculosis, the hemin storage locus and the high-pathogenicity island play an important role. The distinguishing features for $Y$. enterocolitica are less clear; in the past the enterotoxin yst has been suggested (Wren, 2003). Research is currently underway to determine the distribution of virulence factors within the genus, which will also establish descriptors for Y. enterocolitica. For both pathogenic lineages, the acquisition of the virulence plasmid has been crucial in opening a new niche in a warm-blooded host. In $Y$. enterocolitica, this gain has happened after the split of the non-pathgenic, environmental BT 1A. Clustering based on BAPS suggests BT 1A as a separate species cluster, and given the very different nature and environment of $B T 1 A$, this is a reasonable suggestion. Although BT $1 \mathrm{~A}$ is considered non-pathogenic, it is isolated from healthy and ill individuals (Bottone, 1999; Tennant, Grant and Robins-Browne, 2003; Tennant, Skinner, Joe and Robins-Browne, 2005). Research shows though that the ability to cause disease and the mechanism to do so differs markedly from the other pathogenic $Y$. enterocolitica BT, in that survival and hibernation inside macrophages might play a role (McNally, et al., 2006). On the other hand, the pathogenic BTs are not signified by gaining a large number of CDSs, and the nonpathogenic BT shares a considerable number of operons with both high- and lowpathogenic BTs respectively. 


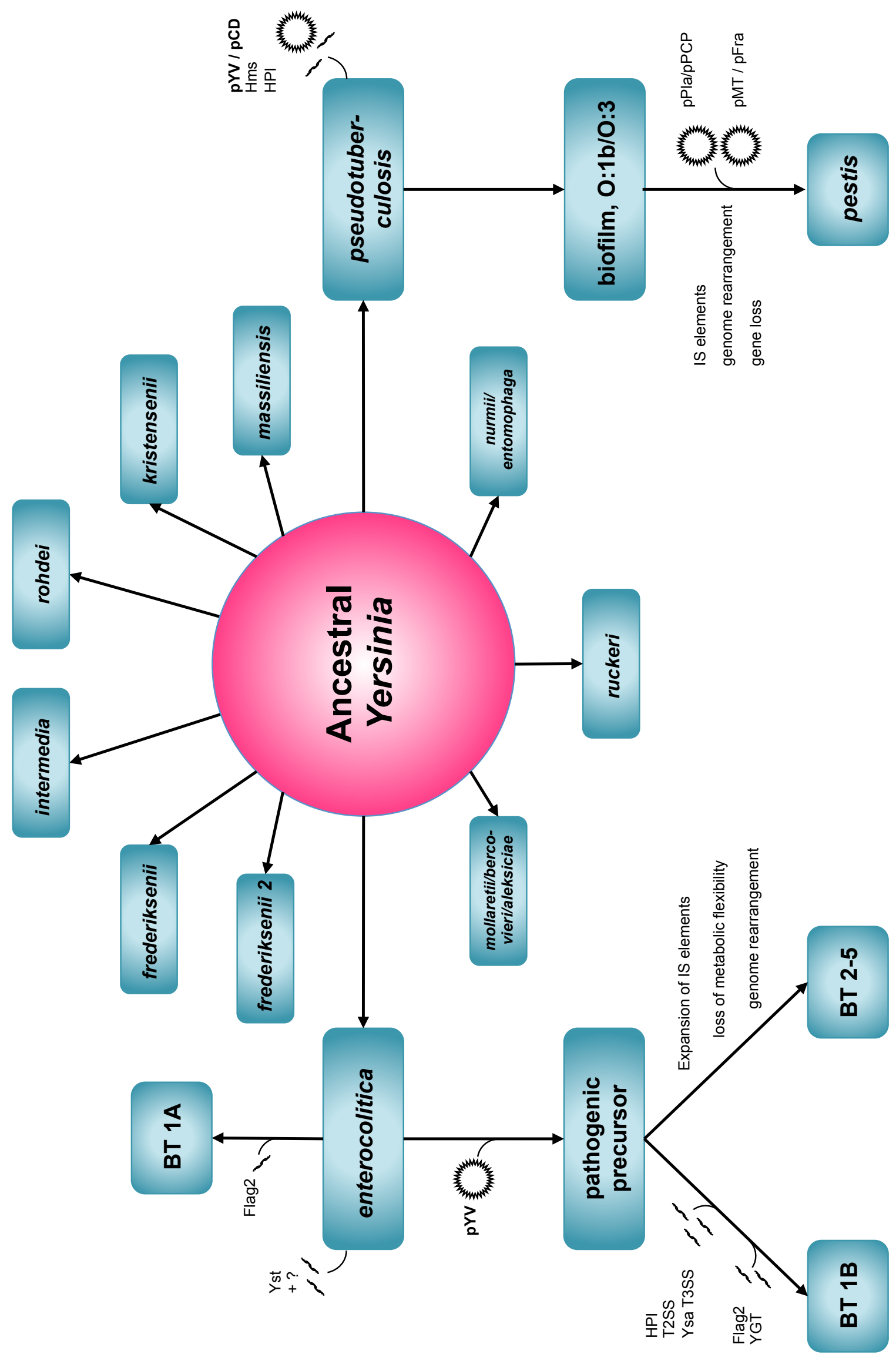

Figure 8.1: Revised model of the evolution of pathogenicity in the genus Yersinia. 


\subsection{Future Work}

"As our circle of knowledge expands, so does the circumference of darkness surrounding it." - Albert Einstein

Now that the evolutionary relationships between the Yersinia spp. have been established, and it was shown that a biotyping approach might not be as suitable for identification as molecular techniques, research is currently underway to determine a set of seven genes for the use in MLST. The genes will be chosen to reflect the lineages identified using BAPS clustering, and will improve speciation in the genus Yersinia. This study should also include the putative species cluster 13, which contains the newly described Y. entomophaga and Y. nurmii, to confirm the initial observation with respect to their relationship to $Y$. ruckeri and as a single species cluster.

A way of improved identification within $Y$. enterocolitica could be the design of a PCR-based approach testing for the presence of O-antigens associated with pathogenic isolates. As only very specific bio-serotype combinations are known to be causing disease, a PCR reaction for genes specific to those serotypes in combination with biochemical data could improve identification of pathogens. The drawbacks are the unknown O-antigen operons that can potentially cross-react. Instead, primers could also be designed to amplify biotype-specific regions, such as the HPI in the high-pathogenic BT 1B, or the tc PAI in low-pathogenic BTs. The caveat for this approach is that a search for important islands and virulence operons is still outstanding. This should determine whether homologous sequences of certain genes are present in the environmental Yersinia species. It would also clarify what defines the species "enterocolitica" as opposed to the environmental species.

In terms of identification, the data from the phenotypic microarray could also be used to potentially define metabolic reactions that reliably distinguish pathogenic and nonpathogenic BTs, but the nutrients identified as different between the BTs would need to be tested in a larger strain collection. A further requirement would be a clear link between phenotype and genotype to avoid past problems of meiotrophy and phenotype reversal due to genes inactivated with revocable mutations.

One interesting region identified during the comparison of the reference genomes of Y. enterocolitica and beyond is the Yersinia Genus T3SS (YGT). The distribution in all Yersinia spp. and the apparently targeted inactivation in humanpathogenic 
lineages of $Y$. pseudotuberculosis/pestis and Y. enterocolitica BT 1B, 4, and 5 might indicate a possible role in environmental survival. This could include interaction with insects such as greater wax moth Galleria mellonella and tobacco hornworm Manduca sexta, nematodes such as roundworm Caenorhabditis elegans or amoeba.

Another open question concerns the essential genes needed during the infection process. As was seen in the comparative analysis, only 30 CDSs plus the virulence plasmid pYV are shared in the pathogenic Y. enterocolitica BTs. It would deepen the understanding of mechanisms of disease to find out about the role of these 30 CDSs in the infection process. One could use RNAseq (Croucher, et al., 2009) to investigate the transcripts of the cell under the experimental condition and give a reflection of active genes and pathways. To complement expression studies, knockout mutants could be examined for their effect, and an interesting advance is the use of TraDIS (Langridge, et al., 2009), the Transposon directed insertion-site sequencing, in which the whole genome is covered with transposon insertions. Genes that are absent of any insertions are thought to be essential under the condition tested (Langridge, et al., 2009). In common to these methods is their global view of the bacterial cell, in trying to understand larger systems and interactions as a whole instead of single entities. This might be important in the case of high- and lowpathogenic BTs that potentially utilize a different combination of genes to establish disease.

Similarly, the phenotypic microarray layout could be used to identify metabolic operons. In this case, only a subset of carbon sources would be studied for a mutant library of one strain. The genes identified under all conditions would represent the normal respiration of a cell, whereas the absence of transposon insertions in some genes under certain conditions only might represent the genes involved specifically in the metabolism of that nutrient. Similarly, RNAseq could identify genes that are generally expressed in carbon metabolism, and also highlight those genes that are specifically upregulated only under certain carbon sources. This would significantly increase annotation precision, and might identify new proteins or assign functions to hypothetical proteins in metabolic pathways.

Further open questions in the phenotypic microarray relate to the role of oxygen saturation in metabolism at $37^{\circ} \mathrm{C}$. The marked reduction of metabolic activity with an increase in temperature might be centrally regulated, and might be triggered in low 
oxygen concentration. A complementation of the phenotypic microarray could be to repeat the experiments under anaerobic conditions at $37^{\circ} \mathrm{C}$. Given the potential roles of insects as vectors and hosts in the environment it could also be of interest to examine the metabolic activities of the BTs at $10-15^{\circ} \mathrm{C}$.

Although the reference genomes of this study have been extensively compared to one another, a comparison to the other recently published genomes would be interesting. The genomes are similar in terms of size, GC content, number of coding sequences, but marked differences were observed in the number of pseudogenes. As the reference genomes presented here are of a high quality draft standard, they could be best compared to the deposited BT 4 0:3 strain Y11 (Batzilla, et al., $2011 a, b)$ of the same quality. The comparison could highlight differences between mainland Europe isolates and UK isolates that relate to their difference in incidence. In this same area, it would also be interesting to take the SNP data one step further than for establishing an evolutionary relationship and relate the SNPs to a biological function. It could be that certain SNPs define human pathogenic isolates despite them not clustering together due to independent SNPs, especially with respect to comparing non-pathogenic and low-pathogenic isolates. It could also be interesting to investigate the SNPs leading to the definition of a lineage, to see whether certain themes relating to membrane proteins and antigen presentation to the host immune system dominate.

Bottone (1999) considered Y. enterocolitica as the "apex of bacterial versatility", but this may also be applied for the whole genus that produced two independent humanpathogenic lineages and a wide range of environmental, underrepresented species. Even nearly 35 years after Bottone's first review of Y. enterocolitica (1977), Yersinia remains an enigmatic and charismatic organism as ever. 


\section{Appendix}

Table 9.1: Y. enterocolitica strain collection.

\begin{tabular}{|c|c|c|c|c|c|}
\hline Strain & Biotype & Serotype & Source & country & Lab \\
\hline 209-36/84 & $\overline{1 \mathrm{~B}}$ & $0: 21$ & "Human & Germany & Fuchs \\
\hline $\mathrm{H} 1527 / 93$ & $1 \mathrm{~A}$ & $0: 5$ & Human & Germany & Fuchs \\
\hline $\mathrm{H} 230 / 89$ & 3 & $0: 5,27$ & Human & Germany & Fuchs \\
\hline H324/78 & 3 & $0: 9$ & Pig & nd & Fuchs \\
\hline $\mathrm{H} 450 / 87$ & 4 & $0: 3$ & Human & nd & Fuchs \\
\hline $\mathrm{H} 469 / 87$ & 4 & $0: 3$ & Pig & nd & Fuchs \\
\hline $\mathrm{H} 608 / 87$ & 4 & $0: 3$ & Human & nd & Fuchs \\
\hline H692/94 & 2 & $0: 9$ & nd & Germany & Fuchs \\
\hline $\mathrm{H} 7580 / 93$ & 3 & $0: 9$ & nd & nd & Fuchs \\
\hline IP10393 & 4 & $0: 3$ & clinical (stool) & France & Carniel \\
\hline IP20322 & 2 & $0: 5,27$ & alimentary & Greece & Carniel \\
\hline IP20851 & 2 & $0: 9$ & clinical (stool) & Spain & Carniel \\
\hline IP21447 & 2 & $0: 9$ & pig (stool) & England & Carniel \\
\hline IP22276 & 4 & $0: 3$ & clinical (stool) & Australia & Carniel \\
\hline IP26249 & 2 & $0: 5,27$ & clinical (stool) & France & Carniel \\
\hline IP26618 & $1 \mathrm{~A}$ & $0: 5$ & alimentary & Italy & Carniel \\
\hline IP26656 & 4 & $0: 3$ & clinical (stool) & France & Carniel \\
\hline IP26766 & 2 & $0: 9$ & clinical (stool) & France & Carniel \\
\hline IP29194 & 3 & $0: 3$ & clinical (stool) & France & Carniel \\
\hline IP29228 & 3 & $0: 5,27$ & clinical (stool) & France & Carniel \\
\hline IP29610 & 4 & $0: 3$ & clinical (stool) & France & Carniel \\
\hline N2118599 & 3 & nd & Human & New Zealand & DuFour \\
\hline N21282756 & 3 & nd & Human blood & New Zealand & DuFour \\
\hline N21383523 & 3 & nd & Human blood & New Zealand & DuFour \\
\hline N2153435 & $1 \mathrm{~A}$ & nd & Human & New Zealand/Fidji & DuFour \\
\hline N21572344 & 4 & $0: 3$ & Animal & New Zealand & DuFour \\
\hline N21622789 & 4 & nd & Dog & New Zealand & DuFour \\
\hline N21873627 & 4 & nd & Human blood & New Zealand & DuFour \\
\hline N22084574 & 4 & nd & Human blood & New Zealand & DuFour \\
\hline N2214757 & 4 & nd & Human blood & New Zealand & DuFour \\
\hline N22232770 & 4 & nd & Human blood & New Zealand & DuFour \\
\hline N22373947 & $1 \mathrm{~A}$ & nd & Sheep & New Zealand & DuFour \\
\hline N2982399 & 2 & nd & Human blood & New Zealand & DuFour \\
\hline NZ1032126 & 2 & nd & Animal meat & New Zealand & DuFour \\
\hline NZ1484423 & 3 & nd & Human & New Zealand & DuFour \\
\hline NZ198109 & 4 & nd & Human blood & New Zealand & DuFour \\
\hline NZ28081 & $1 \mathrm{~A}$ & nd & Human & New Zealand/Asia & DuFour \\
\hline NZ523784 & $1 \mathrm{~B}$ & nd & Human & New Zealand & DuFour \\
\hline NZ761915 & 1B & nd & Human & New Zealand & DuFour \\
\hline NZ832501 & 2 & nd & Human blood & New Zealand & DuFour \\
\hline SZ166/96 & 5 & nd & nd & nd & Fuchs \\
\hline
\end{tabular}




\begin{tabular}{|c|c|c|c|c|c|}
\hline SZ375/04 & 1B & $0: 8$ & Human & Germany & Fuchs \\
\hline SZ506/04 & 1B & $0: 8$ & Human & Germany & Fuchs \\
\hline SZ5108/01 & $1 \mathrm{~B}$ & $0: 8$ & Human & Germany & Fuchs \\
\hline SZ662/97 & $1 \mathrm{~A}$ & $0: 5$ & Human & Germany & Fuchs \\
\hline W22703 & 2 & $0: 9$ & Human & Belgium & Fuchs \\
\hline Y127 & 2 & $0: 9$ & nd & nd & Fuchs \\
\hline Y286 & 1B & $0: 8 b, e, f, i$ & nd & USA & Fuchs \\
\hline YE03/03 & 4 & $0: 3$ & Human faeces & UK & McNally \\
\hline YE04/03 & 3 & $0: 5$ & Human faeces & UK & McNally \\
\hline YE07/03 & 4 & $0: 3$ & Human faeces & UK & McNally \\
\hline YE08/03 & 4 & $0: 3$ & Human faeces & UK & McNally \\
\hline YE09/02 & $1 \mathrm{~A}$ & nd & Sheep & UK & McNally \\
\hline YE09/03 & $1 \mathrm{~A}$ & $0: 6,30$ & Human faeces & UK & McNally \\
\hline YE11/03 & 3 & O:9 & Human case & UK & McNally \\
\hline YE111/02 & 3 & $0: 5,27$ & Sheep & UK & McNally \\
\hline YE119/02 & 2 & $0: 9$ & Sheep & UK & McNally \\
\hline YE13/02 & $1 \mathrm{~A}$ & $0: 6,30$ & Cattle & UK & McNally \\
\hline YE13/03 & $1 \mathrm{~A}$ & O:6,30 & Human faeces & UK & McNally \\
\hline YE17/03 & 3 & nd & Human blood & UK & McNally \\
\hline YE201/02 & 4 & $0: 3$ & Pig & UK & McNally \\
\hline YE204/02 & 4 & $0: 3$ & Pig & UK & McNally \\
\hline YE205/02 & $1 \mathrm{~A}$ & $0: 6,30$ & Pig & UK & McNally \\
\hline YE208/02 & $1 \mathrm{~A}$ & $0: 5$ & Pig & UK & McNally \\
\hline YE213/02 & 4 & $0: 3$ & Pig & UK & McNally \\
\hline YE214/02 & 3 & $0: 9$ & Pig & UK & McNally \\
\hline YE215/02 & 3 & $0: 9$ & Pig & UK & McNally \\
\hline YE218/02 & 3 & $0: 9$ & Pig & UK & McNally \\
\hline YE221/02 & $1 \mathrm{~A}$ & $0: 6,30$ & Pig & UK & McNally \\
\hline YE226/02 & 3 & $0: 5,27$ & Pig & UK & McNally \\
\hline YE227/02 & 4 & $0: 3$ & Pig & UK & McNally \\
\hline YE228/02 & 2 & nd & Pig & UK & McNally \\
\hline YE231/02 & 3 & $0: 5,27$ & Pig & UK & McNally \\
\hline YE232/02 & 3 & $0: 5,27$ & Pig & UK & McNally \\
\hline YE237/02 & 3 & O:9 & Pig & UK & McNally \\
\hline YE24/03 & 3 & $0: 9$ & Human control & UK & McNally \\
\hline YE2702 & $1 \mathrm{~A}$ & $0: 5$ & Sheep & UK & McNally \\
\hline YE30/03 & $1 \mathrm{~A}$ & $0: 5$ & Human case & UK & McNally \\
\hline YE34/03 & $1 \mathrm{~A}$ & $0: 5$ & Human control & UK & McNally \\
\hline Ye3502 & $1 \mathrm{~A}$ & nd & Human case & UK & McNally \\
\hline YE38/03 & $1 \mathrm{~A}$ & O:6,30 & Human case & UK & McNally \\
\hline YE41/03 & $1 \mathrm{~A}$ & O:6,30 & Human case & UK & McNally \\
\hline YE46/02 & $1 \mathrm{~A}$ & nd & Cattle & UK & McNally \\
\hline YE519-36/88 & 5 & nd & nd & nd & Fuchs \\
\hline YE53/30444 & $1 \mathrm{~A}$ & O:7,8 & Pig & Germany & Fuchs \\
\hline YE58/03 & 3 & $0: 9$ & Human control & UK & McNally \\
\hline YE69/03 & $1 \mathrm{~A}$ & $0: 19,8$ & Human case & UK & McNally \\
\hline
\end{tabular}


Table 9.2: Worldwide strain collection of Yersinia spp.

\begin{tabular}{|c|c|c|c|c|c|c|}
\hline Strain & Acc. No & Species & Serotype & Source & Country & Lab \\
\hline ATCC35236 & NZ_ACCB00000000 & aldovae & nd & Drinking water & $\begin{array}{l}\text { Czechoslovaki } \\
\text { a }\end{array}$ & $\mathrm{n} / \mathrm{a}$ \\
\hline $404 / 81$ & & aleksiciae & $0: 16$ & Human stool & Finland & Skurnik \\
\hline $127 / 84$ & & bercovieri & nd & Human stool & Finland & Skurnik \\
\hline $3016 / 84$ & & bercovieri & $0: 58,16$ & Human stool & Finland & Skurnik \\
\hline ATCC43970 & NZ_AALC00000000 & bercovieri & nd & Human stool & France & $\mathrm{n} / \mathrm{a}$ \\
\hline FE80217 & & enterocolitica & nd & Human stool & Finland & Sihvonen \\
\hline 1127 & & enterocolitica & nd & Human & Ireland & Prentice \\
\hline 58735 & & enterocolitica & nd & Human & Ireland & Prentice \\
\hline 2/C/53NMD7 & & enterocolitica & $0: 9$ & Pig & Ireland & Prentice \\
\hline 3430 & & frederiksenii & nd & Human & Ireland & Prentice \\
\hline $112 / 02$ & & frederiksenii & $0: 16$ & Sheep & UK & McNally \\
\hline $120 / 02$ & & frederiksenii & $0: 16$ & Sheep & UK & McNally \\
\hline $22714 / 85$ & & frederiksenii & nd & Human stool & Finland & Skurnik \\
\hline $28 / 85$ & & frederiksenii & nd & nd & Finland & Skurnik \\
\hline $3317 / 84$ & & frederiksenii & $0: 35$ & Human stool & Finland & Skurnik \\
\hline $3400 / 83$ & & frederiksenii & $0: 16$ & Human stool & Finland & Skurnik \\
\hline $38 / 83$ & & frederiksenii & $0: 48$ & Human stool & Finland & Skurnik \\
\hline $498 / 85$ & & frederiksenii & nd & Human stool & Finland & Skurnik \\
\hline ATCC33641 & NZ_AALE00000000 & frederiksenii & nd & Sewage & Denmark & $\mathrm{n} / \mathrm{a}$ \\
\hline BR166/97 & & frederiksenii & nd & nd & Finland & Skurnik \\
\hline FCF208 & & frederiksenii & $0: 16$ & Water & Brazil & Falcao \\
\hline FCF224 & & frederiksenii & $0: 16$ & Water & Brazil & Falcao \\
\hline FCF343 & & frederiksenii & $\mathrm{O}: 2,3$ & Water & Brazil & Falcao \\
\hline FCF467 & & frederiksenii & $0: 16 a, 58$ & Water & Brazil & Falcao \\
\hline FE80151 & & frederiksenii & nd & Human stool & Finland & Sihvonen \\
\hline FE80258 & & frederiksenii & nd & Human stool & Finland & Sihvonen \\
\hline FE80988 & & frederiksenii & nd & Human stool & Finland & Sihvonen \\
\hline IP23047 & & frederiksenii & $\mathrm{O} 3$ & nd & France & Skurnik \\
\hline IP23698 & & frederiksenii & $0: 16$ & nd & France & Carniel \\
\hline IP25924 & & frederiksenii & $0: 46$ & nd & France & Carniel \\
\hline RS-42 & & frederiksenii & nd & Retail meat & Ireland & Prentice \\
\hline 58735 & & intermedia & nd & Human & Ireland & Prentice \\
\hline $109 / 02$ & & intermedia & $0: 16$ & Sheep & UK & McNally \\
\hline $141 / 02$ & & intermedia & rough & Sheep & UK & McNally \\
\hline $182 / 02$ & & intermedia & $0: 52,53$ & Cattle & UK & McNally \\
\hline $821 / 84$ & & intermedia & $0: 52,54$ & Human stool & Finland & Skurnik \\
\hline $9 / 85$ & & intermedia & $0: 16,21$ & nd & Finland & Skurnik \\
\hline $93 / 02$ & & intermedia & O:4,32 & Cattle & UK & McNally \\
\hline $94 / 02$ & & intermedia & nd & Sheep & UK & McNally \\
\hline ATCC29909 & NZ_AALF00000000 & intermedia & nd & Human urine & nd & $\mathrm{n} / \mathrm{a}$ \\
\hline BR16597 & & intermedia & nd & nd & Finland & Skurnik \\
\hline FCF130 & & intermedia & O:4,32 & Sewage water & Brazil & Falcao \\
\hline FCF202 & & intermedia & O:10 & Sea water & Brazil & Falcao \\
\hline FCF335 & & intermedia & $0: 48$ & Polluted water & Brazil & Falcao \\
\hline FCF84 & & intermedia & O:4,32 & Water & Brazil & Falcao \\
\hline IP10066 & & intermedia & O:4,32 & nd & France & Carniel \\
\hline IP10209 & & intermedia & O:4,32 & nd & France & Carniel \\
\hline R148 & & intermedia & nd & Mouse & $?$ & Fukushima \\
\hline Y25 & & intermedia & nd & nd & ? & Fukushima \\
\hline
\end{tabular}




\begin{tabular}{|c|c|c|c|c|c|c|}
\hline 542 & & kristensenii & nd & nd & ? & Fukushima \\
\hline $119 / 84$ & & kristensenii & $0: 12,25$ & Human stool & Finland & Skurnik \\
\hline ATCC33638 & NZ_ACCA00000000 & kristensenii & nd & Human urine & nd & $\mathrm{n} / \mathrm{a}$ \\
\hline FCF221 & & kristensenii & $0: 61$ & Water & Brazil & Falcao \\
\hline FCF324 & & kristensenii & $0: 61$ & Polluted water & Brazil & Falcao \\
\hline FCF326 & & kristensenii & $0: 11,24$ & Polluted water & Brazil & Falcao \\
\hline FCF580 & & kristensenii & $0: 61$ & Oyster & Brazil & Falcao \\
\hline FE80982 & & kristensenii & nd & Human stool & Finland & Sihvonen \\
\hline IP24139 & & kristensenii & Nag & nd & France & Carniel \\
\hline IP6945 & & kristensenii & $0: 16$ & nd & France & Carniel \\
\hline OK6311 & & kristensenii & nd & Marten & $?$ & Fukushima \\
\hline IP27925 & & kristensenii & $\mathrm{O} 3$ & nd & France & Carniel \\
\hline 24070 & & massiliensis & nd & Human & Ireland & Prentice \\
\hline $107 / 02$ & & mollaretii & nd & Sheep & UK & McNally \\
\hline $159 / 02$ & & mollaretii & rough & Sheep & UK & McNally \\
\hline $61 / 02$ & & mollaretii & $0: 1,2 a, 3$ & Sheep & UK & McNally \\
\hline $64 / 02$ & & mollaretii & O? & Sheep & UK & McNally \\
\hline $92 / 84$ & & mollaretii & $0: 59(20,36,7)$ & Human stool & Finland & Skurnik \\
\hline ATCC43969 & NZ_AALD00000000 & mollaretii & nd & Soil & USA & $\mathrm{n} / \mathrm{a}$ \\
\hline FE82747 & & mollaretii & nd & Human stool & Finland & Sihvonen \\
\hline IP22404 & & mollaretii & $\mathrm{O} 3$ & nd & France & Carniel \\
\hline IP25089 & & mollaretii & $\mathrm{O} 20$ & nd & France & Carniel \\
\hline $\mathrm{A} 125 \mathrm{KOH} 2$ & & pekkanenii & nd & Lettuce & Finland & Skurnik \\
\hline Co92 & NC_003143 & pestis Orientalis & na & Fatal human case & USA & Parkhill \\
\hline KIM5 & NC_004088 & pestis Medievalis & na & nd & {$[$ Iran $]$} & Perry \\
\hline Yp91001 & NC_005810 & pestis Microtus & na & Vole & China & Yang \\
\hline 260 & & pseudotuberculosis & $0: 1 a$ & Human & Canada & Fukushima \\
\hline 1231 & & pseudotuberculosis & $0: 4 b$ & Rodent & Russia & Fukushima \\
\hline 2888 & & pseudotuberculosis & $0: 1 a$ & Bird & Italy & Fukushima \\
\hline D585 & & pseudotuberculosis & $0: 3$ & Dog & Japan & Skurnik \\
\hline $\mathrm{IH} 111554$ & & pseudotuberculosis & $0: 1 a$ & Cat & Finland & Siitonen \\
\hline IP31758 & NC_009708 & pseudotuberculosis & $0: 1 b$ & Human stool & former USSR & Carniel \\
\hline IP32463 & & pseudotuberculosis & $0: 5$ & Guinea pig & Switzerland & Carniel \\
\hline IP32544 & & pseudotuberculosis & $0: 3$ & Pig & France & Carniel \\
\hline IP32670 & & pseudotuberculosis & $0: 1$ & Pig & UK & Carniel \\
\hline IP32881 & & pseudotuberculosis & $\mathrm{O}: 2$ & Monkey & Switzerland & Carniel \\
\hline IP32921 & & pseudotuberculosis & $\mathrm{O}: 2$ & Hare & France & Carniel \\
\hline IP32938 & & pseudotuberculosis & $0: 3$ & Cattle & Argentina & Carniel \\
\hline IP32953 & NC_006155 & pseudotuberculosis & O:1 & Human case & France & Carniel \\
\hline IP33038 & & pseudotuberculosis & $0: 1$ & marsupial & Australia & Carniel \\
\hline IP33054 & & pseudotuberculosis & $\mathrm{O}: 2$ & human & Spain & Carniel \\
\hline IP33177 & & pseudotuberculosis & $0: 1$ & cabbage & Russia & Carniel \\
\hline IP33250 & & pseudotuberculosis & $0: 3$ & human & Russia & Carniel \\
\hline MW109-2 & & pseudotuberculosis & $0: 11$ & Water & nd & Fukushima \\
\hline N912 & & pseudotuberculosis & $\mathrm{O}: 2 \mathrm{~b}$ & Rabbit & China & Fukushima \\
\hline N916 & & pseudotuberculosis & $0: 13$ & Rat & Finland & Skurnik \\
\hline No5 & & pseudotuberculosis & $0: 2 b$ & Goat & New Zealand & Fukushima \\
\hline OK5586 & & pseudotuberculosis & $0: 3$ & Racoon dog & Japan & Fukushima \\
\hline OK6088 & & pseudotuberculosis & $0: 10$ & Racoon dog & Japan & Skurnik \\
\hline PB1/+ & NC_010634 & pseudotuberculosis & nd & nd & nd & Nikolich \\
\hline PT682 & & pseudotuberculosis & $0: 2 b$ & Pig & Japan & Fukushima \\
\hline R819 & & pseudotuberculosis & $0: 5 b$ & Mouse & nd & Fukushima \\
\hline
\end{tabular}




\begin{tabular}{|c|c|c|c|c|c|c|}
\hline WP-931201 & & pseudotuberculosis & $0: 15$ & Water & Korea & Fukushima \\
\hline Y428 & & pseudotuberculosis & nd & Badger & Germany & Scholz \\
\hline Y716 & & pseudotuberculosis & $0: 1 a$ & nd & nd & Scholz \\
\hline Y718 & & pseudotuberculosis & $0: 1 b$ & Human & Germany & Scholz \\
\hline Y719 & & pseudotuberculosis & nd & Human & Germany & Scholz \\
\hline Y722 & & pseudotuberculosis & $0: 1$ & Human & Germany & Scholz \\
\hline YPIII & NC_010465 & pseudotuberculosis & nd & nd & nd & Nikolich \\
\hline 3343 & & rohdei & nd & Human & Ireland & Prentice \\
\hline 82589 & & rohdei & nd & nd & Finland & Skurnik \\
\hline $011 / 02$ & & rohdei & $0: 46$ & Sheep & UK & McNally \\
\hline $56 / 02$ & & rohdei & $\mathrm{O} ?$ & Sheep & UK & McNally \\
\hline $65 / 02$ & & rohdei & O? & Sheep & UK & McNally \\
\hline $68 / 02$ & & rohdei & $0: 52$ & Sheep & UK & McNally \\
\hline ATCC43380 & NZ_ACCD00000000 & rohdei & nd & Dog faeces & Germany & $\mathrm{n} / \mathrm{a}$ \\
\hline ATCC29473 & NZ_ACCC00000000 & ruckeri & nd & Trout (clinical) & Idaho_USA & $\mathrm{n} / \mathrm{a}$ \\
\hline OMBL4 & & ruckeri & nd & Fish & Finland & Skurnik \\
\hline RS41 & & ruckeri & nd & nd & Finland & Skurnik \\
\hline Y228 & & similis & $0: 6$ & rabbit & Germany & Scholz \\
\hline Y233 & & similis & $0: 5 b$ & Mole & Japan & Scholz \\
\hline Y252 & & similis & OL5a & Mole & Japan & Scholz \\
\hline
\end{tabular}


Table 9.3: Class key

\section{Functional Group}

1 - Unknown function, no homologues

2 - Cell processes

3 - Protection responses

4 - Transport/binding proteins

\section{5 - Adaptation}

\author{
6 - Cell division \\ 7 - Macromolecule metabolism
}

8 - Macromolecule synthesis, modification

9 - Metabolism of small molecules

\section{Description}

Unknown function, no known homologues

Conserved in E. coli

Conserved in other organism than E.coli

Cell processes

Chemotaxis and mobility

Chromosome replication

Chaperones

Protection responses

Cell killing

Detoxification

Drug/analogue sensitivity

Radiation sensitivity

Transport/binding proteins

Amino acids and amines

Cations

Carbohydrates, organic acids and alcohols; PTS

Anions

Other

Adaptation

Adaptations, atypical conditions

Osmotic adaptation

Fe storage

Nodulation-related

Cell division

Sporulation, differentiation and germination

Macromolecule metabolism

Macromolecule degradation

Degradation of DNA

Degradation of RNA

Degradation of polysaccharides

Degradation of proteins, peptides, glycoproteins

Macromolecule synthesis, modification

Amino acyl tRNA synthesis; tRNA modification

Proteins - translation and modification

RNA synthesis, modification, DNA transcription

tRNA

Basic proteins - synthesis, modification

DNA - replication, repair, restriction/modification

Glycoprotein

Lipopolysaccharide

Lipoprotein

Phospholipids

Polysaccharides - (cytoplasmic)

Protein modification

Metabolism of small molecules

Amino acid biosynthesis

Alanine

Histidine

Isoleucine

Leucine

\section{Classification}

0.0 .0

0.0 .1

0.0 .2

1.0 .0

1.1.1

1.2.1

1.3.1

1.4 .0

1.4 .1

1.4 .2

1.4 .3

1.4 .4

1.5 .0

1.5 .1

1.5 .2

1.5 .3

1.5 .4

1.5 .5

1.6 .0

1.6.1

1.6 .2

1.6 .3

1.6 .4

1.7.1

1.8 .1

2.0 .0

2.1 .0

2.1.1

2.1 .2

2.1 .3

2.1 .4

2.2 .0

2.2.1

2.2.10

2.2.11

2.2.12

2.2.2

2.2 .3

2.2 .4

2.2 .5

2.2 .6

2.2.7

2.2 .8

2.2 .9

3.0 .0

3.1 .0

3.1.1

3.1 .10

3.1.11

3.1.12 


\begin{tabular}{|c|c|c|}
\hline & Lysine & 3.1 .13 \\
\hline & Methionine & 3.1 .14 \\
\hline & Phenylalanine & 3.1 .15 \\
\hline & Proline & 3.1 .16 \\
\hline & Serine & 3.1.17 \\
\hline & Threonine & 3.1.18 \\
\hline & Tryptophan & 3.1 .19 \\
\hline & Arginine & 3.1 .2 \\
\hline & Tyrosine & 3.1 .20 \\
\hline & Valine & 3.1 .21 \\
\hline & Asparagine & 3.1 .3 \\
\hline & Aspartate & 3.1 .4 \\
\hline & Chorismate & 3.1 .5 \\
\hline & Cysteine & 3.1 .6 \\
\hline & Glutamate & 3.1 .7 \\
\hline & Glutamine & 3.1 .8 \\
\hline & Glycine & 3.1 .9 \\
\hline 10 - Biosynthesis of cofactors, carriers & Biosynthesis of cofactors, carriers & 3.2 .0 \\
\hline & Acyl carrier protein $(\mathrm{ACP})$ & 3.2 .1 \\
\hline & Pantothenate, CoA? & 3.2 .10 \\
\hline & Pyridine nucleotide, NAD & 3.2.11 \\
\hline & Pyridoxine & 3.2.12 \\
\hline & Riboflavin & 3.2 .13 \\
\hline & Thiamin & 3.2 .14 \\
\hline & Thioredoxin, glutaredoxin, glutathione & 3.2 .15 \\
\hline & Biotin carboxyl carrier protein (BCCP) & 3.2 .16 \\
\hline & Ferredoxin & 3.2.17 \\
\hline & Biotin & 3.2 .2 \\
\hline & Cobalamin & 3.2 .3 \\
\hline & Enterochelin & 3.2 .4 \\
\hline & Folic acid & 3.2 .5 \\
\hline & Heme, porphyrin, cytochrome biogenesis & 3.2 .6 \\
\hline & Lipoate & 3.2 .7 \\
\hline & Menaquinone, ubiquinone & 3.2 .8 \\
\hline & Molybdopterin & 3.2 .9 \\
\hline 11 - Central intermediary metabolism & Central intermediary metabolism & 3.3 .0 \\
\hline & 2'-Deoxyribonucleotide metabolism & 3.3 .1 \\
\hline & Nucleotide hydrolysis & 3.3.10 \\
\hline & Nucleotide interconversions & 3.3.11 \\
\hline & Oligosaccharides & 3.3.12 \\
\hline & Phosphorus compounds & 3.3 .13 \\
\hline & Polyamine biosynthesis, Putrescine & 3.3.14 \\
\hline & Pool, multipurpose conversions of intermediate metabolism & 3.3 .15 \\
\hline & S-adenosyl methionine & 3.3.16 \\
\hline & Salvage of nucleosides and nucleotides & 3.3.17 \\
\hline & Sugar-nucleotide biosynthesis, conversions & 3.3.18 \\
\hline & Sulfur metabolism & 3.3.19 \\
\hline & Amino sugars & 3.3 .2 \\
\hline & Amino acids & 3.3 .20 \\
\hline & Other & 3.3 .21 \\
\hline & Nitrogen metabolism (urease) & 3.3 .22 \\
\hline & Entner-Douderoff & 3.3 .3 \\
\hline
\end{tabular}




\begin{tabular}{|c|c|c|}
\hline & Gluconeogenesis & 3.3 .4 \\
\hline & Glyoxylate bypass & 3.3 .5 \\
\hline & Incorporation metal ions & 3.3.6 \\
\hline & Misc. glucose metabolism & 3.3.7 \\
\hline & Misc. glycerol metabolism & 3.3.8 \\
\hline & Non-oxidative branch, pentose pwy & 3.3 .9 \\
\hline 12 - Degradation of small molecules & Degradation of small molecules & 3.4 .0 \\
\hline & Amines & 3.4 .1 \\
\hline & Amino acids & 3.4 .2 \\
\hline & Carbon compounds, Cachetuate catabolism & 3.4 .3 \\
\hline & Fatty acids & 3.4 .4 \\
\hline & Other & 3.4 .5 \\
\hline 13 - Energy metabolism, carbon & Energy metabolism, carbon & 3.5 .0 \\
\hline & Aerobic respiration & 3.5.1 \\
\hline & Anaerobic respiration & 3.5.2 \\
\hline & Electron transport, cytochromes + redox & 3.5 .3 \\
\hline & Fermentation & 3.5 .4 \\
\hline & Glycolysis & 3.5 .5 \\
\hline & Oxidative branch, pentose pathway & 3.5.6 \\
\hline & Pyruvate dehydrogenase & 3.5.7 \\
\hline & TCA cycle & 3.5 .8 \\
\hline & ATP-proton motive force & 3.5 .9 \\
\hline 14 - Fatty acid biosynthesis & Fatty acid biosynthesis & 3.6 .0 \\
\hline & Fatty acid and phosphatidic acid biosynthesis & 3.6.1 \\
\hline 15 - Nucleotide biosynthesis & Nucleotide biosynthesis & 3.7 .0 \\
\hline & Purine ribonucleotide biosynthesis & 3.7 .1 \\
\hline & Pyrimidine ribonucleotide biosynthesis & 3.7 .2 \\
\hline & Secondary metabolism & 3.8 .0 \\
\hline & Polyketide synthases & 3.8.1 \\
\hline & Non-ribosomal peptide synthases & 3.8.2 \\
\hline 16 - Cell envelope & Cell envelope & 4.0 .0 \\
\hline & Membrane/exported/lipoproteins & 4.1 .0 \\
\hline & Inner membrane & 4.1 .1 \\
\hline & Murein sacculus, petidoglycan & 4.1 .2 \\
\hline & Outer membrane & 4.1 .3 \\
\hline & Surface polysaccharide \& antigens, EPS & 4.1 .4 \\
\hline & Surface structures & 4.1 .5 \\
\hline & $\mathrm{G}+$ membrane & 4.1 .6 \\
\hline & G+ exported/lipoprotein & 4.1.7 \\
\hline & $\mathrm{G}+$ surface anchored & 4.1 .8 \\
\hline & G+ peptidoglycan, teichoic acid & 4.1 .9 \\
\hline 17 - Ribosome constituents & Ribosome constituents & 4.2 .0 \\
\hline & Ribosomal and stable RNAs & 4.2 .1 \\
\hline & Ribosomal proteins - synthesis, modification & 4.2 .2 \\
\hline & Ribosomes - maturation and modification & 4.2 .3 \\
\hline & Extrachromosomal & 5.0 .0 \\
\hline 18 - Foreign DNA & Laterally acquirred elements & 5.1 .0 \\
\hline & Colicin-related functions & 5.1 .1 \\
\hline & Phage-related functions and prophages & 5.1 .2 \\
\hline & Plasmid-related functions & 5.1 .3 \\
\hline & Transposon-related functions & 5.1 .4 \\
\hline & Pathogenicity island-related functions & 5.1 .5 \\
\hline
\end{tabular}


$\begin{array}{ll}\text { Regulation } & 6.0 .0\end{array}$

Two component system $\quad 6.1 .0$

Sensor kinase $\quad 6.1 .1$

Response regulator $\quad 6.1 .2$

Sensor kinase/response regulator fusion $\quad 6.1 .3$

RNA polymerase core enzyme binding $\quad 6.2 .0$

sigma factor $\quad 6.2 .1$

anti sigma factor $\quad 6.2 .2$

anti sigma factor antagonist $\quad 6.2 .3$

Defined families $\quad 6.3 .0$

AsnC $\quad 6.3 .1$

DeoR $\quad 6.3 .10$

LuxR (GerR) $\quad 6.3 .11$

MerR $\quad 6.3 .12$

ArsR $\quad 6.3 .13$

PadR $\quad 6.3 .14$

LytR $\quad 6.3 .15$

AraC $\quad 6.3 .2$

GntR $\quad 6.3 .3$

ICIR $\quad 6.3 .4$

Lacl $\quad 6.3 .5$

LysR $\quad 6.3 .6$

$\begin{array}{ll}\text { MarR } & 6.3 .7\end{array}$

TetR $\quad 6.3 .8$

ROK $\quad 6.3 .9$

Protein kinases $\quad 6.4 .0$

Serine/threonine $\quad 6.4 .1$

Tyrosine $\quad 6.4 .2$

Others $\quad 6.5 .0$

Not classified (included putative assignments) $\quad 7.0 .0$

DNA sites, no gene product $\quad 7.1 .1$

$\begin{array}{ll}\text { Cryptic genes } & 7.2 .1\end{array}$ 
Table 9.4: Colour-coding key of CDSs.

\begin{tabular}{|c|c|c|c|}
\hline $\begin{array}{l}\text { Colour - } \\
\text { code }\end{array}$ & Colour & Function - Chromosome & Function - Plasmid \\
\hline 0 & White & $\begin{array}{l}\text { Pathogenicity / adaptation / } \\
\text { chaperones }\end{array}$ & Pathogenicity / adaptation \\
\hline 1 & Grey & $\begin{array}{l}\text { Energy metabolism (glycolysis, } \\
\text { electron transport, etc.) }\end{array}$ & Partitioning and maintenance \\
\hline 2 & Red & $\begin{array}{l}\text { Information transfer (transcript- } \\
\text { tion/translation + DNA/RNA } \\
\text { modification }\end{array}$ & Plasmid replication \\
\hline 3 & Green & $\begin{array}{l}\text { Surface (inner membrane, outer } \\
\text { membrane, secreted proteins, } \\
\text { LPS, etc.) }\end{array}$ & $\begin{array}{l}\text { Surface (inner membrane, outer } \\
\text { membrane, secreted proteins, pili, } \\
\text { etc.) }\end{array}$ \\
\hline 4 & Dark blue & Stable RNA & $\begin{array}{l}\text { Inorganic compound / metal } \\
\text { resistance / UV, etc. }\end{array}$ \\
\hline 5 & Bright blue & Degradation of large molecules & Conjugal transfer \\
\hline 6 & Bright pink & Degradation of small molecules & $\begin{array}{l}\text { Antibiotic resistance, surface } \\
\text { exclusion, restriction / } \\
\text { modification systems }\end{array}$ \\
\hline 7 & Yellow & $\begin{array}{l}\text { Central / intermediary / } \\
\text { miscellaneous metabolism }\end{array}$ & Host metabolism \\
\hline 8 & Pale green & Unknown / hypothetical & Unknown / hypothetical \\
\hline 9 & Pale blue & Regulators & Regulators \\
\hline 10 & Orange & Conserved hypothetical & Conserved hypothetical \\
\hline 11 & Brown & $\begin{array}{l}\text { Pseudogenes and partial genes } \\
\text { (remnants) }\end{array}$ & $\begin{array}{l}\text { Pseudogenes and partial genes } \\
\text { (remnants) }\end{array}$ \\
\hline 12 & Pale pink & Phage / IS elements & IS elements \\
\hline 13 & Pale grey & $\begin{array}{l}\text { Some miscellaneous information, } \\
\text { e.g. prosite, but no function }\end{array}$ & $\begin{array}{l}\text { Some miscellaneous information, } \\
\text { e.g. prosite, but no function }\end{array}$ \\
\hline
\end{tabular}




\section{Appendix 9.5: perl script for identification of sets of orthologous genes in seven reference genomes for $Y$. enterocolitica}

\#!/usr/local/bin/perl -w

use strict;

use Bio::PSU::SeqFactory;

my $\$$ file = shift;

my \$seqi = Bio::PSU::SeqFactory->make(-file => \$file,

-format $=>$ 'embl');

my \$obj=Bio::PSU::IO::FTHandler->new;

my (@all_gen_ortho, @gen1_gen2_gen3_gen4_gen5_ortho, @gen1_gen2_gen3_gen4_gen6_ortho,

@gen1_gen2_gen3_gen5_gen6_ortho, @gen1_gen2_gen4_gen5_gen6_ortho, @gen1_gen3_gen4_gen5_gen6_ortho,

@gen2_gen3_gen4_gen5_gen6_ortho, @gen1_gen2_gen3_gen4_ortho, @gen1_gen2_gen3_gen5_ortho,

@gen1_gen2_gen3_gen6_ortho, @gen1_gen2_gen4_gen5_ortho, @gen1_gen2_gen4_gen6_ortho, @gen1_gen2_gen5_gen6_ortho,

@gen1_gen3_gen4_gen5_ortho, @gen1_gen3_gen4_gen6_ortho, @gen1_gen3_gen5_gen6_ortho, @gen1_gen4_gen5_gen6_ortho,

@gen2_gen3_gen4_gen5_ortho, @gen2_gen3_gen4_gen6_ortho, @gen2_gen3_gen5_gen6_ortho, @gen2_gen4_gen5_gen6_ortho,

@gen3_gen4_gen5_gen6_ortho, @gen1_gen2_gen3_ortho, @gen1_gen2_gen4_ortho, @gen1_gen2_gen5_ortho,

@gen1_gen2_gen6_ortho, @gen1_gen3_gen4_ortho, @gen1_gen3_gen5_ortho, @gen1_gen3_gen6_ortho, @gen1_gen4_gen5_ortho,

@gen1_gen4_gen6_ortho, @gen1_gen5_gen6_ortho, @gen2_gen3_gen4_ortho, @gen2_gen3_gen5_ortho, @gen2_gen3_gen6_ortho,

@gen2_gen4_gen5_ortho, @gen2_gen4_gen6_ortho, @gen2_gen5_gen6_ortho, @gen3_gen4_gen5_ortho, @gen3_gen4_gen6_ortho,

@gen3_gen5_gen6_ortho, @gen4_gen5_gen6_ortho, @gen1_gen2_ortho, @gen1_gen3_ortho, @gen1_gen4_ortho, @gen1_gen5_ortho,

@gen1_gen6_ortho, @gen2_gen3_ortho, @gen2_gen4_ortho, @gen2_gen5_ortho, @gen2_gen6_ortho, @gen3_gen4_ortho,

@gen3_gen5_ortho, @gen3_gen6_ortho, @gen4_gen5_ortho, @gen4_gen6_ortho, @gen5_gen6_ortho, @gen1_only_ortho,

@gen2_only_ortho,@gen3_only_ortho, @gen4_only_ortho, @gen5_only_ortho, @gen6_only_ortho, @no_ortho, \$feature );

\# genome1 first comparator genome (gen1), second comparator genome (gen2) are those you are comparing to your genome \$gen1 and $\$ g e n 2$ relate to the identifiers you have used to store the orthologue information. You must also fill in your generic gename pattern $\mathrm{YE}^{\star \star \star *}$ and the qualifier under which it is held eg locus_tag or systematic_id.

\# script extended to generate numbers / groups for comparison of seven different genomes.

my \$gen1= "8081" . "_orthologue";

my \$gen2= "21202" . "_orthologue";

my \$gen3= "5603" . "_orthologue";

my \$gen4= "14902" . "_orthologue";

my \$gen5= "1203" . "_orthologue";

my \$gen6= "3094" . "_orthologue";

my \$genid= "YE5303-";

my \$genqualifier="locus_tag";

\#my \$genqualifier="systematic_id";

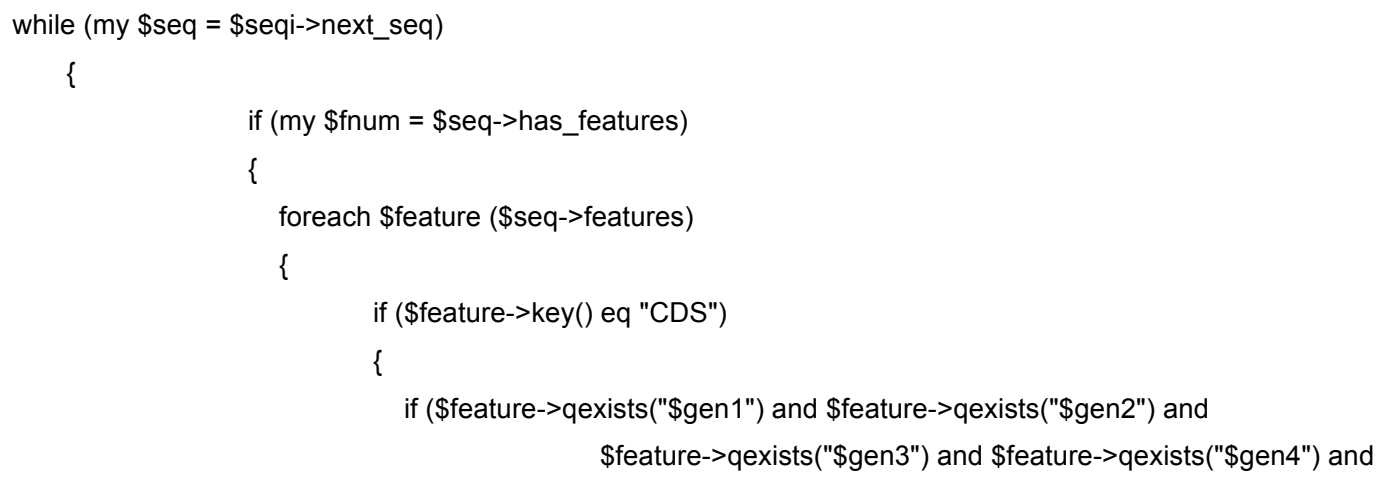


\$feature->qexists("\$gen5") and \$feature->qexists("\$gen6")) \{

push (@all_gen_ortho, \$feature);

\} elsif (\$feature->qexists("\$gen1") and \$feature->qexists("\$gen2") and

\$feature->qexists("\$gen3") and \$feature->qexists("\$gen4") and

\$feature->qexists("\$gen5")) \{

push (@gen1_gen2_gen3_gen4_gen5_ortho, \$feature);

\} elsif (\$feature->qexists("\$gen1") and \$feature->qexists("\$gen2") and

\$feature->qexists("\$gen3") and \$feature->qexists("\$gen4") and

\$feature->qexists("\$gen6")) \{

push (@gen1_gen2_gen3_gen4_gen6_ortho, \$feature);

\} elsif (\$feature->qexists("\$gen1") and \$feature->qexists("\$gen2") and

\$feature->qexists("\$gen3") and \$feature->qexists("\$gen5") and

\$feature->qexists("\$gen6")) \{

push (@gen1_gen2_gen3_gen5_gen6_ortho, \$feature);

\} elsif (\$feature->qexists("\$gen1") and \$feature->qexists("\$gen2") and

\$feature->qexists("\$gen4") and \$feature->qexists("\$gen5") and

\$feature->qexists("\$gen6")) \{

push (@gen1_gen2_gen4_gen5_gen6_ortho, \$feature);

\} elsif (\$feature->qexists("\$gen1") and \$feature->qexists("\$gen3") and

\$feature->qexists("\$gen4") and \$feature->qexists("\$gen5") and

\$feature->qexists("\$gen6")) \{

push (@gen1_gen3_gen4_gen5_gen6_ortho, \$feature);

\} elsif (\$feature->qexists("\$gen2") and \$feature->qexists("\$gen3") and

\$feature->qexists("\$gen4") and \$feature->qexists("\$gen5") and

\$feature->qexists("\$gen6")) \{

push (@gen2_gen3_gen4_gen5_gen6_ortho, \$feature);

\} elsif (\$feature->qexists("\$gen1") and \$feature->qexists("\$gen2") and

\$feature->qexists("\$gen3") and \$feature->qexists("\$gen4")) \{

push (@gen1_gen2_gen3_gen4_ortho, \$feature);

\} elsif (\$feature->qexists("\$gen1") and \$feature->qexists("\$gen2") and

\$feature->qexists("\$gen3") and \$feature->qexists("\$gen5")) \{

push (@gen1_gen2_gen3_gen5_ortho, \$feature);

\} elsif (\$feature->qexists("\$gen1") and \$feature->qexists("\$gen2") and

\$feature->qexists("\$gen3") and \$feature->qexists("\$gen6")) \{

push (@gen1_gen2_gen3_gen6_ortho, \$feature);

\} elsif (\$feature->qexists("\$gen1") and \$feature->qexists("\$gen2") and

\$feature->qexists("\$gen4") and \$feature->qexists("\$gen5")) \{

push (@gen1_gen2_gen4_gen5_ortho, \$feature);

\} elsif (\$feature->qexists("\$gen1") and \$feature->qexists("\$gen2") and

\$feature->qexists("\$gen4") and \$feature->qexists("\$gen6")) \{

push (@gen1_gen2_gen4_gen6_ortho, \$feature);

\} elsif (\$feature->qexists("\$gen1") and \$feature->qexists("\$gen2") and

\$feature->qexists("\$gen5") and \$feature->qexists("\$gen6")) \{

push (@gen1_gen2_gen5_gen6_ortho, \$feature);

\} elsif (\$feature->qexists("\$gen1") and \$feature->qexists("\$gen3") and

\$feature->qexists("\$gen4") and \$feature->qexists("\$gen5")) \{

push (@gen1_gen3_gen4_gen5_ortho, \$feature);

\} elsif (\$feature->qexists("\$gen1") and \$feature->qexists("\$gen3") and

\$feature->qexists("\$gen4") and \$feature->qexists("\$gen6")) \{

push (@gen1_gen3_gen4_gen6_ortho, \$feature);

\} elsif (\$feature->qexists("\$gen1") and \$feature->qexists("\$gen3") and 
\$feature->qexists("\$gen5") and \$feature->qexists("\$gen6")) \{

push (@gen1_gen3_gen5_gen6_ortho, \$feature);

\} elsif (\$feature->qexists("\$gen1") and \$feature->qexists("\$gen4") and

\$feature->qexists("\$gen5") and \$feature->qexists("\$gen6")) \{

push (@gen1_gen4_gen5_gen6_ortho, \$feature);

\} elsif (\$feature->qexists("\$gen2") and \$feature->qexists("\$gen3") and

\$feature->qexists("\$gen4") and \$feature->qexists("\$gen5")) \{

push (@gen2_gen3_gen4_gen5_ortho, \$feature);

\} elsif (\$feature->qexists("\$gen2") and \$feature->qexists("\$gen3") and

\$feature->qexists("\$gen4") and \$feature->qexists("\$gen6")) \{

push (@gen2_gen3_gen4_gen6_ortho, \$feature);

\} elsif (\$feature->qexists("\$gen2") and \$feature->qexists("\$gen3") and

\$feature->qexists("\$gen5") and \$feature->qexists("\$gen6")) \{

push (@gen2_gen3_gen5_gen6_ortho, \$feature);

\} elsif (\$feature->qexists("\$gen2") and \$feature->qexists("\$gen4") and

\$feature->qexists("\$gen5") and \$feature->qexists("\$gen6")) \{

push (@gen2_gen4_gen5_gen6_ortho, \$feature);

\} elsif (\$feature->qexists("\$gen3") and \$feature->qexists("\$gen4") and

\$feature->qexists("\$gen5") and \$feature->qexists("\$gen6")) \{

push (@gen3_gen4_gen5_gen6_ortho, \$feature);

\} elsif (\$feature->qexists("\$gen1") and \$feature->qexists("\$gen2") and

\$feature->qexists("\$gen3")) \{

push (@gen1_gen2_gen3_ortho, \$feature);

\} elsif (\$feature->qexists("\$gen1") and \$feature->qexists("\$gen2") and

\$feature->qexists("\$gen4")) \{

push (@gen1_gen2_gen4_ortho, \$feature);

\} elsif (\$feature->qexists("\$gen1") and \$feature->qexists("\$gen2") and

\$feature->qexists("\$gen5")) \{

push (@gen1_gen2_gen5_ortho, \$feature);

\} elsif (\$feature->qexists("\$gen1") and \$feature->qexists("\$gen2") and

\$feature->qexists("\$gen6")) \{

push (@gen1_gen2_gen6_ortho, \$feature);

\} elsif (\$feature->qexists("\$gen1") and \$feature->qexists("\$gen3") and

\$feature->qexists("\$gen4")) \{

push (@gen1_gen3_gen4_ortho, \$feature);

\} elsif (\$feature->qexists("\$gen1") and \$feature->qexists("\$gen3") and

\$feature->qexists("\$gen5")) \{

push (@gen1_gen3_gen5_ortho, \$feature);

\} elsif (\$feature->qexists("\$gen1") and \$feature->qexists("\$gen3") and

\$feature->qexists("\$gen6")) \{

push (@gen1_gen3_gen6_ortho, \$feature);

\} elsif (\$feature->qexists("\$gen1") and \$feature->qexists("\$gen4") and

\$feature->qexists("\$gen5")) \{

push (@gen1_gen4_gen5_ortho, \$feature);

\} elsif (\$feature->qexists("\$gen1") and \$feature->qexists("\$gen4") and

\$feature->qexists("\$gen6")) \{

push (@gen1_gen4_gen6_ortho, \$feature);

\} elsif (\$feature->qexists("\$gen1") and \$feature->qexists("\$gen5") and

\$feature->qexists("\$gen6")) \{

push (@gen1_gen5_gen6_ortho, \$feature);

\} elsif (\$feature->qexists("\$gen2") and \$feature->qexists("\$gen3") and

\$feature->qexists("\$gen4")) \{ 
push (@gen2_gen3_gen4_ortho, \$feature);

\} elsif (\$feature->qexists("\$gen2") and \$feature->qexists("\$gen3") and

\$feature->qexists("\$gen5")) \{

push (@gen2_gen3_gen5_ortho, \$feature);

\} elsif (\$feature->qexists("\$gen2") and \$feature->qexists("\$gen3") and

\$feature->qexists("\$gen6")) \{

push (@gen2_gen3_gen6_ortho, \$feature);

\} elsif (\$feature->qexists("\$gen2") and \$feature->qexists("\$gen4") and

\$feature->qexists("\$gen5")) \{

push (@gen2_gen4_gen5_ortho, \$feature);

\} elsif (\$feature->qexists("\$gen2") and \$feature->qexists("\$gen4") and

\$feature->qexists("\$gen6")) \{

push (@gen2_gen4_gen6_ortho, \$feature);

\} elsif (\$feature->qexists("\$gen2") and \$feature->qexists("\$gen5") and

\$feature->qexists("\$gen6")) \{

push (@gen2_gen5_gen6_ortho, \$feature);

\} elsif (\$feature->qexists("\$gen3") and \$feature->qexists("\$gen4") and

\$feature->qexists("\$gen5")) \{

push (@gen3_gen4_gen5_ortho, \$feature);

\} elsif (\$feature->qexists("\$gen3") and \$feature->qexists("\$gen4") and

\$feature->qexists("\$gen6")) \{

push (@gen3_gen4_gen6_ortho, \$feature);

\} elsif (\$feature->qexists("\$gen3") and \$feature->qexists("\$gen5") and

\$feature->qexists("\$gen6")) \{

push (@gen3_gen5_gen6_ortho, \$feature);

\} elsif (\$feature->qexists("\$gen4") and \$feature->qexists("\$gen5") and

\$feature->qexists("\$gen6")) \{

push (@gen4_gen5_gen6_ortho, \$feature)

\} elsif (\$feature->qexists("\$gen1") and \$feature->qexists("\$gen2")) \{ push (@gen1_gen2_ortho, \$feature);

\} elsif (\$feature->qexists("\$gen1") and \$feature->qexists("\$gen3")) \{ push (@gen1_gen3_ortho, \$feature);

\} elsif (\$feature->qexists("\$gen1") and \$feature->qexists("\$gen4")) \{ push (@gen1_gen4_ortho, \$feature);

\} elsif (\$feature->qexists("\$gen1") and \$feature->qexists("\$gen5")) \{ push (@gen1_gen5_ortho, \$feature);

\} elsif (\$feature->qexists("\$gen1") and \$feature->qexists("\$gen6")) \{ push (@gen1_gen6_ortho, \$feature);

\} elsif (\$feature->qexists("\$gen2") and \$feature->qexists("\$gen3")) \{ push (@gen2_gen3_ortho, \$feature);

\} elsif (\$feature->qexists("\$gen2") and \$feature->qexists("\$gen4")) \{ push (@gen2_gen4_ortho, \$feature);

\} elsif (\$feature->qexists("\$gen2") and \$feature->qexists("\$gen5")) \{ push (@gen2_gen5_ortho, \$feature);

\} elsif (\$feature->qexists("\$gen2") and \$feature->qexists("\$gen6")) \{ push (@gen2_gen6_ortho, \$feature);

\} elsif (\$feature->qexists("\$gen3") and \$feature->qexists("\$gen4")) \{ push (@gen3_gen4_ortho, \$feature);

\} elsif (\$feature->qexists("\$gen3") and \$feature->qexists("\$gen5")) \{ push (@gen3_gen5_ortho, \$feature);

\} elsif (\$feature->qexists("\$gen3") and \$feature->qexists("\$gen6")) \{ push (@gen3_gen6_ortho, \$feature); 


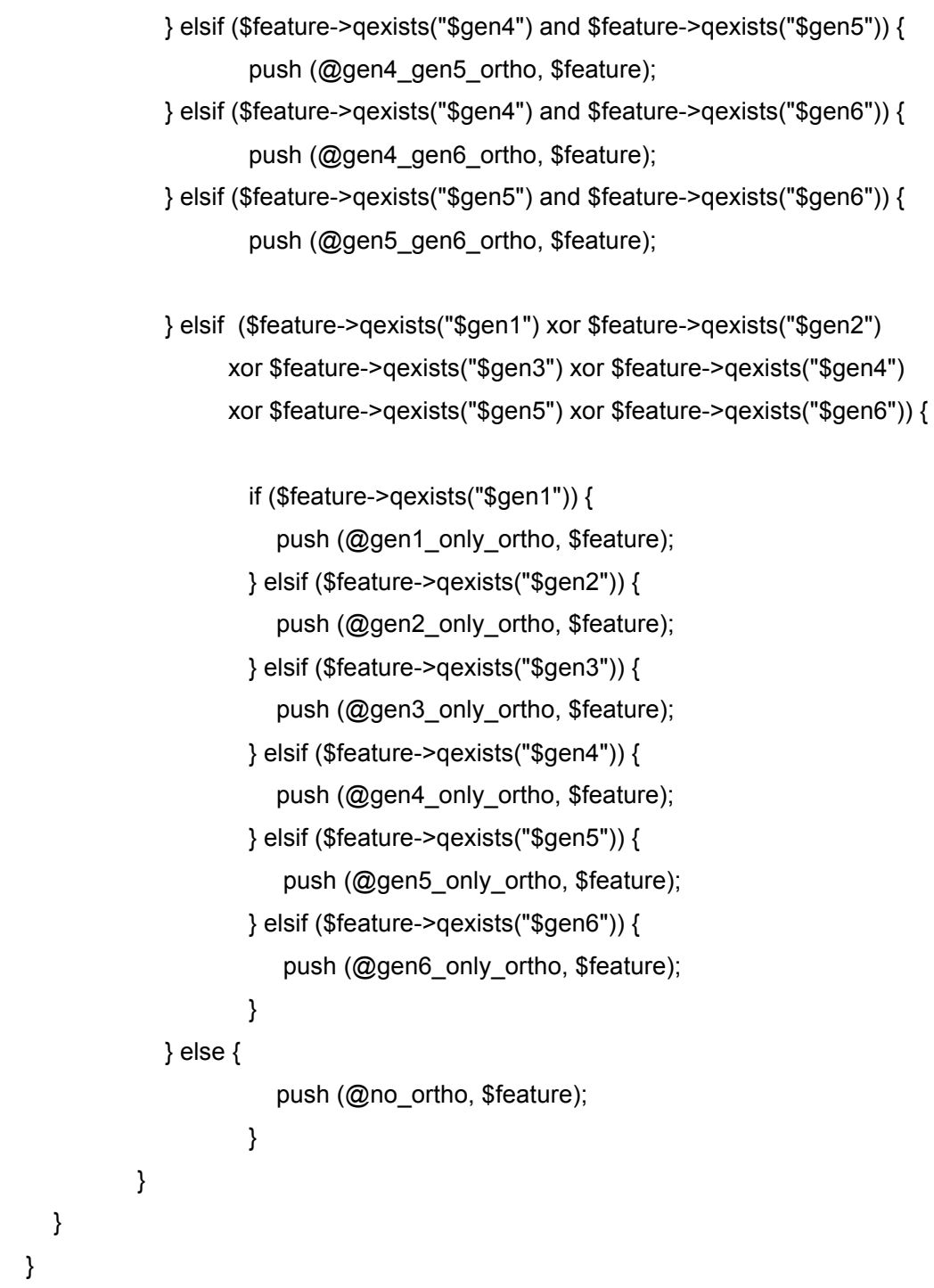

my $\$ n=0$;

my $\$ \mathrm{~m}=0$;

my $\$ 1=0$;

my $\$ y=0$;

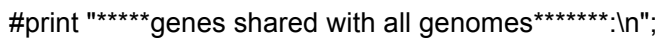

foreach my \$feature1 (@all_gen_ortho) \{

\$feature1->qadd('group', 1);

print \$obj->make_feature_block(\$feature1);\}

\#print "***** genes shared with genome1 (\$gen1), genome2 (\$gen2), genome3 (\$gen3), genome4 (\$gen4) and genome5 (\$gen5) ${ }^{\star * * *: I n " ; ~}$ foreach my \$feature2 (@gen1_gen2_gen3_gen4_gen5_ortho) \{

\$feature2->qadd('group', 2);

print \$obj->make_feature_block(\$feature2);\}

\#print " ${ }^{* \star * \star *}$ genes shared with genome1 (\$gen1), genome2 (\$gen2), genome3 (\$gen3), genome4 (\$gen4) and genome6 (\$gen6) ${ }^{\star * \star *: I n " ; ~}$ foreach my \$feature3 (@gen1_gen2_gen3_gen4_gen6_ortho) \{

\$feature3->qadd('group', 3);

print \$obj->make_feature_block(\$feature3);\}

\#print "***** genes shared with genome1 (\$gen1), genome2 (\$gen2), genome3 (\$gen3), genome5 (\$gen5) and genome6 (\$gen6) ${ }^{\star * * *: I n " ; ~}$ foreach my \$feature4 (@gen1_gen2_gen3_gen5_gen6_ortho) \{ 
\$feature4->qadd('group', 4);

print \$obj->make_feature_block(\$feature4);\}

\#print "*****genes shared with genome1 (\$gen1), genome2 (\$gen2), genome4 (\$gen4), genome5 (\$gen5) and genome6 (\$gen6) ${ }^{* * * *: I n " ; ~}$ foreach my \$feature5 (@gen1_gen2_gen4_gen5_gen6_ortho) \{

\$feature5->qadd('group', 5);

print \$obj->make_feature_block(\$feature5);\}

\#print "***** genes shared with genome1 (\$gen1), genome3 (\$gen3), genome4 (\$gen4), genome5 (\$gen5) and genome6 (\$gen6) ${ }^{\star * * *: I n " ; ~}$ foreach my \$feature6 (@gen1_gen3_gen4_gen5_gen6_ortho) \{

\$feature6->qadd('group', 6);

print \$obj->make_feature_block(\$feature6);\}

\#print "*****genes shared with genome2 (\$gen2), genome3 (\$gen3), genome4 (\$gen4), genome5 (\$gen5) and genome6 (\$gen6) ${ }^{* * * *: I n " ; ~}$ foreach my \$feature7 (@gen2_gen3_gen4_gen5_gen6_ortho) \{

\$feature7->qadd('group', 7);

print \$obj->make_feature_block(\$feature7);\}

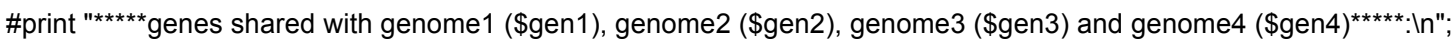

foreach my \$feature8 (@gen1_gen2_gen3_gen4_ortho) \{

\$feature8->qadd('group', 8);

print \$obj->make_feature_block(\$feature8);\}

\#print "*****genes shared with genome1 (\$gen1), genome2 (\$gen2), genome3 (\$gen3) and genome5 (\$gen5) ${ }^{* * * *: I n " ; ~}$ foreach my \$feature9 (@gen1_gen2_gen3_gen5_ortho) \{

\$feature9->qadd('group', 9);

print \$obj->make_feature_block(\$feature9);\}

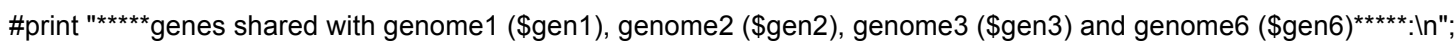
foreach my \$feature10 (@gen1_gen2_gen3_gen6_ortho) \{

\$feature10->qadd('group', 10);

print \$obj->make_feature_block(\$feature10);\}

\#print "***** genes shared with genome1 (\$gen1), genome2 (\$gen2), genome4 (\$gen4) and genome5 (\$gen5) ${ }^{* * * *: \ln " ; ~}$ foreach my \$feature11 (@gen1_gen2_gen4_gen5_ortho) \{

\$feature11->qadd('group', 11);

print \$obj->make_feature_block(\$feature11);\}

\#print "*****genes shared with genome1 (\$gen1), genome2 (\$gen2), genome4 (\$gen4) and genome6 (\$gen6) ${ }^{\star * * *: I n " ; ~}$ foreach my \$feature12 (@gen1_gen2_gen4_gen6_ortho) \{

\$feature12->qadd('group', 12);

print \$obj->make_feature_block(\$feature12);\}

\#print "***** genes shared with genome1 (\$gen1), genome2 (\$gen2), genome5 (\$gen5) and genome6 (\$gen6) ${ }^{* * \star *:}: \ln "$ foreach my \$feature13 (@gen1_gen2_gen5_gen6_ortho) \{

\$feature13->qadd('group', 13);

print \$obj->make_feature_block(\$feature13);\}

\#print "*****genes shared with genome1 (\$gen1), genome3 (\$gen3), genome4 (\$gen4) and genome5 (\$gen5) ${ }^{\star * * *: I n ; ~}$ foreach my \$feature14 (@gen1_gen3_gen4_gen5_ortho) \{

\$feature14->qadd('group', 14);

print \$obj->make_feature_block(\$feature14);\} 
\#print "***** genes shared with genome1 (\$gen1), genome3 (\$gen3), genome4 (\$gen4) and genome6 (\$gen6) $)^{\star * * *: I n " ; ~}$ foreach my \$feature15 (@gen1_gen3_gen4_gen6_ortho) \{

\$feature15->qadd('group', 15);

print \$obj->make_feature_block(\$feature15);\}

\#print "***** genes shared with genome1 (\$gen1), genome3 (\$gen3), genome5 (\$gen5) and genome6 (\$gen6) ${ }^{\star * \star *: I n " ; ~}$ foreach my \$feature16 (@gen1_gen3_gen5_gen6_ortho) \{

\$feature16->qadd('group', 16);

print \$obj->make_feature_block(\$feature16);\}

\#print " ${ }^{* * * * *}$ genes shared with genome1 (\$gen1), genome4 (\$gen4), genome5 (\$gen5) and genome6 (\$gen6) ${ }^{\star * * *: \ln " ; ~}$ foreach my \$feature17 (@gen1_gen4_gen5_gen6_ortho) \{

\$feature17->qadd('group', 17);

print \$obj->make_feature_block(\$feature17);\}

\#print "***** genes shared with genome2 (\$gen2), genome3 (\$gen3), genome4 (\$gen4) and genome5 (\$gen5) ${ }^{\star * * * * * *: I n " ; ~}$ foreach my \$feature18 (@gen2_gen3_gen4_gen5_ortho) \{

\$feature18->qadd('group', 18);

print \$obj->make_feature_block(\$feature18);\}

\#print "***** genes shared with genome2 (\$gen2), genome3 (\$gen3), genome4 (\$gen4) and genome6 (\$gen6) ${ }^{\star * * * * * *:}: \ln " ;$ foreach my \$feature19 (@gen2_gen3_gen4_gen6_ortho) \{

\$feature19->qadd('group', 19);

print \$obj->make_feature_block(\$feature19);\}

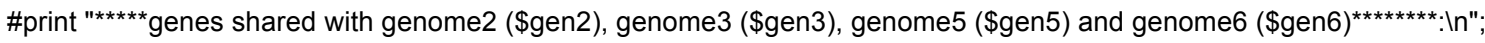
foreach my \$feature20 (@gen2_gen3_gen5_gen6_ortho) \{

\$feature20->qadd('group', 20);

print \$obj->make_feature_block(\$feature20);

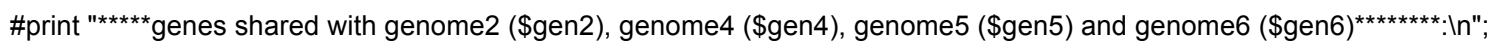
foreach my \$feature21 (@gen2_gen4_gen5_gen6_ortho) \{

\$feature21->qadd('group', 21);

print \$obj->make_feature_block(\$feature21);\}

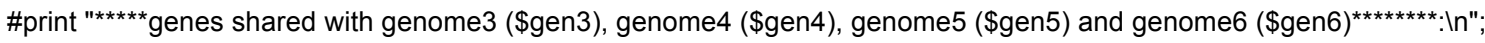
foreach my \$feature22 (@gen3_gen4_gen5_gen6_ortho) \{

\$feature22->qadd('group', 22)

print \$obj->make_feature_block(\$feature22);

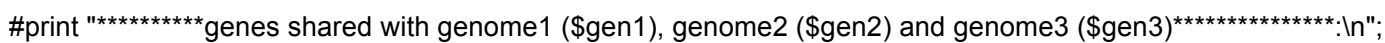

foreach my \$feature23 (@gen1_gen2_gen3_ortho) \{

\$feature23->qadd('group', 23);

print \$obj->make_feature_block(\$feature23);\}

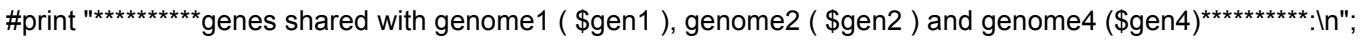

foreach my \$feature24 (@gen1_gen2_gen4_ortho) \{

\$feature24->qadd('group', 24);

print \$obj->make_feature_block(\$feature24);

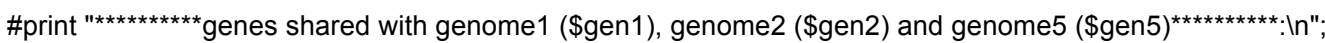

foreach my \$feature25 (@gen1_gen2_gen5_ortho) \{

\$feature25->qadd('group', 25); 
print \$obj->make_feature_block(\$feature25);\}

\#print "********** genes shared with genome1 (\$gen1), genome2 (\$gen2) and genome6 (\$gen6) $)^{\star * * * * * * * *: I n " ; ~}$ foreach my \$feature26 (@gen1_gen2_gen6_ortho) \{

\$feature26->qadd('group', 26);

print \$obj->make_feature_block(\$feature26);\}

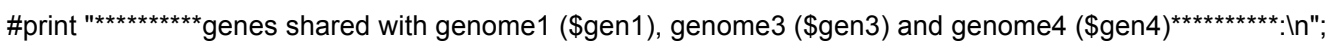
foreach my \$feature27 (@gen1_gen3_gen4_ortho) \{

\$feature27->qadd('group', 27);

print \$obj->make_feature_block(\$feature27);\}

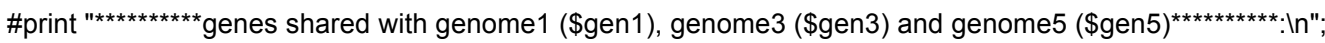
foreach my \$feature28 (@gen1_gen3_gen5_ortho) \{

\$feature28->qadd('group', 28);

print \$obj->make_feature_block(\$feature28);\}

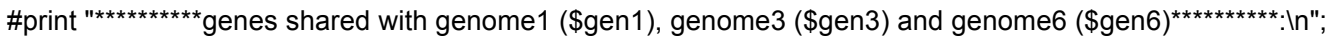
foreach my \$feature29 (@gen1_gen3_gen6_ortho) \{

\$feature29->qadd('group', 29);

print \$obj->make_feature_block(\$feature29);\}

\#print "********** genes shared with genome1 (\$gen1), genome4 (\$gen4) and genome5 (\$gen5) foreach my \$feature30 (@gen1_gen4_gen5_ortho) \{

\$feature30->qadd('group', 30);

print \$obj->make_feature_block(\$feature30);

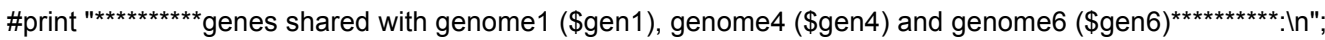
foreach my \$feature31 (@gen1_gen4_gen6_ortho) \{

\$feature31->qadd('group', 31);

print \$obj->make_feature_block(\$feature31);\}

\#print "********** genes shared with genome1 (\$gen1), genome5 (\$gen5) and genome6 (\$gen6) ${ }^{\star * * * * * * * *}: \ln " ;$ foreach my \$feature32 (@gen1_gen5_gen6_ortho) \{

\$feature32->qadd('group', 32);

print \$obj->make_feature_block(\$feature32);\}

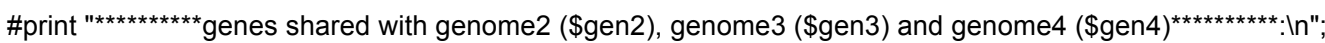
foreach my \$feature33 (@gen2_gen3_gen4_ortho) \{

\$feature33->qadd('group', 33);

print \$obj->make_feature_block(\$feature33);\}

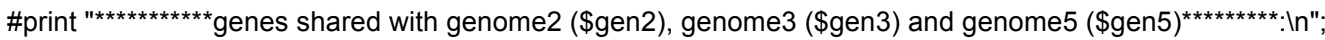
foreach my \$feature34 (@gen2_gen3_gen5_ortho) \{

\$feature34->qadd('group', 34);

print \$obj->make_feature_block(\$feature34);\}

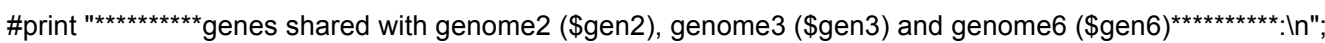
foreach my \$feature35 (@gen2_gen3_gen6_ortho) \{

\$feature35->qadd('group', 35);

print \$obj->make_feature_block(\$feature35);

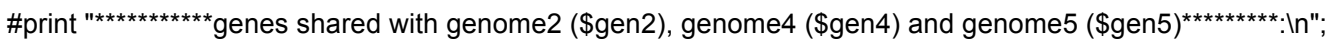


foreach my \$feature36 (@gen2_gen4_gen5_ortho) \{

\$feature36->qadd('group', 36);

print \$obj->make_feature_block(\$feature36);

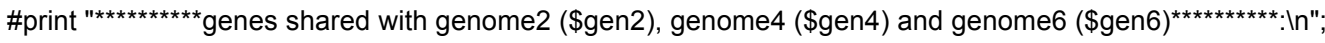

foreach my \$feature37 (@gen2_gen4_gen6_ortho) \{

\$feature37->qadd('group', 37);

print \$obj->make_feature_block(\$feature37);\}

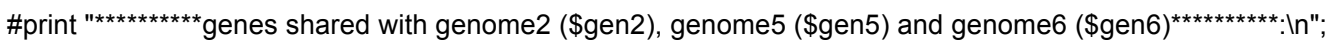

foreach my \$feature38 (@gen2_gen5_gen6_ortho) \{

\$feature38->qadd('group', 38);

print \$obj->make_feature_block(\$feature38);\}

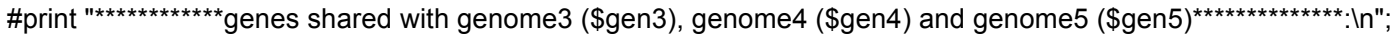
foreach my \$feature39 (@gen3_gen4_gen5_ortho) \{

\$feature39->qadd('group', 39);

print \$obj->make_feature_block(\$feature39);

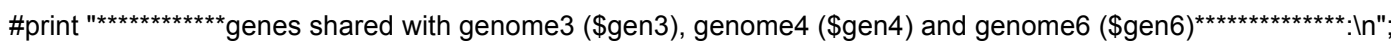

foreach my \$feature40 (@gen3_gen4_gen6_ortho) \{

\$feature40->qadd('group', 40);

print \$obj->make_feature_block(\$feature40);

\#print " "************ genes shared with genome3 (\$gen3), genome5 (\$gen5) and genome6 (\$gen6) ${ }^{* * * * * * * * * * * * *: \ln " ;}$ foreach my \$feature41 (@gen3_gen5_gen6_ortho) \{

\$feature41->qadd('group', 41);

print \$obj->make_feature_block(\$feature41);\}

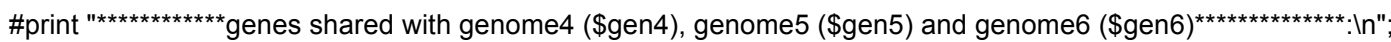

foreach my \$feature42 (@gen4_gen5_gen6_ortho) \{

\$feature42->qadd('group', 42);

print \$obj->make_feature_block(\$feature42);\}

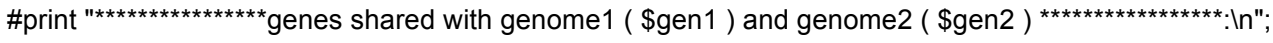

foreach my \$feature43 (@gen1_gen2_ortho) \{

\$feature43->qadd('group', 43);

print \$obj->make_feature_block(\$feature43);\}

\#print "****************** genes shared with genome1 (\$gen1) and genome3 $(\text { ggen3 })^{* * * * * * * * * * * * * * * * * *}: \ln "$;

foreach my \$feature44 (@gen1_gen3_ortho) \{

\$feature44->qadd('group', 44);

print \$obj->make_feature_block(\$feature44);\}

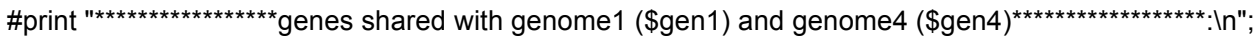

foreach my \$feature45 (@gen1_gen4_ortho) \{

\$feature45->qadd('group', 45);

print \$obj->make_feature_block(\$feature45);\}

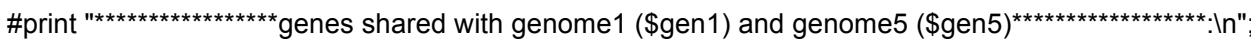

foreach my \$feature46 (@gen1_gen5_ortho) \{

\$feature46->qadd('group', 46);

print \$obj->make_feature_block(\$feature46);\} 


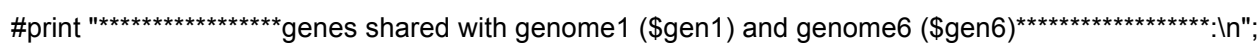
foreach my \$feature47 (@gen1_gen6_ortho) \{

\$feature47->qadd('group', 47);

print \$obj->make_feature_block(\$feature47);

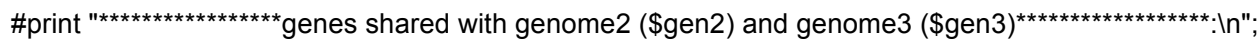

foreach my \$feature48 (@gen2_gen3_ortho) \{

\$feature48->qadd('group', 48);

print \$obj->make_feature_block(\$feature48);

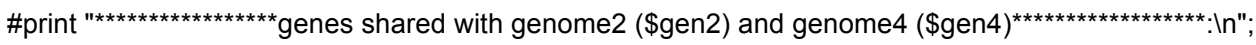

foreach my \$feature49 (@gen2_gen4_ortho) \{

\$feature49->qadd('group', 49);

print \$obj->make_feature_block(\$feature49);\}

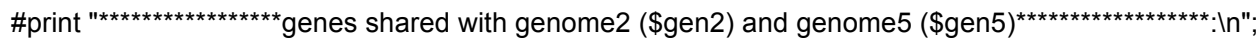

foreach my \$feature50 (@gen2_gen5_ortho) \{

\$feature50->qadd('group', 50);

print $\$$ obj->make_feature_block(\$feature50);

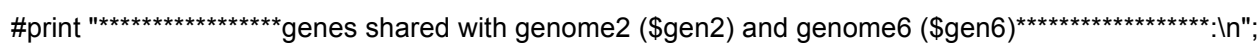

foreach my \$feature51 (@gen2_gen6_ortho) \{

\$feature51->qadd('group', 51);

print \$obj->make_feature_block(\$feature51);\}

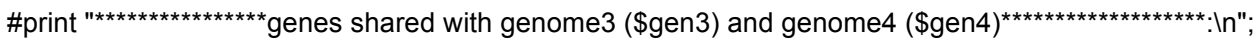

foreach my \$feature52 (@gen3_gen4_ortho) \{

\$feature52->qadd('group', 52);

print \$obj->make_feature_block(\$feature52);

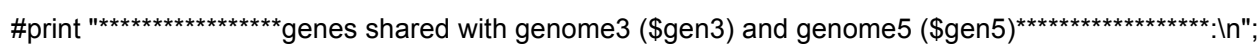

foreach my \$feature53 (@gen3_gen5_ortho) \{

\$feature53->qadd('group', 53);

print \$obj->make_feature_block(\$feature53);

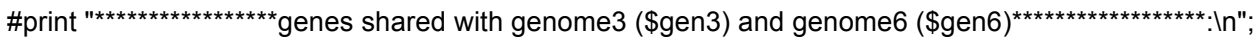

foreach my \$feature54 (@gen3_gen6_ortho) \{

\$feature54->qadd('group', 54);

print \$obj->make_feature_block(\$feature54);

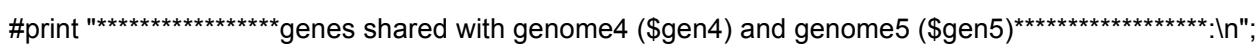

foreach my \$feature55 (@gen4_gen5_ortho) \{

\$feature55->qadd('group', 55);

print \$obj->make_feature_block(\$feature55);\}

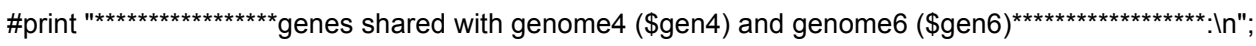

foreach my \$feature56 (@gen4_gen6_ortho) \{

\$feature56->qadd('group', 56);

print \$obj->make_feature_block(\$feature56);

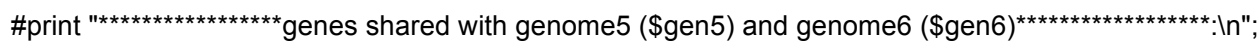

foreach my \$feature57 (@gen5_gen6_ortho) \{ 
\$feature57->qadd('group', 57);

print \$obj->make_feature_block(\$feature57);\}

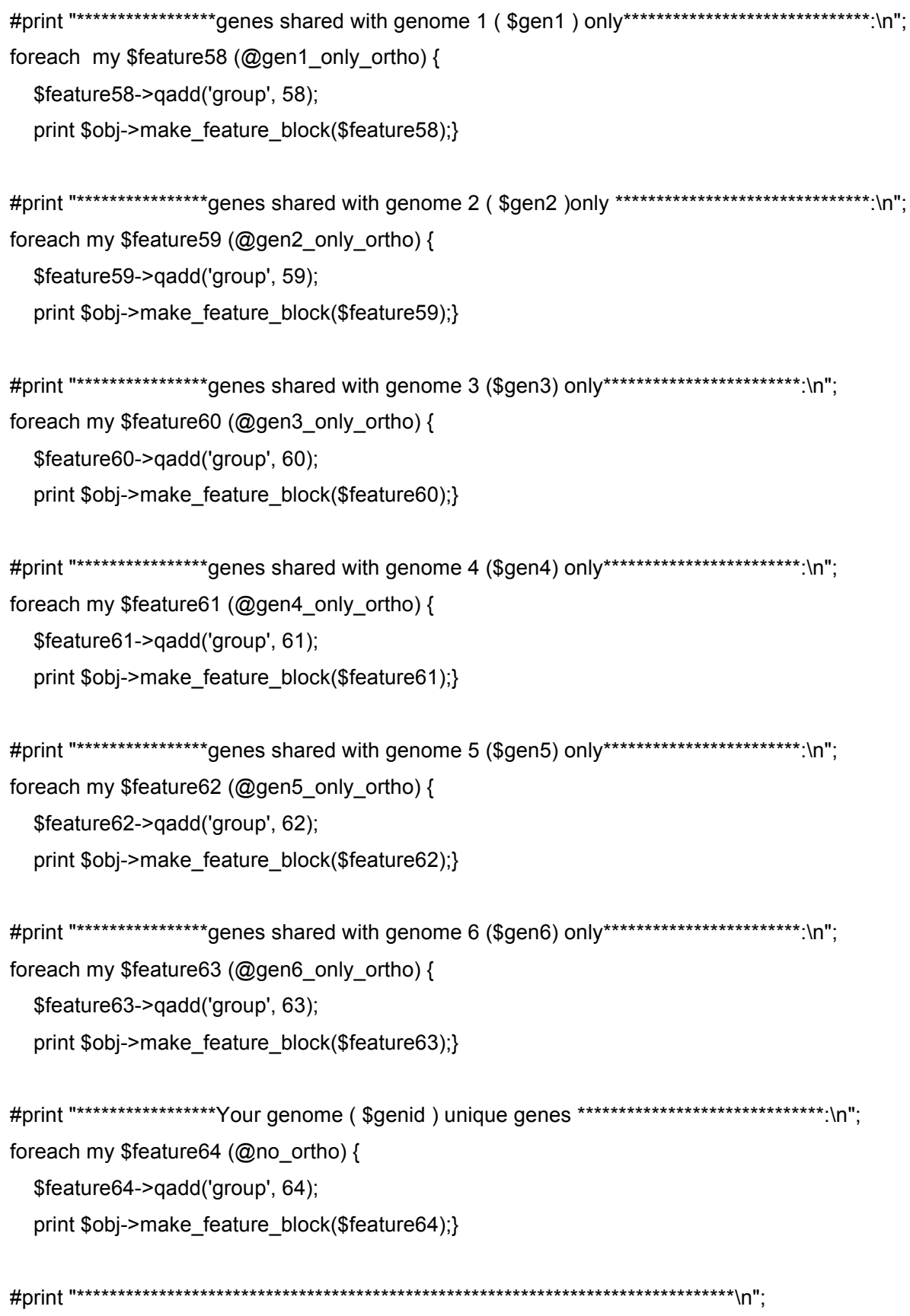


Table 9.6: Matrix of orthologous gene sets.

\begin{tabular}{|c|c|c|c|c|c|c|c|c|}
\hline & & 5303 & 8081 & 21202 & 5603 & 14902 & 1203 & 3094 \\
\hline & genome1 & 8081 & 5303 & 5303 & 5303 & 5303 & 5303 & 5303 \\
\hline & genome2 & 21202 & 21202 & 8081 & 8081 & 8081 & 8081 & 8081 \\
\hline & genome3 & 5603 & 5603 & 5603 & 21202 & 21202 & 21202 & 21202 \\
\hline & genome4 & 14902 & 14902 & 14902 & 14902 & 5603 & 5603 & 5603 \\
\hline & genome5 & 1203 & 1203 & 1203 & 1203 & 1203 & 14902 & 14902 \\
\hline & genome6 & 3094 & 3094 & 3094 & 3094 & 3094 & 3094 & 1203 \\
\hline groups & content & \# CDS & \# CDS & \# CDS & \# CDS & \# CDS & \# CDS & \# CDS \\
\hline 1 & all_gen_ortho & 3306 & 3307 & 3307 & 3305 & 3305 & 3307 & 3306 \\
\hline 2 & gen $1+2+3+4+5$ & 31 & 32 & 32 & 32 & 33 & 33 & 18 \\
\hline 3 & gen $1+2+3+4+6$ & 17 & 18 & 18 & 19 & 18 & 0 & 0 \\
\hline 4 & gen $1+2+3+5+6$ & 0 & 0 & 0 & 0 & 0 & 0 & 0 \\
\hline 5 & gen $1+2+4+5+6$ & 0 & 0 & 0 & 0 & 1 & 0 & 0 \\
\hline 6 & gen $1+3+4+5+6$ & 0 & 0 & 121 & 119 & 118 & 119 & 120 \\
\hline 7 & gen $2+3+4+5+6$ & 118 & 39 & 40 & 40 & 40 & 40 & 42 \\
\hline 8 & gen $1+2+3+4$ & 9 & 8 & 8 & 8 & 8 & 1 & 2 \\
\hline 9 & gen $1+2+3+5$ & 1 & 1 & 1 & 1 & 0 & 0 & 0 \\
\hline 10 & gen $1+2+3+6$ & 2 & 2 & 3 & 2 & 0 & 0 & 0 \\
\hline 11 & gen $1+2+4+5$ & 0 & 0 & 0 & 0 & 0 & 0 & 0 \\
\hline 12 & gen $1+2+4+6$ & 0 & 0 & 0 & 0 & 0 & 0 & 0 \\
\hline 13 & gen $1+2+5+6$ & 0 & 0 & 0 & 0 & 2 & 2 & 2 \\
\hline 14 & gen $1+3+4+5$ & 0 & 0 & 2 & 2 & 2 & 2 & 3 \\
\hline 15 & gen $1+3+4+6$ & 0 & 0 & 3 & 4 & 3 & 0 & 0 \\
\hline 16 & gen $1+3+5+6$ & 0 & 0 & 0 & 0 & 0 & 0 & 0 \\
\hline 17 & gen $1+4+5+6$ & 2 & 2 & 0 & 0 & 0 & 0 & 0 \\
\hline 18 & gen $2+3+4+5$ & 3 & 4 & 4 & 4 & 4 & 4 & 0 \\
\hline 19 & gen $2+3+4+6$ & 3 & 0 & 0 & 0 & 0 & 0 & 0 \\
\hline 20 & gen $2+3+5+6$ & 2 & 0 & 0 & 0 & 0 & 1 & 0 \\
\hline 21 & gen $2+4+5+6$ & 0 & 1 & 0 & 0 & 1 & 0 & 0 \\
\hline 22 & gen $3+4+5+6$ & 0 & 0 & 154 & 153 & 156 & 153 & 155 \\
\hline 23 & gen $1+2+3$ & 0 & 0 & 0 & 0 & 0 & 0 & 0 \\
\hline 24 & gen $1+2+4$ & 0 & 0 & 0 & 0 & 0 & 0 & 0 \\
\hline 25 & gen $1+2+5$ & 0 & 0 & 0 & 0 & 2 & 2 & 0 \\
\hline 26 & gen $1+2+6$ & 0 & 0 & 0 & 0 & 0 & 0 & 0 \\
\hline 27 & gen $1+3+4$ & 0 & 0 & 0 & 1 & 1 & 0 & 1 \\
\hline 28 & gen $1+3+5$ & 0 & 0 & 0 & 0 & 0 & 0 & 0 \\
\hline 29 & gen $1+3+6$ & 0 & 0 & 1 & 1 & 0 & 0 & 0 \\
\hline 30 & gen $1+4+5$ & 2 & 2 & 0 & 0 & 0 & 0 & 0 \\
\hline 31 & $\operatorname{gen} 1+4+6$ & 0 & 0 & 0 & 0 & 0 & 0 & 0 \\
\hline 32 & gen $1+5+6$ & 0 & 0 & 0 & 0 & 0 & 0 & 0 \\
\hline
\end{tabular}




\begin{tabular}{|c|c|c|c|c|c|c|c|}
\hline gen $2+3+4$ & 0 & 1 & 2 & 2 & 2 & 1 & 3 \\
\hline gen $2+3+5$ & 0 & 1 & 1 & 1 & 0 & 0 & 0 \\
\hline gen $2+3+6$ & 0 & 5 & 4 & 5 & 0 & 0 & 0 \\
\hline gen $2+4+5$ & 0 & 0 & 0 & 0 & 0 & 0 & 0 \\
\hline gen $2+4+6$ & 0 & 0 & 0 & 0 & 0 & 0 & 0 \\
\hline gen $2+5+6$ & 0 & 0 & 0 & 0 & 0 & 0 & 0 \\
\hline gen $3+4+5$ & 0 & 0 & 8 & 8 & 9 & 6 & 24 \\
\hline gen $3+4+6$ & 0 & 0 & 22 & 24 & 21 & 15 & 13 \\
\hline gen $3+5+6$ & 0 & 0 & 16 & 16 & 0 & 2 & 0 \\
\hline gen $4+5+6$ & 0 & 0 & 0 & 1 & 0 & 1 & 1 \\
\hline gen $1+2$ & 0 & 0 & 0 & 0 & 0 & 0 & 0 \\
\hline gen $1+3$ & 0 & 0 & 0 & 0 & 0 & 0 & 0 \\
\hline gen $1+4$ & 0 & 0 & 0 & 0 & 0 & 0 & 0 \\
\hline gen $1+5$ & 0 & 0 & 0 & 0 & 0 & 0 & 1 \\
\hline gen $1+6$ & 0 & 1 & 0 & 0 & 1 & 0 & 0 \\
\hline gen2+3 & 0 & 2 & 1 & 1 & 0 & 0 & 0 \\
\hline gen $2+4$ & 0 & 0 & 0 & 0 & 0 & 0 & 0 \\
\hline gen2+5 & 0 & 0 & 0 & 0 & 0 & 0 & 0 \\
\hline gen2+6 & 1 & 0 & 0 & 0 & 0 & 1 & 1 \\
\hline gen3+4 & 0 & 0 & 37 & 37 & 36 & 18 & 8 \\
\hline gen $3+5$ & 0 & 0 & 16 & 16 & 0 & 0 & 0 \\
\hline gen $3+6$ & 0 & 0 & 8 & 8 & 0 & 0 & 0 \\
\hline gen $4+5$ & 0 & 0 & 0 & 0 & 0 & 0 & 0 \\
\hline gen $4+6$ & 1 & 0 & 0 & 0 & 0 & 0 & 0 \\
\hline gen5+6 & 0 & 1 & 0 & 0 & 5 & 8 & 8 \\
\hline gen1 only & 82 & 82 & 0 & 0 & 15 & 2 & 32 \\
\hline gen2 only & 0 & 0 & 0 & 0 & 1 & 1 & 2 \\
\hline gen3 only & 0 & 0 & 106 & 106 & 0 & 2 & 0 \\
\hline gen4 only & 17 & 0 & 0 & 0 & 0 & 2 & 0 \\
\hline gen5 only & 3 & 1 & 2 & 1 & 11 & 11 & 9 \\
\hline gen6 only & 37 & 3 & 0 & 0 & 10 & 25 & 26 \\
\hline no ortho & 728 & 540 & 5 & 0 & 171 & 134 & 274 \\
\hline total CDS & 4365 & 4053 & 3922 & 3917 & 3978 & 3893 & 4051 \\
\hline
\end{tabular}


Table 9.7: Pseudogenes and their orthologs in the Y. enterocolitica reference genomes.

Green - functional gene, light brown - pseudogene with frameshift or stop codon, * - UGA / opal stop codon, dark brown - partial gene (missing N, C, both termini or central part or protein sequence), - no ortholog in this isolate. Numbers given are the unique CDS identifiers. Class assignment as per Table 9.1.

\begin{tabular}{|c|c|c|c|c|c|c|c|c|}
\hline 1B - 8081 & $\begin{array}{l}\text { 1A - } \\
\text { YE5303 }\end{array}$ & $\begin{array}{l}2 \text { - } \\
\text { YE21202 }\end{array}$ & $\begin{array}{l}3 \text { - } \\
\text { YE5603 }\end{array}$ & $\begin{array}{l}3 \text { - } \\
\text { YE14902 }\end{array}$ & $\begin{array}{l}4- \\
\text { YE1203 }\end{array}$ & $\begin{array}{l}5- \\
\text { YE3094 }\end{array}$ & class & product \\
\hline YE0010 & 40761 & 31211 & 20091 & 16071 & 16741 & 00091 & 1.5 .3 & $\begin{array}{l}\text { putative sugar transport system, permease } \\
\text { protein }\end{array}$ \\
\hline YE0012A & 40791 & 31241 & 20061 & 16041 & 16711 & 00121 & 6.1 .1 & ribose operon repressor \\
\hline YE0036 & 43161 & 31491 & 39311 & 38920 & 00251 & - & 6.1 .1 & LuxR family transcription regulatory protein \\
\hline YE0037 & - & 31491 & 39301 & 38921 & 02241 & 00372 & 6.1 .1 & LuxR family transcription regulatory protein \\
\hline YE0048 & 43071 & 31591 & 25351 & 38821 & 00351 & 00471 & 2.2 .3 & putative DNA ligase \\
\hline YE0050 & 43051 & 31611 & 25371 & 38801 & 00371 & 00491 & 4.1 .1 & putative membrane protein \\
\hline- & - & - & - & 38721 & 00471 & - & 5.1 .4 & transposase for IS1668 \\
\hline- & - & 31841 & 25601 & 38561 & 00671 & 00841 & 0.0 .2 & hypothetical membrane protein \\
\hline- & - & 31851 & 25611 & 38551 & 00691 & 00861 & 0.0 .2 & hypothetical membrane protein \\
\hline- & - & - & - & - & 00981 & - & 5.1 .2 & putative phage tail fibre assembly protein \\
\hline- & - & 32081 & 25831 & 38331 & 01201 & 01091 & 1.4 .1 & putative nematicidal protein \\
\hline- & - & 32091 & 25841 & 38321 & 01211 & 01111 & 5.1 .4 & putative transposase \\
\hline- & - & - & - & - & 17351 & 01131 & 5.1 .4 & putative transposase for IS1667 \\
\hline- & - & 30481 & 20801 & 16791 & 17441 & 01221 & 4.1 .0 & putative exported protein \\
\hline YE0098 & 42491 & 32111 & 25861 & 38291 & 01251 & 40591 & 3.3 .0 & putative fructose 1,6-bisphosphatase \\
\hline YE0122 & 42281 & 32330 & 26071 & 38081 & 01481 & 40371 & 1.5 .5 & putative TonB dependent receptor protein \\
\hline YE0123 & 42271 & 32331 & 26081 & 38071 & 01491 & 40361 & 1.5 .5 & hemophore has $\mathrm{A}$ \\
\hline YE0124 & 42261 & 32341 & 26091 & - & 01501 & - & 1.5 .5 & hemophore hasA \\
\hline YE0129 & 42211 & 32391 & 26141 & 38021 & 01561 & 40311 & 4.1 .1 & putative membrane protein \\
\hline YE0130 & - & - & - & - & - & 40301 & 5.1 .4 & transposase for IS1330 \\
\hline- & - & 32671 & 17501 & 36731 & - & - & 1.4 .0 & putative pyocin S2 \\
\hline- & - & 32621 & 17451 & 36681 & 01851 & 40071 & 0.0 .0 & hypothetical protein \\
\hline YE0158 & 41021 & 32721 & 17551 & 36781 & 01951 & 39991 & 4.1 .0 & putative exported protein \\
\hline YE0159 & 41031 & 32731 & 17561 & 36791 & 01961 & 39971 & 4.1 .3 & putative outer membrane usher protein \\
\hline YE0207 & 41481 & 33191 & 18011 & 37251 & 02461 & 39391 & 3.3 .13 & putative lysophospholipase \\
\hline- & - & 33391 & 18221 & 37461 & 02681 & 39191 & 0.0 .0 & hypothetical protein \\
\hline- & - & - & - & 37471 & - & - & 5.1 .4 & putative transposase \\
\hline YE0241 & 41801 & 33541 & 18351 & 37601 & 02831 & 29051 & 1.5 .3 & $\begin{array}{l}\text { sn-glycerol-3-phosphate-binding periplasmic } \\
\text { protein }\end{array}$ \\
\hline- & - & - & - & 37711 & 02951 & - & 5.1 .4 & putative transposase for IS1667 \\
\hline YE0255 & 41941 & 33681 & 18501 & 37751 & 02991 & 38911 & 7.0 .0 & putative DNA recombinatin protein \\
\hline YE0270 & 42091 & 33841 & 18651 & 37901 & 03141 & 38761 & 0.0 .2 & conserved hypothetical protein \\
\hline YE0277 & 43621 & 39341 & 38561 & 40431 & 03431 & 38681 & 7.0 .0 & putative acetyltransferase \\
\hline YE0313 & 30741 & 19591 & 14011 & 25121 & 20801 & 30861 & 4.1.1 & inner membrane protein \\
\hline YE0320 & 30661 & 19661 & 14081 & 25191 & 20891 & 37981 & 2.1 .4 & probable exported Zinc protease \\
\hline YE0322 & 30661 & 19691 & 14091 & 25201 & 20911 & 37951 & 4.1 .1 & putative inner membrane transprt protein \\
\hline YE0324 & 30641 & 19711 & 14121 & 25231 & 20941 & 37931 & 7.0 .0 & putative ATP/GTP-binding protein \\
\hline- & 30531 & - & - & - & - & - & 4.1.0 & putative exported protein \\
\hline- & 30011 & - & - & - & - & - & 0.0 .2 & hypothetical protein \\
\hline- & 30001 & - & - & - & - & - & 0.0 .2 & hypothetical protein \\
\hline- & 29921 & - & - & - & - & - & 2.2 .3 & putative restriction enzyme beta subunit \\
\hline- & 29911 & - & - & - & - & - & 2.2 .3 & putative restriction enzyme alpha subunit \\
\hline YE0344 & - & - & - & - & - & - & 5.1 .1 & putative HlyD-family secretion protein \\
\hline YE0345 & - & - & - & - & - & - & 5.1 .4 & integrase \\
\hline YE0386 & 29481 & 20241 & 14651 & 25771 & 21481 * & 37401 & 7.0 .0 & putative acyl-CoA dehydrogenase \\
\hline- & 29371 & 20332 & 14761 & 25881 & 21581 & 37291 & 1.6 .0 & $\begin{array}{l}\text { regulator of cell morhogenesis and NO } \\
\text { signalling }\end{array}$ \\
\hline YE0409 & 29261 & 20441 & 14871 & 25991 & 21711 & 37171 & 1.1 .1 & methyl-accepting chemotaxis protein \\
\hline YE0416 & 29201 & 20511 & 14931 & 26051 & 21781 & 37111 & 0.0 .2 & conserved hypothetical protein \\
\hline YE0443 & 28931 & 20781 & 14192 & 26312 & 22071 & 36842 & 1.4 .3 & multidrug efflux protein \\
\hline
\end{tabular}




\begin{tabular}{|c|c|c|c|c|c|c|c|c|}
\hline YE0444 & 28921 * & - & - & - & - & - & 1.4 .3 & multidrug efflux protein \\
\hline YE0445 & 28911 & 20791 & 15201 & 26321 & 22081 & 36841 & 4.1 .3 & probable outer membrane efflux lipoprotein \\
\hline YE0453 & 28841 & 20861 & 15271 & 26391 & 22151 & 36771 & 0.0 .2 & conserved hypothetical protein \\
\hline YE0470 & 28701 & 21001 & 15411 & 26531 & 22321 * & 36631 & 3.3.13 & PhnN protein \\
\hline YE0475 & 28651 & 21051 & 15461 & 26581 & 22371 & 36581 & 3.3 .13 & Phnl protein \\
\hline YE0480 & 28601 & 21101 & 15511 & 26631 & 22421 & 36531 & 4.1 .0 & putative exported protein \\
\hline YE0483 & 28571 & 21131 & 15541 & 26661 & 22451 & 36510 & 6.1 .1 & $\begin{array}{l}\text { putative LysR-family transcriptional } \\
\text { regulatory protein }\end{array}$ \\
\hline YE0491 & 28491 & 21202 & 15591 & 26711 & 22501 & 36451 & 1.5 .0 & $\mathrm{ABC}$ transporter, substrate binding protein \\
\hline- & - & - & - & - & 22551 & - & 5.1 .4 & putative transposase for IS 1667 \\
\hline- & - & - & - & - & 22631 & - & 0.0 .1 & conserved hypothetical protein \\
\hline YE0505 * & 28391 & 21301 & 15691 & 26811 * & - & 36341 & 5.1 .4 & integrase \\
\hline- & - & - & - & - & - & 36331 & 5.1 .4 & putative transposase \\
\hline- & - & - & - & - & - & 36321 & 5.1 .4 & putative transposase \\
\hline- & - & - & - & 26831 & - & - & 0.0 .2 & hypothetical protein \\
\hline- & - & - & - & 26851 & - & - & 5.1 .4 & integrase \\
\hline- & - & - & - & 26861 & - & - & 2.1 .1 & $\begin{array}{l}\text { putative type I site-specific } \\
\text { deoxyribonuclease }\end{array}$ \\
\hline- & - & - & - & 26881 & - & - & 0.0 .1 & hypothetical protein \\
\hline- & - & - & - & 27061 & - & - & 5.1 .4 & putative transposase \\
\hline- & - & 21331 & 15721 & - & - & - & 2.2 .3 & $\begin{array}{l}\text { putative type I restriction-modification } \\
\text { system }\end{array}$ \\
\hline- & - & 21361 & 11271 & 27071 & 22691 & - & 2.2 .3 & superfamily I DNA helicase \\
\hline YE0511 & - & - & - & - & - & - & 5.1 .4 & putative phage-related protein \\
\hline YE0517 & - & 21431 & 11312 & - & - & - & 4.1.1 & putative membrane protein \\
\hline- & 28141 & - & - & - & - & - & 0.0 .1 & conserved hypothetical protein \\
\hline YE0518 & 28081 & 21441 & 11321 & 27121 & 22741 & - & 1.5 .0 & putative permease \\
\hline YE0522 & 28051 & 21481 & 11351 & 27151 & 22771 & 36281 & 4.1 .0 & putative exported protein \\
\hline YE0534 & 27941 & 21591 & 11461 & 27261 & 22901 & 36161 & 1.5 .1 & arginine/ornithine antiporter \\
\hline YE0545 & 27841 & 21691 & 11561 & 27361 & 23011 & 36061 & 7.0 .0 & putative oxidoreductase \\
\hline YE0546 & 27831 & 21701 & 11571 & 27371 & 23021 * & 36051 & 0.0 .0 & hypothetical protein \\
\hline YE0559 & 27691 & 21841 & 11711 & 27511 & 23211 & 35911 & 6.1 .1 & LysR-family regulatory protein \\
\hline YE0580 & 27521 & 22011 & 11881 & 27681 & 23411 & 35721 & 6.1 .1 & transcriptional regulator NadR \\
\hline YE0583 & - & 22041 & 11911 & 27711 & 23441 & 35691 & 0.0 .2 & conserved hypothetical protein \\
\hline YE0591 & 27421 & 22121 & 11991 & 27791 & 23531 & 35601 & 0.0 .2 & conserved hypothetical protein \\
\hline- & 27011 & 22522 & 12391 & 28201 & 23971 & 35211 & 5.1 .5 & putative adhesin/invasin protein \\
\hline YE0650 & 26881 & 22661 & 12531 & 28341 & 24161 & 35071 & 3.1 .19 & tryptophanase \\
\hline YE0656 & 26821 & 22721 & 12591 & 28401 & 24231 & 35001 & 7.0 .0 & putative AMP-binding enzyme-family protein \\
\hline YE0688 & 26531 & 23011 & 12881 & 28691 & 24561 & 34711 & 1.5 .5 & putative type II secretion system protein \\
\hline YE0695 & 26471 & 23071 & 12941 & 28751 & 24621 & 34651 & 1.5 .1 & aromatic amino acid transport protein \\
\hline- & 26422 & 23131 & 12991 & 28801 & 24681 & 34591 & 0.0 .2 & hypothetical protein \\
\hline YE0717 & 26211 & 23281 & 13141 & 28951 & 24851 & 34451 & 0.0 .2 & conserved hypothetical protein \\
\hline YE0725 & 26141 & 23351 & 13211 & 29021 & 24921 & 34381 & 6.1 .1 & DnaK suppressor protein homologue \\
\hline YE0787 & - & 25021 & 36720 & 31330 & 38063 & 09069 & 0.0 .0 & hypothetical protein \\
\hline YE0789 & 25511 & 25041 & 36701 & 31311 & 38061 & 09071 & 2.2 .9 & putative thiol:disulfide interchange protein \\
\hline YE0791 & 25491 & 25061 & 36681 & 31291 & 38041 & 09081 & 1.5 .0 & ABC transporter, ATP-binding component \\
\hline- & - & 25141 & 36601 & - & 37961 & - & 5.1 .4 & putative transposase for IS1667 \\
\hline YE0799 & - & 25161 & 35431 & 31211 & 37951 & 09161 & 7.0 .0 & putative aminotransferase \\
\hline YE0807 & 25311 & 25191 & 35451 & 31191 & 37931 & 09181 & 6.0 .0 & putative transcriptional regulatory protein \\
\hline- & 25281 & - & - & - & - & - & 1.4 .0 & putative enterotoxin-like protein \\
\hline YE0813 & 25221 & 25251 & 35511 & 31131 & 37871 & 09251 & 1.5 .3 & $\begin{array}{l}\text { sorbose-specific PTS uptake system } \\
\text { component }\end{array}$ \\
\hline- & 25191 & 25272 & 35541 & 31101 & 37841 & 09281 & 3.5 .0 & conserved hypothetical protein \\
\hline- & 24941 & 25282 & 35641 & 31001 & 37741 & 09381 & 3.5 .2 & $\begin{array}{l}\text { anaerobic dimethyl sulfoxide reductase } \\
\text { chain } \mathrm{A}\end{array}$ \\
\hline- & 24911 & 25285 & 35681 & 30961 & 37711 & 09421 & 1.3.1 & putative oxidoreductase component \\
\hline- & - & 25291 & 35691 & 30951 & 37701 & 09431 & 5.1 .4 & putative transposase for IS1667 \\
\hline YE0840 & 24701 & 25421 & 35801 & 30841 & 37581 & 09531 & 4.1 .1 & putative membrane protein \\
\hline YE0847 & 24631 & 25491 & 35871 & 30771 & 37511 & 09631 * & 4.1 .0 & putative exported protein \\
\hline- & - & 25551 & 35931 & 30711 & 37451 & 09701 & 5.1 .4 & transposase for IS1328 \\
\hline
\end{tabular}




\begin{tabular}{|c|c|c|c|c|c|c|c|c|}
\hline YE0911 & 06591 & 00021 & 38181 & 19541 & 03481 & 32661 & 3.6 .1 & 3-oxoacyl-[acyl-carrier-protein] synthase II \\
\hline YE0915 & 06621 & 00051 & 38151 & 19571 & 03531 & 32621 & 1.5 .5 & MFS-family transporter \\
\hline- & - & 00071 & 38121 & 19601 & 03561 & 32601 & 5.1 .4 & transposase for IS1328 \\
\hline YE0924 & 06701 & 00141 & 38051 & 19671 & 03641 & 32531 & 1.5 .1 & $\begin{array}{l}\text { glycine betaine/L-proline transport system } \\
\text { permease }\end{array}$ \\
\hline YE0933 & 06780 & 00221 & 37951 & 19771 & 03731 & 32441 & 1.6 .1 & putative acid shock protein \\
\hline YE0934 & 06781 & 00231 & 37941 & 19781 & 03741 & 32431 & 0.0 .0 & hypothetical protein \\
\hline YE0935 & 06791 & 00241 & 37931 & 19791 & 03751 & 32421 & 6.1 .1 & AraC-family transcriptional regulator \\
\hline YE0936 & 06801 & 00242 & 37921 & 19801 & 03761 & 32411 & 1.5 .0 & putative MFS family transport protein \\
\hline YE0938 & 06821 & 00271 & 37901 & 19821 & 03791 & 32391 & 4.1 .1 & putative membrane protein \\
\hline YE0941 & 06851 & 00301 & 37871 & 19861 & 03801 & 32351 & 6.1 .1 & AraC-family transcriptional regulator \\
\hline YE0944 & 06881 & 00323 & 37831 & 19891 & 03831 & 32321 & 0.0 .2 & hypothetical protein \\
\hline- & - & 00531 & 22222 & 20091 & 04061 & 32121 & 5.1 .4 & transposase for IS1667 \\
\hline YE0972 & 07131 & 00601 & 22161 & 20161 & 04131 & 32051 & 0.0 .2 & conserved hypothetical protein \\
\hline- & - & 00651 & 22121 & - & - & - & 5.1 .2 & putative prophage integrase \\
\hline- & - & 00691 & 22081 & - & - & - & 0.0 .2 & hypothetical protein \\
\hline- & - & 00771 & 22001 & - & - & - & 2.2 .3 & putative RNA directed DNA polymerase \\
\hline- & 07391 & - & - & - & - & - & 5.1 .2 & prophage regulatory protein \\
\hline YE0974 & - & - & - & - & - & - & 5.1 .2 & prophage integrase \\
\hline YE1005 & 07621 & 00911 & 21861 & 20301 & 04271 & 31931 & 5.1 .5 & phospholipase A \\
\hline- & - & - & - & - & 04301 & 31901 & 5.1 .4 & putative transposase for IS1667 \\
\hline- & - & 00931 & 21841 & 20321 & 04311 & 31891 & 5.1 .4 & transposase for IS1328 \\
\hline YE1023 & 07791 & 01091 & 21681 & 20481 & 04481 & 31731 & 1.5 .3 & putative gluconate transporer \\
\hline YE1034 & 07882 & 01211 & 21582 & 20580 & 04580 & 31632 & 4.1 .1 & putative exported protein \\
\hline YE1036 & 07901 & 01231 & 21571 & 20591 & 04591 & 31621 & 4.1 .0 & putative lipoprotein \\
\hline- & 08181 & 01501 & 21291 & 20871 & 04891 & 31351 & 1.5 .0 & putative autotransporter protein \\
\hline YE1068 & 08211 & 01531 & 21261 & 20901 & 04931 & 31311 & 4.1 .0 & putative exported protein \\
\hline- & - & 01631 & 21161 & $21001 *$ & - & 31211 & 5.1 .4 & putative transposase for IS1667 \\
\hline YE1078 & 08311 & 01651 & 04111 & 21021 & 05051 & 31201 & 4.1 .1 & putative membrane protein \\
\hline YE1085 & 08381 & 01721 & 04041 & 21091 & 05141 & 31131 & 0.0 .0 & hypothetical protein \\
\hline YE1086 & 08391 & 01731 & 04031 & 21101 & 05151 & 31121 & 5.3 .5 & putative cytochrome c \\
\hline- & - & 01741 & 04021 & 21111 & 05161 & 31091 & 5.1 .4 & putative transposase for IS1667 \\
\hline YE1101 & - & - & - & - & - & - & 0.0 .0 & hypothetical protein \\
\hline YE1116 & 08631 & 01971 & 03781 & 21351 & 05511 & 30791 & 4.1.1 & $\begin{array}{l}\text { putative inner membrane protein [region } \\
\text { flipped, protein torn apart on opposite } \\
\text { strands] }\end{array}$ \\
\hline YE1117 & 08641 & 09181 & 03771 & 21361 & 05501 & 30781 & 1.5 .2 & putative divalent cation transport protein \\
\hline- & 08711 & 02021 & 03701 & 21431 & 05521 & 30711 & 1.1 .1 & flagellin lysine- $\mathrm{N}$-methylasec $E C=2.1 .1 .-$ \\
\hline- & - & 02211 & 03511 & 21621 & 05721 & 30531 & 0.0 .2 & hypothetical protein \\
\hline- & - & 02261 & 03471 & 21651 & 05771 & 30501 & 5.1 .2 & transposase for IS1328 \\
\hline- & - & 02271 & 03461 & 21652 & 05801 & 30492 & 5.1 .2 & hypothetical phage-related protein \\
\hline- & - & 02301 & 03431 & 21691 & 05821 & 30481 & 5.1 .4 & transposase for IS1668 \\
\hline YE1158 & 09051 & 02451 & 03291 & 21831 & 05971 & 30341 & 3.5 .3 & cytochrome C-type protein NapC \\
\hline- & - & - & - & - & - & 30421 & 5.1 .4 & putative transposase \\
\hline Ye1165 & 09081 & 02511 & 03252 & 21852 & 06010 & 30302 & 6.1 .1 & $\begin{array}{l}\text { putative LyxR-family transcriptional } \\
\text { regulatory protein }\end{array}$ \\
\hline YE1166 & 09091 & 02521 & 03251 & 21861 & 06011 & 30301 & 6.1 .1 & $\begin{array}{l}\text { putative LysR-family transcriptional } \\
\text { regulatory protein }\end{array}$ \\
\hline YE1184 & - & - & - & - & - & - & 3.3 .15 & putative acetyltransferase \\
\hline- & 09421 & - & - & - & - & - & 7.0 .0 & putative acetyltransferase \\
\hline YE1199 & 09581 & 02721 & 03071 & 22051 & 06191 & 30111 & 1.5 .0 & $\begin{array}{l}\text { putative } A B C \text { transporter, ATP-binding } \\
\text { protein }\end{array}$ \\
\hline YE1228 & 09831 & 02991 & 02821 & 22301 & 06491 & 29851 & 6.1 .1 & two-component system histidine kinase \\
\hline YE1234 & - & 03079 & 02763 & 22359 & 06549 & 29793 & 4.1 .0 & putative lipoprotein \\
\hline YE1236A & 09911 & 03080 & 02762 & 22360 & 06550 & 29792 & 2.1 .4 & 6-phospho-beta-glucosidase \\
\hline YE1238 & 09931 & 03091 & 02751 & 22371 & 06561 & 29781 & 1.5 .1 & putative membrane transport protein \\
\hline YE1246 & 10011 & 03171 & 02671 & 22451 & 06651 & 29701 & 1.5 .0 & MFS-family membrane transport protein \\
\hline YE1251 & 10061 & 03221 & 02621 & 22501 & 06711 & 29652 & 6.1 .1 & putative regulatory protein \\
\hline YE1252 & 10071 & 03231 & 02611 & 22511 & 06721 & 29651 & 4.1 .0 & putative exported protein \\
\hline YE1261 & - & - & - & - & - & - & 5.1 .2 & putative phage integrase \\
\hline
\end{tabular}




\begin{tabular}{|c|c|c|c|c|c|c|}
\hline YE1262 & 10561 & 03341 & 02511 & 22711 & 06821 & 29481 \\
\hline- & - & 03361 & 02501 & - & - & 29461 \\
\hline YE1288 & 10821 & 03611 & 02251 & 22961 & 07081 & 29201 \\
\hline YE1298 & 10921 & 03711 & 02151 & 23061 & 07191 & 29091 \\
\hline YE1304 & 10981 & 03771 & 02091 & 23121 & 07291 & 29031 \\
\hline YE1305 & 10991 & 03781 & 02081 & 23131 & 07301 & 29021 \\
\hline- & 10161 & - & - & - & - & - \\
\hline - & 10211 & - & - & - & - & - \\
\hline- & 10281 & - & - & - & - & - \\
\hline - & 10301 & - & - & - & - & - \\
\hline- & 10321 & - & - & - & - & - \\
\hline- & 10351 & - & - & - & - & - \\
\hline YE1322 & - & 03941 & 01921 & 23291 & 07471 & 28851 * \\
\hline YE1325 & 11171 & 03971 & 01891 & 23321 & 07501 & 28801 \\
\hline - & - & 03991 * & 01871 & 23341 & 07521 & 28771 \\
\hline YE1379 & 11631 & 04441 & 01431 & 23781 & 08031 & 28331 \\
\hline YE1384 & - & - & - & - & - & - \\
\hline- & 11461 & 04271 & 01591 & 23621 & 07841 & 28491 \\
\hline YE1389 & - & 04521 & 01351 & 23861 & 08121 & 28261 \\
\hline YE1391 & 11691 & 04541 & 01331 & 23881 & 08151 & 28230 \\
\hline YE1400 & 11821 & 04631 & 01231 & 23991 & 08251 & 28131 \\
\hline YE1406 & 11881 & 04691 & 01171 & 24051 & 08351 & 28071 \\
\hline YE1409 & 11901 & 04711 & 01151 & 24071 & 08371 & 28051 \\
\hline YE1410 & 11911 & 04721 & 01141 & 24081 & 08381 & 28041 \\
\hline- & 11941 & 04733 & 01122 & 24100 & 08401 & 28011 \\
\hline- & 11981 & - & - & - & - & - \\
\hline- & 12131 & 04780 & 01091 & 24131 & 08451 & 27971 \\
\hline - & - & 04821 & 01061 & 24141 & - & 27951 \\
\hline YE1412 & - & - & - & - & - & - \\
\hline - & 12071 & - & - & - & - & - \\
\hline- & 12651 & - & - & - & - & - \\
\hline YE1432 & 12801 & 04992 & 00880 & 24340 & 08641 & 27762 \\
\hline - & - & 05241 & 00631 & 24581 & 08921 & 27531 \\
\hline YE1488 & 13361 & 05551 & 00321 & 40061 & 09261 & 27211 \\
\hline YE1529 & - & 05881 & 40091 & 40382 & 09611 & 26881 \\
\hline - & - & 05891 & 40101 & 00001 & 09621 & 26871 \\
\hline- & - & - & - & - & 09671 & - \\
\hline YE1576 & 14121 & 06341 & 29351 & 00461 & 10131 & 26431 \\
\hline - & 14371 & 06565 & 29101 & 01121 & 10411 & 26171 \\
\hline - & 14391 & 06567 & 29081 & 01141 & 10431 & 26151 \\
\hline YE1598 & 14401 & 06568 & 29071 & 01151 & 10441 & 26141 \\
\hline- & - & 06631 & 29021 & - & 10491 & 26091 \\
\hline YE1610 & 14501 & 06701 & 19981 & 01261 & 30181 & 26031 * \\
\hline YE1614 & 14541 & 06751 & 19931 & 01311 & 30121 & 25971 \\
\hline YE1617 & 14571 & 06781 & 19901 & 01341 & 30091 & 25921 \\
\hline YE1656 & - & - & - & - & - & - \\
\hline YE1696 & - & 07071 & 19611 & 01631 & 29761 & 25661 \\
\hline YE1697 & - & 07081 & 19601 & 01641 & 29751 & 25651 \\
\hline YE1698 & - & 07091 & 19591 & 01651 & 29741 & 25641 \\
\hline YE1700 & 14841 & 07111 & 19561 & 01681 & 29701 & 25621 \\
\hline YE1716 & 15001 & 07271 & 19391 & 01851 & 29521 & 25441 * \\
\hline - & - & 07381 & 19281 & 01951 & 29391 & 25331 \\
\hline- & - & 07391 & 19261 & 01961 & 29371 & 25321 \\
\hline YE1728 & 15141 & 07442 & 19201 & 02011 & 29301 & 25281 \\
\hline
\end{tabular}

putative inner membrane protein putative transposase for IS1667 conserved hypothetical protein putative flagellar assembly regulatory protein Flk

putative inner membrane protein putative membrane protein putative hemolysin

hypothetical protein hypothetical protein hypothetical protein

putative hemagglutinin/hemolysin putative adhesin/hemagglutinin putative RTX-family protein conserved hypothetical protein putative transposase for IS1667 putative O-succinylbenzoic acid--CoA ligase putative exported protein

hypothetical protein

putative serine protease

putative iron-sulphur binding protein

Putative type I secretion protein putative protease

putative prophage repressor protein probable phage antitermination protein $Q$ putative ATP-binding protein putative bacteriophage coat protein carbonic anhydrase, EC=4.2.1.1 transposase for IS1668

transposase for insertion element IS285 hypothetical phage protein protein umuC

hypothetical protein putative exported protein putative inner membrane protein putative transposase for IS1667

transposase

putative transposase for IS1667 putative 3-oxoacyl-[acyl-carrierprotein]synthase II

putative cytochrome C-type biogenesis protein

cytochrome $\mathrm{c}$ biogenesis protein

nitrite reductase gene remnant

putative transposase for IS1667

putative MFS-family transport protein

tetrathionate reductase complex: sensory

transduction histidine kinase

tetrathionate reductase subunit $\mathrm{A}$

putative transposase

putative $A B C$ transport protein, ATP-binding component

putative exported protein

conserved hypothetical protein

putative shikimate dehydrogenase

conserved hypothetical protein

transposase for IS1668

putative prophage terminase small subunit putative sugar permease 


\begin{tabular}{|c|c|c|c|c|c|c|c|}
\hline YE1729 & 15161 & 07451 & 19181 & 02031 & 29281 & 25261 & 6.1 .1 \\
\hline YE1733 & 15211 & 07491 & 19131 & 02081 & 29221 & 25221 & 1.4 .4 \\
\hline YE1737 & - & - & - & - & - & - & 3.1 .14 \\
\hline- & - & 07541 & 19071 & 02140 & 29141 & 25161 & 0.0 .0 \\
\hline YE1743 & 15431 & 07561 & 19051 & 02151 & 29121 & 25141 & 6.1 .1 \\
\hline- & 15481 & 07621 & 18981 & 02201 & 29071 & 25081 & 5.1 .5 \\
\hline YE1746 & 15741 & 07591 & 19011 & 02191 & 02981 & 25091 & 7.0 .0 \\
\hline YE1748 & 15511 & 07651 & 18951 & 02231 & 29041 & 25051 & 1.5 .0 \\
\hline YE1759 & 15611 & 07731 & 18871 & 02311 & 28921 & 24971 & 0.0 .1 \\
\hline YE1767 & 15681 & 07801 & 18801 & 02381 & 28851 & 24921 & 4.1 .2 \\
\hline Ye1771 & 15721 & 07832 & 18751 & 02431 & 28811 & 24871 & 4.1 .3 \\
\hline YE1803A & - & - & - & - & - & - & 5.1 .2 \\
\hline YE1804 & - & - & - & - & - & - & 5.1 .2 \\
\hline YE1821 & - & - & - & - & - & - & 5.1 .4 \\
\hline YE1823 & - & - & - & - & - & - & 5.1 .4 \\
\hline YE1824 & - & - & - & - & - & - & 0.0 .2 \\
\hline YE1844 & 21032 & 12891 & 15841 & 07601 & - & 19611 & 1.5 .0 \\
\hline - & - & 12881 & 15861 & 07581 & - & 19621 & 5.1 .4 \\
\hline - & - & 12701 & 16041 & 07401 & 13171 & 19811 & 0.0 .0 \\
\hline - & - & 12471 & 16261 & - & 27921 & 20051 & 5.1 .4 \\
\hline YE1845 & 21031 & 12461 & 16271 & 03231 & 27901 & 20071 & 4.1 .1 \\
\hline YE1848 & 21001 & 12431 & 16301 & 03261 & 27871 & 20101 & 7.0 .0 \\
\hline YE1853 & 20971 & 12401 & 16331 & 03291 & 27821 & 20121 & 0.0 .2 \\
\hline YE1855 & 20951 & 12381 & 16351 & 03311 & 27801 & 20151 & 0.0 .2 \\
\hline YE1874 & 20801 & 12231 & 16501 & 03461 & 27641 & 20272 & 2.0 .0 \\
\hline YE1875 & 20791 & 12221 & 16511 & 03471 & 27621 & 20281 & 1.5 .0 \\
\hline - & - & - & - & - & 27571 & - & 5.1 .4 \\
\hline YE1879 & 20751 & 12181 & 16551 & 03511 & 27561 & 20341 & 1.5 .3 \\
\hline YE1895 & 20581 & 12011 & 16741 & 03701 & 27351 & 20531 & 3.6 .0 \\
\hline YE1897 & 20561 & 11991 & 16761 & 03721 & 27331 & 20561 & 1.5 .2 \\
\hline- & - & 11641 & 17111 & 06441 & - & - & 5.1 .4 \\
\hline YE1905 & 20491 & 09121 & 35401 & 03791 & 27252 & 20622 & 0.0 .0 \\
\hline YE1920 & - & - & - & - & - & - & 5.1 .4 \\
\hline YE1925 & - & - & - & - & - & - & 5.1 .4 \\
\hline YE1935 & - & - & - & - & 11451 & 18411 & 5.1 .4 \\
\hline YE1944 & 22921 & 19221 & 25071 & 08971 & 11561 * & 18501 * & 1.5 .0 \\
\hline YE1947 & 22891 & 19251 & 25101 & 08941 & 11601 * & 18541 & 7.0 .0 \\
\hline YE1950 & 22681 & 19281 & 25131 & 08911 & 11631 & 18571 & 3.3 .19 \\
\hline YE1951 & 22851 & 19291 & 25141 & 08901 & 11651 & 18581 & 6.1 .1 \\
\hline YE1953 & - & - & - & - & - & - & 5.1 .2 \\
\hline YE1954 & - & - & - & - & - & - & 5.1 .2 \\
\hline YE1960 & 22801 & 19341 & 25191 & 08851 & 11711 & 18631 & 4.1 .0 \\
\hline- & - & 19311 & 25161 & 08881 & 11681 & 18601 & 5.1 .4 \\
\hline - & - & - & - & - & 11671 & - & 5.1 .2 \\
\hline - & 22711 & - & - & - & 11811 & - & 5.1 .2 \\
\hline - & - & - & - & - & 11821 & - & 5.1 .4 \\
\hline - & - & - & - & 08691 & - & - & 5.1 .2 \\
\hline - & - & - & - & 08391 & - & - & 5.1 .4 \\
\hline - & - & - & - & 08351 & - & - & 5.1 .2 \\
\hline - & - & 11941 & 16811 & - & - & - & 5.1 .4 \\
\hline YE1971 & 18221 & 11481 & 17271 & 06271 & 14451 * & 23231 & 1.5 .0 \\
\hline- & - & - & - & - & 16171 & - & 5.1 .4 \\
\hline YE1984 & 18351 & 11331 & 29911 & 06131 & 16161 & - & 4.1 .1 \\
\hline Ye1997 & 18461 & 11211 & 30031 & 06011 & 16011 & 22581 & 3.3 .15 \\
\hline YE1998 & 18471 & 11201 & 30041 & 06001 & 15991 & 22571 & 1.5 .5 \\
\hline
\end{tabular}

putative sucrose operon repressor (Laclfamily regulator)

putative UV protection and mutation protein possible homoserine synthase

hypothetical protein

putative AraC-family transcriptional

regulatory protein

putative cytolethal distending toxin subunit $A$

hypothetical protein

putative $A B C$ transporter, ATP-binding

protein

conserved hypothetical protein

peptidoglycan synthetase

ferrichrome receptor protein

IS1329 transposase $A$

terminase, ATPase subunit

transposase

IS1400 transposase B

conserved hypothetical protein

sodium/glutamate symport carrier protein

transposase for IS1328

hypothetical protein

transposase for IS1328

putative inner membrane protein

putative transferase

conserved hypothetical protein

conserved hypothetical protein

putative DNA-binding protein

putative transport protein

putative transposase for IS1667

putative transport protein

methylmalonyl-CoA mutase

chelated iron transport system membrane

protein

transposase

hypothetical protein

IS1400 transposase B

putative transposase

transposase for IS1330

putative membrane permease

putative hydrolase

alkanesulfonate monooxygenase

putative TetR-family transcriptional

regulatory protein

bacteriophage late control protein

bacteriophage major tail sheath protein

putative exported protein

putative transposase

putative phage integrase

putative phage integrase

putative transposase subunit

putative phage tail fibre protein

putative transposase

putative phage protein

putative transposase for IS1667 (pseudo)

probable membrane transport protein

putative transposase for IS1667

putative membrane protein

probable formate--tetrahydrofolate ligase

putative RTX-family toxin transporter 


\begin{tabular}{|c|c|c|c|c|c|c|c|c|}
\hline YE1999 & 18481 & 11191 & 30051 & 05991 & 15981 & 22551 & 5.1 .5 & hemolysin transport protein \\
\hline YE2000 & 18491 & 11181 & 30061 & 05981 & 15971 & 22531 & 1.5 .5 & putative toxin transport protein \\
\hline YE2011 & 18601 & 11061 & 30181 & 05861 & 15851 & 22371 & 1.5 .0 & $\begin{array}{l}\text { PTS system, maltose and glucose-specific } \\
\text { Ilabc component }\end{array}$ \\
\hline YE2012 & 18611 & 11051 & 30191 & 05851 & 15841 & 22361 & 6.1 .1 & maltose regulon regulatory protein \\
\hline- & 18621 & 11042 & 30201 & 05841 & 15831 & 22341 & 1.4 .2 & putative acetyltransferase \\
\hline- & - & - & - & - & 15791 & - & 5.1 .4 & putative transposase for IS1667 \\
\hline YE2019 & 18681 & 10991 & 30261 & 05781 & 15761 & 22281 & 1.4 .3 & beta-lactamase precursor \\
\hline YE2025 & 18741 & 10931 & 30331 & 05711 & 15681 & 22211 & 4.1.1 & putative membrane protein \\
\hline YE2026A & 18771 & 10911 & 30361 & 05681 & 15641 & - & 0.0 .2 & conserved hypothetical exported protein \\
\hline YE2027 & 18781 & 10901 & 30371 & 05671 & 15631 & 22210 & 4.1 .0 & putative exported protein \\
\hline YE2030 & 18811 & 10871 & 30401 & 05641 & - & 22200 & 4.1 .0 & putative exported protein \\
\hline YE2035 & 18821 & 10841 & 30421 & 05621 & - & - & 1.5 .0 & putative membrane transport protein \\
\hline- & - & - & - & - & 15461 & - & 5.1 .2 & putative phage-related membrane protein \\
\hline- & - & - & - & - & 15451 & - & 0.0 .2 & conserved hypothetical protein \\
\hline YE2036 & 18841 & 10831 & 30441 & 05601 & 15431 & 22191 & 0.0 .1 & conserved hypothetical protein \\
\hline YE2037 & 18851 & 10801 & 30461 & 05591 & 15421 & 22181 & 4.1 .0 & putative exported protein \\
\hline- & 18871 & 10781 & 30481 & 05571 & 15401 & 22171 & 0.0 .0 & hypothetical protein \\
\hline- & 18881 & 10773 & 30491 & 05561 & 15391 & 22161 & 4.1 .0 & putative exported protein \\
\hline YE2041 & 18950 & 10760 & 30531 & 05511 & 15351 & 22151 & 1.5 .0 & putative amino acid transporter, permease \\
\hline YE2043 & 18951 & 10751 & 30541 & 05501 & 15331 & 22141 & 1.5 .0 & $\begin{array}{l}\text { amino acid transport system permease } \\
\text { protein }\end{array}$ \\
\hline YE2044 & 18961 & 10741 & 30551 & 05491 & - & 22131 & 1.5 .0 & $\begin{array}{l}\text { amino acid-binding periplasmic protein } \\
\text { precursor }\end{array}$ \\
\hline YE2047 & 18981 & 10721 & 30571 & 05471 & 15301 & - & 7.0 .0 & putative hydrolase \\
\hline- & - & 10720 & 30581 & 05421 & - & - & 5.1 .4 & transposase \\
\hline- & - & - & - & - & 15251 & - & 5.1 .4 & putative site-specific recombinase \\
\hline YE2052 & 19001 & 10681 & 30591 & 05411 & 15241 & 22121 & 3.3 .17 & putative AMP nucleosidase \\
\hline YE2055 & 19031 & 10651 & 30621 & 05381 & 15211 & 22091 & 6.1 .1 & putative inner membrane regulatory protein \\
\hline YE2057 & 19041 & 10631 & 39771 & 05361 & 15181 & - & 7.0 .0 & putative virulence factor \\
\hline YE2058 & 19051 & 10621 & 39781 & 05351 & 15171 & 22081 & 7.0 .0 & putative virulence factor \\
\hline YE2059 & 19061 & 10611 & 39791 & 05341 & 15161 & 22071 & 7.0 .0 & putative virulence factor \\
\hline YE2060 & 19071 & 10671 & 28131 & 05311 & 15121 & 22041 & 3.1 .11 & putative acetolactate synthase large subunit \\
\hline YE2064 & 19111 & 10531 & 28171 & 05271 & - & - & 0.0 .0 & hypothetical protein \\
\hline YE2066 & 19131 & 10511 & 28181 & 05261 & 15071 & - & 1.5 .0 & L-fucose permease \\
\hline- & - & 10501 & 28191 & - & - & 22001 & 5.1 .3 & transposase \\
\hline YE2067 & 19141 & 10491 & 28201 & 05251 & 15061 & 21991 & 3.4 .3 & putative carbohydrate kinase \\
\hline YE2069 & 19151 & 10481 & 38211 & 05241 & 15051 & 21981 & 3.3 .13 & putative short chain dehydrogenase \\
\hline YE2073 & 19191 & 10441 & 28251 & 05201 & 15001 & 21931 & 0.0 .2 & conserved hypothetical protein \\
\hline YE2085 & 19311 & 10321 & 28371 & 05011 & 14861 & 21821 & 7.0 .0 & putative carboxypeptidase \\
\hline YE2086 & 19321 & 10312 & 28380 & 05001 & 14851 & 21811 & 1.6 .1 & putative acid shock protein \\
\hline YE2094 & 19391 & - & - & - & - & - & 6.1 .1 & putative transcriptional regulatory protein \\
\hline YE2099 & 19441 & 10211 & 28471 & 04901 & 14741 & 21721 & 4.1 .0 & putative lipoprotein \\
\hline YE2102 & 19471 & 10181 & 28511 & 04861 & 14701 & 21681 & 7.0 .0 & putative pyruvate-flavodoxin oxidoreductase \\
\hline- & - & 10161 & 28521 & 04851 & 14691 & - & 2.2 .7 & putative phospholipase \\
\hline YE2115 & 19581 & 10071 & 28601 & 04771 & 14593 & 21591 & 0.0 .2 & conserved hypothetical protein \\
\hline YE2116 & 19591 & 10061 & 28611 & 04761 & 14592 & 21581 & 0.0 .2 & conserved hypothetical protein \\
\hline- & - & - & - & - & - & 21391 & 5.1 .4 & putative transposase for IS1667 \\
\hline YE2134 & 19771 & 09871 & 28791 & 04561 & 16411 & 21381 & 0.0 .2 & conserved hypothetical protein \\
\hline YE2138 & 19811 & 09831 & 28831 & 04521 & 16461 & 21341 & 3.3 .15 & pyridoxamine kinase \\
\hline YE2141 & 19841 & 09801 & 28861 & 04491 & 16501 & 21311 & 4.1 .0 & putative lipoprotein \\
\hline YE2152 & 19951 & 09691 & 28981 & 04371 & 16631 & 21201 & 7.0 .0 & $\begin{array}{l}\mathrm{NADH} \text { :flavin oxidoreductase / NADH } \\
\text { oxidase family protein }\end{array}$ \\
\hline- & - & 09671 & 29011 & 04351 & 16661 & 21181 & 5.1 .4 & transposase for IS1668 \\
\hline YE2163 & 20061 & 09561 & 34961 & 04231 & 26791 & 21071 & 1.5 .0 & putative transport protein \\
\hline YE2179 & 20212 & 09401 & 35112 & 04072 & 26960 & 20912 & 0.0 .0 & hypothetical protein \\
\hline YE2181 & 20231 & 09381 & 35131 & 04061 & 26971 & 20891 & 1.5 .5 & hemin transport protein \\
\hline YE2182 & 20241 & 09371 & 35141 & 04051 & 26981 & 20881 & 1.5 .5 & hemin-binding periplasmic protein \\
\hline YE2184 & 20261 & 09351 & 35161 & 04031 & 27001 & 20851 & 2.2 .6 & putative lipoate-protein ligase $A$ \\
\hline
\end{tabular}




\begin{tabular}{|c|c|c|c|c|c|c|c|}
\hline- & 17921 & - & - & - & - & - & 3.1 .19 \\
\hline- & - & - & - & - & - & 22791 & 5.1 .2 \\
\hline- & - & - & - & - & - & 22801 & 0.0 .2 \\
\hline \multirow[t]{2}{*}{ - } & - & - & - & - & - & 23091 & 0.0 .1 \\
\hline & & & & & & 23071 & 5.1 .2 \\
\hline- & - & 11571 & 17181 & 06371 & 14361 & 23311 & 0.0 .0 \\
\hline YE2216A & 17891 & 11761 & 16991 & 06561 & 14131 & 23491 & 0.0 .2 \\
\hline- & - & 09081 & 37151 & - & - & - & 4.1 .0 \\
\hline YE2243 & 17641 & 09011 & 37221 & 06801 & 13871 & 23711 & 6.1 .1 \\
\hline YE2245 & 17621 & 08991 & 37241 & 06821 & 13851 & 23731 & 0.0 .0 \\
\hline YE2256 & 17561 & 08921 & 37301 & 06881 & 13761 & 23790 & 4.1 .0 \\
\hline YE2263 & 17481 & 08851 & 37371 & 06951 & 13681 & 23861 & 0.0 .2 \\
\hline- & 17161 & - & - & - & - & - & 5.1 .2 \\
\hline YE2273 & 17021 & 08761 & 37431 & 07011 & 13591 & 23921 & 0.0 .2 \\
\hline YE2278 & 16971 & 08711 & 37491 & 07071 & 13541 & 23971 & 7.0 .0 \\
\hline YE2279 & 16961 & 08701 & 37501 & 07081 & 13531 & 23981 & 1.4 .3 \\
\hline YE2286 & 16891 & 08612 & 37562 & 07142 & 13460 & 24061 & 4.1 .4 \\
\hline- & - & 08611 & 37591 & 03221 & 13401 & 24081 & 5.1 .4 \\
\hline- & - & 08561 & 33631 & 03181 & 27991 & 24121 & 5.1 .4 \\
\hline- & - & 08161 & 34061 & 02751 & 28411 & 24531 & 6.3.11 \\
\hline- & - & 08131 & 34091 & 02711 & 28451 & 24571 & 5.1 .2 \\
\hline- & - & - & - & 02721 & 28441 & - & 5.1 .4 \\
\hline- & - & 08181 & 34021 & 02791 * & 28361 & - & 5.1 .4 \\
\hline- & - & - & - & 07181 & - & - & 5.1 .4 \\
\hline YE2376 & 16771 & 12601 & 16141 & 07301 & 13281 & 19921 & 4.1 .0 \\
\hline- & - & 12981 & 39541 & 07751 & 12811 & - & 0.0 .0 \\
\hline- & - & 13051 & 39471 & 07821 & 12741 & 19391 & 5.1 .4 \\
\hline- & - & 13071 & 39461 & 07831 & 12731 & 19381 & 5.1 .4 \\
\hline YE2410 & 16461 & 13261 & 36551 & 07991 & 12521 & 19181 & 7.0 .0 \\
\hline YE2416 & 16421 & 13301 & 36522 & 08022 & 12491 & 19142 & 4.1 .1 \\
\hline YE2422 & 16371 & 13351 & 36481 & 08071 & 12431 & 19101 & 7.0 .0 \\
\hline- & - & 13661 & 36181 & - & 12111 & 18821 & 5.1 .4 \\
\hline- & - & - & - & - & 12101 & - & 5.1 .2 \\
\hline- & - & - & - & - & 12091 & - & 5.1 .2 \\
\hline- & - & 13791 & 36081 & - & 11971 & - & 5.1 .2 \\
\hline- & - & 13801 & 36071 & - & 11951 & - & 5.1 .2 \\
\hline- & - & 13941 & 35991 & - & - & - & 5.1 .2 \\
\hline- & - & 13771 & 36101 & - & 40661 & 18771 & 5.1 .2 \\
\hline- & - & - & - & - & - & 18761 & 5.1 .2 \\
\hline- & - & 13951 & 40131 & - & - & 18751 & 5.1 .2 \\
\hline- & & 13961 & 40132 & - & - & 18731 & 5.1 .4 \\
\hline- & 16021 & - & - & - & - & - & 0.0 .1 \\
\hline YE2445 & - & - & - & - & - & - & 5.1 .4 \\
\hline- & 21111 & 12924 & 15791 & 07681 & 12921 & 19541 & 1.5 .0 \\
\hline YE2449 & 21141 & 12931 & 15761 & 07711 & 12891 & 19511 & 3.4 .3 \\
\hline YE2450 & 21151 & 12941 & 15751 & 07721 & 12881 & 19491 & 3.4 .3 \\
\hline YE2458 & 21191 & 13001 & 39521 & 07771 & 12791 & 19441 * & 0.0 .2 \\
\hline YE2461 & 21221 & 13031 & 39491 & 07801 & 12761 & 19411 & 3.4 .2 \\
\hline YE2474 & 21341 & 13201 & 39351 & 07941 & 12581 & 19261 & 6.1 .1 \\
\hline - & - & 13211 & 39352 & - & 12571 & 19241 & 5.1 .4 \\
\hline YE2483 & 21341 & 08501 & 33691 & 03121 & 28051 & 24181 & 1.6 .3 \\
\hline YE2484 & 21441 & 08491 & 33701 & 03111 & 28061 & 24191 & 1.6 .3 \\
\hline
\end{tabular}

tryptophan synthase alpha chain,

$\mathrm{EC}=4 \cdot 2 \cdot 1 \cdot 20$

hypothetical phage protein

hypothetical protein

hypothetical protein

putative exinuclease protein

hypothetical protein

conserved hypothetical protein

putative transposase for IS1667

probable response regulator

hypothetical protein

putative exported protein

conserved hypothetical protein

phage replication protein

conserved hypothetical protein

putative phosphodiesterase

putative multidrug resistance protein

acyl-[acyl-carrier-protein]--UDP-N-

acetylglucosamne o-acyltransferase

transposase for IS1328

transposase for IS1668

putative LyxR-family transcriptional

regulatory protein

putative bacteriophage protein

putative transposase

transposase

transposase for IS1328

putative lipoprotein

hypothetical protein

putative transposase subunit

putative transposase for IS1669

putative oxidoreductase

putative inner membrane protein

putative oxidoreductase

IS1400 transposase A

putative phage major capsid protein

putative phage holin

putative variable tail fibre protein

putative phage tail fiber assembly protein

putative bacteriophage tail fiber protein

putative phage baseplate assembly protein

putative phage tail protein

putative bacteriophage late gene regulator

putative transposse for IS1667

conserved hypothetical protein

transposase

putative phoshpotransferase system

enzyme subunit

putative tagatose-6-phosphate ketose /

aldoseisomerase

putative D-tagatose-1,6-bisphosphate

aldolase subunit

conserved hypothetical protein

imidazolonepropionase

putative LysR-family transcriptional

regulatory protein

putative transposase for IS1667

haemin storage system, HmsF protein

haemin storage system, $\mathrm{HmsH}$ protein,

precursor 


\begin{tabular}{|c|c|c|c|c|c|c|c|c|}
\hline YE2487 & 21181 & 12991 & 39531 & 07761 & 12801 & 19451 & 4.1 .0 & hypothetical protein \\
\hline YE2488 & 21481 & 08451 & 33741 & 03071 & 28101 & 24251 & 6.1 .1 & $\begin{array}{l}\text { putative LysR-family transcriptional } \\
\text { regulatory protein }\end{array}$ \\
\hline YE2499 & 21591 & 08341 & 33851 & 02961 & 28211 & 24371 & 4.1 .1 & putative membrane protein \\
\hline YE2502 & 21621 & 08311 & 33881 & 02931 & 28241 & 24401 & 7.0 .0 & putative class II aldolase-family protein \\
\hline YE2508 & 21681 & 08251 & 33941 & 02871 & 28301 & 24461 & 1.5 .1 & L-asparagine permease \\
\hline- & 21761 & - & - & - & - & - & 5.1 .2 & putative phage integrase \\
\hline - & 23022 & 19111 & 24961 & 09081 & 11422 & 18381 & 0.0 .2 & conserved hypothetical protein \\
\hline YE2520 & 23091 & 19031 & 24881 & 09161 & 11341 & 18291 & 2.2 .11 & flagellin lysine- $\mathrm{N}$-methylase \\
\hline - & 23101 & - & - & - & - & - & 1.1 .1 & flagellin \\
\hline YE2521 & 23111 & 19021 & 24871 & 09171 & 11331 & $\begin{array}{l}\text { YE3904- } \\
18281\end{array}$ & 1.1 .1 & flagellin \\
\hline YE2522 & 23121 & 19011 & 24861 & 09181 & - & - & 1.1 .1 & thermoregulated motility protein \\
\hline YE2523 & 23131 & 19001 & 24851 & 09191 & - & 18271 & 1.1 .1 & flagellin \\
\hline YE2531 & - & 18911 & 24771 & 09271 & 11241 & 18204 & 0.0 .0 & hypothetical protein \\
\hline YE2535 & - & 18900 & 24762 & 09280 & 11230 & 18202 & 5.1 .2 & IS1329 transposase B \\
\hline YE2564 & 23481 & 18561 & 24471 & 09571 & 10911 & 17921 & 5.1 .5 & invasin \\
\hline YE2586A & 23691 & 18351 & 24261 & 09780 & 10651 & 17691 & 3.3 .0 & putative coA transferase family protein \\
\hline YE2594 & - & - & - & - & - & - & 5.1 .4 & $\begin{array}{l}\text { transposase for insertion sequence element } \\
\text { IS } 1665\end{array}$ \\
\hline- & - & 18281 & 39880 & 09831 & - & - & 5.1 .4 & transposase \\
\hline YE2597 & 23791 & $18251^{*}$ & 39861 * & 09861 & 10551 & 17621 & 6.1 .1 & $\begin{array}{l}\text { AsnC-family transcriptional regulatory } \\
\text { protein }\end{array}$ \\
\hline YE2598 & 23801 & 18241 & 39851 & 09871 & 10531 & - & 0.0 .1 & conserved hypothetical protein \\
\hline YE2600 & 23821 & 18221 & 39831 & 09891 & 10511 & 17591 & 3.3 .15 & probable histidine acid phosphatase \\
\hline - & 23851 & 18175 & 34831 & 09931 & 30271 & 17551 & 4.1 .5 & $\begin{array}{l}\text { putative outer membrane fimbrial usher } \\
\text { protein }\end{array}$ \\
\hline- & 23861 & 18174 & 34821 & 09941 & 30281 & 17541 & 4.1 .0 & putative exported protein \\
\hline YE2603 & 23891 & 18171 & 34791 & 09971 & 30311 & 17501 & 3.4 .3 & putative sugar kinase \\
\hline YE2609 & 23951 & 18151 & 34781 & 09981 & 30321 & 17491 & 1.5 .0 & $\begin{array}{l}\text { ABC transporter, substrate binding } \\
\text { component }\end{array}$ \\
\hline- & - & - & - & - & 30361 & - & 3.3 .0 & putative hydrolase \\
\hline- & - & - & - & - & 30401 & - & 2.2 .3 & putative antirestriction protein \\
\hline- & - & - & - & - & 30421 & - & 2.2 .3 & putative NTPase \\
\hline YE2611 & - & - & - & - & - & - & 5.1 .2 & integrase \\
\hline YE2624 & - & - & - & - & - & - & 5.1 .4 & $\begin{array}{l}\text { transposase for insertion sequence element } \\
\text { IS } 1328\end{array}$ \\
\hline YE2640 * & 24071 & 18001 & 34641 & 10121 & 30601 & 17341 & 4.1.1 & putative membrane protein \\
\hline YE2644 & 24091 & 17971 & 34621 & 10141 & 30631 & 17321 & 1.5 .3 & $\begin{array}{l}\text { putative C4-dicarboxylate transporter, } \\
\text { periplasmic protein }\end{array}$ \\
\hline YE2646 & 24111 & 17951 & 34601 & 10161 & 30651 & 17291 & 1.5 .3 & $\begin{array}{l}\text { putative C4-dicarboxylate transporter, large } \\
\text { subunit }\end{array}$ \\
\hline YE2652 & 24171 & 17891 & 34541 & 10221 & 30711 & 17221 & 3.3 .15 & citrate lyase acyl carrier protein \\
\hline YE2654 & 24191 & 17871 & 34521 & 10241 & 30731 & 17201 & 6.1 .1 & sensor kinase protein \\
\hline YE2662 & 24271 & 18121 & 34761 & 10001 & 30471 & 17471 & 6.1 .1 & putative two-component response regulator \\
\hline YE2663 & 24281 & 18111 & 34751 & 10011 & 30481 & 17461 & 6.1 .1 & putative virulence sensor protein \\
\hline YE2666 & 24311 & 18081 * & 34721 & 10041 & 30511 & 17421 & 4.1 .5 & probable fimbrial usher protein \\
\hline- & - & 18201 & 34851 & 09911 & 30251 & 17571 & 5.1 .4 & putative transposase for IS 1667 \\
\hline- & - & - & - & - & - & 17191 & 5.1 .4 & putative transposase \\
\hline YE2670 & 24351 & - & - & 10341 & 30841 & 17081 & 6.1 .1 & putative GntR-family regulatory protein \\
\hline YE2671 & 24381 & 17771 & 34431 & 10351 & 30851 & 17072 & 0.0 .0 & $\begin{array}{l}\text { putative short chain dehydrogenase / } \\
\text { reductase family protein }\end{array}$ \\
\hline YE2684 & 31121 & 17641 & 32241 & 10491 & 31001 & 16921 & 0.0 .2 & $\begin{array}{l}\text { conserved hypothetical protein [unresolved } \\
\text { tandem repeat] }\end{array}$ \\
\hline YE2686 & 31141 & 17621 & 32221 & 10511 & 31021 & 16901 & 4.1 .1 & putative OmpA family protein \\
\hline YE2693 & 31211 & 17551 & 32151 & 10581 & 31091 & 16831 & 4.1.0 & outer membrane usher protein \\
\hline YE2705 & 31331 & 17411 & 32031 & 10701 & 31221 & 16701 & 6.1 .1 & putative luxR-family regulatory protein \\
\hline- & 31341 & 17410 & 32021 & 10711 & 31222 & 16691 & 1.5 .0 & xanthosine permease \\
\hline YE2706 & - & - & - & - & - & - & 7.0 .0 & $\begin{array}{l}\text { transcription regulator/permease fusion } \\
\text { protein }\end{array}$ \\
\hline
\end{tabular}




\begin{tabular}{|c|c|c|c|c|c|c|c|c|}
\hline YE2708 & 31391 & 17391 & 32001 & 10731 & 31241 & 16661 & 3.2 .3 & alpha-ribazole-5'-phosphate phosphatase \\
\hline YE2710 & 31411 & 17371 & 31981 & 10751 & 31261 & 16641 & 3.2 .3 & cobinamide kinase and guanylyltransferase \\
\hline YE2719 & 31501 & 17271 & 31881 & 10851 & 31361 & 16541 & 0.0 .2 & conserved hypothetical protein \\
\hline YE2724 & 31571 & 17211 & 31821 & 10911 & 31421 & 16481 & 4.1 .1 & putative membrane protein \\
\hline YE2762 & 31941 & 16831 & 31451 & 11281 & 31811 & 16111 & 1.5 .0 & $\begin{array}{l}\text { putative } A B C \text { transport system ATP-binding } \\
\text { protein }\end{array}$ \\
\hline- & - & - & - & - & 31971 & 15691 & 5.1 .4 & transposase \\
\hline- & - & - & - & - & - & 15951 & 5.1 .4 & putative transposase \\
\hline- & - & - & - & - & 32011 & 15941 & 5.1 .4 & IS1400 transposase B \\
\hline YE2810 & 32391 & 16251 & 10721 & 11731 & 32471 & 15541 & 3.5 .2 & formate dehydrogenase $\mathrm{H}$ \\
\hline YE2812 & 32411 & 16231 & 10710 & 11750 & 32482 & 15512 & 1.5 .3 & putative formate transporter \\
\hline YE2829 & 32581 & 16071 & 10531 & 11921 & 32661 & 15341 & 3.2 .9 & molybdopterin biosynthesis protein \\
\hline YE2832 & 32601 & 16041 & 10511 & 11951 & 32711 & 15301 & 0.0 .0 & hypothetical protein \\
\hline YE2852 & 32801 & 15841 & 10311 & 12151 & 32931 & 15091 & 7.0 .0 & putative peptidase \\
\hline YE2859 & 32871 & 15761 & 10241 & 12221 & 33021 & 15021 & 6.1 .1 & LysR family transcription regulatory protein \\
\hline YE2866 & 32941 & 15691 & 10171 & 12291 & 33111 & 14951 & 4.1 .1 & putative fatty acid desaturase \\
\hline YE2867 & 32951 & 15681 & 10161 & 12301 & 33121 & 14931 & 0.0 .2 & conserved hypothetical protein \\
\hline YE2883 & 33121 & 15521 & 10001 & 12461 & 33291 & 14771 & 3.5 .1 & D-lactate dehydrogenase \\
\hline YE2885 & 33141 & 15491 & 09971 & 12491 & 33331 & 14750 & 0.0 .2 & conserved hypothetical protein \\
\hline YE2900 & 33271 & 15361 & 09841 & 12621 & 33471 & 14621 & 0.0 .2 & conserved hypothetical protein \\
\hline- & - & - & - & - & - & 14361 & 5.1 .2 & putative phage protein \\
\hline- & - & - & - & - & - & 14311 & 5.1 .2 & putative bacteriophage protein \\
\hline YE2922 & - & 15131 & 09611 & 12851 & 33731 & 13941 & 7.0 .0 & putative protease \\
\hline YE2955A & 33811 & 14811 & 09291 & 13171 & 34121 & 13621 & 0.0 .2 & conserved hypothetical protein \\
\hline- & 33861 & 14761 & 09241 & 13221 & 34171 & 13561 & 5.1 .5 & putative cytotoxin RtxA \\
\hline- & 33881 & 14741 & 09221 & 13241 & 34191 & 13541 & 0.0 .2 & putative uncharacterized protein $\mathrm{RtxH}$ \\
\hline YE2966 & 33961 & 14661 & 09141 & 13321 & 34281 & 13461 & 5.1 .5 & putative hemolysin \\
\hline YE3007 & 34461 & 14251 & 08731 & 13731 & 34711 & 13031 & 3.2 .7 & lipoate-protein ligase B \\
\hline YE3014 & 34531 & 14181 & 08661 & 13801 & 34791 & 12962 & 6.1 .1 & putative LyxR-family regulatory protein \\
\hline YE3021 & 34601 & 14111 & 08591 & 13871 & 34871 & 12911 & 7.0 .0 & $\begin{array}{l}\text { putative phenylalanine and histidine } \\
\text { ammonia-lyase }\end{array}$ \\
\hline YE3025 & 24651 & 27571 & 39931 & 13911 & - & 12861 & 6.1 .1 & $\begin{array}{l}\text { putative two-component system sensor } \\
\text { protein }\end{array}$ \\
\hline- & - & 27551 & 39921 & 13921 & - & 12841 & 0.0 .0 & hypothetical protein \\
\hline- & - & - & - & - & 34901 & - & 5.1 .2 & putative phage integrase \\
\hline- & - & 27502 & 39891 & 13951 & 34941 & 12811 & 0.0 .2 & conserved hypothetical protein \\
\hline- & - & - & 39892 & 13961 & 34951 & 12801 & 0.0 .2 & conserved hypothetical protein \\
\hline- & - & 27501 & 23351 & 13991 & 35001 & - & 5.1 .2 & putative phage primase \\
\hline YE3026 & 34661 & - & - & - & - & - & 4.1 .0 & putative exported protein \\
\hline YE3028 & 34681 & - & - & - & - & - & 4.1 .0 & putative exported protein \\
\hline YE3037 & - & - & - & - & - & - & 0.0 .0 & hypothetical protein \\
\hline YE3038 & - & - & - & - & - & - & 5.1 .4 & integrase \\
\hline- & - & 27471 & 23331 & 14041 & 35061 & 12791 & 5.1 .2 & putative integrase \\
\hline- & - & - & - & - & - & 12501 & 5.1 .2 & lysozyme \\
\hline- & - & - & - & - & - & 12281 & 2.2 .3 & $\begin{array}{l}\text { putative adenine-specific modification } \\
\text { methylase }\end{array}$ \\
\hline YE3066 & 35381 & 27281 & $\begin{array}{l}\text { YE5630- } \\
23141\end{array}$ & 14231 & 35261 & 12081 & 0.0 .2 & UDP-sugar hydrolase \\
\hline YE3097 & 35611 & 27111 & 22911 & 14461 & 35541 & 11871 & 1.5 .2 & putative potassium efflux system \\
\hline- & - & 26491 & 22291 & 15081 & 36311 & 11231 & 0.0 .0 & hypothetical protein \\
\hline- & - & 26441 & 22231 & 15141 & 36371 & 11171 & 5.1 .4 & putative transposase for IS1667 \\
\hline YE3168 & 36291 & 26442 & 32811 & 15151 & 36391 & 11161 & 0.0 .2 & conserved hypothetical protein \\
\hline YE3180 & 36381 & 26351 & 32721 & 15241 & 36491 & 11071 & 5.1 .4 & phosphate binding protein \\
\hline YE3190 & 36481 & 26251 & 32621 & 15341 & 36601 & 10961 & 0.0 .1 & conserved hypothetical protein \\
\hline YE3196 & 36541 & 26191 & 32561 & 15401 & 36681 & 10901 & 4.1 .4 & putative cyclic beta $1-2$ glucan synthetase \\
\hline YE3199 & 36571 & 26161 & 32561 & 15431 & 36711 & 10851 & 1.5 .3 & $\begin{array}{l}\text { PTS system, alpha-glucoside-specific IIBC } \\
\text { component }\end{array}$ \\
\hline YE3208 & 36661 & 26071 & 32441 & 15521 & 36821 & 10751 & 0.0 .2 & conserved hypothetical protein \\
\hline- & - & 25861 & - & - & - & - & 5.1 .4 & putative transposase for IS1667 \\
\hline- & - & 25621 & - & - & - & - & 5.1 .2 & putative transposase for IS1667 \\
\hline
\end{tabular}




\begin{tabular}{|c|c|c|c|c|c|c|c|}
\hline- & - & 25601 & 35981 & 40831 & - & 09751 & 5.1 .4 \\
\hline YE3218 & 36761 & 25971 & 32341 & 15621 & 36921 & 10641 & 3.5 .3 \\
\hline YE3225 & 36831 & 25901 & 32271 & 15691 & 37001 & 10561 & 5.1 .5 \\
\hline YE3226 & 36841 & 25891 & 32261 & 15701 & 37021 & 10551 & 5.1 .5 \\
\hline - & - & - & - & - & - & 10131 & 5.1 .2 \\
\hline- & - & - & - & - & - & 10121 & 5.1 .2 \\
\hline- & - & - & - & - & - & 10111 & 5.1 .2 \\
\hline- & 36931 & 25790 & 39021 & 15791 & 37121 & 10001 & 3.4 .2 \\
\hline- & 36941 & 25789 & 39011 & 15801 & 37131 & 09991 & 3.7 .1 \\
\hline YE3285 & 06081 & 34221 & 04451 & 30331 & 26331 & 33001 & 2.2.10 \\
\hline- & - & 24571 & 31431 & 30121 & 38571 & - & 5.1 .4 \\
\hline- & - & 24691 & 30741 & 31661 & 38431 & 08751 & 0.0 .0 \\
\hline- & - & 24651 & 37081 & 31701 & 38471 & 08711 & 3.3 .0 \\
\hline- & - & 24632 & 37100 & 31720 & 38511 & 08701A & 5.1 .2 \\
\hline YE3321 & 05760 & 24441 & - & - & 25941 * & 33332 & 0.0 .1 \\
\hline YE3326 & 05721 & 24401 & 31271 & 29961 & 25901 & 33371 & 4.1 .1 \\
\hline YE3328 & - & - & - & - & 25882 & - & 5.1 .4 \\
\hline YE3338 & 05621 & 24291 & 31171 & 29861 & 25791 & 33491 & 1.1.1 \\
\hline YE3341 & 05591 & 24253 & 31131 & 29821 & 25741 & 33531 & 1.5 .5 \\
\hline YE3342 & 05581 & 24252 & 31121 & 29811 & 25731 & 33541 & 4.1 .0 \\
\hline YE3344 & 05551 & 24241 & $\begin{array}{l}\text { YE6503- } \\
31101\end{array}$ & 29781 & 25711 & 33561 & 1.5 .5 \\
\hline YE3345 & 05531 & 24221 & 31081 & 29761 & 25681 & 33581 & 1.5 .5 \\
\hline YE3347 & 05511 & 24201 & 31061 & 29741 & 25661 & 33601 & 1.5 .5 \\
\hline YE3349 & 05491 & 24181 & 31041 & 29721 & 25631 & 33631 & 1.5 .5 \\
\hline YE3354 & 05421 & 24131 & 30991 & 29671 & 25581 & 33681 & 7.0 .0 \\
\hline YE3358 & 05381 * & 24081 & 30941 & 29631 & - & - & 1.5 .3 \\
\hline- & - & 24041 & 30901 & 29591 & 25551 & - & 5.1 .4 \\
\hline YE3367 & 05271 & 24001 & 30871 & 29551 & 25521 & 33741 & 0.0 .1 \\
\hline- & - & - & - & - & - & 33801 & 5.1 .4 \\
\hline- & - & - & - & - & - & 33861 & 5.1 .4 \\
\hline YE3376 & 04861 & 23831 & 30691 & 29471 & 25431 & 33901 & 2.2 .10 \\
\hline YE3383 & 04801 & 23751 & 13611 & 29411 & 25371 & 33961 & 6.1 .1 \\
\hline - & - & - & - & - & 25321 & 34011 & 5.1 .4 \\
\hline YE3455 & - & - & - & - & - & - & 5.1 .4 \\
\hline YE3456 & - & - & - & - & - & - & 5.1 .4 \\
\hline YE3516 & - & 34811 & 05031 & 32141 & 38971 & 08271 & 5.1 .2 \\
\hline YE3521 & - & - & - & - & - & - & 0.0 .2 \\
\hline YE3522 & - & - & - & - & - & - & 7.2 .1 \\
\hline YE3524 & - & - & - & - & - & - & 5.1 .4 \\
\hline YE3524A & - & - & - & - & - & - & 0.0 .0 \\
\hline YE3527 & 03881 & 34921 & 03881 & 32221 & 39061 & 08201 & 3.3 .20 \\
\hline YE3528 & 03871 & 34931 & 05121 & 32231 & 39071 & 08181 & 4.1 .1 \\
\hline YE3532 & 03831 & 34971 & 05161 & 32271 & 39121 & 08151 & 0.0 .0 \\
\hline YE3580 & - & - & - & - & - & - & 5.1 .4 \\
\hline YE3587 & 03751 & 35058 & 05241 & 32351 & 39221 & 08061 & 7.0 .0 \\
\hline- & 03741 & 35059 & 05251 & 32361 & 39241 & 08051 * & 4.1 .1 \\
\hline- & 03721 & 35062 & 05270 & 32382 & 39280 & 08040 & 3.3 .0 \\
\hline YE3588 & 03711 & 35061 & 05271 & 32381 & 39281 & 08031 & 1.5 .0 \\
\hline YE3590 & 03691 & 35081 & 05291 & 32401 & 39311 & 08001 & 7.0 .0 \\
\hline- & 03641 & 35130 & 05341 & 32451 & 39360 & 07951 & 0.0 .1 \\
\hline YE3595 & 03621 & 35131 & 05351 & 32461 & 39361 & 07941 & 1.4 .3 \\
\hline- & - & 39380 & 38529 & - & 03463 & 38722 & 5.1 .4 \\
\hline- & - & 39381 & 38530 & - & 03462 & 38721 & 5.1 .4 \\
\hline YE3603 & 03531 & 35251 & 05451 & 32561 & 39461 & 07841 & 2.2 .9 \\
\hline
\end{tabular}

transposase for IS1668

$\mathrm{Na}$--translocating $\mathrm{NADH}$-guinone reductase subunit $\mathrm{C}$

putative accessory processing protein putative adhesin

putative phage replication protein

putative DNA replication protein

putative phage replication protein

putative amino acid hydrolase

putative purine catabolism protein

methionine aminopeptidase

putative transposase for IS1667

hypothetical protein

putative oxidoreductase

putative integrase

conserved hypothetical protein

putative membrane protein

putative transposase for IS1667

putative methyl-accepting chemotaxis

protein

prepilin peptidase

putative exported protein

general secretion pathway protein $\mathrm{K}$

general secretion pathway protein I

general secretion pathway protein

general secretion pathway protein $\mathrm{D}$

putative oxidoreductase

putative sugar transporter

putative transposase for IS1667

conserved hypothetical protein

tnpA-like protein

tnpA-like protein

peptide chain release factor 2 - ALL

ORTHOLOGS WITH FS!!! Shown to be

functional

putative histidine kinase sensor

putative transposase for IS1667

transposase for IS3-like insertion sequence

transposase for IS3-like insertion sequence

putative integrase

conserved hypothetical protein

Putative exported protein

putative transposase

hypothetical protein

ornithine decarboxylase, inducible

putative membrane protein

hypothetical protein

putative IS285-like transposase

putative O-methyltransferase

hypothetical membrane protein

putative FMN-binding protein

putative transporter protein

putative hydrolase

conserved hypothetical protein

putative integral membrane efflux protein

putative transposse for IS1669

transposase for IS1328

hydrogenase isoenzymes nickel

incorporation protein 


\begin{tabular}{|c|c|c|c|c|c|c|}
\hline YE3610A & - & - & - & - & - & - \\
\hline YE3611 & - & - & - & - & - & - \\
\hline YE3612 & - & - & - & - & - & - \\
\hline YE3617 & 03401 & 35751 & 05981 & 33081 & - & 07261 \\
\hline YE3622 & 03351 & 35801 & 06031 & 33131 & - & 07211 \\
\hline YE3624 & 03341 & - & - & - & - & - \\
\hline YE3626 & 03331 & - & - & - & - & - \\
\hline - & 03441 & 35729 & 05941 & 33041 & - & 07301 \\
\hline- & 03451 & 35350 & 05541 & 32651 & 39521 & 07751 \\
\hline- & - & 35381 & 05581 & 32691 & 39561 & 07711 \\
\hline- & - & 35431 & 05631 & 32741 & 39641 & 07651 \\
\hline- & - & 35591 & 05801 & 39821 & - & 07451 \\
\hline- & - & 35641 & 05851 & 32951 & - & 07391 \\
\hline- & - & - & - & - & - & 07181 \\
\hline- & 03962 & - & - & - & - & - \\
\hline- & 03971 & - & - & - & - & - \\
\hline- & 03991 & - & - & - & - & - \\
\hline- & 04121 & - & - & - & - & - \\
\hline- & 05041 & - & - & - & - & - \\
\hline- & - & 35831 & 06051 & 33151 & 39831 & 07161 \\
\hline- & & 35850 & 06060 & 33160 & 39851 & 07142 \\
\hline- & - & 35880 & 06092 & 33192 & 39901 & 07103 \\
\hline YE3630 & 03291 & 35921 & 06141 & 33231 & 40010 & 07071 \\
\hline YE3636 & 03241 & 35981 & 06191 & 33281 & 40051 & 07021 \\
\hline YE3671 & 02911 & 36351 & 06531 & 33621 & 40401 & 06681 \\
\hline- & - & 36481 & 06671 & 33761 & 40541 & 06541 \\
\hline- & - & - & - & - & 40841 & - \\
\hline- & - & - & - & 33869 & - & - \\
\hline- & - & 36611 & 06851 & 40775 & - & - \\
\hline- & - & 36621 & 06861 & 33891 & - & - \\
\hline YE3690 & 02721 & 36761 & 07011 & 34031 & 41061 & 06461 \\
\hline YE3694 & 02681 & 36792 & 07042 & 34062 & 41101 & 06430 \\
\hline YE3697 & 02651 & 36811 & 07061 & 34081 & 41111 & 06411 \\
\hline YE3700 & - & 36861 & 07111 & 34121 & 41171 & 06361 \\
\hline YE3722 & 02401 & 37081 & 07331 & 34341 & 41421 & 06141 \\
\hline- & - & - & - & - & - & 06041 \\
\hline- & - & - & - & - & $\begin{array}{l}\text { YE1293- } \\
41551\end{array}$ & 06021 \\
\hline YE3734 & 02291 & 37231 & 07491 & 34501 & 41671 & 05931 \\
\hline YE3735 & 02281 & 37241 & 07501 & 34511 & 41681 & 05921 \\
\hline YE3753 & 02101 & 37421 & 07681 & 34701 & 41891 & 05731 \\
\hline YE3774 & 01911 & 37611 & 07871 & 34891 & 42101 & 05541 \\
\hline YE3776 & 01891 & 37631 & 07891 & 34911 & 42131 & 05501 \\
\hline YE3778 & 01871 & 37661 & 07921 & 34941 & 42181 & 05471 \\
\hline YE3779 & 01861 & 37671 & 07931 & 34951 & 42191 & 05451 \\
\hline- & - & 37771 & 08031 & - & 42291 & 05341 \\
\hline- & 01721 & - & - & - & - & 05301 \\
\hline- & 01711 & - & - & - & - & 05281 \\
\hline- & 01581 & - & - & - & - & 05151 \\
\hline- & 01571 & - & - & - & - & 05141 \\
\hline- & 01501 & - & - & - & - & 05061 \\
\hline- & - & 37861 & 08111 & - & - & - \\
\hline- & - & 37921 & 08171 & - & - & - \\
\hline- & - & 38151 & 08501 & 35141 & 42391 & 04881 \\
\hline- & - & 38171 & 08521 & 35161 & 42411 & 04861 \\
\hline
\end{tabular}

putative flagellar biogenesis protein putative transmembrane transcriptional activator

transposase for IS1330

putative membrane transport protein

enterochelin esterase

gerrichrysobactin receptor precursor putative exported protein, precursor putative flagellar biosynthesis protein flagellar motor protein motB

flagellar hook-length control protein fliK

putative regulatory protein

putative transposase for IS1667

putative flagellar M-ring protein

putative transposase for IS1667

putative exinuclease

putative transposase subunit

putative transposase

putative peptidase

nickel-dependent hydrogenase, b-type cytochrome subunit

putative transposase

putative transcriptional regulatory protein

putative transposase

putative cation efflux system protein

putative secretion system protein

conserved hypothetical protein

putative transposase for IS1667

putative phage terminase

putative phage terminase, ATPase subunit

putative phage protein

putative phage replication protein

photransferase system IIBC component

Putative transcriptional regulator

putative membrane protein

hypothetical protein

probable NADP-dependent alcohol

dehydrogenase

transposase

hypothetical protein

conserved hypothetical protein

glutamate synthase [NADPH] large chain

precursor

putative sodium/calcium exchanger protein

PTS system, trehalose-specific IIBC

component

Mg2+ transporter ATPase

putative exported protein

conserved hypothetical protein

putative transposase for IS1667

hypothetical phage-related protein

hypothetical phage-related protein

putative tail sheath protein

hypothetical phage-related protein

putative portal vertex protein

putative phage tail sheath protein

putative phage tail protein

putative lysR-family transcriptional regulator

putative insecticidal toxin complex protein 


\begin{tabular}{|c|c|c|c|c|c|c|}
\hline - & - & 38181 & 08531 & 35171 & 42421 & 04841 \\
\hline - & - & 38241 & 26241 & - & - & - \\
\hline YE3818 & 01101 & 38481 & 26471 & 35441 & 42711 & 04551 \\
\hline YE3825 & - & - & - & - & - & - \\
\hline YE3828 & $01041^{*}$ & 38541 * & 26512 & 35500 & 42772 & $04502^{*}$ \\
\hline YE3837 & 00971 & 38641 & 26581 & 35561 & 42851 & 04431 * \\
\hline- & - & - & - & 35761 & - & - \\
\hline- & - & - & - & 36081 & - & - \\
\hline YE3860 & - & 38901 & 26831 & 36341 & 43121 & 04171 \\
\hline- & 00061 & 39021 & 26961 & 36471 & 43241 & 04021 \\
\hline- & - & - & - & 36541 & 43302 & 03951 \\
\hline- & - & 00001 & 39951 & 19531 & 03480 & 32671 \\
\hline- & 30761 & 19588 & 13981 & 25091 & 20771 & 38081 \\
\hline- & 30841 & 19580 & 13901 & 25011 & 20691 & 38161 \\
\hline- & 30861 & 19578 & 13881 & 24991 & 20681 & 38181 \\
\hline- & 30891 & 19575 & 13851 & 24961 & 20651 & 38202 \\
\hline- & 31051 & 19482 & 13691 & 24801 & 20491 & 38381 \\
\hline- & - & 27711 & 39571 & 19401 & 20291 & 03831 \\
\hline YE3956 & 37891 & 28341 & 27501 & 18781 & 19651 & 03261 * \\
\hline YE3964 & 37971 & 28421 & 27421 & 18701 & 19561 & 03181 \\
\hline YE3967 & 38001 & 28541 & 27391 & 18671 & 19521 & 03151 \\
\hline YE3970 & 38031 & 28481 & 27361 & 18641 & 19491 & 03111 \\
\hline- & - & 28811 & 27031 & - & 19121 & 02761 \\
\hline YE3989 & 38361 & 28880 & 24151 & 18271 & 19071 & 02711 \\
\hline YE4001 & 38240 & 28721 & 27121 & 18401 & 19221 & 02861 \\
\hline YE4002 & - & 28711 & 27131 & 18411 & 19231 & 02871 \\
\hline YE4026 & 38581 * & 29081 & 23931 & 18051 & 18851 & 02491 \\
\hline YE4041 & 38751 & 29231 & 23771 & 17891 & 18681 & 02331 \\
\hline YE4046 & 38801 & 29281 & 23721 & 17841 & 18631 & 02281 \\
\hline- & - & - & - & - & - & 02271 \\
\hline- & - & - & - & - & - & 02241 \\
\hline- & - & - & - & - & - & 02141 \\
\hline YE4052 & 38861 & 29341 & 23661 & 17781 & 18571 & 02061 \\
\hline - & 39081 & - & - & - & - & \\
\hline YE4062 & 39151 & 29421 & 23581 & 17701 & 18491 & 01971 \\
\hline YE4072 & 39261 & 29521 & 23481 & 17601 & 18381 & 01871 \\
\hline YE4074 & 39291 * & 29551 & 23451 & 17571 & 18351 & 08141 \\
\hline YE4086 & 39411 & 29711 & 38471 & 17411 & 18141 & 39841 \\
\hline- & - & 29751 & 38431 & 17371 & 18091 & 39891 \\
\hline- & 39521 & 29882 & 38921 & 17231 & 17911 & - \\
\hline YE4096 & 39511 & 29921 & 38272 & 17222 & 17902 & - \\
\hline YE4097 & - & 29911 & 38273 & 17223 & 17903 & - \\
\hline YE4101 & 39561 & 29851 & 38331 & 17271 & 17971 & - \\
\hline YE4106 & 39611 & 29801 & 38381 & 17321 & 18021 & 39941 \\
\hline- & - & 29891 & 38281 & - & - & - \\
\hline YE4113 & - & 30041 & 38202 & 13991 & 35001 & 01631 \\
\hline YE4114 & - & 30061 & 38204 & - & - & 01611 \\
\hline YE4115 & - & 30091 & 38205 & - & - & 01601 \\
\hline- & - & - & - & 17181 & 17861 * & - \\
\hline YE4118 & 39741 & 30111 & 21141 & 17131 & 17791 & 01581 \\
\hline YE4123 & 39791 & 30161 & 21091 & 17081 & 17741 & 01531 \\
\hline YE4125 & 39821 & 30191 & 21061 & 17051 & 17711 & 01501 \\
\hline YE4135 & 39901 & 30291 & 20981 & 16971 & 17631 & 01421 \\
\hline
\end{tabular}

putative insecticidal toxin complex protein putative insecticidal toxin complex protein possible regulator invovled in cell signalling transposase for insertion sequence element IS1328

hypothetical protein

putative inner membrane protein

putative phage tail protein

putative phage protein

putative exported protein

hemolysin

putative transposase

transposase

putative type III secretion protein

putative pathogenicity island protein

putative pathogenicity island protein

putative type III secretion apparatus

putative sensor protein

transposase for IS1668

putative membrane protein

cytosine deaminase

putative nitrite transporter

phosphoglycolate phosphatase

putative transposase for IS1667

conserved hypothetical protein

putative MFS-family transport protein

hypothetical protein

myo-inositol 2-dehydrogenase

glutamate mutase

putative sodium-dicarboxylic acid

transporter family protein, precursor

hypothetical protein

hypothetical protein

Tn7-like transposition protein

metalloprotease

putative integrase

putative exported protein

probable $\mathrm{BcsC}$ protein involved in cellulose synthesis

cellulose synthase 1 catalytic subunit[UDP-

forming]

putative exported protein, precursor

transposase

putative fimbrial usher protein

hypothetical protein

hypothetical protein

Putative outer membrane porin

Hypothetical ROK family protein

putative transposase for IS1667

putative phage primase

conserved hypothetical protein

conserved hypothetical protein

transposase subunit ist $A$

putative AraC-family transcriptional

regulator

xylulose kinase

Putative transcriptional regulator

formate dehydrogenase-o, major subunit ALL ORTHOS WITH READ-THROUGH OF STOP CODON 


\begin{tabular}{llllllllll} 
YE4142 & 39961 & 30351 & 20921 & 16911 & 17561 & 01351 & 7.0 .0 & hypothetical protein \\
YE4145 & 39991 & 30381 & 20891 & 16881 & 17531 & 01321 & 4.1 .1 & Putative inner membrane protein \\
YE4167 & 40211 & 30651 & 20631 & 16621 & 17301 & 40661 & 7.0 .0 & pyridoxal-phosphate dependent enzyme \\
YE4174 & 40291 & 30730 & 20561 & 16551 & 17212 & 40722 & 0.0 .0 & hypothetical protein \\
YE4185 & 40401 & 30831 & 20451 & 16441 & 17101 & 40811 & 6.1 .1 & Probable two-component sensor kinase \\
\hline- & 40981 & 32651 & 17481 & 36711 & 01891 & 40041 & 5.1 .1 & putative Colicin
\end{tabular}


Table 9.8: Number of total CDSs, pseudogenes, and partial genes per group for the $Y$. enterocolitica reference genomes.

\begin{tabular}{|c|c|c|c|c|c|c|c|c|}
\hline group & description & 5303 & 8081 & 21202 & 5603 & 14902 & 1203 & 3094 \\
\hline \multirow{3}{*}{1} & $\begin{array}{l}\text { Unknown function, no known } \\
\text { homologues }\end{array}$ & 857 & 662 & 662 & 663 & 686 & 664 & 722 \\
\hline & pseudo & 7 & 3 & 10 & 11 & 11 & 28 & 36 \\
\hline & partial & 8 & 9 & 13 & 12 & 14 & 19 & 14 \\
\hline \multirow{3}{*}{2} & Cell processes & 95 & 84 & 118 & 118 & 118 & 102 & 117 \\
\hline & pseudo & 2 & 0 & 1 & 1 & 1 & 1 & 5 \\
\hline & partial & 7 & 1 & 0 & 1 & 1 & 2 & 0 \\
\hline \multirow{3}{*}{3} & Protection responses & 69 & 54 & 60 & 59 & 57 & 55 & 56 \\
\hline & pseudo & 3 & 0 & 0 & 0 & 0 & 0 & 7 \\
\hline & partial & 3 & 0 & 3 & 1 & 2 & 2 & 1 \\
\hline \multirow{3}{*}{4} & Transport/binding proteins & 533 & 497 & 483 & 483 & 483 & 477 & 472 \\
\hline & pseudo & 4 & 3 & 6 & 5 & 5 & 14 & 23 \\
\hline & partial & 1 & 8 & 10 & 9 & 10 & 14 & 17 \\
\hline \multirow{3}{*}{5} & Adaptation & 52 & 56 & 49 & 49 & 49 & 45 & 49 \\
\hline & pseudo & 0 & 1 & 0 & 0 & 0 & 0 & 3 \\
\hline & partial & 1 & 0 & 3 & 3 & 1 & 2 & 1 \\
\hline \multirow{3}{*}{6} & Cell division & 25 & 24 & 24 & 24 & 24 & 24 & 24 \\
\hline & pseudo & 0 & 0 & 0 & 0 & 0 & 0 & 0 \\
\hline & partial & 0 & 0 & 0 & 0 & 0 & 0 & 0 \\
\hline \multirow{3}{*}{7} & Macromolecule metabolism & 87 & 86 & 89 & 89 & 89 & 88 & 86 \\
\hline & pseudo & 0 & 1 & 0 & 0 & 0 & 1 & 2 \\
\hline & partial & 0 & 1 & 3 & 3 & 4 & 3 & 3 \\
\hline \multirow{3}{*}{8} & $\begin{array}{l}\text { Macromolecule synthesis, } \\
\text { modification }\end{array}$ & 255 & 229 & 233 & 233 & 235 & 232 & 232 \\
\hline & pseudo & 2 & 0 & 2 & 1 & 2 & 2 & 4 \\
\hline & partial & 5 & 0 & 2 & 2 & 1 & 5 & 1 \\
\hline \multirow{3}{*}{9} & $\begin{array}{l}\text { Metabolism of small } \\
\text { molecules }\end{array}$ & 108 & 100 & 100 & 101 & 101 & 100 & 99 \\
\hline & pseudo & 0 & 0 & 1 & 1 & 1 & 2 & 2 \\
\hline & partial & 1 & 1 & 0 & 0 & 0 & 1 & 0 \\
\hline \multirow{3}{*}{10} & $\begin{array}{l}\text { Biosynthesis of cofactors, } \\
\text { carriers }\end{array}$ & 129 & 128 & 128 & 128 & 128 & 128 & 128 \\
\hline & pseudo & 1 & 0 & 1 & 0 & 0 & 1 & 2 \\
\hline & partial & 0 & 1 & 2 & 2 & 3 & 2 & 1 \\
\hline \multirow{3}{*}{11} & $\begin{array}{l}\text { Central intermediary } \\
\text { metabolism }\end{array}$ & 170 & 158 & 159 & 158 & 161 & 160 & 160 \\
\hline & pseudo & 1 & 0 & 0 & 0 & 1 & 4 & 4 \\
\hline & partial & 0 & 2 & 5 & 4 & 5 & 4 & 9 \\
\hline \multirow{3}{*}{12} & $\begin{array}{l}\text { Degradation of small } \\
\text { molecules }\end{array}$ & 124 & 123 & 121 & 120 & 120 & 120 & 118 \\
\hline & pseudo & 2 & 0 & 1 & 1 & 1 & 2 & 3 \\
\hline & partial & 0 & 2 & 1 & 1 & 1 & 2 & 4 \\
\hline \multirow{3}{*}{13} & Energy metabolism, carbon & 159 & 127 & 143 & 143 & 142 & 142 & 142 \\
\hline & pseudo & 1 & 0 & 0 & 0 & 1 & 0 & 6 \\
\hline & partial & 1 & 1 & 2 & 2 & 3 & 3 & 1 \\
\hline \multirow{3}{*}{14} & Fatty acid biosynthesis & 28 & 28 & 23 & 24 & 24 & 24 & 24 \\
\hline & pseudo & 0 & 0 & 0 & 0 & 1 & 1 & 1 \\
\hline & partial & 0 & 0 & 1 & 1 & 1 & 1 & 1 \\
\hline
\end{tabular}




\begin{tabular}{|c|c|c|c|c|c|c|c|c|}
\hline \multirow{3}{*}{15} & Nucleotide biosynthesis & 27 & 26 & 27 & 27 & 28 & 27 & 27 \\
\hline & pseudo & 0 & 0 & 1 & 1 & 0 & 0 & 0 \\
\hline & partial & 0 & 0 & 0 & 0 & 0 & 0 & 1 \\
\hline \multirow{3}{*}{16} & Cell envelope & 648 & 674 & 635 & 636 & 630 & 632 & 624 \\
\hline & pseudo & 8 & 5 & 4 & 1 & 4 & 10 & 26 \\
\hline & partial & 4 & 2 & 10 & 10 & 8 & 15 & 19 \\
\hline \multirow{3}{*}{17} & Ribosome constituents & 67 & 67 & 67 & 67 & 67 & 67 & 67 \\
\hline & pseudo & 0 & 0 & 0 & 0 & 0 & 0 & 0 \\
\hline & partial & 0 & 0 & 0 & 0 & 0 & 0 & 0 \\
\hline \multirow{3}{*}{18} & Extrachromosomal & 370 & 340 & 265 & 260 & 300 & 278 & 380 \\
\hline & pseudo & 6 & 11 & 27 & 24 & 17 & 25 & 43 \\
\hline & partial & 6 & 20 & 26 & 25 & 29 & 37 & 37 \\
\hline \multirow{3}{*}{19} & Regulation & 284 & 280 & 270 & 271 & 272 & 266 & 267 \\
\hline & pseudo & 2 & 0 & 3 & 5 & 3 & 7 & 15 \\
\hline & partial & 0 & 3 & 10 & 8 & 11 & 11 & 13 \\
\hline \multirow{3}{*}{20} & $\begin{array}{l}\text { Not classified (included } \\
\text { putative assignments) }\end{array}$ & 278 & 310 & 264 & 264 & 264 & 262 & 258 \\
\hline & pseudo & 2 & 3 & 4 & 4 & 1 & 11 & 11 \\
\hline & partial & 3 & 6 & 5 & 2 & 3 & 3 & 6 \\
\hline
\end{tabular}


Table 9.9: Signal values for Y. enterocolitica biotypes.

\begin{tabular}{|c|c|c|c|c|c|c|c|c|c|c|c|c|c|}
\hline \multirow{2}{*}{ 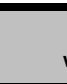 } & \multirow{2}{*}{ well } & \multicolumn{3}{|c|}{ BT 1A 0:5 } & \multicolumn{3}{|c|}{ BT 1B 0:8 } & \multicolumn{3}{|c|}{ BT 2 O:9 } & \multicolumn{3}{|c|}{ BT $30: 9$} \\
\hline & & 5303_1 & 5303_2 & 5303_3 & 8081_1 & 8081_2 & 8081_3 & 21202_1 & 21202_2 & 21202_3 & 5603_1 & 5603_2 & 5603_3 \\
\hline C & PM1-A01 & 0.000 & 0.000 & 0.000 & 0.000 & 0.000 & 0.000 & 0.000 & 0.000 & 0.000 & 0.000 & 0.000 & 0.000 \\
\hline C & PM1-A02 & 241.176 & 200.562 & 193.850 & 184.028 & 175.074 & 163.372 & 196.353 & 194.261 & 144.022 & 208.628 & 210.233 & 151.099 \\
\hline C & PM1-A03 & 258.504 & 185.932 & 195.264 & 172.622 & 122.717 & 94.532 & 246.721 & 214.416 & 107.101 & 239.961 & 216.084 & 188.005 \\
\hline C & PM1-A04 & -0.799 & -2.058 & -6.778 & -1.818 & -8.464 & -1.624 & -0.813 & -2.779 & -2.804 & -3.782 & 2.097 & -1.625 \\
\hline C & PM1-A05 & 199.845 & 220.725 & 191.599 & 161.857 & 157.349 & 139.443 & 156.592 & 66.034 & 159.289 & 11.783 & 174.145 & 6.780 \\
\hline C & PM1-A06 & 272.304 & 261.607 & 255.479 & 243.979 & 255.362 & 249.279 & 271.713 & 260.684 & 223.811 & 250.451 & 268.886 & 198.951 \\
\hline C & PM1-A07 & 248.851 & 261.086 & 206.379 & 211.217 & 197.992 & 192.501 & 180.938 & 11.867 & 183.176 & 9.071 & 205.122 & 1.705 \\
\hline C & PM1-A08 & 52.307 & 6.146 & 5.325 & 17.457 & 66.507 & 47.261 & 105.617 & 67.653 & 57.139 & 7.689 & 65.739 & 8.313 \\
\hline C & PM1-A09 & 44.171 & 33.714 & 18.509 & 76.626 & 76.807 & 71.609 & 3.842 & 2.163 & 4.772 & -1.895 & 1.209 & -6.080 \\
\hline C & PM1-A10 & 165.200 & 126.650 & 133.330 & 135.545 & 101.422 & 107.236 & 173.805 & 147.395 & 86.961 & 179.257 & 166.201 & 126.221 \\
\hline C & PM1-A11 & 178.542 & 88.459 & 94.772 & 105.843 & 77.858 & 79.176 & 168.592 & 152.493 & 124.851 & 167.374 & 170.997 & 93.203 \\
\hline C & PM1-A12 & 8.103 & 7.603 & 7.604 & -0.067 & 3.974 & 6.517 & 52.500 & 12.192 & 7.700 & 1.417 & 6.555 & 10.053 \\
\hline C & PM1-B01 & 271.741 & 269.005 & 275.807 & 2.857 & 3.175 & 0.120 & 157.834 & 0.103 & -0.739 & 2.117 & 5.300 & -0.816 \\
\hline C & PM1-B02 & 236.516 & 211.220 & 209.955 & 156.905 & 138.324 & 131.226 & 212.780 & 189.246 & 103.321 & 177.779 & 177.983 & 142.509 \\
\hline C & PM1-B03 & 210.745 & 147.296 & 146.320 & 161.575 & 133.720 & 120.101 & 112.500 & 113.183 & 82.274 & 94.466 & 113.720 & 77.020 \\
\hline C & PM1-B04 & 4.589 & -7.330 & -3.214 & -0.122 & -4.076 & -2.812 & 3.050 & 0.183 & -0.099 & -1.275 & -2.601 & -4.904 \\
\hline C & PM1-B05 & 292.233 & 290.274 & 288.182 & 216.776 & 237.013 & 219.259 & 282.005 & 274.286 & 227.104 & 250.759 & 266.679 & 213.230 \\
\hline C & PM1-B06 & 301.589 & 292.374 & 294.604 & 225.409 & 248.554 & 224.046 & 285.282 & 284.932 & 240.789 & 276.405 & 283.451 & 216.333 \\
\hline C & PM1-B07 & 208.853 & 213.496 & 210.010 & 96.216 & 116.664 & 93.525 & 207.004 & 177.822 & 105.737 & 165.067 & 195.411 & 111.458 \\
\hline C & PM1-B08 & 242.786 & 190.076 & 195.857 & 204.086 & 183.771 & 171.134 & 194.387 & 184.447 & 152.320 & 204.467 & 200.436 & 143.457 \\
\hline C & PM1-B09 & 8.624 & -1.492 & -0.086 & 4.503 & -6.278 & -2.543 & 85.530 & 0.992 & 0.138 & 3.533 & 1.651 & 1.825 \\
\hline C & PM1-B10 & 72.570 & 90.757 & 78.026 & -5.771 & -2.329 & 0.403 & 8.195 & 13.230 & 0.097 & 1.938 & 9.842 & 6.596 \\
\hline C & PM1-B11 & 224.943 & 187.486 & 194.759 & 137.768 & 108.242 & 111.591 & 171.203 & 168.376 & 82.026 & 197.683 & 150.679 & 136.724 \\
\hline C & PM1-B12 & 68.588 & 76.179 & 55.338 & 62.912 & 82.192 & 90.726 & 137.732 & 133.289 & 137.988 & 13.807 & 124.067 & 18.720 \\
\hline C & PM1-C01 & 260.664 & 249.316 & 240.900 & 220.117 & 212.755 & 199.447 & 254.759 & 263.343 & 227.571 & 240.076 & 261.934 & 194.120 \\
\hline C & PM1-C02 & 48.946 & 118.237 & 117.133 & 0.284 & 1.005 & -2.307 & 0.104 & 1.767 & -3.214 & -1.354 & -2.133 & -0.103 \\
\hline C & PM1-C03 & 202.671 & 216.174 & 201.872 & 177.388 & 159.433 & 137.899 & 190.914 & 179.005 & 136.212 & 4.307 & 247.880 & 3.836 \\
\hline C & PM1-C04 & 218.421 & 171.712 & 168.029 & 174.987 & 144.312 & 139.836 & 154.822 & 148.120 & 100.616 & 183.468 & 187.746 & 97.167 \\
\hline C & PM1-C05 & 49.307 & 45.971 & 23.326 & 8.905 & 4.489 & 10.839 & 12.801 & 8.346 & 5.841 & 4.739 & 6.933 & 8.642 \\
\hline C & PM1-C06 & 2.054 & -6.151 & -1.367 & -2.237 & -8.070 & -3.624 & 3.470 & -2.738 & -2.525 & -2.828 & -1.841 & -3.892 \\
\hline C & PM1-C07 & 120.701 & 58.826 & 64.508 & 79.913 & 52.370 & 61.076 & 186.318 & 159.091 & 76.389 & 220.229 & 144.541 & 151.151 \\
\hline C & PM1-C08 & 193.379 & 160.957 & 161.138 & 113.049 & 102.834 & 114.853 & 139.628 & 124.705 & 82.374 & 137.882 & 151.916 & 87.482 \\
\hline C & PM1-C09 & 176.970 & 99.007 & 115.584 & 85.746 & 53.350 & 59.462 & 224.408 & 196.166 & 71.659 & 213.571 & 174.557 & 162.949 \\
\hline C & PM1-C10 & 208.180 & 155.657 & 162.913 & 173.616 & 162.661 & 161.334 & 205.725 & 179.722 & 160.012 & 189.782 & 199.507 & 156.158 \\
\hline C & PM1-C11 & 2.659 & -7.974 & -6.992 & 0.022 & -5.943 & -4.357 & 84.626 & -1.849 & -0.380 & -2.224 & 0.624 & -2.063 \\
\hline C & PM1-C12 & 42.786 & 19.500 & 20.496 & 11.458 & 0.350 & 6.138 & 1.484 & 11.196 & 13.107 & 13.707 & 7.188 & 11.307 \\
\hline C & PM1-D01 & 192.583 & 187.875 & 169.132 & 154.275 & 129.886 & 128.361 & 9.817 & 8.287 & 148.975 & 2.663 & 103.457 & 0.205 \\
\hline C & PM1-D02 & -1.546 & -8.368 & -6.075 & -6.984 & -9.787 & -9.424 & -3.036 & -10.766 & -3.447 & -6.758 & -10.609 & -13.279 \\
\hline C & PM1-D03 & 0.904 & -4.408 & -7.175 & -3.245 & -8.914 & -5.817 & 1.612 & -3.395 & -0.245 & -3.789 & -4.225 & -8.375 \\
\hline C & PM1-D04 & 3.843 & 0.251 & 4.491 & 3.103 & -1.051 & -1.624 & 4.889 & 2.650 & -1.608 & 0.939 & 4.592 & 0.822 \\
\hline C & PM1-D05 & 106.316 & 4.674 & 118.725 & -0.328 & -1.014 & -0.870 & 4.445 & 3.950 & 1.025 & -0.942 & 1.217 & 0.578 \\
\hline C & PM1-D06 & 0.005 & -7.545 & -4.566 & -6.112 & -7.522 & -6.874 & 1.879 & -1.220 & -5.036 & -3.964 & -9.222 & -4.228 \\
\hline C & PM1-D07 & 0.272 & -5.789 & -7.878 & -6.605 & -8.149 & -11.078 & -4.718 & 3.197 & -8.232 & -3.330 & -1.299 & -5.497 \\
\hline C & PM1-D08 & 2.124 & -7.179 & 1.314 & -1.957 & -2.129 & -3.954 & 3.883 & 1.143 & -2.733 & -3.876 & -0.992 & -1.145 \\
\hline C & PM1-D09 & 203.461 & 116.243 & 138.660 & -2.500 & -6.442 & -2.845 & 1.789 & 0.557 & -0.953 & 0.829 & 1.193 & 1.407 \\
\hline C & PM1-D10 & 90.712 & 122.411 & 80.599 & 0.134 & -3.201 & -1.783 & 3.872 & 2.464 & 1.668 & 0.686 & 0.113 & -4.101 \\
\hline C & PM1-D11 & 111.366 & 68.858 & 79.520 & 79.405 & 64.463 & 71.200 & 207.958 & 143.382 & 60.322 & 174.809 & 86.155 & 147.410 \\
\hline C & PM1-D12 & 158.414 & 150.678 & 153.528 & 39.620 & 20.712 & 21.962 & 144.024 & 124.174 & 41.292 & 83.617 & 136.028 & 31.461 \\
\hline C & PM1-E01 & 56.732 & 62.997 & 20.966 & 5.676 & 8.482 & 6.624 & 6.528 & 53.154 & 106.855 & 3.545 & 89.401 & 5.584 \\
\hline C & PM1-E02 & -1.070 & -2.562 & -2.862 & -5.188 & -2.211 & -8.500 & -0.930 & 0.225 & -2.675 & -8.321 & -8.043 & -1.347 \\
\hline C & PM1-E03 & 258.734 & 254.450 & 250.682 & 207.187 & 195.982 & 190.928 & 253.762 & 235.328 & 193.595 & 223.129 & 248.471 & 185.716 \\
\hline C & PM1-E04 & 246.358 & 235.766 & 233.250 & 187.483 & 196.397 & 183.096 & 258.391 & 244.387 & 199.704 & 220.537 & 254.901 & 135.305 \\
\hline C & PM1-E05 & 14.482 & 5.634 & 7.464 & 27.249 & 4.743 & 28.817 & 7.625 & 8.578 & 4.539 & 3.130 & 11.492 & 1.829 \\
\hline C & PM1-E06 & 18.229 & 20.754 & -5.682 & -3.392 & -4.878 & -2.182 & 8.228 & 4.997 & -2.041 & 1.947 & 0.084 & -2.855 \\
\hline C & PM1-E07 & 2.842 & -8.454 & -0.593 & -0.887 & -5.676 & -5.564 & 0.609 & 0.745 & -2.766 & -1.509 & -2.004 & -3.861 \\
\hline C & PM1-E08 & 208.886 & 132.305 & 150.013 & 182.275 & 157.529 & 155.555 & 193.567 & 190.892 & 159.505 & 192.682 & 176.257 & 138.218 \\
\hline C & PM1-E09 & 2.849 & 0.976 & -0.789 & -1.914 & -8.813 & -7.399 & 2.257 & 2.705 & -3.564 & -0.155 & 2.017 & -4.870 \\
\hline C & PM1-E10 & 169.264 & 98.225 & 110.663 & 143.363 & 127.538 & 128.174 & 191.792 & 159.907 & 130.422 & 190.428 & 169.634 & 139.701 \\
\hline C & PM1-E11 & 224.818 & 224.801 & 231.818 & 121.275 & 131.200 & 133.020 & 200.750 & 191.437 & 121.782 & 181.137 & 194.845 & 139.207 \\
\hline C & PM1-E12 & 233.875 & 231.226 & 224.045 & 126.228 & 136.720 & 109.512 & 236.863 & 208.037 & 143.195 & 191.362 & 212.401 & 136.118 \\
\hline C & PM1-F01 & 215.928 & 194.618 & 192.146 & 92.704 & 83.592 & 97.579 & 191.304 & 16.622 & 38.392 & 20.128 & 178.766 & 13.864 \\
\hline C & PM1-F02 & -2.395 & -6.904 & -2.045 & -3.589 & -3.134 & -5.724 & 1.896 & -3.982 & -1.100 & -6.184 & -9.836 & -8.259 \\
\hline C & PM1-F03 & 2.954 & 76.620 & 1.467 & 215.187 & 220.780 & 202.538 & 242.091 & 218.986 & 197.404 & 220.105 & 240.278 & 191.329 \\
\hline C & PM1-F04 & 0.409 & -9.493 & -6.205 & -2.196 & -8.167 & -6.082 & 3.170 & -4.192 & 0.536 & -3.789 & -7.757 & -8.076 \\
\hline C & PM1-F05 & 246.484 & 260.901 & 239.008 & 177.589 & 170.076 & 110.815 & 42.109 & 21.083 & 155.938 & 3.654 & 27.105 & 2.759 \\
\hline
\end{tabular}




\begin{tabular}{|c|c|c|c|c|c|c|c|c|c|c|c|c|c|}
\hline C & PM1-F06 & 131.317 & 121.233 & 121.243 & 52.467 & 28.437 & -0.378 & 3.900 & 73.403 & 52.876 & 1.183 & 105.816 & 0.237 \\
\hline C & PM1-F07 & -3.200 & -8.238 & -6.449 & 6.495 & -3.780 & -9.079 & 2.588 & 0.925 & -1.112 & 0.822 & 3.929 & -6.934 \\
\hline C & PM1-F08 & 3.441 & -1.024 & 1.270 & 1.654 & 1.214 & -2.553 & -2.139 & 2.661 & 0.442 & 0.826 & 6.375 & 0.474 \\
\hline C & PM1-F09 & -1.070 & -2.696 & -9.886 & -4.407 & -8.366 & -5.534 & -4.295 & -4.047 & -4.329 & -6.207 & -6.197 & -7.439 \\
\hline C & PM1-F10 & -4.728 & -9.846 & -5.589 & -4.451 & -5.372 & -7.003 & -9.161 & -2.942 & -6.586 & -4.695 & -2.911 & -7.832 \\
\hline C & PM1-F11 & 200.979 & 151.621 & 164.954 & 147.455 & 134.616 & 129.005 & 183.676 & 171.095 & 118.855 & 171.130 & 179.690 & 127.735 \\
\hline C & PM1-F12 & 222.025 & 215.132 & 227.580 & 79.664 & 95.016 & 81.447 & 186.547 & 179.491 & 117.062 & 180.424 & 183.278 & 127.030 \\
\hline C & PM1-G01 & 161.858 & 140.783 & 132.726 & 94.113 & 127.093 & 105.617 & 130.611 & 115.337 & 84.855 & 99.676 & 149.724 & 56.808 \\
\hline C & PM1-G02 & -1.700 & -3.057 & -2.516 & -5.451 & -2.922 & -4.451 & 0.816 & -0.632 & -4.159 & -1.671 & -1.441 & -6.296 \\
\hline C & PM1-G03 & 275.480 & 269.484 & 265.546 & 184.238 & 212.125 & 201.120 & 262.463 & 249.742 & 224.174 & 243.280 & 260.899 & 198.788 \\
\hline C & PM1-G04 & 2.021 & -2.509 & -1.804 & 20.045 & 1.109 & 27.195 & 3.620 & 3.926 & 45.403 & 0.988 & -0.307 & -3.179 \\
\hline C & PM1-G05 & 188.217 & 187.704 & 188.710 & 91.826 & 79.745 & 79.626 & 177.689 & 161.100 & 135.155 & 140.301 & 161.792 & 131.428 \\
\hline C & PM1-G06 & 153.349 & 163.438 & 139.703 & 117.095 & 107.917 & 101.040 & 142.672 & 130.771 & 105.701 & 125.588 & 152.766 & 107.493 \\
\hline C & PM1-G07 & 7.843 & 0.762 & 4.750 & 4.421 & 3.950 & 5.205 & 2.149 & 6.100 & 2.020 & 2.967 & 3.789 & 1.620 \\
\hline C & PM1-G08 & 238.850 & 231.224 & 239.439 & 213.174 & 216.525 & 205.396 & 229.687 & 231.812 & 184.976 & 220.921 & 234.486 & 197.307 \\
\hline C & PM1-G09 & 73.941 & 38.395 & 29.055 & 43.491 & 32.000 & 15.042 & 14.271 & 9.174 & 17.396 & 10.647 & 24.113 & 6.082 \\
\hline C & PM1-G10 & 213.525 & 193.962 & 189.749 & 140.216 & 121.899 & 132.945 & 201.913 & 192.164 & 141.628 & 170.509 & 189.950 & 118.196 \\
\hline C & PM1-G11 & 1.841 & 2.491 & 1.612 & 1.055 & 2.312 & 0.463 & -0.338 & 2.732 & 2.426 & -1.970 & -4.178 & 0.343 \\
\hline C & PM1-G12 & 232.237 & 240.071 & 228.122 & 183.451 & 173.880 & 158.120 & 188.845 & 185.821 & 166.749 & 41.950 & 238.621 & 10.413 \\
\hline C & PM1-H01 & 177.061 & 159.684 & 152.147 & 137.889 & 160.524 & 147.657 & 227.857 & 196.172 & 188.199 & 195.671 & 212.283 & 172.714 \\
\hline C & PM1-H02 & 2.654 & 1.342 & 2.979 & -0.462 & 2.875 & -0.821 & 94.688 & 9.586 & 2.499 & 0.325 & 5.324 & 1.738 \\
\hline C & PM1-H03 & -2.028 & 0.428 & 0.317 & 0.296 & -0.296 & -5.263 & 131.853 & 9.445 & 4.001 & -0.218 & -1.724 & -5.779 \\
\hline C & PM1-H04 & 2.080 & 7.314 & 3.737 & -2.126 & 4.441 & -0.700 & 5.333 & 3.417 & 4.158 & -1.245 & 3.561 & 1.271 \\
\hline C & PM1-H05 & 11.886 & 6.453 & 7.900 & 2.979 & 6.096 & 2.575 & 0.499 & 5.136 & 4.757 & -0.254 & 0.043 & 2.741 \\
\hline C & PM1-H06 & 31.442 & 2.022 & 4.642 & 3.908 & -0.300 & -3.692 & -2.587 & -7.930 & -1.130 & -2.300 & -1.575 & -4.601 \\
\hline C & PM1-H07 & 14.878 & -2.236 & 4.293 & 11.078 & 3.796 & 1.161 & -0.780 & 4.087 & 2.743 & 10.392 & 7.054 & 1.964 \\
\hline C & PM1-H08 & 239.428 & 240.089 & 241.874 & 143.795 & 175.682 & 145.436 & 236.333 & 241.818 & 199.788 & 184.316 & 251.008 & 143.707 \\
\hline C & PM1-H09 & 4.289 & 1.017 & 4.814 & 4.296 & 5.084 & 1.207 & 2.129 & 5.292 & 4.228 & 2.378 & 12.146 & 5.634 \\
\hline C & PM1-H10 & 253.942 & 250.847 & 260.285 & 203.979 & 218.941 & 216.903 & 245.039 & 247.797 & 239.274 & 246.408 & 249.561 & 222.618 \\
\hline C & PM1-H11 & -1.345 & -1.134 & 2.116 & -3.558 & 3.141 & -3.250 & -2.478 & 2.271 & 3.711 & -1.153 & 8.046 & 4.313 \\
\hline C & PM1-H12 & -1.774 & 2.711 & 1.474 & -4.511 & 0.049 & -4.057 & -3.461 & 3.107 & 2.446 & -3.024 & 5.055 & 2.803 \\
\hline C & PM2-AA01 & 0.000 & 0.000 & 0.000 & 0.000 & 0.000 & 0.000 & 0.000 & 0.000 & 0.000 & 0.000 & 0.000 & 0.000 \\
\hline C & PM2-AA02 & -4.030 & -8.268 & -8.393 & -2.882 & -2.705 & -4.283 & -7.175 & -6.688 & -5.455 & 3.453 & -7.305 & -4.884 \\
\hline C & PM2-AA03 & -3.608 & -2.251 & -4.446 & -0.516 & -1.305 & -3.113 & -2.345 & -5.796 & -3.608 & 2.643 & -7.445 & -5.066 \\
\hline C & PM2-AA04 & -3.767 & -3.163 & -4.151 & -1.987 & -1.609 & -2.780 & -3.651 & -5.612 & -4.267 & 1.900 & -6.633 & -6.026 \\
\hline C & PM2-AA05 & -4.263 & -5.033 & -10.083 & -2.792 & -2.012 & -5.028 & -5.645 & -9.436 & -5.022 & -1.255 & -8.380 & -6.847 \\
\hline C & PM2-AA06 & 84.113 & 68.784 & 48.717 & 37.413 & 41.446 & 34.183 & 40.901 & 7.401 & 32.172 & 70.026 & 42.251 & 3.570 \\
\hline C & PM2-AA07 & -4.289 & -5.196 & -9.259 & -3.574 & -4.401 & -3.951 & -8.046 & -8.087 & -6.908 & -4.720 & -10.857 & -5.825 \\
\hline C & PM2-AA08 & 1.304 & -0.576 & -1.991 & -2.793 & -1.336 & -1.055 & -1.186 & -5.608 & -1.743 & 5.083 & -9.297 & -6.266 \\
\hline C & PM2-AA09 & -4.476 & -7.682 & -5.775 & -2.143 & -1.324 & -2.184 & -3.971 & -10.668 & -3.920 & -3.263 & -10.550 & -8.114 \\
\hline C & PM2-AA10 & -7.659 & -9.803 & -7.070 & -3.145 & -2.168 & -7.501 & -4.359 & -9.909 & -6.668 & -1.842 & -12.332 & -6.864 \\
\hline C & PM2-AA11 & -3.193 & -1.345 & -3.772 & -3.033 & -2.666 & -1.943 & -2.200 & -8.655 & -2.237 & 2.183 & -9.703 & -7.782 \\
\hline C & PM2-AA12 & 176.596 & 157.680 & 129.262 & 94.907 & 113.217 & 96.012 & 201.807 & 160.011 & 104.447 & 30.587 & 175.884 & 87.991 \\
\hline C & PM2-AB01 & 171.041 & 174.616 & 191.495 & -4.203 & -2.136 & -2.876 & 167.903 & 151.432 & 143.399 & 168.296 & 144.942 & 130.468 \\
\hline C & PM2-AB02 & 241.600 & 246.405 & 243.889 & 185.030 & 206.486 & 182.668 & 236.112 & 219.287 & 210.128 & 223.542 & 227.770 & 187.729 \\
\hline C & РM2-АB03 & -7.157 & -9.030 & -11.143 & -7.830 & -9.433 & -10.793 & -10.172 & -13.784 & -12.589 & -2.733 & -13.689 & -13.642 \\
\hline C & PM2-AB04 & 4.792 & 0.738 & -6.534 & -4.184 & -2.839 & -4.112 & -5.214 & -11.182 & -6.447 & -3.258 & -11.154 & -9.388 \\
\hline C & PM2-AB05 & -8.080 & -7.347 & -10.511 & -6.057 & -5.197 & -7.920 & -9.391 & -15.375 & -10.505 & -0.772 & -12.218 & -11.311 \\
\hline C & PM2-AB06 & 259.628 & 252.371 & 254.628 & -6.722 & -5.959 & -6.609 & -6.737 & -11.676 & -9.305 & -1.430 & -14.500 & -8.783 \\
\hline C & PM2-AB07 & -5.503 & -7.434 & -7.579 & -6.195 & -8.121 & -8.017 & -5.096 & -15.211 & -9.616 & -2.157 & -14.117 & -9.991 \\
\hline C & PM2-AB08 & 194.016 & 190.438 & 199.961 & 135.978 & 136.563 & 157.158 & 38.659 & 7.476 & 2.832 & 5.762 & -2.807 & -3.088 \\
\hline C & PM2-AB09 & 132.229 & 114.605 & 125.655 & 109.992 & 112.372 & 89.586 & 174.718 & 85.866 & 57.663 & 119.925 & 154.543 & 105.616 \\
\hline C & PM2-AB10 & -13.439 & -13.664 & -14.936 & -9.788 & -9.662 & -13.896 & -9.982 & -16.588 & -12.204 & -6.874 & -19.558 & -13.437 \\
\hline C & PM2-AB11 & -9.664 & -9.147 & -11.433 & -6.283 & -8.514 & -7.996 & -10.168 & -13.951 & -8.095 & -5.346 & -15.672 & -10.946 \\
\hline C & PM2-AB12 & 200.166 & 201.497 & 194.883 & 12.466 & 8.143 & 15.305 & 9.742 & 1.541 & 2.114 & 28.141 & -1.271 & 7.220 \\
\hline C & PM2-AC01 & 125.987 & 126.261 & 124.750 & 124.547 & 112.450 & 115.466 & 204.958 & 187.463 & 177.218 & 200.283 & 201.737 & 144.384 \\
\hline C & PM2-AC02 & -4.653 & -4.514 & -9.197 & -5.543 & -7.084 & -8.786 & -6.961 & -11.955 & -8.366 & -1.418 & -10.049 & -6.687 \\
\hline C & PM2-AC03 & -5.147 & -7.275 & -9.809 & -5.455 & -9.629 & -8.562 & -7.293 & -13.876 & -8.754 & -1.842 & -14.384 & -14.755 \\
\hline C & PM2-AC04 & -5.974 & -7.903 & -10.957 & -5.982 & -6.704 & -6.787 & -7.454 & -13.529 & -8.193 & -1.530 & -13.589 & -11.932 \\
\hline C & PM2-AC05 & -5.801 & -7.455 & -7.409 & -4.341 & -9.880 & -6.042 & -4.732 & -10.637 & -6.553 & -0.317 & -11.316 & -8.891 \\
\hline C & PM2-AC06 & -7.238 & -6.963 & -9.838 & -9.421 & -9.534 & -9.334 & -10.822 & -12.462 & -10.276 & 0.533 & -15.599 & -12.032 \\
\hline C & PM2-AC07 & 90.151 & 106.462 & 84.850 & -3.091 & -2.899 & -3.491 & 1.386 & -3.509 & 1.346 & 1.676 & -8.014 & -7.403 \\
\hline C & PM2-AC08 & -4.339 & -4.687 & -7.137 & -5.962 & -8.884 & -8.376 & -7.846 & -11.571 & -10.967 & -1.276 & -14.122 & -9.346 \\
\hline C & PM2-AC09 & -8.334 & -9.972 & -13.882 & -8.978 & -11.393 & -12.009 & -8.051 & -15.539 & -13.253 & -4.134 & -17.767 & -14.687 \\
\hline C & PM2-AC10 & -11.954 & -11.379 & -13.968 & -10.649 & -9.363 & -12.447 & -9.524 & -16.803 & -11.912 & -5.758 & -18.675 & -12.253 \\
\hline C & PM2-AC11 & -7.538 & -1.525 & -9.247 & -5.934 & -4.711 & -7.187 & -8.063 & -10.299 & -9.339 & -2.484 & -14.432 & -8.888 \\
\hline C & PM2-AC12 & -6.859 & -4.755 & -7.418 & -5.124 & -4.287 & -3.736 & -3.076 & -9.955 & -3.486 & 0.766 & -15.296 & -5.970 \\
\hline C & PM2-AD01 & 55.949 & 83.774 & 42.850 & -3.132 & -3.822 & -5.403 & -2.888 & -9.970 & -5.195 & 1.878 & -10.579 & -8.983 \\
\hline C & PM2-AD02 & 213.626 & 214.393 & 219.499 & -6.159 & -4.207 & -6.387 & -0.704 & -11.020 & -8.101 & 1.251 & -10.379 & -10.518 \\
\hline
\end{tabular}




\begin{tabular}{|c|c|c|c|c|c|c|c|c|c|c|c|c|c|}
\hline C & PM2-AD03 & -5.505 & -4.546 & -8.216 & -5.712 & -4.816 & -4.668 & -6.347 & -9.621 & -8.433 & -0.586 & -8.632 & -8.663 \\
\hline C & PM2-AD04 & 161.620 & 165.739 & 170.645 & 115.897 & 118.236 & 101.391 & 158.566 & 123.836 & 115.480 & 123.340 & 122.493 & 87.286 \\
\hline C & PM2-AD05 & -8.207 & -7.603 & -10.432 & -3.793 & -6.464 & -8.033 & -10.317 & -10.003 & -8.150 & -0.543 & -12.987 & -9.108 \\
\hline C & PM2-AD06 & -4.207 & -2.096 & -4.841 & -5.286 & -4.674 & -5.692 & -5.001 & -7.150 & -7.542 & 2.045 & -6.842 & -5.709 \\
\hline c & PM2-AD07 & -2.518 & -9.987 & -11.263 & -3.389 & -8.512 & -5.842 & -6.450 & -12.678 & -4.530 & 0.796 & -10.567 & -10.184 \\
\hline C & PM2-AD08 & -6.091 & -9.770 & -10.933 & -6.392 & -6.566 & -8.053 & -6.587 & -10.253 & -8.013 & -2.668 & -11.937 & -9.139 \\
\hline C & PM2-AD09 & -7.525 & -9.499 & -12.866 & -7.411 & -10.496 & -8.075 & -8.029 & -13.195 & -12.338 & -2.143 & -16.672 & -10.850 \\
\hline C & PM2-AD10 & -7.543 & -7.386 & -9.667 & -8.164 & -8.504 & -8.737 & -7.147 & -13.467 & -8.958 & -3.688 & -15.250 & -8.561 \\
\hline C & PM2-AD11 & -9.529 & -9.611 & -10.613 & -8.559 & -5.564 & -12.454 & -10.811 & -13.391 & -10.799 & -2.824 & -15.403 & -9.547 \\
\hline C & PM2-AD12 & -4.093 & -5.176 & -8.763 & 0.651 & -0.030 & -2.900 & -5.272 & -12.261 & -5.061 & 1.558 & -8.526 & -3.911 \\
\hline C & PM2-AE01 & -11.563 & -9.364 & -22.391 & -16.491 & -12.083 & -16.639 & -17.833 & -13.649 & -14.696 & -7.538 & -17.779 & -15.443 \\
\hline C & PM2-AE02 & -2.020 & -3.533 & -5.378 & -2.847 & -6.058 & -3.762 & -3.334 & -4.884 & -14.187 & 4.717 & -7.607 & -8.282 \\
\hline c & PM2-AE03 & -4.034 & -1.757 & -5.912 & -5.422 & -4.222 & -8.836 & -4.453 & -7.746 & -8.583 & -0.728 & -7.443 & -9.709 \\
\hline C & PM2-AE04 & -7.028 & -6.933 & -7.630 & -6.632 & -8.457 & -8.964 & -6.286 & -8.186 & -12.241 & -1.426 & -6.611 & -13.826 \\
\hline C & PM2-AE05 & 220.189 & 229.026 & 234.767 & 185.068 & 204.612 & 194.866 & 225.476 & 213.812 & 215.143 & 214.036 & 223.161 & 189.596 \\
\hline C & PM2-AE06 & -9.579 & -11.017 & -13.242 & -7.479 & -9.079 & -11.520 & -12.512 & -16.439 & -14.907 & -3.596 & -15.983 & -13.283 \\
\hline C & PM2-AE07 & -6.876 & -5.720 & -8.776 & -6.800 & -5.180 & -8.687 & -6.542 & -12.263 & -12.714 & -2.046 & -12.095 & -9.033 \\
\hline C & PM2-AE08 & -6.062 & -1.829 & -7.134 & -6.342 & -6.059 & -9.526 & -7.312 & -12.621 & -9.291 & 0.146 & -11.842 & -8.583 \\
\hline c & PM2-AE09 & -7.030 & -3.997 & -7.653 & -5.163 & -8.172 & -9.491 & -7.336 & -9.322 & -8.471 & -1.100 & -9.546 & -5.892 \\
\hline C & PM2-AE10 & -8.593 & -8.347 & -10.707 & -6.536 & -3.962 & -11.989 & -7.511 & -14.126 & -11.153 & -3.075 & -15.587 & -11.412 \\
\hline C & PM2-AE11 & -11.699 & -11.404 & -23.445 & -10.225 & -16.689 & -12.711 & -14.475 & -18.568 & -9.662 & -2.405 & -20.230 & -14.899 \\
\hline C & PM2-AE12 & 199.638 & 188.380 & 194.180 & 103.034 & 117.379 & 121.055 & 218.175 & 183.068 & 146.968 & 160.259 & 172.609 & 158.288 \\
\hline C & PM2-AF01 & 0.747 & 2.909 & -2.516 & -1.758 & -1.559 & -2.109 & 1.447 & -2.943 & -5.621 & 6.214 & -2.361 & -1.928 \\
\hline C & PM2-AF02 & -1.324 & -0.821 & -5.959 & -0.271 & 3.647 & -2.654 & -5.013 & -5.030 & -8.099 & 4.297 & -4.163 & -6.041 \\
\hline C & PM2-AF03 & 1.145 & -0.011 & -4.375 & -3.746 & -7.757 & -7.078 & 108.522 & -10.716 & -5.703 & 3.008 & -7.654 & -6.871 \\
\hline C & PM2-AF04 & -4.718 & -2.234 & -7.734 & -5.770 & -3.525 & -5.458 & -8.084 & -8.254 & -6.099 & 0.045 & -6.855 & -8.493 \\
\hline C & PM2-AF05 & -4.589 & -4.247 & -6.649 & -4.117 & -4.868 & -1.747 & -6.670 & -12.146 & -7.012 & -2.553 & -7.416 & -11.616 \\
\hline C & PM2-AF06 & -3.520 & -4.504 & -8.741 & -5.788 & -5.034 & -9.357 & -6.464 & -10.513 & -9.463 & -1.563 & -8.754 & -8.384 \\
\hline C & PM2-AF07 & -5.664 & -1.713 & -8.516 & -4.887 & -4.457 & -5.514 & -8.468 & -9.147 & -9.930 & 1.105 & -10.087 & -7.034 \\
\hline C & PM2-AF08 & -4.896 & -6.557 & -12.197 & -9.246 & -7.825 & -10.705 & -4.153 & -16.007 & -13.738 & -0.650 & -12.251 & -14.064 \\
\hline c & PM2-AF09 & -3.726 & -8.339 & -8.814 & -7.218 & -9.099 & -9.483 & -6.438 & -11.661 & -11.274 & -1.263 & -14.416 & -10.642 \\
\hline C & PM2-AF10 & 31.155 & 47.866 & 21.967 & 23.347 & 27.474 & 17.200 & 14.225 & -5.979 & 14.142 & -4.200 & 2.921 & -5.551 \\
\hline C & PM2-AF11 & -9.280 & -4.542 & -8.229 & -7.463 & -5.750 & -6.884 & -11.221 & -8.778 & -8.817 & -1.884 & -14.501 & -6.461 \\
\hline c & PM2-AF12 & -0.637 & 1.991 & -1.604 & 1.459 & 0.514 & 2.730 & -1.767 & -2.575 & -5.083 & 5.107 & -8.880 & 1.113 \\
\hline C & PM2-AG01 & -0.966 & -2.857 & -4.370 & -4.868 & 2.539 & -1.888 & -6.162 & -3.495 & -4.129 & 1.000 & -3.041 & -3.682 \\
\hline C & PM2-AG02 & -0.184 & -0.889 & -2.307 & -3.012 & -4.832 & -5.764 & 3.787 & -2.391 & -6.068 & 64.716 & -0.839 & -7.200 \\
\hline c & PM2-AG03 & -7.745 & -0.621 & -9.089 & -3.564 & -6.804 & -5.474 & -5.679 & -18.117 & -9.921 & 2.064 & -7.576 & -8.886 \\
\hline C & PM2-AG04 & -4.195 & -4.301 & -7.151 & -3.871 & -3.905 & -7.786 & -5.724 & -11.159 & -7.267 & 0.939 & -11.407 & -10.841 \\
\hline C & PM2-AG05 & -4.632 & -5.700 & -7.241 & -8.745 & -6.564 & -6.789 & -6.045 & -11.500 & -11.407 & 1.626 & -10.196 & -12.030 \\
\hline C & PM2-AG06 & 205.472 & 251.191 & 215.575 & 44.820 & 65.678 & 56.500 & -0.357 & 97.570 & 146.709 & 3.475 & 125.930 & -11.974 \\
\hline C & PM2-AG07 & -8.400 & -4.196 & -10.653 & -8.158 & -7.582 & -9.734 & -11.211 & -14.387 & -12.005 & -1.139 & -15.964 & -9.230 \\
\hline C & PM2-AG08 & -5.279 & -5.243 & -8.133 & -4.446 & -3.751 & -6.925 & -4.204 & -11.449 & -5.729 & 3.784 & -11.082 & -9.189 \\
\hline c & PM2-AG09 & -5.829 & -10.149 & -11.936 & -6.526 & -5.800 & -7.284 & -11.211 & -13.625 & -9.472 & -2.554 & -12.037 & -10.839 \\
\hline C & PM2-AG10 & -14.782 & -20.379 & -17.521 & -5.808 & -8.333 & -6.861 & -14.683 & -26.279 & -8.146 & -5.379 & -18.267 & -8.813 \\
\hline$c$ & PM2-AG11 & -7.305 & -6.612 & -7.445 & -4.937 & -5.409 & -5.784 & -8.086 & -11.124 & -6.442 & -3.441 & -14.904 & -6.971 \\
\hline C & PM2-AG12 & -7.074 & -3.374 & -3.016 & -1.553 & 0.566 & -1.141 & -4.717 & -8.384 & -1.407 & 4.593 & -9.880 & 0.629 \\
\hline C & PM2-AH01 & 0.795 & 1.282 & -4.974 & -0.818 & -3.562 & -5.417 & -0.346 & -1.492 & -3.022 & 2.364 & -5.525 & -5.033 \\
\hline C & PM2-AH02 & 1.804 & 2.949 & -1.278 & -0.276 & -4.580 & -2.386 & -1.520 & -8.658 & 1.914 & 5.626 & -4.850 & -3.833 \\
\hline C & PM2-AH03 & 5.808 & 4.659 & 0.195 & 3.561 & 1.683 & -0.817 & -1.041 & -1.561 & -1.332 & 9.271 & -4.812 & -4.282 \\
\hline C & PM2-AH04 & 0.984 & -2.830 & -4.289 & -3.751 & -5.297 & -3.504 & -5.164 & -5.891 & 0.204 & 5.743 & -4.303 & -6.354 \\
\hline C & PM2-AH05 & -4.429 & -0.883 & -4.559 & -6.897 & 0.408 & -3.684 & -5.843 & -8.516 & -4.272 & -0.607 & -7.324 & -8.992 \\
\hline C & PM2-AH06 & -8.143 & -7.067 & -13.016 & -2.745 & -7.493 & -9.758 & -12.634 & -14.839 & -12.799 & 0.549 & -11.455 & -16.837 \\
\hline C & PM2-AH07 & -1.136 & 0.466 & -0.203 & -0.530 & 2.284 & -2.536 & -0.289 & -1.825 & -0.082 & 4.929 & -4.296 & -4.939 \\
\hline C & PM2-AH08 & 2.080 & 1.636 & -9.158 & -1.609 & 12.557 & -5.976 & -0.087 & -6.730 & 1.292 & 2.129 & -5.295 & -6.171 \\
\hline C & PM2-AH09 & -11.184 & -18.337 & -9.995 & -10.637 & -13.282 & -17.012 & -14.700 & 8.251 & -15.466 & -5.150 & -25.574 & 2.845 \\
\hline C & PM2-AH10 & -7.128 & -4.532 & -8.916 & -2.209 & -5.472 & -4.426 & -5.564 & -10.024 & -2.438 & 1.603 & -11.038 & -6.082 \\
\hline C & $\mathrm{PM} 2-\mathrm{AH} 11$ & 1.699 & -0.083 & -2.720 & 2.505 & 1.814 & -0.647 & -2.943 & -12.826 & -3.097 & 4.401 & -12.154 & -0.145 \\
\hline C & PM2-AH12 & 2.757 & 4.589 & 3.855 & 2.971 & 4.554 & -0.678 & 3.167 & -3.274 & 0.358 & 5.043 & -4.941 & -3.696 \\
\hline $\mathbf{N}$ & PM3-BA01 & 0.000 & 0.000 & 0.000 & 0.000 & 0.000 & 0.000 & 0.000 & 0.000 & 0.000 & 0.000 & 0.000 & 0.000 \\
\hline $\mathbf{N}$ & PM3-BA02 & 229.267 & 231.834 & 221.362 & 115.321 & 128.717 & 109.933 & 196.847 & 208.853 & 174.132 & 191.216 & 201.893 & 148.771 \\
\hline $\mathbf{N}$ & PM3-BA03 & 8.424 & 10.351 & 24.266 & 7.496 & 2.279 & 8.916 & 7.370 & 2.399 & 2.964 & 2.120 & 3.914 & -0.346 \\
\hline $\mathbf{N}$ & РM3-BA04 & 12.258 & 5.925 & 5.892 & 1.825 & -0.983 & 2.457 & 12.053 & 5.572 & 3.714 & 5.537 & 3.536 & -0.825 \\
\hline $\mathbf{N}$ & PM3-BA05 & 244.296 & 250.391 & 234.711 & 105.335 & 106.484 & 84.716 & 184.004 & 213.971 & 149.730 & 160.884 & 205.879 & 111.811 \\
\hline $\mathbf{N}$ & PM3-BA06 & 3.149 & 1.408 & 0.761 & -2.791 & -7.055 & -5.487 & 20.011 & 2.176 & -2.200 & 0.599 & -1.709 & -3.276 \\
\hline $\mathbf{N}$ & PM3-BA07 & 234.904 & 234.387 & 228.783 & 116.053 & 140.055 & 112.147 & 192.301 & 209.433 & 188.767 & 172.126 & 205.260 & 148.236 \\
\hline $\mathbf{N}$ & PM3-BA08 & 226.804 & 225.266 & 211.930 & 121.407 & 129.401 & 108.368 & 146.808 & 151.687 & 138.900 & 111.926 & 162.287 & 96.443 \\
\hline $\mathbf{N}$ & PM3-BA09 & 256.011 & 261.001 & 261.284 & 217.472 & 224.754 & 222.343 & 229.305 & 228.316 & 219.867 & 185.650 & 246.305 & 192.324 \\
\hline $\mathbf{N}$ & PM3-BA10 & 267.053 & 275.892 & 262.553 & 222.835 & 238.580 & 229.550 & 253.667 & 243.185 & 223.863 & 230.828 & 251.049 & 213.784 \\
\hline $\mathbf{N}$ & PM3-BA11 & 270.136 & 261.661 & 268.571 & 20.355 & 284.349 & 280.904 & 67.601 & 276.488 & 274.117 & 270.684 & 277.157 & 260.636 \\
\hline
\end{tabular}




\begin{tabular}{|c|c|c|c|c|c|c|c|c|c|c|c|c|c|}
\hline $\mathbf{N}$ & PM3-BA12 & 220.151 & 224.312 & 216.001 & 188.060 & 201.047 & 203.780 & 203.634 & 171.829 & 191.896 & 127.210 & 185.313 & 118.129 \\
\hline $\mathbf{N}$ & PM3-BB01 & 247.001 & 234.417 & 245.291 & 201.087 & 200.367 & 207.368 & 225.962 & 226.722 & 210.932 & 210.764 & 230.967 & 183.603 \\
\hline $\mathbf{N}$ & РM3-BB02 & 194.325 & 190.395 & 186.763 & 80.071 & 70.300 & 54.984 & 149.954 & 127.783 & 103.188 & 95.389 & 150.404 & 58.524 \\
\hline $\mathbf{N}$ & РМ3-ВB03 & 255.350 & 250.421 & 246.432 & 182.412 & 192.971 & 177.216 & 238.730 & 209.846 & 199.643 & 180.150 & 220.628 & 82.688 \\
\hline $\mathbf{N}$ & PM3-BB04 & 45.514 & 58.003 & 64.130 & 10.711 & 17.546 & 8.629 & 22.497 & 7.738 & 11.263 & 6.421 & 19.138 & 2.489 \\
\hline $\mathbf{N}$ & PM3-BB05 & 128.980 & 123.318 & 121.695 & 10.591 & 7.833 & 4.374 & 80.357 & 91.239 & 22.712 & 57.530 & 107.879 & 21.626 \\
\hline $\mathbf{N}$ & РM3-BB06 & 11.339 & 13.343 & 9.158 & -5.103 & -8.811 & -8.245 & 2.537 & 0.547 & -3.847 & -3.833 & -1.158 & -6.568 \\
\hline $\mathbf{N}$ & РM3-BB07 & 130.651 & 136.630 & 118.395 & 76.354 & 85.739 & 62.229 & 46.575 & 61.645 & 46.800 & 6.530 & 78.866 & -3.437 \\
\hline $\mathbf{N}$ & PM3-BB08 & 120.064 & 122.704 & 116.425 & 46.000 & 41.232 & 37.087 & 38.405 & 22.132 & 28.841 & 0.405 & 46.705 & 0.467 \\
\hline $\mathbf{N}$ & РM3-BB09 & 150.611 & 164.278 & 151.203 & 131.385 & 143.512 & 124.303 & 166.813 & 163.458 & 168.936 & 107.668 & 165.854 & 109.512 \\
\hline $\mathbf{N}$ & PM3-BB10 & 224.437 & 229.861 & 219.447 & 154.407 & 160.149 & 139.991 & 215.267 & 213.295 & 187.872 & 201.732 & 213.437 & 182.480 \\
\hline $\mathbf{N}$ & PM3-BB11 & 97.901 & 136.407 & 119.453 & 55.741 & 63.638 & 64.411 & 51.079 & 88.371 & 66.897 & 3.878 & 100.145 & 6.243 \\
\hline $\mathbf{N}$ & PM3-BB12 & 43.597 & 140.845 & 24.279 & 68.905 & 73.186 & 64.014 & -2.175 & 0.396 & 0.264 & -4.887 & 1.843 & 2.695 \\
\hline $\mathbf{N}$ & РM3-BC01 & -17.313 & -17.409 & -19.088 & -9.376 & -26.143 & -22.383 & -17.351 & -17.978 & -22.497 & -12.412 & -24.442 & -27.728 \\
\hline $\mathbf{N}$ & PM3-BC02 & 40.155 & 54.782 & 49.100 & 12.422 & 25.695 & 6.908 & 7.486 & 22.938 & 20.978 & 3.551 & 30.462 & 0.508 \\
\hline $\mathbf{N}$ & РM3-BC03 & 198.241 & 203.822 & 194.645 & 128.864 & 142.212 & 110.413 & 174.841 & 144.185 & 162.242 & 77.267 & 157.504 & 76.188 \\
\hline $\mathbf{N}$ & РM3-BC04 & 127.761 & 125.671 & 116.353 & 38.403 & 50.868 & 33.389 & 8.600 & 3.349 & 3.564 & 0.508 & 2.343 & -4.612 \\
\hline $\mathbf{N}$ & РM3-BC05 & -4.891 & -5.751 & -5.907 & -9.603 & -8.493 & -9.645 & -6.263 & -3.663 & -6.655 & -8.372 & -5.845 & -7.895 \\
\hline $\mathbf{N}$ & РM3-BC06 & -13.608 & -11.493 & -10.295 & -9.600 & -11.611 & -8.489 & -9.047 & -7.179 & -8.471 & -10.907 & -9.779 & -10.047 \\
\hline $\mathbf{N}$ & РM3-BC07 & -5.291 & -4.983 & -3.087 & -4.801 & -7.100 & -5.905 & -4.317 & -3.671 & -4.363 & -6.432 & -7.459 & -7.109 \\
\hline $\mathbf{N}$ & РM3-BC08 & 203.561 & 198.287 & 221.396 & -6.066 & -7.372 & -5.151 & -7.645 & -0.862 & -4.763 & -3.296 & -3.816 & -3.225 \\
\hline $\mathbf{N}$ & PM3-BC09 & -0.357 & 0.721 & 3.404 & -1.954 & -1.361 & 1.964 & -1.930 & -2.093 & 0.903 & -5.287 & -4.729 & -5.350 \\
\hline $\mathbf{N}$ & PM3-BC10 & 9.113 & 13.707 & 11.197 & -1.171 & 0.947 & 1.753 & 0.638 & -0.751 & -2.282 & -5.572 & -1.266 & -3.901 \\
\hline$N$ & PM3-BC11 & -0.767 & 2.971 & 2.868 & -1.655 & -2.359 & 3.721 & 2.247 & 2.570 & 14.825 & -1.147 & 1.838 & -0.832 \\
\hline $\mathbf{N}$ & PM3-BC12 & 81.571 & 119.576 & 76.972 & 10.205 & 18.908 & 19.463 & 30.482 & 26.599 & 37.162 & 25.486 & 42.246 & 26.609 \\
\hline $\mathbf{N}$ & PM3-BD01 & -7.775 & -6.717 & -3.678 & -0.425 & -2.254 & 0.684 & -1.424 & -3.412 & 0.900 & -4.934 & -6.217 & -4.112 \\
\hline $\mathbf{N}$ & PM3-BD02 & -10.703 & -7.797 & -6.271 & -5.738 & -6.111 & -5.857 & -5.604 & -5.208 & -8.967 & -5.508 & -7.275 & -6.922 \\
\hline $\mathbf{N}$ & PM3-BD03 & 1.182 & 7.349 & 6.000 & 3.533 & 9.284 & 8.643 & 2.095 & 18.333 & 56.093 & -0.918 & 47.792 & -1.950 \\
\hline $\mathbf{N}$ & PM3-BD04 & -5.926 & -6.120 & -4.321 & -3.071 & -4.726 & -1.813 & -1.264 & -4.433 & -1.283 & -4.264 & -6.662 & -5.707 \\
\hline $\mathbf{N}$ & PM3-BD05 & -11.747 & -11.254 & -11.592 & -10.667 & -11.726 & -11.750 & -10.253 & -9.671 & -10.455 & -11.349 & -10.221 & -8.900 \\
\hline $\mathbf{N}$ & PM3-BD06 & -9.137 & -7.779 & -7.288 & -7.057 & -8.970 & -7.979 & -6.257 & -5.495 & -7.391 & -8.989 & -7.088 & -8.562 \\
\hline $\mathbf{N}$ & PM3-BD07 & -4.601 & -3.617 & -1.645 & -5.005 & -5.379 & -4.132 & -3.779 & -3.537 & -0.886 & -7.003 & -5.412 & -5.297 \\
\hline $\mathbf{N}$ & PM3-BD08 & -5.186 & -4.142 & -1.695 & -5.876 & -6.457 & -4.255 & -5.447 & -4.072 & -3.491 & -5.645 & -5.575 & -5.779 \\
\hline $\mathbf{N}$ & PM3-BD09 & -2.278 & -4.745 & -2.016 & -4.678 & -5.828 & -2.764 & -3.978 & -3.593 & -2.912 & -5.967 & -4.143 & -5.484 \\
\hline $\mathbf{N}$ & PM3-BD10 & -8.537 & -7.222 & -3.212 & -6.338 & -3.266 & -0.230 & -0.708 & -5.443 & -0.513 & -5.075 & -6.880 & -5.432 \\
\hline$N$ & PM3-BD11 & -9.946 & -6.201 & -4.570 & -8.111 & -4.770 & -2.075 & -2.288 & -3.638 & -3.882 & -6.214 & -4.396 & -4.909 \\
\hline $\mathbf{N}$ & PM3-BD12 & -5.732 & -1.786 & -0.239 & -2.672 & 0.451 & 6.918 & 9.729 & -1.143 & 3.675 & -3.275 & -0.171 & 0.541 \\
\hline $\mathbf{N}$ & PM3-BE01 & -7.384 & -7.892 & -4.851 & -2.037 & -3.053 & -0.661 & -0.700 & -2.325 & -2.151 & -4.583 & -5.661 & -3.812 \\
\hline $\mathbf{N}$ & PM3-BE02 & -9.261 & -6.420 & -5.120 & -4.307 & -5.408 & -4.937 & -3.442 & -4.718 & -6.211 & -6.758 & -6.726 & -6.012 \\
\hline $\mathbf{N}$ & PM3-BE03 & -7.103 & -4.355 & -1.647 & -3.050 & -4.541 & -4.667 & -2.733 & -2.867 & -5.392 & -5.293 & -5.051 & -5.007 \\
\hline $\mathbf{N}$ & PM3-BE04 & -4.862 & -6.355 & -2.283 & -3.353 & -4.345 & -2.151 & -2.454 & -2.313 & -1.971 & -5.293 & -5.728 & -5.370 \\
\hline N & PM3-BE05 & -5.520 & -3.775 & -0.463 & -2.576 & -2.003 & 2.496 & 2.659 & -2.946 & 3.167 & -2.124 & -4.186 & -4.659 \\
\hline $\mathbf{N}$ & PM3-BE06 & 1.675 & 37.995 & 39.243 & 11.839 & 36.476 & 24.729 & 3.767 & 11.588 & 37.279 & 6.913 & 33.416 & 2.233 \\
\hline $\mathbf{N}$ & PM3-BE07 & 186.483 & 192.587 & 181.737 & -3.036 & -4.543 & -2.645 & 40.607 & 131.799 & 140.792 & 41.811 & 132.867 & 71.121 \\
\hline $\mathbf{N}$ & PM3-BE08 & 212.559 & 178.724 & 204.001 & 145.191 & 135.601 & 126.074 & 219.958 & 184.834 & 149.532 & 188.432 & 192.200 & 167.297 \\
\hline $\mathbf{N}$ & PM3-BE09 & -6.151 & -7.783 & -2.649 & -3.637 & -3.803 & -2.163 & -1.945 & -3.700 & -1.233 & -4.743 & -6.200 & -4.462 \\
\hline $\mathbf{N}$ & PM3-BE10 & -11.628 & -7.789 & -6.816 & -6.978 & -6.341 & -2.271 & -2.345 & -6.478 & -0.571 & -7.374 & -8.108 & -6.634 \\
\hline $\mathbf{N}$ & PM3-BE11 & 81.864 & 83.641 & 85.046 & 80.614 & 78.634 & 40.987 & 203.524 & 171.637 & 20.425 & 186.867 & 170.093 & 164.775 \\
\hline $\mathbf{N}$ & PM3-BE12 & 116.980 & 144.559 & 138.961 & -4.529 & -2.926 & 2.225 & 185.283 & 158.942 & 48.558 & 201.555 & 169.284 & 153.362 \\
\hline $\mathbf{N}$ & PM3-BF01 & 160.178 & 167.407 & 169.228 & 143.738 & 160.400 & 147.682 & 196.414 & 163.137 & 127.899 & 161.417 & 182.276 & 168.263 \\
\hline $\mathbf{N}$ & PM3-BF02 & -11.925 & -17.022 & -16.800 & -5.253 & -20.212 & -12.445 & -3.557 & -12.166 & -11.339 & -10.976 & -13.228 & -7.322 \\
\hline $\mathbf{N}$ & PM3-BF03 & 232.649 & 232.334 & 235.349 & 186.666 & 201.917 & 192.072 & 227.030 & 225.495 & 203.201 & 214.182 & 232.667 & 196.938 \\
\hline $\mathbf{N}$ & PM3-BF04 & 262.188 & 260.393 & 264.663 & 202.088 & 213.946 & 209.576 & 264.713 & 250.355 & 227.530 & 238.472 & 243.296 & 206.818 \\
\hline $\mathbf{N}$ & PM3-BF05 & 26.566 & 33.299 & 39.358 & 34.780 & 50.878 & 31.096 & 1.286 & -2.830 & 1.633 & -4.076 & -5.678 & -5.007 \\
\hline $\mathbf{N}$ & PM3-BF06 & -7.503 & -8.686 & 9.868 & -6.517 & -2.225 & -3.579 & 3.357 & -6.187 & -8.962 & 2.392 & -8.833 & -8.180 \\
\hline $\mathbf{N}$ & PM3-BF07 & -6.900 & -13.882 & -9.741 & -23.397 & -18.687 & -21.324 & -3.661 & -1.582 & -12.287 & -19.661 & -25.003 & -21.537 \\
\hline $\mathbf{N}$ & PM3-BF08 & -3.900 & -0.695 & 5.301 & 9.241 & 14.512 & 11.442 & 1.782 & -4.239 & 5.325 & -2.733 & -4.762 & -3.562 \\
\hline N & PM3-BF09 & 11.936 & 12.153 & 15.879 & 26.180 & 42.683 & 36.103 & -1.263 & -3.388 & 0.026 & -4.208 & -5.030 & -3.793 \\
\hline $\mathbf{N}$ & PM3-BF10 & -0.122 & -0.566 & 5.614 & 6.318 & 9.287 & 9.263 & -0.326 & -5.600 & -0.036 & -4.937 & -5.709 & -6.242 \\
\hline $\mathbf{N}$ & PM3-BF11 & 5.093 & 2.966 & 8.161 & 15.518 & 28.693 & 20.609 & 0.368 & -4.811 & 0.013 & -5.049 & -5.749 & -4.486 \\
\hline $\mathbf{N}$ & PM3-BF12 & -8.408 & -5.809 & -3.268 & -4.909 & -3.417 & 0.001 & -3.170 & -4.788 & -5.038 & -5.459 & -3.250 & -5.347 \\
\hline$N$ & PM3-BG01 & 16.086 & 25.880 & 49.124 & 38.329 & 51.676 & 42.301 & 25.004 & 7.289 & 2.021 & 0.430 & 3.384 & 3.450 \\
\hline $\mathbf{N}$ & PM3-BG02 & 30.061 & 66.441 & 75.593 & 34.841 & 46.445 & 27.287 & 53.353 & 3.900 & -0.383 & 4.387 & 5.739 & 0.937 \\
\hline $\mathbf{N}$ & PM3-BG03 & 42.891 & 44.738 & 51.359 & 8.074 & 13.466 & 7.738 & 25.576 & -18.108 & 13.259 & 11.483 & 14.188 & -5.447 \\
\hline $\mathbf{N}$ & PM3-BG04 & 2.222 & 20.730 & 28.971 & -10.817 & -0.539 & -1.534 & -8.213 & 0.061 & -0.651 & 0.136 & -0.425 & -0.182 \\
\hline $\mathbf{N}$ & PM3-BG05 & 18.064 & 31.039 & 34.395 & -0.397 & 0.836 & 2.204 & 5.691 & 4.541 & 5.709 & 2.046 & 2.949 & 2.697 \\
\hline $\mathbf{N}$ & PM3-BG06 & 6.542 & 30.336 & 32.846 & 3.536 & 8.576 & 14.163 & 12.234 & 3.039 & 12.796 & 3.772 & 2.559 & 2.832 \\
\hline$N$ & PM3-BG07 & 135.647 & 151.072 & 120.501 & 29.226 & 50.822 & 24.483 & 59.413 & 101.007 & 91.701 & 102.792 & 129.683 & 89.440 \\
\hline $\mathbf{N}$ & PM3-BG08 & 78.613 & 102.480 & 113.499 & 46.841 & 72.826 & 51.311 & 103.407 & 28.116 & 47.032 & 18.720 & 70.445 & 8.092 \\
\hline
\end{tabular}




\begin{tabular}{|c|c|c|c|c|c|c|c|c|c|c|c|c|c|}
\hline $\mathbf{N}$ & PM3-BG09 & 71.400 & 68.758 & 99.978 & 45.924 & 63.795 & 51.714 & 97.172 & 11.578 & 36.839 & 8.188 & 48.457 & 5.622 \\
\hline $\mathbf{N}$ & PM3-BG10 & -22.226 & 63.738 & 60.813 & -6.516 & 19.501 & 9.339 & -6.179 & 48.709 & 36.400 & 39.433 & 67.392 & 37.872 \\
\hline $\mathbf{N}$ & PM3-BG11 & -1.888 & -3.324 & 2.089 & -2.545 & 0.804 & 3.943 & 1.142 & -3.618 & 1.401 & -5.189 & -4.324 & -2.832 \\
\hline $\mathbf{N}$ & PM3-BG12 & 90.707 & 96.747 & 73.607 & 12.020 & 28.282 & 8.639 & 37.821 & 48.083 & 27.049 & 27.480 & 48.387 & 17.537 \\
\hline $\mathbf{N}$ & РM3-BH01 & 255.661 & 258.078 & 255.558 & 235.605 & 226.807 & 232.179 & 244.353 & 229.755 & 223.279 & 230.489 & 240.943 & 208.203 \\
\hline $\mathbf{N}$ & PM3-BH02 & 255.439 & 257.039 & 254.549 & 233.830 & 232.986 & 237.488 & 242.246 & 239.364 & 213.350 & 219.700 & 235.766 & 186.961 \\
\hline $\mathbf{N}$ & РM3-ВH03 & 267.121 & 261.945 & 261.837 & 246.993 & 235.889 & 242.178 & 246.612 & 232.163 & 214.690 & 216.989 & 239.764 & 193.236 \\
\hline $\mathbf{N}$ & PM3-BH04 & 238.042 & 234.471 & 234.516 & 174.434 & 191.588 & 156.057 & 220.538 & 215.355 & 195.737 & 203.397 & 215.971 & 172.603 \\
\hline $\mathbf{N}$ & PM3-BH05 & 233.564 & 241.458 & 240.551 & 85.534 & 116.218 & 72.513 & 212.901 & 209.875 & 177.832 & 197.066 & 219.099 & 156.809 \\
\hline $\mathbf{N}$ & РM3-BH06 & 191.786 & 190.587 & 195.028 & 35.559 & 71.217 & 40.875 & 113.051 & 166.722 & 81.730 & 162.934 & 172.310 & 169.217 \\
\hline $\mathbf{N}$ & РM3-BH07 & 226.434 & 232.549 & 225.700 & 119.276 & 147.786 & 131.947 & 205.360 & 204.549 & 188.443 & 193.350 & 204.126 & 185.433 \\
\hline $\mathbf{N}$ & PM3-BH08 & 253.689 & 257.287 & 262.670 & 223.283 & 226.626 & 235.434 & 252.067 & 232.072 & 224.951 & 231.160 & 245.947 & 207.417 \\
\hline $\mathbf{N}$ & РM3-BH09 & 250.718 & 249.621 & 262.222 & 205.005 & 215.826 & 230.007 & 249.824 & 228.407 & 215.096 & 233.074 & 238.574 & 188.368 \\
\hline $\mathbf{N}$ & PM3-BH10 & 212.459 & 218.068 & 225.055 & 176.050 & 197.607 & 202.538 & 229.459 & 196.834 & 200.032 & 184.880 & 205.318 & 157.480 \\
\hline $\mathbf{N}$ & PM3-BH11 & 189.479 & 199.892 & 191.497 & 157.458 & 185.117 & 162.108 & 183.849 & 171.058 & 167.941 & 161.821 & 183.779 & 155.312 \\
\hline $\mathbf{N}$ & РM3-BH12 & 175.339 & 177.297 & 165.995 & 96.542 & 135.014 & 111.375 & 169.728 & 151.255 & 133.986 & 142.395 & 154.121 & 127.382 \\
\hline$P$ & PM4-AA01 & 0.000 & 0.000 & 0.000 & 0.000 & 0.000 & 0.000 & 0.000 & 0.000 & 0.000 & 0.000 & 0.000 & 0.000 \\
\hline$P$ & PM4-AA02 & 169.771 & 151.013 & 142.095 & 71.012 & 57.632 & 57.047 & 86.080 & 117.215 & 86.551 & 141.657 & 76.458 & 89.299 \\
\hline$P$ & PM4-AA03 & 176.870 & 127.400 & 132.272 & 29.771 & 33.168 & 31.166 & 73.180 & 105.661 & 66.651 & 113.853 & 69.074 & 82.437 \\
\hline$P$ & PM4-AA04 & 175.101 & 154.185 & 149.204 & 63.163 & 46.413 & 44.607 & 91.442 & 109.372 & 82.078 & 148.013 & 91.097 & 101.859 \\
\hline $\mathbf{P}$ & PM4-AA05 & 158.262 & 138.125 & 112.416 & 42.437 & 41.912 & 15.708 & 83.071 & 109.250 & 81.516 & 143.863 & 85.343 & 105.230 \\
\hline$P$ & PM4-AA06 & 0.638 & 70.146 & 1.183 & 6.379 & 4.858 & -2.658 & -11.484 & 23.678 & 4.437 & 1.488 & 8.740 & 1.933 \\
\hline$P$ & PM4-AA07 & 40.462 & 86.457 & 36.201 & 23.367 & 9.446 & 17.993 & 18.233 & 25.055 & -22.188 & 17.407 & 14.821 & 0.364 \\
\hline$P$ & PM4-AA08 & 188.903 & 168.974 & 167.263 & 76.759 & 64.079 & 44.000 & 117.312 & 134.178 & 112.571 & 177.226 & 113.259 & 154.004 \\
\hline$P$ & PM4-AA09 & 193.157 & 167.993 & 171.367 & 144.795 & 133.583 & 152.942 & 130.762 & 140.592 & 128.822 & 162.033 & 122.666 & 147.880 \\
\hline$P$ & PM4-AA10 & 195.662 & 29.278 & -55.947 & -23.376 & 136.788 & 164.628 & 140.730 & 136.779 & -19.400 & 173.558 & 117.591 & 141.986 \\
\hline $\mathbf{P}$ & PM4-AA11 & 175.901 & 153.693 & -60.689 & 129.051 & 128.957 & 131.025 & 140.155 & 132.547 & 111.179 & 158.225 & 102.305 & 130.915 \\
\hline$P$ & PM4-AA12 & 172.274 & 146.246 & 146.992 & 119.078 & 116.240 & 118.988 & 122.474 & 119.912 & 90.091 & 153.130 & 87.872 & 115.950 \\
\hline $\mathbf{P}$ & PM4-AB01 & 141.986 & 128.941 & 118.842 & 43.516 & 37.083 & 51.007 & 77.862 & 95.665 & 82.309 & 134.307 & 69.147 & 102.616 \\
\hline$P$ & PM4-AB02 & 184.989 & 142.654 & 133.267 & 76.991 & 74.055 & 67.804 & 81.586 & 105.225 & 96.943 & 140.713 & 84.343 & 107.279 \\
\hline$P$ & PM4-AB03 & 188.987 & 166.732 & 173.688 & 138.464 & 131.296 & 128.671 & 126.796 & 124.280 & 129.088 & 167.309 & 109.017 & 127.395 \\
\hline $\mathbf{P}$ & PM4-AB04 & 170.038 & 150.083 & 142.899 & 130.403 & 133.130 & 134.828 & 95.325 & 112.021 & 113.489 & 141.854 & 89.724 & 111.642 \\
\hline$P$ & PM4-AB05 & 167.928 & 159.449 & 153.458 & 73.358 & 71.840 & 61.616 & 85.775 & 116.138 & 100.399 & 146.970 & 98.975 & 121.478 \\
\hline$P$ & PM4-AB06 & 170.325 & 164.107 & 151.928 & 67.507 & 59.868 & 51.362 & 94.905 & 112.997 & 96.466 & 141.162 & 94.592 & 127.075 \\
\hline $\mathbf{P}$ & PM4-AB07 & 162.272 & 145.925 & 151.158 & 65.154 & 48.643 & 50.351 & 86.253 & 113.568 & 95.054 & 137.089 & 88.653 & 127.661 \\
\hline $\mathbf{P}$ & PM4-AB08 & 198.228 & 189.118 & 192.080 & 159.629 & 159.962 & 172.578 & 135.628 & 145.157 & 159.768 & 179.276 & 124.240 & 167.051 \\
\hline$P$ & PM4-AB09 & 196.692 & 166.224 & 170.084 & 128.051 & 123.826 & 127.026 & 138.317 & 124.355 & 115.926 & 163.716 & 106.897 & 137.104 \\
\hline $\mathbf{P}$ & PM4-AB10 & 180.408 & 156.299 & 161.774 & 119.849 & 125.109 & 127.612 & 123.328 & 124.166 & 112.800 & 152.247 & 104.388 & 143.429 \\
\hline$P$ & PM4-AB11 & 161.580 & 135.026 & 143.292 & 108.357 & 109.013 & 105.204 & 108.120 & 103.115 & 96.183 & 131.778 & 78.107 & 124.751 \\
\hline$P$ & PM4-AB12 & 161.814 & 138.074 & 138.863 & 112.299 & 108.580 & 106.041 & 118.305 & 104.037 & 89.239 & 134.297 & 76.142 & 113.987 \\
\hline $\mathbf{P}$ & PM4-AC01 & 160.741 & 142.263 & 141.432 & 58.232 & 33.918 & 49.026 & 82.557 & 102.486 & 75.562 & 134.337 & 81.924 & 94.009 \\
\hline $\mathbf{P}$ & PM4-AC02 & 156.783 & 143.017 & 129.967 & 57.558 & 63.716 & 47.128 & 81.637 & 102.265 & 84.804 & 131.351 & 79.984 & 109.904 \\
\hline$P$ & PM4-AC03 & 198.455 & 174.930 & 184.367 & 147.111 & 145.250 & 156.414 & 133.442 & 136.953 & 133.899 & 178.766 & 112.178 & 153.429 \\
\hline $\mathbf{P}$ & PM4-AC04 & -33.116 & 189.057 & 195.974 & 159.387 & 158.829 & 171.301 & 127.550 & 148.721 & 144.012 & 182.755 & 127.993 & 142.053 \\
\hline$P$ & PM4-AC05 & -43.782 & -62.450 & -56.796 & -34.496 & -46.871 & -32.782 & -84.195 & -85.870 & -72.649 & -35.949 & -103.054 & -35.004 \\
\hline$P$ & PM4-AC06 & 218.637 & 199.409 & 202.796 & 173.054 & 168.605 & 179.604 & 149.925 & 154.718 & 156.493 & 195.863 & 136.996 & 190.561 \\
\hline $\mathbf{P}$ & PM4-AC07 & 187.546 & 165.962 & 190.197 & 103.111 & 98.966 & 106.574 & 114.816 & 127.965 & 117.974 & 160.814 & 105.142 & 151.175 \\
\hline $\mathbf{P}$ & PM4-AC08 & 214.082 & 175.826 & 174.657 & 148.200 & 129.345 & 121.271 & 147.395 & 145.429 & 132.691 & 179.304 & 128.709 & 161.190 \\
\hline$P$ & PM4-AC09 & 208.482 & 188.193 & -5.517 & 166.092 & 161.887 & 183.034 & 155.633 & 146.313 & 150.155 & 181.101 & 110.670 & 2.980 \\
\hline $\mathbf{P}$ & PM4-AC10 & -53.953 & -54.075 & -57.214 & 141.713 & 143.376 & 152.004 & 126.382 & 134.313 & -65.659 & 163.803 & 91.042 & 156.136 \\
\hline $\mathbf{P}$ & PM4-AC11 & 165.318 & 149.397 & 152.339 & 139.524 & 134.409 & 148.308 & 117.845 & 116.595 & 109.275 & 149.249 & 86.668 & 131.592 \\
\hline $\mathbf{P}$ & PM4-AC12 & 178.737 & 156.151 & 164.099 & 150.443 & 131.666 & 146.554 & 130.474 & 129.004 & 93.358 & 155.030 & 92.125 & 121.603 \\
\hline $\mathbf{P}$ & PM4-AD01 & 182.311 & 166.707 & 176.084 & 144.950 & 124.405 & 139.428 & -79.309 & 127.046 & 110.591 & 158.296 & 109.255 & 140.924 \\
\hline $\mathbf{P}$ & PM4-AD02 & 193.318 & 174.988 & 181.758 & 133.583 & 137.830 & 145.586 & 100.679 & 126.918 & 119.109 & 161.987 & 116.592 & 149.142 \\
\hline $\mathbf{P}$ & PM4-AD03 & 159.467 & 141.251 & 132.180 & 69.466 & 64.032 & 53.834 & 90.822 & 117.449 & 98.814 & 147.057 & 93.883 & 109.967 \\
\hline$P$ & PM4-AD04 & 163.013 & 151.439 & 143.476 & 82.986 & 78.745 & 51.950 & 84.724 & 118.924 & 99.703 & 143.480 & 98.851 & 140.520 \\
\hline $\mathbf{P}$ & PM4-AD05 & 177.836 & 165.179 & 160.246 & 65.221 & 59.940 & 57.130 & 59.512 & 104.263 & 71.818 & 138.396 & 89.550 & 113.157 \\
\hline $\mathbf{P}$ & PM4-AD06 & 186.453 & 169.229 & 158.424 & 97.176 & 89.058 & 78.863 & 99.436 & 126.465 & 119.688 & 157.897 & 99.649 & 156.949 \\
\hline $\mathbf{P}$ & PM4-AD07 & 183.764 & 164.859 & 155.439 & 92.853 & 86.266 & 89.108 & 108.470 & 124.170 & 110.225 & 156.328 & 103.722 & 163.807 \\
\hline $\mathbf{P}$ & PM4-AD08 & 211.716 & 190.347 & 183.880 & 158.443 & -22.224 & 56.339 & -76.228 & 147.507 & -49.147 & 179.711 & 118.471 & 109.384 \\
\hline $\mathbf{P}$ & PM4-AD09 & 189.434 & 188.307 & 184.501 & 161.854 & 121.743 & 159.838 & 142.974 & 152.863 & 133.287 & 184.996 & 119.042 & 165.596 \\
\hline$P$ & PM4-AD10 & -37.411 & 166.842 & 177.183 & 112.257 & 140.337 & 145.661 & -74.699 & 132.857 & 117.509 & 165.820 & 107.418 & 153.436 \\
\hline $\mathbf{P}$ & PM4-AD11 & 181.116 & 155.251 & 168.392 & 136.345 & 134.315 & 135.828 & 133.771 & 122.141 & 101.447 & 160.849 & 92.324 & 136.040 \\
\hline $\mathbf{P}$ & PM4-AD12 & 185.525 & 159.697 & 169.699 & 128.207 & 123.812 & 126.901 & 132.422 & 138.618 & 94.808 & 166.251 & 95.586 & 120.030 \\
\hline $\mathbf{P}$ & PM4-AE01 & 180.497 & 160.776 & 155.392 & 67.754 & 48.826 & 35.917 & 94.944 & 118.567 & 90.532 & -32.691 & 92.179 & 117.408 \\
\hline$P$ & PM4-AE02 & 168.238 & 160.741 & -19.067 & -26.250 & -17.109 & 32.379 & 85.883 & 105.346 & 85.963 & 135.932 & 84.947 & 124.679 \\
\hline $\mathbf{P}$ & PM4-AE03 & 171.230 & 140.921 & 139.332 & 48.786 & 57.805 & 57.101 & 91.703 & 116.116 & 101.034 & 143.842 & 84.668 & 126.688 \\
\hline $\mathbf{P}$ & PM4-AE04 & 167.168 & 139.897 & 133.380 & 39.575 & 51.088 & 45.583 & 64.055 & 103.515 & 75.759 & 127.967 & 71.693 & 118.020 \\
\hline$P$ & PM4-AE05 & 173.305 & 162.484 & 156.238 & 68.704 & 65.532 & 60.511 & 91.601 & 120.453 & 101.617 & 145.297 & 94.225 & 136.413 \\
\hline
\end{tabular}




\begin{tabular}{|c|c|c|c|c|c|c|c|c|c|c|c|c|c|}
\hline $\mathbf{P}$ & PM4-AE06 & 138.951 & 120.834 & 130.096 & -4.822 & -35.978 & 0.428 & 7.940 & 58.238 & 25.535 & 77.525 & 47.200 & 80.609 \\
\hline $\mathbf{P}$ & PM4-AE07 & 168.742 & 165.884 & 150.872 & 90.645 & 88.654 & 76.009 & 34.104 & 115.022 & 94.479 & 99.647 & 84.915 & 86.212 \\
\hline $\mathbf{P}$ & PM4-AE08 & 30.241 & 34.609 & 35.971 & 51.280 & 10.839 & -0.745 & 6.144 & 23.840 & 4.236 & 3.661 & 14.820 & 36.014 \\
\hline $\mathbf{P}$ & PM4-AE09 & 177.717 & 166.597 & 146.934 & 118.805 & 120.271 & 106.979 & 110.065 & 126.492 & 105.137 & 157.559 & 93.601 & 141.396 \\
\hline $\mathbf{P}$ & PM4-AE10 & 183.034 & 170.041 & 170.250 & 127.850 & 125.738 & 136.170 & 131.757 & 133.518 & 124.004 & 158.386 & 99.096 & 146.483 \\
\hline $\mathbf{P}$ & PM4-AE11 & 116.292 & 78.814 & 57.211 & 22.391 & 14.614 & 11.996 & 16.763 & 44.879 & 19.976 & 41.212 & 21.965 & 40.862 \\
\hline $\mathbf{P}$ & PM4-AE12 & 168.721 & 150.424 & 145.276 & 113.659 & 102.649 & 91.151 & 118.732 & 113.813 & 108.214 & 145.083 & 80.379 & 111.383 \\
\hline s & PM4-AF01 & 0.000 & 0.000 & 0.000 & 0.000 & 0.000 & 0.000 & 0.000 & 0.000 & 0.000 & 0.000 & 0.000 & 0.000 \\
\hline $\mathbf{s}$ & PM4-AF02 & 201.892 & 212.070 & 156.468 & 104.555 & 111.920 & 91.537 & 169.459 & 194.714 & 169.449 & 176.132 & 179.867 & 145.058 \\
\hline $\mathbf{s}$ & PM4-AF03 & 218.338 & 215.709 & 164.388 & 115.062 & 135.220 & 112.782 & 177.713 & 209.803 & 187.345 & 195.114 & 191.328 & 157.213 \\
\hline s & PM4-AF04 & 209.433 & 221.001 & 173.284 & 111.133 & 124.529 & 100.472 & 182.434 & 198.475 & 171.189 & 175.259 & 195.680 & 155.063 \\
\hline s & PM4-AF05 & 202.439 & 207.549 & 137.380 & 101.279 & 117.343 & 91.891 & 176.859 & 183.150 & 133.268 & 137.291 & 186.479 & 89.280 \\
\hline $\mathbf{s}$ & PM4-AF06 & 211.268 & 223.201 & 164.865 & 124.850 & 131.055 & 112.843 & 183.842 & 207.013 & 180.955 & 185.279 & 197.820 & 151.692 \\
\hline s & PM4-AF07 & 190.209 & 207.488 & 145.786 & 135.701 & 121.097 & 117.346 & 186.112 & 204.976 & 167.728 & 181.643 & 203.397 & 146.304 \\
\hline s & PM4-AF08 & 197.688 & 215.026 & 160.245 & 95.645 & 110.691 & 92.008 & 180.011 & 204.813 & 179.272 & 182.222 & 198.225 & 166.037 \\
\hline s & PM4-AF09 & 173.325 & 203.530 & 138.392 & 131.867 & 144.567 & 122.972 & 174.776 & 197.854 & 178.003 & 177.799 & 193.640 & 164.405 \\
\hline $\mathbf{s}$ & PM4-AF10 & -5.416 & -2.020 & -44.941 & -5.078 & -0.555 & 1.072 & 1.125 & 4.768 & 2.299 & -2.279 & -2.358 & -0.866 \\
\hline $\mathbf{s}$ & PM4-AF11 & 4.867 & 8.259 & -38.721 & 0.526 & 3.571 & 5.322 & 7.733 & 11.811 & 6.853 & -0.889 & 12.001 & -1.497 \\
\hline $\mathbf{s}$ & PM4-AF12 & 174.599 & 183.018 & 133.028 & 78.801 & 90.520 & 74.347 & 142.538 & 177.578 & 146.255 & 154.154 & 158.408 & 114.725 \\
\hline s & PM4-AG01 & -0.034 & 0.492 & -42.401 & 0.017 & -0.601 & -0.325 & -0.575 & 2.382 & -1.709 & 0.400 & 0.436 & -0.267 \\
\hline $\mathbf{s}$ & PM4-AG02 & 144.496 & 157.539 & 72.561 & 14.726 & 12.536 & 11.037 & 36.678 & 36.295 & 22.370 & 12.555 & 36.772 & 9.262 \\
\hline s & PM4-AG03 & 213.179 & 210.196 & 163.778 & 118.122 & 124.858 & 97.582 & 183.704 & 206.429 & 171.084 & 190.037 & 194.343 & 161.086 \\
\hline s & PM4-AG04 & 219.522 & 218.209 & 166.280 & 130.904 & 138.810 & 110.220 & 184.036 & 211.461 & 186.771 & 190.900 & 198.570 & 167.780 \\
\hline s & PM4-AG05 & 224.939 & 108.299 & 9.030 & 43.261 & 52.968 & 45.713 & 192.778 & 34.947 & 35.642 & 7.742 & 37.176 & -1.299 \\
\hline $\mathbf{s}$ & PM4-AG06 & 48.518 & 66.325 & 3.809 & 0.704 & -1.939 & 1.946 & 65.617 & 104.633 & 23.911 & 78.160 & 99.061 & 83.520 \\
\hline $\mathbf{s}$ & PM4-AG07 & 210.822 & 214.196 & 163.763 & 126.507 & 133.126 & 115.307 & 187.084 & 206.296 & 154.639 & 182.407 & 193.699 & 147.103 \\
\hline $\mathbf{s}$ & PM4-AG08 & 195.808 & 196.134 & 134.475 & 80.905 & 91.124 & 87.404 & 155.655 & 188.838 & 117.692 & 149.512 & 182.253 & 142.038 \\
\hline $\mathbf{s}$ & PM4-AG09 & 204.400 & 211.664 & 171.507 & 135.837 & 144.058 & 128.353 & 194.475 & 204.662 & 178.942 & 183.460 & 194.346 & 144.490 \\
\hline $\mathbf{s}$ & PM4-AG10 & 197.667 & 214.575 & 164.150 & 113.778 & 133.697 & 110.825 & 182.353 & 198.679 & 149.717 & 172.388 & 179.675 & 136.188 \\
\hline $\mathbf{s}$ & PM4-AG11 & 185.571 & 196.441 & 154.888 & 110.997 & 120.989 & 106.947 & 174.549 & 200.316 & 159.314 & 163.437 & 173.263 & 147.916 \\
\hline s & PM4-AG12 & -7.349 & -6.607 & -51.654 & -4.972 & -4.693 & -3.176 & -2.874 & 1.001 & -0.884 & -1.253 & -3.311 & -3.582 \\
\hline $\mathbf{s}$ & PM4-AH01 & 179.675 & 193.851 & 133.603 & 65.882 & 68.696 & 63.163 & 141.809 & 183.928 & 99.768 & 163.578 & 190.909 & 119.515 \\
\hline $\mathbf{s}$ & PM4-AH02 & 42.480 & 85.532 & 173.187 & 30.596 & 42.113 & 32.645 & 27.528 & 31.136 & 23.400 & 34.925 & 47.359 & 22.454 \\
\hline $\mathbf{s}$ & PM4-AH03 & 41.471 & 3.414 & -43.459 & 4.632 & 0.849 & 1.695 & 47.811 & 3.807 & -2.747 & 5.138 & 2.845 & 3.904 \\
\hline $\mathbf{s}$ & PM4-AH04 & 19.962 & 27.491 & -29.754 & 19.770 & 21.975 & 17.605 & 17.588 & 26.612 & 20.558 & 7.986 & 39.953 & 15.009 \\
\hline $\mathbf{s}$ & PM4-AH05 & 34.705 & 32.113 & -19.372 & 10.793 & 25.893 & 11.689 & 24.625 & 22.158 & 15.096 & 13.433 & 22.653 & 12.095 \\
\hline $\mathbf{s}$ & PM4-AH06 & 135.885 & 134.778 & 59.678 & 14.305 & 23.572 & 8.033 & 56.043 & 97.163 & 31.521 & 81.101 & 79.876 & 71.770 \\
\hline $\mathbf{s}$ & PM4-AH07 & 103.414 & 116.996 & 48.015 & 20.811 & 31.099 & 23.537 & 58.300 & 89.329 & 40.255 & 79.308 & 60.666 & 69.120 \\
\hline $\mathbf{s}$ & PM4-AH08 & 13.593 & 11.553 & -39.808 & 6.603 & 5.074 & 5.697 & 2.370 & 6.950 & 7.678 & 5.704 & 6.118 & 3.904 \\
\hline $\mathbf{s}$ & PM4-AH09 & -10.230 & 2.228 & -43.397 & 2.555 & 2.522 & 1.196 & 1.872 & 7.516 & 3.567 & 5.137 & 2.405 & 1.093 \\
\hline s & PM4-AH10 & -1.095 & -1.324 & -43.926 & -5.497 & 2.688 & -0.959 & 2.433 & 3.025 & 2.030 & 2.649 & -3.055 & 0.689 \\
\hline $\mathbf{s}$ & PM4-AH11 & -4.654 & -4.782 & -47.017 & -4.726 & -1.034 & -1.187 & -2.678 & -0.396 & 0.213 & -0.016 & -3.630 & -3.916 \\
\hline $\mathbf{s}$ & PM4-AH12 & -6.896 & -5.213 & -44.375 & -5.551 & 1.204 & -2.603 & -0.788 & -0.555 & 1.022 & -2.078 & -5.601 & -4.636 \\
\hline
\end{tabular}

\begin{tabular}{|c|c|c|c|c|c|c|c|c|c|c|}
\hline & \multirow[b]{2}{*}{ well } & \multicolumn{3}{|c|}{ BT 3 0:5,27 } & \multicolumn{3}{|c|}{ BT 4 O:3 } & \multicolumn{3}{|c|}{ BT 5 O:2a,2b,3 } \\
\hline & & 14902_1 & 14902_2 & 14902_3 & 1203_1 & 1203_2 & 1203_3 & 3094_1 & 3094_2 & 3094_3 \\
\hline $\begin{array}{l}\mathbf{C} \\
\end{array}$ & PM1-A01 & 0.000 & 0.000 & 0.000 & 0.000 & 0.000 & 0.000 & 0.000 & 0.000 & 0.000 \\
\hline C & PM1-A02 & 191.763 & 180.437 & 171.599 & 248.688 & 227.921 & 230.820 & 91.213 & 51.400 & 50.911 \\
\hline C & PM1-A03 & 81.895 & 72.968 & 40.004 & 170.624 & 115.474 & 104.292 & 90.553 & 90.889 & 90.832 \\
\hline C & PM1-A04 & -0.063 & -7.708 & -2.250 & -8.832 & -4.942 & -2.005 & 1.587 & 0.000 & 0.000 \\
\hline C & PM1-A05 & 20.217 & 12.604 & 7.271 & 28.301 & 8.432 & 8.711 & 168.197 & 114.390 & 104.534 \\
\hline C & PM1-A06 & 245.749 & 225.503 & 224.664 & 281.925 & 264.450 & 272.850 & 145.934 & 113.079 & 118.176 \\
\hline C & PM1-A07 & 7.511 & 7.789 & 5.374 & 9.392 & 8.008 & 7.446 & 144.818 & 99.782 & 86.321 \\
\hline C & PM1-A08 & 8.266 & 6.093 & 3.584 & 0.722 & 4.741 & 1.008 & 1.608 & 1.029 & 0.000 \\
\hline C & PM1-A09 & 2.768 & 2.078 & -1.257 & -5.929 & -4.904 & -4.841 & 9.787 & 3.229 & 0.000 \\
\hline C & PM1-A10 & 93.118 & 67.850 & 64.383 & 143.111 & 101.662 & 114.182 & 0.000 & 0.503 & 0.000 \\
\hline C & PM1-A11 & 61.709 & 46.438 & 35.436 & 118.388 & 74.233 & 81.093 & 109.083 & 94.889 & 97.292 \\
\hline C & PM1-A12 & 6.258 & 11.263 & 10.836 & 58.889 & 2.692 & 3.304 & 11.729 & 11.086 & 2.489 \\
\hline C & PM1-B01 & 3.999 & 0.854 & 5.150 & 165.604 & -4.074 & 0.993 & 1.133 & 0.755 & 0.000 \\
\hline C & PM1-B02 & 123.761 & 104.984 & 87.593 & 160.212 & 118.886 & 118.501 & 4.171 & 3.229 & 1.011 \\
\hline C & PM1-B03 & 98.879 & 95.393 & 77.364 & 174.705 & 123.021 & 148.220 & 84.584 & 62.903 & 62.876 \\
\hline C & PM1-B04 & 2.295 & -1.912 & -1.045 & -3.232 & -4.492 & -4.009 & 0.000 & 0.000 & 0.000 \\
\hline C & PM1-B05 & 242.643 & 179.788 & 166.942 & 269.642 & 244.167 & 257.245 & 112.932 & 98.113 & 81.784 \\
\hline C & PM1-B06 & 271.707 & 218.561 & 211.862 & 200.468 & 86.075 & 106.887 & 48.537 & 39.111 & 40.268 \\
\hline C & PM1-B07 & 170.484 & 137.267 & 168.772 & 185.067 & 179.174 & 186.757 & 75.682 & 59.976 & 47.505 \\
\hline C & PM1-B08 & 105.146 & 123.625 & 98.808 & -4.704 & -4.675 & -5.711 & 94.442 & 57.650 & 56.916 \\
\hline C & PM1-B09 & 2.995 & 0.984 & 1.384 & -0.843 & -3.472 & -3.261 & 0.000 & 0.000 & 0.000 \\
\hline C & PM1-B10 & 5.375 & 5.286 & 5.074 & -2.005 & 4.679 & 2.153 & 0.000 & 0.000 & 0.000 \\
\hline C & PM1-B11 & 89.390 & 65.630 & 50.475 & 134.130 & 102.093 & 97.986 & 97.645 & 79.311 & 64.687 \\
\hline
\end{tabular}




\begin{tabular}{|c|c|c|c|c|c|c|c|c|c|c|}
\hline C & PM1-B12 & 9.561 & 13.346 & 14.801 & 131.707 & 115.463 & 136.234 & 20.599 & 27.186 & 19.089 \\
\hline C & PM1-C01 & 253.249 & 185.212 & 181.246 & 241.478 & 211.797 & 231.790 & 11.459 & 10.528 & 9.221 \\
\hline C & PM1-C02 & 3.663 & -0.979 & -1.137 & -4.732 & 0.812 & -3.695 & 0.000 & 0.000 & 0.000 \\
\hline C & PM1-C03 & 8.724 & 5.075 & 17.366 & 4.263 & 4.267 & 0.446 & 89.150 & 63.755 & 58.103 \\
\hline C & PM1-C04 & 79.347 & 84.125 & 75.199 & 195.251 & 180.771 & 159.059 & 60.682 & 44.988 & 44.924 \\
\hline C & PM1-C05 & 11.851 & 5.917 & 9.478 & 5.526 & 5.616 & 2.543 & 4.066 & 3.818 & 2.542 \\
\hline C & PM1-C06 & 1.905 & -5.372 & -0.743 & -7.239 & -4.267 & -7.503 & 0.000 & 0.000 & 0.000 \\
\hline C & PM1-C07 & 52.005 & 46.637 & 40.762 & 95.757 & 64.314 & 60.955 & 49.705 & 45.432 & 37.979 \\
\hline C & PM1-C08 & 53.343 & 74.926 & 84.972 & 122.792 & 105.537 & 110.513 & 38.045 & 39.634 & 37.129 \\
\hline C & PM1-C09 & 56.530 & 47.100 & 37.664 & 106.904 & 65.732 & 72.895 & 79.187 & 64.426 & 46.916 \\
\hline C & PM1-C10 & 150.180 & 137.159 & 133.837 & 193.632 & 175.118 & 177.161 & 5.705 & 7.132 & 1.005 \\
\hline C & PM1-C11 & -0.555 & -0.557 & 1.904 & -2.095 & -6.679 & -7.245 & 0.000 & 0.000 & 0.000 \\
\hline C & PM1-C12 & 20.587 & 13.414 & 17.336 & 13.913 & 9.059 & 13.264 & 37.383 & 32.325 & 19.753 \\
\hline C & PM1-D01 & 7.078 & 0.947 & 1.313 & 7.934 & 4.874 & 0.096 & 44.333 & 27.970 & 20.343 \\
\hline C & PM1-D02 & -3.987 & -4.386 & -4.439 & -4.745 & -9.737 & -14.016 & 0.000 & 0.505 & 0.000 \\
\hline C & PM1-D03 & 2.045 & -3.978 & -0.814 & 83.716 & -6.945 & -6.757 & 0.000 & 0.000 & 0.000 \\
\hline C & PM1-D04 & 1.763 & -0.793 & -0.658 & -0.700 & -0.217 & -3.774 & 0.000 & 0.000 & 0.000 \\
\hline C & PM1-D05 & 3.911 & 2.286 & -1.276 & -1.184 & -1.512 & -2.211 & 0.000 & 0.000 & 0.000 \\
\hline C & PM1-D06 & -0.224 & -2.022 & -5.957 & -5.621 & -5.817 & -8.896 & 0.000 & 0.000 & 0.000 \\
\hline C & PM1-D07 & -1.567 & -0.293 & -6.532 & -8.026 & -3.874 & -1.258 & 0.000 & 0.000 & 0.000 \\
\hline C & PM1-D08 & 0.692 & 0.862 & -1.080 & 56.884 & -2.555 & -5.179 & 0.000 & 0.000 & 0.000 \\
\hline C & PM1-D09 & -1.400 & -0.647 & 5.061 & -2.600 & -6.170 & -7.938 & 3.221 & 3.082 & 0.000 \\
\hline C & PM1-D10 & 1.430 & 1.137 & -0.413 & -0.701 & -0.517 & -1.724 & 4.550 & 4.189 & 0.000 \\
\hline C & PM1-D11 & 128.849 & 130.186 & 111.221 & 94.272 & 60.288 & 57.475 & 56.661 & 49.461 & 52.184 \\
\hline C & PM1-D12 & 59.651 & 69.145 & 69.774 & 185.062 & 135.718 & 112.276 & 47.820 & 44.580 & 33.305 \\
\hline C & PM1-E01 & 6.163 & 0.766 & 2.441 & 7.822 & 6.613 & 8.792 & 2.854 & 3.737 & 1.824 \\
\hline C & PM1-E02 & -3.547 & 0.383 & 0.564 & -7.488 & -0.646 & -6.125 & 0.000 & 0.000 & 0.000 \\
\hline C & PM1-E03 & 226.509 & 185.351 & 184.972 & 250.266 & 239.974 & 244.562 & 113.237 & 89.368 & 80.787 \\
\hline C & PM1-E04 & 211.538 & 88.720 & 170.958 & 253.454 & 215.241 & 225.707 & 0.000 & 0.000 & 0.000 \\
\hline C & PM1-E05 & 1.741 & 5.470 & 3.514 & -0.063 & 3.795 & -4.521 & 0.000 & 0.000 & 0.000 \\
\hline C & PM1-E06 & 2.789 & -2.789 & 0.863 & -3.587 & -0.291 & -3.036 & 0.000 & 0.000 & 0.000 \\
\hline C & PM1-E07 & -2.405 & -3.322 & -3.113 & -6.170 & -5.805 & -5.762 & 0.000 & 0.000 & 0.000 \\
\hline C & PM1-E08 & 118.975 & 118.812 & 96.018 & 222.768 & 161.568 & 156.816 & 0.000 & 0.000 & 0.000 \\
\hline C & PM1-E09 & 1.725 & -6.416 & 0.650 & -0.262 & -2.886 & -5.497 & 0.000 & 0.000 & 0.000 \\
\hline C & PM1-E10 & 128.600 & 112.471 & 111.188 & 187.186 & 156.047 & 157.166 & 18.311 & 16.582 & 9.984 \\
\hline C & PM1-E11 & 167.987 & 169.829 & 173.762 & 208.043 & 207.017 & 199.067 & 88.595 & 56.468 & 59.000 \\
\hline C & PM1-E12 & 222.786 & 225.061 & 223.034 & 233.712 & 216.914 & 224.371 & 129.980 & 104.309 & 103.136 \\
\hline C & PM1-F01 & 25.300 & 8.307 & 12.270 & 170.401 & 143.313 & 141.668 & 14.878 & 16.639 & 10.679 \\
\hline C & PM1-F02 & -1.395 & -5.887 & -3.546 & -2.425 & -3.374 & -4.305 & 0.000 & 0.000 & 0.000 \\
\hline C & PM1-F03 & 192.125 & 193.014 & 190.626 & 130.459 & 114.850 & 142.458 & 10.689 & 9.405 & 5.703 \\
\hline C & PM1-F04 & -1.612 & -4.264 & -2.216 & -5.332 & -5.116 & -6.800 & 0.000 & 0.000 & 0.000 \\
\hline C & PM1-F05 & 4.237 & 3.566 & -0.257 & -0.922 & 0.418 & -1.621 & 118.750 & 86.074 & 69.061 \\
\hline C & PM1-F06 & 0.757 & -1.434 & -2.724 & -1.183 & -1.978 & -4.699 & 57.521 & 39.037 & 30.329 \\
\hline C & PM1-F07 & -2.343 & -2.636 & -6.655 & 3.929 & -1.887 & -6.203 & 0.000 & 0.000 & 0.000 \\
\hline C & PM1-F08 & -2.249 & 2.320 & -2.366 & -5.293 & 0.433 & -3.234 & 0.000 & 0.000 & 0.000 \\
\hline C & PM1-F09 & -2.616 & -4.170 & -2.872 & -12.432 & -6.354 & -8.075 & 0.000 & 0.000 & 0.000 \\
\hline C & PM1-F10 & -6.754 & -1.841 & -5.242 & -6.496 & -3.686 & -8.358 & 0.000 & 0.000 & 0.000 \\
\hline C & PM1-F11 & 132.738 & 128.247 & 116.138 & 143.032 & 129.767 & 123.051 & 103.766 & 85.433 & 65.676 \\
\hline C & PM1-F12 & 155.280 & 167.291 & 174.488 & 215.782 & 197.564 & 190.429 & 124.090 & 96.182 & 80.770 \\
\hline C & PM1-G01 & 80.182 & 68.333 & 73.670 & 110.776 & 99.334 & 102.862 & 14.550 & 8.568 & 6.732 \\
\hline C & PM1-G02 & 2.880 & -1.682 & -0.083 & 173.288 & -1.803 & -5.489 & 0.000 & 0.000 & 0.000 \\
\hline C & PM1-G03 & 226.791 & 212.900 & 210.161 & 240.321 & 239.567 & 244.382 & 128.732 & 113.868 & 109.997 \\
\hline C & PM1-G04 & 2.516 & 0.714 & 0.609 & 5.700 & 5.158 & -5.716 & 8.539 & 3.729 & 2.529 \\
\hline C & PM1-G05 & 53.320 & 91.346 & 47.561 & 133.976 & 122.392 & 109.162 & 19.274 & 6.776 & 4.308 \\
\hline C & PM1-G06 & 99.834 & 110.420 & 107.338 & 123.251 & 121.372 & 112.237 & 15.374 & 16.292 & 15.132 \\
\hline C & PM1-G07 & -2.426 & 5.191 & 2.013 & -3.382 & 2.450 & -1.917 & 6.147 & 0.000 & 0.000 \\
\hline C & PM1-G08 & 195.690 & 178.717 & 190.683 & 223.388 & 223.957 & 204.190 & 76.547 & 59.058 & 47.976 \\
\hline C & PM1-G09 & 7.770 & 8.895 & 3.563 & 2.318 & 7.922 & 2.874 & 46.918 & 42.382 & 34.382 \\
\hline C & PM1-G10 & 172.963 & 131.386 & 128.232 & 193.228 & 155.701 & 164.082 & 54.642 & 53.453 & 46.845 \\
\hline C & PM1-G11 & 2.230 & 4.339 & 4.364 & -6.271 & 2.871 & -1.318 & 3.539 & 4.063 & 0.886 \\
\hline C & PM1-G12 & 11.195 & 34.547 & 28.892 & 67.567 & 24.937 & 35.451 & 138.122 & 114.908 & 102.386 \\
\hline C & PM1-H01 & 163.753 & 158.768 & 139.293 & 189.801 & 187.383 & 167.980 & 99.171 & 77.916 & 55.614 \\
\hline C & PM1-H02 & 7.212 & 4.150 & 3.687 & 3.543 & 3.413 & 0.304 & 2.047 & 2.521 & -0.433 \\
\hline C & PM1-H03 & 5.667 & -0.068 & 2.559 & 0.297 & -0.363 & -13.236 & 6.368 & 5.867 & 0.725 \\
\hline C & PM1-H04 & 5.363 & 3.788 & 2.989 & 86.639 & 4.043 & 4.789 & 1.784 & 2.066 & 0.264 \\
\hline C & PM1-H05 & 6.525 & 4.117 & 4.553 & 0.849 & 7.128 & 3.076 & 4.033 & 3.643 & 0.550 \\
\hline C & PM1-H06 & -1.438 & -0.059 & -0.636 & -3.933 & -3.380 & -4.476 & -4.297 & -2.057 & -3.705 \\
\hline C & PM1-H07 & 8.370 & 5.979 & 6.658 & 1.179 & 6.946 & 2.345 & 12.262 & 8.592 & 3.671 \\
\hline C & PM1-H08 & 112.350 & 107.814 & 117.218 & 177.047 & 187.363 & 187.343 & 66.111 & 53.405 & 54.839 \\
\hline
\end{tabular}




\begin{tabular}{|c|c|c|c|c|c|c|c|c|c|c|}
\hline C & PM1-H09 & 3.014 & 5.463 & 4.312 & -0.712 & 5.962 & 0.864 & 4.201 & 4.479 & 0.000 \\
\hline C & PM1-H10 & 211.090 & 206.628 & 216.346 & 247.755 & 227.932 & 238.630 & 114.328 & 107.280 & 93.709 \\
\hline C & PM1-H11 & -0.393 & 3.639 & 6.571 & 0.267 & 2.796 & 0.628 & 6.208 & 9.012 & 4.737 \\
\hline C & PM1-H12 & 1.888 & 4.182 & 2.980 & -11.988 & 1.109 & -2.572 & 7.188 & 8.050 & 3.971 \\
\hline C & PM2-AA01 & 0.000 & 0.000 & 0.000 & 0.000 & 0.000 & 0.000 & 0.000 & 0.000 & 0.000 \\
\hline C & PM2-AA02 & -3.401 & -3.495 & -3.949 & -2.245 & -5.422 & -6.005 & 0.000 & 0.505 & 1.587 \\
\hline C & PM2-AA03 & -1.651 & -3.647 & -0.601 & -1.045 & -5.276 & -4.689 & 0.000 & 0.000 & 0.000 \\
\hline C & PM2-AA04 & -4.695 & -3.612 & -2.047 & -3.034 & -5.671 & -5.358 & 0.000 & 0.000 & 1.133 \\
\hline C & PM2-AA05 & -6.349 & -5.021 & -4.132 & -3.517 & -7.967 & -8.039 & 0.000 & 0.000 & 0.000 \\
\hline C & PM2-AA06 & 21.405 & 10.274 & 10.242 & 41.074 & 2.139 & 6.449 & 9.282 & 9.839 & 9.711 \\
\hline C & PM2-AA07 & -5.158 & -4.880 & -5.987 & -7.575 & -6.578 & -7.316 & 0.000 & 0.000 & 0.000 \\
\hline C & PM2-AA08 & -4.880 & -2.962 & -2.038 & -1.954 & -5.128 & -5.529 & 0.000 & 0.000 & 1.016 \\
\hline C & PM2-AA09 & -6.929 & -3.568 & -3.791 & -6.580 & -12.062 & -7.664 & 0.000 & 0.000 & 0.000 \\
\hline C & PM2-AA10 & -7.805 & -10.150 & -3.984 & -5.250 & -14.182 & -7.075 & 0.505 & 0.000 & 3.450 \\
\hline C & PM2-AA11 & -4.687 & -3.580 & -1.605 & -2.991 & -5.338 & -4.789 & 1.133 & 0.388 & 1.589 \\
\hline C & PM2-AA12 & 132.471 & 87.495 & 86.693 & 155.650 & 159.637 & 120.025 & 49.370 & 40.067 & 45.863 \\
\hline C & PM2-AB01 & 107.791 & 93.739 & 92.567 & 167.209 & 175.200 & 182.253 & 0.000 & 0.000 & 0.000 \\
\hline C & PM2-AB02 & 211.784 & 174.055 & 170.901 & 219.074 & 204.268 & 204.975 & 74.774 & 61.150 & 47.189 \\
\hline C & РM2-АВ03 & -9.742 & -8.866 & -9.437 & -8.014 & -9.705 & -13.420 & 0.000 & 0.000 & 0.000 \\
\hline C & PM2-AB04 & -5.612 & -4.237 & -4.404 & -6.283 & -8.046 & -8.341 & 0.000 & 0.000 & 0.000 \\
\hline C & PM2-AB05 & -9.288 & -9.450 & -6.341 & -4.711 & -10.172 & -10.325 & 0.000 & 0.000 & 0.000 \\
\hline C & PM2-AB06 & -8.836 & -7.530 & -6.534 & -8.614 & -7.328 & -9.464 & 0.000 & 0.000 & 0.000 \\
\hline C & PM2-AB07 & -10.742 & -8.457 & -8.420 & -6.886 & -12.647 & -12.270 & 0.000 & 0.000 & 0.000 \\
\hline C & PM2-AB08 & 8.389 & 6.632 & 8.354 & 43.276 & 50.824 & 38.955 & 0.000 & 0.000 & 0.000 \\
\hline C & РM2-AB09 & -12.309 & -13.247 & -8.107 & -6.346 & -14.607 & -9.239 & 0.000 & 0.000 & 0.000 \\
\hline C & PM2-AB10 & -14.818 & -8.693 & -10.100 & -12.292 & -11.918 & -13.155 & 0.000 & 0.000 & 0.000 \\
\hline C & PM2-AB11 & -8.742 & -8.241 & -7.670 & -9.512 & -9.975 & -12.546 & 0.000 & 0.000 & 0.000 \\
\hline C & PM2-AB12 & 3.978 & 7.336 & 6.305 & 4.667 & 3.280 & -3.074 & 6.245 & 3.329 & 7.786 \\
\hline C & PM2-AC01 & 157.390 & 126.680 & 112.536 & 187.963 & 168.518 & 186.634 & 0.000 & 0.000 & 0.000 \\
\hline C & PM2-AC02 & -8.675 & -7.271 & -7.211 & -5.434 & -9.132 & -8.729 & 0.000 & 0.000 & 0.000 \\
\hline C & PM2-AC03 & -8.997 & -9.333 & -8.108 & -9.376 & -10.551 & -11.291 & 0.000 & 0.000 & 0.000 \\
\hline C & PM2-AC04 & -8.254 & -9.680 & -7.057 & -9.633 & -9.984 & -10.953 & 0.000 & 0.000 & 0.000 \\
\hline C & PM2-AC05 & -9.649 & -6.974 & -4.905 & -7.907 & -11.547 & -10.830 & 0.000 & 0.000 & 0.000 \\
\hline C & PM2-AC06 & -11.146 & -10.317 & -8.943 & -8.657 & -11.853 & -12.629 & 0.000 & 0.000 & 0.000 \\
\hline C & PM2-AC07 & -7.911 & -5.579 & -2.529 & -2.228 & -2.068 & -4.336 & 9.282 & 0.000 & 0.000 \\
\hline C & PM2-AC08 & -9.586 & -6.495 & -6.464 & -7.329 & -9.759 & -13.446 & 0.000 & 0.000 & 0.000 \\
\hline C & PM2-AC09 & -13.243 & -9.454 & -7.524 & -8.386 & -12.786 & -13.533 & 0.000 & 0.000 & 0.000 \\
\hline C & PM2-AC10 & -13.070 & -8.795 & -8.122 & -12.212 & -12.143 & -11.661 & 0.000 & 0.000 & 0.000 \\
\hline C & PM2-AC11 & -9.925 & -5.689 & -6.047 & -8.068 & -5.825 & -10.774 & 0.000 & 0.000 & 0.000 \\
\hline C & PM2-AC12 & -4.484 & -3.225 & -2.404 & -3.620 & -6.696 & -7.422 & 6.432 & 3.771 & 11.821 \\
\hline C & PM2-AD01 & -3.488 & -5.403 & -3.253 & -3.064 & -7.739 & -8.161 & 1.011 & 2.953 & 1.058 \\
\hline C & PM2-AD02 & -6.778 & -7.499 & -7.034 & -5.813 & -8.757 & -9.245 & 0.000 & 0.000 & 0.000 \\
\hline C & PM2-AD03 & -7.037 & -6.776 & -6.680 & -5.717 & -5.142 & -9.399 & 0.000 & 0.000 & 0.000 \\
\hline C & PM2-AD04 & 124.192 & 115.782 & 102.041 & 122.018 & 109.134 & 118.765 & 33.453 & 18.574 & 24.732 \\
\hline C & PM2-AD05 & -12.451 & -10.746 & -8.336 & -9.254 & -11.811 & -11.913 & 0.000 & 0.000 & 0.000 \\
\hline C & PM2-AD06 & -7.716 & -6.099 & -5.393 & -3.937 & -6.995 & -8.768 & 0.000 & 0.000 & 0.000 \\
\hline C & PM2-AD07 & -7.475 & -10.650 & -5.384 & 5.325 & -11.333 & -10.108 & 0.000 & 0.000 & 0.000 \\
\hline C & PM2-AD08 & -10.453 & -9.224 & -8.055 & -6.726 & -8.179 & -10.174 & 0.000 & 0.000 & 0.000 \\
\hline C & PM2-AD09 & -11.441 & -7.143 & -7.139 & -5.763 & -10.342 & -11.729 & 0.000 & 0.000 & 0.000 \\
\hline C & PM2-AD10 & -10.453 & -6.396 & -8.175 & -7.353 & -9.061 & -9.688 & 0.000 & 0.000 & 0.000 \\
\hline C & PM2-AD11 & -15.142 & -8.063 & -8.255 & -6.704 & -9.095 & -13.974 & 0.000 & 0.000 & 0.000 \\
\hline C & PM2-AD12 & -8.709 & -2.389 & -1.392 & -2.476 & -6.961 & -8.876 & 1.008 & 1.912 & 5.149 \\
\hline C & PM2-AE01 & -18.336 & -12.741 & -12.047 & -13.666 & -19.766 & -23.768 & 1.654 & -0.066 & 2.626 \\
\hline C & PM2-AE02 & -1.395 & -4.921 & -1.661 & -3.028 & -10.761 & -14.150 & 0.000 & 0.000 & 0.000 \\
\hline C & PM2-AE03 & -7.782 & -4.861 & -7.863 & -4.353 & -7.459 & -7.533 & 0.000 & 0.000 & 0.000 \\
\hline C & PM2-AE04 & -12.400 & -9.116 & -8.593 & -4.541 & -8.371 & -11.375 & 0.000 & 0.000 & 0.000 \\
\hline C & PM2-AE05 & 72.595 & 57.013 & 48.062 & 190.467 & 186.547 & 196.622 & 62.211 & 68.892 & 61.211 \\
\hline C & PM2-AE06 & -13.645 & -10.937 & -12.384 & -11.234 & -14.042 & -14.866 & 0.000 & 0.000 & 0.000 \\
\hline C & PM2-AE07 & -9.614 & -7.001 & -7.407 & -3.382 & -6.551 & -9.357 & 0.000 & 0.000 & 0.000 \\
\hline C & PM2-AE08 & -10.997 & -5.861 & -7.789 & -4.646 & -3.839 & -9.825 & 0.000 & 0.000 & 0.000 \\
\hline C & PM2-AE09 & -7.501 & -5.084 & -4.461 & -4.384 & -4.126 & -8.211 & 0.000 & 0.000 & 0.000 \\
\hline C & PM2-AE10 & -10.401 & -6.067 & -6.611 & -6.580 & -9.179 & -9.396 & 0.000 & 0.000 & 0.000 \\
\hline C & PM2-AE11 & -13.258 & -13.859 & -7.850 & -10.741 & -16.114 & -16.692 & 0.000 & 0.000 & 0.000 \\
\hline C & PM2-AE12 & 169.597 & 154.522 & 169.114 & 2.922 & -2.008 & -2.664 & 14.591 & 11.309 & 17.016 \\
\hline C & PM2-AF01 & -1.107 & -1.055 & 1.333 & 1.162 & -1.096 & -3.501 & 0.000 & 0.000 & 0.000 \\
\hline C & PM2-AF02 & -5.537 & -4.695 & -3.988 & -2.483 & -4.321 & -6.712 & 0.000 & 0.000 & 0.000 \\
\hline C & PM2-AF03 & -9.358 & -3.734 & -5.780 & -2.403 & -6.842 & -7.021 & 0.000 & 0.000 & 0.000 \\
\hline C & PM2-AF04 & -8.905 & -4.761 & -8.253 & -3.488 & -5.979 & -7.807 & 0.000 & 0.000 & 0.000 \\
\hline C & PM2-AF05 & -11.291 & -7.301 & -6.587 & -4.614 & -9.864 & -7.511 & 0.000 & 0.000 & 0.000 \\
\hline
\end{tabular}




\begin{tabular}{|c|c|c|c|c|c|c|c|c|c|c|}
\hline C & PM2-AF06 & -12.445 & -7.555 & -8.961 & -2.216 & -7.014 & -9.766 & 0.000 & 0.000 & 0.000 \\
\hline C & PM2-AF07 & -8.600 & -7.375 & -8.872 & -4.588 & -6.168 & -8.258 & 0.000 & 0.000 & 0.000 \\
\hline C & PM2-AF08 & -9.321 & -6.671 & -7.776 & -3.051 & -8.541 & -14.036 & 0.000 & 0.000 & 0.000 \\
\hline C & PM2-AF09 & -4.701 & -4.532 & -8.279 & -0.045 & -11.426 & -13.988 & 0.000 & 0.000 & 0.000 \\
\hline C & PM2-AF10 & -4.986 & -2.183 & -2.807 & 0.454 & -7.196 & -5.830 & 0.000 & 0.000 & 0.000 \\
\hline C & PM2-AF11 & -9.464 & -4.497 & -4.059 & -11.337 & -10.657 & -11.784 & 0.000 & 0.000 & 0.000 \\
\hline C & PM2-AF12 & -8.038 & 0.334 & -3.363 & 1.117 & -1.909 & -4.018 & 0.000 & 0.000 & 0.000 \\
\hline C & PM2-AG01 & -7.250 & -3.616 & -1.017 & -2.841 & -4.300 & -3.988 & 0.000 & 0.000 & 0.000 \\
\hline C & PM2-AG02 & 0.154 & 5.016 & 3.546 & 8.959 & 6.718 & 3.436 & 0.000 & 0.000 & 0.000 \\
\hline C & PM2-AG03 & -7.746 & -5.096 & -6.375 & -4.038 & -5.068 & -7.704 & 0.000 & 0.000 & 0.000 \\
\hline C & PM2-AG04 & -9.297 & -6.229 & -6.816 & -3.295 & -5.995 & -8.670 & 0.000 & 0.000 & 0.000 \\
\hline C & PM2-AG05 & -9.225 & -6.407 & -8.457 & -8.039 & -8.630 & -11.424 & 0.000 & 0.000 & 0.000 \\
\hline C & PM2-AG06 & -5.651 & -6.463 & -7.053 & 22.449 & 6.637 & 26.289 & 0.000 & 0.000 & 0.000 \\
\hline C & PM2-AG07 & -13.030 & -8.218 & -7.916 & -5.839 & -11.261 & -11.184 & 0.000 & 0.000 & 0.000 \\
\hline C & PM2-AG08 & -7.241 & -5.795 & -4.946 & -3.964 & -9.937 & -8.643 & 0.000 & 0.000 & 0.000 \\
\hline C & PM2-AG09 & -9.829 & -7.805 & -7.474 & -8.197 & -12.108 & -11.658 & 0.000 & 0.000 & 0.000 \\
\hline C & PM2-AG10 & -11.628 & -7.264 & -8.749 & -6.543 & -9.071 & -13.870 & 0.000 & 0.000 & 0.000 \\
\hline C & PM2-AG11 & -11.170 & -3.580 & -5.736 & -3.251 & -6.786 & -12.018 & 0.000 & 0.000 & 0.000 \\
\hline C & PM2-AG12 & -2.655 & 0.261 & 0.041 & 4.116 & -7.255 & -1.754 & 0.503 & 0.000 & 0.000 \\
\hline C & PM2-AH01 & -1.488 & -4.400 & -1.599 & 0.276 & -2.261 & -5.932 & 0.000 & 0.000 & 0.000 \\
\hline C & PM2-AH02 & -5.697 & -2.988 & 0.195 & -2.428 & -4.038 & -3.717 & 0.000 & 0.000 & 0.000 \\
\hline C & PM2-AH03 & -0.549 & -0.529 & 0.472 & 1.330 & -0.821 & -5.771 & 0.000 & 0.000 & 0.000 \\
\hline C & PM2-AH04 & -3.408 & -5.280 & -3.200 & -4.197 & -6.333 & -5.346 & 0.000 & 0.000 & 0.000 \\
\hline C & PM2-AH05 & -6.542 & -2.582 & -3.850 & -3.638 & -6.675 & -6.436 & 0.000 & 0.000 & 0.000 \\
\hline C & PM2-AH06 & -14.933 & -10.257 & -14.750 & -10.664 & -12.059 & -15.617 & 0.000 & 0.000 & 0.000 \\
\hline C & PM2-AH07 & -5.742 & -1.449 & -0.253 & -0.251 & -3.014 & -4.989 & 0.000 & 0.000 & 0.000 \\
\hline C & PM2-AH08 & -6.383 & -5.489 & -1.837 & 0.776 & -0.592 & -2.321 & 0.000 & 0.000 & 0.000 \\
\hline C & PM2-AH09 & -17.825 & -13.089 & -10.892 & -13.637 & -17.711 & -14.899 & -2.234 & -7.828 & -6.912 \\
\hline C & PM2-AH10 & -11.055 & -3.274 & -2.751 & -5.909 & -8.213 & -7.055 & 0.000 & 0.000 & 0.000 \\
\hline C & PM2-AH11 & -6.712 & -6.599 & -0.951 & -0.199 & -2.264 & -3.770 & 0.000 & 0.000 & 0.000 \\
\hline C & PM2-AH12 & -5.405 & 0.393 & -1.618 & -0.070 & -1.004 & -2.303 & 1.008 & 0.000 & 1.032 \\
\hline $\mathbf{N}$ & РM3-BA01 & 0.000 & 0.000 & 0.000 & 0.000 & 0.000 & 0.000 & 0.000 & 0.000 & 0.000 \\
\hline $\mathbf{N}$ & PM3-BA02 & 3.214 & 2.604 & -1.039 & 76.260 & 87.670 & 81.564 & 2.520 & 0.505 & \\
\hline $\mathbf{N}$ & РM3-BA03 & -5.457 & -8.203 & -7.330 & 7.789 & 1.591 & 1.884 & 0.000 & 0.000 & 0.000 \\
\hline $\mathbf{N}$ & РM3-BA04 & 2.530 & 3.439 & -0.318 & 1.942 & 1.717 & 5.099 & 0.000 & 1.008 & 0.000 \\
\hline $\mathbf{N}$ & PM3-BA05 & -0.071 & -0.225 & -0.525 & 88.812 & 83.833 & 73.916 & 0.000 & 0.000 & 0.000 \\
\hline $\mathbf{N}$ & PM3-BA06 & -1.897 & -4.399 & -7.591 & 33.075 & 27.350 & 30.286 & 0.000 & 0.000 & 0.000 \\
\hline $\mathbf{N}$ & РM3-BA07 & 15.411 & 37.454 & 21.617 & 74.943 & 77.445 & 69.446 & 0.000 & 0.000 & 0.000 \\
\hline $\mathbf{N}$ & PM3-BA08 & 10.700 & 33.520 & 9.050 & 65.604 & 75.524 & 72.216 & 0.000 & 0.000 & 0.000 \\
\hline $\mathbf{N}$ & PM3-BA09 & 148.580 & 125.410 & 116.953 & 209.146 & 194.426 & 219.753 & 16.308 & 13.895 & 11.200 \\
\hline $\mathbf{N}$ & PM3-BA10 & 191.047 & 142.234 & 136.449 & 227.370 & 207.401 & 220.829 & 31.018 & 20.958 & 20.134 \\
\hline $\mathbf{N}$ & PM3-BA11 & 83.897 & 271.534 & 265.458 & 15.557 & 123.183 & 130.895 & 1.076 & 0.758 & 0.000 \\
\hline $\mathbf{N}$ & PM3-BA12 & 11.368 & 42.712 & 19.737 & 196.725 & 209.700 & 203.838 & 19.203 & 15.112 & 22.011 \\
\hline $\mathbf{N}$ & PM3-BB01 & 10.925 & 35.750 & 79.674 & 187.287 & 180.404 & 193.786 & 11.239 & 12.171 & 14.524 \\
\hline $\mathbf{N}$ & PM3-BB02 & -1.095 & -0.424 & -1.295 & 148.264 & 136.638 & 135.682 & 0.503 & 0.000 & 0.000 \\
\hline $\mathbf{N}$ & РM3-BB03 & 94.876 & 106.493 & 87.307 & 203.116 & 197.520 & 206.133 & 0.000 & 0.000 & 0.000 \\
\hline $\mathbf{N}$ & PM3-BB04 & -1.618 & 1.558 & -3.307 & 34.605 & 25.351 & 30.018 & 0.000 & 0.000 & 0.000 \\
\hline $\mathbf{N}$ & PM3-BB05 & -4.847 & -1.789 & -4.838 & 58.867 & 53.204 & 58.507 & 0.000 & 0.000 & 0.000 \\
\hline $\mathbf{N}$ & PM3-BB06 & -3.016 & -4.441 & -7.951 & 22.833 & 8.100 & 23.128 & 0.000 & 0.000 & 0.000 \\
\hline $\mathbf{N}$ & PM3-BB07 & 2.384 & -3.970 & 7.764 & 25.342 & 27.482 & 46.647 & 0.000 & 0.000 & 0.000 \\
\hline $\mathbf{N}$ & PM3-BB08 & -4.236 & -2.304 & -5.475 & 14.843 & 20.714 & 29.301 & 0.000 & 0.000 & 1.005 \\
\hline $\mathbf{N}$ & PM3-BB09 & 20.412 & 61.350 & 20.705 & 89.749 & 82.811 & 89.843 & 0.000 & 0.000 & 0.000 \\
\hline $\mathbf{N}$ & PM3-BB10 & 77.283 & 98.171 & 117.205 & 163.483 & 152.263 & 161.625 & 0.000 & 0.000 & 0.000 \\
\hline $\mathbf{N}$ & PM3-BB11 & 0.625 & 12.568 & 2.988 & 38.166 & 57.950 & 61.709 & 0.000 & 0.000 & 0.000 \\
\hline $\mathbf{N}$ & PM3-BB12 & 0.068 & 2.339 & 2.800 & -3.641 & -3.426 & -1.883 & 6.814 & 3.305 & 1.599 \\
\hline $\mathbf{N}$ & РM3-BC01 & -15.729 & -14.439 & -28.813 & -15.361 & -23.208 & -25.857 & -12.232 & -13.026 & -6.820 \\
\hline $\mathbf{N}$ & РM3-BC02 & -4.268 & -2.864 & -4.042 & -3.126 & 2.766 & 1.886 & 0.000 & 0.000 & 0.000 \\
\hline $\mathbf{N}$ & РM3-BC03 & -3.080 & -1.279 & -3.187 & 35.074 & 46.653 & 37.657 & 0.000 & 0.000 & 0.000 \\
\hline $\mathbf{N}$ & РM3-BC04 & -2.708 & -1.157 & -3.295 & -1.374 & -1.817 & 1.480 & 0.000 & 0.000 & 0.000 \\
\hline $\mathbf{N}$ & РM3-BC05 & -6.593 & -7.292 & -7.832 & -11.658 & -8.863 & -9.382 & 0.000 & 0.000 & 0.000 \\
\hline $\mathbf{N}$ & РM3-BC06 & -10.034 & -11.051 & -13.059 & -14.216 & -9.666 & -10.625 & 0.000 & 0.000 & 0.000 \\
\hline $\mathbf{N}$ & РM3-BC07 & -7.943 & -6.467 & -8.421 & -9.397 & -7.671 & -5.824 & 0.000 & 0.000 & 0.000 \\
\hline $\mathbf{N}$ & РM3-BC08 & -2.543 & -1.795 & -4.361 & -12.089 & -9.733 & -7.034 & 0.000 & 0.000 & 0.000 \\
\hline $\mathbf{N}$ & РM3-BC09 & -8.339 & -3.707 & -6.432 & -8.233 & -5.587 & -3.364 & 0.000 & 0.000 & 0.000 \\
\hline $\mathbf{N}$ & PM3-BC10 & -3.584 & -2.914 & -3.487 & 3.564 & 1.945 & 16.597 & 0.758 & 0.000 & 0.000 \\
\hline $\mathbf{N}$ & PM3-BC11 & -3.011 & 1.034 & -1.730 & -5.668 & -1.480 & 0.746 & 0.000 & 0.000 & 0.000 \\
\hline $\mathbf{N}$ & PM3-BC12 & 1.958 & 8.863 & 3.361 & 8.393 & 13.297 & 18.930 & 7.529 & 2.267 & 1.562 \\
\hline $\mathbf{N}$ & PM3-BD01 & -4.080 & -2.211 & -4.338 & -2.545 & -1.904 & -1.497 & 0.434 & 1.237 & 2.347 \\
\hline $\mathbf{N}$ & PM3-BD02 & -6.672 & -7.247 & -6.875 & -7.234 & -6.357 & -6.872 & 0.886 & 0.000 & 0.000 \\
\hline
\end{tabular}




\begin{tabular}{|c|c|c|c|c|c|c|c|c|c|c|}
\hline $\mathbf{N}$ & PM3-BD03 & -3.613 & -1.547 & -4.371 & -9.957 & -8.313 & -8.633 & 0.000 & 0.000 & 0.000 \\
\hline $\mathbf{N}$ & PM3-BD04 & -4.568 & -5.293 & -7.949 & -7.571 & -6.009 & -4.474 & 0.000 & 0.000 & 0.000 \\
\hline $\mathbf{N}$ & PM3-BD05 & -11.296 & -11.462 & -11.975 & -14.658 & -12.736 & -12.739 & 0.000 & 0.000 & 0.000 \\
\hline $\mathbf{N}$ & PM3-BD06 & -9.511 & -9.033 & -10.709 & -12.454 & -9.605 & -9.154 & 1.513 & 0.000 & 0.000 \\
\hline $\mathbf{N}$ & PM3-BD07 & -8.458 & -7.054 & -9.493 & -10.143 & -7.612 & -6.447 & 0.000 & 0.000 & 0.000 \\
\hline $\mathbf{N}$ & PM3-BD08 & -9.853 & -7.849 & -6.695 & -10.863 & -8.987 & -6.964 & 0.000 & 0.000 & 0.000 \\
\hline $\mathbf{N}$ & PM3-BD09 & -7.850 & -3.089 & -6.793 & -11.132 & -7.736 & -5.645 & 0.000 & 0.000 & 0.000 \\
\hline $\mathbf{N}$ & PM3-BD10 & -9.307 & -3.087 & -7.976 & -11.038 & -7.424 & -4.759 & 0.758 & 0.503 & 0.000 \\
\hline $\mathbf{N}$ & PM3-BD11 & -8.101 & -3.403 & -6.370 & -13.437 & -7.655 & -6.349 & 0.000 & 0.000 & 0.000 \\
\hline $\mathbf{N}$ & PM3-BD12 & 2.995 & 2.779 & -0.982 & -4.717 & -4.334 & -1.764 & 8.805 & 2.158 & 2.393 \\
\hline $\mathbf{N}$ & PM3-BE01 & -3.468 & -2.420 & -4.675 & -4.009 & -1.891 & -1.562 & 2.829 & 1.024 & 0.633 \\
\hline $\mathbf{N}$ & PM3-BE02 & -6.299 & -5.457 & -7.583 & -8.721 & -5.293 & -5.434 & 0.000 & 0.000 & 0.000 \\
\hline $\mathbf{N}$ & PM3-BE03 & -5.150 & -2.789 & -5.787 & -6.986 & -3.086 & -4.836 & 0.000 & 0.000 & 0.000 \\
\hline $\mathbf{N}$ & PM3-BE04 & -4.854 & -2.105 & -5.942 & -6.776 & -3.999 & -3.546 & 0.000 & 0.000 & 0.000 \\
\hline $\mathbf{N}$ & PM3-BE05 & -4.220 & -1.414 & -6.761 & -6.764 & -5.418 & -3.521 & 0.000 & 0.000 & 0.000 \\
\hline $\mathbf{N}$ & PM3-BE06 & -1.534 & -0.288 & -2.661 & -1.709 & 34.759 & 43.171 & 0.000 & 0.000 & 0.000 \\
\hline $\mathbf{N}$ & PM3-BE07 & -5.426 & -2.189 & -5.801 & 0.662 & -4.083 & 7.063 & 0.000 & 0.000 & 0.000 \\
\hline $\mathbf{N}$ & PM3-BE08 & 22.945 & 15.083 & 10.788 & 155.688 & 99.862 & 100.149 & 0.000 & 0.000 & 0.000 \\
\hline $\mathbf{N}$ & PM3-BE09 & -7.007 & -3.413 & -6.042 & -10.258 & -8.001 & -5.791 & 0.000 & 0.000 & 0.000 \\
\hline $\mathbf{N}$ & PM3-BE10 & -9.367 & -4.667 & -8.862 & -12.254 & -11.495 & -8.972 & 1.438 & 1.391 & 0.000 \\
\hline $\mathbf{N}$ & PM3-BE11 & 13.258 & 22.836 & 9.243 & 9.976 & 31.792 & 13.436 & 2.108 & 0.000 & 0.000 \\
\hline $\mathbf{N}$ & PM3-BE12 & 12.239 & 15.037 & 33.403 & 33.438 & 40.214 & 31.538 & 4.200 & 0.616 & 1.279 \\
\hline $\mathbf{N}$ & PM3-BF01 & 44.629 & 54.203 & 71.850 & 159.137 & 158.530 & 154.349 & 18.609 & 20.430 & 17.250 \\
\hline $\mathbf{N}$ & PM3-BF02 & -7.253 & -14.005 & -9.921 & -10.283 & -12.161 & -13.059 & 1.162 & 1.939 & 0.503 \\
\hline $\mathbf{N}$ & PM3-BF03 & 97.724 & 81.629 & 83.532 & 203.700 & 207.282 & 212.499 & 5.797 & 0.000 & 0.000 \\
\hline $\mathbf{N}$ & PM3-BF04 & 24.972 & 14.761 & 37.278 & 210.474 & 215.101 & 218.675 & 0.000 & 0.000 & 0.000 \\
\hline $\mathbf{N}$ & PM3-BF05 & -4.788 & -1.004 & -4.905 & -6.391 & -4.237 & -2.533 & 0.000 & 0.000 & 0.000 \\
\hline $\mathbf{N}$ & PM3-BF06 & -10.803 & 8.553 & 6.995 & 1.028 & 0.116 & 1.882 & 11.197 & 31.908 & 0.378 \\
\hline $\mathbf{N}$ & PM3-BF07 & -16.862 & -8.600 & -10.432 & -22.251 & -6.025 & -20.605 & -0.171 & 0.899 & 0.000 \\
\hline $\mathbf{N}$ & PM3-BF08 & -6.151 & 0.224 & -6.876 & -6.730 & -7.832 & -1.639 & 1.013 & 0.000 & 0.000 \\
\hline $\mathbf{N}$ & PM3-BF09 & -8.155 & -3.370 & -6.763 & -6.429 & -5.075 & -3.324 & 0.000 & 0.000 & 0.000 \\
\hline $\mathbf{N}$ & PM3-BF10 & -7.387 & -2.097 & -8.179 & -9.653 & -7.870 & -5.207 & 3.595 & 1.047 & 0.000 \\
\hline $\mathbf{N}$ & PM3-BF11 & -7.457 & -0.550 & -5.945 & -10.679 & -6.996 & -4.549 & 1.839 & 0.766 & 0.000 \\
\hline $\mathbf{N}$ & PM3-BF12 & -9.507 & -1.280 & -3.824 & -11.228 & -4.921 & -5.945 & 4.764 & 1.176 & 1.707 \\
\hline $\mathbf{N}$ & PM3-BG01 & 1.192 & 7.437 & -2.416 & 21.658 & 39.843 & 31.586 & -1.587 & -4.286 & -4.729 \\
\hline $\mathbf{N}$ & PM3-BG02 & -2.442 & -0.576 & -3.722 & 32.434 & 35.316 & 34.878 & 0.000 & 0.000 & 0.000 \\
\hline $\mathbf{N}$ & PM3-BG03 & -8.174 & -6.747 & -1.717 & 15.557 & 7.479 & 15.376 & 1.178 & 0.787 & 1.409 \\
\hline $\mathbf{N}$ & PM3-BG04 & -11.886 & -1.545 & -4.433 & -16.462 & 14.554 & 17.436 & 0.195 & 0.255 & 0.000 \\
\hline $\mathbf{N}$ & PM3-BG05 & -2.821 & 0.911 & -4.191 & -0.170 & 4.296 & 14.932 & 0.000 & 0.000 & 0.000 \\
\hline $\mathbf{N}$ & PM3-BG06 & -2.718 & 6.017 & -2.470 & 10.184 & 13.863 & 25.847 & 0.000 & 0.000 & 0.000 \\
\hline $\mathbf{N}$ & PM3-BG07 & -5.309 & -1.114 & -6.567 & -4.579 & 1.678 & 0.596 & 0.000 & 0.000 & 0.000 \\
\hline $\mathbf{N}$ & PM3-BG08 & -3.342 & 5.966 & -2.559 & 39.961 & 40.471 & 46.245 & 0.000 & 0.000 & 0.000 \\
\hline $\mathbf{N}$ & PM3-BG09 & -4.504 & 7.833 & -1.789 & 37.272 & 34.617 & 41.559 & 0.380 & 0.000 & 0.000 \\
\hline $\mathbf{N}$ & PM3-BG10 & -8.921 & -8.099 & -13.216 & -11.597 & 32.679 & 27.226 & 0.592 & 0.680 & 1.021 \\
\hline $\mathbf{N}$ & PM3-BG11 & -5.450 & 2.341 & -2.439 & -4.182 & -3.504 & 0.193 & 5.537 & 3.105 & 1.289 \\
\hline $\mathbf{N}$ & PM3-BG12 & -3.346 & 4.241 & -2.529 & 3.375 & 5.257 & 2.526 & 2.638 & 0.859 & -1.709 \\
\hline $\mathbf{N}$ & PM3-BH01 & 153.988 & 127.255 & 140.574 & 220.393 & 212.479 & 241.221 & 5.342 & 4.114 & 4.378 \\
\hline $\mathbf{N}$ & PM3-BH02 & 157.000 & 169.583 & 155.809 & 230.737 & 222.796 & 219.862 & 0.072 & -0.976 & -1.099 \\
\hline $\mathbf{N}$ & PM3-BH03 & 82.588 & 95.342 & 58.428 & 222.287 & 212.357 & 211.597 & 5.917 & 2.599 & 0.318 \\
\hline $\mathbf{N}$ & PM3-BH04 & 13.321 & 7.857 & 5.157 & 154.760 & 151.651 & 151.280 & 1.247 & 1.001 & 1.142 \\
\hline $\mathbf{N}$ & PM3-BH05 & 1.066 & 4.636 & 2.371 & 198.837 & 195.163 & 207.513 & 2.721 & 0.295 & 0.480 \\
\hline $\mathbf{N}$ & PM3-BH06 & -1.453 & 6.314 & 0.199 & 51.559 & 52.491 & 35.212 & -0.074 & 0.576 & 0.800 \\
\hline $\mathbf{N}$ & PM3-BH07 & 1.809 & 11.108 & 3.595 & 78.410 & 100.365 & 98.976 & 4.061 & 1.774 & 0.000 \\
\hline $\mathbf{N}$ & PM3-BH08 & 66.543 & 116.976 & 112.787 & 223.613 & 203.236 & 226.164 & 27.674 & 29.111 & 27.413 \\
\hline $\mathbf{N}$ & PM3-BH09 & 154.589 & 171.649 & 168.205 & 215.274 & 199.122 & 227.526 & 7.078 & 10.775 & 9.161 \\
\hline $\mathbf{N}$ & PM3-BH10 & 3.176 & 67.501 & 84.997 & 192.207 & 178.104 & 191.779 & 13.649 & 15.672 & 14.242 \\
\hline $\mathbf{N}$ & PM3-BH11 & 148.101 & 193.208 & 154.484 & 117.395 & 126.054 & 134.553 & 4.678 & 3.480 & 1.266 \\
\hline $\mathbf{N}$ & PM3-BH12 & 160.236 & 169.295 & 134.714 & 65.328 & 58.795 & 64.205 & 9.454 & 1.563 & 1.325 \\
\hline $\mathbf{P}$ & PM4-AA01 & 0.000 & 0.000 & 0.000 & 0.000 & 0.000 & 0.000 & 0.000 & 0.000 & 0.000 \\
\hline $\mathbf{P}$ & PM4-AA02 & 1.833 & -1.451 & -1.796 & 28.876 & 49.566 & 28.472 & 0.000 & 0.503 & \\
\hline $\mathbf{P}$ & PM4-AA03 & -8.100 & -3.983 & -3.333 & 48.263 & 43.112 & 21.932 & 5.416 & -3.028 & -2.383 \\
\hline $\mathbf{P}$ & PM4-AA04 & -4.141 & -6.245 & -5.141 & 72.668 & 51.743 & 27.496 & 0.000 & 0.000 & -2.092 \\
\hline $\mathbf{P}$ & PM4-AA05 & -2.966 & -2.068 & -6.054 & 50.533 & 47.061 & 28.000 & 0.000 & 0.000 & -3.100 \\
\hline $\mathbf{P}$ & PM4-AA06 & -7.416 & -5.483 & -5.399 & 35.366 & 16.886 & 3.724 & 0.000 & 0.000 & -3.100 \\
\hline $\mathbf{P}$ & PM4-AA07 & -5.192 & -5.779 & -5.528 & 53.737 & 31.143 & 13.263 & 0.000 & 0.000 & -3.100 \\
\hline $\mathbf{P}$ & PM4-AA08 & 79.878 & 11.118 & 76.608 & 142.714 & 128.746 & 94.054 & 0.000 & 0.000 & -3.100 \\
\hline $\mathbf{P}$ & PM4-AA09 & 2.961 & 64.064 & -10.420 & 150.254 & 138.342 & 137.957 & 0.000 & 1.529 & -3.100 \\
\hline $\mathbf{P}$ & PM4-AA10 & 128.295 & -13.441 & 9.062 & 171.991 & 138.251 & -44.620 & 0.000 & 0.000 & -0.508 \\
\hline $\mathbf{P}$ & PM4-AA11 & 78.501 & -10.454 & -11.696 & 173.700 & 134.224 & 127.139 & 5.316 & 8.507 & 4.268 \\
\hline
\end{tabular}




\begin{tabular}{|c|c|c|c|c|c|c|c|c|c|c|}
\hline $\mathbf{P}$ & PM4-AA12 & 116.257 & 72.225 & 108.387 & 152.426 & 121.161 & 117.635 & 4.745 & 4.475 & 4.814 \\
\hline $\mathbf{P}$ & PM4-AB01 & 153.253 & 139.814 & 117.189 & 42.171 & 17.172 & 3.707 & 0.000 & 0.000 & -3.100 \\
\hline $\mathbf{P}$ & PM4-AB02 & 155.691 & 147.236 & 131.634 & 30.845 & 44.633 & 17.063 & 0.000 & 0.000 & -3.100 \\
\hline $\mathbf{P}$ & PM4-AB03 & 71.584 & 39.787 & 69.897 & 185.683 & 153.341 & 150.558 & 0.000 & 0.000 & -3.100 \\
\hline $\mathbf{P}$ & PM4-AB04 & -7.550 & -7.524 & -7.539 & 145.514 & 128.504 & 112.104 & 0.000 & 0.000 & -3.100 \\
\hline $\mathbf{P}$ & PM4-AB05 & -7.787 & -6.634 & -6.333 & 76.424 & 54.380 & 35.154 & 0.000 & 0.000 & -3.100 \\
\hline $\mathbf{P}$ & PM4-AB06 & -7.332 & -9.682 & -6.376 & 144.939 & 47.901 & 65.557 & 0.000 & 0.000 & -3.100 \\
\hline $\mathbf{P}$ & PM4-AB07 & -10.401 & -8.329 & -9.013 & 59.984 & 47.036 & 25.662 & 0.000 & 0.000 & -3.100 \\
\hline $\mathbf{P}$ & PM4-AB08 & 45.968 & 54.401 & 116.072 & 178.476 & 161.093 & 155.083 & 0.000 & 0.000 & -3.100 \\
\hline $\mathbf{P}$ & PM4-AB09 & 111.197 & 9.807 & 72.122 & 176.326 & 138.757 & 126.225 & 0.000 & 0.000 & -3.100 \\
\hline $\mathbf{P}$ & PM4-AB10 & 44.603 & 34.545 & 88.418 & 168.266 & 133.847 & 118.422 & 0.000 & 0.000 & -3.100 \\
\hline $\mathbf{P}$ & PM4-AB11 & 36.779 & 21.987 & 50.168 & 142.538 & 117.476 & 104.697 & 0.000 & 0.000 & -3.100 \\
\hline $\mathbf{P}$ & PM4-AB12 & 66.301 & 46.818 & 81.196 & 149.766 & 119.530 & 111.557 & 1.011 & 3.299 & 2.301 \\
\hline $\mathbf{P}$ & PM4-AC01 & 0.504 & -7.028 & -0.293 & 63.353 & 48.209 & 30.768 & 0.000 & 0.000 & -3.100 \\
\hline $\mathbf{P}$ & PM4-AC02 & -5.004 & -7.618 & -7.399 & 84.089 & 64.949 & 42.555 & 0.000 & 0.000 & -1.022 \\
\hline $\mathbf{P}$ & PM4-AC03 & 77.170 & 93.295 & 11.189 & 200.322 & 170.576 & 155.272 & 0.000 & 0.000 & -3.100 \\
\hline $\mathbf{P}$ & PM4-AC04 & -14.457 & 72.184 & -7.866 & 160.359 & 154.608 & 127.343 & 0.000 & 0.000 & -3.100 \\
\hline $\mathbf{P}$ & PM4-AC05 & -16.728 & -15.180 & -16.109 & -15.154 & -40.957 & -50.413 & 0.000 & 0.000 & -3.100 \\
\hline $\mathbf{P}$ & PM4-AC06 & 118.199 & 111.345 & 102.521 & 193.542 & 163.750 & 138.772 & 0.000 & 0.000 & -3.100 \\
\hline $\mathbf{P}$ & PM4-AC07 & -8.305 & -9.149 & -7.532 & 87.203 & 56.226 & 33.462 & 0.000 & 0.000 & -3.100 \\
\hline $\mathbf{P}$ & PM4-AC08 & 37.425 & 31.496 & 56.811 & 182.985 & 144.930 & 133.354 & 0.000 & 0.000 & -3.100 \\
\hline $\mathbf{P}$ & PM4-AC09 & 56.242 & 15.011 & -14.243 & 180.695 & 156.453 & -47.107 & 0.000 & 0.000 & -3.100 \\
\hline $\mathbf{P}$ & PM4-AC10 & 18.399 & 4.338 & -14.387 & 143.420 & 106.076 & 99.685 & 0.000 & 0.000 & -3.100 \\
\hline $\mathbf{P}$ & PM4-AC11 & 28.211 & -5.083 & 26.008 & 142.201 & 122.008 & 113.175 & 0.000 & 0.000 & -3.100 \\
\hline $\mathbf{P}$ & PM4-AC12 & 23.734 & 4.524 & 41.229 & 149.410 & 116.479 & 119.822 & 0.503 & 5.136 & 4.154 \\
\hline $\mathbf{P}$ & PM4-AD01 & 20.547 & 80.864 & 97.903 & 165.300 & 152.080 & 144.512 & 0.000 & 0.000 & -3.100 \\
\hline $\mathbf{P}$ & PM4-AD02 & 32.228 & 43.492 & 31.458 & 101.432 & 68.212 & 4.583 & 0.000 & 0.000 & -3.100 \\
\hline $\mathbf{P}$ & PM4-AD03 & 64.347 & 18.309 & 6.949 & 82.603 & 50.101 & 36.943 & 0.000 & 0.000 & -3.100 \\
\hline $\mathbf{P}$ & PM4-AD04 & 30.804 & 8.059 & 14.355 & 26.907 & 35.105 & 13.761 & 0.000 & 0.000 & -3.100 \\
\hline $\mathbf{P}$ & PM4-AD05 & -9.213 & -5.439 & -6.112 & 18.674 & 36.553 & 24.184 & 0.000 & 0.000 & -3.100 \\
\hline $\mathbf{P}$ & PM4-AD06 & -4.666 & -6.372 & -4.432 & 73.634 & 49.218 & 30.346 & 0.000 & 0.000 & -3.100 \\
\hline $\mathbf{P}$ & PM4-AD07 & -5.658 & -5.112 & -5.041 & 90.768 & 54.966 & 30.393 & 0.000 & 0.000 & -3.100 \\
\hline $\mathbf{P}$ & PM4-AD08 & -0.257 & -4.158 & 51.413 & 174.974 & 146.055 & 92.897 & 0.000 & 0.000 & -3.100 \\
\hline $\mathbf{P}$ & PM4-AD09 & -6.779 & 13.499 & 40.021 & 120.787 & 136.029 & 122.782 & 0.000 & 0.000 & -3.100 \\
\hline $\mathbf{P}$ & PM4-AD10 & -12.914 & 14.726 & -13.289 & 110.863 & 127.747 & 116.975 & 0.000 & 0.000 & -3.100 \\
\hline $\mathbf{P}$ & PM4-AD11 & -4.024 & -2.499 & 19.368 & 155.201 & 122.416 & 119.433 & 0.000 & 0.000 & -3.100 \\
\hline $\mathbf{P}$ & PM4-AD12 & 17.738 & 3.399 & 37.078 & 131.062 & 111.778 & 100.167 & 0.000 & 3.966 & 1.014 \\
\hline $\mathbf{P}$ & PM4-AE01 & -1.616 & -1.066 & -8.682 & 80.907 & 55.839 & 35.766 & 0.000 & 0.000 & -3.100 \\
\hline $\mathbf{P}$ & PM4-AE02 & -5.011 & -5.459 & -9.833 & 80.351 & 49.846 & -47.463 & 0.000 & 0.000 & -3.100 \\
\hline $\mathbf{P}$ & PM4-AE03 & -10.816 & -4.861 & -4.325 & 82.941 & 47.021 & 25.553 & 0.000 & 0.000 & -3.100 \\
\hline $\mathbf{P}$ & PM4-AE04 & -7.638 & -7.134 & -8.208 & 34.713 & 21.211 & 5.137 & 0.000 & 0.000 & -3.100 \\
\hline $\mathbf{P}$ & PM4-AE05 & -6.763 & -6.218 & -7.332 & 60.664 & 33.322 & 15.992 & 0.000 & 0.000 & -3.100 \\
\hline $\mathbf{P}$ & PM4-AE06 & -8.330 & -6.582 & -5.336 & -1.916 & -28.666 & -40.380 & 0.000 & 0.000 & -3.100 \\
\hline $\mathbf{P}$ & PM4-AE07 & -7.392 & -7.391 & -6.471 & 70.475 & 40.850 & 20.487 & 0.000 & 0.000 & -3.100 \\
\hline $\mathbf{P}$ & PM4-AE08 & -5.188 & -8.522 & -5.733 & 41.046 & 19.512 & -2.293 & 0.000 & 0.000 & -3.100 \\
\hline $\mathbf{P}$ & PM4-AE09 & -4.916 & -4.358 & -4.967 & 145.704 & 72.922 & 53.525 & 0.000 & 0.000 & -3.100 \\
\hline $\mathbf{P}$ & PM4-AE10 & 47.220 & 9.176 & 54.762 & 176.179 & 140.717 & 130.710 & 0.000 & 0.000 & -3.100 \\
\hline $\mathbf{P}$ & PM4-AE11 & -9.992 & 3.508 & 3.767 & 1.838 & -13.674 & -26.737 & 0.000 & 1.412 & -1.558 \\
\hline $\mathbf{P}$ & PM4-AE12 & 45.934 & 27.799 & 48.125 & 160.713 & 128.400 & 130.667 & 0.000 & 0.000 & -2.597 \\
\hline $\mathbf{S}$ & PM4-AF01 & 0.000 & 0.000 & 0.000 & 0.000 & 0.000 & 0.000 & 0.000 & 0.000 & 0.000 \\
\hline $\mathbf{s}$ & PM4-AF02 & 3.441 & -0.101 & -1.853 & 79.425 & 79.026 & 74.303 & 0.000 & 0.000 & \\
\hline $\mathbf{s}$ & PM4-AF03 & 192.607 & 160.508 & 146.010 & 96.636 & 87.126 & 84.274 & 0.000 & 0.000 & 0.000 \\
\hline $\mathbf{s}$ & PM4-AF04 & 179.574 & 164.603 & 136.870 & 104.876 & 82.816 & 78.193 & 0.000 & 0.000 & 0.000 \\
\hline s & PM4-AF05 & 166.420 & 124.759 & 98.622 & 79.207 & 75.039 & 74.111 & 0.000 & 0.000 & 0.000 \\
\hline s & PM4-AF06 & 180.543 & 157.907 & 139.333 & 73.172 & 95.418 & 81.084 & 0.000 & 0.000 & 0.000 \\
\hline $\mathbf{s}$ & PM4-AF07 & 175.554 & 150.195 & 135.151 & 69.974 & 92.067 & 88.722 & 0.000 & 0.000 & 0.000 \\
\hline $\mathbf{s}$ & PM4-AF08 & 176.199 & 166.482 & 145.654 & 107.866 & 103.375 & 87.049 & 0.000 & 0.000 & 0.000 \\
\hline $\mathbf{s}$ & PM4-AF09 & 163.796 & 163.922 & 147.695 & 19.230 & 76.391 & 62.539 & 0.000 & 0.000 & 0.000 \\
\hline $\mathbf{s}$ & PM4-AF10 & -3.180 & -4.018 & -7.874 & -1.634 & 2.141 & 2.003 & 0.000 & 0.000 & 0.000 \\
\hline s & PM4-AF11 & 4.030 & 8.634 & 0.575 & -0.862 & 16.767 & 3.093 & 0.000 & 0.000 & 0.000 \\
\hline $\mathbf{s}$ & PM4-AF12 & 162.564 & 140.708 & 123.125 & 18.422 & 52.647 & 53.226 & 1.203 & 1.225 & 2.588 \\
\hline s & PM4-AG01 & -0.668 & -1.526 & -1.095 & 105.749 & 1.839 & 0.651 & 1.805 & 1.611 & 0.795 \\
\hline $\mathbf{s}$ & PM4-AG02 & 57.934 & 58.067 & 40.364 & 6.128 & 15.039 & 3.049 & 0.000 & 0.000 & 0.000 \\
\hline $\mathbf{s}$ & PM4-AG03 & 181.575 & 154.942 & 134.641 & 91.770 & 86.261 & 76.926 & 0.000 & 0.000 & 0.000 \\
\hline s & PM4-AG04 & 204.475 & 176.053 & 157.489 & 121.817 & 97.236 & 83.705 & 0.000 & 0.000 & 0.000 \\
\hline $\mathbf{s}$ & PM4-AG05 & 165.355 & 25.330 & 13.507 & 61.733 & 3.982 & -5.797 & 0.000 & 0.000 & 0.000 \\
\hline s & PM4-AG06 & 25.380 & 34.092 & 1.207 & 12.441 & 38.053 & 13.342 & 0.000 & 0.000 & 0.000 \\
\hline $\mathbf{s}$ & PM4-AG07 & 176.850 & 146.238 & 129.578 & 113.667 & 118.142 & 114.450 & 0.000 & 0.000 & 0.000 \\
\hline $\mathbf{s}$ & PM4-AG08 & 138.288 & 125.513 & 105.285 & 67.259 & 89.895 & 69.974 & 0.000 & 0.000 & 0.000 \\
\hline
\end{tabular}




\begin{tabular}{|c|c|c|c|c|c|c|c|c|c|c|}
\hline$S$ & PM4-AG09 & 180.024 & 144.409 & 139.475 & 141.645 & 135.838 & 141.692 & 0.000 & 0.000 & 0.000 \\
\hline $\mathbf{S}$ & PM4-AG10 & 172.629 & 142.817 & 119.516 & 86.661 & 113.526 & 112.212 & 0.000 & 0.000 & 0.000 \\
\hline $\mathbf{S}$ & PM4-AG11 & 159.791 & 137.612 & 123.789 & 93.912 & 103.847 & 103.250 & 0.000 & 0.000 & 0.000 \\
\hline $\mathrm{s}$ & PM4-AG12 & -0.757 & -1.761 & -0.771 & -2.941 & 0.717 & 1.164 & 3.995 & 4.063 & 5.359 \\
\hline $\mathrm{s}$ & PM4-AH01 & 151.968 & 110.154 & 105.784 & 58.324 & 68.024 & 59.249 & 2.803 & 1.788 & 2.216 \\
\hline $\mathbf{s}$ & PM4-AH02 & 27.053 & 32.964 & 24.988 & 20.661 & 32.745 & 12.634 & 0.000 & 0.000 & 1.005 \\
\hline $\mathbf{S}$ & PM4-AH03 & 40.650 & 2.057 & 1.991 & 6.557 & 5.139 & -4.189 & 0.000 & 0.000 & 0.000 \\
\hline s & PM4-AH04 & 11.120 & 9.391 & 7.351 & 1.943 & 15.316 & 0.011 & 0.000 & 0.000 & 0.000 \\
\hline $\mathbf{s}$ & PM4-AH05 & 32.188 & 24.051 & 14.475 & 3.807 & 28.011 & 1.645 & 0.000 & 0.000 & 0.000 \\
\hline $\mathbf{S}$ & PM4-AH06 & 83.996 & 81.445 & 53.807 & -1.164 & 35.829 & 2.042 & 0.000 & 0.000 & 0.000 \\
\hline $\mathrm{s}$ & PM4-AH07 & 86.434 & 80.762 & 58.399 & 17.934 & 43.989 & 13.975 & 0.000 & 0.000 & 0.000 \\
\hline $\mathbf{S}$ & PM4-AH08 & 2.342 & 2.816 & 2.924 & 5.313 & 2.999 & 3.430 & 0.000 & 0.000 & 0.000 \\
\hline $\mathbf{s}$ & PM4-AH09 & 0.362 & 1.220 & 0.653 & -1.712 & 4.525 & 2.905 & 0.000 & 0.000 & 0.000 \\
\hline $\mathbf{S}$ & PM4-AH10 & 3.457 & 1.822 & 1.267 & -6.551 & -0.382 & 1.779 & 0.000 & 0.000 & 0.000 \\
\hline$s$ & PM4-AH11 & -0.238 & -1.630 & -1.691 & -5.391 & -3.683 & -2.222 & 0.000 & 0.000 & 0.503 \\
\hline $\mathbf{S}$ & PM4-AH12 & -0.028 & -2.549 & 0.458 & -5.645 & -0.620 & 1.580 & 3.436 & 4.892 & 8.084 \\
\hline
\end{tabular}


Table 9.10: Positive signal values and significant changes compared to BT 1B strain 8081 for $Y$. enterocolitica biotypes. $p-p$ value; $\Lambda / V$ - significantly higher/lower activity.

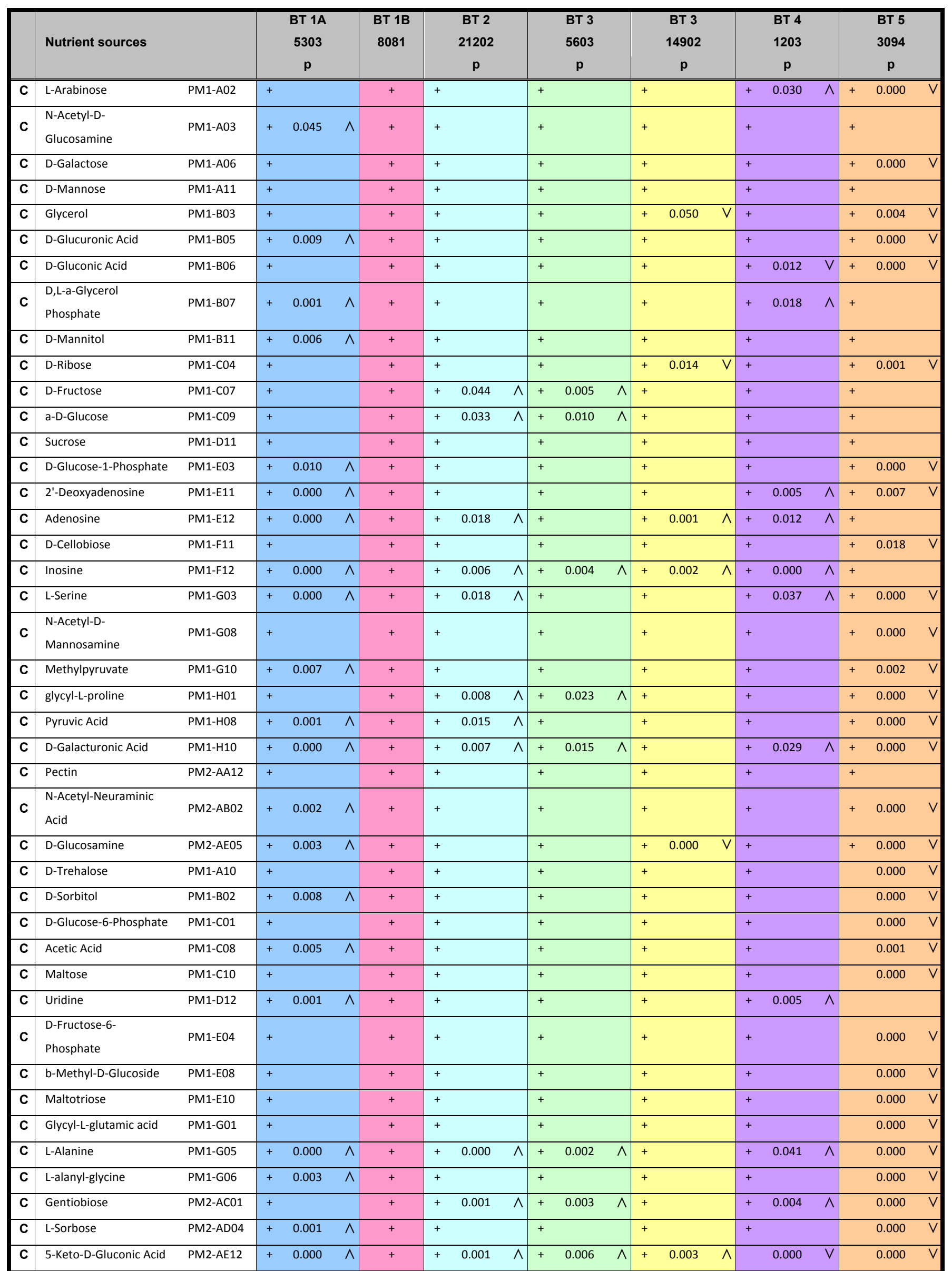




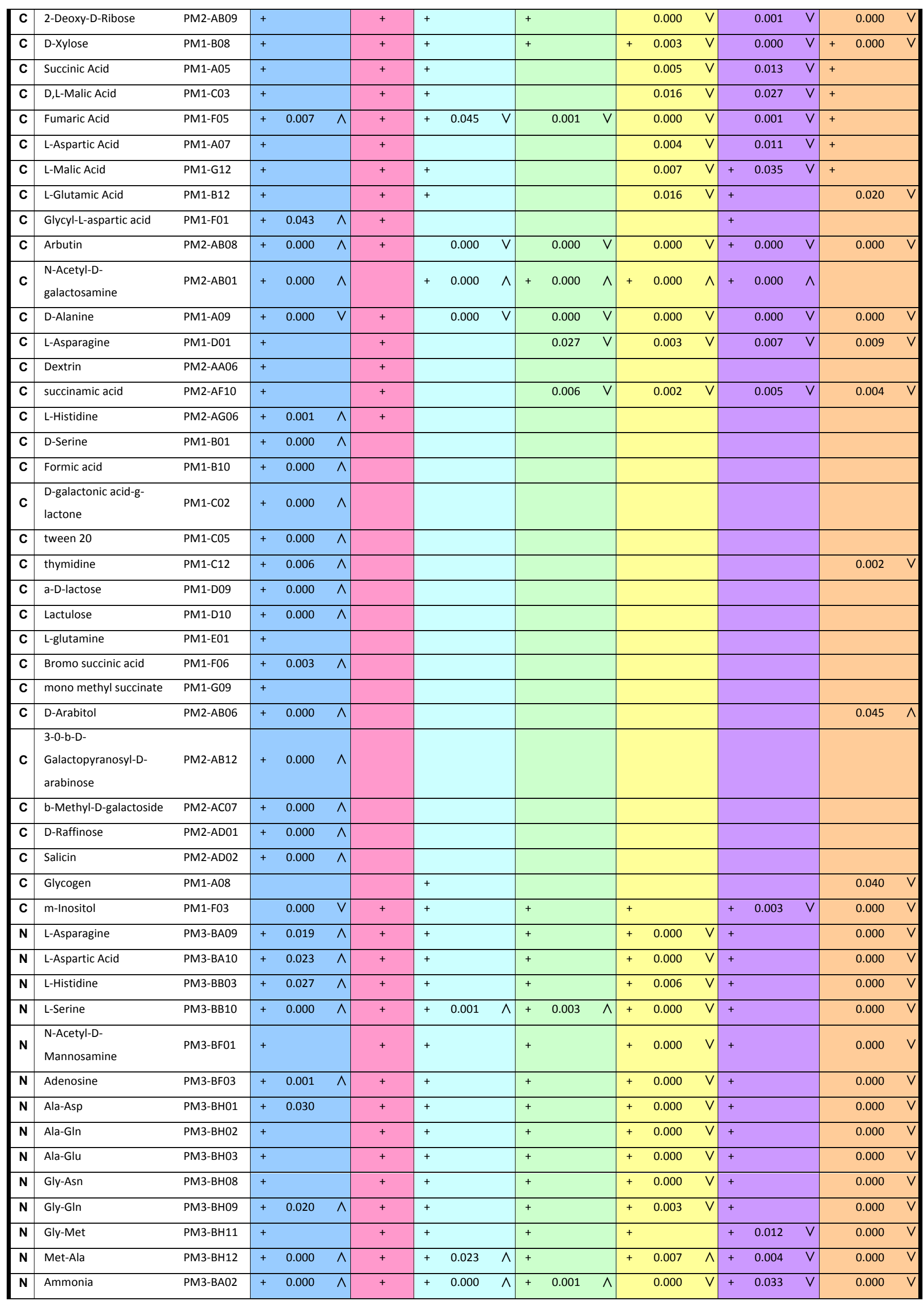




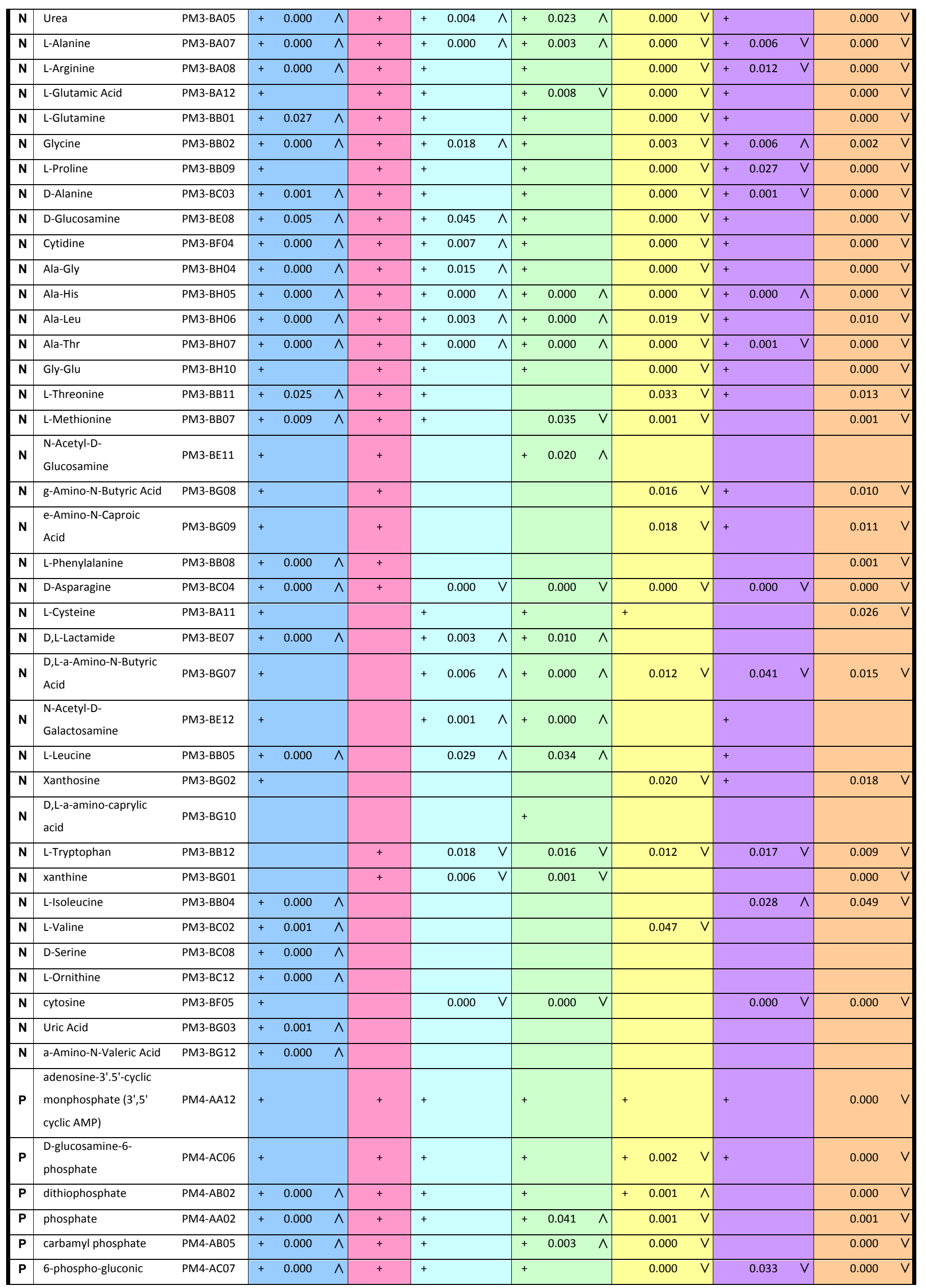




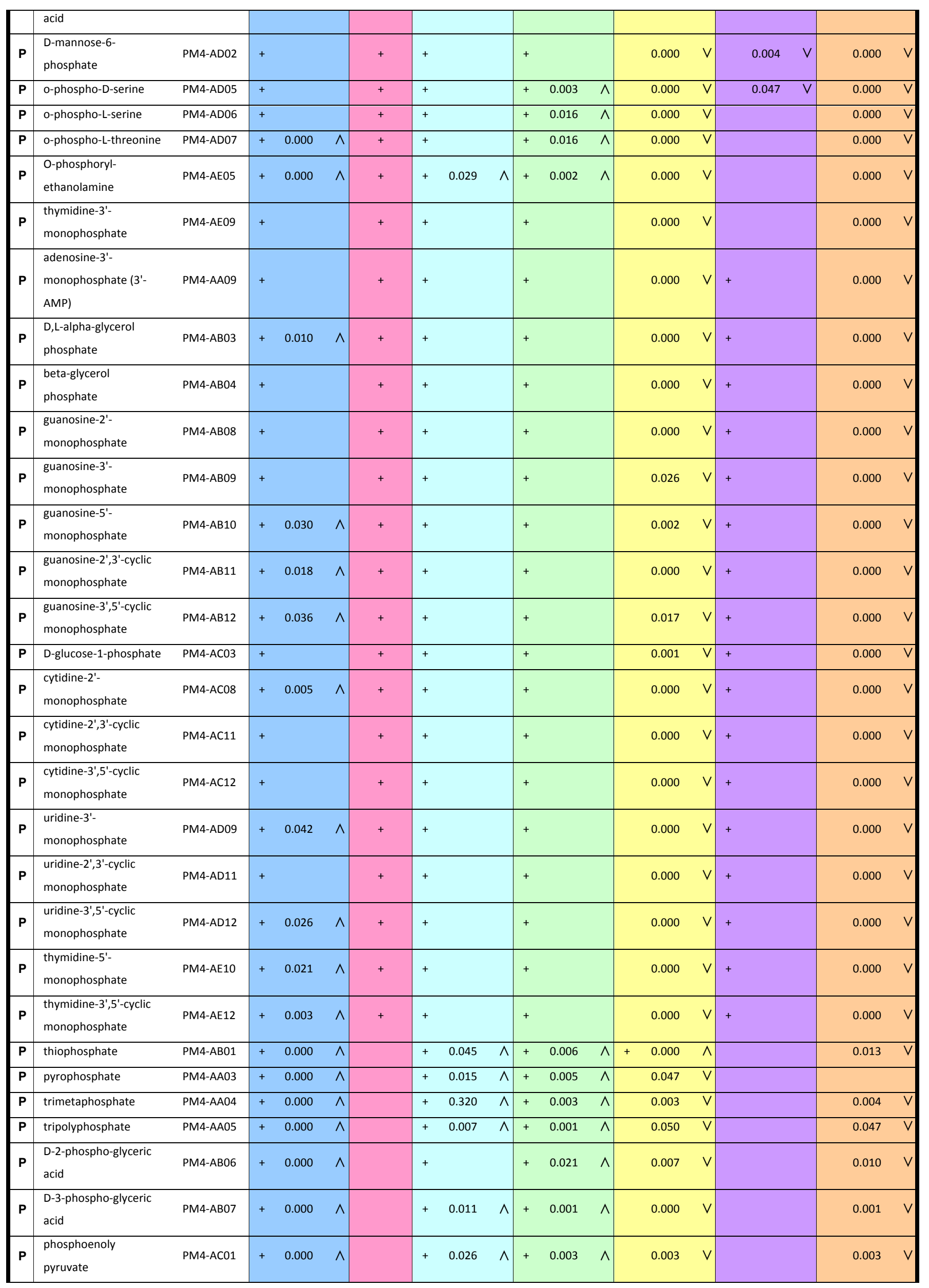




\begin{tabular}{|c|c|c|c|c|c|c|c|c|c|c|c|c|c|c|c|}
\hline \begin{tabular}{l|l}
$\mathbf{P}$ \\
\end{tabular} & phospho-glycolic acid & PM4-AC02 & + & 0.000 & $\wedge$ & & + & & + & 0.005 & $\wedge$ & & $0.000 \mathrm{~V}$ & & $0.001 \quad \mathrm{~V}$ \\
\hline $\mathbf{P}$ & $\begin{array}{l}\text { cysteamine-S- } \\
\text { phosphate }\end{array}$ & PM4-AD03 & + & 0.000 & $\wedge$ & & + & & + & 0.019 & $\wedge$ & & & & $0.003 \mathrm{~V}$ \\
\hline $\mathbf{P}$ & phospho-L-arginine & PM4-AD04 & + & 0.000 & $\wedge$ & & + & & + & 0.004 & $\wedge$ & & $0.002 \mathrm{~V}$ & $0.015 \vee V$ & $0.000 \mathrm{~V}$ \\
\hline $\mathbf{P}$ & phosphocreatine & PM4-AE03 & + & 0.000 & $\wedge$ & & + & $0.028 \wedge$ & + & 0.004 & $\wedge$ & & $0.003 \quad V$ & & 0.005 \\
\hline $\mathbf{P}$ & phosphoryl choline & PM4-AE04 & + & 0.000 & $\wedge$ & & + & & + & 0.003 & $\wedge$ & & $0.005 \mathrm{~V}$ & & 0.008 \\
\hline $\mathbf{P}$ & $\begin{array}{l}\text { adenosine-2'- } \\
\text { monophosphate }\end{array}$ & PM4-AA08 & + & 0.000 & $\wedge$ & & + & $0.032 \wedge$ & + & 0.003 & $\wedge$ & & & $+0.038 \wedge$ & 0.010 \\
\hline $\mathbf{P}$ & O-phospho-D-tyrosine & PM4-AE01 & + & 0.002 & $\wedge$ & & + & & & & & & & & \\
\hline $\mathbf{P}$ & $\begin{array}{l}\text { uridine-2'- } \\
\text { monophosphate }\end{array}$ & PM4-AD08 & + & 0.046 & $\wedge$ & & & & + & & & & & + & \\
\hline $\mathbf{P}$ & $\begin{array}{l}\text { D-mannose-1- } \\
\text { phosphate }\end{array}$ & PM4-AD01 & + & & & + & & & + & & & & & + & $0.006 \mathrm{~V}$ \\
\hline $\mathbf{P}$ & $\begin{array}{l}\text { 2-aminoethyl } \\
\text { phosphonic acid }\end{array}$ & PM4-AE07 & + & 0.001 & $\wedge$ & + & & & + & & & & $0.000 \mathrm{~V}$ & & $0.000 \quad \mathrm{~V}$ \\
\hline $\mathbf{P}$ & $\begin{array}{l}\text { adenosine-2',3'-cyclic } \\
\text { monophosphate }\end{array}$ & PM4-AA11 & & & & + & + & & + & & & & & + & $0.027 \quad \vee$ \\
\hline \begin{tabular}{l|l}
$\mathbf{P}$ \\
\end{tabular} & D-glucose-6-phosphate & PM4-AC04 & & & & + & + & & + & & & & $0.015 \quad \mathrm{~V}$ & + & $0.005 \quad \mathrm{~V}$ \\
\hline $\mathbf{P}$ & $\begin{array}{l}\text { cytidine-3'- } \\
\text { monophosphate }\end{array}$ & PM4-AC09 & & & & + & + & & & & & & & & 0.024 \\
\hline $\mathbf{P}$ & $\begin{array}{l}\text { cytidine-5'- } \\
\text { monophosphate }\end{array}$ & PM4-AC10 & & 0.000 & $\mathrm{~V}$ & + & & & + & & & & 0.007 & + & 0.004 \\
\hline $\mathbf{P}$ & $\begin{array}{l}\text { uridine-5'- } \\
\text { monophosphate }\end{array}$ & PM4-AD10 & & & & + & & & + & & & & & + & 0.039 \\
\hline $\mathbf{P}$ & O-phospho-L-tyrosine & PM4-AE02 & & 0.043 & $\wedge$ & & + & & + & 0.046 & & & & & \\
\hline $\mathbf{P}$ & $\begin{array}{l}\text { adenosine-5'- } \\
\text { monophosphate }\end{array}$ & PM4-AA10 & & & & & & & + & & & & & & \\
\hline $\mathbf{P}$ & phosphono acetic acid & PM4-AE06 & + & 0.000 & $\wedge$ & & & $0.026 \wedge$ & & 0.000 & $\wedge$ & & & & \\
\hline \begin{tabular}{l|l}
$\mathbf{P}$ \\
\end{tabular} & inositol hexaphosphate & PM4-AE11 & + & 0.000 & $\wedge$ & & & & & & & & & & \\
\hline $\mathbf{S}$ & thiosulfate & PM4-AF03 & + & 0.000 & $\wedge$ & + & + & $0.003 \wedge$ & + & 0.007 & $\wedge$ & 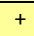 & 0.026 & + & 0.000 \\
\hline $\mathbf{S}$ & tetrathionate & PM4-AF04 & + & 0.000 & $\wedge$ & + & + & $0.002 \wedge$ & t & 0.003 & $\wedge$ & + & 0.012 & + & 0.000 \\
\hline $\mathbf{S}$ & thiophosphate & PM4-AF05 & + & 0.012 & $\wedge$ & + & + & & + & & & + & & + & 0.001 \\
\hline $\mathbf{S}$ & dithiophosphate & PM4-AF06 & + & 0.000 & $\wedge$ & + & + & $0.004 \wedge$ & + & 0.012 & $\wedge$ & + & & + & 0.000 \\
\hline $\mathbf{S}$ & L-cysteine & PM4-AF07 & + & 0.010 & $\wedge$ & + & + & $0.015 \wedge$ & + & 0.029 & $\wedge$ & + & & + & 0.000 \\
\hline $\mathbf{S}$ & D-cysteine & PM4-AF08 & + & 0.000 & $\wedge$ & + & + & $0.000 \wedge$ & t & 0.000 & $\wedge$ & + & $0.001 \wedge$ & + & 0.000 \\
\hline \begin{tabular}{l|l} 
\\
\end{tabular} & cystathionine & PM4-AG03 & + & 0.000 & $\wedge$ & + & + & $0.002 \wedge$ & 7 & 0.003 & $\wedge$ & + & 0.026 & + & 0.000 \\
\hline $\mathbf{S}$ & lanthionine & PM4-AG04 & + & 0.001 & $\wedge$ & + & + & $0.006 \wedge$ & + & 0.010 & $\wedge$ & + & 0.013 & + & 0.000 \\
\hline $\mathbf{S}$ & L-methionine & PM4-AG07 & + & 0.002 & $\wedge$ & + & + & $0.018 \wedge$ & + & 0.036 & $\wedge$ & + & & + & 0.000 \\
\hline $\mathbf{S}$ & D-methionine & PM4-AG08 & + & 0.001 & $\wedge$ & + & + & $0.015 \wedge$ & & 0.008 & $\wedge$ & + & & + & 0.000 \\
\hline $\mathbf{S}$ & glycyl-L-methionine & PM4-AG09 & + & 0.001 & $\wedge$ & + & + & $0.007 \wedge$ & + & & & + & & + & 0.000 \\
\hline $\mathbf{s}$ & $\begin{array}{l}\mathrm{N} \text {-acetyl-D,L- } \\
\text { methionine }\end{array}$ & PM4-AG10 & + & 0.002 & $\wedge$ & + & + & $0.021 \wedge$ & + & & & + & & + & 0.000 \\
\hline S & L-methionine sulfoxide & PM4-AG11 & + & 0.000 & $\wedge$ & + & + & $0.001 \wedge$ & + & 0.006 & $\wedge$ & + & & + & 0.000 \\
\hline $\mathbf{S}$ & L-djenkolic acid & PM4-AH01 & + & 0.001 & $\wedge$ & + & + & $0.018 \wedge$ & + & 0.004 & $\wedge$ & + & 0.049 & + & 0.017 \\
\hline $\mathbf{S}$ & L-cysteinyl-glycine & PM4-AF09 & + & & & + & + & $0.029 \wedge$ & + & 0.045 & $\wedge$ & + & & $0.002 \quad \mathrm{~V}$ & 0.000 \\
\hline $\mathbf{S}$ & L-cystein sulfinic acid & PM4-AF12 & + & 0.000 & $\wedge$ & + & + & $0.003 \wedge$ & + & 0.008 & $\wedge$ & + & $0.005 \wedge$ & & 0.000 \\
\hline $\mathbf{S}$ & sulfate & PM4-AF02 & + & 0.000 & $\wedge$ & + & + & $0.000 \wedge$ & $T_{7}$ & 0.001 & $\wedge$ & & $0.000 \quad V$ & + & 0.000 \\
\hline $\mathbf{S}$ & taurine & PM4-AH06 & + & 0.001 & $\wedge$ & & & & & 0.024 & $\wedge$ & & 0.024 & & \\
\hline $\mathbf{S}$ & S-methyl-L-cystein & PM4-AG02 & + & 0.000 & $\Lambda$ & & & & & & & & & & \\
\hline S & hypotaurine & PM4-AH07 & & 0.003 & $\wedge$ & & & & $t$ & & & 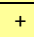 & $\begin{array}{ll}0.017 & \wedge\end{array}$ & & \\
\hline $\mathbf{S}$ & D,L-ethionine & PM4-AG06 & & & & & & & & 0.002 & $\wedge$ & & & & \\
\hline
\end{tabular}




\section{References}

454 LIFE SCIENCES, 2008. 3K long-tag paired-end sequencing with the genome sequencer FLX system. Nature Methods, 5: i - ii.

ACHTMAN, M., ZURTH, K., MORELLI, G., TORREA, G., GUIYOULE, A., and CARNIEL, E., 1999. Yersinia pestis, the cause of plague, is a recently emerged clone of Yersinia pseudotuberculosis. PNAS, 96 (24): 14043 - 14048.

Correction to Achtman, et al., 1999, in PNAS, 97 (14): 8192.

ACHTMAN, M., MORELLI, G., ZHU, P., WIRTH, T., DIEHL, I., KUSECEK, B., VOGLER, A.J., WAGNER, D.M., ALLENDER, C.J., EASTERDAY, W.R., CHENAL-FRANCISQUE, V., WORSHAM, P., THOMSON, N.R., PARKHILL, J., LINDLER, L.E., CARNIEL, E., and KEIM, P., 2004. Microevolution and history of the plague bacillus, Yersinia pestis. PNAS, 101 (51): 17837 - 17842.

AKIBA, M., 2007. Salmonella enterica subsp. enterica serovar Dublin str. L-789 plasmid pMAK2 complete sequence. NCBI accession number: AB366441.1.

ALTSCHUL, S.F., GISH, W., MILLER, W., MYERS, E.W., and LIPMAN, D.J., 1990. Basic local alignment search tool. J Mol Biol, 215 (3): 403 - 410.

ANSORGE, W.J., 2009. Next-generation DNA sequencing techniques. New Biotechnology, 25 (4): 195 - 203.

ASAI, T., ZAPOROJETS, D., SQUIRES, C., and SQUIRES, C.L., 1999. An Escherichia coli strain with all chromosomal rRNA operons inactivated: complete exchange of rRNA genes between bacteria. PNAS, 96: 1971 - 1976.

ASSEFA, S., KEANE, T.M., OTTO, T.D., NEWBOLD, C., and BERRIMAN, M., 2009. ABACAS: algorithm-based automatic contiguation of assembled sequences. Bioinformatics, 25 (15): 1968 - 1969.

AURY, J.M., CRUAUD, C., BARBE, V., ROGIER, O., MANGENOT, S., SAMSON, G., POULAIN, J., ANTHOUARD, V., SCARPELLI, C., ARTIGUENAVE, F., and WINCKER, P., 2008. High quality draft sequences for prokaryotic genomes using a mix of new sequencing technologies. BMC Genomics, 9: 603.

BACH, S., BUCHRIESER, C., PRENTICE, M., GUIYOULE, A., MSADEK, T., and CARNIEL, E., 1999. The high-pathogenicity island of Yersinia enterocolitica Ye8081 undergoes low-frequency deletion but not precise excision, suggesting recent stabilization in the genome. Inf Immun, 67 (10): 5091 - 5099.

BADGER, J.L., and MILLER, V.L., 1998. Expression of invasin and motility are coordinately regulated in Yersinia enterocolitica. J Bact, 180 (4): $793-800$.

BALADA-LLASAT, J.-M., and MECSAS, J., 2006. Yersinia has a tropism for B and T cell zones of lymph nodes that is independent of the type III secretion system. PLoS Pathogens, 2 (9): e86. 
BARTLING, C., WÖLFEL, R., NIKOLAOU, K., PETRY, T., THIEDE, S., HILDEBRANDT, T., FASSBENDER, M., GÖRITZ, F., BLOTTNER, S., SPITTLER, $\mathrm{H}$., and NEUBAUER, H., 2004. Prevalence of anti-Yersinia antibodies in European brown hares in North-Rhine Westphalia, Germany. Dtsch tierärztl Wschr, 111: 259 - 264. [German]

BATZILLA, J., HÖPER, D., ANTONENKA, U., HEESEMANN, J., and RAKIN, A., 2011a. Complete genome sequence of Yersinia enterocolitica subsp. palearctica serogroup O:3. J Bact, 193 (8): 2067.

BATZILLA, J., ANTONENKA, U., HÖPER, D., HEESEMANN, J., and RAKIN, A., 2011b. Yersinia enterocolitica palearctica serobiotype 0:3/4 - a successful group of emerging zoonotic pathogens. BMC Genomics, 12: 348.

BATZILLA, J., HEESEMANN, J., and RAKIN, A., 2011. The pathogenic potential of Yersinia enterocolitica 1A. Int J Med Microbiol [doi: 10.1016/j.ijmm.2011.05.002]

BERRIMAN, M., and RUTHERFORD, K., 2003. Viewing and annotating sequence data with Artemis. Brief Bioinf, 4: 124 - 132.

BLEASDALE, B., LOTT, P.J., JAGANNATHAN, A., STEVENS, M.P., BIRTLES, R.J., and WIGLEY, P., 2009. The Salmonella pathogenicity island 2-encoded type III secretion system is essential for the survival of Salmonella enterica serovar Typhimurium in free-living amoebae. Appl Env Microbiol, 75 (6): 1793 - 1795.

BOCHNER, B.R., GADZINSKI, P., and PANOMITROS, E., 2001. Phenotype microarrays for high-throughput phenotypic testing and assay of gene function. Genome Res, 11: 1246 - 1255.

BOCHNER, B.R., 2003. New technologies to assess genotype-phenotype relationships. Nature Reviews Genetics, 4: 309 - 314.

BOCHNER, B.R., 2009. Global phenotypic characterization of bacteria. FEMS Microbiol Rev, 33: 191 - 205.

BOLAND, A., HAVAUX, S., and CORNELIS, G.R., 1998. Heterogeneity of the Yersinia YopM protein. Micro Path, 25 (6): 343 - 348.

BONFIELD, J.K., SMITH, K., and STADEN, R., 1995. A new DNA sequence assembly program. Nucleic Acids Res, 23 (24): 4992 - 4999. (http://staden.sourceforge.net/)

BOTTONE, E.J., 1977. Yersinia enterocolitica: a panoramic view of a charismatic microorganism. Crit Rev Microbiol, 5 (2): 211 - 214.

BOTTONE, E.J., 1997. Yersinia enterocolitica: the charisma continues. Clin Micro Rev, 10 (20): 257 - 276.

BOTTONE, E.J., 1999. Yersinia enterocolitica: overview and epidemiologic correlates. Microbes Inf, 1: $323-330$. 
BRENDTSEN, J.D., NIELSEN, H., VON HEIJNE, G., and BRUNAK, S., 2004. Improved prediction of signal peptides: SignalP 3.0. J Mol Biol, 340: 783 - 795.

BRESOLIN, G., MORGAN, J.A., ILGEN, D., SCHERER, S., and FUCHS, T.M., 2006a. Low temperature-induced insecticidal activity of Yersinia enterocolitica. Mol Microbiol, 59 (2): $503-512$.

BRESOLIN, G., NEUHAUS, K., SCHERER, S., and FUCHS, T.M., 2006b. Transcriptional analysis of long-term adaptation of Yersinia enterocolitica to lowtemperature growth. J Bact, 188 (8): 2945 - 2958.

BRESOLIN, G., TRČEK, J., SCHERER, S., and FUCHS, T.M., 2008. Presence of a functional flagellar cluster Flag-2 and low-temperature expression of flagellar genes in Yersinia enterocolitica W22703. Microbiology, 154: 196 - 206.

BRINKKÖTTER, A., KLÖß, H., ALPERT, C.-A., and LENGELER, J.W., 2000. Pathways for the utilization of $\mathrm{N}$-acetyl-galactosamine and galactosamine in Escherichia coli. Mol Microbiol, 37 (1): 125 - 135.

BRUBAKER, R.R., 1991. Factors promoting acute and chronic disease caused by Yersiniae. Clin Micro Rev, 4 (3): 309 - 324.

BRUNETEAU, M., and MINKA, S., 2003. Lipopolysaccharides of bacterial pathogens from the genus Yersinia: a mini-review. Biochimie, 85: 145 - 152.

BURROWS, T.W., and GILLETT, W.A., 1966. The nutritional requirements of some Pasteurella species. J gen Microbiol, 45: 333 - 345.

CARNIEL, E., and MOLLARET, H.H., 1990. Yersiniosis. Comp Immun Microbiol infect Dis, 13 (2): 51 - 58.

CARNIEL, E., GUILVOUT, I., and PRENTICE, M., 1996. Characterization of a large chromosomal "High-Pathogenicity Island" in biotype 1B Yersinia enterocolitica. JBact, 178(23): $6743-6751$.

CARNIEL, E., 2002. Plasmids and pathogenicity islands of Yersinia. Curr Top Microbiol Immunol, 264 (1): 89 - 108.

CARNIEL, E., 2003. Evolution of pathogenic Yersinia, some lights in the dark. In: M. Skurnik, ed, 2003. The Genus Yersinia. New York, USA: Klywer Academic I Plenum Publishers, pp. 3 - 12.

CARVER, T.J., RUTHERFORD, K.M., BERRIMAN, M., RAJANDREAM, M.A., BARRELL, B.G., 2005. ACT: The Artemis Comparison Tool. Bioinformatics, 21: $3422-3423$.

CDC (CENTERS FOR DISEASE CONTROL AND PREVENTION), 2005. Information on plague [online]. Available at: http://www.cdc.gov/ncidod/dvbid/plague/info.htm [Accessed 23rd August 2011] 
CDC (CENTERS FOR DISEASE CONTROL AND PREVENTION), 2009. Plague home page [online]. Available at: http://www.cdc.gov/ncidod/dvbid/plague/index.htm [Accessed 23rd August 2011]

CHAIN, P.S.G., CARNIEL, E., LARIMER, F.W., LAMERDIN, J., STOUTLAND, P.O., REGALA, W.M., GEORGESCU, A.M., VERGES, L.M., LAND, M.L., MOTIN, V.L., BRUBAKER, R.R., FOWLER, J., HINNEBUSCH, J., MARCEAU, M., MEDIGUE, C., SIMONET, M., CHENAL-FRANCISQUE, V., SOUA, B., DACHEUX, D., ELLIOTT, J.M., DERBISE, A., HAUSER, L.J., and GARCIA, E., 2004. Insights into the evolution of Yersinia pestis through whole-genome comparison with Yersinia pseudotuberculosis. PNAS, 101 (38): 13826 - 13831.

CHAIN, P.S., GRAFHAM, D.V., FULTON, R.S., FITZGERALD, M.G., HOSTETLER, J., MUZNY, D., ALI, J., BIRREN, B., BRUCE, D.C., BUHAY, C., COLE, J.R., DING, Y, DUGAN, S., FIELD, D., GARRITY, G.M., GIBBS, R., GRAVES, T., HAN, C.S., HARRISON, S.H., HIGHLANDER, S., HUGENHOLTZ, P., KHOURI, H.M., KODIRA, C.D., KOLDER, E., KYRPIDES, N.C., LANG, D., LAPIDUS, A., MALFATTI, S.A., MARKOWITZ, V., METHA, T., NELSON, K.E., PARKHILL, J., PITLUCK, S., QIN, X., READ, T.D., SCHMUTZ, J., SOZHAMANNAN, S., STERK, P., STRAUSBERG, R.L., SUTTON, G., THOMSON, N.R., TIEDJE, J.M., WEINSTOCK, G., WOLLAM, A., GENOMICS STANDARDS CONSORTIUM HUMAN MICROBIOME PROJECT JUMPSTART CONSORTIUM, DETTLER, J.C., 2009. Genomics. Genome project standards in a new era of sequencing. Science 326 (5950): $236-237$.

CHAISSON, M.J., and PEVZNER, P.A., 2007. Short read fragment assembly of bacterial genomes. Genome Research, 18: 324 - 330.

CHAISSON, M.J., BRINZA, D., and PEVZNER, P.A., 2009. De novo fragment assembly with short mate-paired reads: Does the read length matter? Genome Research, 19: 336 - 346.

CHAUDHURI, R.R., SEBAIHIA, M., HOBMAN, J.L., WEBBER, M.A., LEYTON, D., GOLDBERG, M.D., CUNNINGHAM, A.F., SCOTT-TUCKER, A., FERGUSON, P.R., THOMAS, C.M., FRANKEL, G., TANG, C.M., DUDLEY, E.G., ROBERTS, I.S., RASKO, D.A., PALLEN, M.J., PARKHILL, J., NATARO, J.P., THOMSON, N.R., and HENDERSON, I.R., 2010. Complete genome sequence and comparative metabolic profiling of the prototypical enteroaggregative Escherichia coli strain 042. PLoS One, 5 (1): e8801.

CHEN, P.E., COOK, C., STEWART, A.C., NAGARAJAN, N., SOMMER, D.D., POP, M., THOMASON, B., THOMASON, M.P.K., LENTZ, S., NOLAN, N., SOZHAMANNAN, S., SULAKVELIDZE, A., MATECZUN, A., DU, L., ZWICK, M.E., and READ, T.D., 2010. Genomic characterization of the Yersinia genus. Genome Biol, 11: R1.

CHENG, L., CONNOR, T.R., AAENENSEN, D.M., SPRATT, B.G., and CORANDER, J., 2011. Bayesian semi-supervised classification of bacterial samples using MLST databases. BMC Bioinf, 12: 302. 
CH'NG, S.L., OCTAVIA, S., XIA, Q., DUONG, A., TANAKA, M.M., FUKUSHIMA, H., and LAN, R., 2011. Population structure and evolution of pathogenicity of Yersinia pseudotuberculosis. Appl Env Microbiol, 77 (3): 768 - 775.

COLE, S.T., EIGLMEIER, K., PARKHILL, J., JAMES, K.D., THOMSON, N.R., WHEELER, P.R., HONORÉ, N., GARNIER, T., CHURCHER, C., HARRIS, D., MUNGALL, K., BASHAM, D., BROWN, D., CHILLINGWORTH, T., CONNOR, R., DAVIES, R.M., DEVLIN, K., DUTHOY, S., FELTWELL, T., FRASER, A., HAMLIN, N., HOLROYD, S., HORNSBY, T., JAGELS, K., LACROIX, C., MACLEAN, J., MOULE, S., MURPHY, L., OLIVER, K., QUAIL, M.A., RAJANDREAM, M.A., RUTHERFORD, K.M., RUTTER, S., SEEGER, K., SIMON, S., SIMMONDS, M., SKELTON, J., SQUARES, R., SQUARES, S., STEVENS, K., TAYLOR, K., WHITEHEAD, S., WOODWARD, J.R., and BARRELL, B.G., 2001. Massive gene decay in the leprosy bacillus. Nature, 409 (6823): 1007 - 1011.

COLLYN, F., BILLAULT, A., MULLET, C., SIMONET, M., and MARCEAU, M., 2004. YAPI, a new Yersinia pseudotuberculosis pathogenicity island. Inf Immun, 72 (8): $4784-4790$.

CORNELIS, G., LUKE, R.K.J., and RICHMOND, M.H., 1978. Fermentation of raffinose by lactose-fermenting strains of Yersinia enterocolitica and by sucrosefermenting strains of Escherichia coli. J Clin Micro, 7 (2): 180 - 183.

CORNELIS, G., LAROCHE, Y., BALLIGAND, G., SORY, M.-P., and WAUTERS, G., 1987. Yersinia enterocolitica, a primary model for bacterial invasiveness. Rev Infec Dis, 9 (1): 64 - 87.

CORNELIS, G.R., 1994. Yersinia pathogenicity factors. Curr Top Microbiol Immunol, 192: $243-263$.

CORNELIS, G.R., BOLAND, A., BOYD, A.P., GEUIJEN, C., IRIARTE, M., NEYT, C., SORY, M.-P., and STAINIER, I., 1998. The virulence plasmid of Yersinia, an antihost genome. Microbiol Mol Biol Rev, 62 (4): 1315 - 1352.

CORNELIS, G.R., 2002. Yersinia type III secretion: send in the effectors. JCB, 158 (3): $401-408$.

CROUCHER, N.J., FOOKES, M.C., PERKINS, T.T., TURNER, D.J., MARGUERAT, S.B., KEANE, T., QUAIL, M.A., HE, M., ASSEFA, S., BÄHLER, J., KINGSLEY, R.A., PARKHILL, J., BENTLEY, S.D., DOUGAN, G., and THOMSON, N.R., 2009. A simple method for directional transcriptome sequencing using Illumina technology. Nucleic Acids Research, 37 (22): e148.

CROUCHER, N.J., HARRIS, S.R., FRASER, C., QUAIL, M.A., BURTON, J., VAN DER LINDEN, M., McGEE, L., VON GOTTBERG, A., SONG, J.H., KO, K.S., PICHON, B., BAKER, S., PARRY, C.M., LAMBERTSEN, L.M., SHAHINAS, D., PILLAI, D.R., MITCHELL, T.J., DOUGAN, G., TOMASZ, A., KLUGMAN, K.P., PARKHILL, J., HANAGE, W.P., and BENTLEY, S.D., 2011. Rapid pneumococcal evolution in response to clinical intervention. Science, 331: $430-434$. 
DELCHER, A.L., BRATKE, K.A., POWERS, E.C., and SALZBERG, S.L., 2007. Identifying bacterial genes and endosymbiont DNA with Glimmer. Bioinformatics, 23 (6): $673-679$.

DENG, W., BURLAND, V., PLUNKETT III, G., BOUTIN, A., MAYHEW, G.F., LISS, P., PERNA, N.T., ROSE, D.J., MAU, B., ZHOU, S., SCHWARTZ, D.C., FETHERSTON, J.D., LINDLER, L.E., BRUBAKER, R.R., PLANO, G.V., STRALEY, S.C., McDONOUGH, K.A., NILLES, M.L., MATSON, J.S., BLATTNER, F.R., and PERRY, R.D., 2002. Genome sequence of Yersinia pestis KIM. JBact, 184 (16): 4601 - 4611.

DE PAEPE, M., GABORIAU-ROUTHIAU, V., RAINTEAU, D., RAKOTOBE, S., TADDEI, F., and CERF-BENSUSSAN, N., 2011. Trade-off between bile resistance and nutritional competence drives Escherichia coli diversification in the mouse gut. PLoS Genetics, 7 (6): e1002107.

DRANCOURT, M., and RAOULT, D., 2002. rpoB gene sequence-based identification of Staphylococcus species. J Clin Micro, 40 (4): 1333 - 1338.

EBI, 2009. Velvet Sequence assembler for very short reads. [online]. Available at: http://www.ebi.ac.uk/ zerbino/velvet/ [Accessed 25 January 2011]

ELLISON, D.W., LAWRENCE, M.B., and MILLER, V.L., 2004. Invasin and beyond: regulation of Yersinia virulence by RovA. Trends Microbiol, 12 (6): 296 - 300.

ENGLESBERG, E., 1957a. Mutation to rhamnose utilization in Pasteurella pestis. $J$ Bact, 73 (5): 641 - 648.

ENGLESBERG, E., 1957b. Physiological basis for rhamnose utilization by a mutant of Pasteurella pestis. I. Experiments with resting cells; the isolation of lactic aldehyde. J Bact, 74 (1): 8 - 11.

EPPINGER, M., ROSOVITZ, M.J., FRICKE, W.F., RASKO, D.A., KOKORINA, G., FAYOLLE, C., LINDLER, L.E., CARNIEL, E., and RAVEL, J., 2007. The complete genome sequence of Yersinia pseudotuberculosis IP31758, the causative agent of far east scarlet-like fever. PLoS Genetics, 3 (8): e142.

EPPINGER, M., WORSHAM, P.L., NIKOLICH, M.P., RILEY, D.R., SEBASTIAN, Y., MOU, S., ACHTMAN, M., LINDLER, L.E., and RAVEL, J., 2010. Genome sequence of the deep-rooted Yersinia pestis strain Angola reveals new insights into the evolution and pangenome of the plague bacterium. J Bact, 192 (6): 1685 1699.

FANTASIA MAZZOTTI, M., GIRALDI, V., PUGINA, P., MINGRONE, M.G., and BENZI, G., 1985. Survey of some biochemical characteristics of Yersinia sp. Strains. Microbiologica, 8 (1): $43-50$.

FANTASIA MAZZOTTI, M., MINGRONE, M.G., and MARTINI, A., 1987. Fermenting ability of Yersinia spp. strains on esculin, L-fucose and D-arabitol. Microbiologica, 10 (4): $413-416$. 
FARMER III, J.J., CARTER, G.P., MILLER, V.L., FALKOW, S., and WACHSMUTH, I.K., 1992. Pyrazinamidase, CR-MOX agar, salicin fermentation-esculin hydrolysis, and D-xylose fermentation for identifying pathogenic serotypes of Yersinia enterocolitica. J Clin Micro, 30 (10): 2589 - 2594.

FEARNLEY, C., ON, S.L.W., KOKOTOVIC, B., MANNING, G., CHEASTY, T., and NEWELL, D.G., 2005. Application of fluorescent amplified fragment length polymorphism for comparison of human and animal isolates of Yersinia enterocolitica. Appl Env Microbiol, 71 (9): 4960 - 4965.

FERNÁNDEZ, L., MÉNDEZ, J., and GUIJARRO, J.A., 2007. Molecular virulence mechanisms of the fish pathogen Yersinia ruckeri. Vet Microbiol, 125: 1- 10.

FINN, R.D., MISTRY, J., TATE, J., COGGILL, P., HEGER, A., POLLINGTON, J.E., GAVIN, O.L., GUNASEKARAN, P., CERIC, G., FORSLUND, K., HOLM, L., SONNHAMMER, E.L.L., EDDY, S.R., and BATEMAN, A., 2010. The Pfam protein families database. Nucl Acids Res, 38 (1): D211 - D222.

FODOR, I.K., HOLTZ-MORRIS, A.E., and McCUTCHEN-MALONEY, S.L., 2005. Growth curve models for the analysis of phenotype arrays for a systems biology overview of Yersinia pestis. Joint Statistical Meetings. Minneapolis, MN, United States, $6-10$ August 2005. Available from: https://e-reportsext.IInl.gov/pdf/324837.pdf [accessed 13 July 2011].

FOULTIER, B., TROISFONTAINES, P., MÜLLER, S., OPPERDOES, F.R., and CORNELIS, G.R., 2002. Characterization of the ysa pathogenicity locus in the chromosome of Yersinia enterocolitica and phylogeny analysis of the type III secretion systems. J Mol Evol, 55: 37 - 51.

FOULTIER, B., and CORNELIS, G.R., 2003. DNA sequence and analysis of the pYVa127/90 virulence plasmid of Yersinia enterocolitica strain A127/90. Res Microbiol, 154 (8): 553 - 557.

FRASER-LIGGETT, C.M., 2005. Insights on biology and evolution from microbial genome sequencing. Genome Res, 15: 1603 - 1610.

FRECH, G., and SCHWARZ, S., 1998. Tetracycline resistance in Salmonella enterica subsp. enterica serovar Dublin. Antimicrob Agents Chemother, 42 (5): 1288 - 1289.

FUCHS, T.M, BRESOLIN, G., MARCINOWSKI, L., SCHACHTNER, J., and SCHERER, S. , 2008. Insecticidal genes of Yersinia spp.: taxonomical distribution, contribution to toxicity towards Manduca sexta and Galleria mellonella, and evolution. BMC Microbiology, 8: 214.

FUCHS, T.M., BRANDT, K., STARKE, M., and RATTEI, T., 2011. Shotgun sequencing of Yersinia enterocolitica strain W22703 (biotype 2, serotype 0:9): genomic evidence for oscillation between invertebrates and mammals. BMC Genomics, 12: 168. 
FUKUSHIMA, H., GOMYODA, M., ALEKSIC, S., and TSUBOKURA, M., 1993. Differentiation of Yersinia enterocolitica serotype 0:5,27 strains by phenotypic and molecular techniques. J Clin Micro, 31 (6): 1672 - 1674.

FUKUSHIMA, H., MATSUDA, Y., SEKI, R., TSUBOKURA, M., TAKEDA, N., SHUBIN, F.N., PAIK, I.K., and ZHENG, X.B., 2001. Geographical heterogeneity between Far Eastern and Western countries in prevalence of the virulence plasmid, the superantigen Yersinia pseudotuberculosis-derived mitogen, and the high-pathogenicity island among Y. pseudotuberculosis strains. J Clin Micro, 39 (10): $3541-3547$.

GALTIER, N., GOUY, M., and GAUTIER, C., 1996. SEAVIEW and PHYLO_WIN: two graphic tools for sequencing alignment and molecular phylogeny. Comput Appl Biosci, 12: 543 - 548.

GARCIA, J.A., DOMINGUEZ, L., LARSEN, J.L., and PEDERSEN, K., 1988. Ribotyping and plasmid profiling of Yersinia ruckeri. J Appl Microbiol, 85: 949 955.

GARDNER, J., DAUB, J., TATE, J., MOORE, B.L., OSUCH, I.H., GRIFFITHSJONES, S., FINN, R.D., NAWROCKI, E.P., KOLBE, D.L., EDDY, S.R., and BATEMAN, A., 2011. Rfam: Wikipedia, clans, and the „decimal“ release. Nucleic Acids Research [doi: 10.1093/nar/gkq1129]

GHEBREMEDHIN, B., LAYER, F., KÖNIG, W., and KÖNIG, B., 2008. Genetic classification and distinguishing of Staphylococcus species based on different partial gap, 16S rRNA, hsp60, rpoB, sodA, and tuf gene sequences. J Clin Micro, 46 (3): $1019-1025$.

GORSHKOVA, R.P., KALMYKOVA, E.N., ISAKOV, V.V., and OVODOV, Y.S., 1986. Structural studies on O-specific polysaccharides of lipopolysaccharides from Yersinia enterocolitica serovars 0:5 and 0:5,27. Eur J Biochem, 156: 391 - 397.

GOLDBERG, S.M.D., JOHNSON, J., BUSAM, D., FELDBLYUM, T., FERRIERA, S., FRIEDMAN, R., HALPERN, A., KHOURI, H., KRAVITZ, S.A., LAURO, F.M., LI, K., ROGERS, Y.-H., STRAUSBERG, R., SUTTON, G., TALLON, L., THOMAS, T., VENTER, E., FRAZIER, M., and VENTER, J.C., 2006. A Sanger/pyrosequencing hybrid approach for the generation of high-quality draft assemblies of marine microbial genomes. PNAS, 103 (30): $11240-11245$.

GOLUBOV, A., HEESEMANN, J., and RAKIN, A., 2003. Uncovering genomic differences in human pathogenic Yersinia enterocolitica. FEMS Immunol Med Microbiol, 38: 107 - 111.

GOUY, M., GUINDON, S., and GASCUEL, O., 2010. SeaView version 4: a multiplatform graphical user interface for sequence alignment and phylogenetic tree building. Molecular Biology and Evolution, 27 (2): 221 - 224.

GRANT, T., BENNETT-WOOD, V., and ROBINS-BROWNE, R.M., 1998. Identification of virulence-associated characteristics in clinical isolates of Yersinia enterocolitica lacking classical virulence markers. Inf Immun, 66(3): 1113 - 1120. 
GRIFFITHS-JONES, S., BATEMAN, A., MARSHALL, M., KHANNA, A., and EDDY, S.R., 2003. Rfam: an RNA family database. Nucleic Acids Research, 31 (1): 439 441.

GUIYOULE, A., GUINET, F., MARTIN, L., BENOIT, C., DESPLACES, N., and CARNIEL, E., 1998. Phenotypic and genotypic characterization of virulent Yersinia enterocolitica strain unable to ferment sucrose. J Clin Micro, 36 (9): 2732 $-2734$.

GUILVOUT, I., QUILICI, M.L., RABOT, S., LESEL, R., and MAZIGH, D., 1988. $B a m H I$ restriction endonuclease analysis of Yersinia ruckeri plasmids and their relatedness to the genus Yersinia 42- to 47-Megadalton plasmid. Appl Env Microbiol, 54 (10): 2594 - 2597.

HALL, N.,, 2007. Advanced sequencing technologies and their wider impact in microbiology. J Exp Biol, 210 (9): 1518 - 1525.

HALLER, J.C., CARLSON, S., PEDERSON, K.J., and PIERSON, D.E., 2000. A chromosomally encoded type III secretion pathway in Yersinia enterocolitica is important in virulence. Mol Microbiol, 36(6): 1436 - 1446.

HAMMERL, J.A., KLEIN, I, LANKA, E., APPEL, B., and HERTWIG, S., 2008. Genetic and functional properties of the self-transmissible Yersinia enterocolitica plasmid pYE854, which mobilizes the virulence plasmid pYV. J Bact, 190 (3): 991 - 1010.

HARISMENDY, O., NG, P.C., STRAUSBERG, R.L., WANG, X., STOCKWELL, T.B., BEESON, K.Y., SCHORK, N.J., MURRAY, S.S., TOPOL, E.J., LEVY, S., and FRAZER, K.A., 2009. Evaluation of next generation sequencing platforms for population targeted sequencing studies. Genome Biology, 10: R32.

HARRIS, S.R., FEIL, E.J., HOLDEN, M.T.G., QUAIL, M.A., NICKERSON, E.K., CHANTRATITA, N., GARDETE, S., TAVARES, A., DAY, N., LINDSAY, J.A., EDGEWORTH, J.D., DE LENCASTRE, H., PARKHILL, J., PEACOCK, S.J., and BENTLEY, S.D., 2010. Evolution of MRSA during hospital transmission and intercontinental spread. Science, 327 (5964): 469 - 474.

HENSEL, M., 2000. Salmonella pathogenicity island 2. Mol Microbiol, 36 (5): 1015 1023.

HEESEMANN, J., KELLER, C., MORAWA, R., SCHMIDT, N., SIEMENS, H.J., and LAUFS, R., 1983. Plasmids of human strains of Yersinia enterocolitica: molecular relatedness and possible importance for pathogenesis. $J$ Infect Dis, 147 (1): 107 115.

HERTWIG, S., KLEIN, I., HAMMERL, J.A., and APPEL, B., 2003. Characterization of two conjugative Yersinia plasmids mobilizing pYV. In: M. Skurnik, ed, 2003. The Genus Yersinia. New York, USA: Klywer Academic / Plenum Publishers, pp. 38 38.

HEUEL, H., TURGUT, S., SCHMID, K., and LENGELER, J.W., 1997. Substrate recognition domains as revealed by active hybrids between the D-arabinitol and ribitol transporters from Klebsiella pneumoniae. J Bact, 179 (19): 6014 - 6019. 
HINNEBUSCH, B.J., RUDOLPH, A.E., CHEREPANOV, P., DIXON, J.E., SCHWAN, T.G., and FORSBERG, $\AA$, 2002. Role of Yersinia murine toxin in survival of Yersinia pestis in the midgut of the flea vector. Science, 296 (5568): 733 - 735.

HOLTZ-MORRIS, A., 2005. Phenotype array analysis of metabolic differences in Yersinia pestis. The American Society for Microbiology's $105^{\text {th }}$ General Meeting. Atlanta, GA, United States, 5 - 9 June 2005. Available from: https://e-reportsext.IInl.gov/pdf/320376.pdf [accessed 13 July 2011].

HOMANN, O.R., CAI, H., BECKER, J.M., and LINDQUIST, S.L., 2005. Harnessing natural diversity to probe metabolic pathways. PLoS Genetics, 1 (6): e80.

HOWARD, S.L., GAUNT, M.W., HINDS, J., WITNEY, A.A., STABLER, R., and WREN, B.W., 2006. Application of comparative phylogenomics to study the evolution of Yersinia enterocolitica and to identify genetic differences relating to pathogenicity. J Bacteriol, 188: 3645 - 3653.

HULO, N., BAIROCH, A., BULLIARD, V., CERUTTI, L., DE CASTRO, E., LANGENDIJK-GENEVAUX, P.S., PAGNI, M., and SIGRIST, C.J.A., 2006. The PROSITE database. Nucl Acids Res, 34: D227 - D230.

HURST, M.R.H., BECHER, S.A., YOUNG, S.D., NELSON, T.L., and GLARE, T.R., 2011. Yersinia entomophaga sp. nov. isolated from New Zealand grass grub Costelytra zealandica. IJSEM, 61 (4): 844 - 849.

IBRAHIM, A., LIESACK, W., and STACKEBRANDT, E., 1992. Polymerase chain reaction-gene probe detection system specific for pathogenic strains of Yersinia enterocolitica. J Clin Micro, 30 (8): 1942 - 1947.

IBRAHIM, A., GOEBEL, B.M., LIESACK, W., GRIFFITHS, M., and STACKEBRANDT, E., 1993. The phylogeny of the genus Yersinia based on $16 \mathrm{~S}$ rDNA sequences. FEMS Microbiology Letters, 114: 173 - 178.

IBRAHIM, A., LIESACK, W., STEIGERWALT, A.G., BRENNER, D.J., STACKEBRANDT, E., and ROBINS-BROWNE, R.M., 1997. A cluster of atypical Yersinia strains with a distinctive 16S rRNA signature. FEMS Microbiology Letters, 146: $73-78$.

ILLUMINA TECHNOLOGY, 2011. Available at:

http://www.illumina.com/technology/mate_pair_sequencing_assay.ilmn; http://www.illumina.com/technology/paired_end_sequencing_assay.ilmn; http://www.illumina.com/documents/products/datasheets/datasheet_sequencing multiplex.pdf

IRIARTE, M., and CORNELIS, G.R., 1995. MyfF, an element of the network regulating the synthesis of fibrillae in Yersinia enterocolitica. J Bact, 177 (3): 738 744.

IWOBI, A., HEESEMANN, J., GARCIA, E., IGWE, E., NOELTING, C., and RAKIN, A., 2003. Novel virulence-associated type II secretion system unique to highpathogenicity Yersinia enterocolitica. Inf Immun, 71(4): 1872 - 1879. 
JANDA, J.M., and ABBOTT, S.L., 2007. 16S rRNA gene sequencing for bacterial identification in the diagnostic laboratory: pluses, perils, and pitfalls. J Clin Micro, 45 (9): $2761-2764$.

JOHNSON, D.A., TETU, S.G., PHILLIPPY, K., CHEN, J., REN, Q., and PAULSEN, I.T., 2008. High-throughput phenotypic characterization of Pseudomonas aeruginosa membrane transport genes. PLoS Genetics, 4 (10): e1000211.

JOURNET, L., AGRAIN, C., BROZ, P., and CORNELIS, G.R., 2003. The needle length of bacterial injectisomes is determined by a molecular ruler. Science, 302 (5651): $1757-1760$.

KADO, C.I., and LIU, S.-T., 1981. Rapid procedure for detection and isolation of large and small plasmids. J Bact, 145 (3): 1365 - 1373.

KAPPERUD, G., and NESBAKKEN, T., 1987. Restriction endonuclease analysis of 40- to 50-Mdalton plasmids from Yersinia enterocolitica strains of worldwide origin. Contrib Microbiol Immunol, 9: 317 - 323.

KOTETISHVILI, M., KREGER, A., WAUTERS, G., MORRIS, J.G. Jr., SULAKVELIDZE, A., and STINE, O.C., 2005. Multilocus sequence typing for studying genetic relationships among Yersinia species. J Clin Micro, 43 (6): $2674-$ 2684.

KROGH, A., LARSSON, B., VON HEIJNE, G., and SONNHAMMER, E.L.L., 2001. Predicting transmembrane protein topology with a hidden Markov model: Application to complete genomes. J Mol Biol, 305 (3): 567 - 580.

KUO, C.-H., and OCHMAN, H., 2010. The extinction dynamics of bacterial pseudogenes. PLoS Genetics, 6 (8): e1001050.

LANGRIDGE, G.C., PHAN, M.-D., TURNER, D.J., PERKINS, T.T., PARTS, L., HAASE, J., CHARLES, I., MASKELL, D.J., PETERS, S.E., DOUGAN, G., WAIN, J., PARKHILL, J., and TURNER, A.K., 2009. Simultaneous assay of every Salmonella Typhi gene using one million transposon mutants. Genome Res, 19: $2308-2316$.

LARKIN, M.A., BLACKSHIELDS, G., BROWN, N.P., CHENNA, R., McGETTIGAN, P.A., McWILLIAM, H., VALENTIN, F., WALLACE, I.M., WILM, A., LOPEZ, R., THOMPSON, J.D., GIBSON, T.J., and HIGGINS, D.G., 2007. Clustal W and Clustal X version 2.0. Bioinf, 23 (21): 2947 - 2948.

LAROCHE, Y., VAN BOUCHAUTE, M., and CORNELIS, G., 1984. A restriction map of virulence plasmid pYVE439-80 from a serogroup 9 Yersinia enterocolitica strain. Plasmid, 12 (1): 67 - 70.

LEPKA, D., KERRINNES, T., SKIEBE, E., HAHN, B., FRUTH, A., and WILHARM, G., 2009. Adding to Yersinia enterocolitica gene pool diversity: two cryptic plasmids from a biotype $1 \mathrm{~A}$ isolate. J Biomed Biotechnol, 2009: 398434. 
LESEL, R., LESEL, M., GAVINI, F., and VUILLAUME, A., 1983. Outbreak of enteric redmouth disease in rainbow trout, Salmo gairdneri Richardson, in France. $J$ Fish Dis, 6: $385-387$.

LEWIN, A., STRAUCH, E., HERTWIG, S., HOFFMANN, B., NATTERMANN, H., and APPEL, B., 1996. Comparison of plasmids of strains of Yersinia enterocolitica biovar $1 \mathrm{~A}$ with the virulence plasmid of a pathogenic $Y$. enterocolitica strain. Zentralblatt Bakt, 285 (1): 52 - 63.

LI, H., and DURBIN, R., 2009. Fast and accurate short read alignment with BurrowsWheeler Transform. Bioinformatics, 25: 1754 - 1760.

MAHAN, M.J., SLAUCH, J.M., and MEKALANOS, J.J., 1993. Selection of bacterial virulence genes that are specifically induced in host tissues. Science, 259 (5095): $686-688$.

MAIDEN, M.C.J., BYGRAVES, J.A., FEIL, E., MORELLI, G., RUSSELL, J.E., URWIN, R., ZHANG, Q., ZHOU, J., ZURTH, K., CAUGANT, D.A., FEAVERS, E.M., ACHTMAN, M., and SPRATT, B.G., 1998. Multilocus sequence typing: A portable approach to the identification of clones within populations of pathogenic microorganisms. PNAS, 95 (6): 3140 - 3145.

MARCEAU, M., 2004. Transcriptional regulation in Yersinia: an update. In: E. Carniel, B.J. Hinnebusch, eds, 2004. Yersinia - Molecular and cellular biology. Norfolk, UK: Horizon Bioscience, pp. $109-148$.

MARDIS, E.R., 2008a. The impact of next-generation sequencing technology on genetics. Trends in Genetics, 24 (3): 133 - 141.

MARDIS, E.R., 2008b. Next-generation DNA sequencing methods. Annu Rev Genomics Hum Genet, 9: 287 - 402.

MARGULIES, M., EGHOLM, M., ALTMAN, W.E., ATTIYA, S., BADER, J.S., BEMBEN, L.A., BERKA, J., BRAVERMAN, M.S., CHEN, Y.J., CHEN, Z., DEWELL, S.B., DU, L., FIERRO, J.M., GOMES, X.V., GODWIN, B.C., HE, W., HELGESEN, S., HO, C.H., IRZYK, G.P., JANDO, S.C., ALENQUER, M.L., JARVIE, T.P., JIRAGE, K.B., KIM, J.B., KNIGHT, J.R., LANZA, J.R., LEAMON, J.H., LEFKOWITZ, S.M., LEI, M., LI, J., LOHMAN, K.L., LU, H., MAKHIJANI, V.B., MCDADE, K.E., MCKENNA, M.P., MYERS, E.W., NICKERSON, E., NOBILE, J.R., PLANT, R., PUC, B.P., RONAN, M.T., ROTH, G.T., SARKIS, G.J., SIMONS, J.F., SIMPSON, J.W., SRINIVASAN, M., TARTARO, K.R., TOMASZ, A., VOGT, K.A., VOLKMER, G.A., WANG, S.H., WANG, Y., WEINER, M.P., YU, P., BEGLEY, R.F., and ROTHBERG, J.M., 2005. Genome sequencing in microfabricated high-density picolitre reactors. Nature, 437 (7057): $376-380$.

MARTIN, L., LECLERCQ, A., SAVIN, C., and CARNIEL, E., 2009. Characterization of atypical isolates of Yersinia intermedia and definition of two new biotypes. $J$ Clin Micro, 47 (8): $2377-2380$.

McNALLY, A., CHEASTY, T., FEARNLEY, C., DALZIEL, R.W., PAIBA, G.A., MANNING, G., and NEWELL, D.G., 2004. Comparison of the biotypes of Yersinia enterocolitica isolated from pigs, cattle and sheep at slaughter and from 
humans with yersiniosis in Great Britain during 1999 - 2000. Lett Appl Microbiol, 39: $103-108$.

McNALLY, A., DALTON, T., LA RAGIONE, R.M., STAPLETON, K., MANNING, G., and NEWELL, D.G., 2006. Yersinia enterocolitica isolates of differing biotypes from humans and animals are adherent, invasive and persist in macrophages, but differ in cytokine secretion profiles in vitro. J Med Microbiol, 55: 1725 - 1734.

McNALLY, A., LA RAGIONE, R.M., BEST, A., MANNING, G., and NEWELL, D.G., 2007. An aflagellate mutant Yersinia enterocolitica biotype 1A strain displays altered invasion of epithelial cells, persistence in macrophages, and cytokine secretion profiles in vitro. Microbiology, 153: 1339 - 1349.

McQUISTON, J.R., FIELDS, P.I., TAUXE, R.V., and LOGSDON JR, J.M., 2008. Do Salmonella carry spare tyres? Trends in Microbiology, 16 (4): 142 - 148.

MERHEJ, V., ADÉKAMBI, T., PAGNIER, I., RAOULT, D., and DRANCOURT, M., 2008. Yersinia massiliensis sp. nov., isolated from fresh water. IJSEM, 58 (4): 779 $-784$.

METZKER, M.L., 2010. Sequencing technologies - the next generation. Nature Reviews Genetics, 11: 31 - 46.

MILLER, V.L., and FALKOW, S., 1988. Evidence for two genetic loci in Yersinia enterocolitica that can promote invasion of epithelial cells. Inf Immun, 56(5): 1242 $-1248$.

MILLER, V.L., FARMER III, J.J., HILL, W.E., and FALKOW, S., 1989. The ail locus is found uniquely in Yersinia enterocolitica serotypes commonly associated with disease. Inf Immun, 57(1): 121 - 131.

MILLER, J.M., and RHODEN, D.L., 1991. Preliminary evaluation of Biolog, a carbon source utilization method for bacterial identification. J Clin Micro, 29 (6): 1143 1147.

MORELLI, G., SONG, Y., MAZZONI, C.J., EPPINGER, M., ROUMAGNAC, P., WAGNER, D.M., FELDKAMP, M., KUSECEK, B., VOGLER, A.J., LI, Y., CUI, Y., THOMSON, M.R., JOMBART, T., LEBLOIS, R., LICHTNER, P., RAHALISON, L., PETERSEN, J.M., BALLOUX, F., KEIM, P., WIRTH, T., RAVEL, J., YANG, R., CARNIEL, E., and ACHTMAN, M., 2010. Yersinia pestis genome sequencing identifies patterns of global phylogenetic diversity. Nature Genetics, 42 (12): 1140 $-1143$.

MOTIN, V.L., GEORGESCU, A.M., FITCH, J.P., GU, P.P., NELSON, D.O., MABERY, S.L., GARNHAM, J.B., SOKHANSANJ, B.A., OTT, L.L., COLEMAN, M.A., ELLIOTT, J.M., KEGELMEYER, L.M., WYROBEK, A.J., SLEZAK, T.R., BRUBAKER, R.R., and GARCIA, E., 2004. Temporal global changes in gene expression during temperature transition in Yersinia pestis. J Bact, 186 (18): 6298 $-6305$.

MÜLLER-LOENNIES, S., RUND, S., ERVELÄ, E., SKURNIK, M., and HOLST, O., 1999. The structure of the carbohydrate backbone of the core-lipid A region of the 
lipopolysaccharide from a clinical isolate of Yersinia enterocolitica 0:9. Eur $J$ Biochem, 261: 19 - 24.

MURROS-KONTIAINEN, A., FREDRIKSSON-AHOMAA, M., KORKEALA, H., JOHANSSON, P., RAHKILA, R., and BJÖRKROTH, J., 2010a. Yersinia nurmii sp. nov. IJSEM [doi:10.1099/ijs.0.024836-0].

MURROS-KONTIAINEN, A., JOHANSSON, P., NISKANEN, T., FREDRIKSSONAHOMAA, M., KORKEALA, H., and BJÖRKROTH, J., 2010b. Yersinia pekkanenii sp. nov. IJSEM [doi:10.0199/ijs.0019984-0]

NAKAJIMA, H., INOUE, M., MORI, T., ITOH, K.-I., ARAKAWA, E., and WATANABE, H., 1992. Detection and identification of Yersinia pseudotuberculosis and pathogenic Yersinia enterocolitica by an improved polymerase chain reaction method. J Clin Micro, 30 (9): 2484 - 2486.

NAVID, A., and ALMAAS, E., 2009. Genome-scale reconstruction of the metabolic network in Yersinia pestis, strai 91001. Mol BioSyst, 5: 368 - 375.

NELSON, K.M., YOUNG, G.M., and MILLER, V.L., 2001. Identification of a locus involved in systemic dissemination of Yersinia enterocolitica. Inf Immun, 69(10): $6201-6208$.

NEUBAUER, H., SAUER, T., BECKER, H., ALEKSIC, S., and MEYER, H., 1998. Comparison of systems for identification and differentiation of species within the genus Yersinia. J Clin Micro, 36 (11): 3366 - 3368.

NEUBAUER, H., HENSEL, A., ALEKSIC, S., and MEYER, H., 2000. Identification of Yersinia enterocolitica within the genus Yersinia. System Appl Microbiol, 23: 58 62.

NEUBAUER, H., ALEKSIC, S., HENSEL, A., FINKE, E.-J., and MEYER, H., 2000. Yersinia enterocolitica 16S rRNA gene types belong to the same genospecies but form three homology groups. Int J Med Microbiol, 290 (1): 61 - 64.

NEUBAUER, H.K.J., and SPRAGUE, L.D., 2003. Epidemiology and diagnostics of Yersinia-infections. In: M. Skurnik, ed, 2003. The Genus Yersinia. New York, USA: Klywer Academic / Plenum Publishers, pp. 431 - 438.

NEYT, C., IRIARTE, M., THI, V.H., and CORNELIS, G.R., 1997. Virulence and arsenic resistance in Yersiniae. J Bact, 179 (3): 612 - 619.

NIELSEN, H., ENGELBRECHT, J., BRUNAK, S., and VON HEIJNE, G., 1997. Identification of prokaryotic and eukaryotic signal peptides and prediction of their cleavage sites. Protein Engineering, 10: $1-6$.

NING, Z., COX, A.J., and MULLIKIN, J.C., 2001. SSAHA: a fast search method for large DNA databases. Genome Res, 11: 1725 - 1729.

OBERHETTINGER, P., SCHÜTZ, M., RADDATZ, G., KELLER, H., and AUTENRIETH, I.B., 2011. The sequence of the pYV virulence plasmid from 
Yersinia enterocolitica strain WA-314 biogroup 1B serotype O:8. Plasmid, 65 (1): $20-24$.

OERTELT, C., LINDNER, B., SKURNIK, M., and HOLST, O., 2001. Isolation and structural characterization of an R-form lipopolysaccharide from Yersinia enterocolitica serotype O:8. Eur J Biochem, 268: 554 - 564.

OTTO, T.D., SANDERS, M., BERRIMAN, M., and NEWBOLD, C., 2010. Iterative correction of reference nucleotides (iCORN) using second generation sequencing technology. Bioinformatics 26 (14): $1704-1707$.

PARKHILL, J., WREN, B.W., THOMSON, N.R., TITBALL, R.W., HOLDEN, M.T.G., PRENTICE, M.B., SEBAIHA, M., JAMES, K.D., CURCHER, C., MUNGALL, K.L., BAKER, S., BASHAM, D., BENTELY, S.D., BROOKS, K., CERDEÑO-TÁRRAGA, A.M., CHILLINGWORTH, T., CRONIN, A., DAVIES, R.M., DAVIS, P., DOUGAN, G., FELTWELL, T., HAMLIN, N., HOLROYD, S., JAGELS, K., KARLYSHEV, A.V., LEATHER, S., MOULE, S., OYSTON, P.C.F., QUAIL, M., RUTHERFORD, K., SIMMONDS, M., SKELTON, J., STEVENS, K., WHITEHEAD, S., and BARRELL, B.G., 2001a. Genome sequence of Yersinia pestis, the causative agent of plague. Nature, 413 (6855): 523 - 527.

PARKHILL, J., DOUGAN, G., JAMES, K.D., THOMSON, N.R., PICKARD, D., WAIN, J., CHURCHER, C., MUNGALL, K.L., BENTLEY, S.D., HOLDEN, M.T., SEBAIHIA, M., BAKER, S., BASHAM, D., BROOKS, K., CHILLINGWORTH, T., CONNERTON, P., CRONIN, A., DAVIS, P., DAVIES, R.M., DOWD, L., WHITE, N., FARRAR, J., FELTWELL, T., HAMLIN, N., HAQUE, A., HIEN, T.T., HOLROYD, S., JAGELS, K., KROGH, A., LARSEN, T.S., LEATHER, S., MOULE, S., O'GAORA, P., PARRY, C., QUAIL, M., RUTHERFORD, K., SIMMONDS, M., SKELTON, J., STEVENS, K., WHITEHEAD, S., and BARRELL, B.G., 2001b. Complete genome sequence of a multiple drug resistant Salmonella enterica serovar Typhi CT18. Nature, 413 (6858): 848 - 852.

PAYNE, S.H., HUANG, S.-T., and PIEPER, R., 2010. A proteogenomic update to Yersinia: enhancing genome annotation. BMC Genomics, 11: 460.

PEDERSEN, K.B., WINBLAD, S., and BITSCH, V., 1979. Studies on the interaction between different O-serotypes of Yersinia enterocolitica and HeLa cells. Act path microbiol scand Sect B, 87: 141 - 145.

PEPE, J.C., and MILLER, V.L., 1993. Yersinia enterocolitica invasin: a primary role in the initiation of infection. PNAS, 90 (14): $6473-6477$.

PERRY, R.D., and FETHERSTON, J.D., 1997. Yersinia pestis - etiologic agent of plague. Clin Microbiol Rev, 10 (1): 35 - 66.

PIERSON, D.E., and FALKOW, S., 1990. Non-pathogenic isolates of Yersinia enterocolitica do not contain functional inv-homologous sequences. Inf Immun, 58 (4): 1059 - 1064.

PIERSON, D.E., and FALKOW, S., 1993. The ail gene of Yersinia enterocolitica has a role in the ability of the organism to survive serum killing. Inf Immun, 61 (5): 1846 $-1852$. 
POP, M., and SALZBERG, S.L., 2008. Bioinformatics challenges of new sequencing technology. Trends in Genetics, 24 (3): 142 - 149.

PORTNOY, D.A., MOSELEY, S.L, and FALKOW, S., 1981. Characterization of plasmids and plasmid-associated determinants of Yersinia enterocolitica pathogenesis. Inf Immun, 31 (2): 775 - 782.

PORTNOY, D.A., and FALKOW, S., 1981. Virulence-associated plasmids form Yersinia enterocolitica and Y. pestis. J Bact, 148 (3): 877 - 883.

PORTNOY, D.A., WOLF-WATZ, H., BOLIN, I., BEEDER, A.B., and FALKOW, S., 1984. Characterization of common virulence plasmids in Yersinia species and their role in the expression of outer membrane proteins. Inf Immun, 43 (1): 108 - 114.

PRIOR, J.L., HITCHEN, P.G., WILLIAMSON, E.D., REASON, A.J., MORRIS, H.R., DELL, A., WREN, B.W., and TITBALL, R.W., 2001. Characterization of the lipopolysaccharide of Yersinia pestis. Microbial Pathogenesis, 30: 49 - 57.

PUJOL, C., and BLISKA, J.B., 2003. The ability to replicate in macrophages is conserved between Yersinia pestis and Yersinia pseudotuberculosis. Inf Immun, 71 (10): $5892-5899$.

REEVES, P.R., HOBBS, M., VALVANO, M.A., SKURNIK, M., WHITFIELD, C., COPLIN, D., KIDO, N., KLENA, J., MASKELL, D., RAETZ, C.R.H., and RICK, P.D., 1996a. Bacterial polysaccharide synthesis and gene nomenclature. Trends in Microbiology, 4 (12): 495 - 503.

REEVES, P.R., HOBBS, M., VALVANO, M.A., SKURNIK, M., WHITFIELS, C., COPLIN, D., KIDO, N., KLENA, J., MASKELL, D., RAETZ, C.R.H., and RICK, P.D., 1996b. Application of a new nomenclature for bacterial surface polysaccharide genes. Available at:

http://sydney.edu.au/science/molecular_bioscience/BPGD/big_paper.pdf

[Accessed 21 June 2011]

REIZER, J., RAMSEIER, T.M., REIZER, A., CHARBIT, A., and SAIER JR., M.H., 1996. Novel phosphotransferase genes revealed by bacterial genome sequencing: a gene cluster encoding a putative $\mathrm{N}$-acetylgalactosamine metabolic pathway in E. coli. Microbiology, 142: $231-250$.

REVELL, P.A., and MILLER, V.L., 2001. Yersinia virulence: more than a plasmid. FEMS Micro Lett, 205 (2): 159 - 164.

ROBINS-BROWNE, R.M., MILIOTIS, M.D., CIANCIOSI, S., MILLER, V.L., FALKOW, S., and MORRIS, G. JR., 1989. Evaluation of DNA colony hybridization and other techniques for detection of virulence in Yersinia species. J Clin Micro, 27 (4): 644 -650 .

ROCHE, 2011. 454 Sequencing - Products \& Solutions. [online] Available at: http://www.454.com/products-solutions/analysis-tools/gs-de-novo-assembler.asp [Accessed 25 January 2011] 
ROGGENKAMP, A., GEIGER, A.M., LEITRITZ, L., KESSLER, A., and HEESEMANN, J., 1997. Passive immunity to infection with Yersinia spp. Mediated by anti-recombinant $\mathrm{V}$ antigen is dependent on polymorphism of $\mathrm{V}$ antigen. Inf Immun, 65 (2): 446 - 451.

ROHMER, L., HOCQUET, D., and MILLER, S.I., 2011. Are pathogenic bacteria just looking for food? Metabolism and microbial pathogenesis. Trends Microbiol, 19 (7): $341-348$.

RYCKAERT, J., BOSSIER, P., D'HERDE, K., DIEZ-FRAILE, A., SORGELOOS, P., HAESEBROUCK, F., and PASMANS, F., 2010. Persistance of Yersinia ruckeri in trout macrophages. Fish Shellfish Immunol, 29: 648 - 655.

SALZBERG, S.L., DELCHER, A.L., KASIF, S., and WHITE, O., 1998. Microbial gene identification using interpolated Markov models. Nucl Acids Res, 26 (2): $544-548$.

SAMUEL, G., and REEVES, P., 2003. Biosynthesis of O-antigens: genes and pathways involved in nucleotide sugar precursor synthesis and O-antigen assembly. Carbohydrate Research, 338: 2503 - 2519.

SANGER, F., NICKLEN, S., and COULSON, A.R., 1977. DNA sequencing with chain terminating inhibitors. PNAS, 74 (12): 5463 - 5467.

SANGER, F., 1988. Sequences, sequences, and sequences. Ann Rev Biochem, 57: $1-28$.

SCHIEMANN, D.A., and DEVENISH, J.A., 1982. Relationship of HeLa cell infectivity to biochemical, serological, and virulence characteristics of Yersinia enterocolitica. Inf Immun, 35 (2): 497 - 506.

SCHOULS, L.M., SCHOT, C.S., and JACOBS, J.A., 2003. Horizontal transfer of segments of the 16S rRNA genes between species of the Streptococcus anginosus group. J Bact, 185 (24): 7241 - 7246.

SCHUBERT, S., FISCHER, D., and HEESEMANN, J., 1999. Ferric enterochelin transport in Yersinia enterocolitica: Molecular and evolutionary aspects. JBact, 181 (20): $6387-6395$.

SHEA, J.E., HENSEL, M., GLEESON, C., and HOLDEN, D.W., 1996. Identification of a virulence locus encoding a second type III secretion system in Salmonella typhimurium. PNAS, 93: 2593 - 2597.

SHEHEE, M.W., and SOBSEY, M.D., 2004. Development of a L-rhamnose and Darabitol supplemented MacConkey agar to identify pathogenic Yersinia enterocolitica among environmental Yersinias in swine production wastes. J Microbiol Methods, 57: 289 - 292.

SHENDURE, J., and JI, H., 2008. Next-generation DNA sequencing. Nature Biotechnology, 26 (10): 1135 - 1145. 
SIGRIST, C.J.A., CERUTTI, L., HULO, N., GATTIKER, A., FALQUET, L., PAGNI, M., BAIROCH, A., and BUCHER, P., 2002. PROSITE: a documented database using patterns and profiles as motif descriptors. Brief Bioinform, 3: 265 - 274.

SIRISENA, D.M., and SKURNIK, M., 2003. Mutations in the genes for synthesis of the outer core region of the lipopolysaccharide of Yersinia enterocolitica $0: 3 . J$ Appl Microbiol, 94: 686 - 692.

SKURNIK, M., VENHO, R., BENGOECHEA, J.A., and MORIYÓN, I., 1999. The lipopolysaccharide outer core of Yersinia enterocolitica serotype 0:3 is required for virulence and plays a role in outer membrane integrity. Mol Microbiol, 31 (5): 1443 $-1462$.

SKURNIK, M., and BENGOECHEA, J.A., 2003. The biosynthesis and biological role of lipopolysaccharide O-antigens of pathogenic Yersiniae. Carbohydrate Res, 338: $2521-2529$.

SKURNIK, M., BIEDZKA-SAREK, M., LÜBECK, P.S., BLOM, T., BENGOECHEA, J.A., PÉREZ-GUTIÉRREZ, C., AHRENS, P., and HOORFAR, J., 2007. Characterization and biological role of the O-polysaccharide gene cluster of Yersinia enterocolitica serotype 0:9. J Bact, 189 (20): 7244 - 7253.

SMALLA, K., WACHTENDORF, U., HEUER, H., LIU, W.-T., and FORNEY, L., 1998. Analysis of BIOLOG GN substrate utilization patterns by microbial communities. Appl Env Microbiol, 64 (4): 1220 - 1225.

SMYTH, G.K., 2004. Linear models and empirical bayes methods for assessing differential expression in microarray experiments. Stat Appl Genet Mol Biol, 3 (1): Article 3.

SNELLINGS, N.J., POPEK, M., and LINDLER, L.E., 2001. Complete DNA Sequence of Yersinia enterocolitica Serotype 0:8 Low-Calcium-Response Plasmid Reveals a New Virulence Plasmid-Associated Replicon. Inf Immun, 69 (7): 4627 - 4638.

SONG, H., HWANG, J., YI, H., ULIRCH, R.L., YU, Y., NIERMAN, W.C., and KIM, H.S., 2010. The early stage of bacterial genome-reductive evolution in the host. PLoS Pathogens, 6 (5): e1000922.

SOUZA, R.A., PITONDO-SILVA, A., FALCÃO, D.P., and FALCÃO, J.P., 2010. Evaluation of four molecular typing methodologies as tools for determining taxonomy relations and for identifying species among Yersinia isolates. $J$ Micro Methods, 82: 141 - 150 .

SOUZA, R.A., FALCÃO, D.P., and FALCÃO, J.P., 2011. Emended description of the species Yersinia massiliensis. IJSEM, 61 (5): 1094 - 1097.

SPRAGUE, L.D., and NEUBAUER, H., 2005. Yersinia aleksiciae sp. nov. IJSEM, 55: $831-835$.

SPRAGUE, L.D., SCHOLZ, H.C., AMANN, S., BUSSE, H.-J., and NEUBAUER, H., 2008. Yersinia similis sp. nov. IJSEM, 58 (4): 952 - 958. 
STAINIER, I., BLEVES, S., JOSENHANS, C., KARMANI, L, KERBOURCH, C., LAMBERMONT, I., TÖTEMEYER, S., BOYD, A., and CORNELIS, G.R., 2000. YscP, a Yersinia protein required for Yop secretion that is surface exposed, and released in low $\mathrm{Ca}^{2+}$. Mol Microbiol, 37 (5): 1005 - 1018.

STAMATAKIS, A., 2006. RAXML-VI-HPC: maximum likelihood-based phylogenetic analyses with thousands of taxa and mixed models. Bioinformatics, 22 (21): 2688 $-2690$.

STOCK, I., HENRICHFREISE, B., and WIEDEMANN, B., 2002. Natural antibiotic susceptibility and biochemical profiles of Yersinia enterocolitica-like strains: Y. bercovieri, Y. mollaretii, Y. aldova and 'Y. ruckeri'. J Med Microbiol, 51: 56 - 69.

STRALEY, S.C., and PERRY, R.D., 1995. Environmental modulation of gene expression and pathogenesis in Yersinia. Trends in Microbiology, 3 (8): 310 - 317.

SULAKVELIDZE, A., 2000. Yersiniae other than Y. enterocolitica, Y. pseudotuberculosis, and Y. pestis: the ignored species. Microbes Inf, 2: 497 513.

SWAMINATHAN, B., HARMON, M.C., and MEHLMAN, I.J., 1982. A review Yersinia enterocolitica. J Appl Bacteriol, 52: 151 - 183.

TENNANT, S.M., GRANT, T.H., and ROBINS-BROWNE, R.M., 2003. Pathogenicity of Yersinia enterocolitica biotype 1A. FEMS Immunol Med Microbiol, 38: 127 137.

TENNANT, S.M., JOE, A., and ROBINS-BROWNE, R.M., 2004. LPS genes of a serotype 0:5 strain of Yersinia enterocolitica biotype 1A. NCBI Accession number: AY653208.

TENNANT, S.M., SKINNER, N.A., JOE, A., and ROBINS-BROWNE, R.M., 2005. Homologues of insecticidal toxin complex genes in Yersinia enterocolitica biotype $1 \mathrm{~A}$ and their contribution to virulence. Inf Immun, 73 (10): 6860 - 6867.

THOMSON, N.R., HOWARD, S., WREN, B.W, HOLDEN, M.T., CROSSMAN, L., CHALLIS, G.L., CHURCHER, C., MUNGALL, K., BROOKS, K., CHILLINGWORTH, T., FELTWELL, T., ABDELLAH, Z., HAUSER, H., JAGELS, K., MADDISON, M., MOULE, S., SANDERS, M., WHITEHEAD, S., QUAIL, M.A., DOUGAN, G., PARKHILL, J., and PRENTICE, M.B., 2006. The complete genome sequence and comparative genome analysis of the high pathogenicity Yersinia enterocolitica strain 8081. PLoS Genet, 2: e206.

THOMSON, N.R., CLAYTON, D.J., WINDHORST, D., VERNIKOS, G., DAVIDSON, S., CHURCHER, C., QUAIL, M.A., STEVENS, M., JONES, M.A., WATSON, M., BARRON, A., LAYTON, A., PICKARD, D., KINGSLEY, R.A., BIGNELL, A., CLARK, L., HARRIS, B., ORMOND, D., ABDELLAH, Z., BROOKS, K., CHEREVACH, I., CHILLINGWORTH, T., WOODWARD, J., NORBERCZAK, H., LORD, A., ARROWSMITH, C., JAGELS, K., MOULE, S., MUNGALL, K., SANDERS, M., WHITEHEAD, S., CHABALGOITY, J.A., MASKELL, D., HUMPHREY, T., ROBERTS, M., BARROW, P.A., DOUGAN, G., and PARKHILL, J., 2008. Comparative genome analysis of Salmonella Enteritidis PT4 and 
Salmonella Gallinarum 287/91 provides insights into evolutionary and host adaptation pathways. Genome Res, 18 (10): 1624 - 1637.

TOBBACK, E., DECOSTERE, A., HERMANS, K., RYCKAERT, J., DUCHATEAU, L., HAESEBROUCK, F., and CHIERS, K., 2009. Route of entry and tissue distribution of Yersinia ruckeri in experimentally infected rainbow trout Oncorhynchus mykiss. Dis Aquatic Org, 84: 219 - 228.

TOMPKINS, D.S., HUDSON, M.J., SMITH, H.R., EGLIN, R.P., WHEELER, J.G., BRETT, M.M., OWEN, R.J., BRAZIER, J.S., CUMBERLAND, P., KING, V., and COOK, P.E., 1999. A study of infectious intestinal disease in England: microbiological findings in cases and controls. Commun Dis Pub Health, 2 (2): 108 $-113$.

TORANZO, A.E., BARJA, J.L, COLWELL, R.R., and HETRICK, F.M., 1983. Characterization of plasmids in bacterial fish pathogens. Inf Immun, 39 (1): 184 192.

VIRDI, J.S., and SACHDEVA, P., 2005. Molecular heterogeneity in Yersinia enterocolitica and 'Y. enterocolitica-like' species - Implications for epidemiology, typing and taxonomy. FEMS Immunol Med Microbiol, 45: 1 - 10.

UNIPROT, 2011. UniProt Protein knowledgebase BLAST server. [online] Available at: http://www.uniprot.org/ [Accessed 25 January 2011]

WAGNER, S., SORG, I., DEGIACOMI, M., JOURNET, L., PERARO, M.D., and CORNELIS, G.R., 2009. The helical content of the YscP molecular ruler determines the length of the Yersinia injectisome. Mol Microbiol, 71 (3): 692 - 701.

WANG, X., LI, Y., JING, H., REN, Y., ZHOU, Z., WANG, S., KAN, B., XU, J., and WANG, L., 2011. Complete genome sequence of a Yersinia enterocolitica "Old World" (3/O:9) strain and comparison with the "New World" (1B/O:8) strain. J Clin Micro, 49(4): 1251 - 1259.

WAUTERS, G., KANDOLO, K., and JANSSENS, M., 1987. Revised biogrouping scheme of Yersinia enterocolitica. Contrib Microbiol Immunol, 9: 14 - 21.

WELLCOME TRUST SANGER INSTITUTE, 2011. Scientific resources - Software ACT: Artemis Comparison Tool - User Manual. [online] Available at: ftp://ftp.sanger.ac.uk/pub/pathogens/software/arte-mis/extra/big_blast.pl [Accessed 25 January 2011].

WREN, B.W., 2003. The Yersiniae - a model genus to study the rapid evolution of bacterial pathogens. Nat Rev Micro, 1 (1): $55-64$.

WUTHE, H.H., and ALEKSIC, S., 1997. Yersinia enterocolitica serovar 2a, wb, 3:b,c biovar 5 in hares and sheep. Berl Munch Tierarztl Wochenschr, 110 (5): 176 177. [German]

YOUNG, G.M., and MILLER, V.L., 1997. Identification of novel chromosomal loci affecting Yersinia enterocolitica pathogenesis. Mol Microbiol, 25(2): 319 - 238. 
ZERBINO, D.R., and BIRNEY, E., 2008. Velvet: algorithms for de novo short read assembly using de Bruijn graphs. Genome Res, 18: 821 - 829 .

ZHANG, L., TOIVANEN, P., and SKURNIK, M., 1996. The gene cluster directing Oantigen biosynthesis in Yersinia enterocolitica serotype O:8: identification of the genes for mannose and galactose biosynthesis and the gene for the O-antigen polymerase. Microbiology, 142: 277 - 288.

ZHANG, L., RADZIEJEWSKA-LEBRECHT, J., KRAJEWSKA-PIETRASIK, D., TOIVANEN, P., and SKURNIK, M., 1997. Molecular and chemical characterization of the lipopolysaccharide $\mathrm{O}$-antigen and its role in the virulence of Yersinia enterocolitica serotype O:8. Mol Microbiol, 23 (1): 63 - 76.

ZHOU, L., LEI, X.-H., BOCHNER, B.R., and WANNER, B.L., 2003. Phenotype microarray analysis of Escherichia coli K-12 mutants with deletions of all twocomponent systems. J Bact, 185 (16): 4956 - 4972. 